


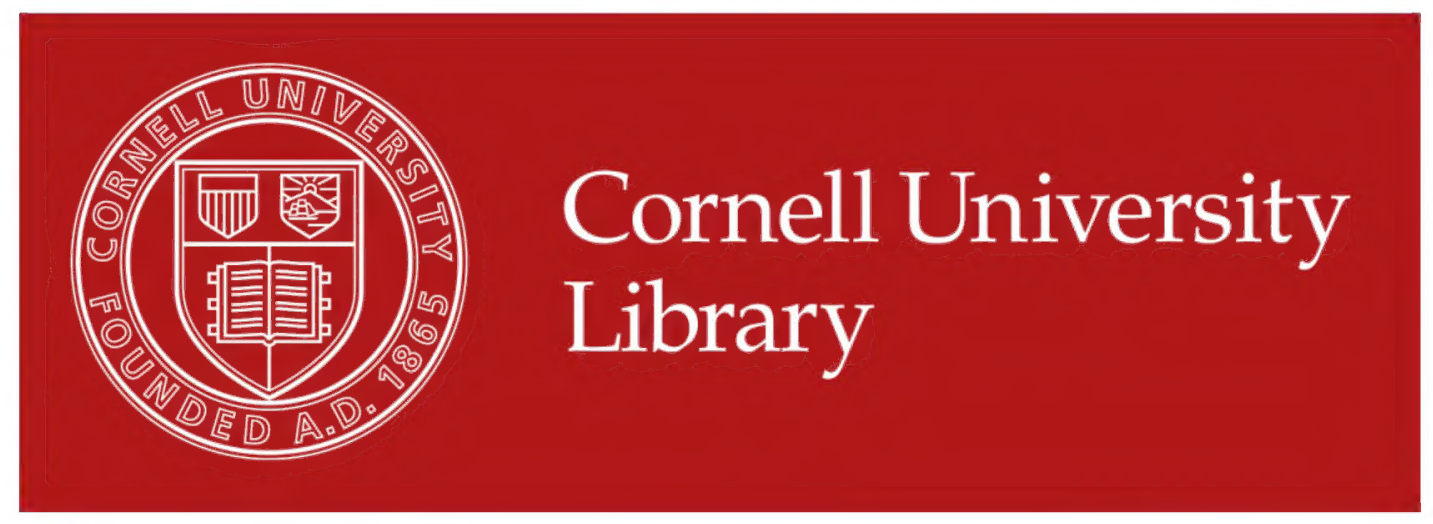

The original of this book is in
the Cornell University Library.

There are no known copyright restrictions in the United States on the use of the text.

http://www.archive.org/details/cu31924000615397 




\author{
SYSTEMATIC CENSUS \\ $-A$ \\ OF \\ AUSTRALIAN PLANTS, \\ WI'II
}

- Chromologic, 迆iterary and Geographic Anmotations;

BY

BARON, FERDINAND VON MUELLER,

K.C.M.G., M.D., Pн.D., F.R.S.,

F.T.S., F.G.S., F.R.G.S., C.M.Z.S., H.F.R.H.S. \&C.

GOVERNMENT BOTANIST FOR THE COLONY OF VICTORIA.

PART I.-VASCULARES.

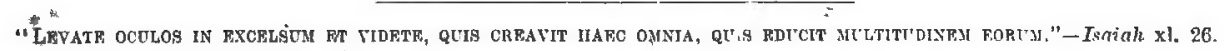

(4)

MELBOURNE :

PRINTED FOR THE VICTORIAN GOVERNMENT

By M'Carron, Bird \& Co., 37 Flinders lane West.*

1882. 


$$
\begin{aligned}
& \text { PK } 431 \\
& \text { m } 94
\end{aligned}
$$


To

GEORGE BENTHAM, EsQ., F.R.S., V.P.L.S.,

To

SIR JOSEPH HOOKER, K.C.S.I., C.B., F.R.S., V.P.L.S.

AND TO

M. ALPHONSE DE CANDOLLE, F.M.R.S., F.M.L.S.,

WHO AS HEIRS OF GREAT NAMES WORTHILY SUSTAIN WORLD-WIDE ANCESTRAL FAME, AND WHO AS LEADERS IN PHYTOGRAPHY DURING MIORE THAN A GENERATION'S TINE WILL BE PRE-EMINENT IN BIOMORPHIC SCIENCE THROUGH ALL AGES,

\section{THESE PAGES}

ARE OFFERED AS AN APPRECIATIVE TRIBUTE

FROM A YOUNG COLONY IN AXTIPODAL REMOTEXESS. 
$\stackrel{Q}{Q K 431}$

$\mathrm{Ma}_{4}$

@38730 
THIS work needs hardly to be introduced by prefatory objervations to the notice of professed naturalists, who will recognise its scope and object at a glance. To those however, who may be less conversant in phytographic science, and who may use these pages, some explanatory preface would be needful, in order that they may fully understand the views, which guided the author through the details of this enumerative essay on Australian indigenous plants. Again, to all recipients it may be of some interest, to be informed of the causes, which gave rise to the present literary issue. Of the seven volumes of the "Flora Australiensis," elaborated by Mr. Bentham, mainly through the aid of the Botanic Departments of Kew and Melbourne, the earlier ones were written at a time, when a large portion of Australia remained yet untraversed by any explorers. Since then the territorial occupation as a whole bas become nearly doubled, while new lines of geographic research have opened up vast additional tracts of lands for future settlements. Thus, the Flora of our islandcontinent became also more rerealed, with the result, of about 850 species of vascular plants having been added already to those described in the "Flora Australiensis"; but, irrespective of this, we have learned in later years much more concerning the regional distribution of the species formerly recorded. All these additional data, accumulated gradually during the last two decennia, though mainly kept together in the volumes of the "Fragmenta Phytographiae Australiae", became necessarily much disconnected and dispersed during the progress of discoveries and the early records connected therewith, so much so, that much difficulty was experienced of late, to command a clear and easy view orer these scattered literary fragments. To collect and rearrange them into one or more volumes, conformous with those of the "Flora Australiensis," and supplementary to that work, proved beyond the range of operations of the venerable savant, who spent much of his precious time during fully sixteen years for graudly systematising throughout the native regetation of these great parts of the British Dominions,-because his unrivalled experience and scientific valour were called more pressingly into channels of research, by which not only the realm of plants of Australia, but indeel that of the whole globe, became drawn both comprehensively and connectedly into the cycle of his observations. Perhaps it may fall yet to the share of the writer of the present pages, to furnish supplements to the "Flora Australiensis"; indeed, he has it under contemplation, to add one rolume, solely to be devoted to a descriptive enumeration of all the additional species, and to be arranged in consonance with Bentham's views, whereas another volume would be rendered exclusively the vehicle of record of all new localities, from whence any species of the "Flora Australiensis" were obtained, when perhaps also such alterations, as largely augmented material may suggest, could be made to the descriptive text. But to complete the "Flora" in this manner, so far as our present state of knowledge will admit of it, would involve the thorough revision of the whole vast collections now stored in our local Botanical Museum; therefore, the connected re-examination of at least 80,000 specimens, from which the pages of the "Flora" largely, and those of the "Fragmenta" solely, have emanated. Such a task, to be performed with conscientious care, would need continued and close application throughout several years. Under these circumstances it seemed best, to pave the way for subsequent purposes through the issue of a preliminary publication, by which anyhow the use of both works, above referred to, would be facilitated. A postponement of the mork for supplementary volumes, thus arising, would bring with it one advantage also, that of gaining time for carrjing the augmentation of the Flora still further, as we would meanwhile profit from phytologic searches of travellers and settlers in wide regions of Australia, as yet in reference to vegetation but scantily investigated or altogether unexplored.

By deferring therefore the elaboration of supplemental volumes, we could aim more at future exhaustiveness, whereas the work as left by its illustrious author will meet mainly all present wants, and must remain the foundation for all future systematic study of the regetation peculiar to this part of the globe. In offering now a separate statistic volume, the writer has availed himself of the opportunity, to place on recoril independently his views on preferable systematic sequences of orders, wherever occasionally his opinions differed from those enunciated by other" phytographers. He furthermore could draw the limits of some genera and wany species in accordance with original observations of his own; besides he was able to apply more rigorously the rules for systematic naming in strict application of chronologic data. But to aroid misapprehensions, the author at the very outset endeavours to anticipate objections, which may perhaps be raised against some portion of the nomenclature adopted in these pages. If, for instance, Haller is regarded as the earliest indicator of Cyperaceae, it must be conceded, that his wording of the order was "Cyperi" in 1742, as well shown in Pfeiffer's extensire work. If however in accordance with the more exact limitation of this ordinal group of plants we ascribe it under the name of 
Cyperaceae to De Candolle, as defined in 1805, or to St. Hilaire as circumscribed in the same year, or to A. L. De Jussieu, who already in 1789 adopted the same order under the appellation Cyperoideae, then we evidently act unjustly in not recognising prior claims; indeed, so far as wording is concerned, we lose the substance for the shadow, especially as no necessity exists, to strive for etymologic uniformity at the sacrifice of fair priority. To overcome difficulties or ambiguities of these kinds, we could use the prepositiou "from" (to be rendered nach in German and d'après in French) for indicating the originator of an order, genus or species, after the quotation of any later accepted authority. Indeed this priuciple was adopted already for a list of Australian plant-genera, published by the Royal Society of New South Wales in 1881; and had the scope assigned to the present pages admitted of it, the same rule would have been extended to the species so far as applicable.

To accomplish the object of rendering this publication, as a commentary index, one for easy reference, the schematic form appeared the most eligible. But to bring it within the means available for issue, all specific synonymy and any secondary notations had to be abandoned. Iudeed for these, in quoting the "Flora" and the "Fragmenta" throughout, all needful indications are afforded. The geographic notes had to be limited to the initial letters, indicative of our main colonial territories, in which respect a plan was followed, precisely the same as that adopted in 1866 for a geographic list of nearly one thousand species of trees, then already known to constitute the very varied arboreous vegetation of Australia (see volume of the Intercolonial Exhibition of 1866, and of the second Paris "Exposition," 1867.) On that occasion also the terms Choripetaleae and Synpetaleae were already used, to distinguish the chief divisions of the Dicotyledoneae, while the sequence of orders, effected then, was also mainly the same as that followed in the present pages, the Apetaleae of Jussieu or Monochlamydeae of De Candolle having been merged into the petaliferous divisions. But the system, built up by these two great masters in botanic science, is in its main features so genuinously natural, that no subsequent research could bring about very material changes, except in the one particular direction above indicated, a design more or less happily carried out from the time of the earlier writings of Brogniart to that of the latest essays of Jean Mueller, because it was felt, that so long as the Monochlamydeae remained isolated and associated with the Gymnospermeae, so long would we have an imperfect natural system. To proceed in chief alterations further than this, would give a less acceptable system. Even among the Thalamiflorae and Calyciflorae, represented in Australia, as placed together by Bentham, we had alrearly not less than 58 genera, which are entirely apetalous or contain species, in which the corolla remains undeveloped, leaving out of consideration numerous absolutely extra-Australian genera wholly or partly apetalous. Again in Haloragis, Myriophyllum, Cotula, Soliva and some other genera the corolla is alosent in the non-antheriferous flowers, while even the very commencement of the Candollean arrangement is made with the apetalous genera Clematis, Thalictrum and Anemone, soon followed by the equally well-known Caltha. Furthermore the Euphorbiaceae, which in the train of their alliances must carry with them always the Urticeae, count among their numerous genera more than one third provided with petals. Besides, in Proteaceae the floral envelope may be regarded as homologous to that of the closely allied Loranthaceae, with an absence of a calyx, comparable to the suppression of that organ in Diplolaena, Asterolasia and few other thus far exceptional genera. Again, a firm thread runs uninterruptedly through all the orders of Curvembryonatae or Amyliferae, whether placed in Thalamiflorae or Calyciflorae or Monochlamydeae or even as regards Plumbagineae into Corolliflorae; nor is there any real difficulty of finding for the rest of the Monochlamydeae proper places among naturally allied orders of supposed higher organisation. Nevertheless affinity is variously radial, not altogether uniserial, as beautifully demonstrated already by Linné in his map of ordinal alliances of plants, published by Fabricius and Giseke, or as lucidly exhibited as long ago as the middle of the last century by Bernard de Jussieu in the Royal Garden of Trianon, through arranging the plants in a class-ground, a method adopted in our Botanic Garden here also already in 1857. In using the term Calyceae for the three main-divisions of the Monocotyledoneae, the author wishes it to be understood, that it remains an open question, whether the flowers of two of these divisons are to be regarded as mono- or di-chlamydeous. But if in the latter case a petaleous development is attributed to the flowers, we are then bound by homology to carry the term of petals also to Junceae even and Restiaceae, and a change in the naming of the three great sections would be needful accordingly. In suppressing throughout the epigynous divisions for unison with the perigynous, into which they often pass so gradually, simplification is aimerl at, without causing any disarrangement, or without attempting alterations in the great received systems except nomiually. Furthermore we should always regard the apocarpic orders as those of the highest development, because the fruit-the final effort of all growth-whicn multiplying into separate and complete carpic elcments, exhibits the most advanced vegetable organisation.

Much could it have been wished, to allot in this systematic census also columns of citation to those of the larger works, which are exclusively devoted to the Australian Flora. But Robert Brown's ever-memorable "Prodromus" records only vascular Acotyledoneate, Mlonocotyledineae, some Apetaleae. 
and a portion of the Sympetaleae, as far as known in 1810. The tro important rolumes, issued by Lehmann and his coadjutors as "Plantae Preissianae," refer solely to South-West Australian species. Sir Joseph Hooker's grand "Flora Tasmaniae" became necessarily" limiterl to the plants of that island, although prefaced by an extensive general essay.

A future re-edition of this census from augrnentin material, in ampler paginal form, might afford space also for noting these works. The "Flora Tasmaniae" will replace however the "Flora Lustraliensis" in the quotation-column of the second part of this census, in which all the "Eracculares" if Australia are to be enumerated, the total of them having risen already to about 3750 recorded species. For, be it understood, that the genial and learned Director of the Royal Botanic Garten of Kem has laid a firm foundation for the srstem of evascular Acotylerloneae of All-Australia in his Tasmanian Flora, that work being the first, in which any extensire and connectedly elaborated account of Jlosses, Lichens, Fungs and Algs of Australia has been presented.

It was beyond the scope of these pages, to extend the statistics here given; otherwise the writer would have gladly assigned geographic columns also to Europe, Asia, Africa, Forth and South America for indicating the respective range of many Australian species of plants into other regions of the globe. Whoerer may be interesterl in such phytogeographic subject is referred to an al,le article, written by Dr. Engler, Director of the Botanic Garden of Kiel, for the rolume of 1 b $\$ 1$ of his "Jahrbuicher," or" to the print of a discourse, delivered a few months ago before the School of Mines at Ballarat. To sum up some of the results of this statistic rolune, talule, are appended, setting forth the number of the species in each order as well as the grand totals arriver at, clearly immigrater plants being excluded from these pages throughout. Future phrtological explorations are not likely, to add beyond sereral hundred specific form, consilered in conservative limitations, to those now adduced; but extensive additions are sure to be made yet to the records of regional distribution. Inteerl even while this first part of the work went through the press, si many new localities became known, as to render an appendix now alrearly necescary. For these addenda I am largely indebted eitler in notations or material to the Rer. Dr. Woolls, Professor 'late, the Rev. B. Scortechini and Mr. F. M. Bailey, while the supplemental notes concerning $T_{\text {est }}$ Australian plants manated chiefly from gatherings, which we owe to the Hon. J. Forrest, C.II.G. It should however he olwerved, that the whole of the ra-t additional collections, which accumulated in our Botanic JIuseum since the rolumes of the "Flora Australien is" successirely appeared, could not receive in all instances timely close and critical attention for the elaboration of the present work, though its author had the advantaye of departmental help from Mr. G. Luehmann in preliminary sorting and comparing of much of the supplemental material; and here it is an apt place, to recognise likewise the aid, afforderl by Jlr. Léon Henry, in mriting out primarily the requisite notes from the "Flora" and the "Fragmenta." Nearly one hundred species more might have been taken into account already, for estimating the number of species in various orders; but the samples hitherto secured did not admit of obtaining accurate specitic data, though the ordinal position could be recogniserl. Thus we know, that already some access to Anonaceae, Menispermaceae, Lauraceae, Euphorbiaceae, Rutaceae and a few other orders could be recorded.

The chronologic references for species, here more extensively carried out, than ever before, amount to nearly 10,000, including those for genera, in this first part of the present publication. The bibliographic works of Pritzel, Pfeiffer and Jackson have done excellent services in these particular inquiries. The position, given to sereral of the orders on this occasion, is to some extent assailahle; thus-as well-known-leasons exist for moving Piperaceae to Nymphaeaceae, Aristolochieae and Nepenthaceae to Sarraceniaceae, Tiniferae to Araliaceae, Thymeleae to Rhamnaceae, Balanophoreae ancl Podostemoneae to Halorageae, Droseraceae to Saxifrageae, Rubiaceae to Loganiaceae, Fluviales to HJdrocharideae, Casuarineae to Coniferae, although the two latter are neither anatomically nor morphologically approaching each other, notwithstanding some deceptive external resemblance, in which howerer Exocarpus also shares, not to speak of a few other genera. But in considerations like these we should not insist in a dogmatic spirit on the full acceptance of hitherto recognised systematic arrangement: particularly as not yet all the forms of the world's regetation are known, and as at any moment the discovery of a new plant may turn the scale in weighing the affinity of its allies.

In incurring the responsibility of restoring some generic names, which had sunk almost into oblivion, the writer has been guided by the impartial rules of strict priority, feeling assureil, that phytographers will become quite as quickly accustomed to changes from Stylidium to Candollea, or Ionidium to Hybanthus, or Chenolea to Bassia, as they became reconciled to the long forgotten appellations of Trema, Hevea, Centipeda, Galeola, Floriscopa, Nunnezharia and others, thereby discarding names, sanctioned by lengthened use and familiar to all of us, with a readiness as surprising as universal. To alterations or restitutions of specific names shall only be alluded here with a few words. Why Lamarck for an Eleusine selected designedly the appellation E. cruciata, or why Schleilen called a Wolffia purposely $\Pi$. Michelii, can be as easily perceived as the advisability of withdrawing the specific name antarctica from a Titis, Cjmodocea or Dicksonia. Where authors bestowed several names 
at the same time on a species, it should be free to those, who effect the reductions, to choose a collective designation for the consolidated species; cases in point are afforded by Euphrasia Brownii, Fimbristylis communis, Pappophorum commune, Danthonia penicillata.

As to the circumscription of species themselves, it will require yet much of assiduous research of future generations for arriving at a full understanding of the diagnostic value, which has to be attached respectively to the thousands of specific and therefore original forms in the Australian and any other vegetation. Of the plants, enumerated as species in the present pages, a considerable number may prove, that they owe their particularity to hybridism. In such cases they should occupy a subordinate position, and should also here, as elsewhere, receive their double parental name; ;thus Lasiopetalum Tepperi would require to have its appellation changed into Lasiopetalum Baueri $x$ discolor; but as the instances of ascertained hybridism among our native plants are as yet so very few, it was deemed best to admit at present the very limited number of known bastards under ordinary specitic rank.

The geographical limitations in this work coincide with the political boundaries of the colonial territories, except that the tropic of Capricorn eastward to the 138th degree separates what is here called Northern Australia (N.A.), from the South- and West-Australian extratropic possessions. Such geographic segregations are necessarily quite arbitrary, though they serve our present purpose of assigning to each of the colonial divisions of Australia its number of specified plants; the limitation is the same as that adopted in the "Flora Australiensis," and as regards abbreriations also identical with the method of indications, chosen for the list of Australian trees in 1866. Here may it further be remarked, that the plants of the small and isolated insular spot, called Norfolk-Island, have been counted with those of N.S.W., to whose dominions this isle as well as Lord Howe's Island politically belongs. To draw the species into physiographic and regional complexes must be the work of future periods, when climatic and geologic circumstances throughout Australia shall have become more extensively known. The geographic columns in these pages indicate simply the occurrence of plants within any of the colonial areas, but hare been extended even to such spécies, which merely may pass boundary-lines. In this way are noted for W.A. and S.A. such tropical plants, as reach not beyond Shark-Bay or the vicinity of the MacDonnellRange; in the same manner Victoria is credited with those plants of New South Wales, which barely advance into East-Gippsland, while South-Australia obtains credit for several Tasmanian plants, which are confined to very limited stretches of country, chiefly in the extreme south-east of that province; and again a few of the New England plants are recorded for Queensland, although they pass merely on to the nearest adjacent ranges across the boundary. At the whole however it can be foreseen, that many a species, still accepted as genuine for these lists, will in the course of further and more facilitated research be eliminated from any future editions of this work, though still larger arrays, containing many a novelty, will have to be mustered for systematic rolls. The lines of demarkation between truly indigenous and more recently immigrated plants can no longer in all cases be drawn with precision; but whereas Alchemilla vulgaris and Veronica serpillifolia were found along with several European Carices in untrodden parts of the Australian Alps during the author's earliest explorations, Alchemilla arvensis and Veronica peregrina were at first only noticed near settlements. The occurrence of Arabis glabra, Geum urbanum, Agrimonia Eupatoria, Eupatorium cannalinum, Carpesium cernuum and some others may therefore readily be disputed as indigenous, and some questions concerning the nativity of various of our plants will probably remain for ever involved in doubts. While concluding these introductory remarks, it is incumbent on the writer, to acknowledge in grateful terms the consideration, shown him by the Hon. James Macpherson Grant, M.L. A., the Ministerial Chief of the Department, in allowing this work to be issued under the auspices of the Victorian Government and at departmental expenditure. Encouraged by generous support, the author will cheerfully continue through such time, as may still be allotted for his worldly career, to devote his strength and resources also in future mainly for the furtherance of Australian phytography, on which much of his efforts were concentrated through more than a thiril of a century; and as these enquiries are largely carried on without worldly gain, he feels free, to claim the co-operation of any educated and high-minded colonists in these great dominions of the British Crown for promoting studies, which cannot fail to be of great industrial advantage at all future time, which are calculated to advance continuously the cause of education in this part of the world also, which must far and wide contribute to intellectual enjoyments, and above all should lead to religious reverence of that Supreme Godly power, so gloriously revealed in nature's wondrous works. 


\title{
DICOTYLEDONEAE.
}

\author{
Ray, Method. Plant. nova 2 (1652).
}

\section{CHORIPETALEAE HYPOGYYAE.}

F. v. Mueller, native plants of Victoria I, 1 (1879).

\section{RANONCULACEAE.}

A. L. de Jussien, Rec. de l'Acad. des sc. (17/3) from B. de Jnssien (1759).

CLEMATIS, Linné, gen. pl. 163 (1737) from l'Ecluse (1576).

C. aristata, R. Brown in De Candolle, syst. veg. I, $147(1818) \ldots$-.. S.A. T. V. .....W. Q. - B.fl.I,6

C. pubescens, Huegel, emum. pl. Nor. Holl. austr. occ. I (1837) W.A. - - - - - - B.fl.I.6

C. glycinoides, De Candolle, syst. veg. I, $145(1818) \quad \ldots \quad \ldots \quad-\quad-\quad--$ N.S. IT. Q. N.A. B.f.I,

C. microphylla, De Candolle, syst. reg. I, 147 (1818) $\ldots . . \ldots$. W.A. S.A. T. V. N.S.T. Q. - B.fl.I,7

C. Fawcettii, F. v. Mueller, fragm. phytogr. Austr. X, I (1976) - - - - N.S.W. - - AMEMIONE, Tournefort, inst. rei herb. 275, t. 147 (1700) from Hippocrates, Theophrastos and Dioscorides.

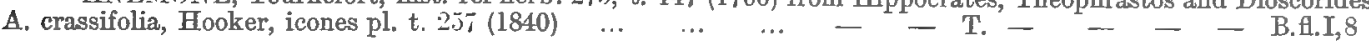
MYYOSURCS, Dillenius, catal. pl. Gie-s, 106, t. 4 (1719) from Tabemaemontanus (1.59s). (Myosuros).

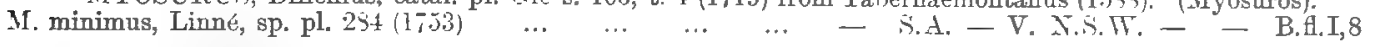
RANUACLLL's, Tournefort, inst. 285, t. 149 (1700) from Bock (1552).

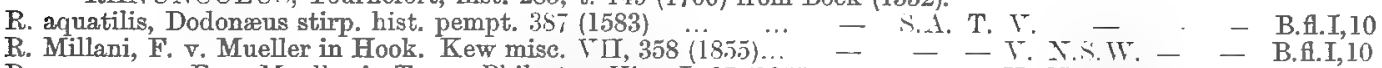

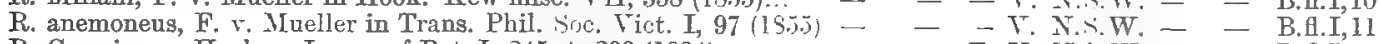

R. Gunnianus, Hooker, Journ. of Bot. I, 24.5, t. 233 (1834) ․ - - - T. T. N.S.II. - - B.fl.11

R. lappaceus, Smith in Rees's Cyclopædia, XXLX (1815) … W.A. S.A. T. T. Х...T. Q. - B.H.I,12

R. Muelleri, Bentham, Fl. Austr. I, $13(1863) \quad \ldots \quad \ldots . \quad \ldots \quad-\quad-\quad-$ Y

R. rivularis, Bks. \& Sol. in G. Forst. fl. ins. austr. prodr. $90(1-56)-$ - S.A. T. V. N.S.TT. Q. - B.H.I,

R. hirtus, Bks. \& Sol. in G. Forst. f. ins. austr. prodr. $90(1,56)$ W.A. - - T. X.S.T. Q. - B.I.I, 13

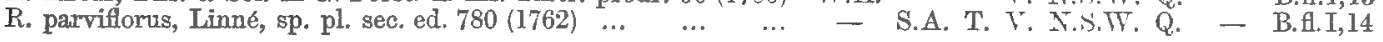
CALTHA, Ruppius, flora Jenensis 119 (1;18) from Bock (1552). (Trollius).

C. introloba, F. r. Mueller in Trans. Phil. Soc. Vict. I, 98 (1855) - - T. Y. N.S.W. - - B.fl.I,15

\section{NYMPHAEACEAE.}

Salisbury in Koenig \& Sims's Ann. of Bot. II. 70 (1805).

CABOMBA, Aublet, Hist. pl. Guian. I, 321, t. 124 (1775). (Brasenia, Hydropeltis).

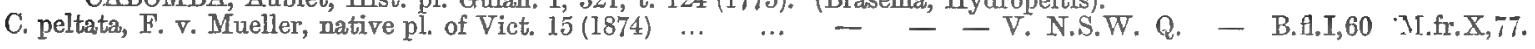
NYMPHAEA, Tournefort, inst. 260, t. 1378 (1700) from Theophrastos and Dioscorides.

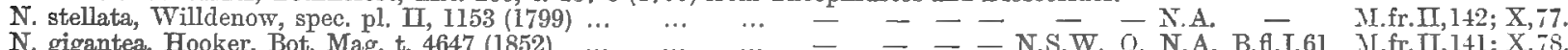

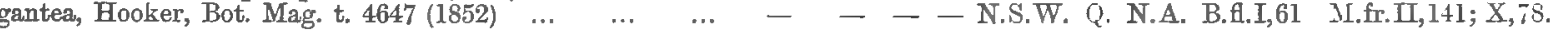
NELUMBO, Tournefort, inst. 261 (1700). (Nelumbium).

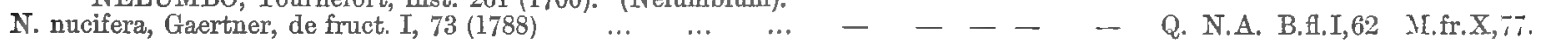

\section{DILLENIACEAE.}

Salisbury, Parad. Lond. I, 73 (1801).

TTORMIA, Rottboell, Nye Saml. Vid. Selsk. Skrivt. II, 532 (1783).

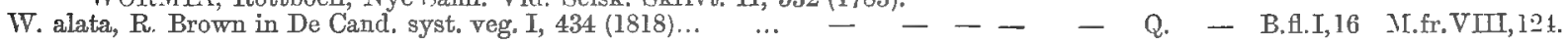

HIBBERTIA, Andrews, Bot. Reposit. t. 126 (1800). (Candollea, Pleurandra, Hemistemma, Adrastaea, Ochrolasia, Hemistephus, Huttia.)

H. Banksii, F. v. Mueller, pl. of Vict. I, I4, implied (1860)

H. Brownii, Bentham, Fl, Austr. I, 21 (1863)

H. dealbata, F. v. Mueller, pl. of Vict. I, I4, implied (1860)
H. candicans, Bentham, Fl. Austr. I, $21(1863) \ldots$

H. Benthami, H. angustifolia, Benth. fl. Austr. I, 21 (10̈63)

H. Muelleri, Bentham, Fl. Austr. I, 21 (1863)

H. ledifolia, Bentham, Fl. Austr. I, 22 (1863)

H. verrucosa, F. v. Mueller, pl. of Vict. I, 14, implied (1860) ...

H. spicata, F. จ. IIueller, fragm. II, $1(1860)$

H. polystachya, Bentham, Fl. Austr. I, 22 (1863)...

H. rhadinopoda, F. v. Mueller, fragm. XI, 91 (1880)

H. furfuracea, Bentham, Fl. Austr. I, 23 (1683)

H. hypericoides, F. v. IIueller, pl. of Vict. I, 13, implied (1860)

H. microphylla, Steudel in Lehmann pl. Preiss. I, 273 (1844) ...

H. recurvifolia, Bentham, Fl. Austr. I, 24 (1863).

H. lineata, Steudel in Lehmann pl. Preiss. I, 272 (1844)...

H. acerosa, Bentham, Fl. Austr. I, 24 (I863)

H. aurea, Steudel in Lehmann pl. Preiss. I, 272 (1844) $\ldots$

H. crassifolia, Bentham, Fl. Austr. I, $25(1863) \ldots$
H. nitida, F. v. Mueller, pL of Vict. I, $15(1860) \ldots$

H. bracteata, Bentham, Fl. Anstr. I, 26 (1863) $\ldots$
H. densiflora, F. v. Mueller, pl. of Vict. I, $15(1860)$

H. hirsuta, Bentham. Fl. Austr. 26 (1863)

H. synandra, F. v. Mueller, fragm. IV, $151(1864) \quad$... $\quad \ldots$

$H$. stricta, $R$. Brown in De Candolle, syst. veg. I, 422 (1818)

H. humifusa, F. v. Mueller, pl. of Vict. I, 16, t. Suppl. I (1860)

$$
\begin{aligned}
& - \\
& - \\
& - \\
& - \\
& - \\
& - \\
& \text { W.A. } \\
& \text { W.A. } \\
& \text { W.A. } \\
& \text { W.A. } \\
& \text { W.A. } \\
& \text { W.A. } \\
& \text { W.A. } \\
& \text { W.A. } \\
& \text { W.A. }
\end{aligned}
$$

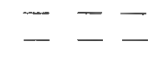$$
\text { - }
$$$$
-\quad-
$$$$
-\quad-\cdots
$$$$
\begin{aligned}
& \text { W.A. }--- \\
& \text { W.A. - - }
\end{aligned}
$$$$
\text { W.A. - - }
$$$$
\text { W.A. - - - }
$$$$
\text { W.A. - - - }
$$$$
\text { W.A - - - }
$$

W.A. - - -

W.A. - .. -

‥ W.A. - - -

W.A. - - -
W.A. - $-~$

-

Q. - B.fl.I,2I

Q. B. B.fl.I,21

N N.A. B.fl.I, 21

- $\quad$ - N.A. B.f.I,21

- - N.A. B.fi.I,21

- - N.A. B.fl.I,21

- $\quad$ - B.f1.I, 22

- B.f.I,22

- B.Al.I, 22

- - - B.⿴囗I,22

- - - B.fi.I,23

- - - B.

- - - B.fl.1,23

- $\quad$ - B.fl.I.24

- - - B.fl.I,24

- - - B.f.I. B.,24

- - - - B.fl.I,24

- - - B.ff.I, 25

- - - - N.S.T. - - B.fl.I.25

- - - - N.S.II. - - B.fl.I,25

- S.A. T. V. N.S.W. - - B.f.I,26

- - T. - - - - B.H.I,26

W.A. S.A. T. V. N.S.T. Q. -
II.fr.II, $1 ; \mathrm{XI}, 92$.

NI.fr.II, 1 .

II.fr.II, 1.

M.fr.II, 1 .

M.fr. XI, 92

M.fr.II, $1 ;$ XI, 20,92.

M.fr.XI, 92

MI.fr. XI, 91

iI.fr.I.161; XI, 92.

M.fr. $1,217$.

II.fr, XI, 92.

II.fr.XI,92

M.fr.XI,92.

\section{M.fr. VII, 125; XI,92.}

M.fr.IV, 151, 
H. Billardieri, F, v. Mueller, pl. of Vict. I, 14 (1860)

H. gracilipes, Bentham, Fl. Austr. I, 28 (1863)

H. acicularis, F. v. Mueller, pl. of Vict. I, $17(1860)$

$\mathrm{H}$. mucronata $\mathrm{F}$. Mueller

H. hermannifolia, De Candolle, syst. veg. I, 431 (1818) ...

H. velutina, R. Brown in Bentham, Fl. Austr. I, 30 (1863)

H. oblongata, R. Brown in De Candolle, syst. veg. I, 431 (1818)

H. melhanoides, F. v. Mueller, fragm. IV, 116 (1864)

H. tomentosa, R. Brown in De Candolle, syst. veg. I, 432 (1818)

H. cistifolia, $R$. Brown in De Candolle, syst. veg. I, 431 (1S18)

H. echiifolia, R. Brown in Bentham, Fl. Austr. I. 31 (1863)

H. scabra, R. Brown in Bentham, FI. Austr. I, 31 (I863)

H. lepidota, $R$. Brown in De Candolle, syst. veg. I, 432 (1818) ...

H. vestita, Cunningham in Bentham, Fl. Austr, I, 31 (1863) ...

H. serpyllifolia, R. Brown in De Cand. syst. veg. I, 430 (1818)

H. pedunculata, R. Brown in De Cand. syst. veg. I, 430 (1818)

H. ochrolasia, Bentham, Fl. Austr. I, 32 (1863)

H. angustifolia, Salisbury, parad. Lond. 73 (1807)

H. fasciculata, R. Brown in De Candolle, syst. veg. I, $42 \ddot{2}$ (181 13 )

H. virgata, R. Brown in De Candolle, syst. veg. I, 428 (1818) ...

$\dot{H}$. inclusa, Bentham, Fl. Austr. I, 34 (1863)

H. rostellata, Turcz. in Bull. Soc. Mosc. XXII, part III, \& (1849)

H. glomerata, Bentham, Fl. Austr. I, 34 (1863)

H. argentea, Steudel in Lehmann, pl. Preiss. I. 268 (1844)

H. pilosa, Stendel in Lehmann, pl. Preiss. I, 272 (1844)...

H. montana, Steudel in Lehmann, pl, Preiss. I, 270 (1844)

$H$. linearis, $R$. Brown in De Candolle, syst. veg. I, 428 (1818) ...

$H$. diffusa, R. Brown in De Candolle, syst. veg. I, 429 (1818) ...

H. saligna, R. Brown in De Candolle, syst. veg. I, 427 (1818) ...

$H$. volubilis, Andrews in Bot. Reposit, t 126 (is0o)

H. grossularifolia, Salisbury, parad. Lond. t. 73 (1806) $\ldots .$.

$H$. dentata, $R$. Brown in De Candolle, syst. veg. $I, 426$ (1818) ...

H. Cunninghamii, Aiton in Hook. Bot. Mag. t. 318 (1832)

H. perfoliata, Huegel, enum. pl. Nov. Holl. austr. occ. 3 (1837)

H. bracteosa, Turczaninow, in Bull. Soc. Mosc. XXV, 140 (1852)

H. longifolia, F. v. Mueller, fragm. IV, 115 (1S64)

H. oenotheroides, F. จ. Mueller, fragm. VII, 37 (1870) ...

H. glaberrima, F. v. Mueller, fragm. III, 1 (1862)

H. Mylnei, Bentham, FI. Austr. I, 39 (1863)

H. lasiopus, Bentham, Fl. Austr. I, 40 (1863)

H. potentillifiora, F. V. Mueller in Bentham, Fl, A ... ... ...

H. pungens, Bentham, Fl. Austr. I, 40 (1863)

H. nutans, Bentham, Fl. Austr. I, 40 (1863)

H. leptopus, Bentham, Fl. Austr. I, 41 (1863)

H. obcuneata, Salisbury, parad. Lond. t. $73(\mathbf{1 8 0 7}) \quad \ldots . \quad \ldots$ W.A.

F. glomerosa, F.v. M.; Candollea glomerosa, Benth.Fl.Aus.I, $43(1363)$ W. A.

H. teretifolia, F. v. Mueller, fragm. IV, $117(1864) \quad \ldots . \quad \ldots$ W.A.

H. desmophylla, F. v. Mueller, fragm. XI, 95 (1880) $\ldots . . .$. W.A.

H. helianthemoides, F. v. M. Candollea helianthemoides, Turcz. in Bull. Mosc. XXII, part III, 8 (1849) .

H. depressa, Steudel in Lehm. pl. Preiss. I, 268 (1844)

H. Huegelii, F. v. Mueller, fragm. XI, 95 (1880) ...

H. subvaginata, F. v. Mueller, fragm. XI, 95 (1880)

$H$. vaginata, $F$. v. Mueller, fragm. XI, 96 (1880)...

H subexisa, Steuclel in Lehmann

H. squamosa, Turczaninow in Bull. Soc. Mosc. XXII, partIII, $9(1849)$ W.A.

H. uncinata, F, v. M. ; Candollea uncinata, Benth. Fl. Aus. I, 46 (1863) W.A.

H. salicifolia, F. v. Mueller, fragm. I, 161 (1859) ...

H. Huttii, F. v. Mueller, fragm. VII, $123(1870) \ldots$

H. Goyderi, F. v. Mueller, fragm. VII, 123 (1870)

TETRACERA, Linné, gen. pl. 345 (1737).

T. Nordtiana, F. v. Mueller, fragm. V, 1 (1865)

T. Daemeliana, F. v. Mueller, fragm. V, 191 (1866)

T. Wuthiana, F. v. Mueller, fragm. X, 49 (1876) ...

DILLENIA, Linné, gen, pl. 162 (1737).

D. Andreana, F, v. Mueller, fragm. V, 175 (1866)

PACHYNEMA, R. Brown in De Candolle, syst. veg. I, 412 (1818).

P. junceum, Bentham, Fl. Austr. I, 47 (I863)

P. complazatum, R. Brown in De Cand., syst. veg. I, 4.12 (1818)

P. dilatatum, Bentham, Fl. Austr. I, 48 (1863)
S.A. T. V. N.S.W, Q.

S.A. T. V. N.S.W. Q.

Q. -

B. Al.I,28

B.fl,I,28

B.fl. I, 29

B.fl. 1,29

B.f.I, 30

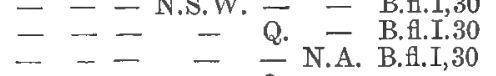

- - - - Q. N.A. B.F.I,30

- - - - - N.A. B.f.I,30

- - - - - N.A. B.A.I,31.

- - - - N.A. B.fl.I,31

- - - - N.A. B.A.I,31

- - - N.S.W. Q. - B.fl.I,31

- T. V. N.S.W. - - B.fl.I,32

- - V. N.S.W. - - B.f.I,32

- $\overline{\text { T. V. }}$ - - - B.fl.I,32

$\begin{array}{lll}\text { S.A. T. V. N.S.W. - } & - \text { B.H.I,33 } \\ \text { B.I., } 33\end{array}$

S.A. T. V. N.S.W. - - B.f.I,34

W.A. - - - - - - B.f.I,34

W.A. - - - - - B.fl.I,34

W.A. - - - - - B.fl.I,35

W.A. - - - - - - B.fl.I,35

W.A. - - - - - - B.fl.I,35

- - - V. N.S.W. Q.

- - V. N.S.W. -

- - - N.S.W.

S

A.

.

- -7

-

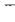

B.fl.I, 36

- B.fl.I,36

- B.fl.I,37

- B.fl.I, 37

B.fl.I,37

- B.fl.I,38

- B.fl.I,39

- B.fl.I,38

- B.fl.I,38

Q.

$$
\text { 二 }
$$

B.

$-$

B. 1,139

- B.fl.I,40

- B.fl.I, 40

B. I 40

- B.fl. 40

- B.fl.I, 41

- B.fl.I,41

- B.H.I,42

- B.ff.I.43

- B. $1 . I, 43$

B.H.I,43

M.fr. VII, 125;XI, 93.

M.fr.XI, 94 .

M.fr.XI, 93 .

M.fr.XI, 94 .

M.fr.XI,94.

M.fr.IV, 116;VII, 125; XI, 94.

M.fr.XI, 94.

M.fr.VII, 125;XI, 94.

M.fr.XI, 93 .

M.fr.XI, 93.

M.fr.XI, 94.

M.fr.IV,115.

M.fr.VII, 37.

M.fr.IUI, $1 ; \mathbf{X} I, 94$.

M.fr.XI, 94.

M.fr.XI, 94 .

M.fr.XI, 93.

M.fr.XI, 94 .

M.fr.IV, 117;XI,95.

M.fr.XI, 95 .

W.A. - - - - - B.fl.I,43

W.A. - - - - - - B.fl.I,44

W.A. - - - - - B.f.I,44

B.f., I, 45

B.fl.I, 45

B.fi.I, 46

B. fl. I, 46

W.A. - - - N.S.W. Q. - B.H.I,46
W.A. - - B.I,46
- - - B.f.I,47

M.fr.XI, 95.

M.fr.XI, 95.

M.fr.XI,95.

M.fr.XI, 96 .

M.fr.XI, 95.

M.fr.I, $161 ; \mathrm{VII}, 124 ; \mathrm{XI}, 94$.

M.fr. VII, 123;: II, 20 .

M.fr.VII, 12:3.

M.fr. V, l.

M.fr. V, 191.

M.fr.X, 49 .

M.fr.V,175.

M.fr. X, 124.

M.fr. $\mathrm{X}, 124$.

M.fr.X, 124 .

\section{MAGNOLIACEAE.}

J. de St. Hilaire, Expos. fam. nat. II, 74, t. $83-84$ (1805).

DRIMYS, R. \& G. Forster, char. gen. 83, t. 42 (1776). (Tasmania).

D. aromatica, F. v. Mueller, pl. of Vict. I, 20 (1860)

D. membranea, F. v. Mueller, fragm. V, 175 (1866)

D. dipetala, F. v. Mueller, pl. of Vict. I, 21 (1860)

D. Howeana, F. v. Mueller, fragm. VII, 17 (1870) $-\quad-$ T. V. N.S.W. - Q. B.f.I,49
$--\quad-$ - - S.W. Q.
$-\quad$ B.f.I,49
$-\quad-$ N.S.W. -
M.fr.VII, 18.

M.fr. V, 175; VII 18.

M.fr.VII, 18. 
ANONACEAE.

A. L. de Jussieu, Gen, 283 (1; 79 ) from B. de Jussieu (1;,59).

UVARIA, Linné, Amoen. acad. 404 (1747).

U. membranacea, Bentham, Fl. Austr. I 51 (1Si:3

U. Goezeana, F. v. Mueller, fragm. VII, 125 (15:1) FITZALANIA, F. r. Mueller, fragm. IV, 33 (1863).

F. heteropetala, F. v. Mueller, fragm. IV, 33 (1863)

CANANGA, Rumphius, herb. Amboin. II, 195, t. 65 (1741).

C. odorata, J. Hooker and Thomson, fl. Indic. 130 (1855)

ANCANA, F. v. Mueller, fragm. $\nabla, 27$ (1865).

A. stenopetala, F. v. Míller, fragm. $T$, 27 (1865)

POLYAITHIA, Blume, Fl. Jar. Anon. 68 (1829).

P. nitidissima, Bentham, Fl. Austr. I, 51 (1863).

POPOWLA, Endlicher, Gen. 831 (1839).

P. australis, Bentham, Fl. Austr. I, 52 (1863) $\quad \ldots \quad \ldots \quad \ldots \quad \ldots \quad-\quad-\quad-\quad-\quad \ldots \quad-\quad$ N.A. B.fl.I,52 MELODORLM, Loureiro, Fl. Cochinch. I, 351 (1790).

I. Leichhardtii, Bentham, Fl. Austr. I, 52 (1863)

M. Ľhrii, F, r. Mueller, fragm. VI, 2 (186i)

M. Maccreai, F. T. Mueller, fragm. VI, 176 (1868)

MUIUSIA, Leschenanlt in Mém. Crenèv. V, 213 (1832),

Species undetermined ...

SACCOPETALUM, Bennett in Horfs.- pl. Jav. rar. 115, t. 35 (1838).

S. Bidwilli, Bentham, Fl. Austr. I, 53 (1863) ... ... ... -

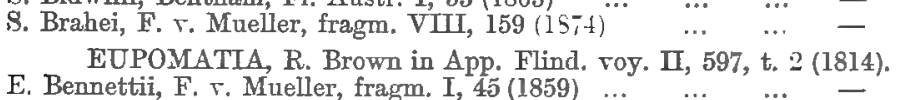

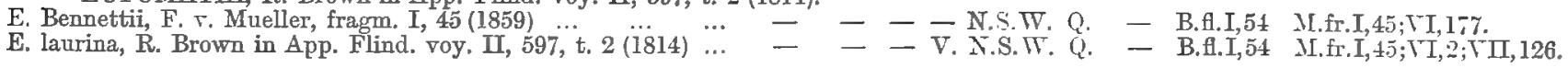

\section{MONIMIEAE.}

A. L. de Jussien in Ann. du Mus. XTT, 30 (1809).

DORYPHORA, Endlicher, Gen. 3015 (1837) (Learosa).

D. sassafras, Endlicher, iconogr. gen. pl., t 10 (1838) ... … - - - - N.S.W. A THEROSPERMA, Labillardière, jov, Holl. pl. spec, II, 74, t. 224 (1806).

A. moschatum, Labillardiere, Nor. Holl. pl. spec. II, 74, t. 224(1806) - - T. V. N.S. T. DAPHNA.TRRA, Bentham, Fl. Austr. Y', 285 (15;0).

D. micrantha, Bentham, Fl. Austr. V, $285(1870) \ldots \quad \ldots \quad \ldots \quad-\ldots \quad-\quad-\quad$ N.S.W. Q.

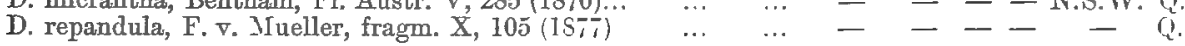
MOLLINEDIA, Ruiz \& Pavon, prodr. 83, t. 15 (1794). (Kibara, Tilkiea).

II. Huegeliana, Tulasne in Ann. sc. nat. sér. quatr. III, 45 (I855) - - - N.S.T. Q.

M. Wardellii, F. F. Mueller, fragm. F, 1.5.5 (1866)

M. Ioxocarya, Bentham, Fl. Austr. V, 25: $(1870)$...

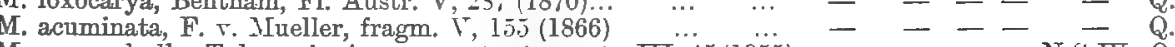

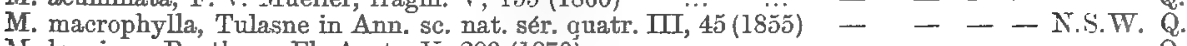

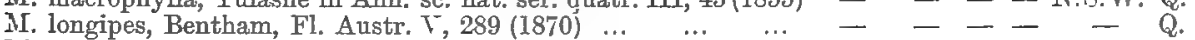

M. Iaxiflora, Bentham, Fl. Austr. V, $289(1870)$
M. pubescens, Bentham, Fl. Austr. V, $290(1870)$

HEDYCARYA, R. \& G. Forster, char. gen, 127, t. $64(1 ; 76)$.

H. Cunninghami, Tulasnein Archiv. du Mus. hist. nat.VIII,408(1855) - - - V. N.S.W. Q. - B.fl.V,291

PALMERIA, F. v. JIucller, fragm. IV, 151 (1864).

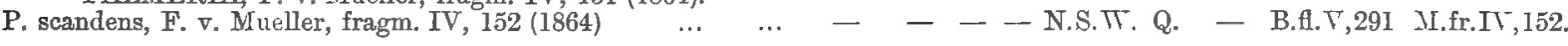
PIPTOCALYX, Oliver in Bentham's FI. Austr. V, 292 (1870).

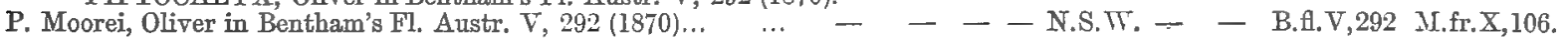

\section{MYRISTICEAE.}

R. Brown, prodr. 399 (1810) from A. L. de Jussieu (1806).

MYRISTICA, Linné, gen. pl. ed. IT, 524 (1742).

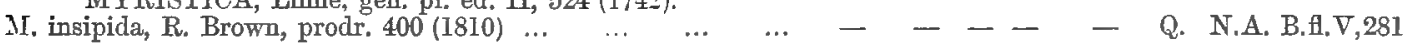

\section{IAURACEAE.}

Tentenat, Tabl. I, 245 (1799).

CRYPTOCARYA, R. Brown, prodr. 402 (1810). (Caryodaphne).

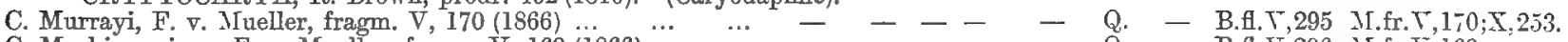

C. Mackinnoniana, F, v. Mueller, fragm. V, 169 (1866) ... … - - - - - Q. - B.f.V,296 II.fr.V,169.

C. patentinervis, F. v. Mueller in De Candolle, prodr.XV, 508(1864) - - - - N.S.T. Q. - B.fl.V,296 iI.fr.V,166.

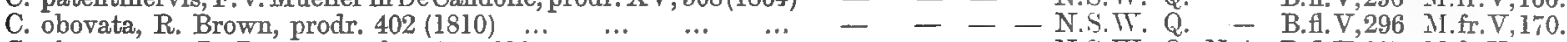

C. glaucescens, R. Brown, prodr. $402(1810) \quad \ldots \quad \ldots \quad \ldots \quad-\quad-\quad-\quad$ N.S.T. Q. N.A. B.A.V,297 I.,fr.T.165,I66.

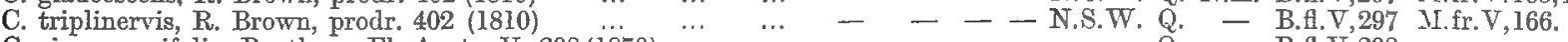

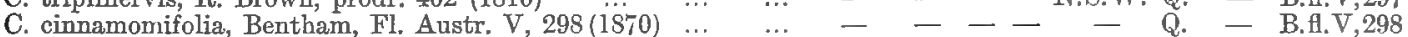

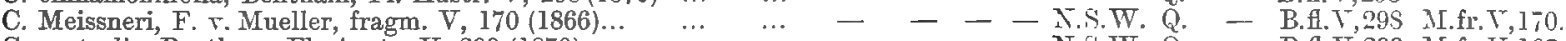

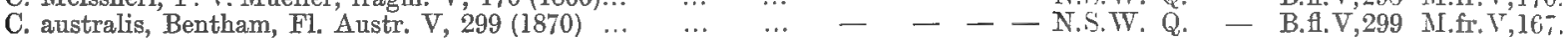

-BEILSCHMIEDIA, Nees in Wallich, pl. Asiat. rarior $\amalg$, 69 (1831). (Nesodaphne).

B. obtusifolia, Bentham, in B. \& H. gen. III, 1521 (1880) ‥ - - - - T.S.W. Q. - B.t.T,299 II.fr.T,166. 
ENDIANDRA, R. Brown, prodr. 402 (1810)

E. glanca, R. Brown, prodr. 402 (1810) ... ...

E. hypotephra, F. v. Mueller, fragm. V, $106(186 \ddot{6})$

E. discolor, Bentham, Fl. Austr. V, 301 (1870) ...

E. Sieberi, Nees, Syst. Laturin. 194 (1836) ...

E. virens, F. v. Mueller, fragm. II, 90 (1860)

E. Muelleri, Meissner in De Candolle, prodr. XV," 509 (1864)

E. pubens, Meissner in De Candolle, prodr. XV, 509 (1864) CINNAMOMUM, Burman, fl. zeil. 62 (1737).

C. Tamala, C. G. \& T. F. Nees, Syst, Laurin, 56 (1836) ..

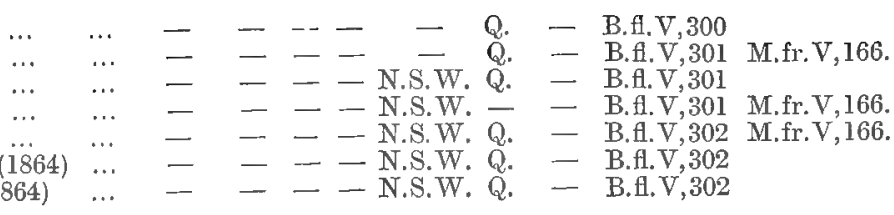

LITSEA, Lamarck, Diction. III, 574 (1789). (Glabraria 1771, Tetranthera, Cylicodaphne, Litsaea).

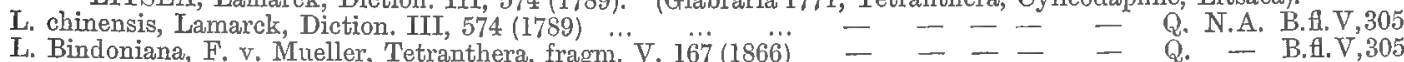

L. Hexanthus, A. L. de Jussieu in Annal, du Mus. VT, $212(1805) \quad-\quad-\quad-\quad-\quad$ Q. - B.fl.V,305

L. reticulata, Bentham in B.\& J.H.gen.pl. III, partI, 161(1880) - - - - _ Q. - B.fl.V,306

L. Zeylanica, C. G. \& T. F. Nees, in Amoen. Bonn. I, 58t.5(1823) - - - - - Q. - B.fi.V,307

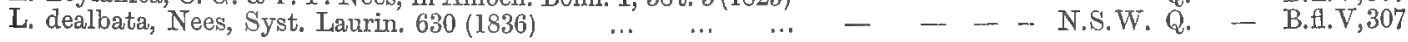
CASSYTHA, Osbeck in Linné, sp. pl. 35 (1753).

C. nodiflora, Meissner in De Candolle, prodr. XV, 252 (1864) $\ldots$

C. glabella, R. Brown, prodr. 404 (1810)

C. flava, Nees in Lehmann, pl. Preiss. I, 620 (1845)

C. pubescens, R. Brown, prodr. 404 (1810)... ...

C. phaeolasia, F. v. Mueller, fragm. V, 167 (1866)

C. paniculata, R. Brown, prodr, 404 (1810)
C. filiformis, Linné, sp. pl. 35 (1753)

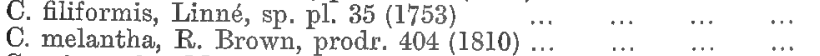

C. micrantha, Meissner in De Candolle, prodr. XV, 256 (1864) ...

C. racemosa, Nees in Lehmann, pl. Preiss. I, 621 (1845)..

C. pomiformis, Nees in Lehmann, pl. Preiss. I, 620 (1845) HERNANDIA, Plumier, Gen. 6, t. 40 (1703).

H. peltata, Meissner in De Candolle, prodr. XV, $263(1864) \quad \ldots \quad-\quad-\quad-\quad-\quad$ Q. $\quad-\quad$ B.f.V,314

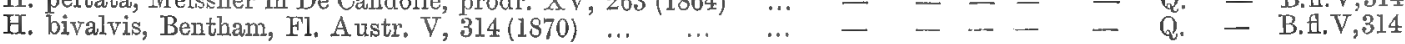

\section{MENISPERMEAE.}

A. L. de Jussieu, Gen. 284 (1789).

TTNOSPORA, Miers in Ann. and Mag. of nat. hist. sec. ser. VII, 35 (1851).

T. smilacina, Bentham in Journ. Linn. Soc. V, suppl. II, $2(1861)-L_{-}-$Q. N.A. B.fl.I,55 M.fr.IX,82.

T. Walcottii, F. v. Mueller in Bentham's El. Austr. I, 56 (1863) - - - - - - N.A. B.fl.I,56 FAWCETTYA, F. v. Mueller, fragm. X, 93 (1877).

F. tinosporoides, F. v. Mueller, fragm. X, $93(1877) \quad \ldots \quad \ldots \quad-\quad-\quad-\quad-$ N.S.W. - - - COCCULUS, De Candolle, sys. veg. I, 515 (1818) from C. Bauhin (1623). (Pericampylus, Leguephora).

C. Moorei, F. v. Mueller, fragm. I, $162(1859) \quad \ldots \quad \ldots \ldots \ldots \ldots$ HYPSERPA, Miers in Ann. and Mag. of nat. hist. sec. ser. VII, 36 (1851). (Selwynia).

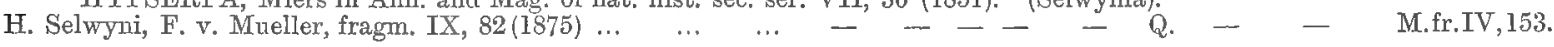
TRISTICHOCALYX, F. v. Mueller, fragm. IV, 27 (1863).

T. pubescens, F. v. Mueller, fragm. IV, $27(1863)$
T. diffusus, Miers, contrib. to Bot. III, $286(1871)$
I. SARCOPETALUM, F. v. Mueller, pl, of Vict. I, 26 (1860).

S. Harveyanum, F. v. Mueller, pl. of Vict. 27 and $221(1860) \ldots-\ldots-1$ - - V. N.S.IV. Q. - B.tl.I,57 M.fr.IX,S3. LEICHHARDTIA, F. v. Mueller, fragm. X, 67 (1876).

L. clamboides, F. v. Mueller, fragm. X, 68 (1876) STEPHANIA, Lonreiro, Fl. Cochinch. II, 608 (1790).

S. hernandifolia, Walpers, report bot. syst. I, $96(1842) \ldots \ldots \ldots-\ldots-\ldots-$ - V. N.S.W. Q. N.A. B.Al.I.57 M.fr.X.94. PACHYGONE, Miers in Amm. and Mag. nat. hist. sec. ser. VII, 37 (1851).

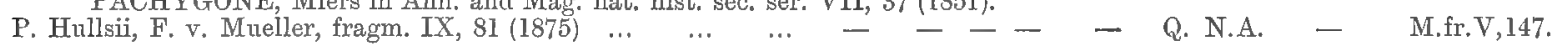
PLEOGYNE, Miers in Taylor's Ann. and Mag. of nat. hist, sec. ser. VII, 37 (1851). (Microclisia).

P. Cunninghami, Miersin Ann.\& Mag.of nat. hist, sec.ser. VII,37(1851) - - - - - Q. - B.fl.I,59 M.fr. IX, 82. CARRONIA, F. v. Mueller, fragm. IX, 84 (1875).

C. multisepalea, F, v. Mueller, fragm. IX, $171(1875) \quad \ldots \quad \ldots \quad-\quad-\quad-\quad$ - N.S.W. - - - - M.fr.IX,S4. ADELIOPSIS, Bentham \& J. Hooker, Gen. pl. I, 436 (1862).

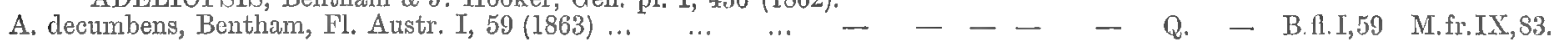

\section{PAPAVERACEAE.}

A. L. de Jussicu, Gen. 235 (1789) from B. de Jussicu (1759).

PAPAVER, Tournefort, inst. 237, t. $119(1700)$.

P. aculeatum, Thunberg, Fl. Capensis $431(1813) \ldots \ldots \ldots \ldots$... $\quad$ - S.A. T. V. N.S. IV. Q. - B.f.I,63

\section{CAPPARIDEAE.}

Tentenat, Tabl. III, 118 (1799).

CLEOME, Linné, syst. nat. 9 (1735); Linné, gen. 200 (1737). (Polanisia).

C. oxaliclea, F. v. Mueller, fragm. I, 69 (1858)

C. tetrandra, Banks in De Candolle prodr. I, 240 (1824)...

C. visoosa, Linné, sp. pl. $672(1753)$...

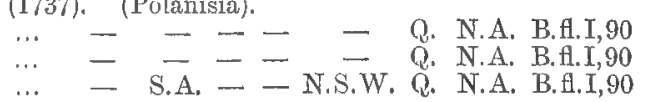

M.fr. I,69,

M. fr. VIX, 40;IX, 174. 
ROEPERLA, F. v. Mueller in Hooker's Kew Misc. IX, 15 (1857). (Tetratelia).

R. clemoides, F, Yueller in Hooker's Ker Yisc IX, 15 (15'

EMBLINGLA, F. v. Mrueller, fragm. ПI, 2 (1860).

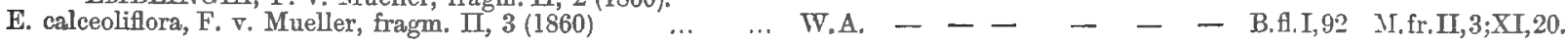

CADABA, Forskael, Fl. Aeg. Arab. 67 (1775).

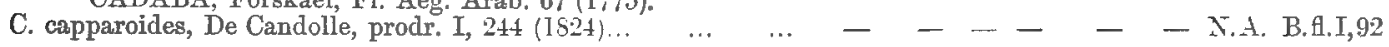

CAPPARIS, Tournefort, inst. 261, t. 139 (1700) from Theophrastos, Dioscorides and Plinius. (Busbeckea, Busbecquea).

C. umbellata, R. Brown in De Candolle, prodr. I, $24 i$ (1824) ...

C. lasiantha, R. Brown in De Candolle, prodr. I, 247 (1824) ... - S.A. - - N.S.W. O. N.A. B.Al. I,94

C. quiniflora, De Candolle, prodr. I, $247(1824)$... $\ldots$...

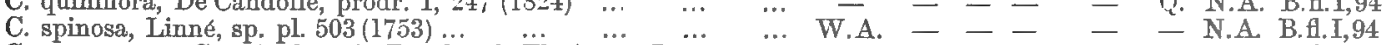

C. sarmentosa, Cunningham in Bentham's Fl. Austr. I, 95 (1563) - - - - o. - B.t1.I,95

C. ornans, F. v. Mueller in Bentham's Fl, Austr. I, 95 (1863) .. - $---C$ C. B.fl.I,95

C. nobilis, F. v. Nueller in Bentham's FI. Austr. I, 95 (1863) ... $-\quad-\quad-$ N.S.T.. . $\quad-$ B.A.I,95

C. canescens, Banks in De Candolle, prodr. I, $246(1824) \quad \ldots \quad-\quad-\quad-\quad-\quad$ Q. - B.fl.I,96

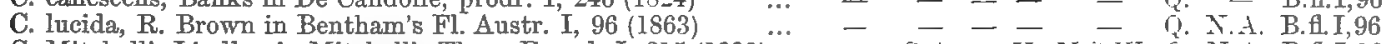

C. Mitehelli, Lindley in Mitchell's Three Exped. I, 315 (1838)... - S.A. - T. N.S.W. O. N.A. B.fl.I,96

C. loranthifolia, Lindley in Mitchell's Trop. Austr. 220 (1848) ... - - - - N.S.W. r. - B.fl. I,9

C. umbonata, Lindley in Mitchell's Trop. Austr. 257 (1848) $\ldots= \pm-Z=-$ C. N.A. B.A.I, 97

C. uberiflora, F. v. Mueller, fragm. IX, 172 (18i-j)

C. Shanesiana, F. v. Mueller, fragm. X, 94 (15:7)

C. humistrata, F. v. Mueller, fragm. $\nabla, 156(1 \mathrm{~S} 66)$

C. Thozetiana, F. v. Mueller, fragm. V. 104 (1866)

- -

二

8

- -

Q.

MI.fr.IX, 173.

II.fr.IX.1-3.

M. fr.I, $14 ; \%$ T $104 ; \mathrm{IX}, 173$.

MI.fr. $T^{\prime}, 104 ; \mathrm{LX}, 173$.

iI.fr.LX, 173.

M. fr. I, $163 ; T, 104$.

M.fr.IX.173.

I.f. I, 163; T, 104;IX,1;3.

M.fr.IX, 173 .

M.fr.IX,173.

M.fr. MY 172 .

M.fr.X. 94.

M. fr. Y, 156 .

APOPHYLLUM, F. r. MIueller in Hooker's Kew Misc. IX, 307 (1857).

A. anomalum, F. F. Mueller in Hooker's Kew Misc. IX, 307 (185\%) - - N.S. W. Q. N.A. B.f.I,97 M.fr.IX, 173;XI, 27.

\section{CRUCIFERAE.}

A. L. de Jussieu, Gen. 237 (1789) from B. de Jussieu (1759).

NASTURTIUI, R. Brown in W. T. Aiton's hortus Kewensis T, 110 (1812) from Linné, syst. nat. $9(1 ; 3.3)$.

N. terrestre, R. Brown in Aiton's hort. Kewens. $T^{\prime}, 110$ (1812) - S.A T. V. N.S. W. Q. - B.fl. I,66 M.fr.XI, 60 .

BARBARAEA, Beckmann, Lex. botan. 33 (1801). (Barbarea).

B. vulgaris, R. Brown in De Candolle, prodr. I, 140 (1S24) ‥ - - T. T. N.S. W. - - B.A.I,66 Y.fr. VIII,I42;II,6,60. ARABIS, Linné, gen. pl. 198 (1737) from Dalechamps (1554). (Turritis).

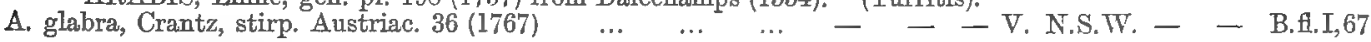

CARDALINEE, Tournefort, inst. 224, t. 109 (1700) from l'Ecluse (1576).

C. stylosa, De Candolle, regn. veg. syst. nat. II, $24(1821) \ldots \ldots$ - _ - T. V. N.S. IV. Q. - B.t.I,68

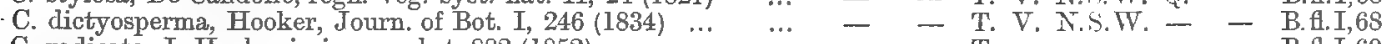

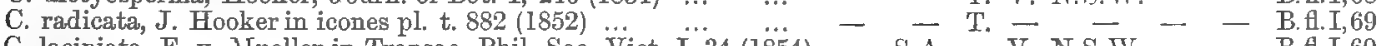

C. laciniata, F. v. Mueller in Transac. Phil. Soc. Vict. I, 34 (1854) - S.A. - V. N.S.W. - - B.H.I,69

C. hirsuta, Linné, sp. pl. 655 (1753)... … … … … TT.A. S.A. T. T. X.S.TF. Q. - B.fl., I,

C. eustylis, F. v. Mueller in Transact. Vict. Inst. I, $114(1854)$ - S.A. - V. N.S.T. Q. N.A. B.A.I, II ALISSCM, Tournefort, inst. 217 (1700) from l'Ecluse (1576). (Meniocus, Alysson).

A. minimum, Pallas, Reise Prov. Russ. Reich. III, $746(176)$ W.A. S.A - T. N.S. I. Q. N.A. B.fl.I,71 WILKIA, Scopoli, introd. hist. nat. 317 (17i). (Malcolmia).

W. Africana, F. v. ILuller, native plants of Vict. I, $33(1879) \ldots$ SISYMBRIUM, Tournefort, inst. 225, t. 109 (1700). (Blennodia).

S. filifolium, F. v. Mueller in Transact. Phil. Soc. Vict. I, $34(1854)-$ S.A. -- N.S. T. $-\quad-$ B.fl.I,73

S. trisectum, F Mueller in Transact. Vict

S. nasturtioides, F. v. Mueller in Transact. Tjet. Inst. I, 115 (1854) - S.A. - V. N.গ. W. - - B.fl.I,74

S. Richardsii, F. v. Mueller, fragm. X, 105 (187\%) $\ldots$... $\quad$ W.A. S.A. - - - -

S. eremigenum, F. T. Mueller, fragm. II, 143 (1861) $\quad \ldots \quad \ldots \quad-\quad-\quad-\quad$ N.S.W. Q.

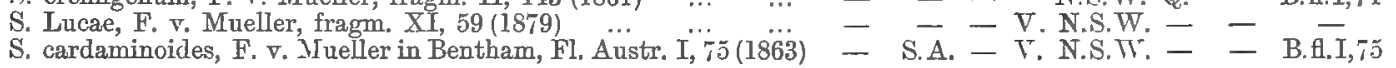
ERYSIMUM, Linné, gen. pl. 198 (1737). (Blennodia).

E. curvipes, F. v. Mueller in Linnaea XXV, 368 (1852) ... ‥ - S.A. - V. N.S.IV. -

E. brevipes, F. v. Mueller in Linnaea XXV, 367 (1852) ... $\quad$... T.A. S.A. - V. N.S.T. -

E. blennodioides, F. v. Yueller in Linnaea XXV, 367 (1852) $\ldots$ - - S.A. - V. N.S.W. Q.

E. Cunninghamii, Bentham, Fl. Austr. I, $76(1863) \quad \ldots \quad \ldots-\ldots--\quad-\quad$ N.N.W. Q.

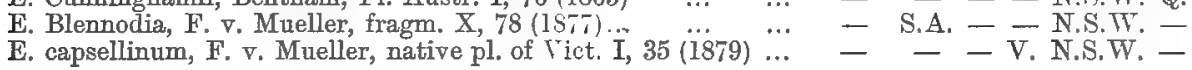

STENOPETALUM, R. Brown in De Candolle, Mém. du Mus. VI, 239 (1821).

S. velutinum, F. v. Mueller, pl. Vict. I, $49(1860) \quad \ldots \quad \ldots \quad-$ S.A. - V. N.S.T. Q.

S. lineare, R. Brown in De Candolle, syst. veg. I, 513 (1821) $\ldots . .$. W.A. S.A. T. V. N... II. Q.

S. sphaerocarpum, F. v. Mueller in Trans. Ph. Soc. Vict. I, 35 (1854) W.A. S.A. - V. N.S.W.

S. nutans, F. v. Mueller, fragm. III, 27 (1862) $\ldots \cdots \cdots$

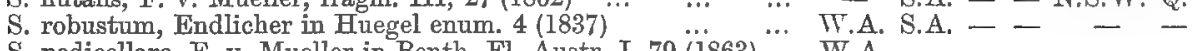

S. pedicellare, F. v. Mueller in Benth. Fl. Austr. I, 79 (1863) $\ldots$... W.A.
S. croceum, Bunge in Lehmann, pl. Preiss. I, 258 (1844) $\quad$... GEOCOCCUS, Drummond and Harvey in Hook. Kew Misc. VII, 52 (I855).

G. pusillus, Drumm. \& Harv. in Hook. Kew Misc. VII, 52 (1855) W.A. S.A. - V. N.S.W. MENKEA, Lehmann, Ind. sem. hort. bot. Hamb. 8 (1843).

M. australis, Lehmann in Ind. sem. hort. bot. Himb. 8 (1843) ... W.A. S.A. - V. N.S.T. -

M. draboides, J. Hooker in Benth. Fl. Austr. I, 80 (1863)

M. sphaerocarpa, F. v. Mueller, fragm. VIII, 223 (1874)

W.A. S.A. - V. N.S.W. -
W.A.

- B.tl.I,75

B.fl.I, 75

- B.Al.I, 76

- B.tl.I, 76

- B.fl.I,76

B.fl.I,77

B.fl.I, 78

B.fl.I,78

B. $1 . I, 78$

B.fl.I, 79

B.fl.I,79

B.fl.I, 79

-

B.Al.I, 80

B. Al. I, 80

B.H.I, S0
M.fr. VII, 19; XI, 60.

M.fr.VII, $19 ; X, 6,60$.

M.fr.XI, 6 .

I.fr.XI, 27,60

M.fr. VII, 19;XI, 60.

M.fr. TII, 19;XI, 60 .

M.fr. XI, 27,60 .

M.fr. VII, 18.

MI.fr. VII, 20;XI, 60.

M.fr.X, 105.

I.fr.П. 143

MI.fr.XI, 59 .

M. fr. XI, 27,59 .

II.fr. VU, 20.

II. VII, 20 ; XI, 6 .

M.fr.VII, 20;XI, $27,60$.

II.fr.VII, $20 ; \mathrm{X}, 78$.

II.fr. VII, $20 ; \mathrm{XI}, 27$

M.fr. VII. 20;XI, 6 .

M.fr.VII, 20 .

M.fr.II, 27;VII, 20;XI, 6 .

M.fr.VII, $20 ; \mathrm{XI}, 5,60$.

M.fr. VIT, 20

II.fr, XI, 6 .

II.fr. VII, 19; XI,6,26.

M.fr.II, 142.

M.fr.II,142; XIT, 19.
M.fr. VII, 223. 
CAPSELLA, Medicus, Pflanzen-Gattungen 85 (1792). (Hutchinsia partly, Thlaspi partly, Microlepidium).

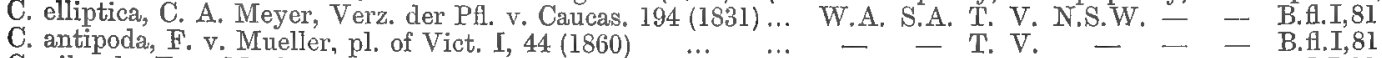

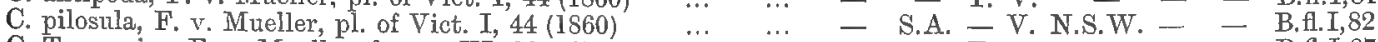

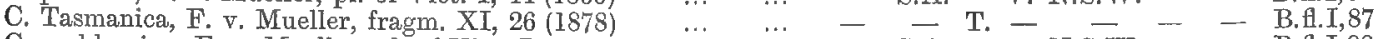

C. cochlearina, F. v. Mueller, pl. of Vict. I, 51 (1860) $\ldots-\ldots, \ldots-$ S.A. - - N.S.W. - - B.fl.I,88

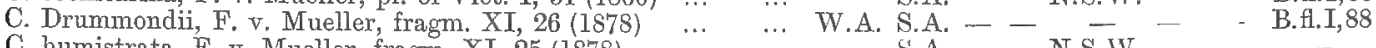

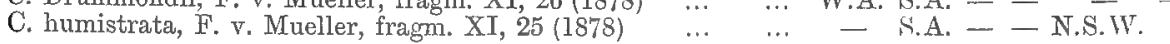

SENEBIERA, De Candolle in Mém. soc. hist. nat. Par. I, 140 (1799).

S. integrifolia, De Candolle in Mérn. soc. his. nat. Par. I, 144(1799) - - - - — Q. - B.f.I,82

LEPDDIUM, Tournefort, inst. 215, t. 103 (1700) from Diosc. and Plinius. (Tberis partly, Lepia, Monoploca).

L. strongylophyllum, F. v. Mueller in Benth. Fl. Austr. I, 84 (1863) - S.A. - - - Q. N.A. B.fl.I,84

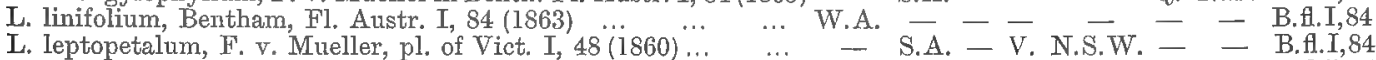

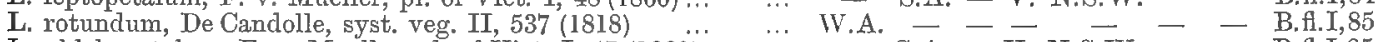

L. phlebopetalum, F. v. Mueller, pl. of Vict. I, $47(1860) \quad \ldots \quad-$ S.A. - V. N.S.W. - - B.H.I,85

L. pedicellosum, F. v. Mueller, fragm. XI, $27(1878)$
L. monoplocoides, F. . . Mueller in Trans. Phil. Soc. Vict. 35(1854) $\cdots-$ S.A. - V. N.S.W. - N.A. - B.A.I,85

L. monoplocoides, F. v. Mueller in Trans. Phil. Soc. Vict. 35(1854)
L. papillosum, F. V. Mueller in Linnaea XXV, 370 (1852) $\quad$... W.A. - W.A. S.A. - V. N.S.W. - N.S.W. - - - B.f.I,85

L. foliosum, Desvaux, Journ, de Bot. III, 164 et 180 (1813) $\quad \ldots$ W.A. S.A. T. V. N.S.W. - - B.H.I,86

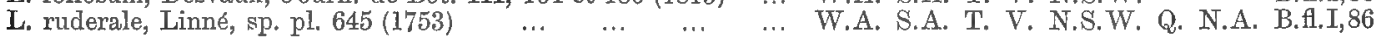
CAKLE, Tournefort, coroll. 49, t. $483(1700)$.

C. maritima, Scopoli, Fl, Carniolica, 344 (1760)

- S.A. T. V. N.S.W. - - -

M.fr.VII,19;XI.60.

M.fr.XI, 60 .

M.fr.XI, 26.

M.fr.VII,90;XI, 26 .

M.fr.XI, 25.

\section{M.fr.XI, 6 .}

M.fr.VII, 20;XI, 6 .

M.fr.VII, $20 ; \mathrm{XI}, 6$.

M.fr. VII, $19 ; \mathrm{XI}, 60$.

M.fr.XI, 27.

M.fr.XI, 27 .

M.fr.VII, $19 ; \mathrm{XI}, 6,28$.

M.fr. VII, 19.

M.fr.VII, $19 ; X I, 27,61$.

M.fr.VII, $19 ; \mathrm{XI}, 6,28,60$.

\section{VIOLACEAE.}

De Candolle, Fl. Franc. IV, 801 (1805).

VIOLA, Tournefort, inst. 419, t. 236 (1700) from Plinius.

V. betonicifolia, Smith in Rees's Cyclopedia XXXVII (18I7) ... - S.A. T. V. N.S.W. Q. - B.fl.I,99

V. hederacea, Labillardière, Nov. Holl. pl, spec. I, 66, t.91(1804) - S.A. T. V. N.S.W. Q. - B.A.I,99

V. Cunninghamii, J. Hooker, Fl. N. Zel. I, $16(1853)$... .. - - - T. - - - - B.fl.I,100

V. Caleyana, G. Don, gen. syst. dichlam. pl. I, $329(1831) \quad \ldots .-1$ - $\quad$ T. V. N.S.W. - - B.f.I,100 HYBANTHUS, Jacquin, Stirp. Amer. hist. 77, t. 75 (1763). (Ionidium, Pigea, Vlamingia).

H. floribundus, F. v. Mueller, nat. pl. of Vict. I, 45 (1879) … W.A. S.A. - V. N.S.W. -

H. filiformis, F. v. Mueller, native pl. of Vict. I, $44(1879) \quad \ldots---\quad-$ V. N.S.W. Q. - B.A.I,103

H. Tatei, F. v. Mueller in Trans. R. Socy. of S. Aust. IV, $102(1882)-$ S.A. - - - - - -

H. debilissimus. F, v. Mueller, fragm. XI, 4 (1878)

H. calycinus, F. v. Mueller, fragm. X, 81 (1877)

$\cdots \quad \cdots \quad$ W.

W.A. - - - - - - - W.

‥ - S.A. - - N.S.W. Q. N.A. B.A.I,10I

HYMENANTHERA, R. Brown in Tuck. Cong. 442 (1818).

H. Banksii, F. v. Muellex, first. gen, report 9 (1853)

— T. V. N.S.W. - - - B.fl.I,104

M.fr.X, 82 .

M.fr.X, 82 .

M.fr.X, $\$ 2$.

\section{FLACOURTIEAE.}

Cl. Richard in Mém. du Mus. I, 366 (1815).

COCHLOSPERMUM, Kunth, Malvac. 6 (1822).

C. Fraseri, Planchon in Hook. Lond. Journ. VI, 307 (1847) $\ldots-\ldots-z_{-}-\quad$ - N.A. B.fi.I.106

C. heteronemum, F. v. Mueller in Hook. Kew. Misc. IX, 15 (1857) - - - - - - N.A. B.f.I.106

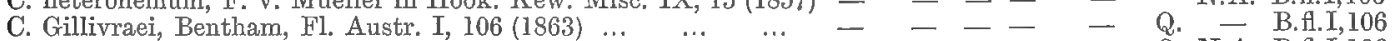

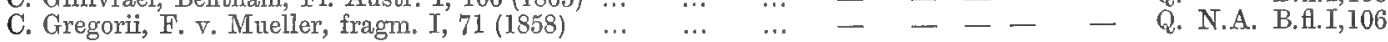

M.fr.IX, 61.

SCOLOPIA, Schreber, Gen, 335 (1789). (Phoberos).

S. Brownii, F., v. Mueller, fragm. III, 11 (1862) $\ldots \quad \ldots \quad \ldots \quad-\quad-\ldots \quad-$ N.S.W. Q. $\quad-$ B.Al.I,107

M.fr.IX,61.

XYLOSMA, G. Forster, prodr. 380 (1786).

X. ovata, Bentham, Fl. Austr. I, $108(1863) \quad \ldots \quad \ldots \quad \ldots \quad-\quad \ldots \quad-\quad$ - N.S.W. Q. - B.fl.I,108 M.fr.IX,60. STREPTOTHAMNUS, F. v. Mueller, fragm. III, 28 (1862).

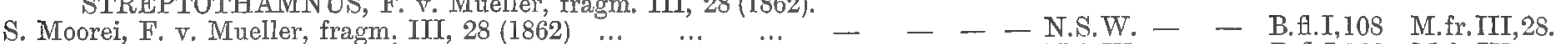

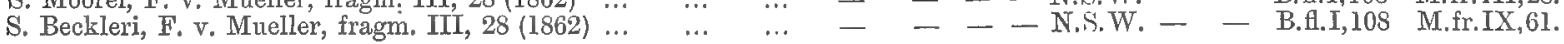

\section{SAMYDACEAE.}

J. Gaertner, de Fruct. III, 328 (1805).

CASEARIA, Jacquin, Stirp. Amer. hist. 132, t. 85 (1763).

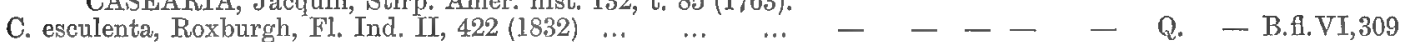

C. Dallachii, F. v. Mueller, fragm, V, $107(1866) \ldots \quad$.. $\quad \ldots \quad-\ldots \quad-\quad$ - $\quad$ - Q. - B.f.III,309 M.fr.VI,252.

HOMALIUM, Jacquin, Stirp. Amer. hist. 170, t. 183 (1763). (Blackwellia).

H. Vitiense, Bentham in Journ. Linn. Soc. IV, $36(\mathbf{1 8 6 0 )}, \ldots+-\quad-\quad-\quad$ Q. $\quad$ B.fl.III,310

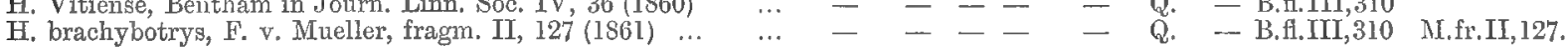

\section{PITTOSPOREAE.}

R. Brown in Flinders' voy. 542 (1814).

PITTOSPORUM, Banks in Gaertner, de Frutet. I, 286, t. 59 (1788).

$P$, rhombifolium, Cunningham in Hook. icon, pl. t. 62l (1844)...

P. melanospermum, F. v. Muellcr, fragm. I, 70 (1858) (1844)... -

P. undulatum, Andrews, Bot. reposit. 383 (1800).

P. revolutum, Aiton, Hort. Kew, sec. ed. II, 27 (1811) ...

P. venulosum, F. v. Mueller, fragm. VI, 186 (1868)

P. ferrugineun, Aiton, Hort. KeW, sec. ed. II, 27 (1811)
- - N.S.W. Q. - B.A.I,110

- - - $-\overline{\text { N }}$ N.A. B.fi.I,111

Q. - B. I.I,Ill

- - V. N.S.W. Q. - B.f.I,111

.. - - - - - Q. Q -

-

B. f. I. 112
M.fr.XII,4.

M.fr. YI, $167: X I I, 4$

M.fr.VT,167; XII,4.

M.fr. VI, $167 \& 1 \& 6 \div V I T, 140$.

M.fr. VII, $140 ; \mathrm{XII}, 4$.

M.fr.II, 78;III, 165;VI, 167 


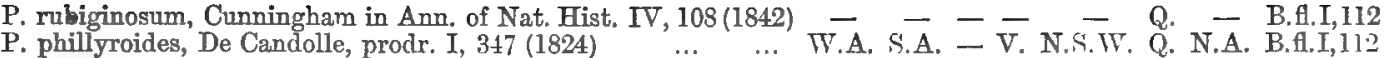

P. bicolor, Hooker, Journ. Bot. I, $249(1834) \quad \ldots \quad \ldots . \quad \ldots \quad-\quad$ - T. V. N.N.W. - - B.t.I,113

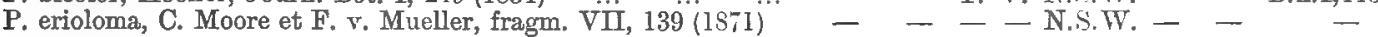

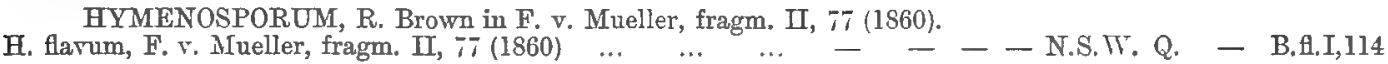
BURS.ARIA, Cavanilles, icon, et descr. pl. IV, 30, t. $350(1 ; 97)$.

B. spinosa, Cavanilles, Icon. pl. IV, 30, t. 350 (1797) … … W.A. S.A. T. V. N.S.W. Q. N.A. B.A.I,11ว MARIANTHUS, Huegel, enum. pl. Nor. Holl. 8 (1S37). (Oncosporum, Rhytidosporum, Calopetalon).

M. procumbens, Bentham, Fl. Austr. I, 117 (1863)

M. microphyllus, Bentham, FI. Austr. I, I17 (1863)
M. villosus, Bentham, Fl. Austr. I, I17 (1863) $\ldots$

M. microphyllus, Bentham, FI. Austr. I, I17 (1863)
M. villosus, Bentham, Fl. Austr. I, I17(1863) $\ldots$ ‥

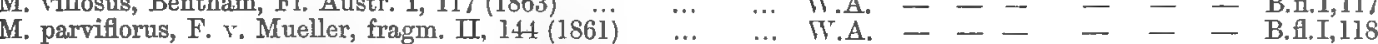

M. bignoniaceus, F. v. Mueller in Trans. Phil. Soc. Vict. 6 (1854)

M. Drummondianus, Bentham, Fl. Austr. I, 119 (1863) ...

M. coeruleo-punctatus, Klotzsch in Link and Otto, icon. pl, 2. t. $12(1841)$

M. candidus, Huegel, enum. pl. Nov. Holl, 8 (183̈7)

M. erubescens, Putterlick in Endl. nov. stirp. dec, 60 (1339)

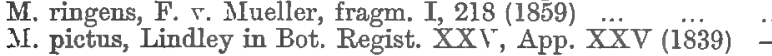
CITRIOBATUS, Cunningham, in Loudon's hort. Brit. supp. I, 585 (1832). (Ixiosporum).

C. multiflorus, Cunningham in Loud. hort. Brit. I, 585 (1832) ... - - - N.S.W. Q BILLARDIERA, Smith, Sp. Bot. New Holl. I, t. 1 (1793). (Pronaya, Campylanthera).

B. longiflora, Labillardière, Nof. Holl. plant. spec. I, 64, t. $89(1804)-\quad-$ T. V. N.S.W. - - B.H.I,123

B. scandens, Smith, Spec. bot. New Holl. I, t. I (1793) .. … - S.A. T. T. X.S.T. Q. - B.fl. I 123

B. coriacea, Bentham, Fl. Austr. I, 124 (1863) $\ldots . \quad \ldots \quad \ldots$ W.A $-\quad$ - $-\quad-\quad-\quad$ B.fl.I,124

B. cymosa, F. r. IIueller in Transact. Vict. Inst. $\dddot{I}, 29$ (1855) … - S.A. - T. N.S.W. - - B.H.I,124

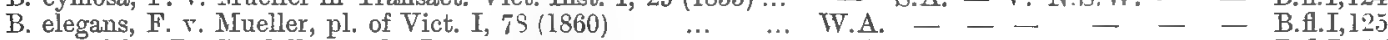

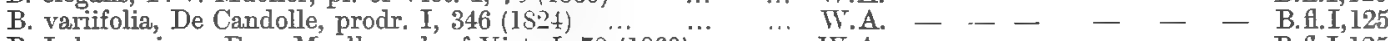

B. Lehmanniana, F. v. Mueller, pl. of Vict. I, is (1860) $\quad$ … IT.A. - - - - - - B.H.I,125

B. floribunda, F. г. MLueller in South Sc. Record II, 1 (1882) $\ldots$ W.A. - - - - - - B.fl.I,120 SOLLYA, Lindley, Bot. Regist. STW, t. 1460 (1831). (Xerosollya.)

S. heterophylla, Lindley, Bot. Regist. XVII, t. 1466 (183I) ... W.A. - - - $-\quad-\quad$ B.H.I,126

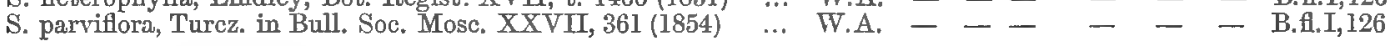
CHEIRANTHERA, Cunningham, in Brongn. Bot. voy. Coquille, t. 77 (1829).

C. linearis, Cunningham in Bot. Regist. 1719 (1834) $\ldots \ldots \ldots$ - S.A. - V. N.S.T. Q. - B.f.I, 127

C. filifolia, Turczaninow in Bull. Soc. Mose. XXVII, $364(1854)$ T.A. - - - - - - B.fl.I,I27

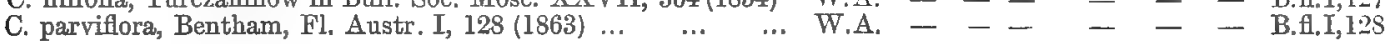

\section{DROSERACEAE.}

Salisbury, Parad. Lond. 95 (1809).

ALDROVANDA, Monti in Act. Bonon. $\Pi$, 404, t. $12(174 \bar{j})$.

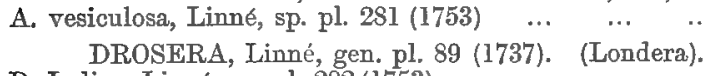

D. Indica, Linné, sp. pl. 282 (1753) ... 154 (1864) ...

D. Areturi, Hooker, Journ. of Bot. I, 247 (1834) ...

D. glanduligera, Lehmann, pugill. VIII, 37 (1844)

D. pygmaea, De Candolle, prodr. I, 317 (1824)

D. platystigma, Lehmann, pugill. TIII, $37(18+4) \ldots$

D. pulchella, Lehmann, pugill. VIII, 38 (1844)

D. leucoblasta, Bentham, Fl. Austr. II, 458 (1864)

D. nitidula, Planchon in Ann. des sc. nat. IX, 285 (1949)

D. paleacea, R. Brown in De Candolle, prodr. I, 318 (1824)

D. parvula, Planchon in Ann. des sc. nat. IX, 2ST (1848)

D. Burmanni, Vahl, Symbol. III, 50 (1794)

D. spathulata, Labillardière, Nov. Holl. pl. spec. I, 79 , t. 106 (1804)

D. Drummondii, Lehmann, pl. Preiss. II, 235 (1847) $\ldots$

D. petiolaris, R. Brown in De Candolle, prodr. I, 318 (1824)

D. binata, Labillardière, Nor. Holl. pl. spec. I, 78, t. 105 (1804)

D. zonaria, Planchon in Ann. des sc. nat. IX, 303 (1848)

D. bulbosa, Hooker, icon. pl. t. 375 (1841)...

D. rosulata, Lehmann, pugill. TIII, 36 (1814)

D. Whittakerii, Planchon in Ann, des sc. nat. IX, 302 (1848)

D. macrophylla, Lindley, Bot. Regist. XTT, App. XX, (1839)...

D. squamosa, Bentham, Fl. Austr. II, 463 (1864)

D. erythrorrhiza, Lindley, Bot. Regist. XXY, App. XX (1839)...

D. stolonifera, Endlicher in Hueg. enum. 5 (1837)

D. humilis, Planchon in Ann, des sc. nat. IX, 300 (1848) $\quad \ldots$

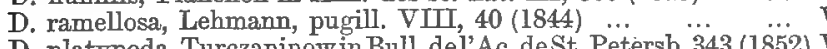

D. platypoda, Turczaninow in Bull. del'Ac. deSt. Petersb. 343 (1852)
D. auriculata, Backhouse in Ann. des sc. nat. LX, 295 (1848) ...

D. auriculata, Backhouse in Ann. des sc. nat. LX, 295 (1848)

D. lunata, Buchanan in De Cand. prodr. I, $319(1824)$
D. peltata, Smith in Willd. spec. pl. I, $1546(1797)$
W.A. S.A. - V. N.S.TI. Q. N.A. B.fl.I, 456 - - T. V. N.S.W. Q. -

T.A. S.A. - T. N.S.IT. - - B.fl.M, 4.57

- S.A. T. V. N.S.T. - - B.fl.II,457

W.A. - - - - - - B.H.II,457

W.A. - - - - - - B.f.II,458

W.A. - - - - - - B.fl.II,458

W.A. - - - - - - B.f.II, $45 \mathrm{~S}$

W.A. - - - - - - B.f.П,458

W.A. - - - - - - B.H.II,459

- S.A. - T. V. N.S.T. Q. N.A. B.A. I, 459

W.A. - - - - - - B.H.II,460

W.A. - - - - - - B.H.II,460

- - - - Q. N.A. B.f.II,460

- S.A. T. V. N.S.W. Q. - B.H.I, 461

W.A. - - - - - B.fl.II,462

W.A. - - - - - - B.fl,II,462

II.A. $--\bar{V}--$ - B.H.M,462

- S.A. - V. - - - B.fl.II,462

W.A. - - - - - - B.fl.I, 463

W.A. - - - - - B.fl.II,463

W.A. - - - - - - B.H. II,463

W.A. - - - - - - B.fl.II,463

W.A. - - - - - B.f.II,464

W.A. - - - - - B.fl.II,464

- S.A. T. F. N.S.I. -

- S.A. T. V. N.S.W. Q.

B.fl. $\Pi, 464$

B.H.I, 465
M.fr.VI, 167;FII, 140;IX,

M.fr. VI, 167.

M.fr. VII, 142; XII, 4 .

M.fr. VI, 139 .

M.fr.II,77; VI, I6s;VIII,

M. fr, XШ, 3.

M.fr.XП.2.

M.fr.II, 145 .

MI.fr.II, 144; XI, 2 .

M.fr. XI,,

M.fr. XII, 2.

MI.fr.XII, 2 .

M.fr.XI, 2 .

M.fr.XII,?

M.fr.I,2ls;XII, 2 .

M.fr. I, 217;II, 150; XI, 2 .

II.fr.II, 77 ;YI, 168 ;XII,3. M.fr.II, $; 6 ;$ VI 168;VII,180.

M.fr.XI, 123 .

M.fr.XI,

MI.fr. TI, 168; VII, 140 .

M.fr.XU,2.

M.fr.

MI. fr. XII, 2.

M.fr.XI, 1 .

M.fr.XII, 3,

II. fr. XII, 3 ,

XI.fr.I,97;II, 180;IX,168.

M.fr.I, $97 ; \Pi, 79 ; \mathrm{IX}, 168$.

M.fr.XII, 3.

II.fr. TI, 104;X, 79.

M.fr. $\Gamma, 79$.

M.fr.X, 80 .

M.fr. X, 80 .

$M . f r . X, 80$.

M.fr.X, 80 .

M.fr. X, 80 .

M.fr.X, 80 .

M.fr. $X, 80$.

MI. fr.VIII, 185; X, 85 .

M.fr. X, 80 .

Il.fr. X, SO.

II.fr. X, 80 .

M.fr. TIII, 185; X, 80 .

iI.fr.X, 80 .

M.fr.X, 80 . 
D. Neesii, Lehmann, pugill. VIII 42 (1844)

D. gigantea, Lindley, Bot. Regist. XXV, App. X X (1839)

D. myriantha, Planchon in Ann. des sc. nat. IX, 291 (1848)

D. pallida, Lindley, Bot. Regist. XXV, App. XX (1839)

D. penicillaris, Bentham, Fl. Austr. II, 467 (1864)

D. Huegelii, Endlicher in Hueg. enum. 6 (1837) $\ldots$.

D. macrantha, Endlicher in Hueg. enum. 6 (1837) …

D. Menziesii, R. Brown in De Candolle, prodr. I, 319 (1824)

D. calycina, Planchon in Ann. des sc. nat. IX, 299 (1848)

D. Banksíi, R. Brown in De Candolle, prodr. I, 319 (1824) BYBLIS, Salisbury, Parad. Lond. t. 95 (1808).

B. liniflora, Salisbury, Parad. Lond. t. 95 (180S)

B. gigantea, Lindley Bot. Regist. XXV, App. XXI (1839)
D. filicaulis, Endlicher in Hueg. enum. 6 (1837) ...

D. heterophylla, Lindley, Bot. Regist. XXV, App. XX (1839)...

\begin{tabular}{|c|c|c|c|c|c|c|c|c|}
\hline W.A. & - & - & - & & & $-\cdot$ & B.ff.II, 466 & \\
\hline W.A. & - & - & - & - & - & 一 & B.fl.II, 466 & \\
\hline W.A. & - & - & - & - & - & - & B.fl.IX, 466 & \\
\hline W.A. & - & & - & - & - & - & B. H.II, 467 & \\
\hline W.A. & - & - & - & - & - & - & B.fl.II, 467 & \\
\hline W.A. & - & - & - & - & - & - & B.fl.II, 467 & \\
\hline W.A. & - & - & - & - & 一 & - & B.fl.II,467 & M.fr. VIH, $180 ; \mathrm{X}, 81$ \\
\hline W.A. & - & - & $\bar{T}$ & - & - & - & B. fl.11,468 & \\
\hline W.A. & S.A. & T. & V. & N.S.W. & - & - & & \\
\hline W.A. & - & 一 & - & - & - & - & $\begin{array}{l}\text { B.H.II,468 } \\
\text { B.fl.II, } 469\end{array}$ & \\
\hline W.A. & - & - & - & - & $\overline{\mathrm{Q}}$ & - & B.fl.II, 469 & \\
\hline - & - & - & - & - & 0. & N. A. & B.Al.1 & M.fr.X,81. \\
\hline$R T A$ & - & - & -- & -- & - & - & B.fl.II, 470 & \\
\hline
\end{tabular}

ELATINEAE.

Cambessèdes in Mém. du Mus. XVIII, 225 (1829).

ELATINE, Linné, gen. pl. 118 (1737).

E. Americana, Arnott in Edinb. journ. nat. sc. I, 431 (1830) ‥ W.A. S.A. T. V. N.S.W. Q. - B.H.I,178 BERGIA, Linné, mantiss, II, 152 (1771).

B. ammannioides, Roth, nov. pl. spec. $219(1821) \ldots \ldots \ldots \ldots-\ldots-\ldots-$ - $\quad$ V. N.S.W. Q. N.A. B.fl.I, 180

B. pedicellaris, F. v. Mueller in Benth. FI. Austr. I, $180 \ddot{(1863)}$ - - - - - - N.A. B.f.I, I80

B. perennis, F. v. Mueller in Benth. Fl. Austr. I, $180(1863) \ldots \ldots-\ldots-\ldots$ - - - N.A. B.A.I,181

\section{HYPERICINAE.}

J. de St. Hilaire, Expos. fam. II, 23, t. 75 (1805).

HYPERICUM, Tonrnefort, inst. 254, t. 131 (1700), from Dioscorides and Plinius.

H. Japonicum, Thunberg, Fl. Jap. 295 , t. 31 (1784) $\ldots . \quad \ldots$ W.A. S.A. T. V. N.S. W. Q. N.A. B.AlI, 182

\section{GUTTIFERAE.}

CALOPHYLLUM, Linné, gen. 154 (1737).

A. L. de Jussieu, Gen. 243 (1789).

C. inophyllum, Linné, sp. pl. 513 (1753)

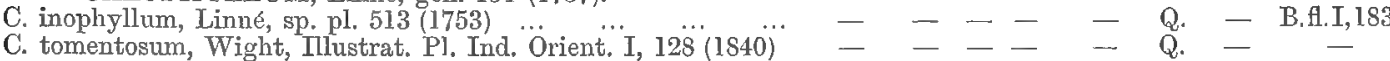

M.fr.IX, 175

M.fr.IX,174.

\section{POLYGALEAE.}

A. L. de Jussieu, in Ann. du Mus. XIV, 386 (1809).

SALOMONIA, Loureiro, Fl. Cochinch. I, 14 (1790).

S. oblongifolia, De Candolle, prodr. I, $334(\mathbf{1 8 2 4 ) \ldots} \quad \ldots \quad \ldots \quad-\quad-\quad-\quad-$ N.S.W. Q. $\quad$ - B.fl.I, 138 POLYGALA, Toumefort, inst. 174, t. 79 (1700).

P. Sibirica, Linné, sp. pl. 702 (1753)

P. leptalea, De Candolle, prodr. I, 325 (182̈)

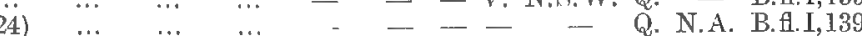

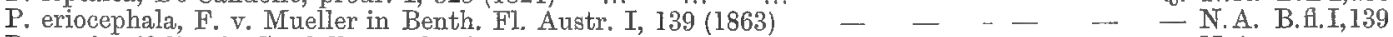

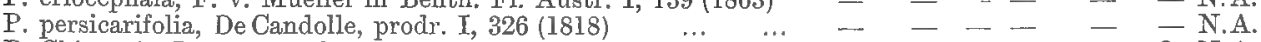

P. Chinensis, Linne, sp. pl. 704 (1753)

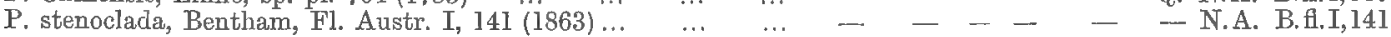
COMESPERMA, Labillardière, Nov. Holl. pl. spec. II, 21 (1806).

C. sphaerocarpum, Steetz in Lehm. pl. Preiss. II, 314(1847) ... - - - - N.S.IV. Q. - B.fl.I,143

C. scoparium, Steetz in Lehm. pl. Preiss. II, $309(1847) \ldots \quad$... W.A. S.A. - V. N.S.W. - - B.fi.I,143

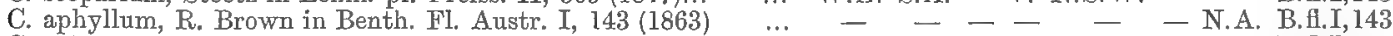

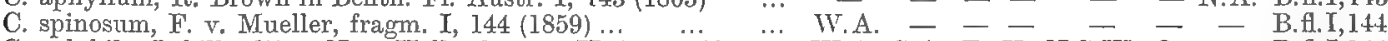

C. volubile, Labillardière, Nov. Holl. pl. spec. IT, 24, t. 163 (1806) W.A. S.A. T. V. N.S.W. Q. - B.fl.I, 144

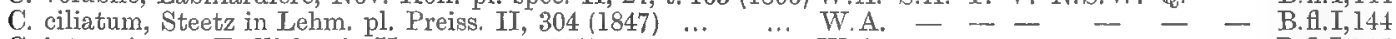

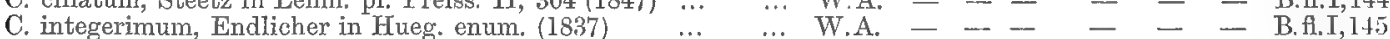

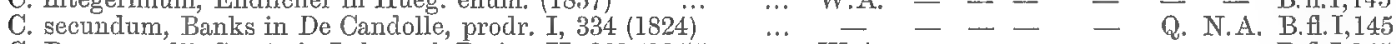

C. Drummondii, Steetz in Lehm. pl. Preiss. II, 301 (1847) $\quad \ldots$ W.A. - - - - - - - B.H.I,145

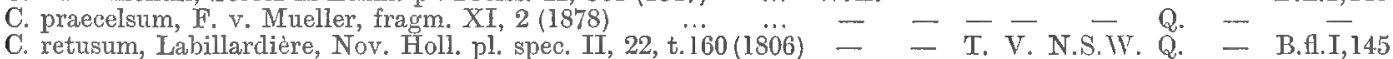

C. retusum, Labillardière, Nov. Holl. pl. spec. II, 22, t.160(1806) - - T. V. N.S. W. Q.
C. sylvestre, Lindley in Mitch. Trop. Austr. $342(1848) \ldots$

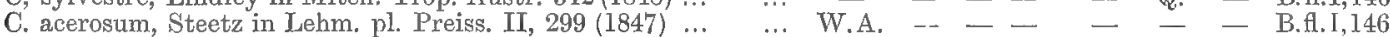

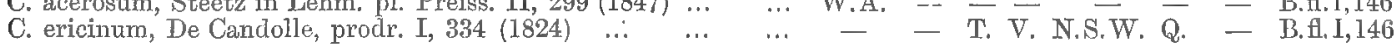

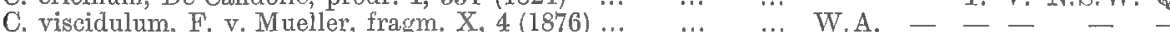

C. confertum, Labillarclière, Nov. Holl.pl. spec. II, 23, t. 161 (1806) W.A. - - - - - - B.fl.I,147

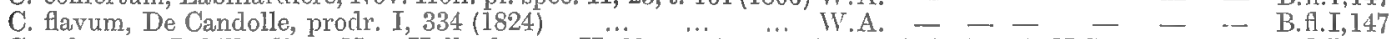

C. calymega, Labillardière, Nov. Holl. pl. spec. II, 23, t. 162 (1806) W.A. S.A. T. V. N.S.IV. - - B.A.I,147

C. rhadinocarpum, F. v. Mueller, fragm. XI, I (1878) … … W.A. - - - - - -

C. defoliatum, F. v. Mueller, pl. of Vict. I, 189 (1862) ... ‥ - - T. T. N.S.W. Q. - B.f.I,148

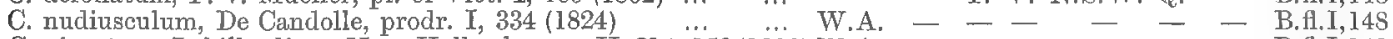

C. virgatum, Labillardière, Nov. Holl. pl. spec. II, 21 t. 159 (1806) W.A. - - - - - - B.f.I,149

C. polygaloides, F. v. Mueller in Trans. Phil. Soc. Vict. I, 7(1854) W.A. S.A. - V. - - - B.fl.I,149

XANTHOPHYLLUM, Roxburgh, Pl. Corom. III, 81, t. 284 (1819).

X. Macintyrii, F. v. Mueller, fragm. V, 57 (1865)
M.fr.XI,27.

M.fr.II, 147.

M.fr.II, 145 .

M.fr.II, 146.
M.fr.XI, 4.

M.fr.XI,4. M. fr.XI, 4 .

M.fr. XI, 4 . M. fr.XI, 4 .

M.fr. XI, 3.

M.fr.I, 144 . M.fr.XI, 3 . M. fr. X, 3 . M.fr.XI, 3 .

M.fr. XI, ?. M.fr.XI, 2 . M.fr. XT, 2 . M. fr. I, 49;:I, 2. M.fr.XI, 3. M fr. I, 48 ;XI, 2 . $\mathrm{MI}, \mathrm{fr}, \mathrm{X}, 4: \mathrm{XI}, 2$. M.fr.XI, 3 . MI.fr. XI, B. M. fr. XI, 3. MI.fr. XI, 1 . M.fr. X $I, 1$. M.fr.XI, 3. M.fr. XI, 4 . MI.fr. XI, 2. M.fr. XI, 4 .

M. fr. $\mathrm{V}, 57 ; \mathrm{XI}_{2} 4$. 
TREMANDREAE.

R. Brown in Flind. roy. II, 551 App. (1814).

PLATYTHECA, Steetz in Lehmann, pl. Preiss. I, 2:20 (1840).

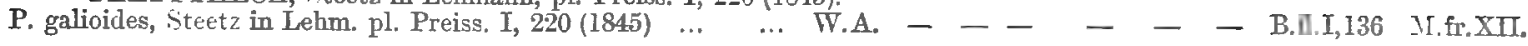

TETRATHECA, Smith, Sinec. Bot. New Holl. I, t. 2 (1793).

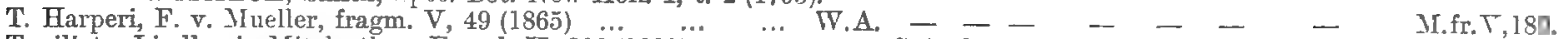

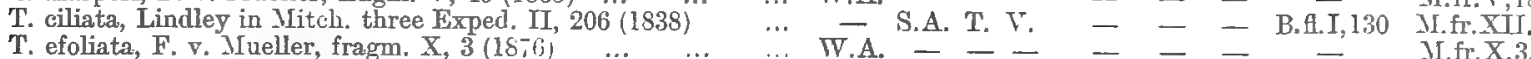

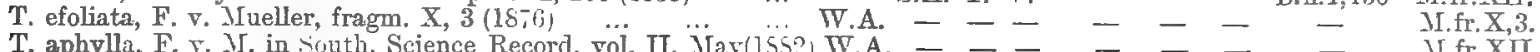

- S. S. T. T. T. T. T. - - II.fr.XI.

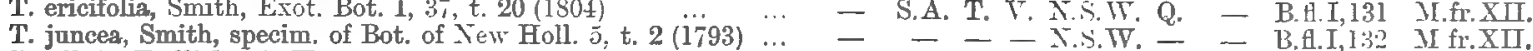

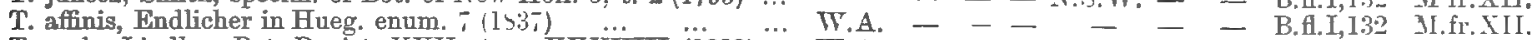

T. nuda, Lindley, Bot. Regist. XXT, App. XXXVIII (1839) ... TT.A. - - - - - - P.fl.I,I33 M.fr.XII

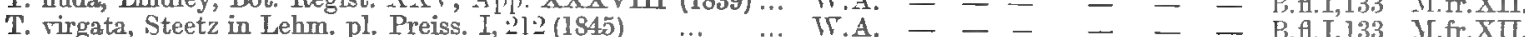

T. confertifolia, Steetz in Lehm. pl. Preiss. I, $214(1840) \quad \ldots$ W.A. - - - - - - B.f.I,133 M.fr.XII

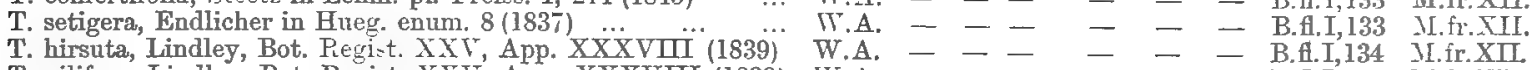

T. pilifera, Lindley, Bot. Regist. XXT, App. XXXVIII (1839) W.A - - - - - - B.fl.I,135 II.fr.II.

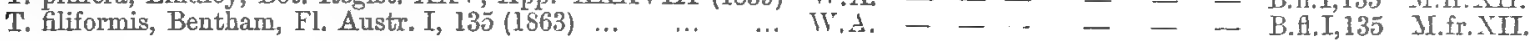

TRENIANDRA, R. Brown in App. Flind, voy. II, 54 (1814).

T. stelligera, R. Brown in De Candolle, prodr. I, $344(1524)$.. Tr.A. - - - - - - P.fl.I.136 M.fr.XI.

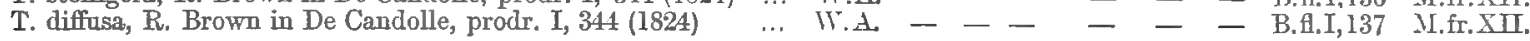

\section{MELIACEAE.}

Ventenat, Tabl. III, 159 (1799).

HEDRAIANTHERA, F. v. Mueller, fragm. V, 58 (1865).

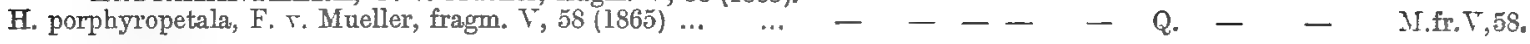

TURRAEA, Linné, mantiss. II, 1.50 (1:-i1).

T. pubescens, Hellenius in K. Sr. Vent. Acad. Handl, 26, t.10(1788)- - - - T.S.T. Q. N.A. B.A.I,379 M.fr. T,1H.

IFELLA, Linné, gen. pl. 127 (173i).

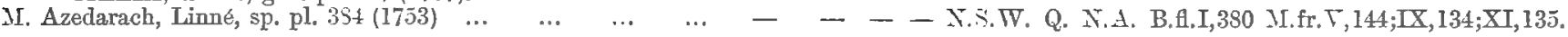

DYSOXILTM, Blume, Bijdrag. 172 (1825). (Dysoxylon, Epicharis, Hartigshea).

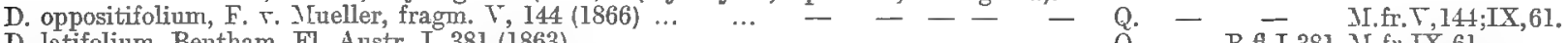

D. latifolium, Bentham, Fl. Austr. I, 381 (1863) ... $\quad \ldots \quad \ldots \quad-\quad-\quad-\quad-\quad$ - Q. $\quad$ - B.A.I.381 M.fr.IX,61.

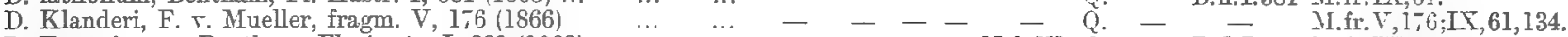

D. Fraserianum, Bentham, Fl, Austr. I, 381 (1863) … ‥ - - - K.S.W. Q. - B.fl.I,381 Y.fr.IX, 61.

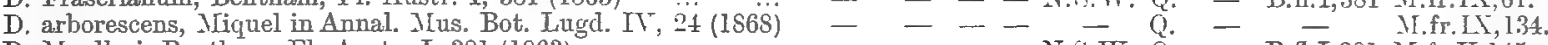

D. Muelleri, Bentham, Fl. Austr. I, 381 (1863) $\ldots \quad \ldots \quad \ldots \quad-\ldots \quad-\quad-\quad$ N.S.W. Q. - B.H.I,3S1 MI.fr.V,145.

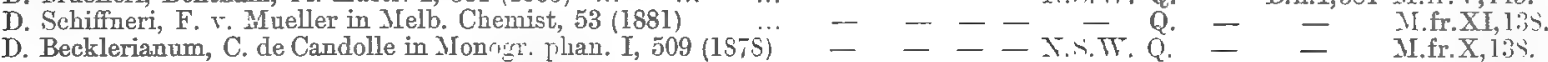

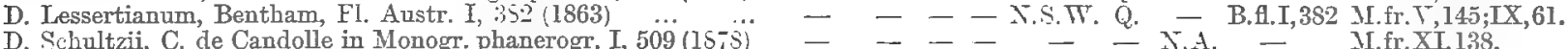

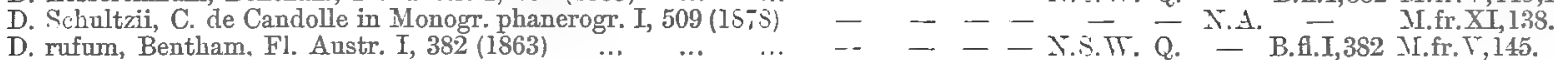

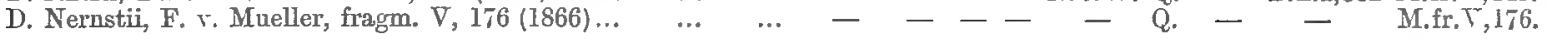
AMOORA, Roxbargh, pl. Corom. III, 54, t. 258 (1819).

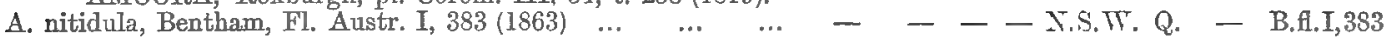
SYNOUM, Adr, de Jussien in Mím. du MIns. XIX, 226, t. 15 (1830).

A. glandulosum, de Jussieu in XI'm. du XIns. XIX, 22-, t. 15 (1830) - - - N.S.W. Q. - B.A.I,384 MI.fr. T, 14., , IX, 134.

A. Muelleri, C. de Candolle in Monogr. phanerogr. I, 593 (1878) - - - - Q. - - M.fr. T, 11.5;.II,138. OWETIA, F. v. Mueller in Hook. Kew Misc. IX, 303 (185̃7).

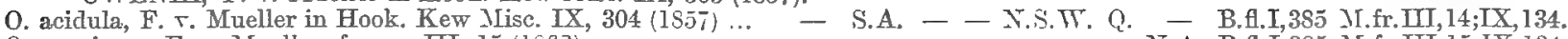

0. vernicosa, F. v. IIneller, fragm. III, IJ (1962)

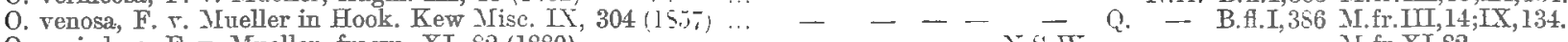

O. cepiodora, F. v. Mueller, fragm. XI, S2 (1880). … ‥ - - - N.S.W. - - - M.fr,XI,82.

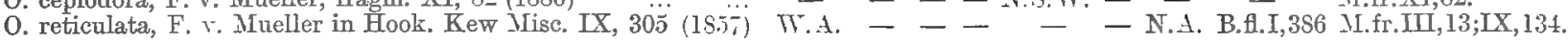
AGLAI A, Loureiro, Fl. Cochinch. I, 173 (1790), non Allemand (17/0). (Milnea, Nemedra).

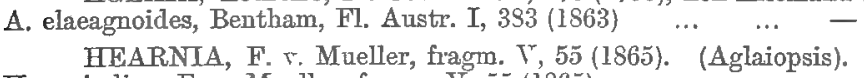

H. sapindina, F. r. Mueller, fragm. V, 55 (1865) …

CARAPA, Aublet, Fist. pl. Guian. II, Suppl. 32, t. 35 (17/5). (Xylocarpus).
C. Moluccensis, Lamarck, Encycl. meth. Bot. I, 621 (I783) CEDRELA, P. Browne, Nat. hist. of Jamaica $15 \% 8(1756)$.

C. australis, F. v. MIueller, fragm. I, 4 (1858) … … $\quad \ldots$ FLINDERSIA, R. Brown in Flind. voy. II, 595 (1814). (Oxleya, Strzeleckia).

F. australis, R. Brown in Flind. roy. II, 59a, t. 1 (1814)

F. Bourjotiana, F. v. Mueller, fragm. IX, 133 (1875)

F. Schottiana, F. v. Mueller, fragm, III, 25 (1862)

F. Oxleyana, F. F. MIueller, fragm. I, 65 (1858) … …

F. Pimenteliana, F. T. Mveller, fragm. IX, 132 (1875) ...

F. Bennettiana, F. v. Mueller in Benth. Fl. Austr. I, 389 (1863)

F. Ifflaiana, F. v. Mueller, fragm, X, 94 (1577) ..

F. Brayleyana, F. F. Mueller, fragm. T, 143 (1866)

F. Strzeleckiana, F. v. MIueller, fragm. I, 65 (1858)

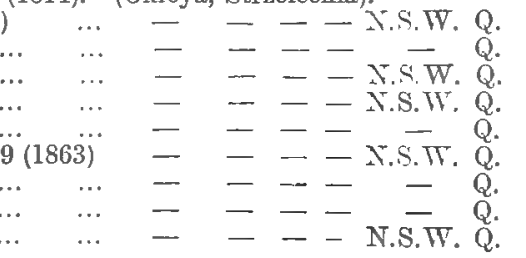

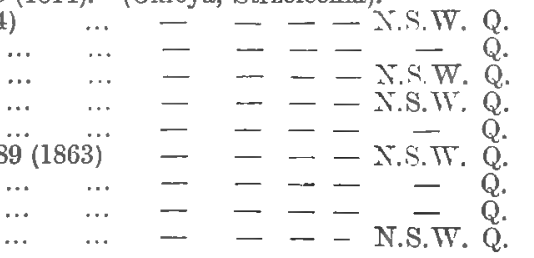

- N.A. B.fl.I,3\$3 II.fr. T,145;IX,134.

Q. - - M.fr. T, כ็ .

Q. N.A. B.A.I.85; M.fr.T, 145;LX, 134 .

Q. - B.fl.I,3ST II.fr.LX, 133;XI, 82 .

B.fl.I,3S8 M.fr.I,65;II, $17 \pm ;$ TX, 133 .

I.fr.IX, I33.

B.Al.I,3S8 \I.fr.ITI,25; II I 143;IX, 133.

B. $1 . I, 389$ II.fr.I, $6 \overline{5} ; I I I, 25 ; L X, 133$.

II.fr.LX, 132 .

B.A.I,389 II.fr.IX.131;XI,135.

II.fr. $\mathrm{X}, 94$.

M.fr.I, 143; గI, 252. 


\section{OCHNACEAE.}

De Candolle in Ann. du Mus. XVII, 398 (1811).

BRACKENRIDGEA, Asa Gray, Bot. Wilk. Expl. Exped. 361, t. 42 (1854).

B. Australiana, F. v. Mueller, fragm. V, 29 (1865) … ㄴ..

\section{RUTACEAE.}

A. L. de Jussieu, Gen, 296 (1789).

ZIERIA, Smith in Transact. Linn. Soc. IV, 216 (1\%98).

Z. laevigata, Smith in Rees's Cyclopaedia, XXXIX (1819)

Z. pilosa, Rudge in Transact. Linn. Soc. X, 293, t. 17 (1809) .'.

Z. obcordata, Cunningham in Field. N.S. Wales, 330 (1625)

Z. cytisoides, Smith in Rees's Cyclopaedia, XXXIX (1819)

Z. Smithii, Andrews' Bot. Rep. t. 606 (1810)

Z. granulata, C. Moore in Benth. Fl. Austr. I, 307 (1863)

Z. veronicea, F. v. Mueller in Trans. phil. Soc. Vict. I, 11 (1854) BORONIA, Smith, Tracts relat. nat. hist. 285 (179S).

B. grandisepala, F. v. Mueller, fragm. I, 66 (1858)

B. artemisifolia, F. v. Mueller, fragm. I. 66 (1858)

B. affinis, R. Brown in Benth. Fl. Austr. I, 311 (1863) "

B. filicifolia, Cunningham in Benth. Fl. Austr. I, 311 (1863) ...

B. alata, Sinith in Transact. Linn. Soc. VIII, 283 (1807)

B. algida, F. v. Mueller in Transact. phil. Soc. Vict. I, 100 (1855)

B. Edwardsii, Bentham, Fl. Austr. I, 312 (1863) ...

B. ternata, Endlicher, nov, stirp. dec, 6 (1839) $\ldots \ldots$

B. ericifolia, Bentham, Fl. Anstr. I, 313 (1863) ... ...

B. inconspictua, Bentham, Fl. Austr. I, 313 (1863)

B. alulata, Solander in Benth. Fl. Austr. I, 313 (1863) ...

B. ledifolia, J. Gay, monogr. des Lasiopetalées 20 (1S21)

B. lanceolata, F. r. Mueller, fragm. I, 66 (1858) ...

B. Bowmanii, F. v. Mueller, fragm. IV, 135 (1S64)

B. Fraseri, Hooker, Bot. Mag. t. 4052 (1844)

B. mollis, Cunningham in Lindl. Bot. Reg. 47 (1841)

B, heterophylla, F. v. Mueller, fragm. II, 98 (186I)

B. elatior, Bartling in Lehm, pl. Preiss. I, 170 (1844) ...

B. tetrandra, Iabillardière, Nov. Holl. pl. spec. I, 98, t. I2.5 (180

B. crassifolia, Bartling in Lehm. pl. Preiss. I, 169 (1844)

B. albiflora, R. Brown in Benth. Fl. Austr. I, 317 (1863)

B. lanuginosa, Eudlicher in Hueg. enum. 16 (1837)

B. pulchella, Turczaninow in Bull. Soc. Mosc. XXV, 162 (1552)

B. gracilipes, F. r. Mueller, fragm. II, 99 (1861).

B. microphylla, Sieber in Spreng. syst. cur. post. $148(1827) \quad \ldots$

B. pinnata, Smith, Tracts relat, nat. hist, 290, t. 4 (1798)

B. pilosa, Labillardiere, Nor. Holl. pl. spec. I, 97, t. 124 (1804)

B. spinescens, Bentham, Fl. Austr. I, 319 (1863).

B. baeckeacea, F, v. Mueller, fragm. IX, 112 (1875)

B. coerulescens, F. V. Mueller in Trans. phil. Soc. Vict. 11 (1854)

B. defoliata, F. r. Mueller, fragm, IX, 113 (1875)

B. Busselliana, F. v. Mueller, fragm. IX, $113(1875) \quad \ldots . \quad \ldots$

B. subcoemulea, F. v. Mueller, fragm. II, $100(1860) \quad \ldots \ldots$

B. polygalifolia, Smith, Tracts relat, nat. hist. 297, t. 7 (1798)

B. falcifolia, Cunningham in Lindl. Bot. Reg. 47 (1841)...

B. penicillata, Bentham, Fl. Austr. I, 322 (1863)..

B. crassipes, Bartling in Lehm. pl. Preiss, I, 168 (1844)

B. subsessilis, Bentham, Fl. Austr. I, 3:2 (1863) ...

B. capitata, Bentham, Fl. Anstr. I, 323 (1863)

B. nematophylla, F, v. Mueller, fragm. II, 100 (1861)

B. crenulata, Smith in Transact. Linn. Soc. VIII, 284 (1807)

B. serrulata, Smith, Tracts relat, nat. hist. 292 (1798) ..

B. rhomboiclea, Hooker icon. pl. t. 722 (1848)

B. parviflora, Smith, Tracts relat. nat. hist. 295 , t. 5 (1798)

B. riminea, Lindley, Bot. Regist. XXV, App. XVII (1839)

B. filifolia, F. v. Mueller, fragm. I, 3 (1858)
B. clavellifolia, F. v. Mueller in Trans. phil. Soc. Vict. I. 12 (1854)

B. scabra, Lindley, Bot. Regist. XXV, App. XVII (I839)

B. thymifolia, Turcz, in Bull. Soc. Mose. XXV, partII, 165 (1852)

B. ovata, Lindley, Bot. Regist. 47 (1841)

B. Barkeriana, F. v. Mueller, fragm. XI, 96 (1880)

B. denticulata, Smith in Transact. Linn. Soc. VIII, 284 (1807)

B. Machardiana, F. r. Mueller, fragm. IX, 115 (1875)

B. spathulata, Lindley, Bot. Regist. XXV, App. XVII (1839)...

B. juncea, Bartling in Lehm. pl. Preiss. I, 166 (1S44) ... ...

B. cymosa, Endlicher in Hueg. enum. 16 (1837) ...

...
B. megastigna, Nees in Lehm. pl. Preiss. II, 227 (1847)

$$
\begin{aligned}
& -\quad-\quad-\text { V. N.S.W. Q. } \\
& -\quad-\quad-\text { N.S.W. } \\
& -\quad-\text { - } \\
& -\quad-\text { T.S.T. Q. N.S.T. Q. } \\
& \text { - - T. V. N.S.W. Q. } \\
& \text { - S.A. T. V. N.S.W. Q. }
\end{aligned}
$$

Cyanothamnus).

$$
\begin{aligned}
& \therefore \text { - }- \text { - } \\
& -\quad-\quad- \\
& \text { - } \\
& \text { W.A. }-\overline{-} \text { - V. N.S.W. } \\
& \text { … W.A. - - - - - } \\
& \text { TV A } \\
& \text { W.A. } \\
& \text {.. - - - - - - Q } \\
& \text {. - - - - N.S.IY.Q. } \\
& \text { - }
\end{aligned}
$$$$
\text { - - }
$$$$
\text { . }
$$$$
\begin{aligned}
& \overline{-} \\
& \text { W.A. } \\
& \text { W.A. }
\end{aligned}
$$$$
\text { W.A. }
$$$$
\text { 04) W.A. }
$$$$
\text { W.A. }
$$

W.A.

W.A.

W.A.

W.A.

- $=-\overline{\mathrm{N}} \mathrm{N} \mathrm{W} . \overline{\mathrm{O}}$

- S.A.T. Y. N.S.W. Q.

W.A. - T. V. - -

IV.A. $-\bar{A}=\overline{\mathrm{V}}$ N.S.WY

W.A. S.A. V. N.S.W. -

Wr.A.

W.A.

$\overline{S A}, \bar{T}, \bar{V}, \mathrm{~N}, \overline{\mathrm{S}} \mathrm{W}, \overline{\mathrm{D}}$

- - - - N.S.W. Q.

W.A. - - - - -

W.A. ---
W.A. --

W.A.

W.A.

W.A.

E- - - .

- S.A. T. $\overline{\text { T. N.S.W. }} \overline{\mathrm{Q}}$

W. A.
$-\overline{-}--$

S.A. - V. N. $\overline{\text { S.W. }}$ -

W.A.

W.A.

W.A.

IV.A.

- - - -

W.A.

IV.A.

IV.A.

IV.A.

ERIOSTEMON, Smith in Transact. Linn. Soc. IX, 221 (1798), Geleznowia, Urocarpus, Sandfordia, Actinostigma).

E. virgatus, Cunningham in Hooker's Journ. of Bot. II, 417 (1840)

E. nodifiorus, Lindley, Bot. Regist. XXV, App. XVII (1839) ..

\author{
TV
}

- - - - -
- B.fl.I,304 M.fr.IX,I16.
- B.fl.I,305 M.fr.I,100;IX, 116
B.fl.I,305 M.fr.I, 101;IX, 116.
- B.fl.I,306 M.fr.IX,116.
B. $1 . I, 306$ M.fr.I, 100;IX, 116.
- B.fl.I,307 M.fr.IX,116.
- B.f.I,305 M.fr.IX,116.

N.A. B.fl. I, 311 M.fr. I, $66 ; I I, 179$.

N.A. B.fl.I,311 M.fr. I, $66 ; 1 X, 111$.

N.A. B.f.I, 311

N.A. B.f.I, 311

- B.fl.I,312 MI,fr.IX,111.

B.fl.I.312 M.fr.IX,111.

B.A.I, 312

- B.fl.I,312 M.fr.IX, 111.

- B.fl,I,313 M.fr.IX,111.

- B.H.I,313

- B.f.I,313

B.fl. I, 313

B.fl.I,314 M.fr.IX,111.

N.A. B.fl.I,314 M.fr.I, 66;VII,3S;IX,112;XI,

- M.fr.VII,38.

B.fl.I,315 M.fr.IX,111.

B.A.I,315 M.fr.IX,112

- B.fl.I,31J M.fr.II,97;IV,112;IX,112.

- B.fl.I,31J M.fr.II,9S;IX, 112 .

B.fl.I,316 MIfr.II,98;IX, 122; XI, 97 .

B.H.I,316

- B.fl.I,316 M.fr.IX,112.

- B.fl. I,317 M.fr.IX, 114 .

B.fl.I,317 M.fr.IX,114.

B.A.I,318 M.fr.IX, 11.

- B.fl.I,318 M.fr.IJ,99;IX, 114.

- B.fl.I,318

- B.fl.I,318 M.fr.IX,114.

- B.fl.I,319

- B.fl.I,319

- M.fr.IX, 112.

- B.t. I, 320 M.fr. IX, 112.

- M.fr.IX, II3.

- M.fr.IX, 113.

B.fl.I, 320 M.fr.II, 100;IX, $112 \& 19$ S.

B.Al.I, 320 M.fr.IX, 114 .

B.fl.I,32.2 M.fr.IX, 114 .

B. $1.1,3,2.2$

B.H.I,3322 M.fr.IX, 116 .

B.fl.I, 322

13.f.I, $3 \geq 3$ MI.fr. IX, 116.

- B.A.I,:3:3 M.fr.II, 100.

- B.fl.I,32:3 M.fr.XI, $115 \& 19$.

B.A.I, 323 M.fr. IX, 115.

B.Hl, I, 3:24

- B.A.I,304 M.fr.IX, 115.

B.fl. I, 3:24

li.f. I, 3:.5 M, fr, I, 3I;IX, 114 .

B.H.I,:3:- M.fr.I,99;IX, $114 \& 198$.

- B.fl.I, $3: 06$

- B.H.I,326 M.fr.I,99;II,99,LX, 116 .

- B.fl.I,320 M.fr.IX,116.

- $\quad$ II fr. XI,96.

B. $1 . \overline{1}, 327$ M.fr.IX,113.8198.

B. A. M. IX, 115 .

B.fl.I, 327 M.fr. IX, 115.

- B.fl.I,:327 M.fr.IX,115.

B.fl.I, 3:2S M.fr.II, $101 ; \mathrm{IX}, 116$.

(Crowea, Phebalium, Asterolasia, Microcybe, Pleurandropsia,

T. - - - - B.fl.I,332 M.fr.IX,111. 
E. spicatus, A. Richard, sert. Astrolab. 76, t. 27 (1833)

E. pungens, Lindley in Mitch. Three Exped. II, 156 (1838)

E. montanus, F. v. M., pl, of Vict. I, 129 (1860)

E. phylicifolius, F. v. M., fragm. I, 105 (1859)

E. umbellatus, Turcz, in Bull. Soc. Mosc. XXII, part III, $15(18$

E. Ralstoni, F. v. M., fragm. II, 101, t. 14 (1861)

E. Hillebrandi, F. v. M, in Transact, phil Soc. Vict 1 ,

E lamprophyllus F, $r$ M in Journ pharm Soc. Viot. II 43

E. elatior, F. v. M., fragm. I, 181 (1859).

E. ambiens, F. v. M., fragm. VI, 166 (1868)

E. Oldlfieldii, F. v. M., fragm. I, 3 (1858)

F. rotundifolius, Cumningham in Hueg. enum. 15 (1837)...

E. microphyllus, F, v. M. in Transact. phil. Soc. Vict. I, 99 (1854)

E. phylicoides, F. v. MI., fragm. I, 107 (1859)

E. ozothamnoides, F. v. M., fragm. I, 103 (1859)

E. Mortoni, F. v. M., fragm. IX, 108 (1875)

E. seditlorus, F. v. M., fragm. I, 102 (1859)

E. Nottii, F. v. M., fragm. VI, 22 (I867)

E. lepidotus, Sprengel, syst. II, 302 (1825)

E. alpinus, F. v. M., fragm. I, 103 (1859) ...

E. tuberculosis, F. v. M., pl. of Vict. I, 130 (1862)

E. Benthami, F. v. M., fragm. IX, 108 (1875)

E. Maxwelli, E. v. M., fragm. IX, 108 (1875)

E. filifolins, F. v. M., fragm. IX, 108 (1875)

E. squameus, Labillardiere, Nov. Holl. pl. spec. I, i11, t. 141 (1804)

E. anceps, Sprengel, syst. II, 322 (1825)

E. ovatifolius, F. v. M., fragm. I, 103 (1859)

E. bilobus, F. v. M., fragm. I, 102 (1859) ...

E. Beckleri, F. v. M., fragm. IX, 109 (1875)

E. capitatus, F. v. M., fragm. I, 116 (1859)

E. Geleznowii, F. v. M., fragm. I, 107 (1859)

E. correifolius, F. v. M., fragm. I, 105 (I859)

E. Cunninghami, F. v. M., fragm. IX, 107 (1875)

E. mollis, Asterolasia mollis, Benth. Fl, Austr. I, 351 (1 363 )

E. pleurandroides, F. v. M., fragm. I, 106 (1859)

E. trymalioides, F. v. M., fragm. I, 106 (1859)

E. Hookeri, F. v. M., fragm. I, 104 (1S59)

E. pallidus, Asterolasia pallida, Benth. Fl. Austr. I, 352 (1863)

E. Drummondii, F. v. M., fragm. I, 105 (1859)

E. grandiflorus, F. v. M., fragm. I, 105 (1859)

E. Crowei, F. v. Mueller, pl. of Vict, I, 119 (1860)

E. Turczaninowii, F. v. Mueller, pl. of Vict. I, 120 (1860)

E. lanceolatus, K. F. Gaertner, de fruct. III, 154, t. 210 (1807)

E. Banksii, Cunningham in Hueg, enum. 15 (1837)

E. trachyphyllus, F. v. M. in Transact. phil. Soc. Vict. I, 99 (1 1854

E. nyoporoides, De Candolle, prodr. I, 720 (1824)

E. hispidulus, Sieber in Spreng. syst. cur. post. 164 (1S27)

E. buxifolius, Smith in Rees's Cyclop. XIII, 2 (1809)

E. obovalis, Cunningham in Field's N. S. Wales, 331 (1825)

E. scaber, Paxton, Mag. of Bot. XIII, 127 (1846)...

E. Brucei, F. v. M., fragm. VII, 38 (1869)

E. linearis, Cunningham in Hueg, enum. 16 (1837)

E. difformis, Cunningham in Hreg. enum. 15 (1837)

E. parvifolius, R. Brown in Benth. Fl. Austr. I, 335 (18̈63)

E. ericifolius, Cinningham in Benth. Fl. Austr. I, 335 (1863)

PHILOTHECA, Rudge in Transact. Linn. Soc. XI, 298,

P. australis, Rudge in Transact. Linn. Soc. XI, 298, t. 21 (1815)

P. ericoides, F. v. M., fragm. I, 107 (1859)

P. calida, F. v. M., fragm. VII, 21 (1869) ...

CORREA, Smith in Transact. Linn. Soc. IV, 219 (1798).

C. aemula, F. v. M., fragm. I, 3 (1859)

C. alba, Andrews, Bot. Reposit. t. 18 (1797)

C. speciosa, Andrews, Bot. Reposit. t, 653 (1811).

C. Iewren

C. decumbens, F. v. M. in Transact. phil. Soc. Vict. I, 30 (1854) ...

NEMATOLEPIS, Turczaninow in Bull. Soc. Mosc. XXV, part III, 158 (1852).

N. phlebalioides, Turczaninow in Bull. Soc. Mosc. partIII, 158(1852) W.A.

N. Euphemiae, F. v. M., fragm. III, $149(1863) \ldots \ldots \ldots \ldots$... W.A. $\quad$ - -

CHORILAENA, Endlicher in Hueg. enum. 17 (1837).

C. quercifolia, Endlicher in Hueg. enum. it (1837)

C. hirsuta, Bentham, Fl. Austr. I, 357 (1863)

DIPLOLAENA, R, Brown in Append. Flind. voy. II, 546 (1814).

D. Dampieri, Desfontaines in Mém. du Mus. 452, t. 20 (1817) ... TV.A.

BOSISTOA, F, v. M. in Benth. Fl. Austr. I, 359 (1863).

B. sapindiformis, F. v. M. in Bentl. Fl. Austr. I, 359 (1863)

B. euodiformis, F. v. M., fragm. IX, $174(1575) \ldots$
B.fl.I,336 M.fr.IX, 110 .

B.f.I. 338 M.fr.IX, 110

B.fl.I,338 M.fr.IX, 109 .

B.fl. I, 339 M.fr.I,105.

B.fl.I,339 M.fr.I, 104;IX,109.

B.fl.I,339 M.fr.III, I $6 ; 5 ; \mathrm{IX}, 109$.

B.fl.I,340 M.fr.I, $4 ; I X, 109$.

B.fl.I, 340 M.fr.IX, 109 .

B. $1 . I, 340$ M.fr.IX, 109 .

M.fi. VI, $166 ; \mathrm{IX}, 110$.

B. A.I, 340 M.fr.IX, 109 .

B. fl. I,341

B.fl.I,341 M.fr.IX, 109

B.fl, I,341 M.fr. IX, 107.

B.H.I,342 MI.fr.IX, 107.

B.fl.I,342 M.fr.IX, 108 .

B. fl. I, 342 M.fr.I, 102.

- M.fr.VI, 22

B. fl.I, 343 M.fr.I, 104;IX, 107. M.fr.I. 103 .

B.fl.I,343 M.fr.IX, 108 .

B.fl.I, 343 M.fr.IX, 108 .

M.fr.IX, 103 .

B.fl.I.344 M.fr.IX, 108.

B.fl.I,344 M.fr.I, 104;IX, 107.

B.fl. I,34, M.fr.I,103;IX, 108.

B.f1.I, 345 M.fr.I, 103 .

B. Al.I,345 MI.fr.IX,10S.

M.fr.IX, 108.

B. H.I, 346 M.fr.I, 106;IX, 107.

B.f.I,347 M.fr.IX, 107.

B.A.I,350 M.fr.IX, 107 .

B.A.I,351 M.fr.IX, 107 .

B.fl. I, 349

B.fi.I,349 M.fr.IX,107.

B.fl.I,351 M.fr.I, 106.

B. fl. I, 3.12 M.fr. VII, 22;IX, 107.

W.A. - - - - - - B.H.I,3,2

W.A. - - - - - - B.fl.I,352 M.fr. YII, 2:2.

B.fl.I,35.3 M.fr.VI,2l.

B.fl.I,329 M.fr.IX, 106.

- B.丹.I,330 M.fr.IX, 106.

- B.fl.I,331 M.fr.IX,110.

- B.fl.I,332

- B.fl.I,332 M.fr.IX,110.

B.ff.T,333 M.fr.IX,110.

B.f.I,333 M.fr.IX,110.

B.fl.I,333 M.fr.IX,111.

B.fl.I,334 M.fr.IX, 110.

B.H.I,334 MI.fr.IX,111.

B.fl.I, 334

B.fl.I, 335 M.fr.IX, 110.

B.A.I, 335

- B.fl.I,335

- B.fl.I,348 MI.fr.VII,20,141.

- B.fl.I,349 M.fr.VII,21.

M.fr.VII, 38 .

B.H.I, 353 II.fr.IX, 117.

B.H.I,3.5 M.fr.IX,117.

B.fl.I, 354 M.fr.IX, 117 .

B.fl.I,355 دI.fr. VII, 142;IX, 11;.

B.fl.I, 356

(Symphyopetalum).

- - B.fi.I,356 M.fr.IX, 106.
$-\quad-$ B.H.I,345 M.fr.IX,106.

W.A. - - - - - - B.fl.I,357 M.fr.IX, 106.

- - - B.fl.I,357 M.fr.IX,106.

- B.fl.I,358 MI.fr.IX, 106.

- B.fl.I,359 M.fr.IX.103.

- - M.fr.IX, 17t. 
ACRADENIA, Kippist in Transact. Linn. Soc, XXI, 207 (1855) ankliniae, Kippist in Transact Linn. Soc. XXI, 207 (1855) EUODIA, R. et G. Forster, char. gen. 13, t. 7 (1776)

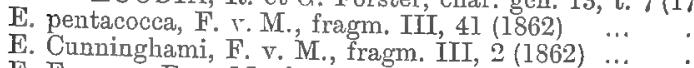

E. erythrococca, F. v. M., fragm. I, 26 (1858)

E. contermina, Moore \& F. v. M., fragm. VII, 1444 (187i)

E. octandra, F. v. M., fragm. II, 102 (1861)

E. micrococca, F. v. M. , fragm. I, $144(1859)$

E. xanthoxyloides, F. v. II., fragm. IV, 155 (1864)

E. Bonwickii, F. V. I., fragm. V, 56 (1865) … $\quad \ldots$

E. alata, F. v. II., fragm. VII, $142(1871)$

E. accedens, Blume, Bijclr, 246 (1825)

E. littoralis, Endlicher, prodr. FL. Norfolk. 86 (1833) ...

E. polybotrya, Moore \& F. v. M., fragm. VII, 143 (1871)

E. vitillora, F. v. M., fragm. VII, 144 (1871) BROMBY A, F. v. MI., fragm. V, 4 (1865).

B. platynema, F. v. .I., fragm. V, 4 (1865) ... PAGETIA, F. v. M., fragm. V, 178 (1866).

P. medicinalis, F. v. M., fragm. V, 178 (1866) .. BOUCHARDATIA, Baillon in Adans. VII, 350, t. 10 (1867).

B. neurococca, Baillon in Adans. IX, 110 (I869) ... ... $\ldots$. XANTHOXYLUM, Catesby in Limné, hort. Cliff. 487 (1737). (Zanthoxylum, Blackburnia)

X. brachyacanthum, F. T. M., pl. of Vict. I, 108 (1862)

X. Blackburnia, Bentham, Fl. Austr. I, 363 (1863)

X. parviflorum, Benthan, El. Austr. I, 363 (1863)

X. torvum, F. v. .IL., fragm. VII, 140 (1871) GEIJERA, Schott, Rntac. 4, t. 7 (1834). (Coatesia).

G. salicifolia, Schott, Rutac. t. 4 (1834) … …

G. parviflora, Lindley in Mitch. Trop. Austr. 102 (1848) PLEIOCOCCA, F. r. Mueller, fragm. IX, 117 (1875).

P. Wilcoxiana, F. v. M., fragm. IX, $117(1875)$... ... ACRONYCHIA, R. \& G. Forster, char. gen. 53, t. 27 (1776). (Cyminosma).

A. Baneri, Schott, Rutac. t. 3 (1834) … 18 .. $\quad \ldots \quad-\quad-\quad--$ N.S.W. Q.

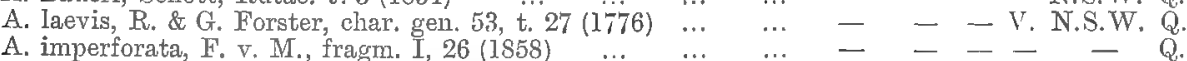

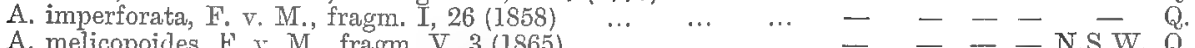

A. acidula, F. r. M., fragm. IV, 154 (1864)

A. vestita, F. r. M., fragm. IT, 155 (1864)

A. Endlicheri, Schott, fragm, bot. III, t. 2

A. tetrandra, F. T. M., fragm, IX, 104 (1875) HALFORDIA, F. v. Mueller, fragm. V, 43 (1865).

H. drupifera, F. .. M., fragm. V, 43 (1865) ... ...

H. scleroxyla, F. V. M., fragm. VII, 142 (1871) ... ...
GLYCOSiIS, Correa in Ann, du Mus. VI, $384(1805)$

G. pentaphylla, Correa in Annal. du Mus. VI, 384 (1805) MICROMIELUM, Blume, Bijdrag. 137 (1825).

II. pubescens, Blume, Bijdrag. 137 (1825) ... MURRAYA, Koenig in Linné, mant. alt. 563 (1771). (Chalcas, 1767).

I. exotica, Koenig in Linné, mant. alt. 563 (1771)

II. crenulata, Oliver in Journ. Linn. Soc. V, suppl. II, 29 (1861) CLAUSENA, Burman, Fl. Indica index \& t. 29 (1768).

C. brevistyla, Oliver in Journ. Linn. Soc. V, Suppl. II. 31 (1861) ATALANTIA, Correa in Ann. du Nus. VI, 383 (1805).

A. glauca, J. Hooker in B. \& H. Gen. pl. I, 305 (1862) ...

A. recurva, Bentham, Fl. Austr. I, 370 (1863) ... ...
CITRUS, Linné, gen. pl. $230(1737)$, from Plinius. CITRUS, Linné, gen. pl. 230 (1737), from Plinins.
anchonii, F. v. M., Report Intercol. Exhib. 23 (IS67)

C. Planchonii, F. v. M., Report Intercol, Exhib. 23 (IS67) $\quad . \quad-\quad-\quad-$ N.S.W. Q.

C. Australasica, F. v. M., fragm. I, $26(1858) \quad \ldots \quad \ldots \ldots+\ldots-$ PENTACLRAS, J. Hooker in B. \& H. Gen. pl. I, 298 (1862).

P. anstralis, J. Hooker in B. \& H. Gen. pl. I, 298 (1862)
E. Fareana, F. v. M., fragm. IX, $101(1875) \quad \ldots$.

r. Muelleri, Bentham, Fl. Austr. I, 364 (1863) ‥ …
.. - - - N.S.IT. Q. - B.fl.I,370 M.fr.XI,105. .. - - - N.S.IT. Q. - B.fl.I,365 M.fr.IX,104.
- M.fr.III, 168;IV, 175.

B.fl.I,362 M.fr.I, $27 ; \mathrm{IX}, 102$.

MI.fr.IX, 101.

B.fl. I, 360 M.fr.II, 103, 178;IX, 102.

II.fr. VII, 144.

B.fl.I, 360 MI.fr.II, 102;IX, 102.

B.fl.I,361 M.fr.II, 103, 180;IX, 102.

M.fr.IV, 155 .

M.fr.V, 179 .

M.fr.VII, 142 .

II.fi, V, 4,56; VII, 22;IX, 102.

M.fr.IX, 102 .

H.fr.TX, 193.

M.fr. FII, 144.

II.fr.V, $56 ;$ IX, 102.

M.fr. V,178;IX, 103.

B. Al.I, 360 MI.fr.I,28;II,163,178;IX, 103.

B.fl.I,363 MI.fr. VTI, 141;IX, 104.

B.fl. I, 363

B. Al. I, 363 MI. fr. VII, 141 ;IX, 104 .

M.fr.VII, 140;IX, 104.

A. Al.I,364 MI.fr.I,26;IX, 104.

- B.fl.,364 M.fr.VII. 22;IX, 105;XI,134.

- B.H.I,364 …fr. VII, 22;XI, 105;XI, 139.

B.f.I,366 XI.fr.I,26:IX, 103.

- B.fl.I,366 M.fr.I, 27;IX, 104.

- B.fl.I,367 M.fr,I,26;IX, 104.

- M.fr. IV,117;VII, I4J.

M.fr.IV,154.

M.fr.IV, 155;IX, 104.

M.fr.IX, 103.

M.fr. $T, 1 ; 9 ;$ IX, 104 .

MI.fr. T, 5,$189 ;$ IX, 103 .

M.fr. VII, 14?;IX, 103.

Q. - B.fl.I,367 M.fr.IX, 105.

Q. N.A. B.A.I,36S N.fr.I,25;IX, 105.

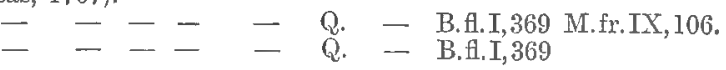

Q. - B.fl.I,369 M.fr.IX, 106.

N.A. B.t.I,370 M.fi. VII, 142 .

SIMARUBEAE,

De Candolle in Aun. du Mus. XVII, 32:3 (1811).

ATLANTUS, Desfontaines, in Act. Acad. Paris, 265 (1786).

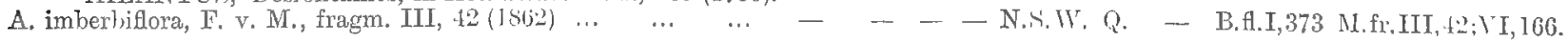
BRUCTA, J. S. Miller, icon. plantar. t. 25 (1780).

B. Sumatrana, Roxburgh, Fl. Indic. I, $469(1820) \quad \ldots \quad \ldots \quad-\quad-\quad-\quad-\quad$ - N.A. B.,A.I,:373 M.fr.IX, 166.

H Y PTIANDRA, J. Hooker in B. \& H. Gen. j1. 29. (1862).

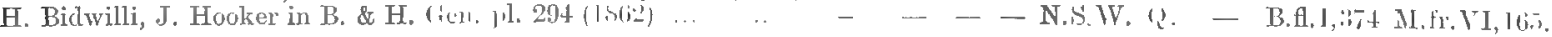


CADELLIA, F. r. Mueller, fragm. II, 25 (1860).

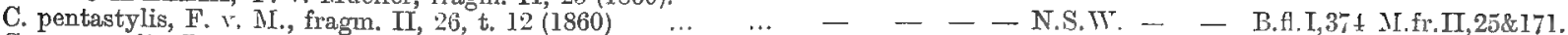

C. monostylis, Bentham, Fl. Austr. I, 375 (1863)... $\quad \ldots \quad \ldots \quad \ldots \quad-\quad-\quad-\quad$ N.S.W. Q. $\quad$ - B.fl.I,37. M.fr.IV,166;VIII,34.

SURLANA, Plumier, nov. plant. Amer, gen. 37, t. 40 (1703).

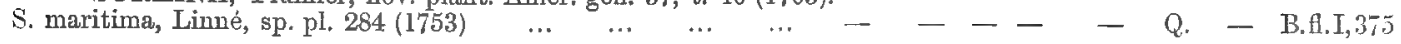

HARRISONIA, R. Brown in Mém. du Mus, XII, $517(1825)$. (Ebelingia).
H. Brownii, A. L. deJuss. in Mém. du Mus. Par. XII, 530, t.28(182う) - - - N.A. B.tl. I,376

ZYGOPHYLLEAE.

R. Brown in App. Flind. voy. II, 545 (1814).

NITRARIA, Linné, syst. nat. X, 1044 (17\%9).

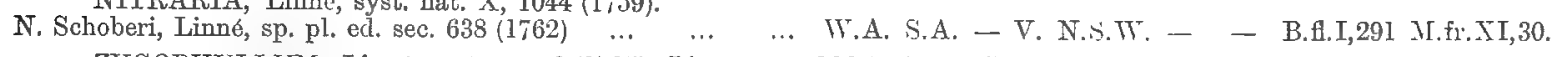

ZXGOPHYLLUMI, Linné, syst. nat. 8 (1735), Linné, gen. 126 (173-). (Sarcozygium).

Z. apiculatum, F. r. M. in Linnaea XXV, $373(1852) \quad \ldots \quad \ldots \quad-$ S.A. T. Y. N.S.W. Q.

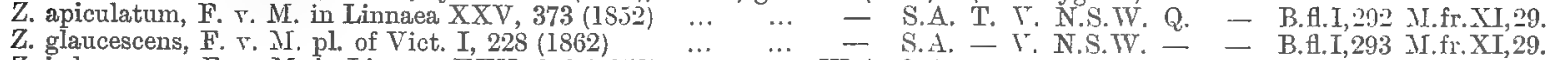

Z. prismatothecum, M. in Limnaea XXV, $372(1852) \quad \ldots \quad \ldots$ W.A. S.A. - T. N.S.W. - - B.fl.I,293 M.fr.XI,29.

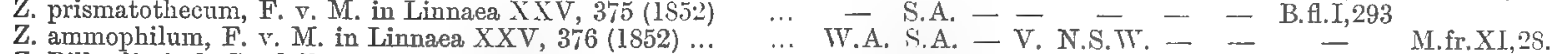

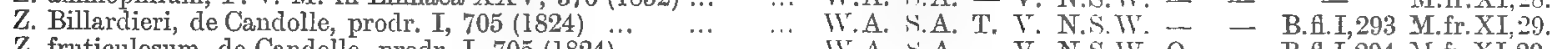

Z. fruticulosum, de Candolle, prodr. I, 705 (1824) $\quad \ldots . \quad \ldots$ W.A. S.A. - V. N.S.W. Q. - B.A.I,294 II.fr.XI, 29.

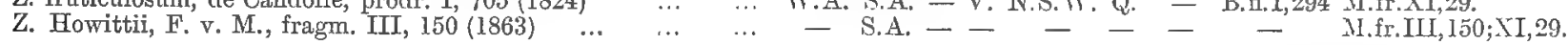

TRIBULUS, L'Obel, Icon. II, 84 (1581), from Theophr. Diosc. \& Plinins. (Tribulopsis).

T. terrestris, L'Obel, stirp. icones II, 84 (1581) $\ldots \quad \ldots \quad \ldots$ W.A. S.A. - V. N.S.W. Q. N.A. B.fl.I,288 M.fr.XI,30.

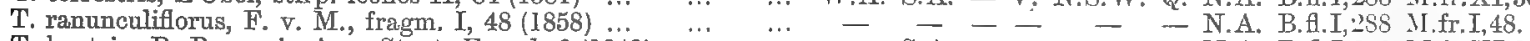

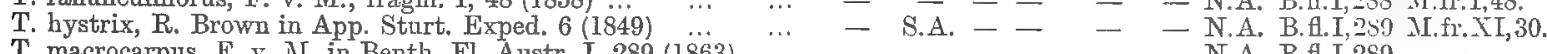

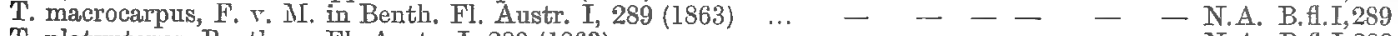

T. platypterus, Bentham, FI. Austr. I, 289 (1863) $\quad \ldots \quad$ …

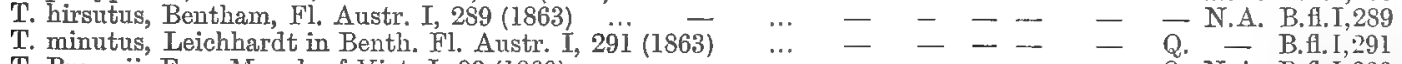

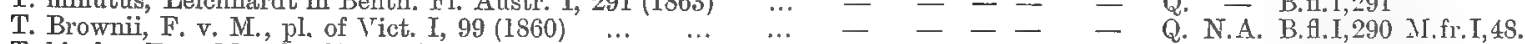

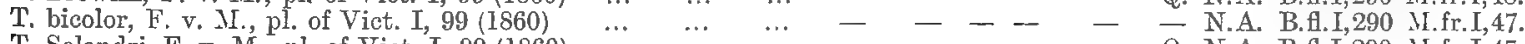

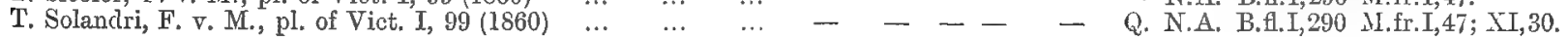

\section{LINEAE.}

HUGONIA, Linné, gen, pl. 134 (1737).

H. Jenkinsii, F. จ. M., fragm, V, 7 (1865)

De Candolle. Théor. élém. bot. 214 (1813).

ERYTHROXYLUII, P. Brown, Hist. pl. of Jamaica 278 (1756), from Theophr. Diosc. \& Plinius. (Erythroxylon).

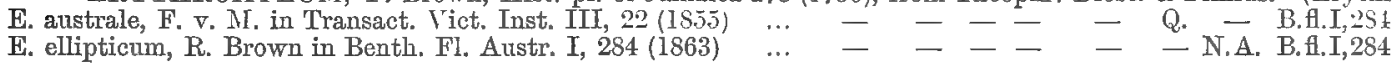

LINUM, Tournefort, inst. 339, t. 176, (1700), from Theophr. Diosc. \& Plinius.

L. marginale, Cunningh, in Hook. Lond. Jour. Bot. VII, 169 (1848) W.A. S.A. T. V. N.S.T. Q. - B.H.I,283

\section{GERANIACEAE.}

A. L. de Jussieu, Gen. 268 (1789), from B. de Jussien (1759).

GERANIUM, Tournefort, inst. 266, t. $142(1 ; 00)$, from Diosc. \& Plinius.

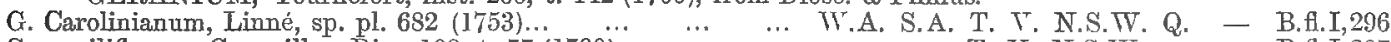

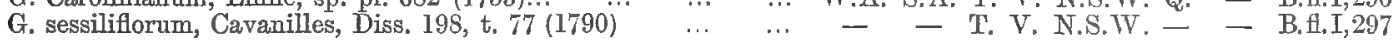

ERODIUM, L. Héritier, Geraniologia, t. I $(1 ; 8 ;)$.

E. cygnorum, Nees in Lelm. pl. Preiss. I, $162(1844) \quad \ldots \quad$... W.A. S.A. - T. N.S.W. Q. N.A. B.fl.I,297 M.fr.XI, 20.

PELARGONIUM, L'Héritier, Geraniologia, t. 7 (1787).

P. australe, Willdenow, sp. pl. III, 675 (1800)
P. Rodneyanum, Mitchell, three Exped. II, 144 (1838)

OXALIS, Linné, gen. pl. 134 (1737), from Plinins.

O. Magellanica, G. Forster in Comm. Goetting. IX, $33(1789) \ldots-\ldots-$ T. T. $-\quad-\quad-$ B.fl.I,300

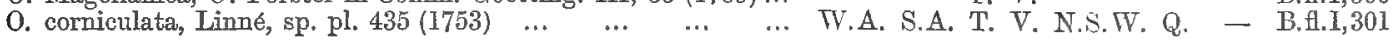

\section{MALVACEAE.}

Adanson in Mém. Acad. fr. 224 (1761).

LAVATERA, Tournefort in Act. Acad. Par. 86, t. 3 (1706).

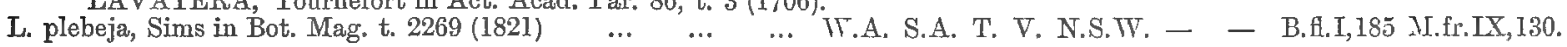

MALVASTRUM, Asa Gray in IIém. Amer. Acad. IV, 21 (1S49). (Malva partly).

M. spicatum, A. Gray in Mém. Amer. Acad. IV, 21 (1849) ‥ - S.A. - - N.S.TT. Q. N.A. B.fl.I,187 MI.fr. TI,170;IX,130.

M. tricuspidatum, A. Gray in Mém. Amer. Acad. IV, 21 (1849) - - - - N.S.W. Q. - B.Al.I,187

PLAGIANTHUS, R. \& G. Forster, char. gen. 85, t. 43 (1776). (Asterotrichon, Blepharanthemum, Lawrencia, Halothamnus).

P. sidoides, Hooker, Bot. Mag. t. 3396 (1835) … … ‥ - T. - - - - B.fl.I,1S8 MI.fr. TI,169.

P. pulchellus, A. Gray, Bot. Amer. Expl, Exped. I, I81 (1854) $\cdots-$ - T. V. N.S.T. - - - B.I.I,189 M.fr.IX,130.

P. spicatus, Bentham in proceed. Linn. Soc. VI, 103 (1862) … W.A. S.A. T. V. N.S.TV. - - B.t.I,189 MI.fr.TI,170;IX,130.

P. glomeratus, Bentham in proceed. Linn. Soc. VI, 103 (1862) … W. W. S.A. - - N.S. I. Q. - B.t.I,190 II.fr.IX,130.

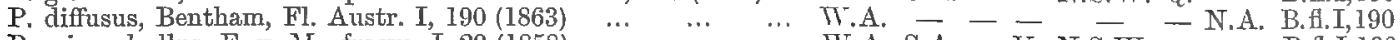

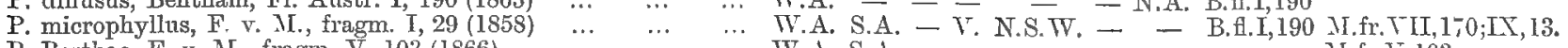

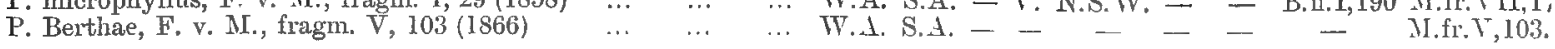


SIDA, Linné, gen. pl. 205 (1737), from Theophrastos.

S. corrugata, Lindley in Mitch. Three Exped. II, 13 (1838)

S. intricata, F. v. M. in Transact. phil. Soc. Vict. I, 19 (I860)...

S. macropoda, F. v. M. in Benth. Fl. Austr. I, 193 (1863)

S. virgata, Hooker in Mitch. Trop. Austr. 361 (1848) ...

S. echinocarpa, F, v. M., fragm. XI, 62 (1879) ...

S. cryphiopetala, F. v. M., fragm. III, 4 (1862) ... ...

S. petrophila, F. V. M. in Linnaea XXV, 381 (1852) $\ldots$
S. calychymenia, J. Gay in de Candolle, prodr. I, 462 (1824)

S. physocalyx, F. ז. M., fragm. III, 3 (1862)

S. subspicata, F. v. M. in Benth. Fl. Austr. I, 195 (1863)

S. pleiantha, F. v. M. in Benth. Fl. Austr. I, 195 (1863)

S. spinosa, Linné, sp. pl. 683 (1753).

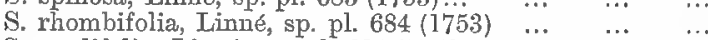

S. cordifolia, Linné, sp. pl. 684 (1753)

S. platycalyx, F. v. M. in Benth. Fl. Austr. I, $19 \ddot{97}$ (1863)

S. cleisocalyx, F. v. M., fragm. X, 73 (1876)

S. inclusa, Bentham, Fl. Austr. I, 197 (1863)

S. cardiophylla, F. v. M., fragm. VIII, $242(1874)$

S. Hookeriana, Miquel in Lehm. pl. Preiss. I, 242 (1844)

S. lepida, F. v. M., fragm. VI, 168 (1868) ...

ABUTILON, Tournefort, inst. 99, t. 25 (1700), from Camerarius (15S6)

A. tubulosum, Hooker in Mitch. Trop. Austr. 390 (1848) ‥ - S.A. - - N.S.W. Q. - B.fl.I,200 M.fr.IX,I31.

A. amplum, Bentham, FI. Austr. I, 200 (1863)

A. leucopetalum, F. v. M. in Benth. Fl. Austr. I, 200 (1863)

A. Mitchelli, Bentham, Fl. Austr. I, 201 (1863)

A. micropetalum, F. r. M. in Benth. Fl. Austr. I, 201 (1863)

A. cryptopetalum, F. v. M. in Benth. Fl. Austr. I, 201 (1863) ...

A. geranioides, Bentham, Fl. Austr. I, 202 (1863)

A. otocarpum, F. v. M. in Transact. phil. Soc. Vict. I, 13 (1854)

A. longilobum, F. v. M., fragm. IX, $130(1875)$.
A. subviscosum, Bentham, Fl. Austr. I, $202(1863)$

A. Indicum, G. Don, gen. syst. I, 504 (1831)

A. auritum, $(:$. Don, gen. syst. I, 500 (1831)

A. Avicennae, Gaertner, de fruct. II, 251, t. 135 (1791)

A. graveolens, Wight \& Arnott, Prodr. Fl. Pen. Ind. or. I, 56 (1840)

A. oxycarpum, F. v. M. in Benth. Fl. Austr. I, 204 (1863)

A. muticum, G. Don, gen. syst. I, 502 (1831)

A. Cunninghamii, Bentham, Fl. Anstr. I, 205 (1863)

A. exonemum, F. v. M., fragm. XT, 63 (1879)

A. Fraseri, Hooker in Walpers, annal. II, 158 (1851)

A. halophilum, F. v. M. in Linnaea XXV, 381 (1852)

A. macrum, F. v. M., fragm. IX, 59 (1875)

A. crispum, G. Don, gen. syst. I, 502 (1831)

$\cdots$

URENA, Dillenits, Hort. Eltham. 430, t. 319 (1732).

U. lobata, Linné, sp. pl. 692 (1753) .

U. Armitiana, F. v. M., fragm. X, 78 (1877) PAVONIA, Cavanilles, monadelph. dissert. II, App. II (1786). (Greevesia).

P. hastata, Cavanilles, clissert. III, 138, t. 47 (1786) $\ldots . .$.
HOWITTIA, F. v. Mueller in Transact. Vict. Inst. I, 116 (1855).

H. trilocularis, F. v. M, in Transact. Vict. Inst. I, 116 (1855) ... HIBISCUS, Linne, syst. nat. (1735); Linne, gen. pl. 207 (1737).

H. ficulneus, Linné, sp. pl. 605 (1753)

H. Abelmoschus, Linné, sp. pl. 696 (1753)...

H. rhodopetalus, F. v. M. in Benth. Fl. Austr. I, 209 (1863) ..

H. Manihot, Linné, sp. pl. 696 (1753)

H. Notho-Manihot, F.

H. Trionum, Linné, sp. pl. 697 (1753)

H. brachysiphonius, F. v. M., fragm. I, 67 (1858)

H. Drummondii, Turczan, in Bull. Soc. Mosc. XXXI, 195 (1858)

H. microlaenus, F. v. M., fragm. II, 116 (1861)

H. Pinonianus, Gaudichaud in Freyc. voy. Bot. 476, t. 100 (1826) W.A.

H. cannabinus, Limne, sp. pl. ed. sec., 979 (1763)..

H. divaricatus, Graham in Edinb. phil. Joum. (1830)

H. Fitzgeraldii, F. v. M., fragm. VIII, 242 (1874)

H. Elsworthii, F. v. M., fragm. VIII, 241 (1874)...

H. heterophyllus, Ventenat, jard. de la Malm., t. 103 (1804)

H. diversifolius, N. Jacquin, icon. pl. rar. 551 (1789)

H. splendens, Frasex in Elinb. phil. journ. (1830)

H. zonatus, F. v. M., fragm. I, 221 (1859)...

H. Coatesii, F. v. M., fragm. III, 5 (1862)...

H. leptocladlus, Bentham, Fl. Austr. I, 214 (1863)

H. setulosus, F. v. M., fragm. I, 221 (1859)

H. Golclsworthii, F. v. M., fragm. XI, 30 (1878) ...

H. pentaphyllus, F. v. MI., fragm. II, 13 (1860)

H. geranioides, Cunningham in Benth. Fl. Austr. I, 215 (1863)

H. vitifolins, Linne, sp. pl. 696 (1753)

H. panduriformis, N. Burman, Fl. Ind. 15i, t. $47^{\prime \prime}(1768)$

H. Normani, F. v. M., fragm. II, 4 (1862)
W.A. S.A. - V. N.S.W. Q. N.A. B.f.I, 192

W.A. S.A. - - N.S.W. - N.A. B.fl.I, 193

S.A. - - N.S. W. Q. N.A. B.fl.I, 194

- - - - - N.A. - M.fr.XI,62.

W.A. S.A. - - - - N.A. B.f.I, 194

- N.A. B.fl.I, 195 M.fr.III,3.

- - - N.S.TV. Q. N.A. B.A.I,195

Q. N.A. B.fl,I,195 M.fr.VI, 169.

- - - N.S.T. Q. Q. N.A. B.A.I.I, B.f1.I, 196

- _ - Q. N.A. B.f.I,196

- - - - - N.A. B.fl.I,197

- - N.A. B.fl.I,197 M.fr,IX,181;XI,32

W.A. S.A. - - - - N.A. $-{ }_{\text {M.fr.VIII, } 242 .}$

- S.A. - - N.S.W. Q. - - B.fl.I, 200 M.fr.IX,I3
- S.A. - - N.S.W. - N.A. B.fl.I,200 B.fl.I,200 M.fr.II,12.

- S.A. - - N.S.W. - N.A. B.Al.I,200

- - - - N.S.W. Q. - B.f.I,201 M.fr.VI, 169 S.;IX.131;XI,

W.A. S.A. - - N.S.W. - N.A. B.fl.I,201 M.fr.II, Il;IX, I3. [32.

- - - - - - N.A. B.f.I,202

- S.A. - V. N.S.W. Q. N.A. B.fl. I, 202 M.fr.XI,32.

W.A. - $--=\overline{\mathrm{Q}}=$ B.fl.I,202 M.fr.IX,130.

- - - Q Q. N.A. B.fl.I,202

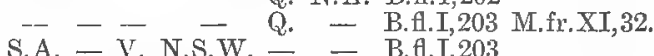

- S.A. - V. N.S.W. $\bar{Q}$ - B.f.I, 203

W.A. S.A. - - N.S.W. Q. N.A. B.fl.I,204 M.fr.II,12;IX,131;XI,32.

- - - - Q. - B.fl.I,204

- - - - Z Q. N.A. B.1.1,205

T.A. S.A. - - N.S.W. Q. N.A. B.AlI, 20.0

W.A. S.A. - - N.S.IV. - - B.fl.I,206 M.fr.XI,27.

- S.A. - - - - - M.fr.IX,59.

- - - - - - N.A. B.H.I,206 MI,fr.VI,169.

- - N.S.WV. Q. - B.fl.I, 207 M.fr.IX, 130.

S.A. - V. N.S.W.

B.fl.I, 198 MI.fr.VI, 169.

(Fugosia partly, Abelmoschus, Paritium).

- - - - Q. N.A. B.fl.I,209 M.fr.I,67.

- - - - - - N.A. - M.fr.VI, 169.

- - - - N.S.TV. Q. N.A. B.H.I,209 M.fr.IX,229;XI,31.

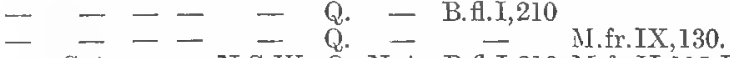

- S.A. - - N.S.TW. Q. N.A. B.f.I.I,210 M.fr.II,11,5:IX,129;XI,31.

S.A. - - N.S.W. Q. - B.fl.I, 210 N.fr.I,67;VI,169.

W.A. - - - - - - B.fl.I,211 M.fr.I,220;IX,129;:I, 31.

W.A. S.A. - - - - N.A. B.A.I,211 M.fr.III, $5 ; \mathrm{IX}, 129$.

- N.A. B.fl.I,211 M.fr.II,116. [IX,129:XI,3I.

- Q. N.A. B.f.I, 212 M.fr.II,117;III,166;VI,269;
- - - N.S.W. Q. - B.f.I,2l2 MI.fr.IT,118;IX,129;XI,31.

- - - - Q. - - M.fr.VIII,242.

- - - Q Q. - - M.fr.VIII,24l,

- - - N.S.W. Q. - B.A.I,212 M.fr.YI, 170;XI, 31

- - N.S.W.Q. - B.fl.I,213 M.fr.II,117;VI, 169.

- - - N.S.W. Q. - B.fl,I,213 M.fr. YI, 170;XI,31\&123.

- - N.A. B.f.I,213 M.fr. VI, 170.

- N.A. B.fl.I.2l4 M.fr.III,T.

- N.A. B.fl.I.214 M.fr. YI, 160;IX, 129.

- N.A. B.fl.I, 214 M.fr.I,22l;XI,3l.

- N.A. - M.fr.XI, 30 .

- N.A. B.fl.I,214 M.fr. YI, 169:XI,32.

- N.A. B.fl.I,215

Q. - B.f.I,215 M.fr.II, 114;YI,169.

- N.A. B.fl.I,215 M. fr.II, 115; YI, 169;IX, 129.

(.) - B.H.I,216 M.fr,III, 4; VI, 169 .
W.A. S.A. - - N.S.W. Q. N.A. N.A. B.f.I, 194
S.A. - - N.S.

W.A. - - - - - B.E.I,197 
H. Krichauffi, F. v. M., Rop. Babb. Expert, 7 (1858)

H. Tarragei, F. V. M., fragm. VIII, 241 (1874) $\ldots$

H. phyllochlaenus, F. v. M., fragm. IX, $128(1875)$

H. Huegelii, Endlicher in Hueg. enum. 10 (1837)...

H. Wrayae, Lindley, bot. Regist. t. 69 (1840) ...

H. tiliaceus, Linné, sp. pl. 694 (1753)

H. cuneiformis, de Candolle, prodr. I, 454 (1824)....
LAGUNARIA, G. Don, gen. syst. dichlam. pl. I, 485 (1831).

L. Patersoni, G. Don, gen. syst. I, 485 (1831) … $\ldots$ THESPESIA, Solander in Ann. du Mus. IX, 290 (1807).

T. populnea, Solander in Anu, du Mus. IX, 290, t. 8 (1807) ...

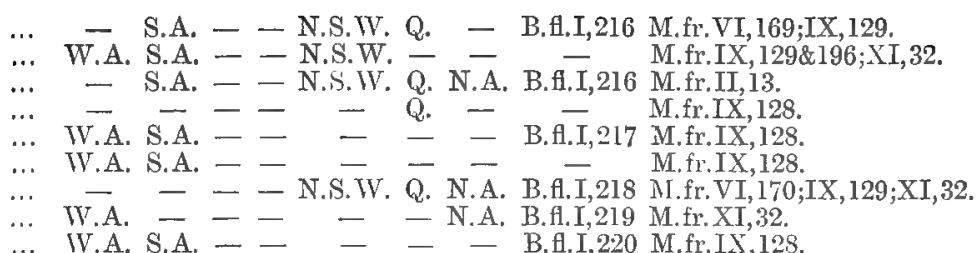

GOSSYPIUM, Linne, gen. pl. 206 (1737), from Camerarins (1586). (Fugosia partly, Sturtia).

G. Sturtii, F. V. M., fragm. III, 6 (1862) ...

G. australe, F. v. MI., fragm. I, 40 (1858) ..

G. thespesioides, F. v, M., fragm. IX, 127 (1875)

G. Cunninghamii, Todaro, Monogr. Gen. Gossyp. 7 (1s $7 \ddot{8}$ )

G. costulatum, Toctaro, Monog1. Gen. Gossyp. 7 (1878) ...

G. flaviflorum, F. v. M., fragm. IX, $127(1875) \ldots$
G. populifolium, F. v. M., fragm. IX, $127(1875) \ldots$

G. Robinsoni, F. v. M., fragm. IX, 126 (1875) ... ADANSONIA, Limé, sp. pl. I, 1190 (1753).

A. Gregorii, F. v. M. in Hook. Kcw misc. IX, 14 (1857) CAMPTOSTEMON, Masters in J. Hook, icon. pI. XII, 18, t. 1119 (1876).

C. Schultzii, Masters in J.'Hook. icon. pl. XII, 18, t. 1119 (1876) BOMBAX, Linné, sp. pl. I, 511 (1753).

B. Malabarica, de Candolle, prodr. I, 479 (1824) ...

$$
\begin{aligned}
& \text {... - S.A. - N.S.W. } \\
& \text {... - S.A. - - } \\
& \text { … }- \pm-
\end{aligned}
$$

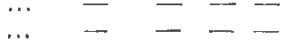

$$
\begin{aligned}
& \text { ‥ - }-2 \\
& \text { … T.A. }=-- \\
& =
\end{aligned}
$$

- B.fl.I, 222 M.fr. III, $174 ;$ VI, $169 \& 2.5$;

$[\mathrm{IX}, 127 ; \mathrm{XI}, 63$.
$4 ; \mathrm{VI}, 169 \& 251$

Q. N.A. B.fl.I, 220 M.fr. III, $6 ;$ IX, $127, X I, 32 \& 63$

- N.A. B.Hl. I,2:10 M.fr.V.177;VI.169;XI,32\&63

- N.A. B.fl,I,221 M.fr.IX,127;XI,32\&63. [63.

- N.A. - M.fr.XI,63.

- N.A. B.A.I,22I M.fr.XI $32 \& 63$.

N.A. - M.fr.XI,63.

- N.A. B.fl.I, 223 M.fr.VII,40;XI,32.

- N.A. - M.fr. XI,32,

Q. N.A. B.fl.I,223 M.fr.XI,32.

\section{STERCULIACEAE.}

Ventenat, Jard, de la Malm. II, 91 (1804).

STERCULIA, Linne, Fl. Zeyl. 166 (1747).

S. quadrifida, R. Brown in Benn. pl. Jav. rar. 233 (1852) ‥ - - - - - N.S.W. Q. N.A. B.f.I, 227 M.fr.VI,173.

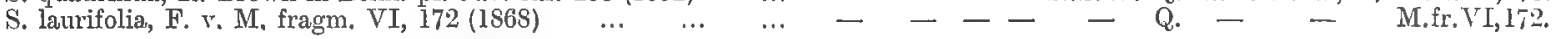

BRACHYCHITON, Schott \& Endlicher, Melat, bot. 34 (1832). (Trichosiphon, Poecilodermis, Delabechea).

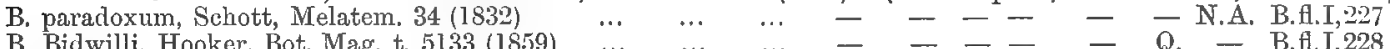

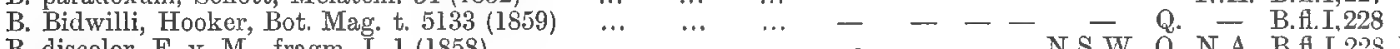

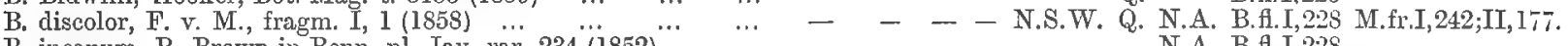

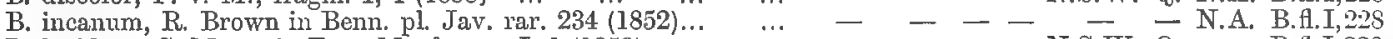

B. luridum, C. Moore in F. v. M., fragm. I, 1 (1858) $\ldots \quad \ldots-\quad-\quad-\quad-$ N.S.W. Q. - B.甘.I,228 M.fr.I,I;II,177.

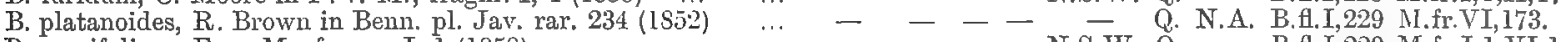

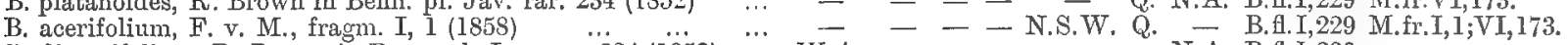

B. diversifolium, R. Brown in Benn. pl. Jav, rar. 234 (1852) $\ldots$ W.A. - - - - - N.A. B.fl.I,230

B. populneum, R. Brown in Benn. pl, Jav. rar. $234(1852) \quad \ldots-\ldots-$ - - V. N.S.W. Q. - B.fl., 229

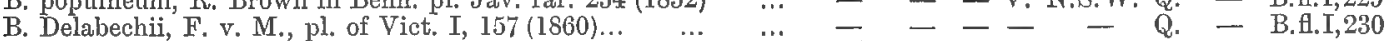

B. Gregorii, F. v. M. in Hook. Kew misc. IX, 199 (1857) … W.A. S.A. - - $\quad-\quad-\quad-\quad-\quad$ M.fr.IX,137. TARRIETIA, Blume, Bijdr. 227 (1825). (Argyrodenclron).

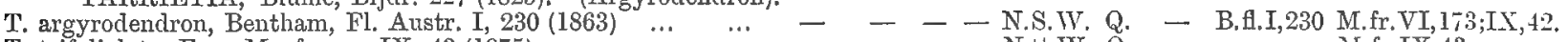

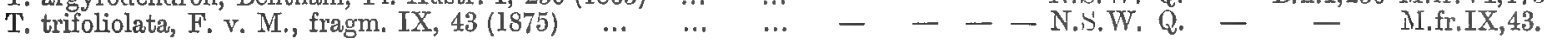
HERITIERA, Dryander in Aiton, Hort. Kew III, 546 (1789).

H. littoralis, Dryander in Aiton, Hort. Kew III, $546(1789) \quad \ldots \quad-\quad-\quad-\quad$ - Q. N.A. B.fl.I,231 M.fr.VI,I73. UNGERIA, Schott \& Endlicher, Melat. 27-31 (1832).

U. floribunda, Schott \& Endlicher, Melat. 27, t. 4 (1832) HELICTERES, Plukenet Phytogr. 181, t. 245 (1692).

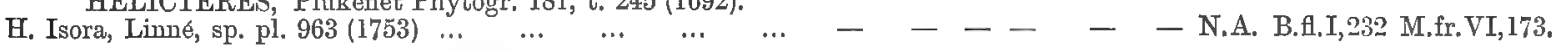
METHORIUM, Schott \& Endlicher, Melat. 29-30 (1832).

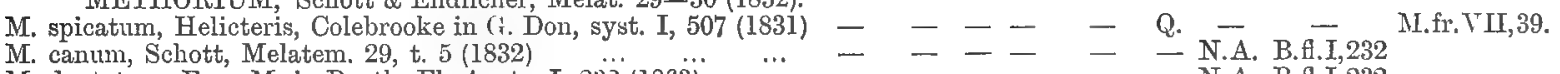

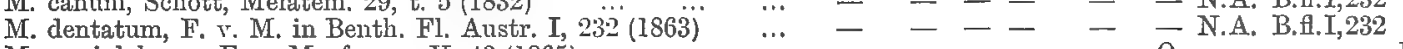

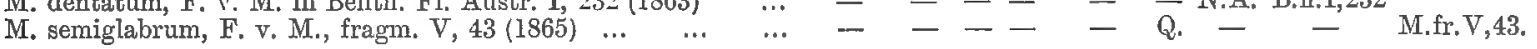
MELHANIA, Forskael, Fl. Aeg. Arab. 64 (1775).

M. incana, Heyne in Wight \& Arn. prodr. 68 (1834) ... MELOCHIA, Dillenius, Hort. Eltham. 221, t. 176 (1732). (Riedleya).

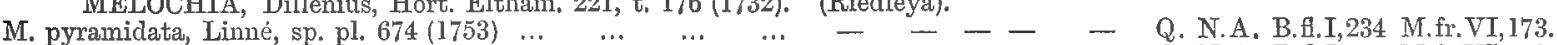

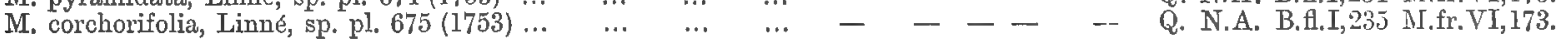
HERMANNIA, Toumefort, inst. 656, t. 432 (1700). (Gilesia, Mahernia partly).

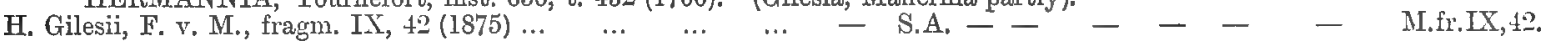
DICARPIDIUM, F. v. Mueller in Hook. Kew misc. IX, 302 (1857).

D. monoicum, F. v. M., in Hook. Kew misc. IX, 302 (1857) .. - - - - — - N.A. B.fl.I,235 WALTHERIA, Limne, gen. pl. 203 (1737).

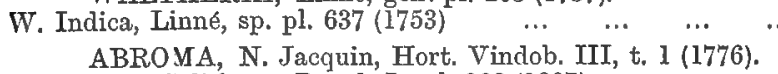

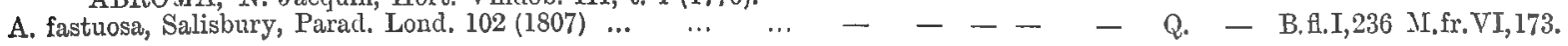


COMMERCONIA, R. \& G. Forster, char, gen. 43, t. 22 (1776). (Ruelingia, Achilleopsis).

C. salvifolia, Bentham, Fl. Austr. I, 238 (1863)

C. dasyphylla, Andrews, Bot. Reposit. t. 603 (1804)

C. rugosa, Rulingia, Steetz in pl. Preiss. II, 352 (1847) ...

C. Preissii, Stendel in Lehm. pl. Preiss. I, 237 (1844) ...

C. cinerea, Steudel in Lehm. pl. Preiss. I, 238 (1844)

C. cygnorum, Steudel in Lehm. pl. Preiss. I, 237 (1844)...

C. platycalyx, Rulingia, Benth. fl. Austr. I, 240 (1863)

C. parvitlora, Rulingia, Endlicher in Hueg. enum. I.2 (1837)

C. hermanniaefolia, Rulingia, Steetz in pl. Preiss. II, 353 (1847)

C. Kempeana, F. v. M., fragm. XI, 113 (1881)

C. laxophylla, F. r. MI., fragn, X, 22 (1876

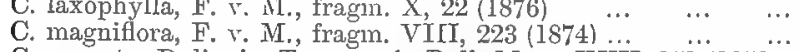

C. cuneata, Rulingia, Turczan. in Bull. Mosc. XXV, $15 \dddot{1}(1852)$

C. densillora, F. r. M., fragm. X, 21 (1876)

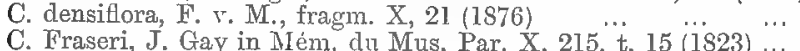

C. Leichhardtii, Bentham, Fl Austr I $210(1863)$

C. echinata, R. \& G. Forster, char, gen. 43, t. $22(1776) \ldots$

C. Gaudichaudi, J. Gay in Mém. du Mus. Par. X, 213, t. $14(1823)$

C. crispa, Turczaninow in Bull. Soc. Mosc. XIX, 501 (1846)

C. melanopetala, F. V. M., fragm. X, 2 I (1876)

C. pulchelia, Turczaninow in Bull. Soc. Mosc. XIX, 502 (1846)

C. microphylla, Bentham, Fl, Austr. I, 244 (1863)

C. craurophylla, F. v. M., fragm. IX, 59 (1875) ...

HANNAFORDIA, F. v. Mueller, fragm. II, 9 (1860).

H. quadrivalvis, F. v. M., fragm. II, 9 (1860)

H. Shanesii, F. v. M., fragm. VI, 175 (1868)

H. Bissillii, F. v. M., fragm. X, 95 (1877) ...

$\begin{array}{ll} & \\ \cdots & \cdots \\ \cdots & \cdots\end{array}$

SERINGEA, Sprengel, Anleit. II, 649 (1818). (Kerandrenia).

S. platyphylla, J. Gay in Mém. du Mus. Par. VII, 443 (1821) ... -

S. lanceolata, Steetz in Lehm. pl. Preiss. II, 349 (1847) ..

i. corollata, Steetz in Lehm. pl. Preiss. II, 330 (1847)

s. Hillii, Keraudrenia, F. v. M. in Benth. FI. Austr. I, 246 (1863)

S. nephrosperma, F. v. M. in Hook. Kew misc. IX, 15 (1857) ...

S. microphylla, F. v. M., fragm. II, 5 (1860)

S. adenolasia, F. v. M., fragm. X, 96 (1877)

S. integrifolia, F. v. M., fragm. II, 5 (1860)

T. macrocarpa, Huegel in Endlicher, nov. stirp. dec. 32 (1839) ...

T. xugosa, Turczaninow in Bull. Soc. Mose. XIX, 501 (1846) ...

T. montana, Steudel in Lehm. pl. Preiss. I, 230 (1844) ...

T. tenuivestita, F. v. M., fragm. II, 7 (1860)

T. solanacea, J. Gay in Mrém. du Mus. VII, 456, t. 21 (1821)

T. brachystachys, Turczan. in Bull. Soc. Mosc. XXV, 143 (1852)

$T$. discolor, Stendel in Lehm. pl. Preiss. I, 233 (1844)

T. quercifolia, J. Gay in Mém. du Mus. VII, 459, t. 21 (1821) ...

T. foliosa, J. Gay in Mém. du Mus. VII, 454, t. 22 (1821) ...

T. triloba, Turczaninow in Bull. Soe. Mose. XIX, 500 (1846) ...

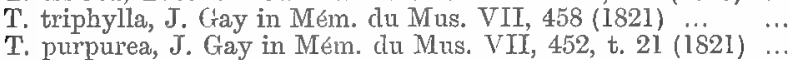

T. macrocalyx, Steudel in Lehm. pl. Preiss. I, 230 (1844)

T. pauciflora, Lindley, Bot. Regist. XXV, App. XVIII (I839)...

$T$. rhynchocarpa, Turez. in Bull. Soc. Mosc. XXV, 142 (1852) ...

T. grandiflora, Lindley, Bot. Regist. XXV, App. XVIII (1839)

T. cognata, Steudel in Lehm. pl. Preiss. I, 232 (1844)

T. rulingioides, Steudel in Lehm. pl. Preiss. I, 232 (1844)

T. angustifolia, Steudel in Lehm. pl. Preiss. I, 232 (1844)

T. petalocalyx, F. v. M. in Transact. phil. Soc. Vict. I, 35 (1854)

T. Sarotes, Turczaninow in Bull, Soc. Mose. XXV, 145 (1852).. GUICHENOTIA, J. Gay in Mém. đlu Mus. VII, 448, t. 5

G. Iedifolia, J. Gay in Mém. du Mus. VII, 449, t. 5 (1821)

G. macrantha, Turczaninow in Pull. Soc. Mosc. XIX, 500 (1846)

G. semihastata, Bentham, Fl. Austr. I, 258 (1863)

G. Sarotes, Bentham, Fl. Austr. I, 258 (1863)

G. micrantha, Bentham, Fl. Austr. I, 258 (1863) ... $\ldots . . .$. W.A. LASIOPHTALUM, Smith in Transact. Linn. Soc. IV, „216 (1798)

L. laxiflorum, F. v, M., fragm. XI, 112 (1881) ... ... ... IV.A.

L. pymactum, Bentham, Austr. I, 256 (1863) $\quad . .6 \quad \ldots . \quad \ldots$. IV.A.

L. stelligerum, Bentham, Austr. I, 256 (1863) $\quad \ldots .6 \ldots .7$.

L. glutinosum, F, v. M., fragm. XI, $113(1881)$... ... ... W.A.

L. discolor, Hooker, Comp. to Bot. Mag. I, 276 (1835) … …

L. dasyphyllum, Sieber in Hook, journ, of bot. II, 414 (1840) ...

L. indutum, Steudel in Lehm. pl. Preiss, I, 235 (1844) $\ldots . \quad \ldots$ W.A.

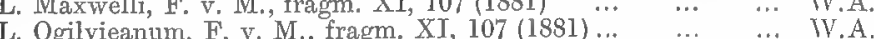

I. Behrii, F, v. M., in Transact. phil. Soc. Vict. I, 36 (1854)

L. parviflorum, Rudge in Transact. Linn. Soc. X, 297, t. 19 (181i)

L. parvuliflorum, F. v. M., fragm. VI, 174 (1868)

L. Fitzgibbonii F. v. M. in South. Sc. Record II, Apr. (1882)...

$$
\begin{aligned}
& \text { - - - Q Q - B.fl.I,238 } \\
& \text { - - V. N.S.W. Q. - B.fl.I,238 M.fr.X,22. } \\
& \text { - - - N.S.W. - - B.f. } 238 \\
& \text { B.f.I, } 239 \\
& \text { B.H.I, } 239 \\
& \text { B.fl. I, } 239 \\
& \text { B. H.I, } 240 \\
& \text { B.fl.I, } 240
\end{aligned}
$$

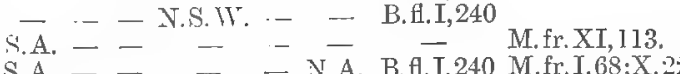

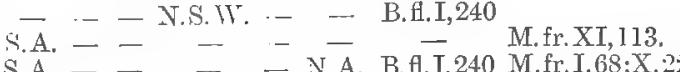

$$
\begin{aligned}
& \text { S.A. - - - N.A. B.fl.1, M.fr.X, 22;XI,114 } \\
& \text { - B.Al.T,241 } \\
& \text { B.fl. I, 24I } \\
& \text { B.H.I,241 M.fr.VI, 17J;XI,I14. } \\
& \text { B.fl. I, } 242 \\
& \text { B.fl. I, } 2+2 \text { M.fr.VI, } 17 ; ; \mathrm{X}, 22 ; \mathrm{XI}, 114 \text {. } \\
& \text { B f.I, } 24 \% \text { M.fr.VI, } 175 ; \mathrm{X}, 22 \text {. } \\
& \text { B. fl. I, } 24: 3 \\
& \text { - } \quad \text { - } \quad \text { B.f.I,243 M.fr. X,21. } \\
& \text { W.A. - - - - - - B.fl.I,244 M.fr.X,2.2. } \\
& \text { A. - - - - - B.fl.I,244 }
\end{aligned}
$$

W.A.

\section{B. Al. I,248 M.fr.XI, $20 \& 115$. M.fr. VI, 175 .

- M.fr.XI,115.

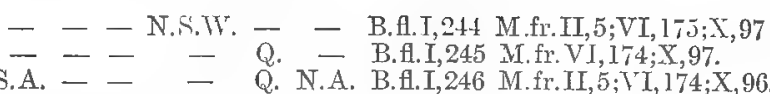

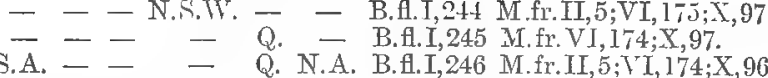

- - N.S.IV. Q. - B.H.I,246 M.fr.VI, 174;X,97.

N.A. B.fl. I, $2+6$ M.fr.II, $5 ; X, 97$.

A. - - - - $\overline{\text { N.A }}$ B.H.I,247 M.fr.II,5.

(Leucothamnus, Rhychostemon).

W.A.

W.A.

W.A.

TV.A.

W.A.

W.A.

WT.A.

W.A.

W.A.

IV.A.

W.A.

IV.A.

W.A.

IV.A.

(1821).

W.A.

cothamnus, Rhychostemon).
- - - - - - -
- - - -

B. A. I, $2 \pm 7$ MI.fr.II, $5 ; \mathrm{YI}, 17 \bar{J} ; \mathrm{X}, 97$

B. f. I, $2 \widetilde{J} 0$

B.Al.I, 250

B. H. I, 250

B.fl. I, 201 M.fr. II, 7

B. Al. $1,2.51$

B. f.. .2 .21

B.fl.I,25I

B. fl. 1,2522

B.fi. $1,2 \pi 2$

B.fl.I, 252

B.tl. I, 2502

- B.fi.I, 2. ir

B.fl.I, 253 גI.fr. VI, 175 .

B. $1 . I, 2,53$

B.A.I, 254 M.fr.II, 8;VI,17.J

- B.fl.I,254

B.fl. $I, 254$

B. Al. I, 255

- B.fl.1,255

B.fl.I, 2,

- B.A.I, 2.5.5

B.f.I,:is ML.fr. YII,174;XI,114. B. f.. I, 2.58

B.H.I,2.S M.fr.II, 4.

B.fl.I, 258

B-fl.I, 258 M.fi:II, 7.

(Corethrostylis, Asterochiton)

W.A. S.A

- T. V. N.S.W.

IV.A.

- S.A. - V. N.S.W -

- - - T. N.S.W. -

W.A.
B.A.I,256 MI.fr. X I, Il2. I. fr. XI, 113.

II.fr. XI, 113.

M.fr. XI,113.

13.fl.I, 260 M.fr. XI, 110 .

li.fl, I, M.

13.fl.I, 261 MI.fr. XI, 110 .

M.fr. XI, 107.

B.f. I,, 201

B.H. I, 262 M.fr. TI, 17.

M.fr. VI, 174

M.fr.XII. 
L. oppositifolium, F. v. M., fragm. II, $5(1860) \quad \ldots \quad \ldots \quad \ldots \quad \ldots \quad$ W A

L. micranthum, J. Hooker, Fl. Tasman. I, 51 (1860)

L. macrophyllum, Graham in Bot. Mag. t. 3098 (1844) ...

L. Baneri, Steetz in Lehm. pl. Preiss. II, 339 (1847)

L. Tepperi, F. v. M., fragm. XI, 109 (1881)

L. rufum, R. Brown in Benth. Fl. Anstr. I, 263 (ï63)

L. ferrugineum, Smith in Bot. Reposit. t. 308 (1799)

L. acutiflorum, Turczaninow in Bull. Soc. Mosc. XXV, 145 (18

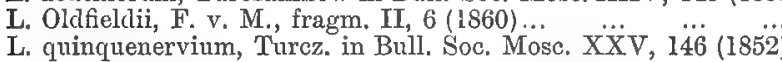

L. Drummondii, Bentham, Fl. Austr. I, 264 (1863)

L. rosmarinifolium, Bentham, FI. Austr. I, 264 (1863) ...

L. cordifolium, Endlicher in Hueg, enum. 10 (1837)

L. Schulzenii, F. v. M., pl. of Vict. I, 145 implied (1861)

L. floribundum, Bentham, Fl. Austr. I, 265 (1863)

L. molle, Bentham, Fl. Austr. I, 265 (1863)

L. membranaceum, Bentham, Fl. Austr. I, 266 (1863) .

L. bracteatum, Bentham, Fl. Austr. I, 266 (1863)

LYSIOSEPALUM, F. v. Mueller, fragm. I, 143 (1859).

L. Barryanum, F. v. M., fragm. I, 143 (1859)

L. rugostum, Bentham, Fl. Austr. I, 267 (1863)

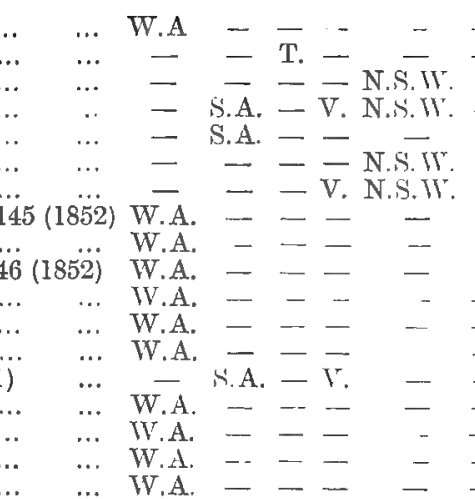

B.fl.I,262 M.fr.XI, 112 .

B.f.I. I, 26:2

B.f.I.I,262

B. fl. I, 263 MI.fr. VI, I7t;XI, 110 .

B.f. I. 263

B.f.I, 263

B.Æ.I,264 M.fr.XI, 109

M.fr.IT,6.

M.fr. XI, 100 .

B.f.I, 264 M.fr.VI, 174;XI, 112.

B.f.I,264 M.fr.XI,112.

B.f.I, 26.$)$ M.f.VI, 174;XI, 112.

B.fl. I, 2(5.)

- B.fl.I,265 M.fr.XI,112.

- B.fl.I,265 M.fr.XI,111.

- B.fl.I,266 M.fr.XI,112.

- B. $1, I, 266$

W.A. - - - - - B.fl.I, 267 M.fr.VI, 174.

TILIACEAE.

A. L. de Jussieu, Gen. 289 (1789).

BERRYA, Roxburgh, Pl. Coromand. III, 60, t. 264 (1819).

B. ammonilla, Roxburgh, pl. Coromand. III, 60, t. 264 (1819) ... -

GREWIA, Linné, syst. nat. 9 (1735); Limne, gen. pl. 276 (1737).

G. orientalis, Linné, sp. pl. 964 (1753)

G. breviflora, Bentham, Fl. Austr. I, 270 (1863)

G. latifolia, F, v. M. in Benth. Fl. Austr. I, 271 (1863) ...

G. polygama, Roxburgh, Fl. Ind. LI, 588 (I832).

G. xanthopetala, F. v. M. in Benth. Fl. Austr. I, 271 (1363)

G. scabrella, Bentham, Fl. Austr. I, 272 (1863)

G. orbifolia, F. v. M. in Benth. Fl. Austr. I, 272 (1863)...

G. pleiostigma, F. v. M., fragm. VIII, 4 (1872) ... ...

TRIUMFETTA, Plumier, nov. gen. 40, t. 8 (I703).

T. procumbens, G. Forster, prodr. fl. insul. Austr. 35 (1786) ..

T. appendiculata, F. v. M., fragm. III, 7 (1862) ...

T. chactocarpa, F. v. M., fragm. XI, 61 (1879)

T. glauscescens, R. Brown in Benth. Fl. Austr. I, 273 (18̈63)

T. denticulata, R. Brown in Benth. Fl. Austr. I, 273 (1863)

T. micracantha, F. v. M., fragm. III, 7 (1862)

T. leptacantha, F. v. M., fragm. XI, 62 (1879)

T. plumigera, F. v. M., fragm. I, 69 (1858)

T. pilosa, Roth, nov. pl. spec. 223 (1821)

T. parviflora, Bentham, Fl. Austr. I, 274 (1863) '..'

NETTOA, Baillon in Adans. VI. 238, t. 7 (1866).

N. chrozophorifolia, Baillon in Adans. VI, 238, 242 (1866)

CORCHORUS, Tournefort, inst. 259, t. 135 (1700).

C. echinatus, Bentham, Fl. Austr, I, 276 (1863) … 270 (1863)

C. Cunninghamii, F. Y. M., fragm. III, 8 (1862) $\ldots . . \ldots$

C. tridens, Linne, mantiss. alter. 566 (177i)

C. acutangulus, Lamarck, encycl. meth. II, 104 (1786) ...

C. fascicularis, Lamarck, encycl. meth. II, 104 (1786) ...

C. pumilio, Bentham, F1. Austr. I, 277 (1863)

C. vermicularis, F. v. M., fragm. III, 10 (1862) ...

C. tomentellus, F. v. M., fragm. III, 10 (1862)

C. sidoides, F. V. M., fragm. III, 9 (1862) ... …

C. elachocarpus, F. v. M., fragm. VIII, 6 (1872) ..

C. Walcottii, F. v. M., fragm. III, 9 (1862)

C. trilocularis, Linné, mantiss. 77 (1771)

SLOANEA, Linné, hort. Cliffort. 210 (1737). (Echinocarpus.)

S. Australis, F. v. M., fragm. IV, 91 (1864)

S. Langii, F. v. M., fragm. V, 28 (1S65)

S. Macbrydei, F. v. M., fragm. VI, 170 (1868)

S. Woollsii, F. v. M., fragm. VI, 171 (1868)

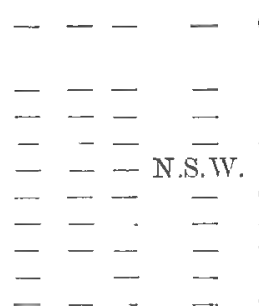

Q. - B.fl.I,268

Q. N.A. B.fl.I, 270

Q. N.A. B.fi. I, 270

- N.A. B.A.I, 270

Q. B. B.I, 271

- N.A. B.fl.I,27l

Q. - B.fl.I,272

- N.A. B.H.I, 272

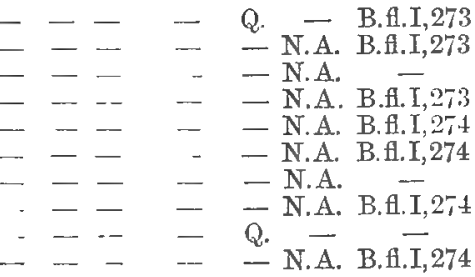

M.fr.III, 8;VI, 172 .

$$
\text { - N.A. }
$$

$\begin{array}{llll}- & - \\ - & - & \text { N.A. B.fl.I, } 276 \\ - & \text { Q. } & \text { B.f.I, } 276 \\ \text { - N.W.W. Q. } & \text { B.H.I, } 276\end{array}$

$\begin{array}{llll}- & - & \text { N.A. B.fl.I, } 276 \\ - & - & \text { Q. } & \text { B.f.I,276 } \\ - & - \text { N.S.W. Q. } & \text { B.f.I, } 276\end{array}$

- N.A. B.H.I, 276

- N.A. B.fl.I, 276

Q. N.A. B.fl.I, 277

- N.A. B.fl.I.2T

- N.A. B $\mathrm{H} .1,277$

- N.A. B.H.I, 277

Q. - B.fl.I, 278

Q. N.A. B.fl. I, 278

- N.A. B.H.I, 278

- N.A.

- N.A. B.t.I, 278

Q. - -

- - N.S.W.Q. - B.fl.I, 279

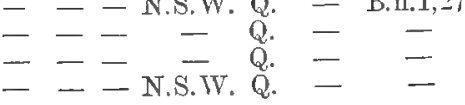

ARISTOTELIA, L'Héritier, stirp. II, 21, t. 16 (1784). (Friesia.)

A. peduncularis, J. Hooker, Fl. Nor. Fel. I, 33 (1853) ...

A. Australasica, F. v. M., fragm. II, 79 (1860)

A. megalosperma, F. v. M., fragm. IX, 84 (1S75)...

ELAEOCARPUS, Burman, thesaur. Zeil. 39, t. 40 (1737).

E. holopetalus, F. v. M., fragm. II, 143 (1861)

E. Arnhemicus, F, v. M., Docum. intercol. Exhib. 24 (1867) ...
-

- T. - N.S.W. - - B.fl.I,280

(Elaiocarpos, Dicera.)

- - - V. N.S.W. - - B.fl.I,281
M.fr. VIII,4.

M.fr.VIII, 5 .

M.fr.XI,6l.

M.fr.VIII, $5 ; \mathrm{XI}, 62$.

M.fr.XI, 62.

M. fr.III, $3 ; \mathrm{VI}, 172$.

M.fr.IV,28;VIII, 5 .

M.fr.VI, 172.

M.fr. VI, 172;VIII, 4.

M.fr.III, 8;VIII, 5 .

M.fr. VIII, 5 .

M.fr. 167;VI, 172.

M.fr.VIII, 5 ,

M.fr. VIII, 6.

M.fr.VIII, 5 .

M.fr.III, 10

M.fr.VIII, 6. $[62$

M.fr.VI, 172;VIII, 6; XI.

M.fr. VIII, 5.

M.fr. VI,172;VIII,2.

M.fr. VIII,2.

M.fr. VI, 170 .

M.fr. VIII, 2 .

M.fr. YIII, 2.

M.fr.VIII, 2.

M.fr. IX, 84 .

M.fr.IV,173;VI, 172; [VIII. M.fr. X, 4 .
M.fr.VII, $6 ; \mathrm{XI}, 62$. 
E. oboratus, G. Don, gen. syst. dichl. pl. I, 599 (1831)

E. cyaneus, Aiton, Epit. hort. Kew, addend. 367 (1814)

E. grandis, F. Y. M., fragm. II, $81(1860)$..

E. foveolatus, F. v. M., fragm. V, 157 (1866)

E. ruminatus, F. v. M., fragm. VIII, $1(1872)$

E. Grahami, F. v. M., fragm. X, 3 (1876) ...

F. sericopetalus, F. v. M., fragm. VI. 171 (1868) ...

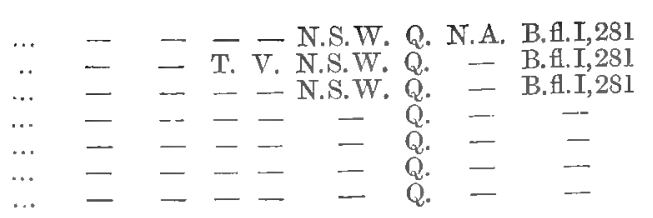

EUPHORBIACEAE.

A. L. de Jussieu, Gen. 384 (1789) from B. de Jussieu (1759).

CALYCOPEPLUS, Planchon in Bull, de la Soc. Bot. cle Fr. VIII, 31 (1861).

C. ephedroides, Planchon in Bull. Soc. Bot. Fr. VIII, 31 (1861) W.A. - - _ _ - - B.fl.VI,53

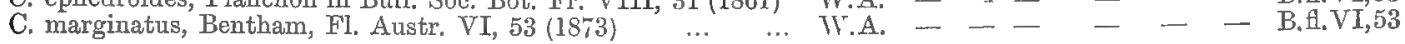
EUPHORBIA, Linné, gen. pl. 152 (1737) from Plinins.

E. Atoto, G. Forster, florul. ins. Austr. prodr. $36(1786) \quad \ldots \quad-\quad-\quad-\quad-\quad-\quad$ Q. N.A. B.fl.VI,46

E. Sparmanni, Boissier, Cent. Euphorb. 5(1860) - - - N.S.W. - - B.fl.VI,46

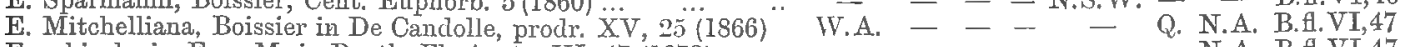

E. schizolepis, E. v. M. in Benth. Fl. Austr. VI, 47 (1873) .. - - - - - - - N.A. B.H.VI,47

E. Schultzii, Bentham in Fl. Austr. VI, $47(1873)$ - _ _ _ _ N.A. B.tl.VI,47

E. Armstrongiana, Boissier in De Candolle, prodx. XV, $\ddot{47}(1866)--\quad-\quad-\quad$ - N.A. B.f.VI,48

E. erythrautha, F. v. M., fragm. II, 152 (1861) ... ... .. - S.A. - - N.S.W. Q. N.A. B.A.VI.48

E. Muelleri, Boissier in De Candolle, prodr. XV, $27(1866) \quad \ldots+-\quad-\quad-$ N.A. B.fl.VI,48

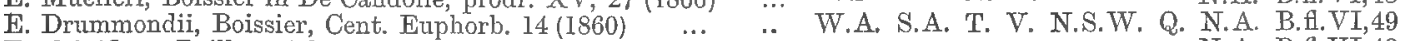

E. alsiniflora, Baillon, Adans. VI, $288(1866) \quad \ldots \quad \ldots . \quad \ldots \quad-\quad-\quad-\quad-\quad-\quad-$ N.A. B.fl.VI,49

E. Wheeleri, Baillon, Adans. VI, 286 (1866) $\quad \ldots \quad \ldots . \quad \ldots$ - S.A. - - _ - N.A. B.fl.VI,49

E. myrtoides, Boissier in De Candolle, prodr. XV, 15 (1866) $\cdots$ W.A. - - - - - - N.A. B.f.VI,50

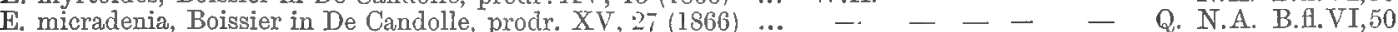

E. Macgillivrayi, Boissier in De Candolle, prodr. XV, $26(1866)-z_{-}-$N.S.T. Q. - B.fl.VI,50

E. serrulata, Reinwardt in Blum. Bijdrag. 635 (1826) $\ldots . . . \quad-\quad-\quad-\quad-\quad-\quad$ Q. N.A. B.f.VI,51

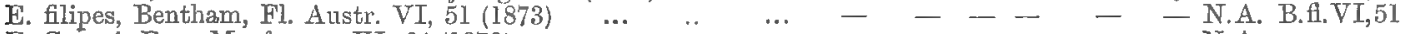

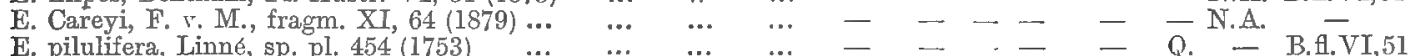

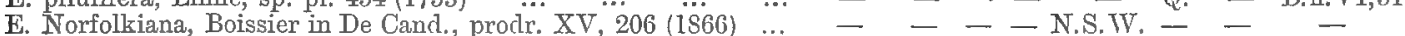

E. eremophila, Cunningham in Mitch. Trop. Austr. 348 (1848)... W.A. S.A. - V. N.S.W. Q. N.A. B.A.VI,52 MONOTAXIS, Brongniart in Duperr. Voy. Bot. 223, t. 49 (1829). (Hippocrepandra.)

M. macrophylla, Bentham, Fl. Austr. VI, 79(1873) .. ‥ - - - - N.S.W. Q. - B.fl.VI, 79

M. linifolia, Brongniart in Duperr. Voy. Bot. 223 , t. $49(1829) \ldots-. . \quad-\quad-$ N.S.IT. - - B.fl.VI,79

M. occidentalis, Endlicher in Hueg. enum. 19 (1837) $\quad \ldots \quad$... W.A. $\quad$ W $\quad$ - $\quad-\quad$ - $\quad$ - B.f.VI,79

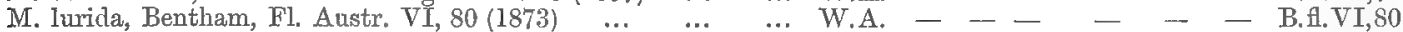

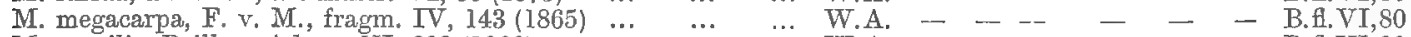

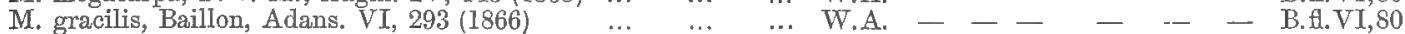

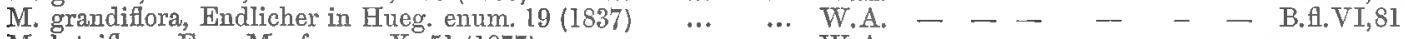

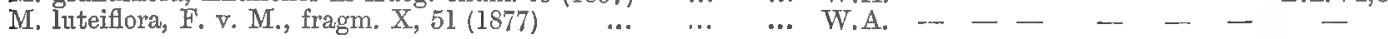

PORANTHERA, Rudge in Transact. Linn. Soc. X. 302, t. 22 (1811).

P. ericifolia, Rudge in transact. Linn. Soc. X, 302, t. 22 (1811) - - - N.S.W. - - B.A.VI,55

P. ericoides, Klotzsch in Lehm. pl. Preiss. II, 239 (1847) ... W.A. S.A. - . Y. _ _ - - B.fl. VI,55

P. Huegelii, Klotzsch in Lehm. pl. Preiss. II, 231 (1847) … W.A. - - - - - - B.fl.VI,55

P. corymbosa, Brongniart in Duperr. Toy. Cog. Bot. 219, t. 56(1826) - - - V. N.S.W. - - B.fl.VI,56

P. microphylla, Brongniart in Duperr. Voy. Cog.Bot.218,t.50(1826) W.A. S.A. T. V. N.S.W. Q. N.A. B.fl.VI,56 MICRANTHEUM, Desfontaines in Mém. du Mus. hist. nat. IV, 253 (1818). (Caletia.)

M. ericoides, Desfontaines in Mém. du Mus. IV, 253, t. 14(1818) - - - - N.S.VV. Q. - B.fl.VI,57

M. hexandrum, J. Hooker in Lond. Journ. VI, 283 (1847) ... - S.A. T. V. N.S. W. - - B.fl.VI,57

PSEUDANTHUS, Sieber in Sprengel, syst. veg, cur. post. 25 (1827). (Stachystemon.)

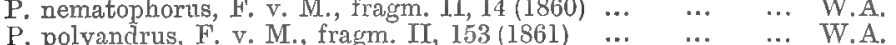

P. pimeloides, Sieber in Sprengel, syst. veg.cur. post. 25 (1827)

P. micranthus, Bentham, Fl. Austr. VI, $59(1873)$

P. divaricatissimus, Bentham, Fl. Austr. VI, 60 (1873) ...

$\mathrm{P}$, orientalis, F. V. M., fragm. II, I4 (1860)

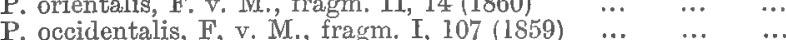

P. nematoporas, F. M. fragm. II $14(1860)$

P. brachyphyllus, F. v. M. in Transact. R. Soc. N. S. Wales 11 (1881) W.A.

P. vermicularis, F. v. M. in Transact. R. Soc. N.S.W. 11 (1881) W.A.

7). (Stachystemon.)

- - T. T. N.S.W. -

- $\quad-$ N.S.W. Q.

$--$

$-\ldots$

$-$

BEYERIA, Miquel in Amn. sc. nat. sér. trois. I, 350 (1844). (Calyptrostigma, Beyeriopsis.)

B. viscosa, Miquel in Ann. des. sc. nat. sér. trois. I, 350, t. 15 (1844) W.A. S.A. T. V. N.S. \. Q.

B. lasiocarpa, F. v. M. in Benth. Fl. Austr. VI, 65 (1873)

B. opaca, F. v. M. in Transact. phil. Soc. Vict. I, 16 (1854)

B. uncinata, F. v. M. in Benth. Fl. Austr. VI, 65 (1873)

B. Latifolia, Baillon, Adans. VI, 304 (1866)

B. cygnorum, Baillon, Adans. VI, 309 (1866)

B, cinerea, Baillon, Adlans. VI, 309 (1866) ...

B. cyanescens, Bentham, Fl. Austr. VI, 66 (1873)

B. lepidopetala, F. v. M., fragm. I, $230(1859)$

B. similis, Baillon, Adans. VI, 309 (1866) ...

B. brevifolia, Baillon, Adans. VI, 309 (1866)

B. tristigma, F. v. M., fragm. VI, 181 (1868)
- A. - T. N.S.T. -

$-$

B. Drummondii, J. Mueller in Linnaea XXXIV, $\ddot{58}$ (1865)

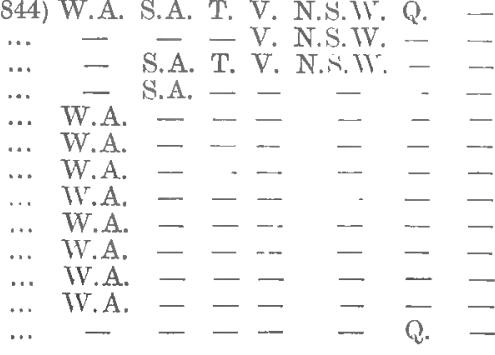
B.f. YI 64 B.f. Y. Y, 1.5 B. A. YI, $(0$. B. fl. VI, 6.5

B. fl. YT, 66 B.fl.VI, 66 B. fl. VI, 66 B.A. VI,66 B.f. Y I,67 B.fl. $Y I, 67$ B.fl.VI, 67 B.fl. VI, 68 B. fl. VI, 68
M. fr.II, 80;VI, 172 .

M.fr.II.175;VI.172; VIII,

M. fr. VI, 172; VIII,2.

M.fr.X, 3 .

M.fr.VI, 171 .
M.fr. VI, 172;VIII, 2. [12.

M.fr.X,4.

M.fr.VI, 182.

M.fr.XI, 64 .

M.fr. VI, 182 .

M.fr. XI, 64 .

M.fr. IX, 169 .

M.fr.IV, 143 .

M.fr. X, 51.

M.fr. VI, 183 .

M.fr.XI,20.

MI.fr.II, I4.

M.fr.I, 107.

MI.II, 14.

M.fr.II, $153 ;$ IV, 173

M fr. I, 230 .

NIf. Y Y I 18:.

M.fr. YI, 152 .

M.fr.I,230;III, 164.

M.fr.VI, 181. 
RICINOCARPUS, Desfontaines in Mém. du Mus. III, 459, t. 22 (1817). (Roeperia.)

R. pinifolius, Desfontaines in Mém. du Mus. III, 459, t. 22 (1817)

$\mathrm{R}$. tuberculatus, J. Mueller in Linnaea XXXIV, 60 (1865)

$\mathrm{R}$. cyanescens, J. Mueller in Linnaea XXXIV, 60 (1865)

R. psilocladus, Bentham, Fl. Austr. VI, 7 I (1873)

R. glaucus, Endlicher in Hueg, enum. is (1837) ...

R. major, J. Mueller in Linnaea XXXIV, 59 (I865)

R. Bowmanii, F. v, M., fragm. I, 181 (1859)

$\mathrm{R}$, ledifolins $\mathrm{F}, \mathrm{M}$, fram. $\mathrm{T}$ (1858)

R. rosmarinifolius, Bentham, FI. Anstr. VI, 72 (1873)

R. marginatus, Bentham, Fl. Austr. VI, 73(1873)

R. speciosus, J. Mueller in De Candolle, prodr. XV, 204 (1S63)...

R. trichophorus, J. Mueller in Limnaea XXXIV, 60 (1865) ...

R. muricatus, J. Mueller in Limnaea XXXIV, 61 (1865)

$R$, velutinus, $F$, V. NI, fragm. IX, 2 (1875)

BERTYA, Planchon in Hook. Lond. Journ. Bot. IV, 472, t. 16 (1845).

B. gummifera, Planchon in Hook. Lond. Journ. IV, 473(1845) - - - - N.S.W. - -- B.fl.VI,75

B. pinifolia, Planchon in Hook. Lond. Journ. IV, $473(1845) \ldots \ldots-$ - - N.S.W. Q. - B.fl.VI,75

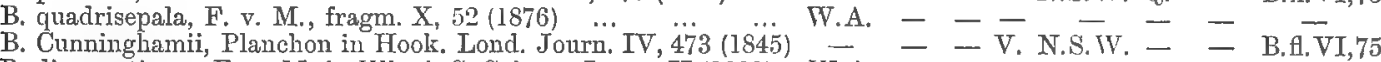

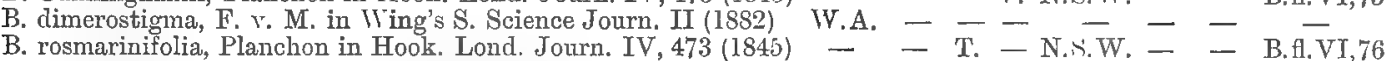

B. rosmarinifolia, Planchon in Hook. Lond, Journ. IV, $473(1845)-$ - T. - N.N.W. - - B.fl.VT,76
B. Mitchelli, J. Mueller in Linnaea XXXIV, 63 (1865)

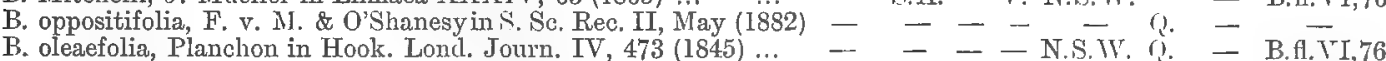

B. oleaefolia, Planchon in Hook. Lond. Journ. IV, 473 (1845) ... - - - - N.S.TV. r. - B.fl.TI,76

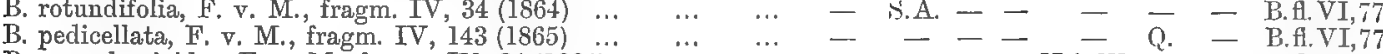

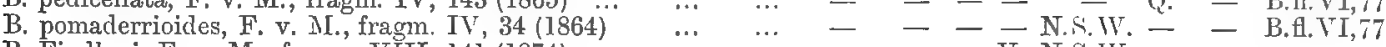

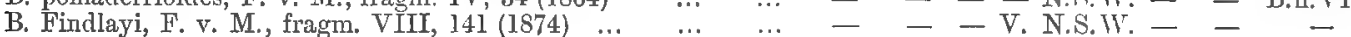
AMPEREA, Adr. de Jussieu, Euphorb. gen. 35, t. 10 (1824).

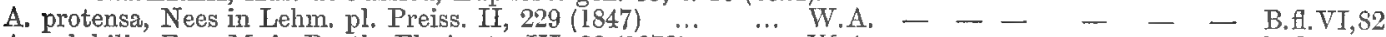

A. volubilis, F. v. M. in Benth. Fl. Austr. VI, $82(1873) \quad \cdots$ W.A. - - - - - - B.fl.VI,82

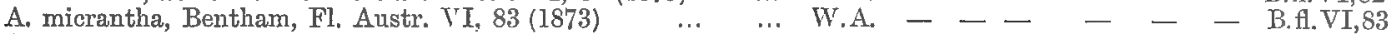

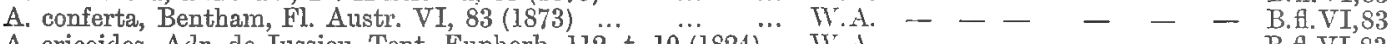

A. ericoides, Adr. de Jussieu, Tent. Euphorb. 112, t. 10 (1824) ${ }^{\text {W.A. }}$ - - - - - - B.Al.VI,S3

A. spartioides, Brongniart in Duperr. Voy. Cog. 221, t. $49(1826)$ - S.A. T. V. N.S.W. Q. - B.H.VI,84 ANTIDESMA, Linné, Fl. Zeyl. 169 (1747).

A. Ghaesembilla, Gaertner, de Fruct. I, 189, t. 39 (1788) ‥ - - - - - - N.A. B.H. YI,85

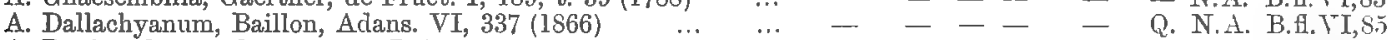

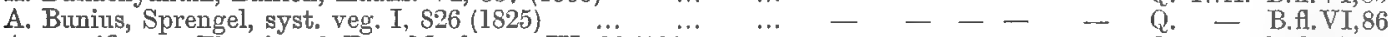

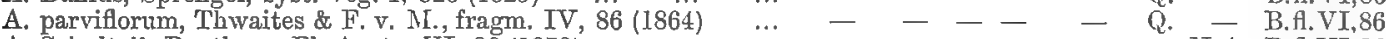

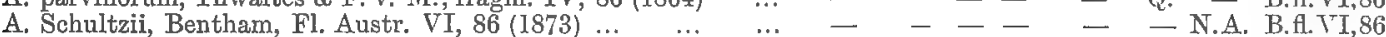

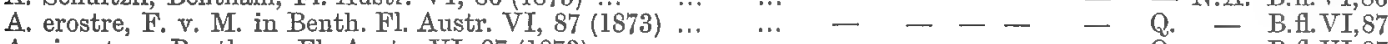

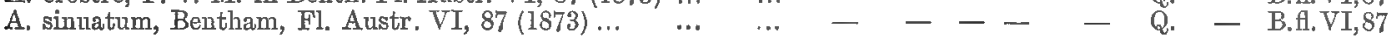
ANDRACHNE, Linné, syst. nat. 9 (1735); Linné, gen. pl. 287 (1737).

A. Decaisnei, Bentham, Fl. Austr. VI, 88 (1873)... ... ... W.A. ACTEPHILA, Blume, Bijdr. XII, 581 (1825).

A. grandifolia, Baillon, Adans. VI, $330 \& 360$, t. 10 (I866)

A. Mooreana, Baillon, Adans. YI, 330 \& 336 (1866)

A. latifolia, Bentham, Fl. Austr. VI, 89 (1873)

A. petiolaris, Bentham, Fl. Austr. VI, 89 (1873) ...

A. sessilifolia, Bentham, Fl. Austr. VI, 90 (1873) DISSILIARIA, F. v. M. in Baillon, Adans. VII, 356, pl. 1 (1S67). (Choriceras.)

D. balloghioides, F. v. M. in Baillon, Adans. VII, 356 (1867)

D. Muelleri Baillon, Adans VII $359, t, 1(1867)$

D. tricornis, Bentham, El. Austr. VI, 91 (1873) $\ldots . \quad \ldots . \quad \ldots$. PETALOSTIGMA, F. v. M. in Hook, Kew Misc. IX, 17 (1857).

P. quadriloculare, F. v. M. in Hook. Kew Mise. IX, 77 (15.57) PHYLLANTHUS, Linné, gen, pl. 282 (1737), from J. Commelyn.

P. Ferdinandi, J. Mueller in Regensb. Flora, 379 (1865)

P. lobocarpus, Bentham, Fl. Austr. VI, 97 (1873)

P. ditassoides, J. Mueller in Regensb. Flora, 487 (1864)

P. Adami, J. Mueller in De Candolle, prodr. XV, 327 (1866) ..

$\mathrm{P}$. thesioides, Bentham, Fl. Austr. VI, 9S (1873)

P. hirtellus, J. Mueller in De Candolle, prodr. XV, 326 (1866)

P. rigens, J. Mrueller in Regensb. Flora, 513 (1864)

P. ochrophyllus, Bentham, Fl. Austr. VI, 99 (1873) $\cdots$

$P$. rigidulus, $F$. v. $M$. in Limnaea XXXIV, $72(1865) \quad \ldots$.

$P$. ramosissimus, J. Mueller in Linnaea XXXIV, 70 (1865)

P. Tatei, F. V. M. in Wing's South. Science Record II, 55 (1882)

P. rhytidospermus, F. v. M. in Linnaea XXXIV, 70 (1S6อ̃) ...

$P$.

P. crassifolius, J. Nueller in Regensb. Flora, 513 (1864)...

P. elachophyllus, F, v. M. in Benth. FI. Austr. VI, 101 (1873)...

P. uberiflorus, F. v. M. in Adansonia, 343 (1S66)...

P. reticulatus, Poiret, encycl. meth. V, 298 (1804)

P. baccatus, F. v. M. in Benth. Fl. Austr. VI, 102 [(1873)

$\ldots$
$\ldots$
$\ldots$
$\ldots$
$\ldots$
$\ldots$
$\ldots$
$\ldots$
$\ldots$
$\ldots$
$\ldots$
$\ldots$
$\ldots$

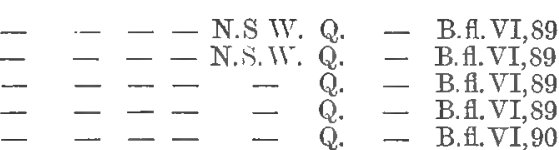

- - - Q. - B.fl.VI,90

- - - - Q. Q. N.A. B.fl.VI,91

(Hylococcus.)

- - N.S.W. Q. N.A. B.H.YI,92

(Glochiction, Synostemon, Bradleia.)

- - - N.S.W. Q. N.A. B.Al.VI, 96

- - - - - N.A. B.f.TI,97

- - - - N.A. B.fl.VI,97

- - - N.S.W. Q. - B.A.VI, 98

- - - - Q. - B.f.Y.Y,99

- - N.S.W. Q. - B.fl.VI,99

- _ - - - N.A. B.f. TY,99

- - - - N.A. B.fl.TI,99

- - - N.S.T. Q. N.A. B.H.VI, 100

S.A. - -

- - - - N.A. B.E.VI, 100

- - - Q Q - B.A.VI,100

A.

(1. - B.fl.VI,101

Q - B.T.T, 101

- N.A. B.ff. YI, 101

- N.A. B.A.TI, 102
M.fr.VI, 182 .

M.fr. VI, 182.

M.fr.I, $1>1$.

M.fr.I, 76 .

M.fr.IX,2.

M.fr. X,jo.

M.fr. X, 5,?.

MI.fr.XII.

M.fr.XII.

M.fr.IV, 34 .

M.fr. IV, 143 .

M.fr. $\Gamma, 34$.

M.fr. VIII, 141.

M.fr. TI, 182.

M.fr. TI, 183.

M.fr. Y I, 183.

M.fr. IV, $86 ; V^{\prime} I, 183$.

M.fr, VI, 183.

MI.fr. VI, 182 .

M.fr. TI,183.

M.fr.III,s9. 
P. Urinaria, Limné, sp. pl. 992 (1753)

P. trachygyne, Bentham, Fl. Austr. VI, 103 (1873)

P. Maderaspatanus, Linné, sp. pl. 982 (1753)

P. Mitchelli, Bentham, Fl Austr. VI, 103 (1873) “"

P. Gastroemii, J. 入Iueller in De Cand, prodr. X

P. Dallachyanus, Bentham, Fl. Austr. VT, 104 (1873)

P. subcrenulatus, F. v. .I., fragm. I, 108 (1859)

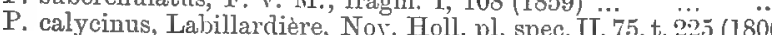

P. flagellaris, Bentham, Fl. Austr. VI, 106 (1873)

$P$. similis, J. Mueller in Limnaea XXXIV, 71 (1865)

P. microcladus, J. Mueller in Limnaea XXXIV, 71 (1865)

P. grandisepalus, F. v. M. in Linnaea XXXIV, 72 (1863)

P. Carpentariae, J. Nueller in Linnaea XXXIV, $72(1863)$

P. Fuermrohrii, F. v. M. in Transact. phil. soc. Vict. I, 15 (1854)

P. hebecarpus, Rentham, Fl. Austr. VI, 108 (1873)

P. lacunarius, F. r. II. in Transact. phil. Soc. Vict. I, $\dddot{14}$ (1854)

P. trachyspermus, F. .r. NI. in Transact. phil. Soc. Vict. I, 14 (1SJ゙)

P. australis, J. Hooker in Loncl. Journ. VI, 284 (1847) ..

P. thymoides, Sieber in Linnaea XXVIII, 566 (1866)

P. scaber, Klotzsch jn Lehm. pl. Preiss. I, 179 (1844) ...

P. indigoferoides, Bentham, Fl. Austr. VI, $110(1873)$...

P. aridus, Bentham, Fl. Austr. VI, $110(1873) \quad \ldots$
P. Gunnii, J. Hooker in Lond. Journ. VI, $284(1847) \quad \ldots$

P. simplex, Retzius, observ. V, 29 (1789)

P. filicaulis, Bentham, Fl. Austr. VI, 111 (1873).

P. minutiflorus, F. F. M. in Linnaea XXXIV, 75 (1863).

P. Armstrongii, Bentham, Fl. Austr. VI, 112 (1873)

P. buxifolius, Reinwardt in Blume, catal, plant. Buitenz. $106\left(1{ }^{2} 23\right)$ BREYNIA, R. \& G. Forster, char. gen. 145, t. 73 (1776).

B. cernua, J. Mueller in De Candolle, prodr. XV, 439 (1866) ..

B. oblongifolia, J. Mueller in De Candolle, prodr. XV, 110 (1866)

B. stipitata, J. Mueller in De Candolle, prodr. XV, 442 (1866) ..
B. rhynchocarpa, Bentham, Fl. Anstr. VI, $114(1873) \ldots$. SECURINEGA, A. L. de Jussieu, Gen. 388 (1789). (Flneggea.)

S. Abyssinica, A. Richard, tentam. fl. Abyss. II, 256 (1851) ... -

S. Lencopyrus, J. Mueller in De Cand., prodr. XV, 451 (1866).. NEOROEPERA, F. \& J. Mueller in De Candolle, prodr. XV, 489 (1866).

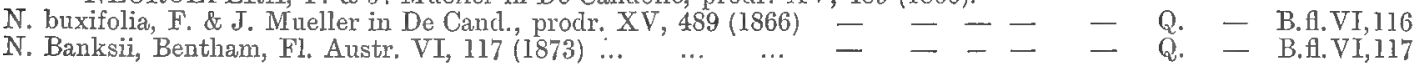

N. Banksii, Bentham, Fl. Austr. VI, 117 (1873) : BISCHOFFIA, Blume, Bijclr. 1168 (1825).

B. Javanica, Blume, Bijdr. 1168 (1825) HEMICYCLIA, Wight \& Arnott in Ediub. phil. Journ. XIV, 297 (1833).

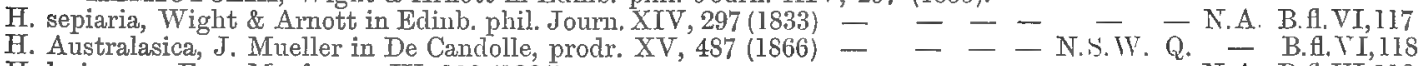

H. lasiogyna, F. v. M., fragm. IV, $119(1864) \quad \ldots \quad \ldots \quad \ldots \quad-\quad-\quad-\quad-\quad--\quad$ N.A. B.fl.VI,118 APOROSA, Blume, Bijdrag. 514 (1824).

A. Australiana, F. v. M., fragm. XII ined. BRIDELIA, Tilldenow, Spec, IV 978 (1805).

B. exaltata, F, v. M., fragm. III, 32 (1862)

B. ovata, Decaisne in nouv. ann. du Mus. V, 484 (1835)..'

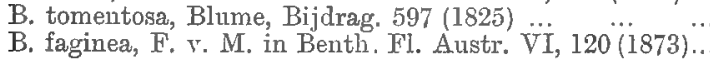
CLEISTANTHUS, J. Hooker, icon, pl, t. 779 (1847).

C. Cunninghamii, J. Mueller in De Cand, , prodr. XV, 506 (1866)

C. apodus, Beutham, Fl. Austr. VI, 122 (1873)

C. Dallachyanus, Baillon in Benth. FI. Austr. VI, 122 (1873) ...

C. semiopacus, F. v. M. in Benth. Fl. Austr. VI, 123 (1873) ... CROTON, Linné, gen. pl. 288 (1737).

C. Schultzii, Bentham, FI, Austr. VI, $12 \frac{1}{(1873) . .}$

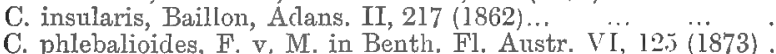

C. opponens F. . M. M. in Benth. H. Austr. 125 (1S73)

C. tomentellus, F, v. M., fragm. IV, 141 (1864) ...

C. Verreauxii, Baillon, Etud. Euph. $3.57(1858)$... $\quad \ldots$

C. acronychioides, F. v. M., fragm. IV, $142(1864)$

C. triacros, F. v. M., fragm. VI, $185(1867)$
C. Arnhemicus, F. v. M. in Linnaea XXXIV, $112(1865)$ ALEURITES, R. \& G. Forster, char. gen. 111, t. 56 (1776).

A. triloba, R. \& G. Forster, char. gen, 111, t. 56 (1776)...

CLAOXYLON, Adr. de Jussieu, Euphorb. gen. 43, t. 14 (18:4).

C. angustifolium, J. Mueller in Limaea XXXIV, 165 (1865) ... -

C. tenerifolium, F, v. M. in Baillon, Aclans. VI, 323 (1866) $\ldots$.

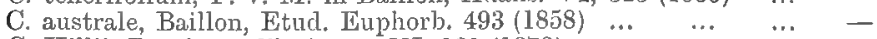

C. Hillii, Bentham, FI. Austr. VI, 131 (1873) ... ... ... ACALYPHA, Royen in Linné, hort. Cliffort. 495 (1737).

A. nemorum, F. . . X, in Linnaca XXXIV, 38 (1865) ... ... -

- N.A. B.fl. TI, 102

- - - N.S.W. - - B.f.TI,119

- - N.S.W. Q. - B.H.VI,1:2

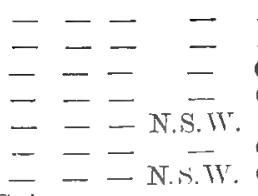

N.A. B.fl. YI, 103

Q. N.A. B.f.VI, 103

- B.f.VI,104

- - N.W.W. Q. - B.fl.VI,104

- - B. B. VI, 105

- - - - Q. - B.f.VI,106

- - N.S.IT. Q. - B.fl.VI,106

- - - - N A. B.fl.VI,106

- - - - N.A. B.fl.VI, 107

- - - - G. N.A. B.f.VI, 108

S.A. - Y. N.S.II. - - B.f.VI, 108

S.A. - V. N.S.W. - - B.fl.VI, 108

- T. - N.S.W - - B.fl VI,108

B. Al. VI, 109

N.A. B.fl.VI,110

- N.A. B.fl.VI, 110

S.A. T. V. N.S.W. - - B.f.VI,110

- - - T Q Q B.fl.VI,111

- - - Q. N.A. B.fl.VI,112

- - - - N.A. B.f.VI,112

thesa, Melanthesiopsis.)

- - - N. - Q. N.A. B.fl.VT,113

- - - - Q. N.A.B.fl.VI,114

- - - - N.A. B.f.VI,114

- - - - Q. N.A. B.f.VI,116

- - - Q. - -

- - - - - N.A. B.fl.VI, 120

Q. N.A. B.fl.VI, 120
Q. - B. I.VI, 120

Amanoa.)

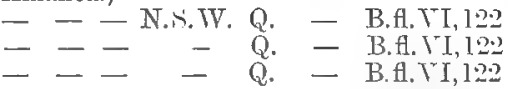

- - - - Q. Q B.fl.YI,122

- - - - - N.A. B.fl. VI, 124

- - - N.S.IT. Q. - B.f.VI,124

- - - N.S.W. Q. - B.fl. YI, 1.5

- - - Q. - B.flYI, I25

- - - - - N.A. B.f.YI, 126

-- - N.S.W. Q. N.A. B.fl.VI,126

$-\quad-\quad-$ Q. - B.flYI,

- - - Q. - B.f.रI, I.2

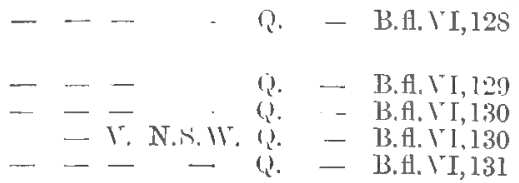

II. fr. IV, 140.

M.fr. IV $1+1$

M. fr.I I $141 ; Y^{\top} I, 186$.

II fr.TI, 186 .

M.fr. VI, 18.5.

M.fr, YI, 186 .

M.fr. YI, 13:.

MI.fi. YT, 183.

M.fr, IV, I t?. 
A. eremorum, F. v. M. in Regensb. Flora 440 (1864) $\quad \ldots \quad \ldots \quad-\quad-\quad-\quad-\quad-\quad$ Q. $\quad$ - B.A.VI, 132

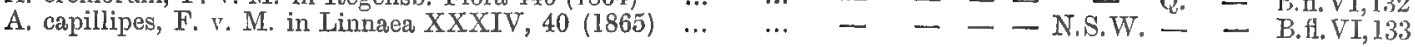
ADRIANA, Gaudichand in Ann. se, nat. V, 223 (1825). (Trachycaryon.)

A. tomentosa, Gaudichaud in Aun, sc. nat. V, 2223 (1825) … W.A. S.A. - V. N.S.W. O. N.A. B.A.VI,134

A. quadripartita, Gaudichaud in Freyc. voy, Bot. 489 (1826) … W.A. S.A. - V. ALCHORNEA, Solander in Swartz, prodr. 698 (1788). (Coelebogyne, Cladodes.)

A. ilicifolia, J. Mueller in Limnaea XXXIV, $170(1865) \ldots \ldots$.... - - - N.S.W. . . - B.G.VI,136

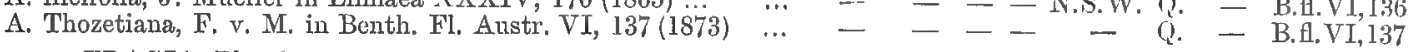
TRAGIA, Plumier, gen. 14, t. T22 (1703).

T. Novae Hollandiae, J. Mueller in Linnaea XXXIV, 180 (1865) - - - - - Q. - B.Al.VI,137 MALLOTUS, Loureiro, Fl, Cochinch. II, 635 (1790). (Echinus, Rottlera, Echinocroton.)

M. ricinoides, J. Mueller in Linnaea XXXIV, IS7 (1865) ‥ - - - - - B.f.VI,140

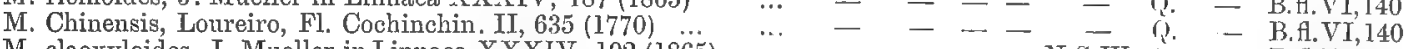

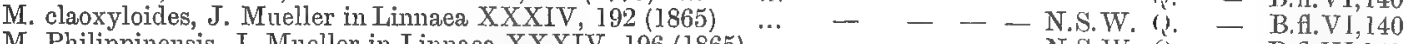

M. Philippinensis, J. Mneller in Limnaea XXXIV, 196 (1865) … - - - - N.S.W. .. - B.H.VI,141

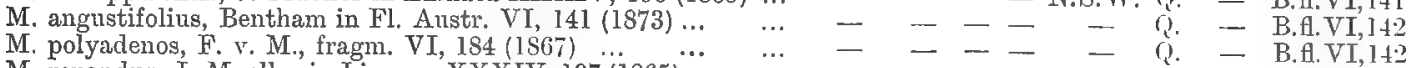

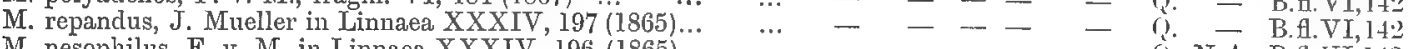

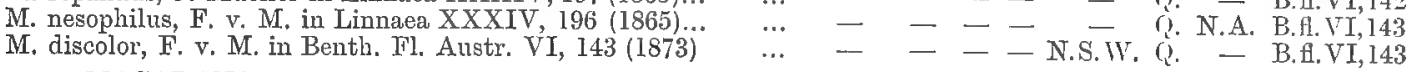
MACARANGA, Petit-Thouars, Gen. Madag. n. 88 (1809). (Mappa.)

M. Dallachyi, F. r. M. in Benth. Fl. Austr. VI, 144 (1873) ‥ - - - - Q. - B.fl.VI,144

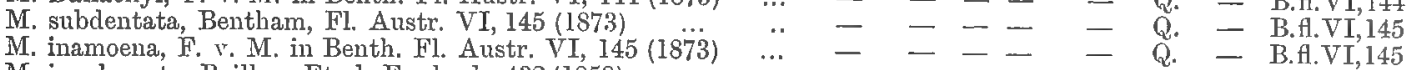

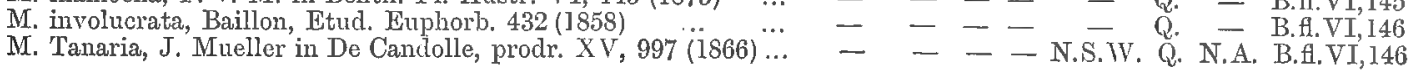
CODIAEUM, Rumphins, herbar. Amboin. IV, 65, t. 25 (1743).

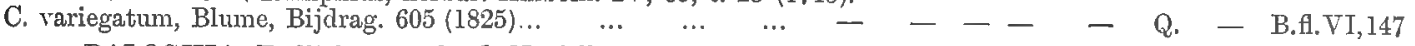
BALOGHIA, Endlicher, prodr. fl. Norfolk, 84 (1833).

B. lucida, Endlicher, prodr. A. Norfolk. 84 (1833) $\quad \ldots \quad \ldots \quad-\quad-\quad-\quad$ N.S.W. Q. $\quad-$ B.甘.VI,148 FONTAINEA, Heckel, thése inaugurale, Montpell. (1870).

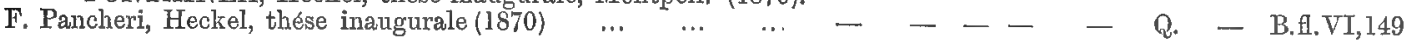
OMALANTHUS, Adr. de Jussieu, Euphorb. gen. 50, t. 16 (1824). (Carumbium, Wartmanuia.)

O. populifolius, Graham in Bot. Mag. 2780 (1827) ... ... - - - V. N.S.W. Q. - B.A.VI, 150

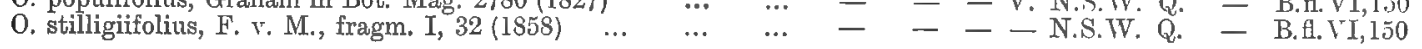
SEBASTIANTA, Sprengel, Neue Entd. II, 118 (1821). (Microstachys, Elachocroton.)

S. chamelaea, J. Mueller in De Cand., prodr. XV, 1175 (1866)... - - - Q N.A. B.f.VI,151 EXCAECARIA, Linne, syst. veg. ed. decim. 1288 (1759).

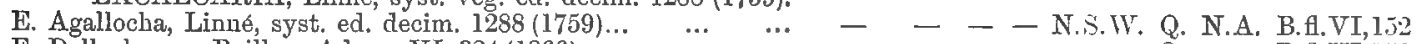

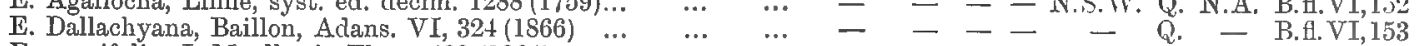

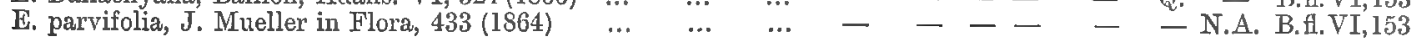

\section{URTICACEAE.}

Ventenat, Table III, 524 (1799).

CELTIS, Tournefort, inst. 612, t. 383 (1700), from Camerarius (1586). (Solenostigma.)

C. Philippinensis, Blanco, FI, de Filipinas. 197 (1837) … … - N. B. A.VI,156

C. paniculata, Planchon in Ann. sc. nat. sér. trois. $X, 305(1848)=-Z-$ N.S.W. Q. N.A. B.H.VI, $=$

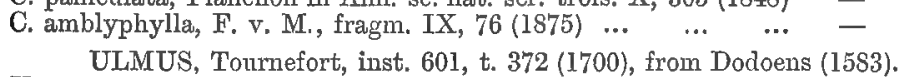

U. parvillora, N. Jacquin, pl. rar. hort. Schoenbr. descr, et icon.

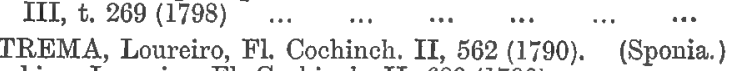

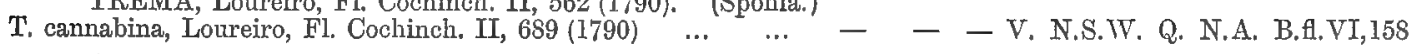
APHANANTHE, Planchon in Ann, sc, nat. X, 265 (1848).

A. Philippinensis, Planchon in Ann. sc. nat. X, $337(1848)$ ‥ $-\quad-\quad$ N.S.W. Q. - B.fl.VI,160 FICUS, Tournefort, inst. 662 (1700), from Plinius. (Urostigma, Covellia.)

F. colossea, F. T. M. in Benth. Fl. Austr. VI, 163 (1873)

F. pilosa, Reinwardt in Blume, Bijdr. 446 (1825)...

F. nesophila, F. v. M., Docum. Interc. Exhib. 26 (1866) $\ldots$

F. Cunninghamii, Miquel in Ann. Mus. Lugd, Bot. III, $286(1867)$
F. Henneana, Miquel in Ann. Mus. Lugd. Bot. III, $216(1867) \ldots$

F. validinervis, F. v. M. in Benth, Fl. Austr. VI, 166 (1573) ...

F. retusa, Linné, mantissa plant. 129 (1767)

F. engenioides, F. v. M., Docum. Interc. Exhib. 26 (1866) $\quad \ldots$

F. Benjaminea, Linne, mantissa plant. 129 (1767)

F. Muelleri, Miquel in Ann. Mus. Lugd. Bot. III, 287 (1867) $\ldots$

F. leucotricha, Miquel in Ann. Mus. Lugd. Bot. III, 285 (1867)

F. rubiginosa, Desfontaines, catal. pl. hort. Paris. 209 (1804) ...
F. columnaris, F. v. M. \& Moore in proc. AccI. Soc. Vict. III,

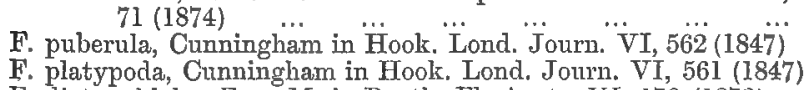

F. platypoda, Cunningham in Hook. Lond. Journ. VI, 561 (1847)

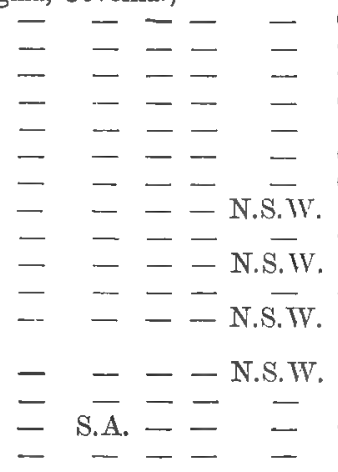

Q. - B.fl.VI, 163

Q. - B.fl.VI, 164

Q. N.A. B.f.VI, 164

Q. - B.Al.VI, 165

Q. N.A. B.H.VI, 165

Q. - B.H.VI, 166

Q. N.A. B.H.YI, 166

Q. N.A. B.A.YI, 166

Q. - B.fl. II, 167

- - B.fl.VI,167

- N.A. B.H.YI,167

Q. - B.f.TI, 168

- N.A. B.A. VI, 169

Q. N.A. B.fl.VI, 169

Q. - B.f.VI, I,0
M.fr. IX,2.

M.fr. YI, 183.

M. fr.IV, 138;VI, 1S4.

M.fr. VI, 184 .

M.fr.VI, 184.

M.fr. VI, 183.

M.fr. VT, 184.

M.fr. TI, 185

M.fr. TI, 184 .

M.fr.IV, 139 .

M.fr.VI, 184.

M.fr. TV, 139; TI, 183. M.fr.VI, 183 .

M.fr.VI, IS?.

MI.fr, VI, 182 .

M.fr.I, 32 .

II. fr. I, $; 2$.

M.fr. XI, 64 .

ML.fr.IV, 88 .

M.fr. IX, 76 .

M.fr. VI, 101;XI, 133.

M.fr. VIII, 247.

NI.fr. VI, 195.

M.fr.VI, 195 .

M.fr. VI, 195;VIII, 246.

MI.fr. TI, 195.

M.fr. TI, 195.

M.fr.VI, 195.

M.fr.VI, 195; VIII, 2403.

MI.fr. VI, 195.

M.fr. VI, 194; VIII, 247.

M.fr. VIII, 247.

M.fr.VI, 194; VIII, 247. 
F. macrophylla, Desfontaines, catal. pl. hort. Paris. 209 (1S04)

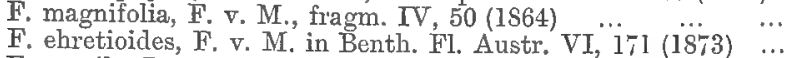

F, pumila, Linné, spec. plant, $1060(1753)$

F. coronulata, F. v. M. in Journ. Bot. Neerland 241 (1861) $\quad \ldots$

F. leptoclada, Bentham, Fl. Anstr. TT, $172(1873)$

F. depressa, Bentham, Fl. Austr. VI, 172 (1873)...

F. Philippinensis, Miquel in Hooker, Lond. Journ. VII 435 (1848)

F. mollior, F. v. M. in Benth. Fl. Austr. VI, 173 (1873)

F. stenocarpa, F. v. M. in Benth. Fl. Austr. V I, 174 (1873)

F. scabra, G. Forster, florul. ins. Austr. prodr. 76 (1786)

F. orbicularis, Cunningham in Hook. Lond. Journ. VII, 426 (184S)

F. aculeata, Cunningham in Hook. Lond. Journ. VII, 426 (1848)

F. opposita, Miquel in Hook. Lond. Journ. VII, 426 (1848)

F. scobina, Bentham, Fl. Austr. TI, 176 (1873) ... $\quad . . \quad$..

F. hispida, Linne, fil. suppl. 442 (1781) …
F. fasciculata, F. v. M. in Benth. Fl. Austr. TI, 17 (1873) $\quad \ldots$

F. casearia, F', v. M. in Benth. Fl. Austr. VI, 177 (1873)

F. glomerata, Willdenow, spec. pl. IV, 1148 (1S06)

F. pleurocarpa, F. v. MI., fragm. VIII, 246 (1874) CUDRANIA, Trécul in Ann. des. sc. nat. VIII, 122 (1847).

C. Jaranensis, Trécul in Ann. des. sc. nat. VIII, 123 (1847) … ANTIARIS, Leschenault in Ann. du Mus. XVI, 476 (1810).

A. macrophylla, R. Brown in Flind. voy. II, 602, t. 5 (1814) ... MALAISIA, Blanco, Fl. Filip. 789 (1837).

M. tortuosa, Blanco, El. Filip. 789 (1S37) ... PSEUDOMORUS, Burean in Ann. des sc. nat. sér. cinq. XI, 372 (1872).

P. Brunoniana, Bureau in Ann. des sc, nat. sér. cinq. XI, $372(1872)$ - - - N.S.T. Q. - B.fl.VI,181 FATOUA, Gaudichaud in Freyc. voy. Bot. 509 (1826).

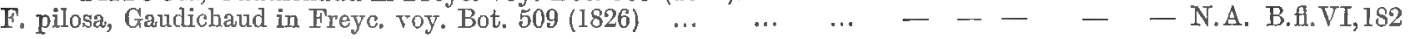
ELATOSTEMMA, R. \& G. Forster, char. gen. 105, t. $53(1 ; 76)$.

E. reticulatum, Weddell in Ann, des sc. nat. sér. 4, I, $188(1854)$ -

E. stipitatum, Weddell in Ann. des sc. nat. sér. 4, I, 190 (1854) PROCRIS, Commerçon in A. L. de Jussieu, gen. 403 (1789).

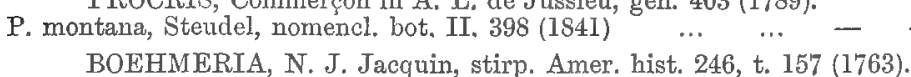

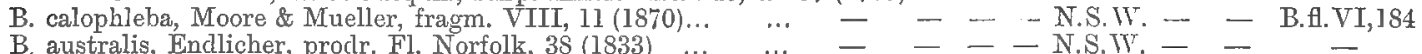

B. australis, Endlicher, prodr. Fl. Norfolk, 38 (1833) $\ldots \ldots \ldots$ …
PIPTURUS, Weddell in Ann. des sc. nat. sér. quatr. I, 196 (1S54).

P. propinquus, Weddell in Archiv. du Mus. 447, t. 15 (1856) ... - - - - N.S.W. Q. - B.A.VI,185 POUZOLZIA, Gaudichand in Freyc. voy. Bot. 503 (1826). (Memorialis, Hyrtanandra.)

P. Indica, Gaudichand in Frejc. voy. Bot. 503 (1826) $\quad \ldots \quad \ldots \quad-\quad-\quad-\div-$ Q. N.A. B.fl.VI, 186

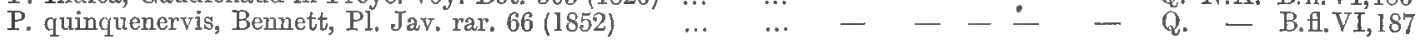
PARIETARIA, Tournefort, inst. 509, t. 289 (1700), from C. Bauhin (1623). (Freirea.)

P. debilis, G. Forster, florul. ins. Austr. prodr. 73 (1786) ‥ TT.A. S.A. T. V. N.S.W. Q. N.A. B.f.VI, 1S8 AUSTRALINA, Gaudichaud in Freyc. voy. Bot. 497 (1826).

A. pusilla, Gaudichaud in Freyc. voy. Bot, 505 (1826) ... . FLEURYA, Gaudichaud in Freyc. voy. Bot. 497 (1826).

F. intermpta, Gaudichaud in Freyc. voy. Bot. 497 (1826) $\ldots$
URTICA, Tournefort, inst. 534, t. 308 (1700), from Plinius.

U. incisa, Poiret, Encycl. meth. suppl. IV, 223 (1817) ... ... LAPORTEA, Gaudichaud in Freyc, voy. Bot. 498 (1826).

L. gigas, Weddell in Arch. du Mus. IX, 129, t. 3 \& 4 (1856) ...

L. photiniphylla, Weddell in Arch. du Mus. IX, 138 (1856)

L. moroides, Weddell in Arch. du Mus. IX, 142 (1856) ...

\section{.}

- $\quad$ - T. V. N.S.W. -- - B.fl.VI,189

$-\quad-\quad-$ Q. - - M.fr.X,114.

- S.A. T. V. N.S.Wr. Q. - B.fl.VI,190

- $\quad$ - - N.S.W. Q. - B.f.TT,192

... - - - - N.S.W. Q. - B.fl.VI, 192

\section{CUPULIFERAE.}

L. Cl. Richard, Anal. de fr. 32 \& 92 (1808).

FAGUS, Tournefort, inst. 584, t. 351 (1700), from Camerarius (1586).

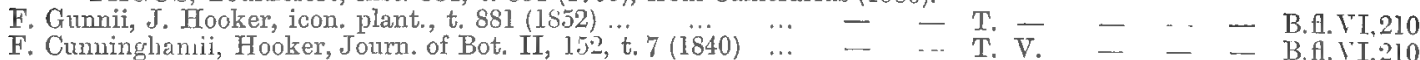

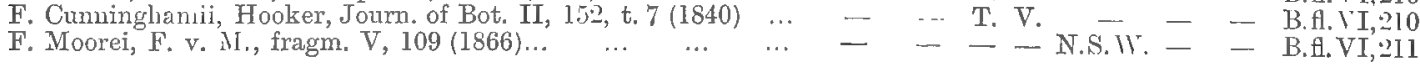

BALANOPS, Baillon, Hist. des pl. VI, $237 \& 258$ (1876).

B. Australiana, F. v. M., fragm. X, $114(1877)$

\section{CASUARINEAE.}

Mirbcl in Am. du Mus. XTI, 451 (1S10).

CASUARINA, Rumphius, herb. Amboin. III, 87, t. 58 (1748).
C. quadrivalvis, Labill., nov. Holl. pl. spec. II, 67, t. 218 (1S06)
C. trichodon, Miquel in Lehm. pl. Preiss. I, 641 (1845) ...
C. lepidophloia, F. v. M., fragm. X, 115 (1877)
W.A.
A.A. T. V. N.S.W. -
C. glauca, Sieber in Sprengel, syst. III, 803 (1826)
- B.fl. I I,10.5 B.fl. VI, 196 B. fl. VT, 196
M.fr. VT,18\& $19 ; \mathrm{X}, 62$,
Mi.fr, YI, 17.
M.fr. X, 115.

M.fr.VII, 247 .

M.fr. T'III,248.
M.fr. V, 109 .

MI.fr.X, 114.

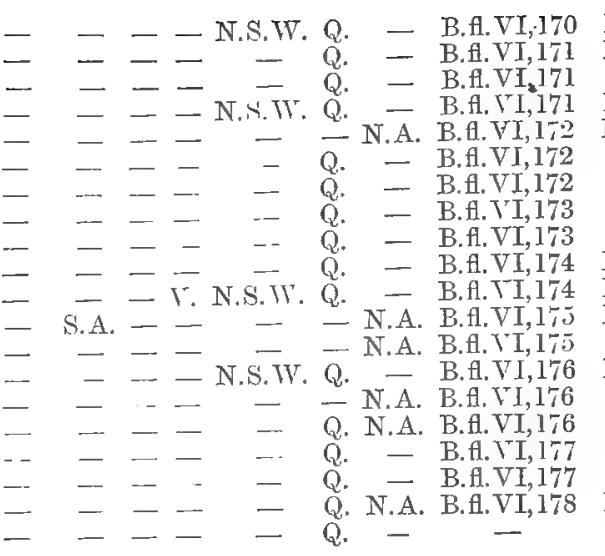

M.fr. VI, 194; VIII, 247.

M.fr.IV,50\& $177 ; V I, 196$.

M.fr. VI, 196.

[246.

M.fr.IV, 49; VI, 196;VIII,

M.fr.IX, 152.

[114.

If.VI.196;IX, 152;X,

M.fr.VI, 196.

M.fr. VI, 196;VIII, 246.

M.fr. VI, 195

M.fr. VITI, 246.

M.fr. VIII, 247.

M.fr. VI, 194.

M.fr.VI, 193\&255;VIII,

M.fr.IX, I69.

M.fr. VIII, 11

M.fr.IX, 169.

M.fr.VI, 197.

M. fr.IV,87.

M.fr.XI, 20 . 
C. Huegeliana, Miquel in Lehm. pl. Preiss. I, 640 (1845)

C. equisetifolia, R. \& G. Forster; char, gen. 103, t. 52 (1776)

C. suberosa, Otto \&-Dietrich, allgem. Gartenzeit. 155 (1841) .

C. Cuminghamiana, Miquel, Revis. Casuarin. 56, t. 6(1848) ..

C. distyla, Ventenat, pl. dans le jard. de Cels. t. 62 (1800)

C. Fraseriana, Miquel, Revis. Casuarin. 50, t. 6 (1848) ...

C. nana, Sieber in Sprengel, syst. III, 804 (1826)...

C. humilis, Otto \& Dietrich, allgem. Gartenzeit. 163 (184i)

C. torulosa, Aiton, hort. Kew. III, 320 (1789)

C. decussata, Bentham, Fl. Austr. VI, 200 (1873) ...

C. Drummondiana, Miquel, Revis. Castar, 26, t. $\dddot{1}$ (1848)

C. microstachya, Miquel in Lehm. pl. Preiss. I, 642 (1845)

C. acutivalvis, F. v. M., fragm. $X, 61$ (1876)

C. bicuspidata, Bentham, FI. Austr. VI, 202 (1873)

C. thuyoides, Miquel in Lehm. pl. Preiss. I, 641 (1845) ...

C. acuaria, F. v. M., fragm. VI, 16 (1868)...
C. inophloia, F. y. M. \& Bailey in Melb. Chemist, April (1882)

C. Decaisneana, F. r. M., fragm. I, 61 (1858)

C. corniculata, F. v. M., fragm. X, $62(1876)$

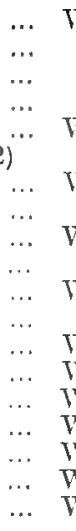

IV.A. - - - - N.S.W. Q. . N.A. B.f.VI, BI,197

- - T. V. N.S.W. O. - B.A.VI,197

- - - N.S.W. Q. - B.fl.VI,198

W.A. S.A. T. V. N.S.IV. - - B.fl.VI,19S

V.A. - - - Q. - B.f.

- - - - N.S.W. - - B.fl.VI,199

W.A. - - - - - - B.A.VI,200

- - - - N.S.IV. . - B.fl.VI, 200

V.A. - - - - - B.f.VI,200

- S.A. - - - - N.A. B.fl.VI,201

IT.A. - - - - - B.fl.VI,201

IV.A. - - - - - - B.H.VI,201

W.A. - T - - -

W.A. - - - - - B.f.VI,202

W.A. --

$\div-$

PIPERACEAE.

L. Cl. Richard in Humb. Bonpl. \& Kunth, Nov, gen, I, 46 (1815).

PIPER, Linné, gen. pl. 333 (1737), from C. Bauhin (1623). (Macropiper.)

P. subpeltatum, Willdenow, spec. plant. $V, 166$ (1810) ...

P. excelsum, G. Forster, florul. ins. Austr. prodr. 5 (1786)

P. Novae Hollandiae, Miquel in Medd. Alrad. Vetensk. Amsterd. sec. ser. II, 8 (1866)

P. Banksii, Miquel in Medd. Akad. Amst. sec. ser. II, $\dddot{9}(1866)$

P. triandrum, F. v. M., fragm. V, $197(1866) \quad \ldots \quad \ldots$

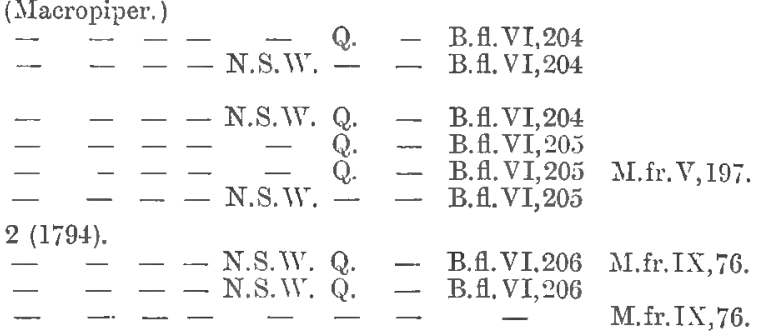

$P$. hederaceum, Cumningham in De Cand. prodr. XVI, 366 (1869)

PEPEROMIA, Ruiz \& Pavon, fl. Peruv, \& Chil. prodr. s, t 2 (1794)

P. leptostachya, Hooker \& Arnott, Bot. Beech. 96 (1841)

P. reflexa, A. Dietrich, spec, plant. I, I80 (1831) ...

P. Urvilleana, Ach. Richard in d'Urrille, voy, Bot. 356 (1832)...
M. fr. VI, $19 ; \mathrm{X}, 62$.

M.fr. VI, 17.

M.fr. VI,20\&254.

MI.fr, VI, 20 .

M.fr.XII.

II.fr. VI, 19.

M.fr.VI, 18.

M.fr.VI, 17 .

I.fr.I, $61 ; X, 62$.

M.fr. VI, I7; $\mathrm{X}, 62$.

M.fr.X, 61 .

MI.fr. VI, 17.

M.fr.X, 62 .

M.fr. VI, $16 \& 250$.

\section{PODOSTEMONEAE.}

L. Cl. Richard in Humb. Bonpl. \& Kunth, Nov. gen. I, 246 (1S15).

Genus and species not yet determined

\section{NEPENTHACEAE.}

Reichenbach, Conspectus regni vegetabilis 45 (182S).

NEPENTHES, Linné, syst. nat. 9 (1735); Linné, gen. pl. 273 (17.37).

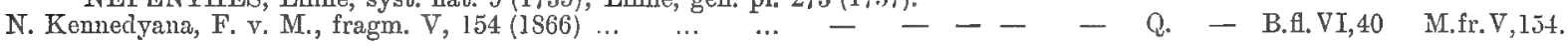

\section{ARISTOLOCHIEAE.}

A. L. de Jussieu, in Ann. du Mus. V, 221 (1801).

ARISTOLOCHIA, Tournefort, inst. 162, t. 71 (1700), from Hippocrates, Theophrastos \& Dioscorides.
A. deltantha, F. v. M., fragm. VI, 179 (1869)
A. praevenosa, F. v. M., fragm. II, 166 (1861)
A. pubera, R. Brown, prodr. 349 (1810)
$\begin{array}{llll}\ldots & \ldots & \ldots & - \\ \ldots & \ldots & \ldots & - \\ & \ldots & \ldots & - \\ \ldots & \ldots & \ldots & - \\ \ldots & \ldots & \ldots & -\end{array}$
$-\quad-$ N.S.W. Q.
$-\quad-$ N.S.W. Q.
$-\quad-\quad$ Q
B.fl. Y 1,208
B.fl. VI,205
A. Thozetii, F. v. M., fragm. II, 167 (1861)
A. Indica, Limné, spec. plant. 960 (1753) ...
MI.fr.VI, 1798255. M.fr. VI, 180 .
II.fr.II, 167.

\section{BALONOPHOREAE.}

L. Cl, Richard in Mém. du Mus. VIII, 404 \& 429 (1822).

BALONOPHORA, R. \& G. Forster, char. gen. 99, t. 50 (1776).

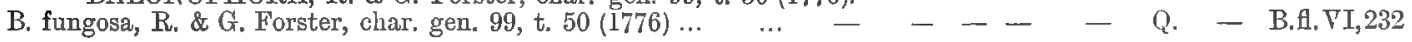

\section{VINIFERAE.}

J. de St. Hilaire, Exp. fam. II, 48, t. 79 (1805).

VITIS, Tournefort, inst. 613, t. 384 (1700), e Latinis. (Cissus.)

V. Baudiniana, F. v. M., fragm. IV, $136(1864)$...

V. oblonga, Bentham, FI. Austr. I, 447 (1863)

V. cordata, Mrallich in Bentham's flora Hongk. 54 (186i)

V. adnata, Wallich in Wight \& Amott, prodr. 126 (1834)

V. nitens, F. V. M., fragm. II, 73 (1860)

V. brachypoda, F. v. M., fr. IX, 125 (1875)

V. saponaria, Seemann, Bonplandia, 254 (1859) ...

V. acris, F. v. M., fragm. II, 75 (1860)

V. trifolia, Linné, spec. plant. 203 (1753) ...

V. clematidea, F. v. M., fragm. II, 74 (1860)

V. arcetosa, F. v. M., plant. of Vict. I, 94 (1860)

V. hypoglauca, F. v. M., pl. of Vict. I, 95 (1860)...

V. sterculifolia, F. v. M. in Benth. Fl. Austr. I, 450 (1863)

V. penninervis, F, v. M., fr. VI, 177 (1868)

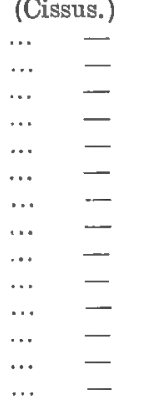


F. opaca, F. . M. in Benth. Fl. Austr. I, 450 (1863) .

T. augustissima, F. v. M., fragm. I, 141 (1859) ...

LEEA, Linné, mantiss. 17 (1767).

L. staphylea, Roxburgh, hort. Beng. 18 (1814) ...

L. aculeata, Blume, Bijdrag. 197 (1825)

$$
\begin{aligned}
& \text {.. - - - - N.S.W. Q. - B.H.I,450 } \\
& \text { B.f. I, } 450
\end{aligned}
$$

M.fr. VI, $17 \% ; I X, 125$

M.fr. I, 244; II, 180; IX, 126.

\section{SAPINDACEAE.}

\section{A. L. de Jussieu, Gen. 246 (1789).}

CARDIOSPERMUN, Linné, syst. nat. (1735); Linné, gen. pl. 117 (1737).

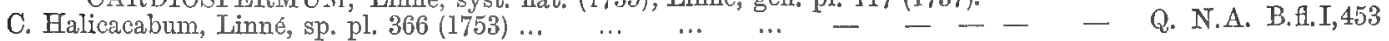
GANOPHYLLUM, Blume, Mus. Bot. Lugd. I, 230 (1850).

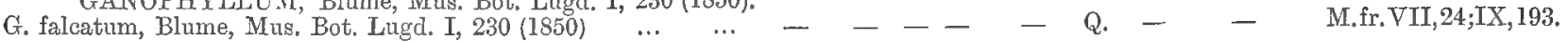
ATALAYA, Blume, Rumphia III, 186 (1847). (Pseudatalaya, Sapindus partly.)

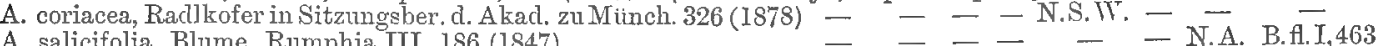

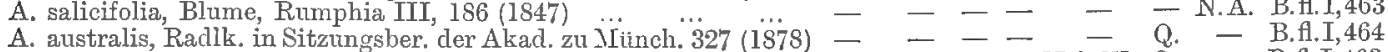

A. multiflora, Bentham, Fl. Austr. I, 463 (1863) ... ... ... - - - - N.S.W. Q. - B.f.I, 463

A. hemiglanca, F. v. M. in Benth. Fl. Austr. I, 463 (1863) $\ldots . .-$ S.A. - - N.S.W. Q. N.A. B.A.I,463

A. variifolia, F. v. M. in Benth. Fl. Austr. I, $463(1863)$ ‥ - - - - - - N.A. B.fl., 463 DIPLOGLOTTIS, J. Hooker in H. \& B. gen. pl. 395 (1862). D. Cunninghamii, J. Hooker in H. \& B. gen. pl. 395 (1862) … -
ERIOGLOSSUM, Blume, Bijdr. 229 (1829). (Pancovia partly.)

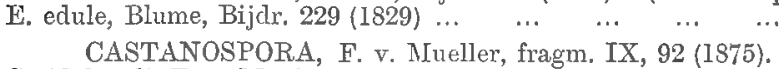
C. Alphandi, F.. M., fragm. IX, $92(1875) \ldots \ldots \ldots \ldots$
ALLOPHYLUS, Linne, amoen. acad. 124 (1747).

A. ternatus, Loureiro, Fl. Cochinch. ed. Willd. $286(1790)$ ‥ - - - - - Q. N.A. B.fl.I,455 M.fr.IX,90. CUPANIA, Plumier, Gen. 45, t. 19 (1703). (Guioa, Ratonia, Aryteria, Elattostachys, Mischocarpus, Euphoria partly.)

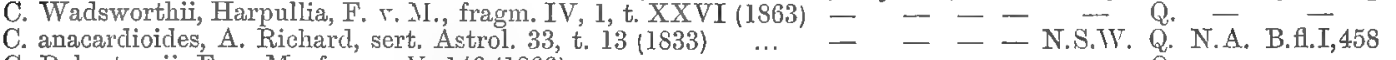

C. Robertsonii, F. v. M., fragnı. V, $146(1866)$...

-C. serrata, E. V. M., fragm. III, 43 (1862)...

C. foveolata, F. v. MI., fragm. IX, 95 (1875)

C. tomentella, F. v. M. in Benth. Fl. Austr. I, $4 \ddot{5} 8$ (1863)

C. pseudorhus, A. Richard, sert. Astrol. 34, t. 14 (1833)

C. xylocarpa, Cunningham in Benth. Fl. Austr. I, 459 (1863) ...

C. erythrocarpa, F. v. MI., fragm, V, 7 (1865)

C. Mortoniana, F. v. M., fragm. V, 177 (1866)

C. lachnocarpa, F. v. M., fragm. IV, 157 (1864) ...

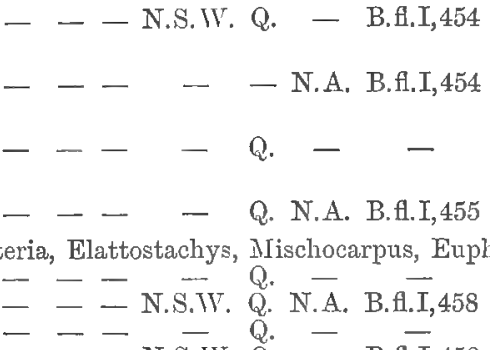

… - - - - N.S.W. Q. - B B.I,45s

‥ - - - - - - Q Q.

- - - N.S.W. Q

- - - N.S.W. Q. - B.fl.I,459

- - - - Q

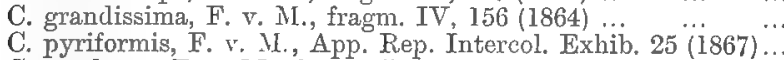

C. anodonta, F. v. M., fragm. TI, 76 (1860)

C. stipitata, F. v. M., fragm. II, 75 (1860)

C. punctulata, F. V. II., fragm. IIT, 12 (1862)

C. exangulata, F. v. M., fragm. IV, 156 (1864) ...

C. O'Shanesiana, F. .. M. fragm. IX, 96 (1875) ...

C. Daemeliana, F. r. iI., fragm. IX, 96 (1875) ..

C. Martyana, F. v. M., fragm. V, 6 (1865)

C. Cordieri, F. v. M., fragm. IX, 93 (1875)

C. tenax, Cunningham in Benth. Fl. Austr. I, 461 (1863)

C. semiglauca, F. v. M. in Benth. Fl. Austr. I, 457 (1863)

NEPHELIUM, Linné, mantiss. 18 (1767). (Euphoria partly, Alectryon, Spanoghea.)

N. connatum, F. r. M. in Benth. Fl. Austr. I, 465 (1863) - - N.S.W. Q.

N. semicinereum, F. v. M. fra. IV, 158 (1864)

N. subdentatum, F. v. M. in Benth. Fl. Austr. I, 465 (1̈̈63)

N. tomentosum, F. v. M. in Transact. Vict. Inst. II, 64 (1858)

N. coriaceum, Bentham, Fl, Austr. I, 466 (1863)

N. foveolatum, F. v. M. in Benth. Fl. Austr. I, 466 (1863)

N. leiocarpum, F. v. M. in Transact. phil. Inst. Vict. III, 25 (1858) -

N. Beckleri, Bentham, Fl. Austr. I, 467 (1863)

N. divaricatum, F. v. II. in Benth. Fl. Austr. 467 (1863)

N. microphyllum, Bentham, Fl. Austr. I, 468 (1863)

N. Leichhardtii, F. v. MI, App. Fe'p. Tntercol. Exhib. 20 (1867)

N. clistyle, F. v. M., fragm. IX, 99 (1875)...

$$
\text { Iím. du Mus. IV, 8, t. } 3(1818) \text {. }
$$

$$
\text { HETERODENDRON, Desfontaines in Mím. du Mus. IV, 8, t. } 3 \text { (1818). }
$$

H. oleaefolium, Desfontaines in Mém. du Mus. IV, 8, t. 3 (1818) W.A. S.A. - V. N.S. IV. Q. N.A. B.fl.I.419

H. diversifolium, F. v. M., fragm. I, 46 (1858) .. ... ...
HARPULLIA, Roxburgh, Fl. Ind. ed. Carey II, 44 (1826).

H. alata, F. v. M., fragm. II, 103 (1861)

H. Hilli, F. v. M. in Transact. Vict. Inst. III, 26 (1859)

H. Leichhardtii, F. v. M. in Benth. Fl. Austr. I, 470 (1863)

H. pendula, Planchon in Transact. Vict. Inst. III, 26 (1859)

AKANIA, J." Hooker in B. \& H, rien. pl. 409 (1862).

A. Hillii, J. Hooker in B. \& II. Gen. pl. 409 (IS62)
( $\bar{I}_{45 S}$

.

B. $\mathrm{H} I, 458$

M.fr. I, $1 ; I X, 89,197$.

M.fr.IX,91.

I. fr. V, $146 ; \mathrm{IX}, 94$

M.fr.III, $43 ; \mathrm{IX}, 94$

M.fr.IX, 95 .

M.fr.IX,92.

M.fr.IX, 95 .

M.fr. $V, 7 ; \mathrm{IX}, 91$

M.fr. V, 177;IX,94.

M.fr.IV,156;IX,91.

M.fr.IV,I56;IX, 91 .

B.fl. $\bar{x}, 461$

M.fr. I, $2 ; I T, 76 ; \mathrm{IX}, 90$.

B.fl.I, 461 MI.fr. I, $2: I I, 76 ; I X, 90$.

B.tl.I,461 M.fr.II,7);IX,91

B. fl. I, 458

M.fr.III, $12 ; 1 X, 91$.

M.fr.IV, $156 ; I X, 91$.

M.fr.IX, 96.

M.fr.IX, 96 .

M.fr. T, $6 ; \mathrm{IX}, 94$.

M.fr.IX, 93 .

B.fl.I,461 M.fr.IX,94.
B.fl.I,457 M.fr.IX,97.

B. Al. I, 465

B. fl. I 465

B.fl. I, 466

B. H.I, 466

B. A. I, 466

B.f. I, 467

- N.S.W. $\frac{1}{\text { O }}$ - B.f.I,467

- Q. - B.H.I,467

- Q. - B.f.I,465

B.f..I, 468

M.fr.IX, 98 .

II.fr.Ir, Iss.

M.fr.IX, 99.

M.fr.IX, 99 .

M.fr.IX,99.

M.fr.IX, 98 .

M.fr.IX,9S.

MI.fr.IX, 99 .

M. fr. IX, 99

M.fr.IX, $90:$ X. 82

M.fr.I, $46 ; \mathrm{II}, 90 ; \mathrm{X}, 82$,

- N.S.W. Q. - B.f.I, 46 ?

- N.S.W. 1. - B.f.I,470

M.fr.IT, 103;IX, 59 .

- N.A. B.H.I,470

N.S.W. Q. - B.A.I,471 M.fr.IX, S9\&1S7.

- N.S.WV. Q. - B.H.I,47I

M.fr.III, 44;IX, 898197 
DIPLOPELTIS, Endlicher in Huegel, enum. 13 (1837).

D. Huegelii, Endlicher in Hueg, enum. 13 (1837)...
D. Stuartii, F. v. M., fragm. III, 12 (1862) DODONAEA, Linné, gen. pl. 341 (1737). (Empleurosma).

D. triquetra, Wendland, Bot. Beobacht. p. $4 \pm$ (1798) $\ldots \ldots \ldots$... $\quad-\quad-$ V. N.S.W. Q.

D. lanceolata, F. จ. M., fragm. I, 73 (1858)

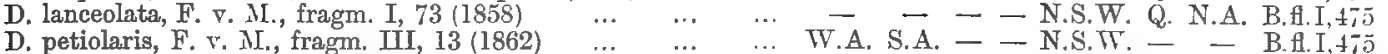

D. viscosa, Linne, mantissa X, altera 149 \& 228 (i771) $\cdots \quad$ W.A. S.A. T. V. N.S.T. O. NA B.fl. I, tio

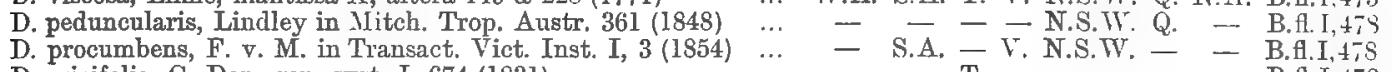

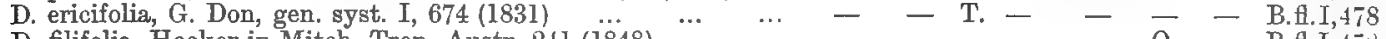

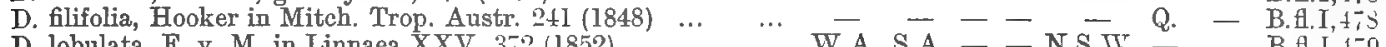

D. lobulata, F. v. M. in Linnaea XXV, 372 (1852) … … W.A. S.A. - - N.S.T. - - B.f.I, 470

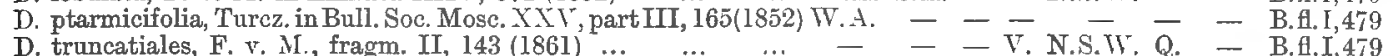

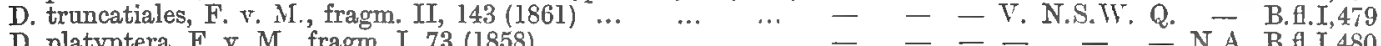

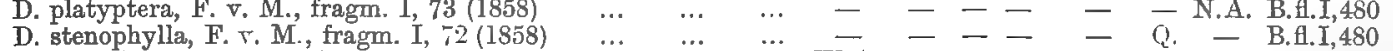

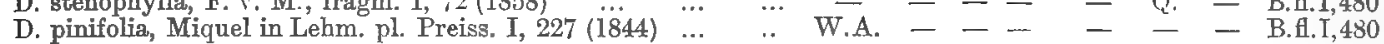

D. ceratocarpa, Endlicher in Hueg. enum. 13 (1837) $\quad$... $\quad$... W.A. W - $\quad$ - $\quad$ - $~-~ B . f l . I, 481$

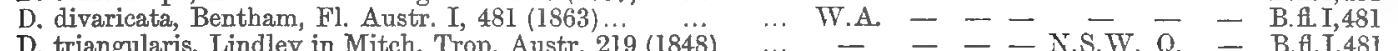

D. triangularis, Lindley in Mitch. Trop. Austr, 219 (1848) $\quad \ldots \quad$ T. $-Z=$ N.S.W. Q. - B.H.I,48I
D. aptera, Miquel in Lehm. pl. Preiss. I, 225 (1844) $\ldots$.

D. bursarifolia, Behr \& F. v. M. in Transact. Vict. Inst. I, 8 (1855) T.A. S.A. $-\overline{\mathrm{V}}$. N.S.T. - - B.H.I.,482

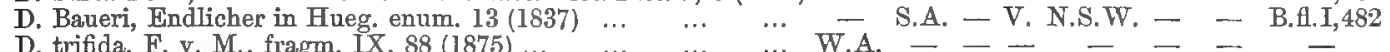

D. trifide F. Y. M from. IX, 88 (1875)

D. humifusa, Miquel in Lehm. pl. Preiss. I, 266 (1844) ...

D. hexandra, F. V. M. in Transact. Vict. Inst. I, 117 (1855)

D. exicoides, Miquel in Lehm. pl. Preiss. I, 227 (1844) ...

D. polyzyga, F. v. M. in fragm. I, 74 (I858)

D. megazyga, F. T. M. in Benth. Fl, Austr. I, 483 (1863)

D. macrozyga, F. V. M., fragm. IV, 135 (1864) ...

D. physocarpa, F. v. M, fragm. I, 74 (1858)

D. vestita, Hooker in Mitch. Trop. Austr. 265 (1848)

D. pinnata, Smith in Rees' Cycl. XII (1809)

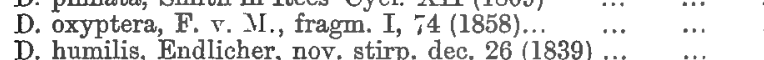

D. Macrossani, F. v. M. \& Scortechini in Melb. Chemist Jan.

D. microzyga, F. v. M. annual Rep. bot. Gard. 12 (1863)

D. boronifolia, G. Don, gen. syst. I, 674 (1831) ...

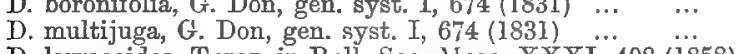

D. larraeoides, Turcz. in Bull. Soc. Mosc. XXXI, 408 (1858)
D. inaequifolia, Turcz in Bull. Soc. Mosc. XXXI, 408 (1858)

D. inaequifolia, Turcz in Bull. Soc. Mosc. XXXI, 408 (1858) ...
D. adenophora, Miquel in Linnaea XTIII, 95 (1845) $\ldots . .$.

D. adenuifolia, Lindley in Vitch. Trop. Austr. 248 (1848)
D. stenozyga, F. . M. M., fragm. I, 98 (1859) $\quad \ldots$

D. concinna, Bentham, Fl. Austr. I, 487 (1863)

DISTICHOSTEMON, F. v. Mueller in Hook. Kew misc. IX, 306 (1857).

D. phyllopterus, F. v. MI. in Hook. Kew misc. IX, 306 (1857) ...
BLEPHAROCARYA, F. v. Mueller, fragm. XI, 15 (1878).

B. involucrigera, F. v. M., fragm. XI, 16 (1878) ...

... П.A. - - - - - - B.f.I, 482

- S.A. - - - - - B.A.I,483

.. W.A. - - - - - B.fl.I,483

... - - - - - - N.A. B.fl.I,483

... - - - N.S.W. - - B.H.I.483

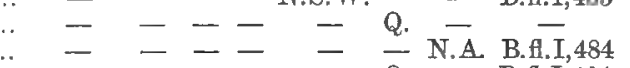

... - - - - Q. - B.H.I,484

- - - - N.S.W. - - B.H.I,484

.. - - - - - - N.A. B.fl.I,484

- S.A. - - - - N.A. B.H.I,485

$\dddot{1882}$

-..

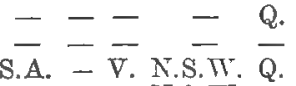

W.A. - - - N.S.W. - = B.H.I,485

- - - - B.f.I.486

W.A. - - N.S.W. Q. - B.A.I,486

W. A. S.A. $=\overline{\mathrm{V}} \cdot \mathrm{N} \cdot \overline{\mathrm{S} . \mathrm{W} .}=-$ B.A.I. 486

MI.fr.IX, 87 .

fr.

F. IX, 87.

M fr.IX, 88 .

II.fr.IX, 88.

I.fr.I, 74 .

I.fr.IX, 86

M.fr.IX, 86 .

M.fr.I, 74 .

M.fr. IX, 86.

M.fr. IX 86

M.fr.IX,86.

M.fr.I, 98 .

I. fr.I, $73 ; \mathrm{IX}, 88$.

M.fr.I, 73 .

.fr.IX, 89

I.fr.IX, so.

II.fr.IX, 87 .

II.fr.I.71.

M.fr.IX, s?

M.fr.I, $97 ; \mathrm{LX}, 67$.

r.IX, 89 .

fr.IX, 87 .

$M$ fr. IY 87.

M.fr.IX, 88 .

M.fr. I, $;+\mathrm{IX}, 86$.

M.fr.I,219;IX,89.

I.

M.fr.IX, 89.

MI.fr.XI, $16 \& 137$

\section{MALPIGHIACEAE}

Ventenat, Tabl. III, 131 (1799).

RYSSOPTERYS, Blume in Delessert ic. sel. III, 21, t. 35 (1837).

R. Timorensis, Blume in Deless. ic. sel. III, 21, t. 35 (1837) ... - - - - - Q. - B.fl.I,285 TRISTELlateia, Petit-Thouars in Roemer. Collect. bot. 206 (1809).

T. Australasica, A. Richard, Sert. Astrol. 38, t. 15 (1833) ‥ - - - - — Q. - B.fl.I,286

\section{BUESERACEAE.}

Kunth in Ann. des sc. nat. II, 346 (1824).

GARUGA, Roxburgh, Pl. of Coromandel III, 5, t. 208 (1819).

G. floribunda, Descaisne, in nouv, annal. du Mus. III, 477 (1834) - $\quad$ - - _ - N.A. B.A.I,377

CANARIUMI, Rumphius, herb, Amboin. II, 145, t. 47, 49, 54 (1741). (Sonzaya.)

C. Australianum, F. v. M., fragm. III, $15(1860) \ldots$

\section{ANACARDIACEAE.}

R. Brown in Tuckey's Expedit. Congo 431 (1818).

RHUS, Tournefort, Inst. 611, t. 381 (1700), from Hippocr. Theophr. Diosc. \& Plin. (Rhodosphaera.)

R. rhodanthema, F. v. M. in Journ. pharm. soc. Vict. pl. I, 43(1858) - - - - N.S.W. Q. - B.A.I,489 M.fr.VII, 23.

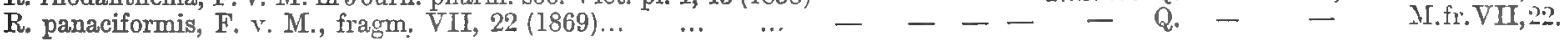

EUROSCHINUS; J. Hooker in B. \& H. Gen. pl. I, 422 (1862).

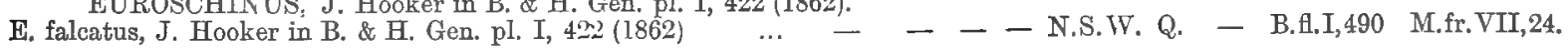

BUCHANANIA, Sprengel in Schrader's Journ. II, 234 (1800).

B. angustifolia, Roxburgh, Pl. Corom. III, 68, t. 262 (1819) … -

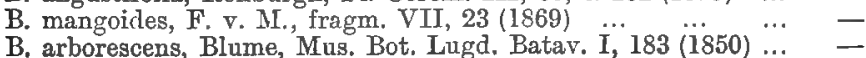

二 二 二 - Q. N.A. B.H.I,490

M.fr. VII, 23.

MI.fr. VII, 23.

M.fr. VII, 23. 
SEMECARPUS, Linne fil., suppl. 25 (1781).
S. Anacardium, Linné fil, suppl. 182 (1781)
SPONDIAS, Linné, gen. pl. 365 (1737).
S. Solandri, Bentham, Fl. Austr. I, 492 (1863)
S. pleiogyna, F. v. M., fragm. IV, 78 (1864)

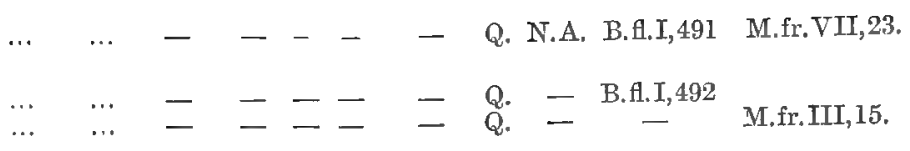

CELASTRINEAE.

R. Brown in App. Flind, Voy. 554 (1814).

EUONYMUS, Tournefort, inst. 617, t. 388 (1700), from Theophr. \& Plinius. (Evonymus.)

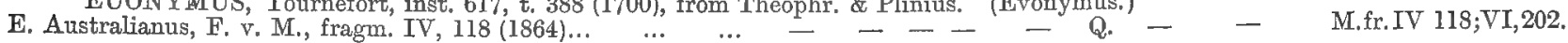
CELASTRUS, Linné, gen. pl. 59 (1737).

C. Australis, Harvey \& F. v. M. in Trans. phil. Soc. Vict. I, $41(1854)--\quad-$ V. N.S.W. Q. - B.fl.I,398

C. Muelleri, Bentham, Fl, Austr. I, $399(1863)$.. ‥ ‥ - - - - - - N.A. B.fl.I,399

C. dispermus, F. v, M. in Transact. phil. Inst. Vict. III, 31 (18̈9) - - - - - Q. - B.fl. I,399

C. bilocularis, F. v. M. in Transact. phil. Inst. Vict. III, 31 (1859) - - - - _ Q. - B.t.I,399

C. Cunninghamii, F. v. M. in Trans. phil, Inst. Vict. III, 30(1859) - - - - N.S.W. Q. N.A. B.A.I,399 GYMNOSPORIA, Wight \& Arnott, prodr. fl. paenius. Ind. I, 159 (1834.)

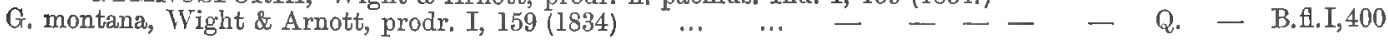
LEUCOCARPUM, A. Richard, sert. Astrolabe 46 (1834). (Denhamia, Leucocarpon.)

L. Oleaster, F. .. M. in Transact. Roy. Soc. of N.S.W. 15 (1881) - - - - - Q. - B.fl.I,401

L. obscurum, A. Richard, sert. Astrol. 46, t. $18(1834) \ldots \ldots$...
L. pittosporoides, F. v. M. in Trans. Roy. Soc. of N.S.W.15 (1881) - - - - - N.S.TV. Q. N.A. B.f.I,401 ELAEODENDRON, J. F. Jacquin in Nov. Act. Helv. I, 36 (1787).

E. Australe, Ventenat, Jard, Malm. t. $117(1804) \quad \ldots \quad \ldots \ldots$ _.. $\quad \ldots$ N.S.W. Q. N.A. B.fl.I,402 CARYOSPERMUM, Blume, Mus. bot. Lugd. I, 175 (1850).

C. arborescens, F. v. M., fragm. VI, $202(1868) \ldots \quad$... $\quad \ldots \quad-\quad-\quad-\quad$ - $\quad$ Q. - - - M.fr.VI,202. SIPHONODON, Griffth in Calc. journ, of Nat. Hist. IV, 150 (1843).

S. Australe, Bentham, Fl. Austr. I, 403 (1863) $\ldots$... $\quad \ldots \quad-\quad-\quad-\quad-$ N.S.W. Q. - B.fl.I,403 M.fr.VI,204. HIPPOCRATEA, Linné, gen. pl. 363 (I737).

H. obtusifolia, Roxburgh, flor. Ind. edit. Carey, I, $170(1820) \ldots-$ - - - - N.S.W. Q. - B.f.I,404 SALACLA, Linné, mantiss. II, 159 (1771).

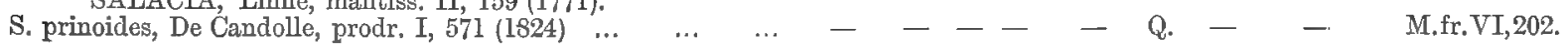

STACKHOUSIEAE.

R. Brown in App. to Flind, Voy. 555 (1814).

STACKHOUSIA, Smith in Transact. Linn. Soc. IV, 218 (1798). (Tripterococcus, Plokiostigma.)

S. pulvinaris, F. v. M. in Transact. phil. Soc. Vict. I, 101 (1855) - - T. V. N.S. IV. - - B.f.I,405
S. linarifolia, Cunningham in Field's N.S. Wales $365(1825) \ldots .$.

S. pubescens, A. Richard, sert. Astrol. 89, t. 33 (1834)... … W.A. - - - - - — B.fl.I,407

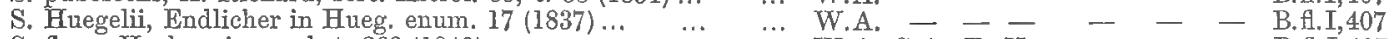

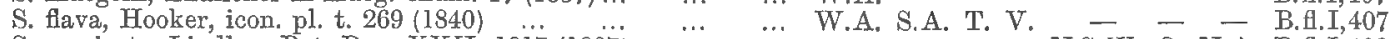

S. muricata, Lindley, Bot. Reg. XXII, 1917 (1837) $\ldots . \cdots \cdots-$ -

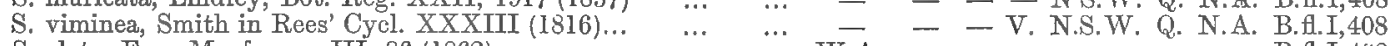

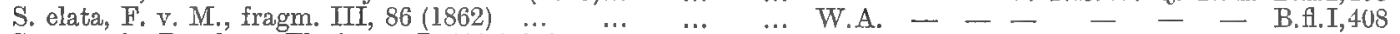

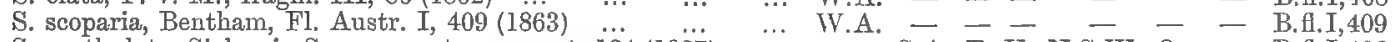

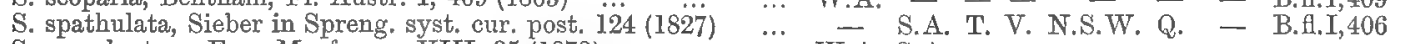

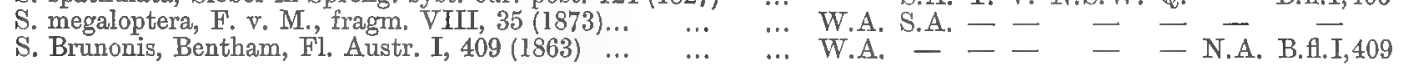
MACGREGORIA, F, v. Mueller in Caruel, Giorn. Bot. Italian. 128 (1873).

M. racemigera, F. v. M. in Caruel, Giorn. Bot. Ital. $128(1873)$ - S.A. - - - - - - M.fr, VIII,161;XI,134.

\section{FRANKENIACEAE.}

A. de St. Hilaire in Bull. de la Soc. Philom. 22 (1815).

FRANKENIA, Linné, gen. pl. 92 (1737).

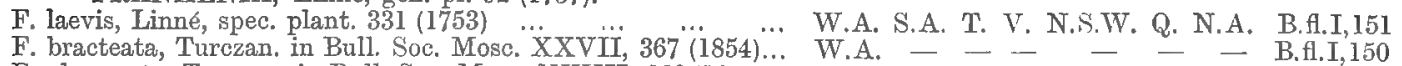

F. glomerata, Turczan, in Bull. Soc. Mosc. XXVII, $368(1854)$ W.A. - - - - _ - B.f.I,151

F. parvula, Turczan. in Bull. Soc. Mosc. XXVII, 368 (1854) ... W.A. - - _ - - - B.A.I, - $15 \%$

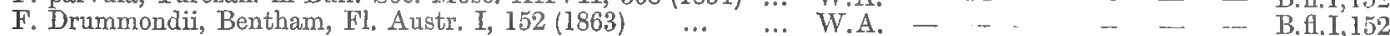

F. tetrapetala, Labillard., nov. Holl. pl, spec. I, 88, t. 114 (1804) W.A. - - _ _ _ _-- - B.fi.I,152

F. punctata, Turczan. in Bull. Soc. Mosc. XXVII, 367 (1854) ... W.A. - _ - _ _ _ B.A.I,153

M.fr.III, 88.

M.fr.III,87;XI.27.

M.fr.VIII, 36.

M.fr.VIII, 36.

M.fr.III, 86 .

M.fr.III, 86 .

M.fr.X, 119.

M.fr.VIII, 36.

\section{PLUMBAGINEAE.}

PLUMBAGO, Tournefort, inst. 140, t. 58 (1700).

A. L. de Jussicu, gen. plant. $92(1 ; 89)$.

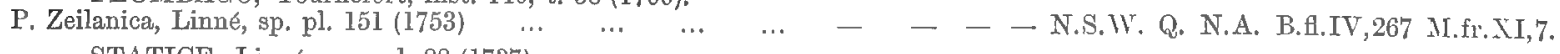

STATICE, Linné, gen. pl. 88 (1737).

S. Taxanthema, Roemer \& Schultes, syst. veg. VI, $798(1820) \ldots-\ldots-$ - T. V. N.S.W. Q. - B.fl.IV,267 M.fr.XI,7.

A

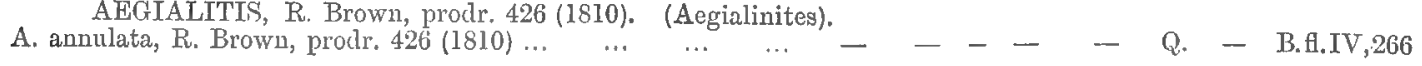




\section{PORTULACEAE,}

A. L. de Jussieu, Gen. 312 (1789).

PORTULACA, Tournefort, inst. 236, t. 118 (1700), from 1 Obel (1581).

P. oleracea, Linné, spec. plant. $445(1753) \ldots \ldots \ldots$... ... ... W.A. S.A. - V. N.S.T. Q. N.A. B.f.I.169

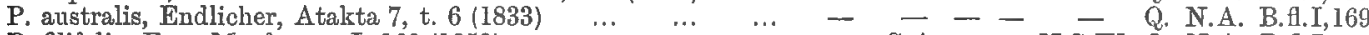

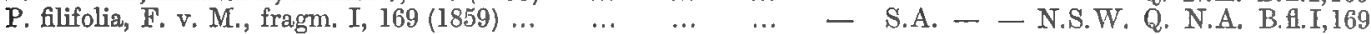

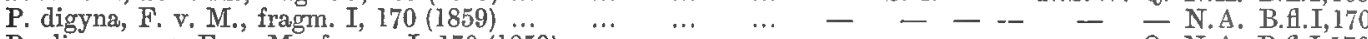

P. bicolor, F. v. M., fragm. I, $171(1859)$...

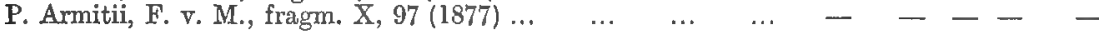

CLAYTONIA, Gronovius in Linné, gen. pl. 339 (1737). (Calandrinia, Talinum.)

C. spergularina; Calandrinia, F. v. M., fragm. I, 175 (1859)

C. strophiolata, F. v. M. fragm. XI, S2 (1880)

C. Lehmanni, F. v. M.; Calandr., Endl. in Lehm. pl. Preiss. III, $235(1847)$

C. uniflora, F. v. M.; Calandr. in Trans. Inst. Vict. MI, 41 (1859)

$-\quad-\quad-$ Q. N.A. B.A.I,176

W.A. S.A. 二 = $=\frac{\text { Q. }}{\text { N.A. }} \overline{-}$

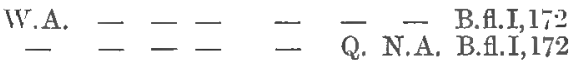

- S.A. - - N.S.W.Q. Ansis, F. v. M.; Calandrinia, Lindley in Mitch. Trop.

C. polyandra, F. v. M.; Calandr., Benth. Fi. Austr. I, 172 (1863) W.A. S.A. - - N.S.W.

C. pleiopetala; Calandrinia, F. v. M., fragm. X, 70 (1877)

C. quadrivalvis; Calandrinia, F. v. M., fragm. I, 176 (1859)

C. liniflora, F. v. M.; Calandr., Fenzl. in Hueg. enum. 52 (183

- B.ff.I, $1: 2$

- B.A.I,172

C. polypetala, F. v. M.; Calandr., Fenzl. in Hueg. enum. 51(1837) W.A. _ _ _ _ - B.fl.I,174

C. Pickeringi, F. v. M.; Calandr., A. Gray, Bot. Wilkes' Exped.

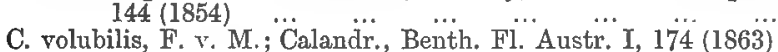

C. calyptrata, F. v. M., fragm. III, 89 (1862)

W.A. S.A. $=$ - N.S.W.

W.A. S.A. $-\overline{\text { N.S.W }}$ W. Q
W.A. S.A. T. V. N.S.W.

C. pumila; Calandrinia, F. V. M., fragm. X, 68 (1877) … ...

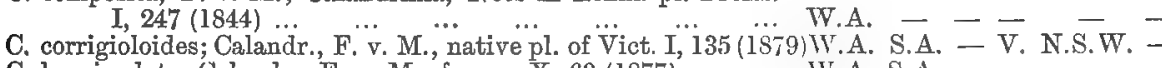

C. brevipedata; Calandr., F. v. M., fragm. X, 69 (1877)

C. pogonophora; Calandrinia, F. v. M., fragm. X, $69(1877)$
C. pygmaea, F. v. M., fragm. III, 89 (1862)

C. Australasica J Hooker icon, plant, to $293(1840)$

MONTTA, Micheli, nov. pl. gen. 17, t. 13 (1729).

M. fontana, Linné, spec. plant. 87 (1753) ...

$$
\text { ... - - T. - - - - B.A.I,177 }
$$

P. napiformis, F. v. M. in Benth. Fl. Austr. I, 169 (1863) ‥ - - - - $\quad$ - Q. N.A. B.fl.I,169

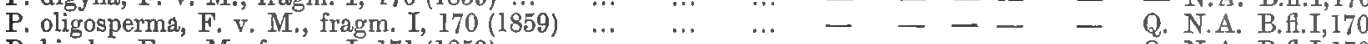

- S.A. - - - Q.

C. gracilis, F. v. M.; Calandrinia, Benth. Fl. Austr. I, $173(1863)-{ }_{-}-C_{-}$N.A. B.fl.I,173

C. composita, F. v. M.; Calandrinia, Nees in Lehm. pl. Preiss,

C. granulifera, F. v. M.; Calandr., Benth. Fl. Austr. I, 176 (I863) W.A. - - - - -
- B.A.I. 174

- B.fl.I,17t

B.A.I, 175

- B.fl.I,1\%

二 B.A.I.I,176

- B.

二 B.H.I. 176

B.H.I, 177
M.fr.X,, 71 .

M.fr.X. 72

II.fr. X, T.

II.fr.III, $164 ; \mathrm{X}, \mathbf{2}$,

M.fr.I, 170

II.fr.III, $164 ; \mathrm{X},-2 \cdot$

MI.fr. III, $161 ; \mathrm{X},-2$.

M.fr.X, 97 .

M.fr.I, I75;X, 71 ,

M.fr.IV, 137;X, 70

M.fr. XI, 82 .

M.fr. X, 71 .

M. fr. $X, 71$.

MI.fr.X,7].

Mifr.X, 71 .

M.fr. X, 70

II fr. $I, 170$.

M.fr.X,71.

M. fr. X, 69;XI, 136 .

M.fr.X, $68 ; \mathrm{XI}, 27$.

M.fr.IV, $175 ; \mathrm{X}, 68$.

M.fr.X, 68 .

MI.fr.X, 69

XI.fr.X, 69

II.fr.X,69;XI, 136 .

M.fr.X, 69

M.fr.I, $175 ; X, 71$.

M.fr.III, $89 ; X, 71$.

\section{CARYOPHYLLEAE.}

Linné, philosoph. bot. 31 (1751).

STELLARIA, Linné, spec. plant. 421 (1753).

S. pungens, Brongniart, Voy. sur la Coquille t. 78 (1826)

S. glauca, Withering, Arr. veg. in Great Brit. I, 420 (1776)

S. flaccida, Hooker, Comp. Bot. Mag. I, 275 (1835)

S. multiflora, Hooker, Comp. Bot. Mag. I, 275 (1835) ...
… - S.A. T. V. N.S.W. Q.

… - - T. V. N.S.W. -
N. - S.A. T. V. N.S.W. -

… W.A. S.A. T. V. N.S.W. -

SAGINA, Linné, syst. nat. 8 (1735); Linné, gen. pl. 118 (1737).

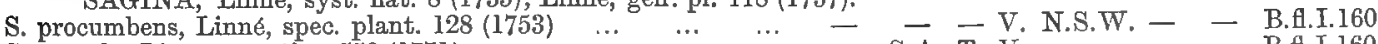

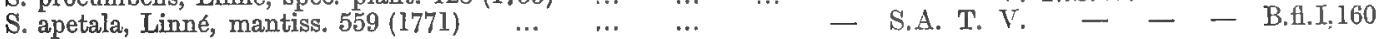
COLOBANTHUS, Bartling, Reliq. Haenk. II, 13, t. 49 (1830).

C. Benthamianus, Fenzl in Ann. Wien. Mus. I, $49(1838) \quad \ldots \quad-\quad-\quad$ - V. N.S.W. - - B.fl.I,160

C. Billardieri, Fenzl in Ann. des Wien. Mus. I, 48 (1838) _.. _ - S.A. T. V. — - - B.A.I,161 SCLERANTHUS, Linné, gen. plant. 130 (1737). (Mniarum.)

S. pungens, R. Brown, prodr, 412(1810) ... ... .. … - S.A. - V. N.S.W. - - B.f.V.260

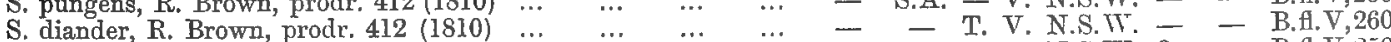

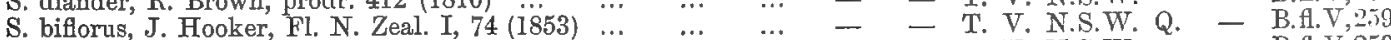

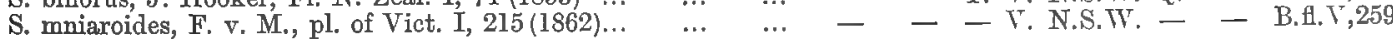
SAPONARIA, Linné, gen. plant. 130 (1737), from Camerarius (1586). (Gypsophila.)

S. tubulosa, F, v. M., native pl. of Vict. I, 136 (1879) .. .. - S.A. - V. N.S.W. - - B.f.I,155 SPERGULARIA, Persoon, synops. pl. I, 504 (1805). (Lepigonum, Arenaria partly.)

S. rubra, Cambesseddes in St. Hilaire, Fl. Brazil. II, 179 (1829)... W.A. S.A. T. V. N.S.W. - - - B.A.I,162 M.fr.XI,27 DRYMARIA, Willdenow in Roemer \& Schultes, syst. V, 406 (1819).

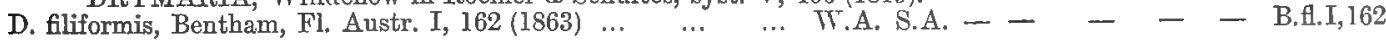
POLYCARPON, Loefling, iter Hispanic. 7 (1758).

P. tetraphyllum, Loefling in Linne, syst. nat. ed. X, 881 (1759) T.A. S.A. T. V. N.S.TV. Q. - B.A.I,163 POLYCARPAEA, Lamarck in Journ. d'hist. nat. II, 8, t. 25 (1792). (Aylmeyera.)

P. longiflora, F. r. M., Rep. of Babb. Exped. 8(1858) ... ... - - - - -

P. spirostylis, F. Y. M., Rep. of Babb. Exped. 8 (1858)

P. violacea, Bentham, Fl. Austr. I, 165 (1863)

P. Indica, Lamarck in journ. d'hist. nat. IT, \& (1792) ..

$\cdots$
$\cdots$
$\cdots$
$\cdots$
$\cdots$

- N.A. B.H.I, 164

Q. N.A. B.fl.I, 165

Q. N.A. B. $A \cdot I, 165$

Q. N.A. B.H.I, 165

Q. N.A. B.A.I, 166
M.fr.XI, 27. 
P. breviflora, F. v. M., Rep. of Babb. Exped. 9 (1858) ... P. spicata, Arnott in Ann. of nat. hist. III, 91 (1839) ... P. involucrata, F. v. M., Rep. of Babb. Exped. 9 (1858)

$$
\begin{aligned}
& \text { ․ - - - - - Q. N.A. B. A.I,166 } \\
& \begin{array}{l}
\ldots-\text {-. } \\
\cdots
\end{array}-\text { - }- \text { - N. N.A. B.H.I, B.H.I,167 }
\end{aligned}
$$

\section{AMARANTACEAE.}

A. L. de Jussieu, Gen. 87 (1789).

GQMPHRENA, Limné, geu. plant. 69 (1737). (Philoxerus.)

G. canescens, R. Brown, prodr. 416 (1810) ...

G. flaccida, R. Brown, prodr. 416 (1810)

G, affinis, F. Y, M. in Benth. Fl. Austr, V, 254 (1870) $\cdots \quad$.. - - - -

G. humilis, R. Brown, prodr. 416 (1810)

nil, Moquin in De Cand. prodr. XIII, 2, 397 (1849)

G. brachystylis, F. V. M., fragm. III, 124 (1862) ...

G. leptoclada, Bentham, Fl. Austr. V, 255 (1870)

G. Maitlandi, F. v. M., fragm. III, ]24, t. 23 (1862)

G. pusilla, Bentham, Fl. Austr. V, 256 (1870)

G. tenella, Bentham, FI. Austr. V, 256 (1870)

G. conica, Sprengel, syst. I, 824 (1825)

G. conferta, Bentham, Fl. Austr. V, 257 (1870) ...

G. diffusa, Sprengel, syst. I, S24 (1825) …

G. parviflora, Bentham, Fl. Austr. V, 257 (1870) ...

ALTERNANTHERA, Forskael, flor, Aegypt. Arab. 28 (1775). (Telanthera.)

A. triandra, Lamarck, encycl. meth. I, 95 (1783) ... $\ldots . \ldots \ldots$ W.A. S.A. T. V. N.S.W. Q. N.A. B.fl.V,249

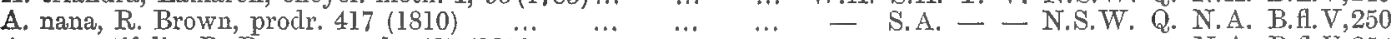

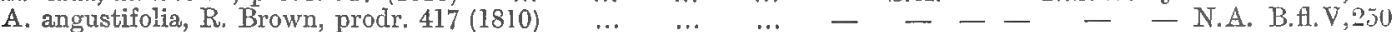

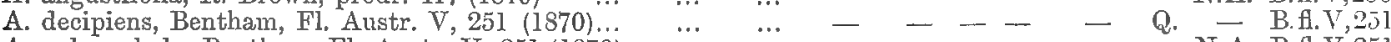

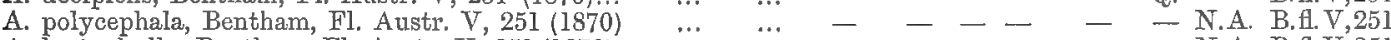

A. leptophylla, Bentham, Fl. Austr. V, $251(1870) \quad \ldots . \quad \ldots \quad-\quad-\quad-\quad$ - - N.A. B.fl.V,251

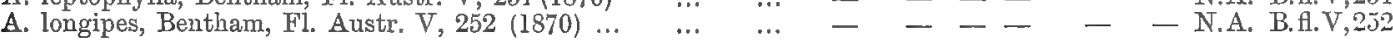
ACHYRANTHES, Linné, gen. plant. 34 (1737).

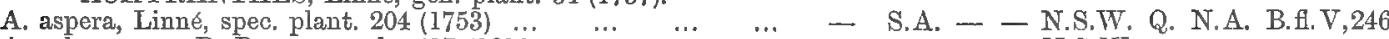

A. arborescens, R. Brown, prodr. 417 (1810) … NYSSANTHES, R. Brown, prodr. $418(1810)$.
cta, R. Brown, prodr. 418 (1810) $\ldots$

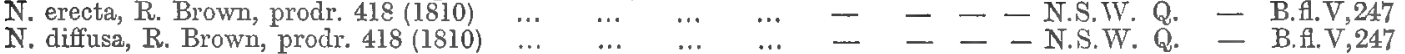
PTILOTUS, R. Brown, prodr. 415 (1810). (Trichinium, Psilotrichum, Goniotriche, Hemisteirus, Arthrotrichum.)

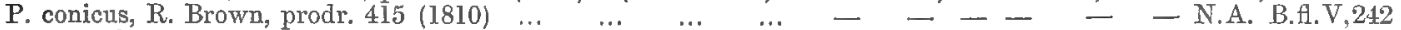

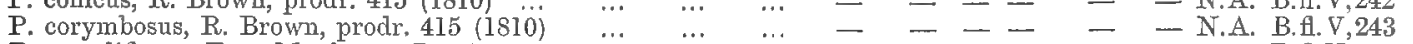

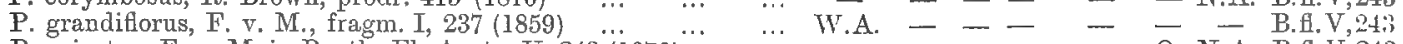

P. spicatus, F. v. M. in Benth. Fl. Austr. V, $243 .(1870) \ldots \ldots \ldots-\ldots-\ldots$ - $\quad-\quad$ Q. N.A. B.fl. Y,243

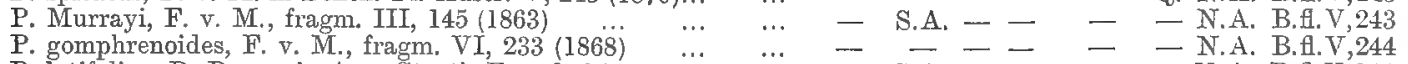

P. latifolius, R. Brown in App. Sturt's Exped. $25(1849) \quad \ldots-$ - S.A. - - $-\quad$ - N.A. B.A.V,244

P. macrotrichus, F. v. M., fragm. IV, $90(1864) \ldots \quad \ldots \quad \ldots$. W.A. $\quad$ - $\quad$ - $\quad$ - $\quad$ - $\quad$ - N.A. B.fl.V, 244

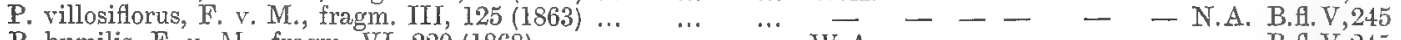

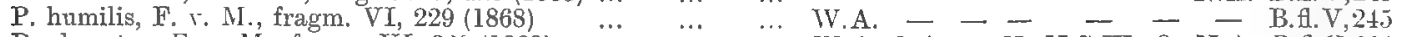

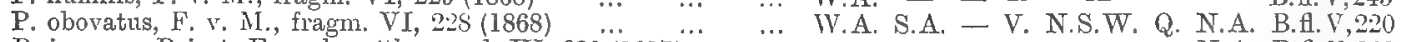

P. incanus, Poiret, Encycl. méth. suppl. IV, 620 (1817) ‥ - - - - -- - N.A. B.fl.V,221

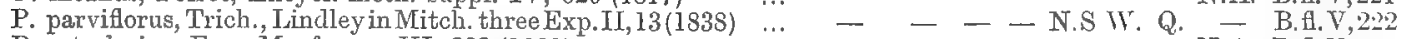

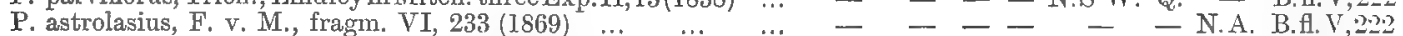

P. rotundifolius, F. v. M., fragm. VI, $230(1868) \ldots \quad \ldots \quad \ldots \quad-\ldots-\ldots-\ldots$

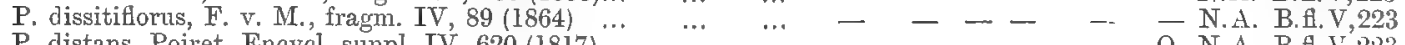

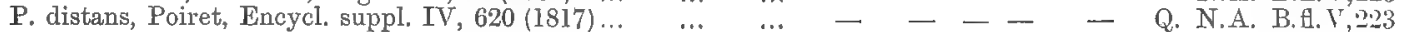

P. alopecuroideus, F. v. M., fragm. VI, $227(1868) \quad \ldots . \quad \ldots .6$ W.A. S.A. - V. N.S.W. Q. N.A. B.fl.V, 224

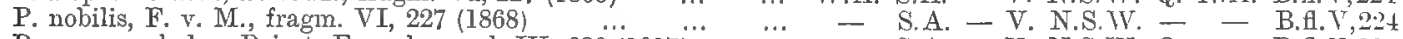

P. macrocephalus, Poiret, Encycl. suppl. IV, $620(1817)$... - S.A. - V. N.S.TV. Q. - B.fl. Y.22J

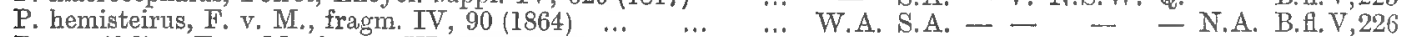

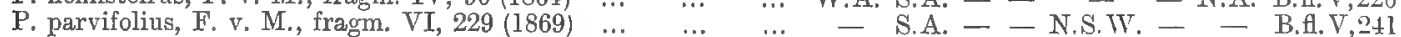

P. exaltatus, Nees in Lehm. pl. Preiss, I, $630(1845) \quad \ldots . \quad \ldots$. W.A. S.A. - V. N.S. W. Q. N.A. B.f. Y,227

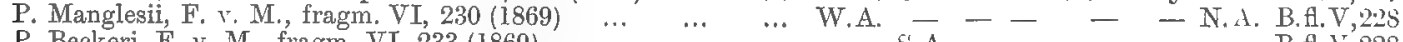

P. Beckeri, F. v. M., fragm. VI, 233 (1869) … … … - S.A. - - - - B.H.V,22S

P. gomphrenoides, Moquin in De Cand., prodr. XIII, 2, $287(1849)$ - S.A. - - - - - B.f.V,2.29

P. esquamatus; Trichinium, Bentham, FI. Austr. V, $229(1870)$ W.A. - - - - - - B.H.V.229

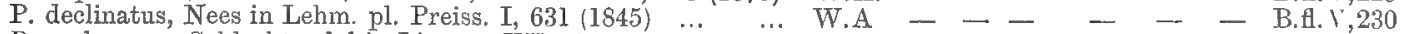

P. erubescens, Schlechtendal in Linnaea XX, $575(1847) \quad \ldots \ldots-$ S.A. - V. N.S.W. - - B.A.V,230

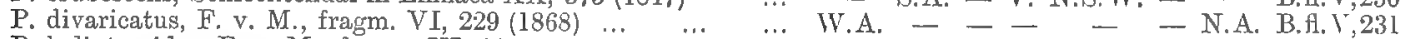

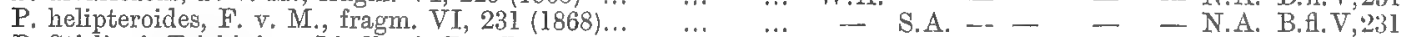

P. Stirlingi; Trichinium, Lindley in Bot. Reg. under n. 28 (1839) W.A. - - - - - N.A. B.f. Y,232

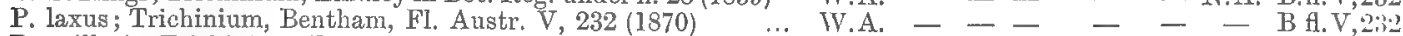

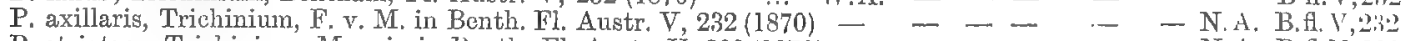

P. striatus; Trichinium, Moquin in Beuth. Fl. Austr. V, $233(1870)----1-$ - N.A. B.f.V,233

P. auriculifolins; Trichinium, Cunningham in De Cand, prodr. XIII, part II, 287 (I849)

P. sericostachyus, F. v. M., fragm, $\dddot{V} I, 230$ (1868)

IV.A. - - -

- $\quad$ - N.A. B.f.1

P. roseus; Trichin., Moq. in De Cand, prodr. XIII, p.II, $284(1849)$ W.A. - - - - - E B.A.

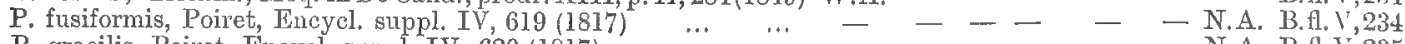

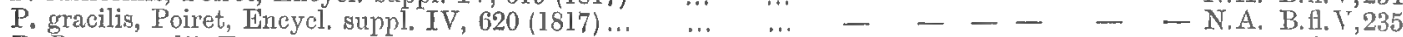

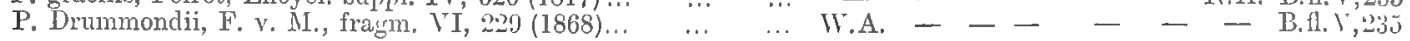

M.fr.III, 123.

M.fr.III, 124.

MI.fr. VI, 234 .

M.fr.III, 125.

M.fr.IX, 169 .

M.fr.III, 125.

M.fr.I, 237.

M.fr.III, 145.

M.fr. VI, 232.

M.fr. VI, 232 .

M.fr.IIf, 125 .

M. fr.III, 161; VI, 229.

M.fr. $\mathrm{I} I, 27$.

M.fr. VI, $2: 2 \mathrm{~S}$.

M. fr. VI, 233.

M.fr.III, 122.

M.fr.IV, 89.

M.fr. VI, 228.

M. fr. VI, 227.

MI.fr. VI,228; XI, 27.

M.fr. VI, 231 .

M.fr. VI, 229.

M.fr. VI, 230.

M.fr. II, 229.

M.fr. VI, 229.

I.fr.III, $122 \& 161$.

M.fr. VI, 230

M.fr. TI, 228.

M.fr. $\backslash 1,2=20$ 
P. calostachyus, F. v. M., fragm. VI, 231 (1868) ..

P. Fraseri ; Trichinium, Cunningham in De Cand., prodr. XIII, part II, 295 (1849) ...

P. spathulatus, Poiret, Encyci suppi. IV $\dddot{6} 620(18177) \quad \cdots \quad \cdots$

W.A. S.A. T. V. N.S.TV. - - B.A.V,236

... W.A. - - - - - - B.A.V,237

P. lanatus, Cunningham in De Cand, prodr. XIII, p. II, 281 (18̈49) - - - - - - N.A. B.A.V,238

P. leucocoma ; Trichinium, Moquin in De Cand., prodr. XIII, part II, 292 (1849)

P. brachyanthus; Trichin., F. v. M. in Benth. Fl. Austr. V, $639(1870)$

P. arthrolasius, F. v. M., fragm. VI, 232 (1868)

P. Forrestii, F. v. M., pl. of N. W. Austr. 7 (188i)

P. aervoides, F. v. M., fragm. VI, 231 (1868) $\quad \ldots \quad \ldots, \quad \ldots$

P. Roei; Trichinium, F. v. M. in Benth. Fl. Austr. V, $240(1870)$

P. Hoodii, F. v. M., fragm. V III, 232 (1874)

P. caespitulosus, F. v. M., fragm. VI, 232 (1868)..'

P. helichrysoides, F. v. M., fragm. VI, 231 (1868)

P. psilotrichoides, F. v. M., fragm. VI, 231 (1869) EUXOLUS, Rafinesque, Fl. Tell. 42 (1836).

E. Mitchellii, F. v. M. in Giles, geogr. trav. Centr. Austr. $214(1875)-$ S.A. - - N.S.W. Q. - B.fl.V,214

E. interruptus, Moquin in De Cand, prodr. XILI, part II, 275(1849) - - - - - Q. N.A. B.fl.V,2l.J

E. viridis, Moquin in De Cand., prodr. XIII, part II, 273(1849) W.A. - - - N.S.W. Q. - B.fl.V,215

E. macrocarpus; Amarantus, Benth. Fl. Austr. V, 216(1870) ‥ - - - V. N.S.W. Q. - B.fl.V,216 AMARANTUS, Dodoens, stirp. hist. pempt. 185 (1583):

A. leptostachyus, Bentham, Fl. Austr. V, $214(1870) \quad \ldots \quad \ldots \quad-\quad-\quad-\quad-\quad$ Q. N.A. B.fl. V,213

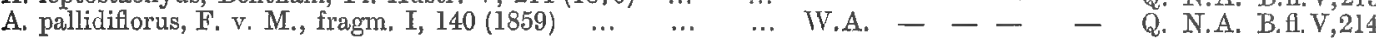
POLYCNEMON, Linné, gen. pl. ed. aec. 21 (1742). (Hemichroa.)

P. pentandrum, F. v. M., native pl. of Vict. I, 162 (1879) $\quad$.. W.A. S.A. T. V. N.S.W. - $\quad$ - B.fl.V,211 P. diandrum, F. v. M., native pl. of Vict. I, $163(1879) \ldots \quad$... W.A. S.A. - V. N.S.W. - N.A. B.A.V,21I

P. mesembrianthemum, F. v. M., fragm. VIII, 38 (1873)

- S.A. - - - - -

DEERINGIA, R. Brown, prodr. 413 (1810). (Lestibudesia partly, Celosia partly, Lagrezia.)

D. celosioides, R. Brown, prodr. $413(1810) \quad \ldots . \quad \ldots \quad \ldots \ldots$

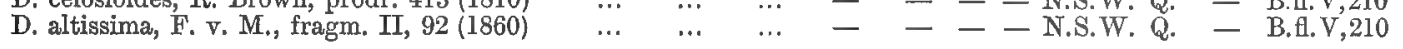

\section{SALSOLACEAE.}

Linné, class. pl. 507 (1738).

RHAGODIA, R. Brown, prodr. 408 (1810).

R. Billardieri, R. Brown, prodr. 408 (1810)

R. parabolica, R. Brown, prodr. 408 (1810)

R. dioica, Nees in Lehm. pl. Preiss. I, 636 (1845)...

R. Gaudichaudiana, Moquin, Chenop. monogr. enum. 11 (1s40)

R. crassifolia, R. Brown, prodr. 408 (1810)

R. Preissii, Moquin in De Cand, proch. XIII, part II, $4 \ddot{9}$ (1849)

R. obovata, Moquin, Chenop. monogr. enum. 10 (1840)..

R. spinescens, R. Brown, prodr. 408 (1810)

$\mathrm{R}$. hastata, R. Brown, prodr. $408(1810) \ldots \ldots$

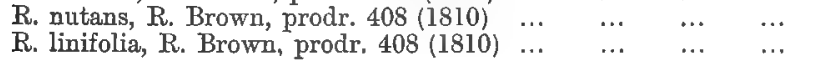

CHENOPODIUM, Tournefort, inst. 506, t. 288 (1700).

CHENOPODIUM, Tournefort, inst. 506, t. 288 (1700). (Blitum, Ambring.)
C. nitrariaceum, F. v. M. in Benth. Fl. Austr. V, $158(1870)$... W.A. S.A. - V. N.S.IV.
C. auricomum,

C. anricomum, Lindley in Mitch. Trop. Austr. 94 (1848) … - S.A. - V. N.S.W. Q. N.A. B.fl.V,159

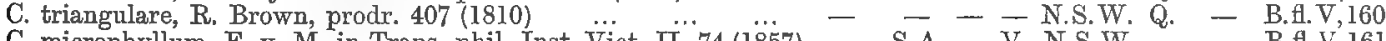

C. microphyllum, F. v. M. in Trans. phil. Inst. $\dddot{V}$ ict. II, $74(18 \ddot{8} 5)$ - S.A. - V. N.S.W. - - B.H.V,161

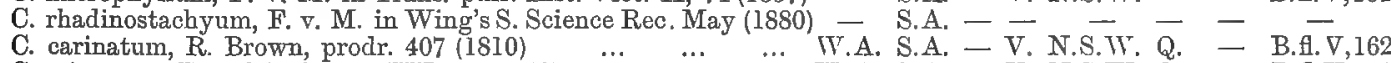

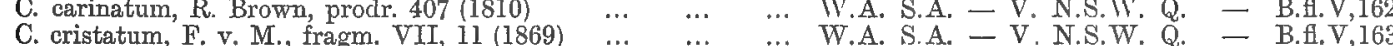

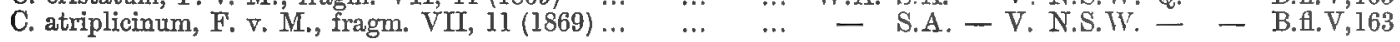
DYSPHANIA, R. Brown, prodr. 411 (1810).

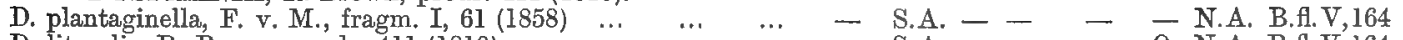

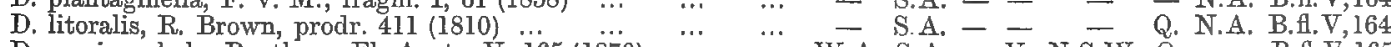
D. myriocephala, Bentham, Fl. Austr. V, 165 (1870) $\quad \ldots . \quad \ldots$. W.A. S.A. - V. N.S.W. Q. - B.fl.V,165 ATRIPLEX, Tournefort, inst. 506, t. 288 (1700), from l'Obel (1581). (Obione, Theleophyton.)

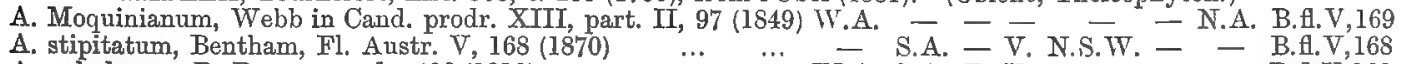

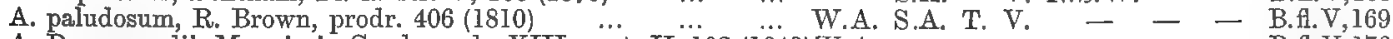

A. Drummondii, Moquin in Cand. prodr. XIII, part. II, 102 (1849) W.A. - - - - - - B.H.V,170

A. isatideum, Moquin, Chenop. monogr. enum. 63 (1840) ... W.A. - - - - - - B.H.V,170

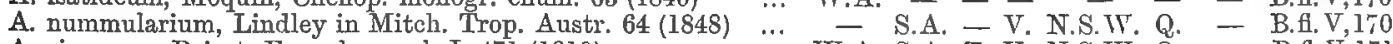

A. cinereum, Poiret, Encycl. suppl. I, 471 (1810) $\quad \ldots \quad \cdots \quad$ W.A. S.A. T. V. N.S.W. Q. - B.fl.V,171

A. rhagodioides, F. v. M. in Trans. phil. Inst. Vict. II, 74 (1857) W.A. S.A. - V. N.S.W. - - B.t.V,17.

A. incrassatum, F. v. M., Rep. Babb. Exped. 20 (1858)... ‥ - S.A. - - - - - B.fl.V,172

A. vesicarium, Heward in Benth. Fl. Austr. V, $172(1870) \quad \ldots-$ - S.A. - V. N.S.W. Q. - B.f.V,17.2

A. hymenothecum, Moquin in Cand. prodr. XIII, part. II, $101(1849)$ W.A. - - - - - - B.f.V,I73

A. humile, F. v. M., fragm. IV, $48(1866) \ldots$

A. fissivalve, F. v. M., fragm. IX, 123 (1875)

A. velutinellum, F. v. M., Rep. Babb. Exped. $20(1858)$

A. angulatum, Bentham, Fl. Austr. V, 174 (1870)

.. -

- - - - N.A. B.fl.V,174
S.A. - V. N.S.W. -
- S.A. - - N.S.T. -
M.fr.I, 140.

M.fr. VI, 234

M.fr.VI, 234

M.fr. VIII, 38.

M.fr.II, 92 .

M.fr. VI, 251.
M.fr. VI, 228

M.fr. VI, 230

M.fr. VI, $2 ; 2$.

M.fr. III, 123; VI, 231 .

M.fr. VIII, $2: 322$

IIf.

M.fr.XI, 97 . 
A. semibaccatum, R. Brown, prodr. $406(1810) \ldots$

A. exilifolium, F. V. MI, fragm. VII, 9 (1870) ..

A. Muelleri, Bentham, Fl. Austr, V, 17j (1870) ...

A. elachophyllum, F. v. M., fragm. TII, S (1870)

A. microcarpum, Bentham, Fl. Austr. T, 176 (1870)

A. prostratum, R. Brown, prodr. 406 (1810)

A. pumilio, R. Brown, prodr. 406 (1810)

A. campanulatum, Bentham, FI. Austr. V, $17 \%$ (1870)

A. leptocarpum, F. F. M. in Trans. phil. Inst. Vict. II, 74 (1857)

A. limbatum, Bentham, F1. Aust. V, 17s (1870) ...

A. halimoicles, Lindley in Mitch. Three Exped, I, 282 (̈̈

A, holocarpum, F. r. II., Rep. Babb. Exped. 19 (1858)...

A. spongiosum F r M in Trans. Vict phil Tnst. II $74(1857)$

A. crystallinum, J. Hooker in Lond, journ. of Bot. VI, 279 (1847) KOCHIA, Roth in Schrader's Journ. Bot. I, 303 (1799).

K. fimbriolata, F. r. M., fragm. IX, 75 (1875)

K. lobiflora, F. v. M. in Benth. Fl. Austr. V, $184(1870)$

K. lanosa, Lindley in Mitch. Trop. Austr. $88^{\prime}$ (1848)

K. triptera, Bentham, Fl. Anstr. V, 185 (1870)...

K. oppositifolia, F. r. M. in Transact. Tiet. Inst. 134 (1855) ..

K. brevifolia, R. Brown, prodr. 409 (1810)

K. pyramidata, Bentham, Fl. Austr. V, $186(1870)$

K. eriantha, F. v. M., Rep. Babb. Exped. 20 (1858)
K. villosa, Lindley in Mitch. Trop. Austr. 91 (1848)

K. planifolia, F, v. M., fragm. I, 213 (1859)

K. sedifolia, F. r. M. in Transact. Vict. Inst. I, $\dddot{1} 34$ (1855)

K. humillima, F. r. M., fragm. IX, 168 (1875)

K. microphylla, F. v. NI., fragm. VII, 148 (1874)

K. dichoptera, F. v. M., fragm. VIII, 37 (1873) ...

K. decaptera, F. v. M., fragm. IX, 75 (1875)

K. ciliata, F. .. M., Riep. of Babb. Exped. 20 (1858)

K. brachyptera, F, v. M., second gen. Rep. 15 (1854)

K. stelligera, F. v. M. , fragm. VII, 13 (1869) … DIDYMANTHUS, Endlicher, nov. stirp. decad. 7 (1839) BABBAGIA, F. v. M., Rep, on pl. of Babb. Exped. 21 (1858),

B. dipterocarpa, F. v. M., Rep. of Babb. Exped. 21 (1858) ‥ - S.A. - - N.S.W. Q. - B.fl.V,192 M.fr.VII,11. BASSIA, Allioni in Misc. Taurin. III, 117, t. 4 (1766). (Chenolea, Sclerolaena, Anisacantha, Echinopsilon, Kentropsis, Dissocarpus, Eriochiton, Osteocarpum, Coilocarpus.)

B. carnosa; Chenolea, Benth. Fl. Austr. V, $190(1870)$

B. Dallachyana; Chenolea, Benth. Fl. Austr. V, 191 (1870)

B. tricornis; Chenolea, Benth. Fl. Austr. V, 191 (1870)

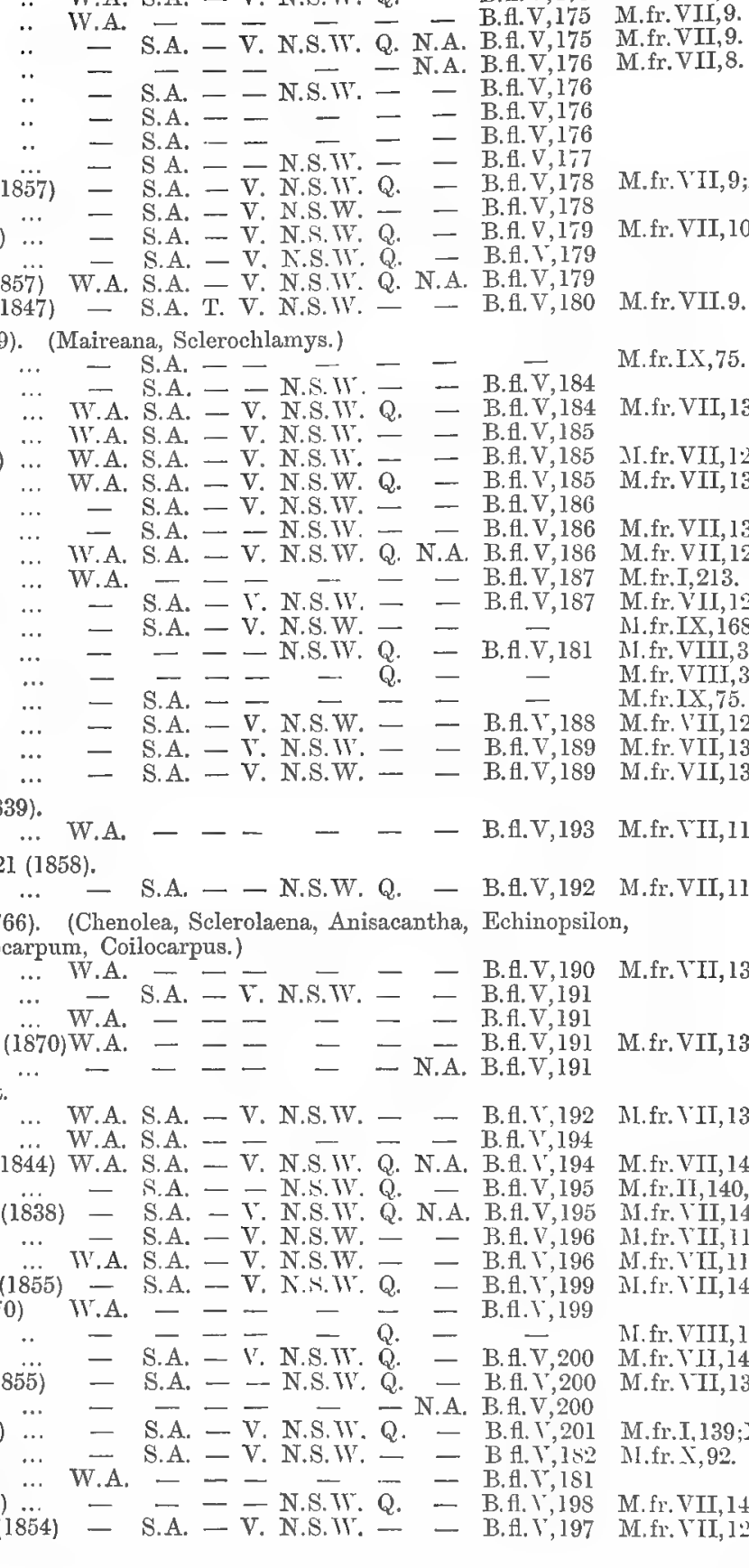

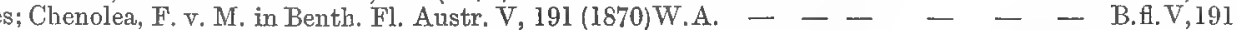

B. Muelleri; Chenolea, Benth. FI. Austr. V, 191 (1870)...
B. sclerolaenoicles; Echinopsilon, F. v. M. in Tr, phil. Inst. Vict. II, 75 (1857)

B. unifiora: Sclerolaena, $\mathrm{R}$ Brown $\cdots \cdots$

diacantha; A … W.A. S.A. - - $-\overline{-}-\bar{T}-$ B.fl. T,194

B. Ianicuspis; Anisacantha, F. v. M., fragm. II, 170 (1861) ‥ - S.A. - - N.S.WV. Q. - B.fl.V,I95

B. bicornis; Sclerolaena, Lindl. in Mitch. Three Exp. II. $47(1838)-$ S.A. - V. N.S.W. Q. N.A. B.fl.V, 195

B. biflora; Sclerolaena, R. Brown, prodr. $410(1810)$. $\quad \ldots \quad-$ S.A. - V. N.S.W. — - B.fl.V,196

B. paradoxa; Sclerolaena, R. Brown, prodr. $410(1810)$ … $\quad \ldots$. W.A. S.A. - V. N.S.W. - - B.fl. ., 196

B. quinquecuspis; Anisac., F. r. M. in Tr. Tict. Inst. I, 134 (1855) - S.A. - V. N.A.W. Q. - B.H.V,199

B. Drummondii; Anisacantha, Benth. Fl. Austr. V, $199(1870)$ W.A. - - - - - - B.fl.V,199

B. Birchii; Anisacantha, F, v. M, fragm. VIII, 163 (1874)

B. divaricata; Anisacantha, R. Brown, prodr. 410 (1810)

B. bicuspis; Anisac., F. v. M. in Trans. Vict. Inst. I, $133(1855)$

B. glabra; Kentropsis, F. v. M., fragm. I, 139 (1859)

B. echinopsila; A nisacantha, F. v. M., fragm. VII, 14 (1870) $\ldots$

B. enchylaenoides; Chenolea, F. V. M., fragm. X, $92(1876) \quad \ldots$

B. micrantha; Enchylaena, Benth. FI. Austr, V. 181 (1870)

B. brevicuspis; Anisacantha, F. v. M., fragm. IV, 150 (1864)
B. salsuginosa; Osteocarpum, F. v. M., second gen. Rep. 15 (1854) ENCHYLAENA, R. Brown, prodr. 408 (1810).

E. tomentosa, H. Brown, prodr, 408 (1810)

(1810) THRELKELDIA, R. Brown, prodr. 409 (1810).

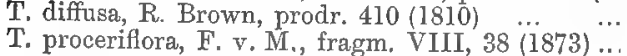
SALICORNIA, Tournefort, coroll. 5l (1703).

S. robusta, F. v. M., fragm. VI, 251 (1868)

S. arbuscula, R. Brown, prodr. 411 (1810)

S. cinerea, F. v. M., fragm. VI, 251 (1869)

S. leiostachya, Bentham, Fl. Anstr. V, 203 (1870)

S. bidens, Bentham, Fl. Austr. T, 204 (18,0)

S. tenuis, Bentham, Fl. Austr. V, 204 (1870)

S. australis, Solander in G. Forster, prodr. 88 (1786) SUAEDA, Forskael, F]. Aegypt. Arab. 69 (1779)

S. maritima, Dumortier, Fl. Belg. 22 (1827)

\author{
$\cdots$
}

...

... M.fr. VII, 9 . M.fr.VII, 8 .

M.fr.VII.9.

M.fr.IX,75.

1.fr. VII, 13.

II.fr. VII, 12.

M.fr. VII, 13.

M.fr. VII, 12.

fr.1,213.

IX, 168.

12.

M.fr. VII, 13.

M.fr.VII, 13 .

\section{M.fr. TrII, 11.}

\title{
M.fr. VII, 9;XI 27 \\ M.fr.VII, 10;XI,2
}

M.fr. VIII, 37, 163.

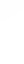

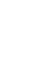


SALSOLA, Linne, gen. pl. 67 (1737), from Cisalpini.

S. Kali, Linné, spec. plant. 222 (1753)

... W.A. S.A. - V. N.S.W. Q. N.A. B.f.V,207 M.fr.XI,20,27.

\section{FICOIDEAE.}

A. L. de Jussieu, Gen. 315 (1789).

MESEMBRIANTHEMUM, Breyne, prodr. fasc. rar. pl. II, 67 (1689).

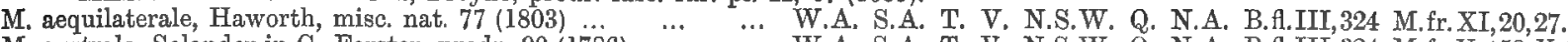

M. australe, Solander in G. Forster, prodr. 90 (1786) $\quad \ldots . \quad \ldots$ W.A. S.A. T. V. N.S.W. Q. N.A. B. I.III,324 M.fr.V, I58;X,S3;XI,20,27 TETRAGONIA, Linné, syst. nat. 9 (1735); Linné, gen. pl. 144 (1737). (Tetragonella.)

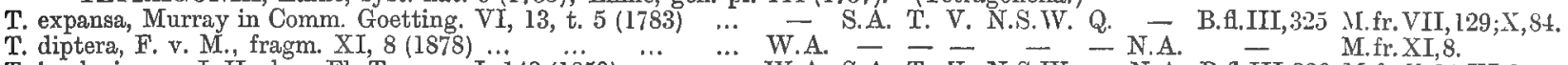

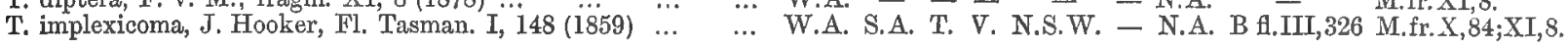
AIZOON, Linné, gen. pl. 161 (1737). (Aizoum.)

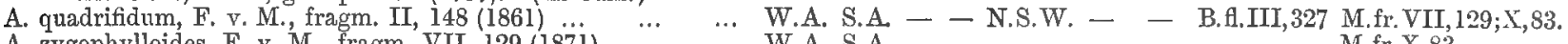

A. zygophylloides, F, v. M., fragm. VII, 129 (1871) $\quad \ldots \quad$... W.A. S.A. $\quad-\quad-\quad-\quad-\quad-$

GUNNIA, F. v. M., Rep. on pl, of Babb. Exped. 9 (1858).

G. septifraga, F. v. M., Rep. of Babb. Exped. $9(1858) \ldots \quad \ldots$... - S.A. - - — - - B.A.III,327

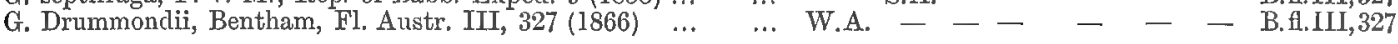
SESUVIUM, Linné, syst. ed. decim. 1058 (1759).

S. portulacastrum, Linné, syst. ed. dec. $1058(1759) \quad \ldots \quad \ldots \quad-\quad-\quad-\quad$ - N.S.W. Q. N.A. B.fl.III,328 M.fr.VII,129;X,83. ZALEXA, N. L. Burmann, Fl. Indic. 110, t. 31 (1768).

Z. decandra, N. L. Burmann, Fl. Ind. 110, t. $31(1768) \ldots \ldots$... - S.A. - - N.S.W. Q. N.A. B.A.III,329 M.fr.I,172;X,83. TRIANTHEMA, Sauvage, Méth. fol. 127 (1751). (Ancistrostigma.)

T. turgidifolia, F. v, M., fragm. X, 83 (1876)

T. crystallina, Vahl, symbol. I, $32(1790) \ldots$

T. glaucifolia, F. v. M., fragm. I, $172(1859)$

T. pilosa, F. v. M., fragm. I, $174(1859) \ldots \ldots$

T. oxycalyptra, F. v. M., fragm. I, $173(1859) \quad \ldots$

T. rhynchocalyptra, F. v. M., fragm. I, 174 (1859)

T. cypseloides, Bentham, Fl, Austr. III, 331 (1866) POMATOTHECA, F. v. M., fragm. X, 72 (1876).

P. humillima, F. v. M., fragm. X, $72(1876) \quad \ldots \quad \quad \ldots$ MACARTHURIA, Huegel. enum. pl. Nov. Holl. Austr. occ. 11 (1837).

M. apetala, Harvey in Hook. Kew. misc. VII, 55 (1855) $\quad$... W.A. $\quad$ W. - - $-\quad$ - - B.AlIII.332

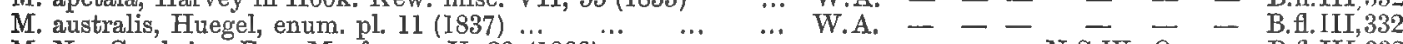

M. Neo.Cambrica, F, v. M., fragm. V, 28 (I866)... $\quad \ldots \quad \cdots \quad \ldots \quad-\quad-\quad-\quad$ N.S.W. Q. $\quad$ - B.H.III,332 MOLLUGO, Linné, gen. plant. 336 (1737). (Glinus, Trigastrotheca.)

M. Ginus, A. Richard, tentam. f. Abyss. I, 48 (1847) ... … W.A. S.A. T. V. N.S.W. Q. N.A. B.fl.III,333

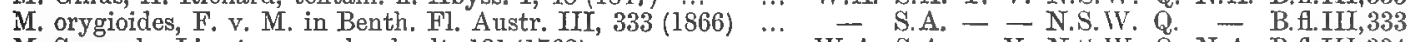

M. Spergula, Linné, spec. pl. ed. alt. $131(1762) \ldots \ldots \ldots$... W.A. S.A. - V. N.S.WV. Q. N.A. B.H.III,334

M. trigastrotheca, F. v. M., pl. of Vict. I, $201(1860) \ldots$.
M. Cerviana, Seringe in De Cand., prodr. I, $392(1824) \ldots \quad$... $\quad$ - S.A. - V. N.S. W. - - - B. B.III,334

\section{POLYGONACEAE.}

A. L. de Jussieu, gen. pl. 82 (1789), from B. de Jussien (1759).

EMFX, Necker, elem. bot. II, 214 (1790). (Perhaps immigrated.)

E. australis, Steinheil in Ann. des sc.nat. ser.2, IX, 195, t.7(1839) W.A. S.A. - - _- - - B.f.V,262 RUMEX, Linne, gen. pl. 105 (1737), from Plinius.

R. Brownii, Campdera, Monogr. Rum. 81 (1819)... ... .. - S.A. T. V. N.S.W. Q. - B.fl.V,263

R. flexuosus, Solander in G. Forst., f. ins. Austr. prodr. $90(1786)-$ S.A. - - N.S.W. - - B.fl.V,264

R. crystallinus, Lange, ind. sem. hort. Hafn. 28 (1861) ... .. - S.A. - V. N.S.W. Q. N.A. B.A.V,265

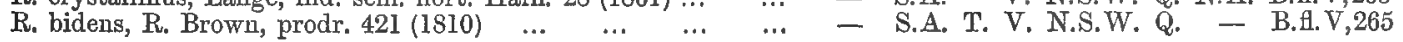
POLYGONUM, l'Obel, stimp. hist. 228 (1576), from Dioscorides and Plinius.

P. plebejum, R. Brown, prodr. $420(1810)$...

P. strigosum, R. Brown, prodr. $420(1810) \ldots$

P. prostratum, R. Brown, prodr. 419 (1810)

P. hydropiper, Linné, spee. plant. 361 (1753)

P. minus, Hudson, F'. Anglica I, 148 (1762)

P. subsessile, R. Brown, prodr. $419(1810) \ldots$

P. barbatum, Linné, spec. plant. 362 (1753)

P. articulatum, R. Brown, prodr. 420 (1810)

P. lapathifolium, Linne, spec. plant. 360 (1753)

P. orientale, Linné, spec. plant. $362(1753) \ldots$
P. attenuatum, R. Brown, prodr. 420 (1810)

$\ldots$
$\ldots$
$\ldots$
$\ldots$
$\ldots$
$\ldots$
$\ldots$
$\ldots$
$\ldots$
$\ldots$
$\ldots$ MUEHLENBECKIA, Meissner, gen. pl. vasc. 316 (1840)

M. adpressa, Meissner, gen. pl. comm. 227 (1843)

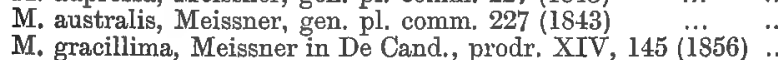

M. rhyticarya, F. v. M., fragm. V, 92 (1866) ...

M. axillaris, J. Hooker in Lond. Journ. VI, 278 (ï847) ...

M. polybotrya, Meissner in Lehm, pl. Preiss. I, 623 (1845)

M. polygonoides, F. v. M., fragm. V, 73 (1865) ...

M. stenophylla, F. v. M., fragm. I, $138(1859) \ldots$

M. Cunninghamii, F. v. M., fragm. V, 91 (I865) ... $\ldots$
$\ldots$
$\ldots$
$\ldots$
$\ldots$
$\ldots$
$\ldots$
$\ldots$
$\ldots$
$\ldots$
$\ldots$
$\ldots$
$\ldots$
$\ldots$
$\ldots$
$\ldots$
$\ldots$

- S.A. - V. N.S.W. Q. N.A. B.fl.V,267

- - T. V. N.S.W. Q. - B.f.V,268

W.A. S.A. T. V. N.S.W. Q. - B.H.V,268

- S.A. - V. N.S.W. Q. - B.H.V,269

W.A. S.A. T. V. N.S.W. Q. - B.H.V,269

- - T. V. N.S.W. Q. - B.f.V,269

-. - - - N.S.W. Q. - B.t.V,270

- - - - N.S.W. Q. - Q B.f.V,270

- S.A. T. V. N.S.W. Q. - B.f.V.,270

- - - - N.S.W. Q. - B.fl.V,271

- S.A. - - N.S.W. Q. N.A. B.H.V,272

W.A. S.A. T. V. N.S.W. - - B.A.V,273

- $\quad-\quad-$ - N.S.W. $\overline{\text { Q. }}$ - B.f. $\bar{V}, 2,4$

- - - - N.S.W.Q. - B B.V,2I

$\therefore$ W.A. - T. V. N.S.W. - - B.f.V.,275

... $\quad$ - S.A. - V. N.S.W. - - B.fl.V,275

.. - - - V. - - - B.fl.V,275

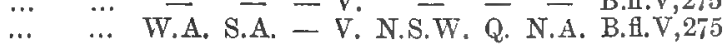

M,fr.II, 148.

M.fr.X, 83 .

M.fr.I, 171-2.

M.fr. I, 172 .

M.fr. $X$, s.3.

MI.fr. I, 173 .

M.fr. $[, 174$.

M.fr.X, 83 .

M.fr. $X, 72$.

M.fr. XI, 20.

M.fr. V,28.

M.fr.XI, 20 .

M.fr. IX, 169.

M.fr. $\Gamma, 130$.

M.fr. V, 73 .

M.fr.I, 138.

I.fr, V, 95. 
PHYTOLACCEAE.

R. Brown in Tuck. Exped. Cong. App. V, 454 (1818).

MONOCOCCUS, F. v. M., fragm. I, 47 (1858).

M. echinophorus, F. v. M., fragm. I, $47(1858) \quad \ldots \quad \ldots . \quad \ldots \quad-\quad-\quad-\quad$ N.S.W. Q. $\quad-\quad$ B.fl.V, 144

M.fr,I,47.

DIDYMOTHECA, J. Hooker in Lond. Journ. Bot. VI, 278 (1847). (Cyclotheca.)

D. thesioides, J. Hooker in Lond. Journ. V, 279 (1847) .. $\quad$... W. W. S.A. T. - _ - - B.f.V,I45

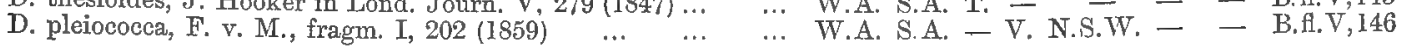

M.fr.I,202.

GYROSTEMON, Desfontaines in Mém. du Mus, VI, 16, t. 6 (1820).

G. brachystigma, F. v. M. in Benth Fl. Austr. V, $146(1870) \ldots$ W.A. - - - - - - - B.A.V., 146

G. ramulosus, Desfontaines in Mém. du Mus. V, 17, t. 6 (1820)

CODONOCARPUS, Cunningham in Hook. Bot. Misc. I, 244 (1830). (Hymenotheca.)

C. pyramidalis, F. v. M., plants of Vict. I, 201 (1862) ... … - S.A. - - - - - B.fl.V,I48

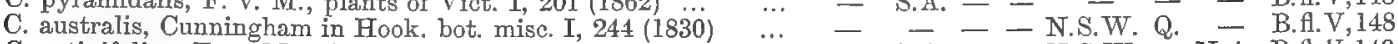

C. cotinifolius, F. v. M., pl. of Vict. I, $200(1862) \quad \ldots . \quad \ldots . .7$ W.A. S.A. - V. N.S.W. - N.A. B.A.V.149

TERSONIA, Moquin in De Cand., prodr. XIII, part sec. 40 (1849).

T. brevipes, Moquin in De Cand., prod. XIII, part II, 40 (1849) W.A. - - - - - - B.f.V,I49

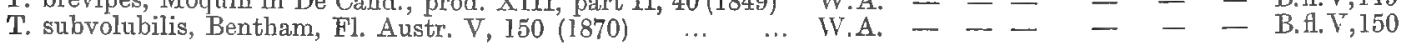

CYPSELOCARPUS, F. v. M., fragm. VIII, 36 (1873).

C. haloragoides, F. v. M., fragm. VIII, 36 (1873)

W.A. - -
NYCTAGINEAE.

A. L. de Jussieu, Gen. 90 (1789).

BOERHAAVIA, Vaillant, serm. de struct. fl. 50 (1718).

B. diffusa, Linné, spec. fl. Zeylan. 4 (1747)

B. repanda, Willdenow, sp. pl. I, 22 (1;97)

$\begin{array}{lll}\cdots & \cdots & \cdots \\ \cdots & \cdots & \cdots\end{array}$

7, t. $11(1703)$.

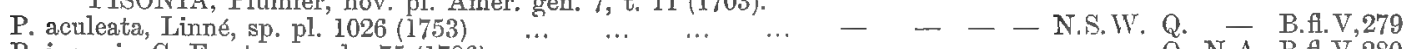

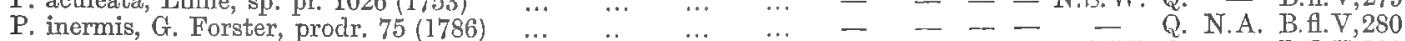

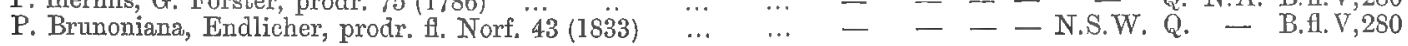

M.fr.VI, 197. M.fr.VI, 197. M.fr.I,20;IV, 169 .

\title{
CHORIPETALEAE PERIGINAE.
}

\author{
F. v. M. in Woolls pl, of the neighb. of Sydney 18 (1880).
}

\section{CONNARACEAE.}

R. Brown, Narr. exped. Cong. App. V, 431 (1878).

ROUREA, Aublet, Hist, des pl. de la Guian. I, 467, t. 187 (1775).

ROUREA, Aublet, Hist, des pl, de la Guian. I, 467, t. 1
R. brachyandra, F. v. M., fragm. VIII, 6 (1872) ... $\ldots$
TRICHOLOBUS, Blıme, Mus. Bot. Lugl. I, 237 (1850).
T. connaroides, F. v. M., fragm. VIIY, 224 (1874)

\section{LEGUMINOSAE.}

Haller, enum. stirp. Helv. II, 565 (1742).

JANSONIA, Kippist in Gard. Chron. 19 (1847). (Cryptosema.)

J. formosa, Kippist in Transact. Linn. Soc. XX, 384, t. 16 (1851) W.A. - - - - - - - B.fl.II,8

BRACHYSEMA, R. Brown in Ait. hort. Kew. sec. edit. III, 10 (1811), (Leptosema, Kaleniczenkia, Burgesia.)

B. praemorsum, Meissner in Lehm. pl. Preiss. I, 25 (1844) ... IV A. - - - - - - B.f.II,10

B. Ianceolatum, Meissner in Lehm. pI. Preiss. I, 24 (1844) … W.A. - - - - - - B.f.II,10

B. latifolium, R. Brown in Ait. hort. Kew. sec. ed. III, 10 (181i) W.A. - - - - - - - B.f.II,10

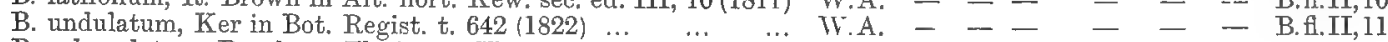

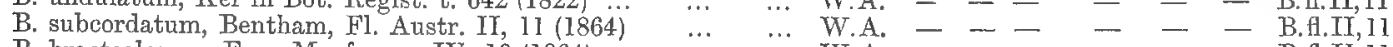

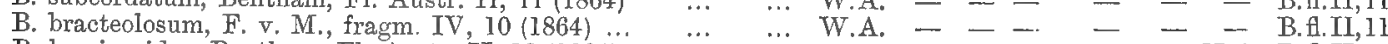

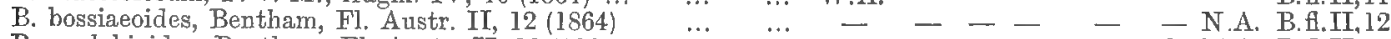

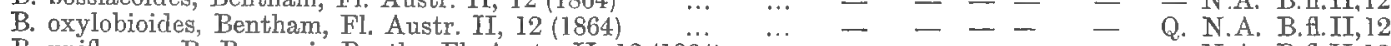

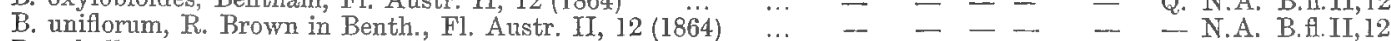

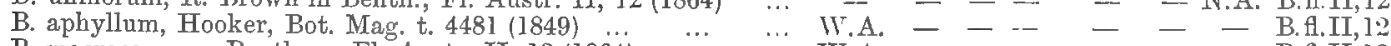

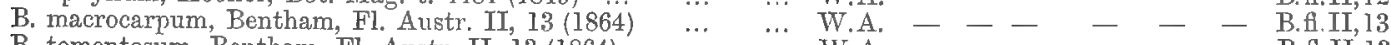

B. tomentosum, Bentham, Fl. Austr. II, 13 (1864) $\quad \ldots .6$... W.A. - - - - - - - - B.fl.II,13

B. Chambersii, F. v. M. in Benth., Fl. Austr. II, 13 (1864) $\quad \ldots \quad-$.

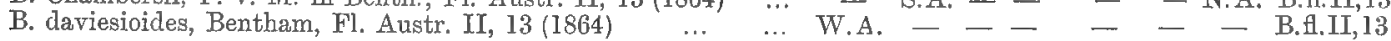

OXYLOBIUM. Andrews, Bot. Reposit. t. 492 (1809). (Chorizematis subgenus, Callistachys, Podolobium.)

o. Callistachys, Bentham, F. Austr. II, 16 (1864) ‥ W. W. - - - - - - B. H.II, 16

O. ellipticum, R. Brown in Ait. hort. Kew. sec. ed. III, $\dddot{10}$ (I81i) - - T. T. N.S.I. Q. - B.fi.II,16

O. alpestre, F. v. M. in Transact. phil. Soc. Vict. I, 38 (1854) .. - - - V. N.S.W. - - B.f.II,17

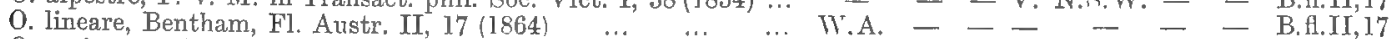

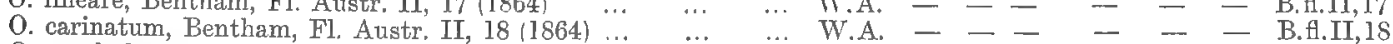

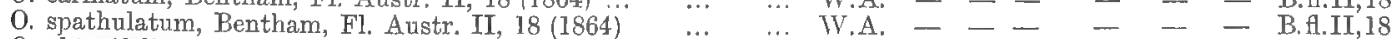

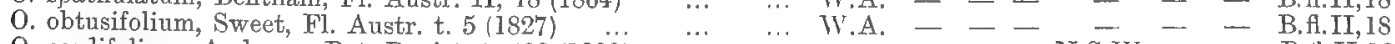

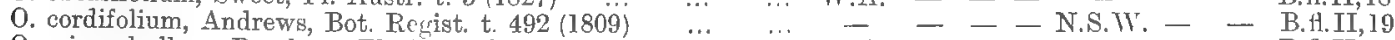

O. microphyllum, Bentham, FI. Austr. II, 19(1864) $\quad \ldots \quad$... W.A. - $\quad$ W - - - - B.fl. II,19

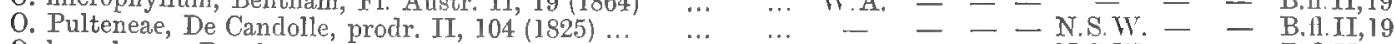

O. hamulosum, Bentham in A. Gray, Bot. Will. Exped. t. $379-2-$ - - N.S.W. - - B.H.II,20

O. scandens, Bentham in Ann. Wien. Mus. II, 70(1839)... ... - - - - N.S.T. Q. - B.fl.II,20

O. procumbens, F. v. M. in Transact. phil. Soc. Vict. I, 37 (1854) - - - V. N.S.W. - - B.f.II,20

0 . tricuspidatum, Meissner in Lehm. pl. Preiss. I, $30(1844)$.. W.A. - - - - - - B.H.II,21

M.fr.IT, I1.

M.fr.IV, 10 .

M.fr. I, 222,

M.fr.IT, IS.

II.fr.IY, 17 .

M.fr.IV,I7.

M.fr.IV, 19 . 
0. spectabile, Endlicher, nov. stirp. dec. 2 (1839)

0. atropurpureum, Turczanin. in Bull. Soc. Mosc. XXVI, 250 (1853) W. A

0 . retusum, $R$. Brown in Bot. Regist. t. 913 (1825)

O. virgatum, Bentham, Fl. Austr. II, 22 (1864) ...

O. reticulatum, Meissner in Lehm. pl. Preiss. I, 29 (1844)

O. capitatum, Bentham in Hueg. enum. 28 (1837)

O. cuneatum, Bentham in Bot. Regist. XXV, App. XII (1839)

o. acutum, Bentham, Fl. Austr. II, 24 (1864)

O. parviflorum, Bentham in Bot. Regist. XXV, App. XII (1839)

0 . heterophyllum, Bentham, Fl. Austr. II, 25 (1864)

0 . aciculiferum, F. v. M., fragm. I, 75, implied (1859) ...

-.. CHORIZEMA, Labillardière, Voy. I, 405 (1799). (Orthotropis.)

C. Dicksonii, Grabam in Maund's Botanist, t. 106 (184I)

C. nervosum, Th. Moore in Gard. Companion (1852)

C. varium, Bentham in Bot. Regist. XXV, t. 49 (1839) ...

C. cordatum, Lindley, Bot. Regist. t. 10 (1838)

C. ilicifolium, Labillardière, Voy. I, 405, t. 21 (1799) ... … IV.A.

C. rhombeum, R. Brown in Ait. hort. Kew. sec. ed. III, 9 (1811) W.A.

C. diversifolium, A. de Candolle, Pl. rar. jard. Genèv. 44, t. 8 (1836)W.A.

C. angustifolium, Bentham in Hueg. enum. 28 (1837)

C. reticulatum, Meissner in Lehm. pl. Preiss. I, 34 (1844)

C. trigonum, Turcz. in Bull. de la Soc. Mosc. XXVI, 254 (1853)

C. humile, Turcz. in Bull. de la Soc. Mose. XXVI, 254 (1853) ..

C. parviflorum, Bentham in Ann, des Wien. Mus. II, 71 (1838)

C. cytisoides, Turczaninow in Bull. Soc. Mosc. XXVI, 256 (1853)

C. Henchmanni, R. Brown in Bot. Regist. t. 986 (1826)...

C. ericifolium, Meissner in Lehm. pl. Preiss. II, 209 (1847)

GASTROLOBIUM, R. Brown in Ait. hort. Kew. sec. edit.

… W.A. - - -

G. pulchellum, Turez, in Bull. de la Soc. Mosc. XXVI, 274(1853) W.A. - - -

G. stipulare, Meissner in Lehm. pl. Preiss. II, 218 (1847) ... W.A. - - -

G. Brownii, Meissner in Lehm. pl. Preiss. I, 71 (1844) ... ... W.A. - -

G. reticulatum, Bentham, Fl. Austr. II, 99 (1864)

, M.

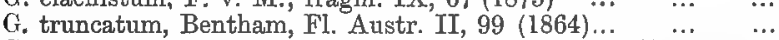

G. spathulatum, Bentham in Bot. Regist. XX, App. XIV (1839)

G. plicatum, Turczanin. in Bull. Soc. Mosc. XXVI, 274 (1853)

G. tricuspidatum, Meissner in Lehm. pl. Preiss. I, 66 (1844)

G. obovatum, Bentham in Bot. Regist. XXV, App. XIV (1839)

G. epacridioides, Meissner in Lehm. pl. Preiss. I, 72 (1844)

G. trilobum, Bentham in Bot. Regist. XXV, App. XIIT (1839)...

G. ilicifolium, Meissner in Lehm, pl. Preiss. I, 67 (1844)

G. villosum, Bentham in Bot. Regist. XXV, App. XIII (1839)...

G. polystachyum, Meissner in Lehm. pl. Preiss. II, 217 (1847)...

G. ovalifolium, Henfrey in Gard. Comp. I, 41 (1852)

G. grandiflorum, F. v. M., fragm. III, 17 (1862) $\ldots . \quad \ldots \quad \ldots$

G. pycnostachyum, Bentham, Fl. Austr. II, 103 (1864)

G. spinosum, Bentham in Bot. Regist. XXV, App. XIII (1839)

G. rotundifolium, Meissner in Lehm. pl. Preiss. II, 216 (1847)...

G. microcarpum, Meissner in Lehm. pl. Preiss. I, 70 (1844)

G. oxylobioides, Bentham in Bot. Regist. XXV, App. XIII (1839)

G. calycinum, Bentham in Bot. Regist. XXV, App. XIII (1839)

G. Callistachys, Meissner in Lehm. pl. Preiss. II, 216 (1846)

G. stenophyllum, Turczanin. in Bull. Soc. Mosc. XXVI, 275(1853) W

G. crassifolium, Bentham, Fl. Austr. II, I05 (1864) ... ... W

G. parvifolium, Bentham, Fl. Austr. II, $106(1864) \quad \ldots$
G. hamulostum, Meissner in Lehm. pl. Preiss. II, 218 (1847) $\ldots$

G. hamulostum, Meissner in Lehm. pl. Preiss. II, 218 (1847) $\ldots$

G. bidens, Meissner in Regensb. Bot. Zeit. 29 (1855)

G. bilobum, R. Brown in Ait. hort. Kew. sec. edit. III, $\dddot{16}$ (181i)

G. seorsifolium, F. v. M., fragm. X, 35 (1876)

ISOTROPIS, Bentham in Hrueg. enum. 28 (1837).

I. striata, Bentham in Hueg. enum. 28 (1837) $\ldots$

I. Drummondii, Meissner in Lehm. pl. Preiss. I, 31 (1844)
I. juncea, Turczanin. in Bull. Soc. Mosc. XXVI, 251 (1853)

I. canescens, F. $v$. M., fragm. X, 51 (1876).

I. atropurpurea, F. v. M., fragm. III, I6 (1862) $\quad \ldots \quad$ $\quad \ldots$

I. filicaulis, Bentham in Ann. des Wien. Mus. II, 71 (1838) $\cdots$

I. parviflora, Bentham in Ann. des Wien. Mus. II, 71 (1838) ...

I. Wheeleri, F. v. M. in Benth. Fl. Austr. II, 40 (1864)

MIRBELIA, Smith in Koenig \& Sims, Ann. of Bot. I, 511

M. dilatata, $R$. Brown in Ait. hort. Kew. sec. ed, III, 21 (1811)

M. racemosa, Turczanin. in Bull. Soc. Mosc. XXVI, 282 (1853)

M. grandiflora, Aiton in Bot. Mag, t. 2771 (1827)

M. subcordata, Turczanin. in Bull. Soc. Mosc. XXVI, $282(1853)$

M. ovata, Meissner in Lehm. pl. Preiss. I, 77 (1844)

W.A. - - -

Y
- - -

W.A.

W.

IV.A.

W.A. - - -

- S.A. - -

W.A. - - -

W.A. $\rightarrow-$

W.A. - - -

W.A. - - -

W.A. - - -

W.A. - - -

W.A. - - -

W.A. - - -

W.A. - - -

W.A. - - -

$\overline{W A}---$

W.A - - -

W.A. - - -

W.A.

W.A.

W.A.

W.A.

W.A.

W.A. - - -

W.A. - - -

W.A. - -

W.A. - - -

W.A. - - -

W.A. - - -

W.A. - - -

W.A. - - -

W.A. - - -

- S.A. - -

二 S.A. - - - - N.A. B.fl.II,40

- S.A - - N.S.W - N.A. B.f.II, 40

(1805). (Dichosema, Oxycladium.)

W.A. - - - - - - B.fl.II, 33

W.A. - - - - - - B.fl.II,33

- - - - N.S.W. - - B.f.II, 34

M.fr.IV,18.

M.fr.I,75.

M.fr.IV, 19 .

M.fr. X, 35 .

M. fr, X, 35 .

M.fr.IX, 67.

M.fr.X, 35 .

M.fr.III, I7;IV, 174; X, 35 .

M.fr.X, 35 .

M.fr.X,35.

M.fr.X, 35

M.fr.X, 35 .

M.fr.X, 3 อ̃.

M.fr.III, I6.

M.fr.X. 51 .

M.fr.X, 5I.

M.fr.III, $16 ; \mathrm{X}, 51$.

M.fr. IV,20.

W.A. - - - - - B.fl.M,34

V.A. - - - - - B.円.II,34 
M. oxylobioides, F. v. II, fragm. II, 154 (1861) ...

M. reticulata, Smith in Koen. \& Sims, Amn. of Bot. I, $5 \dddot{11}$ (180̈)

M. aotoides, F. v. M. in Transact. phil. Inst. Vict. III, 53 (1859)

M. pungens, Cunningham in G. Don, gen. syst. II, 126 (1832) ...

M. speciosa, Sieber in De Cand. prodr. Ir, 115 (1824)

M. floribunda, Bentham in Bot, Regist. XXV, App. XII (1839)
M. spinosa, Bentham, Fl. Austr. II, $36(1864)$... ..

M. multicaulis, Bentham, Fl. Austr. II, 37 (1864)

M. microphylla, Bentham, Fl. Austr. II, 37 (1864)

M. daviesioides, Bentham, Fl. Austr. II, 37 (1864)

M. oxyclada, F'. v. M., fragm. IV, 12 (1864)

GOMPHOLOBIUM, Smith in Transact. Linn. Soc. IV, 220 (1798)

G. ovatum, Meissner in Lehm. pl. Preiss. I, $35(1844) \ldots \ldots$ W.A.

G. amplexicaule, Meissner in Lehm. pl. Preiss. I, 36 (1844) ... W. A.

G. Huegelii, Bentham in Hueg. enum. 29 (1837) ...

G. polymorphum, R. Brown in Ait. hort. Kew, sec. ed. III, 11 (18i1) W.A.

G. obcorclatum, Turczanin. in Bull. Soc. Mosc. XXVI, 258 (1853) W.A.

G. marginatum, R. Brown in Ait. hort. Kew, sec. ed. III, 11 (1811)W.A.

G. grandiflorum, Smith, Exot. Bot, t. 5 (1804)

G. virgatum, Sieber in De Cand. prodr. II, 105 (1824) $\quad \ldots \quad \ldots$

G. minus, Smith in Transact. Linn. Soc. IX 251 (1808)

G. uncinatum, Cunningham in Annal. des Wien. Mus. II, 72 (1838) -

G. Baxteri, Bentham, Fl. Austr. II, 46 (1864)

G. Baxteri, Bentham, $\ldots$. $\ldots$ W.A.

G. burtonioicles, Meissner in Lehm. pl. Preiss. I, 37 (1844) … W.A.

G. capitatum, Cunningham in Bot. Regist. t. 1563 (1839) $\ldots .$. W.A.

G. tomentosum, Labillard., Nov. Holl. pl. sp. I. 106, t. 134 (1804) W.A.

G. Preissii, Meissner in Lehm. pl. Preiss. I, 40 (1844) . $\quad \ldots$ W.A.

G. viscidulum, Meissner in Lehm. pl. Preiss. I, 39 (1844)

G. glabratum, De Candolle, prodr. II, 106 (1824) ...

G. nitidum, Solander in Benth. Fl. Austr. II, 48 (1864)

G. pinnatum, Smith in Transact. Linn. Soc. IX, 251 (1808)

G. Shuttleworthii, Meissner in Lehm. pl. Preiss. I, 39 (1844)

G. venust BURTONIA, R. Brown in Ait. hort. Kew, ed. sec. III, 13 (1811).

B. subulata, Bentham, Fl. Austr. II, 50 (1864) ... ... ... -

B. foliolos, Benthem,

B. polyzyga, Benthan, Fl. Austr. II, 5l (1864) ...

B. villosa, Meissner in Lehm. pl. Preiss. I, 4l (184t)

B. Hendersonii, Bentham, Fl. Austr. II, 51 (IS64)

B. gompholobioides, F. . . M., fragm. X, 34 (1876)

B. scabra, R. Brown in Ait. hort. Kew. sec. ed. III, 12 (is11) $\cdots$

B. conferta, De Candolle, prodr. II, 106 (1825) JACKSONIA, R Brown in Ait, hort. Kew.

J. dilatata, Bentham in Ann. des Wien. Mus. II, 74 (1838)

J. densiflora, Bentham in Bot. Regist. XXV, App. XIII (1839)

J. carduacea, Meissner in Bot. Zeit. 25 (1855)

J. floribunda, Endlicher in Ann des Wien. Mus. II 197 (1838*.

J. odontoclada, F, v. M. in Benth. Fl. Austr. II, 55 (1864)

J. ramosissima, Bentham in Mitch. Trop. Austr. 258 (1848)

J. foliosa, Turcz. in Bull. de la Soc. Imp. de Mosc. XXVI, 260 (18

$J$. spinosa, R. Brown in Ait. hort. Kew, sec. ed. III, 13 (1811)

J. stricta, Meissner in Regensb. Bot. Zeit. 27 (1855)

J. hakeoides, Meissner in Lehm. pl. Preiss. I, 45 (1844) ...

J. furcellata, De Candolle, prodr. II, 107 (1824) ...

J. horrida, De Candolle, prodr. II, 107 (1824)

$\mathbf{J}$. sericea, Bentham in Hueg. enum. 31 (1837)

J. Sternbergiana, Huegel, Bot. Archiv. t. 3 (1837)

J. Sterner.

J. vernicosa, F. v. M. in Benth. FL. Austr. II,
J. rhadinoclona, F. v. M., fragm. X, 37 (1876)

J. thesioides, Cunningh. in den Ann. des Wien. Mus. II, 74 (1838)

J. compressa, Turcz. in Bull. de la Soc. de Mosc. XXVI, 260 (1853)
J. scoparia, R. Brown in Ait. hort. Kew. sec. ed. III, I3 (1811)

J. cupulifera, Meissner in der Bot. Zeitung 27 (1855)

J. restioides, Meissner in Lehm. pl. Preiss. I, 46 (1844) ...

J. velutina, Bentham, Fl. Austr. II, 60 (1864)

J. Lehmanni, Meissner in Lehm. pl. Preiss. I, 46 (1844) ...

J. racemosa, Meissner in Lehm. pl. Preiss. II, 212 (1847)

J. umbellata, Turczaninow in Bull. Soc. Mosc. XXVI, 261 (1553)

J. nematoclada, F. v. M., fragm. X, 50 (1876)

J. rhadinoclada, F. v. M., fragm. X, $38(1876)$

J. capitata, Meissner in Lehm. pl. Preiss. I, 45 (1844)

J. alata, Bentham in Hueg, enum. 30 (1837)

J. angulata, Bentham, Fl. Austr. II, 62 (1864)

J. Staplo

J. pteroclada, F. v. M., frarm. X, 37 (1876)

J. macrocalyx, Meissner in der Bot. Zeit. 26 (1855)

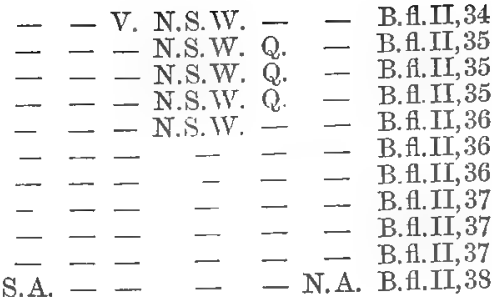

M.fr.IV 12 .

M.fr.IV,11. M.fr.IV,12.

\begin{tabular}{|c|c|c|c|c|c|}
\hline - & - & - & - & - & B.fl.II, 42 \\
\hline - & - & - & & - & \\
\hline & & S.W. & Q. & - & B.fl.II, 42 \\
\hline T. & V. & N.S.W. & - & - & B.fl.II, 43 \\
\hline - & - & - & - & - & B.fl.II, 43 \\
\hline - & - & - & - & 一 & B. Al. II, 44 \\
\hline - & - & - & $\ldots$ & - & B.fl.II, 44 \\
\hline- & 一 & N.S.W. & $\cdots$ & - & B.fl.II, 44 \\
\hline - & - & N.S.W. & $Q$. & 一 & B. fl. II, 45 \\
\hline - & V. & N.S.W. & - & 一 & B.fl.II, 45 \\
\hline - & - & N.S.W. & - & - & B fl, II, 46 \\
\hline - & 一 & - & - & - & В.А.II, 46 \\
\hline - & 一 & - & - & - & B.fl.II, 46 \\
\hline 一 & - & - & 一 & 一 & B.Al.IT, 46 \\
\hline - & - & - & - & - & B. $\mathrm{A} . \mathrm{II}, 47$ \\
\hline - & - & - & - & - & B.fl.IX, 47 \\
\hline - & - & - & 一 & - & B.fl.II, 17 \\
\hline 一 & 一 & - & - & 一 & B. $1 . \mathrm{II}, 48$ \\
\hline - & 一 & N.S.W. & 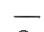 & - & B.l.II, 48 \\
\hline 一 & - & - & Q. & - & B.fl.II, 48 \\
\hline 一 & - & N.S.IV. & $Q$. & - & B.fl.II, 48 \\
\hline - & 一 & - & - & - & B. 囵.II, 49 \\
\hline - & $\ldots$ & - & - & - & B.fl.II, 49 \\
\hline - & 一 & - & - & 一. & B.fl.II, 49 \\
\hline
\end{tabular}

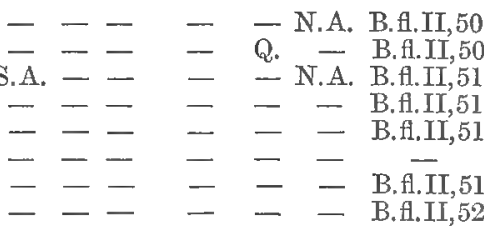

(Piptomeris.)

- - - - - - N.A. B.fi.II, 51

W.A. - - - - - - B.A.II,54

W.A. - - - - - - B.H.II,55

W.A. - - - - - B.f.II,55

- N.A. B. 1 l.II,55

Q. - B.fl.II,56

- - B.fl.II,56

- - B.fl.II,56

- - B.fl.II,56

- $\quad$ - B.fl.II,57

- - B.fl.II,57

- - B.A.II,57

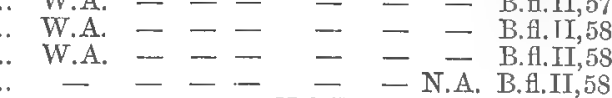

W.A. - - - - - - - B.f.II,58
W.A. - - - - B.f.II,58
-. - B.H.II,58

- - - - N.S.W.

Q. -

.

W.A. - - - - Q. N.A. D.fl.II,59

W.A. - - N.S.W. Q. - B.fl.II,59

W.A. - - - - - - B. -

W.A. - - - - - - B.f.II,60

W.A. - - - - - - B.f.II,60

VV.A - - - - - B.

W.A. - - - - - B.ff.II,61

W.A. - - - - - -

W.A. - - - - - - B.A.II,

W.A. - - - - - - B.f.II,61

B.H.II, 62

-

- - - N.S.W. Q.$$
\text { - }
$$

二

D.f. II, 62
M.fr.III,30;X,35.

M.fr.III, $29 ; \mathrm{X}, 34$.

MI.fr.X, 35 .

M.fr.X, 34 .

M.fr. X, 35.

M.fr.X.35.

M.fr.X, 37 .

M.fr.X,38.

MI.fr.X, 37.

M.fr.X,a7.

M.fr. X, 38\&39.

M.fr.X, 39 .

M.fr.X,39.

M.fr.X, 39 .

MI.fr. X, 37 .

M. fr. X, 37 .

M.fr.IV, I61;X, 37 .

M.fr. X,3s.

M.fr. X, 37.

II.fr. X, 36 .

M.fr. X, 38 .

M. fr. $X, 50$.

Mi.fr. $\mathrm{X}, 38$,

I.fr. X, 38 ,

M.fr. X, 3s.

M.fr. XI, 138

M.fr, X, 37 . 
J. purpurascens, F. v. M., fragm. IV, 61 (1864)..

J. piptomeris, Bentham, Fl. Austr. II, 62 (1864)...

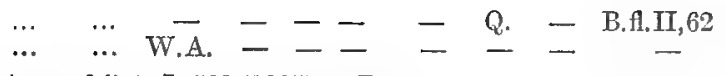
SPHAEROLOBIUM, Smith in Koenig \& Sims, Ann, of Bot. I, 509 (1805). (Roea.)

S. linophyllum, F. v. M., fragm. I, 167 (1859)

S. foliosum, F, v. M., fragm. I, 166 (1859)...

S. gracile, Bentham, Fl. Austr. II, 64 (1864)

S. racemulosum, Bentham, FI. Austr. II, 64 (1864)

... W.A. W - - - -

... ‥ TV.A. $\quad--\quad-\quad-$ B.fl.II,64

... $\quad$... W.A. - W - - - - - B.f.II. 64

… $\quad \cdots$ W.A. - - - - - - B.fl.II,65

S. alatum, Bentham in Hueg. enum. 32 (1837) $\quad \ldots \quad \ldots . \quad \ldots$. W.A. $-\quad--\frac{\ldots}{-}-\quad-$ B.H.II,65

S. vimineum, Smith in Koen. \& Sims, Ann. of Bot. I, 509 (1805)...

S. grandiflorum, R. Brown in Benth. Fl. Austr. II, 66 (1864) ...

S. fornicatum, Bentham in Hueg. enum. 32 (1837)

S. medium, R. Brown in Ait. hort. Kew. sec. ed. III, 14 (1811)

S. scabriusculum, Meissner in Lehm. pl. Preiss. II, 214 (1849)...

S. macranthum, Meissner in Lehm. pl. Preiss. II, 213 (1847) ...
S. daviesioides, Turczaninow in Bull. Soc. Mose. XXVI, 266 (1853) VIMINARIA, Smith, Exotic Botany 51, t. 27 (1804).

V. denudata, Smith, Exot. Bot. 5l, t. 27 (1804) ... $\ldots . \quad$... W.A. S.A. T. V. N.S.W. Q. - B.A.II,68 DAVIESIA, Smith in Transact. Linn. Soc. TV, 222 (1798).

D. ovata, Bentham, Fl. Austr. II, 72 (1864) ... ...

D. crenulata, Turczanin. in Bull. Soc. de Mosc. XXVI, 265 (1853)

D. oppositifolia, Endl. in den. Ann. des Wien. Mus. II, 199 (1838)

D. alternifolia, Endlicher in Ann. Wien. Mus. II, 199 (1838) .

D. elongata, Bentham, Fl. Austr. II, 74 (1864)

D. pedunculata, Bentham in Bot. Regist. XXV, Äpp. XIV (1839)

D. mollis, Turczaninow in Bull. Soc. Mosc. XXYI, 263 (1853) $\cdots$

D. umbellulata, Smith in Koen. \& Sims, Ann. of Bot. I, $507(\dddot{1005})$ -

D. Wyattii, Bailey in pap. Gardn. Assoc. of S. Austr. (1880)

D. Iatifolia, R. Brown in Ait. hort. Kew. sec. ed. III, 20 (1811)

D. corymbosa, Smith in Koen. \& Sims, Ann. of Bot. I, 507 (1805)

D. horrida, Meissner in Lehm. pl. Preiss. I, 54 (1844)

D. reclinata, Cunningham in Benth. Fl. Austr. II, 77 (1964) …

D. obtusifolia, F. v. M., fragm. II, 104 (1861)

D. obovata, Turczaninow in Bull. Soc. Mosc. XXV̈I, 261 (1853)

D. longifolia, Bentham in Bot. Regist. XXV, App. XTV (1839)

D. chordophylla, Meissner in Lehm. plant. Preiss. I, 48 (1844)...

D. nematophylla, F. v. M. in Benth. Fl. Austr. II, is (1864) ...

D. daphnoides, Meissner in Lehm. plant. Preiss. I, 54 (1844) ...

D. nudiflora, Meissner in Lehm. plant. Preiss. I, 53 (1844) $\ldots$
D. rhombifolia, Meissner in Lehm. plant. Preiss. I, 56 (1844) $\ldots$

D. rhombifolia, Meissner in Lehm. plant. Preiss. I, 56 (1844) ...

D. filipes, Bentham in Mitch. Trop. Austr. 363 (1848) ... ...

D. cardiophylla, F. v. M., fragm. II, 105 (1861) ...

D. squarrosa, Smith in Koen. \& Sims, Ann, of Bot. I, 507 (1805)

D. ulicina, Smith in Koen. \& Sims, Ann. of Bot. I, 506 (1805) ...

D. acicularis, Smith in Koen. \& Sims, Ann. of Bot. I, 506 (1805)

D. pachyphylla, F. v. M., fragm. IV, 15 (1864)

D. teretifolia, R. Brown in Benth. Fl. Austr. II, 82 (1864)

D. genistifolia, Cunningham in Annal. Wien. Mus. II, 75 (1838)

D. hakeoides, Meissner in Lehm. plant. Preiss. I, 47 (1844) …

D. colletioides, Meissner in Lehm. plant. Preiss, I, 48 (1844) ...

D. reversifolia, F. v. M., fragm. I, 145 (1859)

D. incrassata, Smith in Transact. Linn. Soc. IX, 255 (1808) $\ldots$

D. brevifolia, Lindley in Mitch. Three Exped. II, 201 (1838) ...

D. arcanthoclona, F. v. M., fragm. X, 32 (1876)

D. Preissii, Meissner in Lehm. plant. Preiss. I, 50 (1844)

D. spinosissima, Meissner in Lehm. plant. Preiss. I, 51 (1844) ...

D. pachylina, Turczaninow in Bull. Soc. Mosc. XXVI, 263 (1853)

D. quadrilatera, Bentham in Bot. Regist. XXV, App. XIV (1839)

D. striata, Turczaninow in Bull. Soc. Mosc. XXVI, 264 (1853)

D. polyphylla, Bentham in Bot. Regist. XXV, App. XIV (1839)

D. microphylla, Bentham, Fl. Austr. II, 86 (1864)

D. flexuosa, Bentham in Hueg. enum. 32 (1837)

D. pectinata, Lindley in Mitch. Three Exped. II, I5l (1838) ...

D. trigonophylla, Meissner in Lehm. plant. Preiss. II, 213 (1847)

D. epiphylla, Meissner in Regensb. Bot. Zeit. 27 (1855) ... $\quad \ldots$
D. euphorbioides, Bentham, Fl. Austr. II, 88 (I864) $\ldots$.

D. divaricata, Bentham in Hueg. enum. $31(1837)$
D. paniculata, Bentham in Hueg. enum. $31(1837)$
D.

D. paniculata, Bentham in Hueg. enum. $31(1837) \quad \ldots$
D. aphylla, F. v. M. in Benth. Fl. Austr. 88 (1864) $\quad \ldots$

D. juncea, Smith in Transact. Linn. Soc, IX, 260 (1808) $\quad \ldots$

D. alata, Smith in Transact. Limn. Soc. IX, $259(1808) \ldots$
D. anceps, Turczaninow in Bull. Soc. Mose. XXVI, 266 (1853)...
AOTUS, Smith in Koenig' \& Sims, Ann. of Bot. I, 504 (1805) AOTUS, Smith in Koenig \& Sims, Ann. of Bot. I, 504 (1805).

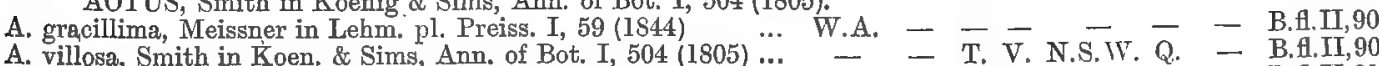
A. villosa, Smith in Koen. \& Sims, Ann. of Bot. I, 504 (1805) ...
A. mollis, Bentham in Mitch. Trop. Austr. $236(1848) \ldots \ldots$...

- T. V. N.S.W. -

W.A. - - - - - - B.H.II, 88
S.A. T. V. N.S.W. $\bar{Q}$.

W.A. - - - - - - - B.A.II,66
W.A.II,66

W.A. - - - - - B.f.II, 66

W.A. - - - - - - B.fl.II, 66

IV.A - - - - - B.II,67

M.fr.IV, 161.

M.fr.I, 167.

M.fr.I, 166 .

.A. - - - - - - B.fl.II,72

IV.A. - - - - - - B.f.II, ,2

W.A. - - - - - B.fl.II,72

W.A. - - - - - - B.fl.II, 3

W.A. - - - - - B.fl.II,74

V.A. - - - - - - B.fl.II,7t

W.A. S.A. - - - - - B.f. II, 74
W.A. -

- - N.S.W. Q. - B.fl.II,75

- - N.S.W. Q. - B.fl.II,75

- $\quad-$ - V. N.S.W. Q.

- S.A. - V. N.S.W. Q.

- B.fl.II, 76

- B. H.II, 76

IT.A. - - - - - - B.fl.II,77

- _ - - - N.A. B.H.II,77

W.A. - - - - - B.fl.II,77

W.A. - - - - - B.fl.II, 78

W.A. - - - - - - B.fl.II,78

W.A. - - - - - B.f.II,78

W.A. - - - - - B.fl.II,78

W.A. - - - - - - B.A.II,79

W.A. - - - - - B.fl.II,79

W.A. - - - - - - B.fl.II,79

W.A. - - - - - - B.fi. II,80

W. - - N.S.W.Q. - B.f.I., 79

- B.ti.II,80

- S.A. T. - N.S.W. Q. Q - B.H.II.81

- - - - N.S.W. - — B.f.II,81

W.A. - - - - - - B.fl.II,82

W.A.
S.A. - V. N.S.T.
Q.

W.A. - - - - - B.H.II, 82

W.A. - - - - - - B.A.II,83

W.A. - - - - - - B.fl.II, 83

W.A. S.A. - - - - - B.fl.II 83

W.A. S.A. - V. N.S.W. - - B.H.II,84

W.A. - - - - - B.H.II, 84

W.A. - - - - - - B.H.II,84
W.A. - - - II,85

W.A. - - - - - B.H.II,85

W.A. - - - - - - B.H.II,85

W.A. - - - - - B.H.II,85

W.A. - - - - - B.fl.II, 86

W.A. - - - - - - B.H.II, 86

W.A. - - - - - B.fl.II,86

W.A. S.A. - T. N.S.W. - - B.A.II,87

W.A. - - - - - - B.H.II,87

W.A. - - - - - B.f.II,87

W.A. - - - - - B.f.II,88

W.A. - - - - - B.fl.II, 88

W.A. - - - - - - B.H.II, 88

W.A. - - - - - - B.fl.II, SS

- - - N.S.W - - B.H.II,S9

B.HI.II, 89

M.fr.IT, 16.

M.fr. VIII, 225.

MI.fr. X, 138 .

MI.fr.II, 104.

M.fr.II, 105.

M.fr.IV,15.

M.fr.I, 145.

M.fr. X, 32 .

M.fr. II, 105. 
A. Preissii, Meissner in Lehm plant Preiss II 214 (1846) . IV A A. phyllit. A. phylicoides, F. v. M, in Benth. Fl. Austr. II, 72 (1864) … A. genistoides, Turczaninow in Bull. Soc. Mosc. XXVI, 268 (1853) A. carinata, Meissner in Lehm. plant. Preiss. II, 215 (1849) A, passerinoides, Meissner in Lehm. plant. Preiss. I. 61 (1844)... A. Tietkensii, F. r. M., fragm. X, $33(1870)$

A. cordifolia, Bentham., in Hu. X, 33 (1876) ...

PHYLLOTA, De Candolle, prodr. II, 113 (1825). (Urodon. P. barbata, Bentham in Hueg. enum. 33 (1837) ... ... ... W. A P. gracilis, Turczaninow in Bull. Soc. Mosc. XXVI, 267 (1853) W.A.

P. Sturtii, Bentham, Fl. Austr. II, 95 (1864)

P. phylicoides, Bentham in Ann. des Wien. Mus. II, 77 (1838)...

P. humifusa, Bentham, Fl. Austr. II, 95 (1861) $\quad \ldots \quad$... $\quad \ldots$

P. diffusa, F. v. M., fragm. I, 8 (1858)

P. pleurandroides, F. v. M. in Transact. phil. Inst. Vict. I, 38 (1854)

P. Luehmanni, F. v. M., fragm. X, 33 (1876)

P. Urodon, F. v. M. in papers of the Roy. Soc. of Tasm. 120 (1877) W.A. P. daphnoides, Wendland, Bot. Beob. 49 (1798)

P. stricta, Sims, Bot. Mag. 1588 (1813) … $\quad \cdots \quad \ldots$

P.

P. Benthamii, F. v. M. in Transact. phil. Inst. Vict. I, 38 (I854)

P. pycnocephala, F. v. M. in Benth. FI. Austr. II, 114 (1864) ...

P. myrtoides, Cunningham in Annal. des Wien. Mus, II, 81 (1838)

P. mucronata, F. v. M., fragm. I, 8 (1858)...

P. polifolia, Cunningham in Field's N. S. Wales 346 (182̈5)

$P$. petiolaris, Cunningham in Ann. des Wien. Mus. II, 82 (1838)

P. paleacea, Willdenow, spec. plant. II, 506 (1799)

P. Gunnii, Bentham in Ann, des Wien. Mus. II, S2 (1839) $\ldots$

P. scabra, R. Brown in Ait. hort. Kew. sec. ed. III, 18 (1811)...

P. Hartmanni, F. v. M., fragm. VIII, 166 (1874)...

P. microphylla, Sieber in De Cand. prodr. II, 11 (1825) ... ...

P. Skinneri, F. v. M., fragm. VIII, 166 (1874)

P. Drummondii, ILeissner in Lehm. plant. Preiss. II, 219 (1847)

P. pinifolia, Meissner in Lehm. plant. Preiss. II, 220 (1847) ...

P. pedunculata, Hooker, Bot. Magaz. t. 2859 (1828)

P. conferta, Bentham, Fl. Austr. II, 118 (1S64)

P. aciphylla, Bentham in Hueg. enum. 35 (1837) ...

P. ochreato Meissner in Lehm plant. Preiss. I 73 (18

P. aspalathoides, Meissner in Lehm. plant. Preiss. I. 73 (1844)

P. obcordata, Bentham, Fl. Austr. II, 120 (1864) ...

P. rotundifolia, Bentham, Fl. Austr. IT, 121 (1864)

P. calycina, Bentham, Fl. Austr. II, 121 (1864)

P. spinulosa, Bentham, Fl. Austr. II, 121 (1864) ...

P. tenella, Bentham, FI. Austr. II, $122(1864)$

P. ternata, F. v. M., fragm. I, 8 (1858)

P. styphelioides, Cunningham in G. Don, gen. syst. II, $124(1832)$

P. altissima, F. v. M. in Bentham, Fl. Austr. II, 123 (1864) ...

P. obovata Bentham Fl Austr. II $129(1864)$

P. incurvata, Cunningham in Field's N.S. Wales 346 (1825)

P. subumbellata, Hooker, Bot. Mag. t. 3254 (1833)

P. stipularis, Smith, Specim. of the Bot. of New Holl. 35, t. 12 (1793)

P. glabra, Bentham, Fl. Austr. II, 124 (1864)

P. dentata, Labillardière, Nov. Holl. pl. spec. I, 103 , t. 131 (1804)

$P$. aristata, Sieber in De Cand, prodr. II, 112 (1825)

P. plumosa, Sieber in De Cand. prodr. II, 111 (1825)

P. viscosa, $R$. Brown in Benth. Fl. Austr. II, 127 (1864)

P. echinula, Sieber in De Cand. prodr. II, 112 (1825)

P. hibbertioides, J. Hooker, Fl. Tasm. I, 89 (1860)

P. rosea, F. v. M., fragm. II, 15 (1860)

P. mollis, Lindley in Mitch. Three Exped. İ̈, 260 (1838)

P. strobilifera, Meissner in Lehm. pl, Preiss. I, 75 (1844)

P. ericifolia, Bentham in Bot. Regist. XXV, App. XIII (1839)

$P$. verruculosa, Turcz in Bull. Soc. Mosc XXVI, 278 (1853)

P. empetrifolia, Meissner in Lehm. plant. Preiss. 1, 76 (1844) ...

P. adunca, Turczaninow in Bull. Soc. Mosc. XXVI, 279 (1853) ...

P. neurocalyx, Turcz. in Bull. Soc. Mose. XXVI, 281 (1853) ...

P. rigida, R. Brown in Benth. Fl, Austr. II, 130 (1864) ...

P. juniperina, Labillardière, Nov. Holl. pl. spec. I, 102, t. 130 (1804)

P. acerosa, R. Brown in Benth. Fl. Austr. II, 131 (1864)

P. humilis, Bentham in J. Hook. Fl. Tasm. I, 9l (I860) ...

P. parvillora, Sieber in De Cand. prodr. II, 111 (1825) ...

P. setulosa, Bentham, Fl. Austr. IT, 132 (1864)

P. vestita, R. Brown in Ait. hort. Kew. sec. ed. III, 19 (1811)

P. procumbens, Cunningham in Field's N. S. Wales $347(1838) \ldots$

P. hispidula, R. Brown in Bentham, Fl. Austr. II, 133 (1864) ...

P. laxifiora, Bentham, Fl. Austr. I, 133 (1864)

P. largiflorens. F. v. M. in Benth. Fl. Austr. II, 134 (1864) ...

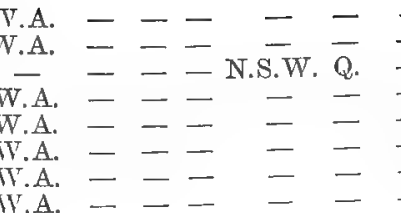

B.fl.II, 91

B.fl.II, 92

- B.Al.II,92

B. H.II, 92

B.fl.II,93

B. .I.II, 93

B. . $\overline{I I}, 93$

M.fr.X, 33 .

B. fl.II, 94

B.f.II, 94

S.A. - - N.S.TV. $\bar{Q}$ - B.H.II,95

B.fl.II,95

B.H.II, 119

- - - N.S.W. -

S.A. $-\overline{\text { T. }}$.

- -

B.fl.II, 96

B.由. $\overline{\text { II, }} 124$

M.fr.X, 33 .

M.fr.I, 8.

M.fr.X,33.

M.fr.X, 33 .

t. 12 (1793). (Euchilus, Spadostyles, Bartlingia.)

- S.A. T. V. N.S.W. -

- S.A. T. V. N.S.W. $\overline{\text { Q. }}$

- - V. N.S.IV. -

$\overline{\mathrm{A}}=\overline{\mathrm{V}}-\mathrm{Q}$.

- - - N.S.W. -

- - - $\overline{\mathrm{V}} \mathrm{Q} \mathrm{Q}$.

- T. V. N.S.W. -

- - V. N.S.W. -

- - N.S.W. Q.

- - N.S.W. Q.

W.A. - - - - -

W.A. - - - - -

W.A. $\overline{\text { S.A. T. }} \overline{\text { V. N.S.W. }}$

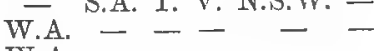

W.A. - - - - -

W.A.

W.A.

W.A.

W.A.

W.A.

- - N S.W

- - - N.S.W. -

- T. V. N.S.W. -

- - N.S.W. -

- - N.S.V. -

- - V. N.S.W. -

- - N.S.W.Q.

- T. V. N.S.W. -

- - V

S.A. - V.

W A

IV.A.

W.A.

TV.A.

W.A.

II.A.

二

-

-

- -

$--$

-

$-\ldots-1$

$--$

S.A. T. V. - -

S.A. T. T. N.S.W. -

S.A. T. Y. N.S.W. -

- - V. N.S.IV. W.A. S.A. - - N.S.W. -

S.A. - V. N.S.W. -

B.fl.II,112

B. H.II, 113

- B.fl.II, 113

B.fl.II, 114

B. H.II, 114

- B.fl.II, I14

- B.fl.II,115

B.fl.II,115

- B.fi.II, 115

- B.fl.II, 116

- B.fl.II,116

— B.fl. $\overline{I I}_{1} 117$

-

B.fl.II, 118

B.fl.II, 118

B. fl. II, 118

B.fl.II, 119

- B.fl.II,120

- B.fl.II, 120

- B.fi.II, 120

- B.A.II, 121

- B.fl.II, 1:1

B.fl.II, 12i

B.fl.IX, 122

B. Al. II, 122

B. H. II, 12:

B.fl.II, 123

B. fi.II, 123

B.H.II, 124

- B.fl.II, 124

- B.fl.II, 12j

B.fi.II, 125

- B.H.II, 125

- B.fl.II, 126

- B.A.II,126

- B.fl.II,127

- B.fl.II, 127

- B.H.II,127

- B.fl.II, 125

- B.f.II,12S

- B.fi.II,12S

- B.fl.II, 129

- B.fl.II, 129

- B.fl.IT,129

- B.fl.II, 130

- B.fl.II, 130

B. A.II, 130

- B.fl.II,131

- B.f.II,131

B.f.II, 131

B.fl.II, $15: 2$

B. fl.II, 1;2

- B.fl.II,132

B. fl.II, 133

B.fl.II, 133

- B.fl.IT,133

- B.fl.II,134
M.fr.I, 8 .

M.fr. VIII, 166.

M.fr. VIII, 166.

M. fr.I, 145 .

M.fr.I, $8 ; \Gamma, 10$.

M.fr.II, 15 . 
P. villosa, Willdenow, spec. plant. II, 507 (1799)...

P. foliolosa, Cunningham in Ann. des Wien. Mus. II, 83 (1838)

P. flexilis, Smith in Koen. \& Sims, Ann. of Bot. I, 502 (1805) ...

P. enchila, De Canilolle, prodr. II, 112 (1825)

P. selaginoides, J. Hooker, Fl. Tasm. I, 87 (1860)

P. densifolia, F. v. M. in Transact. Vict. Inst. 119 (1854)

P. elliptica, Smith in Transact. Linn, Soc. IX, 246 (1808)

P. subspicata, Bentham, Fl. Austr. II, 137 (1864)

P. villifera, Sieber in De Cand. prodr. II, 111 (1825)

P. involucrata, Bentham, Fl. Austr. II, 138 (1864)

P. Muelleri, Bentham, Fl. Austr. II, 138 (1864)

P. prostrata, Bentham in J. Hook. Fl. Tasm. I, 89 (1860)

P. canaliculata, F, v. M. in Transact. Vict. Inst, 119 (1854)

P. fasciculata, Bentham in Ann. des Wien. Mus. II, 82 (1838) ...

P. tenuifolia, R. Brown in Bot. Mag. t. 2086 (1819)

LATROBEA, Meissner in Lehm. plant. Preiss. II, 219 (1847)

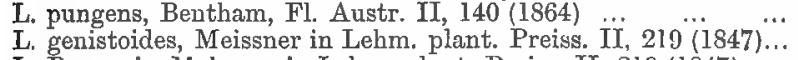

L. Brunonis, Meissner in Lehm. plant. Preiss. II, 219 (1847) ...

L. tenella, Bentham, Fl. Austr. II, 141 (1864)

L. hirtella, Benthan, Fl. Austr. II, 142 (1864)

L. diosmifolia, Bentham, FI. Austr. II, 142 (1864)

E. cuneata, Meissner in Lehm. plant. Preiss. I, 65 (1844) $\ldots$ W.A
W.

E. myrtifolia, R. Brown in Ait, hort. Kew. sec. ed. III, 16 (18ii)

E. epacridoides, Meissner in Lehm. plant. Preiss. I, 64 (1844) ...

E. virgata, Bentham in Hueg. enum. 34 (1837) $\ldots$ X
E. densifolia, Turczaninow in Bull. Soc. Mose. XX 271 (1853)

E. dillwynioicles, Meissner in Lehm. plant. Preiss. I, 63 (1844)

E. parvifolia, Bentham in Hueg. enum. 34 (1837)...
E. empetrifolia, Scblechtendal, Linnaea XX, 667 (1847)

DILLWYNIA, Snith in Koenig \& Sims, Ann. of Bot.

D. hispida, Lindley in Mitch. Three Exped. II, 251 (1838)

D. ericifolia, Smith in Koen. \& Sims, Ann. of Bot. I, 510 (1805)

D. floribunda, Smith in Koen. \& Sims, Ann. of Bot. I, 510 (1805)

D. Preissii, Bentham, Fl. Austr. II, 149 (1864)

D. brunioides, Meissner in Lehm. pl. Preiss. I, 62 (1844)

D. juniperina, Sieber in Hueg. enum. pl. N. Holl. occ. 33 (1837)

D. pungens, Mackay in den Annal. des Wien. Mus. II, 79 (1838)

D. cinerascens, R. Brown in Bot. Mag. t. 2247 (1821) ..

D. divaricata, Bentham, Fl. Austr. II, 151 (1864)

D. patula, F. v. M., fragm. IV, 16 (1864) ... EUCHILOPSIS, F. v. M. in Melbourne Chemist, June (1882).

E. linearis, F. v. M. in Melb. Chem., Jume (1882) ... ... W.A. PLATYLOBIUM, Smith in Transact. Linn. Soc. II, 350 (1794).

P. formosum, Smith in Transact. Linn. Soc. II, $350(1794) \ldots \ldots$
P. obtusangulum, Hooker, Bot. Mag. t. 3258 (1833) $\ldots$.

P. obtusangulum, Hooker, Bot. Mag. t. 3258 (1833)
P. triangulare, R. Brown in Ait. hort. Kew. seo. ed.III, $266(1811)-$ BOSSTAEA, Ventenat, Jardin de Cels I, 7, t. 7 (1800).

B. dentata, Bentham, Fl. Austr. II, 156 (1864)

B. Aquifolium, Bentham, Fl. Austr. II, 157 (1864)

B. cordigera, Bentham in J. Hook. Fl. Tasm. I, 95, t. 16 (1860)...

B. lenticularis, Sieber in De Cand, prodr. II, 117 (1825)

B. Kiamensis, Bentham, Fl. Austr, II, 158 (1864)

B. ornata, F. T. M. fragm. IV, 13 (1863)

B. eriocarpa, Bentham in Hueg. enum. 36 (1837) ...

B. divaricata, Turczaninow in Bull. Soc. Mosc. XXVI, 285 (1853)

B. calyoing Bentham El. Austr. II, 159 (1864)

B. foliosa, Cunningham in Field's N. S. Wales, 347 (1825)

B. cinerea, R. Brown in Ait. hort. Kew. sec. ed. IV, 268 (1812)

B. biloba, Bentham in Hueg. enum. 36 (1837)

B. Preissii, Meissner in Lehm, pl, Preiss. I, $82(1844) \quad \cdots \quad \cdots$

B. concinna Bentham Fl Austr. II, 161 (1864)

B. carinalis, Bentham in Mitch. Trop. Austr. 290 (1848)

B. rupicola, Cunningham in Benth. Fl. Austr. II, 162 (1864)

B. linophylla, R. Brown in Ait. hort. Kew. sec. ed. IV, 268 (1812) IV.

B. disticha, Lindley, Bot. Regist. t. 55 (1841)

B. prostrata, $R$. Brown in Ait. hort. Kew. sec. ed. $\Pi$, $\dddot{268}(1812)$

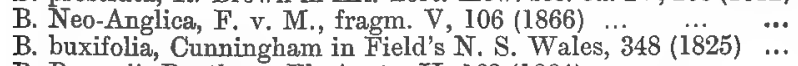

B. Brownii, Bentham, FI. Austr. II, 163 (1864)

B. rhombifolia, Sieber in De Cand, prodr. II, $117^{(1825)}$

B. pulchella, Meissner in Lehm. pl. Preiss. I, 84 (1844)..

B. microphylla, Smith in Transact. Linn. Soc. IX, 303 (1808) ...

B. peduncularis, Turczaninow in Bull. Soc. Mosc. XXVI, 287 (1853)

B. heterophylla, Ventenat, Jardin de Cels t. 7 (1800)

B. rufa, R. Brown in Ait. hort. Kew. sec. ed. III, 267 (1812) ...
- - - V. N.S.W. Q.

- $\quad-\quad$ V. N.S.IV. -

- - N.S.W. -

$-\bar{T}-$ N.S.W. Q.

S.A. - - - -

- - - N.S.W. -

S.A. - V. N.S.IV. -

S.A. - -

- - V.

S.A. T. V. N.S. IV. -

S.A. - V.

T. V. N.S. W. -

Leptocytisus.)

W.A. - - -

W.A. - - -

W.A. - - -

W.A.

W.A.

W.A.

W.A.

IV.A.

W.A. - - - - -

W.A. S.A. - V. N.S.W. -

- S.A. - V. N. V.S.W. Q. T.A. S.A. T. V. N.S.W. Q.

- - - - N.S.W. 二

$-\quad-$ - V. N.S.W. Q.

W.A. S.A. T. V. N.S.W.

W.A. S.A. - V. N.S.W. -

(Cheilococca.)

- T. V. N.S.W. Q. S.A. T. V. S.A. T. V.

- -

(Lalage, Scottia.)

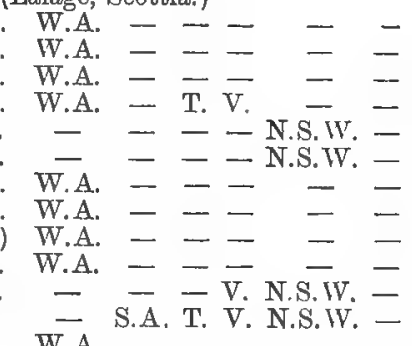

W.A. - - -

W.A.

-

IV.A.

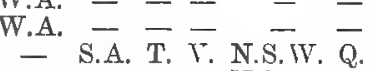

- - - - - N. N.S.W.W. -

- - - - N.S.W. Q.

W.A. - - - N.S.W. Q.

- - V. N.S.W. -

W.A. $=-\bar{V} \cdot$ N.S.W.
$\overline{\text { W.A. }}$ - - -
B. A.II, 134

B.fi.II, 135

B.fl.II, 135

B.fl.II, 135

B.f.II, 136

L.f.II, 136

B.A.II, 136

B.fl.II, 137

B.fl.II, 137

B.fl.II, 138

B.fi.II, 138

B. f..II, 138

B. fl. II, 139

- B.t.II, 139

B. fl. II, 139

II.fr.I,9.

M.fr. II, 106.

M.fr.I, 7 .

B.fl.II, 147

B.fl. II, I 77

- B.fl.II,149

- B.fl.II, 149

B. fl. II, 150

B fl.II, 150

B. fl. II, 150

- B.H.II, 151

B. H.II, 151

B. A.II, 151

M.fr. IV, 16.

B. A.II, 67

- B.fl.II, 153

- B.fl.II, 153

- B.f.II, 152

M.fr.IX, 157.

M.fr.IX, 158 .

B.fl.II, 156

B.fl.II, 157

B. fl.II, 157

B.fl.II, 1.7

B.fl.II, 157

B.fll.II, 158

B.fl.II, 1.58

B. fl. II, 159

B.fl.II, 159

B.fl.II, 159

B. H. II, 160

B.H.II, 160

B.fl.II, 160

B fl.II, 16

B.fl.II, 161

B. 1. II, 161

B.fl.II, 162

B.fl.II, 162

B.H. I, 162

B.H.II, 162

B.fl. $\overline{\mathrm{II}}, 163$

B.ff.II, 163

B.A.II, 164

B.fl. $\Pi, 164$

B.A.II, 164

B.A.II, 165

B. ㄱ. IT, 165

B.fl. II, 165
M.fr. III, 100;IX, 4. .

MT.fr.IX, $4 \pi$

M.fr.IX, 45 .

MI.fr.IV,12.

II.fr.III, 100;IX, 45.

M.fr.UII, 98 .

M.fr.IX, 45.

M.fr. IX, 45 .

MI.fr.IX, 45.

M.fr.IX, 45.

I. fr. III, 97.

M. fr. V, 106.

II. fr. I, $9 ; \mathrm{IX}, 4$.

II.fr. IS, $4 \overline{3}$.

M.fr. III, 99.

M. fr. II, 97.

M.fr.IX, 45 . 
B. bracteosa, F. r. M. in Benth. Fl. Anstr. II, 166 (1864)

B. riparia, Cunningham in Bentl., Fl. Austr. II, 166 (1864)

B. ensata, Sieber in De Cand. prodr. II, 117 (1825)

B. scolopendria, Smith in Transact. Limn Soc. IX, 307 (1808) $\cdots$

B. Talkeri, F. V. M., fragm. II, 120 (1861)

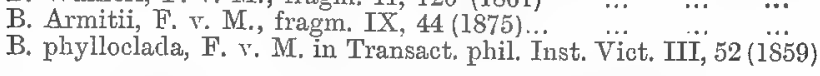

TEMPLETONIA, R. Brown in Ait. hort. Kew. sec. ed, 269 (1812)

T. retusa, R. Brown in Ait. hort. Kew. sec. el. IV, 269 (1872) ... W.A.S.A. - - — - - B.fl.II,169

T. Drummondii, Bentham, Fl. Austr. II 169 (1864)

T. Muelleri, Bentham, Fl. Austr. II, 169 (1864) … … - _ - - V. N.S.IV. Q. - B.f1.II, 169

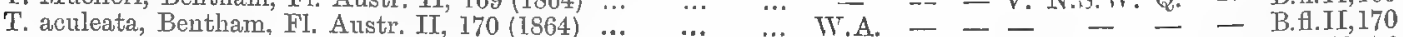

T. egena, Bentham, FI. Austr. II, $170(1864) \quad \cdots$ W.A. S.A. - V. N.S.W. - N.A. B.Al.II, I70

T. sulcata, Bentham, Fl. Austr. II, 171 (1864) $\quad . . \quad$... $\quad$... W.A. S.A. - V. N.S.W. - - B.fl.II,17l

HOVEA, R. Brown in Ait. hort. Kew. sec. ed. IV, 275 (1812). (Poiretia, Plagiolobium, Platychilum.)

H. linearis, R. Brown in Ait. hort. Kew, sec, ed. IV, 275 (1812) - - - N.S.W. - - B.fl.II, 172

H. heterophylla, Cunningh. in J. Hook. Fl. Tasm. I, 93, t. $15(1860)$ - S.A. T. V. N.S.W. Q. - B.fl.II,172

H. longifolia, R. Brown in Ait. hort. Kew. sec. ed. IV, $275(1812)$ - S.A. T. V. N.S. W. Q. N.A. B.f.II, 172

H. acutifolia, Cunningham in G. Don, gen, syst. II, 126(1832) .. - - - - N.S. IV. Q. - B.fl.II,174

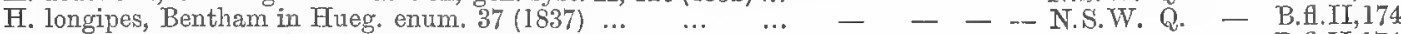

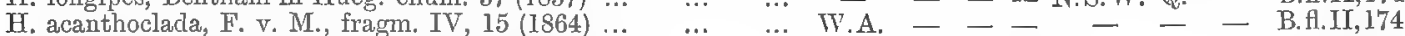

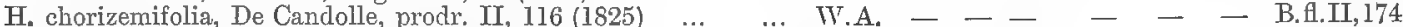

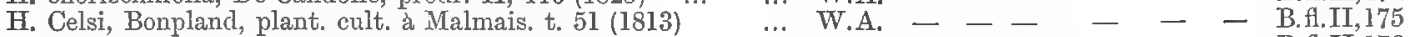

H. trisperma, Bentham in Hueg. enum. 37 (1837) $\ldots .1 \ldots$ W.A. - - - - - - B.fl.II,176

H. stricta, Meissner in Lehm, pl. Preiss. I, 79 (1844) $\ldots . \quad$ IV.A. - - -

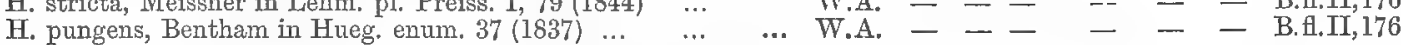

NEMATOPHYLLUM, F. v. M. in Hook. Kew miscell. IX, 20 (1857).

N. Hookeri, F. v. M. in Hook. Kew misc. IX, 20 (1857)

GOODIA, Salisbury, paraclis. Londin. t. 41 (1806).

G. Iotifolia, Salisbury, parad. Lond. t. 41 (1806) ... ...

G. medicaginea, F. V. M., Second Gen. Rep. 11 (1854) ...

CROTALARIA, Hermann, hort. acad. Lugd. Bat. 196-202, t. 197-203 (1687).

C. verrucosa, Linné, spec. plant. 715 (1753)

… … - $\quad$ - $\quad$ - Q. N.A. B.fl.II, 179

C. crispata, F. v. M. in Benth. Fl. Austr. II, $179(1864) \quad \cdots \quad-\quad-\quad-\quad-\quad-$ N.A. B.fl.II, 179

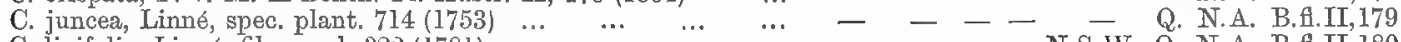

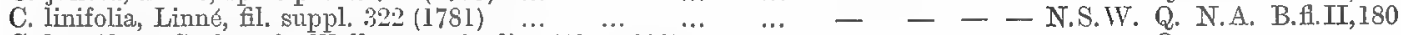

C. humifusa, Graham in Wall. numeric. list 5421 (1828)

C. calpcina, Schranck, pl. rar, hort. Monac. t. 12 (1819)

C. retusa, Linné, spec. plant. 715 (1753)

C. Mitchelli, Bentham in Mitch. Trop. Austr. 120 (1848)

C. Novae-Hollandiae, De Candolle, prodr. II, 127 (1825)

C. crassipes, Hooker, icon, plant, t. 830 (1852)

C. Chasn, Ha - - - - -

C. Cunninghamii, B. Brown, App. to Sturt's Centr. Austr. 8(1849) W.A. S.A. - - - Q. N.A. B.A.II,182

C. medicaginea, Lamarck, encycl. meth. II, 201 (1786) ...

C. incana, Limné, spec. plant. 716 (1753)

C. dissitiflora, Bentham in Mitch. Trop. Austr. 386 (1848)

C. Iaburnifolia, Linné, spec. plant. 715 (1753)

C. quinquefolia, Linné, spec. plant. $716(1753) \quad \ldots \quad \ldots$

C. alata, Hamilton in D. Don, prodr. Fl. Nepal, $\ddot{2} 11$ (1825)

WESTONIA, Sprengel, syst. veg. III, 152 (182̇6).

W. trifoliata, Sprengel, syst. veg. III, 230 (1826)

TRIGONELLA, Linné, gen. pl. 351 (1737).

T. suavissima, Lindley in Mitch. Three Exped. I, 255 (1838) ... W.A. S.A. - V. N.S.W. - - B.f.II,187

LOTUS, Tournefort, inst. 402, t. 227 (1700), from C. Bauhin (1623).

L. corniculatus, Linne, spec. plant. 775 (1753) $\ldots \quad \ldots . \quad \ldots \quad-$ S.A. T. V. N.S.W. - - B.fl.II,IS8

L. australis, Andrews, Botanist's Reposit. t. 624 (1810) ${ }^{\cdots} \quad \ldots$. W.A. S.A. T. V. N.S.W. Q. N.A. B.H.II, 188

PSORALEA, Linné, gen. ed. sec. 358 (1742).

P. badocana, Bentham, Fl. Austr. II, 190 (1864) ...

P. cephalantha, F. v. M., fragm. IV, 35 (1863) ...

P. Martini, F y M fragm. V 11 (1865)

P. Archeri, F. v. M., fragm. IV, 21 (1863)

P. balsamica, F. v. M. in Transact. Vict. Inst. III, 55 (1858) $\cdots$

P. plumosa, F. v. M., fragm. IV, 22 (1864)

P. pustulata, F. v. M. in Transact. Vict. Inst. III, 54 (1858) $\cdots$

P. lachnostachys, F. v. M., fragm. III, 105 (] 863)

P. eriantha, Bentham in Mitcl.. Trop. Austr. 131 (1848)...

P. patens, Lindley in Mitch. Three Exped. II, 9 (1838)...

P. cinerea, Lindley in Mitch. Three Exped. II, 65 (1838)

P. leucantha, F. v. M. in Transact. Vict. Inst. III, 54 (1859)

P. parva, F. v. M. in Transact. Vict. Inst, I, 40 (1854) ...

P. tenax, Lindley in Mitch. Three Exped. II, 10 (1838)...

$P$, adscendens, F. v. M. in Transact. V ict. Inst. I, 40 (1854)

P. Schultzii, F. v, M., fragm. IX, 155 (1875)

P. Testariae, F. v. M., fragm. V, 45 (1865)
... - - - - - N.S.W. Q. N.A. B.H.I, 183

.. - S.A. - - N.S.W. Q. N.A. B.H.II, 184

.. - - - - - Q. - B.H.II,184

.. - - - - - Q. - B. A.II,184

.. $\quad-\quad-\quad-\quad$ N.A. B.f.II, 185
- - - - N.S.W. Q. - B.H.II,193
M.fr.XI, $27 \& 123$

M.fr.III, 95 .

M.fr.II, $120 ; I X, 45 ; X I, 27$.

M.fr.IX,44.

M.fr.IX,158.

M.fr.I, $9 ; \mathrm{II}, 178 ; \mathrm{IX}, 158$.

Mi.fr.II, 120.

M.fr.III, $94 ;$ IX, 158.

MI. fr. III, 94.

M.fr. IX, 158.

M.fr.IX, 158 .

M.fr.IX, 158 .

M.fr.IX,158.

MI.fr.IV, 15.

M. fr. IX, 158 .

M.fr.IX, 158 .

M.fr.IX, 158 .

M.fr.IX, 15s.

M.fr.I, 169;IV, 170;IX,157

M.fr.I, 10;IX, 157.

M.fr.I.10;IX, 157.

M.fr.III, 54;IX, 156 .

M.fr.III, 55 .

NI.fr.III, 51 ; IX, 156.

M.fr.III, $55 ; \mathrm{IX}, 156$.

M.fr. VI, 225;IX, 156 .

M.fr.IX, 156 .

M. fr.III, $51 ; \mathrm{IX}, 156$.

M.fr.III, 50;IX, 156 .

II.fr.IX, 156 .

M.fr.III, $52 ; I X, 156$. M.fr.III, $56 ; 1 X, 157$.

M.fr.IX, 157 .

M.fr.IX, 156 .

M. fr.III, 52;IX, 157.

M.fr.IX, 157.

M.fr.XI, 138.

M.fr.XI, $20 \& 27$.

MI.fr. IX, 156.

II. fr. IT 35 .

M.tr.T, 11 .

M.fr, IV, 21 .

M.fr.IV, $2 \Omega$

MI.fr.III, 105.

M.fr. IX, 155.

W.A. S.A. - T. N.S. W'. Q. - B.flIT,

W.A. S.A. - V. N.S.W. Q. N.A. B.f.II, 192

IV.A. - - - N.S.IV. - N.A. B.A.II,192

W.A. S.A. - - - Q. N.A. B.flII,193

- S.A. - V. N.S.IV. - - B.fl.II,194

- S.A. T. V. N.S.W. - - B.f.II,193

- - - - - - - -

M.fr.IX, 156 .

M.fr. IX, 155 .

NI.fI. IX, 155 .

MI.fr, 1N, 155 .

M.fr.IX, 155.

M.fr. V, 45,189 
INDIGOFERA, Royen, pl. hort. Lugd. 372 (1740), from Linné (1737).

I. linifolia, Retzius, observ. bot. IV, $29(1786) \ldots \ldots \ldots \ldots$ … S.A. - - N.S.W. Q. N.A. B.fl.II,19J I. cordifolia, Heyne in Roth, nov, pl. spec. 357 (1821) $\ldots$... - - - - - - N.A. B.fl.II, I96

I. enneaphylla, Linne, mantissa altera $272(1771) \quad \ldots \quad \ldots \quad-$ S.A. - - N.S.IV. Q. N.A. B.H.II,196

I. glandulosa, Willdenow, spec. plant. III, $1227(1800) \ldots \quad \ldots \quad-\ldots \quad-\quad-\quad-\quad$ Q. - B.fl.II, 196

I. haplophylla, F, v. M., fragm. III, $102(1863)$
I. trifoliata, Linne, amoen. acad. IV, $327(1759)$
$\ldots$

I. trita, Linné fil., suppl. plant, 335 (1781)

I. parviflora, Heyne in Wall. numeric. list 5457 (I828) .

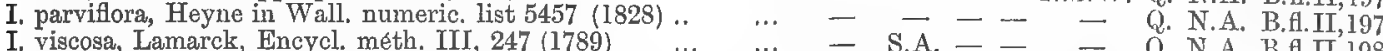

... - S.A. - - - Q. N.A. B.fl. 198

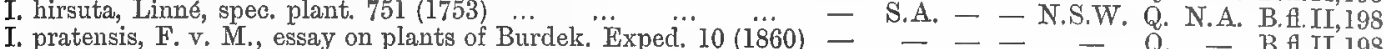

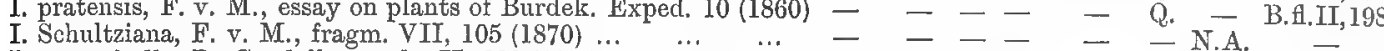

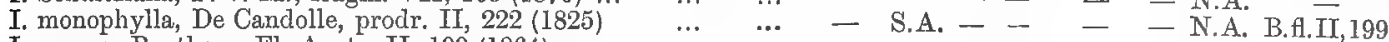

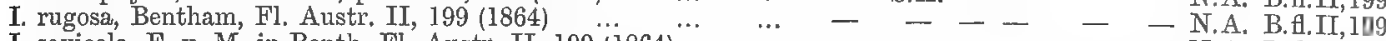

I. saxicola, F. v. M. in Benth. Fl. Austr. II, 199 (1864) ... $\ldots-\ldots----\overline{-}-$ N.A. B.fl.II, 199

I. australis, Willdenow, spec. plant. III, 1235 (1800) $\ldots . \quad \ldots .$. W.A. S.A. T. V. N.S.IV. Q. $\quad$ - - . B.fl.II, 199

I. brevidens, Bentham in Mitch. Trop. Austr. 385 (1848) ... W.A. S.A. - - N.S. IV. Q. N.A. B.f.II, 200

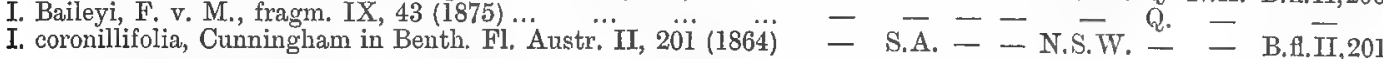
PTYCHOSEMA, Bentham in Lindl. Bot. Regist. XXV, App. XVI (1839).

P. pusillum, Bentham in Lindl. Bot. Regist. XXV, App. XVI(1839) W.A. - - — - - - B.A.II,201

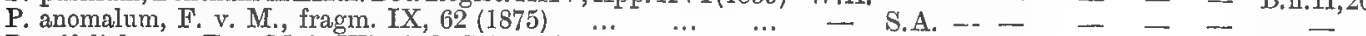

P. trifoliolatum, F. v. M. in Wing's S. Science Record II, 72 (1882) W.A. - - - - - - - LAMPROLOBIUM, Bentham, Fl. Austr. II, 202 (1864).

L. fruticosum, Bentham, Fl. Austr. II, $202(1864)$ ․ $\quad \cdots \quad-\quad-\quad-\quad-\quad$ - Q Q. $\quad-\quad$ B.fl.II,202 TEPHROSIA, Persoon, synops, pl. II, 328 (1807).

T. coriacea, Bentham, Fl. Austr. II, 204 (1864) ...

T. lamprolobioides, F. v. M., fragm. IX, $64(1875) \quad \ldots$

T. Forrestiana, F. v. M., fragm. XI, 98 (1880)
T. flammea, F. v. M. in Benth. Fl. Austr. II, 204 (1864)

T. flammea, F. v. M. in Benth. Fl. Austr. II, 204 (1864)
T. reticulata, Bentham, Fl. Austr. II, 205 (1864) ...

T. crocea, Bentham Tin Austr. IT, 205 (1864)

T. oblongata, Bentham, Fl. Austr. II, 205 (1864) ...

T. porrecta, Bentham, Fl. Austr. II, 206 (1864) ㄱ .

T. polyzyga, F. v. M. in Benth. FI. Austr. II, 206 (1864)

T. graminifolia, F, v, M. in Benth. Fl. Austr. II, 206 (1864)

T. simplicifolia, F. v. M. in Benth. Fl. Austr. II, 206 (1864)

T. nematophylla, F. v. M., fragm. IX, 63 (1879) ... ... ...

T. leptoclada, Bentham, Fl, Austr. I, 207 (1864) $\ldots$

T. brachycarpa, F. v. M. in Benth. Fl. Austr. II, 207 (1864)

T. uniovulata, F. v. M., fragm. XI, 70 (1879)

T. Stuartii, Bentham, Fl, Austr. II, 207 (1864)

T. conferta, F. v. M., fragm. IX, 65 (1879)

T. phacosperma, F. v. M. in Benth. FI. Austr. II, 208 (Ï64; $\cdots$

T. astragaloides, Bentham, Fl. Austr. II, 208 (1864) $\ldots . .$.

T. juncea, Bentham, Fl. Austr. II, 208 (1864)

T. filipes, Bentham, Fl. Austr. II, 208 (1864)

T. remotiflora, F. v. M. in Benth. Fl. Austr. II, 209 (180̈4)

T. oligophylla, Bentham, Fl. Austr. II, 209 (1864)

T. macrocarpa, Bentham, Fl. Austr. II, 209 (1864)

T. purpurea, Persoon, synops, plant. II, 329 (1807)

T. Bidwilli, Bentham, Fl. Austr. II, 210 (1864)

T. rosea, F. v. M. in Benth. Fl. Austr. II, 211 (1864) ... WISTARIA, Nuttall, Gen. of N. Amer. pl. II, 115 (1818),

W. megasperma, F. v. M., fragm. I, ad hist. natur. 10 (1858) ... SESBANIA, Scopoli, Introd, 308 (1777). (Agati.)

S. grandiflora, Persoon, synops. pl. II, 316 (1807)

... - - - - - - N.A. B.fl.II, 212

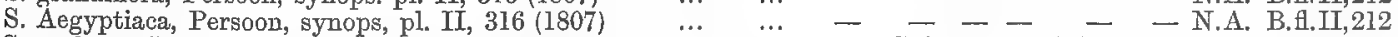

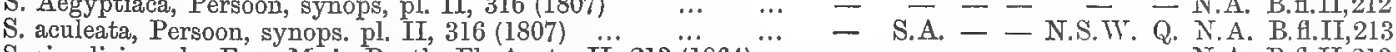

S. simpliciuscula, F. v. M. in Benth. Fl. Austr. II, 213 (1864) .. - - - - - - N.A. B.H.II, 213

S. brachycarpa, F. v. M., fragm. XI, $32(1878) \quad \ldots \quad$.. $\quad \ldots$ CARMICHAELIA, R. Brown in Bot. Regist. XI, 912 (1825).

C. exsul, F. v. M., fragm, VII, 126 (1871) ... CLIANTHUS, Banks \& Solander in G. Don, dichlam. pl. II, 468; indicative (1832), (Donia.)

C. Dampieri, Cunning. in Trans, hort. soc. Lond. sec. ser. I, 522(1835)W.A. S.A. - - N.S.W. - N.A. B.fl.II, 214 STREBLORRHIZA, Endlicher, prodr, fl. Norfolk. 92 (I833).

S. speciosa, Endlicher, prodr. fl. Norf. 93 (1833) $\ldots \quad$... $\quad \ldots \quad-\quad-\quad$ - N.S. Y.

SWAINSONIA, Salisbury, parad. Londin. t. 28 (1806). (Cyclogyne, Diplolobium.)

S. Greyana, Lindley, Bot. Regist, t. 66 (1846) … … - - S.A. - V. N.S.W. Q. - B.fl.II,2I6 S. galegifolia, R. Brown in Ait. hort. Kew. sec. ed. III, 327 (18il) - - - - N.S.W. Q. - B.fl.II,217

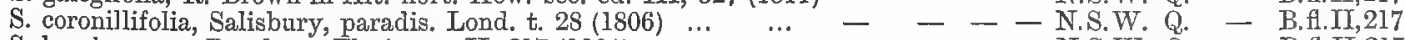

S. brach carpa, Bentham, Fl. Austr. II, 217 (1864)

S. phacoides, Bentham in Mitch. Trop. Austr. 363 (1848)

S. Burkittii, F. v. M. in Benth. Fl. Austr. II, 218 (1864)
M.fr.III, 101;IX, 44.

M.fr.III, $102: 1 \mathrm{X}, 44 . \quad[44$.

H.fr.IV, $22 ; \mathrm{VII}, 106 ; \mathrm{IX}$,

II. fr. IX, 41 .

M.fr.III, 104.

M. fr.III, 103 .

iI.fr.III, 103;IX, 44.

II.fr. III, 104:IX, 44 .

M.fr.III, 105;IX, 44 .

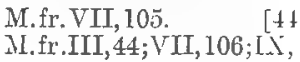

M.fr.IX, 43 .

M.fr.IX, 62.

MI.fr.IX, 68.

M.fr.IX, 67 .

M.fr. IX, $65 \& 66$.

M. fr. IX, 64

M.fr.XI, 98 .

M.fr.IX, 64 .

MI.fr.IX, 66.

M.fr.IX,63.

M.fr.XI,, 0 .

M.fr.IX, 75 .

M.fr. IX, Bt.

II.fr.I, I0.

M.fr. II, 88, XI, 33.

M.fr.XI, 33.

I.fr.XI, 33 .

M.fr.IX, 32 .

M.fr. VII, I26, 193.

NI.fr.IX, 169.

MI.fr. IX, $1 \tilde{\jmath} 4 ; \mathrm{X}, 7$.

M.fr.XI,70.

II.fr.IX, 155. 
S. Burkei, F. v. M, in Benth. Fl, Austr. II, 218 (1864) . $\ldots$ - S.A. - - - - N.A. B.fl.II,218 S. oligophylla, F. V. M. in Benth. Fl. Austr. II, 219 (1864) ‥ - S.A. - - N.S. IT. - N.A. B.fl.II, 219 S. campylantha, F. . M. in Gregory's pap rel. to Leichh. Exp.6(1858) - S.A - - N.S.TV. Q. - B.fl.II,219 S. occidentalis, F. V. M. fragm. III $46(1862) \quad$ W.A. - - _ - _ N.A. B.fl.II,219

S. stenodonta, F. v. M., fragm. XI, 70 (1879)

S. gracilis, Bentham, Fl. Austr. II, 220 (1864)

S. plagiotropis, F. v. M., fragm. IN, 153 (1875) ...

S. procumbens, F. v. M., fragm. III, 46 (1863)

S. Drummondii, Bentham, FI. Austr. II, 220 (1864)

S. canescens, F. v. M., fragm. III, 46 (1863)

S. phacifolia, F. v. M. in South Austr. Register (1850) …

S. oroboides, F. v. M. in Benth. Fl. Austr. II, 2202 (1S64)

S. unifoliolata, F. v. M., fragm. VIII, 226 (1874) ...

S. lessertiifolia, De Candolle, prodr. II, 271 (1825)

S. luteola, F. v. M., fragm. I, 75 (1858)

S. parvillora, Bentham, Fl. Austr. II, 223 (1864) ..

S. monticola, Cunningham in A. Gray's Bot. Wilk. Exp. I $_{411} \ldots \ldots$

S. min

S. laxa, R. Brown in Sturt's Centr. Austr. App. 18 (1849)

S. colutoides, F. T. M., fragm. X, $6(1876)$...

S. Fraseri, Bentham, Fl. Austr. II, 224 (1864)

S. Macullochiana, F. v. M., fragm. VII, 25 (1869)

$$
\begin{gathered}
\ldots \\
\ldots \\
\ldots \\
\ldots \\
\ldots \\
\ldots \\
\ldots \\
\ldots \\
\ldots \\
\ldots \\
\ldots \\
185 \\
\ldots \\
\ldots \\
\ldots \\
\ldots \\
\ldots
\end{gathered}
$$

$\begin{array}{ll}\ldots & \\ \ldots & \\ \ldots & W \\ \ldots & \\ \ldots & W \\ \ldots & W \\ \ldots & \\ \ldots & \\ \ldots & \\ \ldots & \\ \cdots & \\ \cdots & \\ 85 & \\ \ldots & \\ \ldots & \\ \ldots & \end{array}$

-

A. - V. N.S.TV. $\overline{\text { N.S.TV. }}$

N.A.

-

- S.A. - V. N.S.TT. Q. - B.fl. $\overline{I I}, 220$

A. - - - - B.fl.II,220

S.A. - - V. N.S.W. - - B.fl.II,221

- S.A. - - N.S.W.Q. - B.H.II,222

A. S.A. - - V. N.S.W. - - B.A.II, 222

S.A. T. V. N.S.W. - - B.A.II, 222

- - - - Q Q. - $\mathrm{Q}$ B.H.II,223

- - - N.S.W. Q. - B.fl.II, 223

S.A. - V. N.S.W. Q. - B.fl.II, 223

S.A. - V. N.S.IT. - - B.fl.II,224

S.A. - - N.S.W. Q. - B.A.II, 224
B.fl. $\overline{\text { II }, 2: 20}$

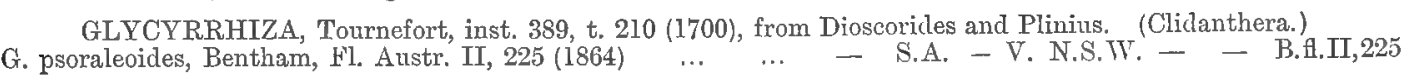

ORMOCARPUM, Palisot, Fl. d'Ovar. I, 95, t. 58 (1805).

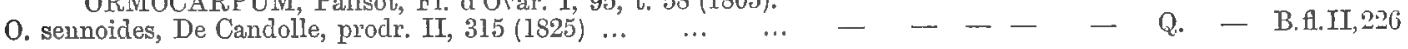
AESCHYMOMFNE, Linné, gen. pl. 350 (1737).

A. Indica, Linne, spec. plant. 713 (1753)

A. falcata, De Candolle, prodr. II, $322(1825)$

A. Americana, Linné, spec. plant. 713 (1753)

SMITHIA, Aiton, hort. Kew. IIT, 496 (1789).

S. conferta, Smith in Rees, Cyclop. XXXIII (1816)

S. sensitiva, Aiton, hort. Kew. III, 496, t. 13 (1789)

ZORNIA, Gmelin, Syst. Nat. 1076 (1791).

Z. diphyllar, Persoon, synops. II, 318 (1807)

Z. chaetophora, F. v. M. in Transact. phil. Inst. Vict. III, $56(1857)-\ldots-\ldots-$ - - N.A. B.H.II,229

DESMODIUM, Desvaux, Journ. de Bot. III, 122 (1813). (Dicerma, Phyllorlinm, Pteroloma.)

D. umbellatum, De Candolle, prodr. IX, 325 (1825)

D. pulchellum, Bentham, FI. Hongk. 83 (1861)

D. biarticulatum, F. v. M., fragm. II, 121 (1861)..

D. acanthocladum, F. v. M., fragm. II, 122 (1861)

D. triquetrum, De Candolle, prodr. II, 326 (182.5)

D. Gangeticum, De Candolle, prodr. II, 327 (1825)

D. brachypodum, A. Gray, Bot. Wilk. Exped. 434 (1854)

D. varians, Endlicher in Ann. des Wien. Mus. I, 185 (1839)

D. flagellare, Bentham, Fl. Austr. II, 23.3 (1864) ...

D. rhytidophyllum, F. v. M. in Benth. Fl. Austr. II, 223 (1864)

D. campylocaulon, F. v. M. in Benth. Fl. Austr. II, 233 (1864)

D. nemorosum, F. v. M. in Benth. FI. Austr. II, 234 (1864) ...

D. neurocarpum, Bentham, El. Austr. II, 234 (1864)

D. trichostachyum, Bentham, Fl. Austr. II, 234 (1864) ...

D. polycarpum, De Candolle, proctr. II, 334 (182.)

D. trichocaulon, De Candolle, prodr. II, 335 (1825)

D. Muelleri, Bentham, Fl. Austr. II, 235 (1864) ...

D. reniforme, De Candolle, prodr. II, $327(18: 5)$...

D. parvifolium, De Candolle, prodr. II, 334 (1825)

D. dependens, Blume in Miq. Fl. Ind. Batav. I, 249 (1855)

PYCNOSPORA, R. Brown in Wight \& Arnott, prodr. I, 197 (1834).

P. hedysaroides, R. Brown in Wight \& Arn., prodr. 197 (1834)

URARIA, Desvaux, Journ. de bot. III, 122 (1813).

U. picta, Desvaux, Journ. de Bot. III, 122 (1813)

U. cylindracea, Bentham, Fl. Austr. II, 237 (1864)

U. lagopoides, De Candolle, prodr. II, 324 (1325)

LOUREA, Necker, elem. bot. III, I7 (1790).

L. obcordata, Desvaux, Journ. de Bot. III, 122 (1813) ...

ALYSICARPUS, Necker, elem. bot. III, 15 (1790).

A. vaginalis, De Candolle, prodr. II, 353 (1825) ...

A. longifolius, Wight \& Arnott, prodr. 223 (1834) …

, Desvaux in Annal. Soc, Linn. Par, 301 (1825)

L. cumeata, G. Don, gen. syst. II, 307 (1832)

CLITORIA, Limné, gen. plant. 216 (1737), from Petiver.

C. australis, Bentham, Fl. Austr. II, 212 (1864) ...

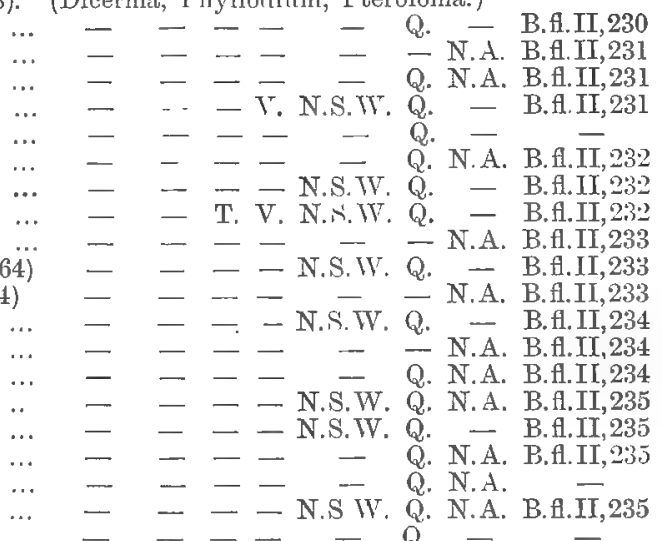

- $\quad$ - - N.S.W. Q. - B fl.IT,237

- - - - Q. N.A. B.fl.II, 237

Q. - B.H.II,237

$-\quad-\quad-\quad-\quad-$ N.A. B.A.II,238

- - - - - Q. - B.H.II,239

- - - - - Q. N.A. B.H.II,239

- - - - Q. N.A. B.A.II,239

- - - V. N.S.W.Q. - B.fl.II, 240

- - - - - - N.A. B.A.II, 242
M.fr.IX, 155.

M.fr.IX, 154 .

M. fr. III, 46 .

M.fr.XI, 70 .

M.fr.IX, 143. M.fr.IX, 154;XI,27.

M.fr.IX, 154;XI, 70.

M.fr.IX, 154 .

M.fr. VIII, 226.

M.fr.IX, 154 .

M.fr.I,75;XI, 70 .

M.fr.IX, 155.

M.fr.IX, 155.

M.fr.X, 6 .

M.fr.IX, 154.

M.fr.III, 45 .

M.fr. VII, 27;IX, 193.

M.fr.IX, 66 .

M. fr. IX, 66 .

M.fr.IX, 66 .

M.fr.IX, 66 .

M.fr.IX, 66 .

M.fr.XII.

Mifr.IX, 66 [X, 120 .

M.fr.VIII, $225 ; L X 66 \$ 190$ 
GLYCINE, Linné, gen, pl, 349 (1737). (Leptolobium, Leptocyamus.)

G. falcata, Bentham, Fl. Austr. II, $243(1864) \quad \ldots \quad \ldots \quad \ldots-$... $\quad$ S.A. - - N.S.W. Q. N.A. B.fl.II, 243

G. clandestina, Wendland, Bot. Reob. $54(1798)$... $\quad \ldots \quad$... TT.A. S.A. T. V. N.S.W. Q. - B.f.II, 243

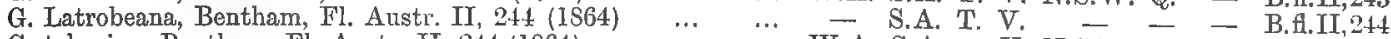

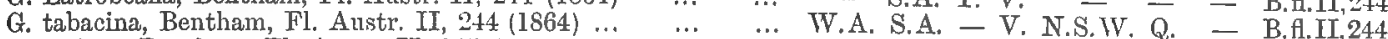

G. sericea, Bentham, Fl. Austr. II, 245 (1864) $\ldots \quad \ldots . \quad \ldots \quad-$ S.A. - V. N.S.W. $-\quad-$ B.Al.II,245

G. tomentosa, Bentham, Fl. Austr. II, $245(1864) \quad \ldots \quad \ldots-$ - S.A. - - N.S.W. Q. N.A. B.f.II,245 KENNEDYA, Ventenat, Jardin de la Malmaison II, t. 104, 106 (1804). (Caulinia, Moench 1802, Hardenbergia, Physolobium, Zichya,
Amphodus.)

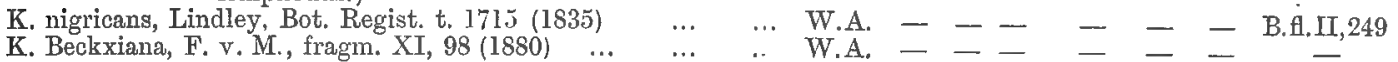

K. rubicunda, Ventenat, Jard. de la Malm. t. $10 \dddot{4}(1804) \quad \cdots \quad-. \quad-\quad-\bar{V}$. N.S. IV. Q. - B.f.II, 249

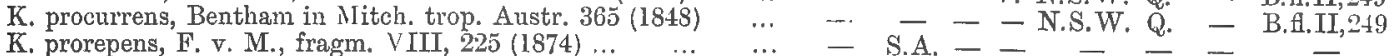

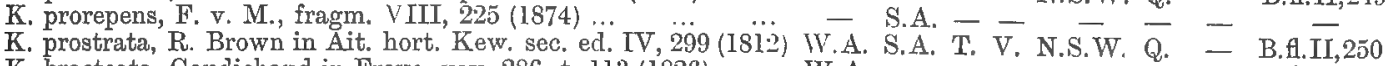

K. bracteata, Gaudichand in Freyc. voy. 2S6, t. $113(1826) \quad \ldots$ W.A. - - - - - - B.fl.II,250

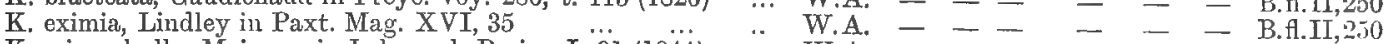

K. microphylla, Meissner in Lehm. pl. Preiss. I, 91 (1844) $\quad$ … W.A. - - - - - - B.fl.II,251

K. parvifora, Meissner in Lehm. pl. Preiss. I, 91 (1844) … W.A. - - - - - - B.H.II,251

K. Stirlingii, Lindley, Bot. Regist. t. $1845(\mathbf{1 8 3 7}) \quad \ldots \quad \ldots$. W.A. $\quad$ - - - $\quad-\quad-\quad-$ B.fl.II,252

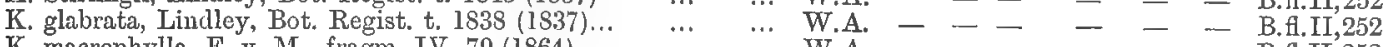

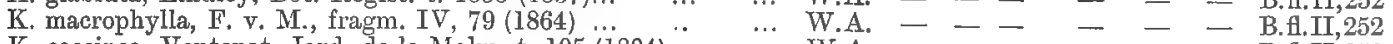

K. coccinea, Ventenat, Jard, de la Malm. t. 105 (1804) ... ... W.A. - - - _ - - B.fl.II,250

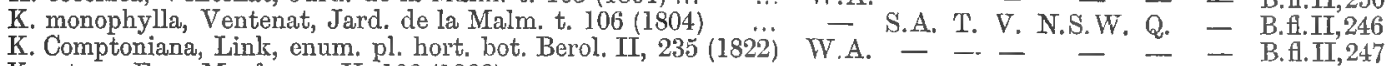

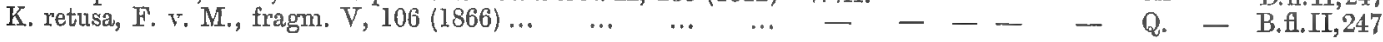
ERYTHRINA, Limné, gen. pl. 216 (1737).

E. vespertilio, Bentham in Mitch. Trop. Austr. 218.(1848) ‥ - S.A. - - — Q. N.A. B.fl.I,253

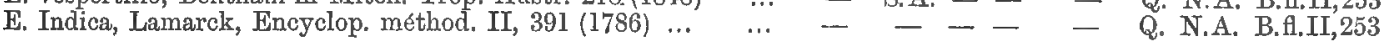
STRONGYLODON, T. Vogel in Linnaea X, 585 (1836).

S. ruber, T. Vogel in Linnaea X, 585 (1836) MUCUNA, Marcgraf, hist. nat. Brazil. 18 (1648).

M. gigantea, De Candolle, prodr. II, $405(1825) \ldots \quad \ldots \quad \ldots \quad-\ldots-\ldots$ GALACTIA, P. Browne, Civil \& Nat. Hist. of Jamaica 298 (1756).

G. tenuiflora, Wight \& Arnott, prodr, fl. penins. Ind. 206 (1834) - S.A. - - N.S.IV. Q. N.A. B.Al.II,255

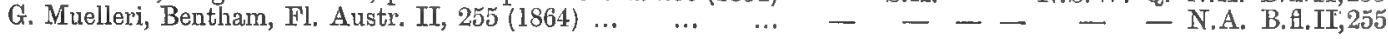
CANAVALIA, De Candolle, prodr. II, 403 (1825). (Canavali.)

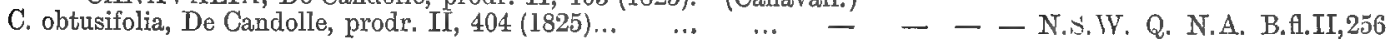
PHASEOLUS, Tournefort, inst. 412, t. 232 (1700), from Dioscorides \& Columella.

P. vulgaris, de l'Obel, stirp. icon, 59 (1581) ‥

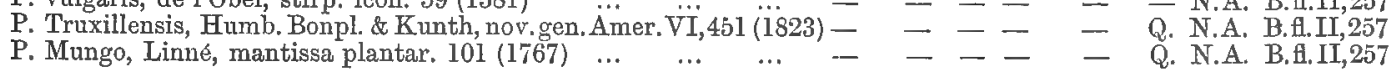
VIGNA, Savi, Dissert. 6 (1S24). (Callicystus.)

V. vexillata, Bentham in Mart. Fl. Bras. Papil. 193, t. $50(1862) \quad-\quad-\quad-$ N.S.W. Q. N.A. B.fl.II, 258

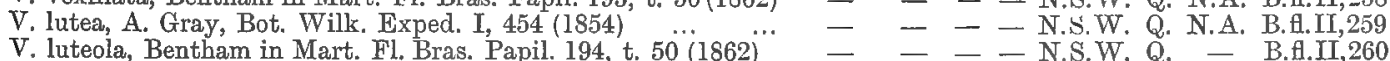

V. lanceolata, Bentham in Mitch. trop. Austr. 350 (1848) $\ldots-$ - S.A. - - N.S.W. Q. N.A. B.fl.II,260 DOLICHOS, Linné, gen. plant. 222 (1737).

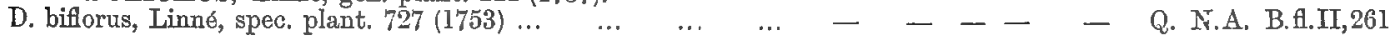
DUNBARIA, Wight \& Arnott, prodr. I, 258 (1834).

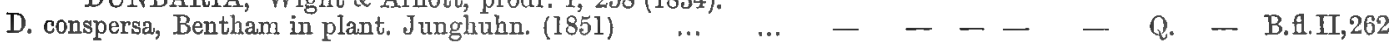
CAJANUS, De Candolle, Catal. hort. Monsp. 85 (1813). (Cajan, Atylosia.)

C. marmoratus, F. v. M.; Atylosia, Benth., Fl. Austr. II, 263(1S64) - - - - — Q. N.A. B.H.II,263

C. scarabeoides, F. v. M.; Atyl., Benth. in pl. Jungh. I, $242(1851)--1--$ - Q. - B. B.II,263

C. reticulatus, F. v. M.; Atylosia, Benth., Fl. Austr. II, $263(1864)-\quad-\quad-\quad$ - Q. N.A. B.H.II,263

C. grandifolius, F. v. M., Essay on pl. Burdekin Exped. 9 (1S60) - - - - - Q. N.A. B.H.II,264

C. confertiflorus, F. v. M., Essay on pl. Burdekin Exped. 9 (1860) - - - - - Q. - B.f.II,264

C. cinereus; Atylosia, F. v. M. in Benth. Fl. Austr. II, 264 (1864) - - - - - - N.A. B.f.II,264 RHYNCHOSIA, Loureiro, FI. Cochinch. II, 460 (1790).

R. rhomboidea, F. v. M. in Benth. Fl. Austr. II, $265(1864)$.. $-\quad-\quad-\quad-\quad$ - N.A. B.fl.II,265

$R$. acutifolia, Bentham, Fl. Austr. II, $266(1864) \ldots \quad \ldots \quad \ldots \quad-\ldots-2-\ldots-1-$ N.A. B.fl.II, 266

R. rostrata, Bentham, Fl. Austr. II, $266(1864)$
R. Cunninghamii, Bentham, Fl. Austr. II, $266(1864)$
.

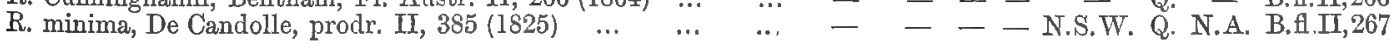
ERIOSEMA, De Candolle, prodr. II, 388 (1525).

E. Chinense, T. Vogel in Meyen's Beitr. zur Bot. 31 (1843)

FLEMINGIA, Roxburgh in Ait. hort. Kew. sec. ed. IV, 349 (1S12).

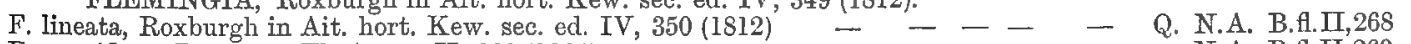

F. paucillora, Bentham, Fl, Austr. II, 269 (1864)... ... ..

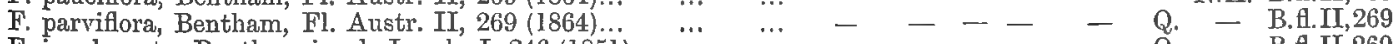

F. involucrata, Bentham in pl. Jungh. I, 246 (1851) $\quad \cdots \quad \cdots \quad \ldots \quad-\quad-\quad-\quad-\quad-\quad$ Q. $\quad$ - B.f.II,269

ABRUS, Rauwolf in Dalechamps, hist. general. plant. append. 193 (1587).

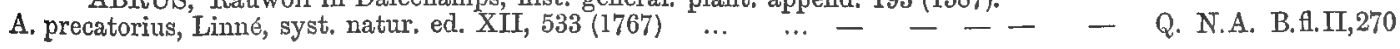

M.fr.VII, I28.

M.fr.IV, 79.

M.fr.VII, 128 .

M.fr.VII, 128 .

M.fr. VII, I28.

M.fr. VII, 128 .

M.fr.XI, 98 .

II.fr. VII, 127.

M.fr. VIII, 225.

M.fr. VII, 128.

.


DALBERGIA, Linne, fil. suppl. 52 (17 $\$ 1)$.

D. densa, Bentham in Hook. "Lond. Journ. II, $211^{\circ}(1843) \quad \ldots \quad-\quad-\quad-\quad-\quad$ Q. - B.fl.II, 271

LONCHOCARPUS, Humboldt, Bonplaand \& Kunth, nor. gen. VI, $383(1823)$.
N.S.W. Q. - B. A.II, 272

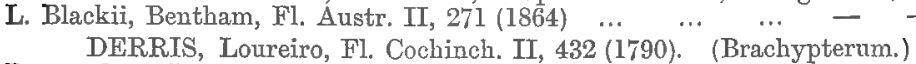

D. scandens, Bentham in Journ. Linn. soc. IV, suppl. $103(1860)-\ldots-$ N.S.W. Q. T. B.Al.II,272

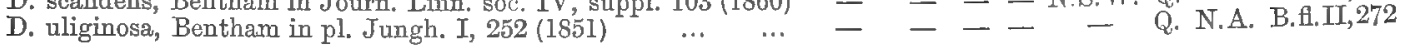
PONGAMIA, Lamarck in Ventenat. Jardin de la Malmaison, t. 2s (1803).

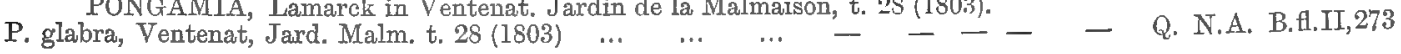
SOPHORA, Linne, gen. plant. 125 (1737).

S. tomentosa, Linné, spec. plant. $373(1753)$
S. Fraseri, Bentham, Fl. Austr. II, $274(1864)$

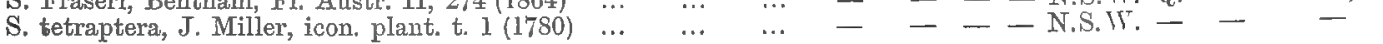

M.fr.VII,27.

M.fr.V,31;VII,27.

PODOPETALUM, F. F. M. in Melbourne Chemist, June (1882).

P. Ormondi, F. v. M., fragm. X III (inedit.)

CASTANOSPERMUM, Cunningham \& Fraser in Hook. bot. misc. I, 241, t. 51 (1830).

C. Australe, Cunningh. \& Fraser in Hook. bot. misc. I, 241, t.51(1830) - - - N.S.W. Q. - B.H.II, 275 BARKLYA, F. v. M., fragm. I, 109 (1859).

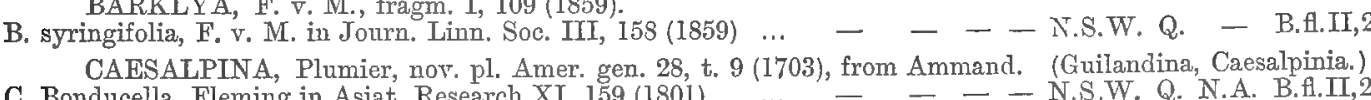

C. Bonducella, Fleming in Asiat. Research XI, 159 (1801) ‥ - - - N.S.W. Q. N.A. B.A.II, 276

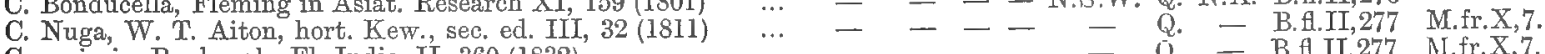

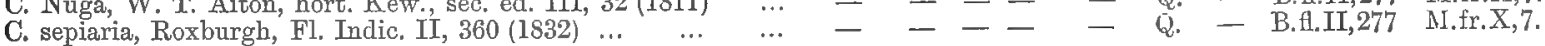
MEZONEURON, Desfontaines in Mém. du Mus. IV, 245, t. 10 et 11 (1818).

M. brachycarpum, Bentham, Fl. Austr. II, 278 (1864)... - - - N.S.W. Q. - B.A.II,278 II.fr.X,7.

M. Scortechinii, F. v. M. in Wing's South. Sc. Record:II, 73 (1882) - - - N.S.W. Q. - PTEROLOBIUM, R. Brown in Salt. Abyssin. 65 (1814).

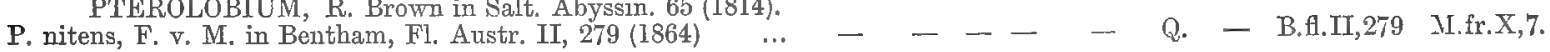
PELTOPHORUM, Vogel in Linnaea XI, 406 (1837).

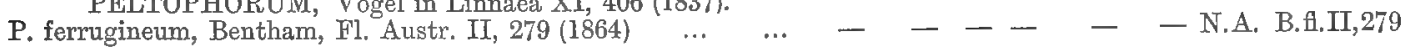
CASSIA, Tournefort, inst. 619, t. 392 (1700), from Plumier (1693). (Cathartocarpus.)

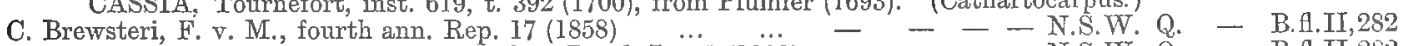

C. laevigata, Willdenow, enum. pl. hort. bot. Berol. I, 441 (1809) - - - - N.S.W. Q. - B.H.II, 282

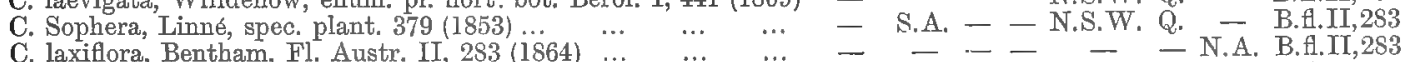

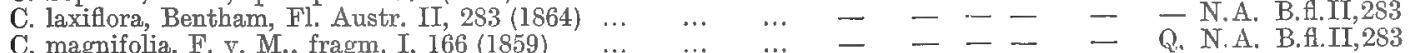

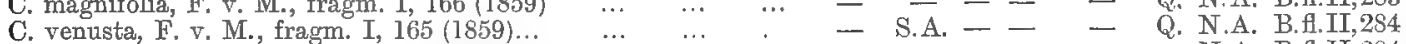

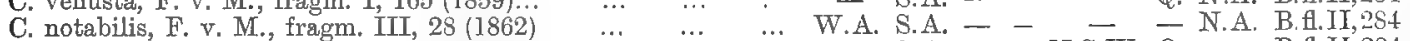

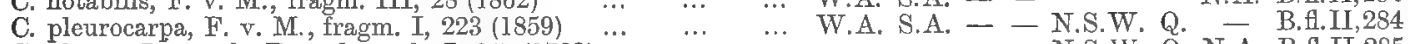

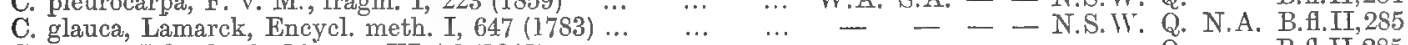

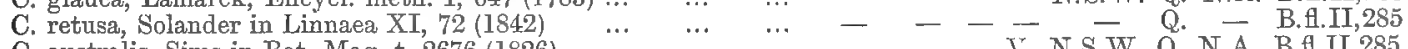

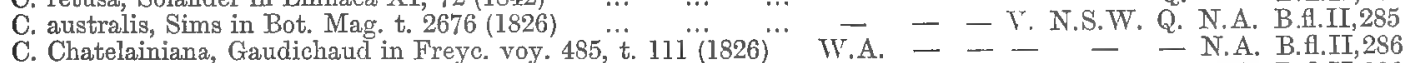

C. Chatelainiana, Gaudichaud in Freyc. voy. 485, t. 111 (1826)
C. glutinosa, De Candolle, prodr. II, 495 (1825) $\ldots$
C...

C. pruinosa, F. v. M., fragm. III, $48\left(\begin{array}{llll}(1862) & \ldots & \ldots & \ldots\end{array}\right.$

C. circinata, Bentham in Mitch. trop. Austr. 384 (1848)... $\ldots$ - $\ldots$ - S.A. - - N.S.W. Q. - B.A.II,2S6

C. phyllodinea, R. Brown, App. to Sturt's Centr. Austr. 15 (1849) - S.A. - T. N.S.W. - - B.tl.II,287

C. eremophila, Cunningh. in T. Vogel, gen. Cass. synops. $47(1837)$ W.A. S.A. - V. N.S.W. Q. - B.fl.II,2S7

C. artemisioides, Gaudichaud in De Cand. prodr. II, $495(1525)$ W.A. S.A. - V. N.S.W. Q. - B.fl.II,288

C. Sturtii, R. Brown, App. to Sturt's Centr. Austr. 14 (1849) … W.A. S.A. - V. N.S.T. Q. - B.t.II,2s8

C. desolata, F. v. M. in Linnaea XXV, $387(1852) \quad \ldots \quad \ldots$. W.A. S.A. - V. N.S.W. Q. N.A. B.A.II, 259

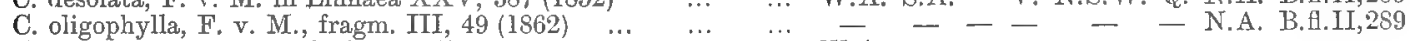

C. cardiosperma, F. v. M., fragm. X, $50(1877)$
C. oligoclada, F. v. M., fragm. III, $49(1862)$
C.

C. heptanthera, F. v. M., fragm. X, \& $(1876)$
C. leptoclada, Bentham, Fl. Austr. II, 290 (1864)...
C.

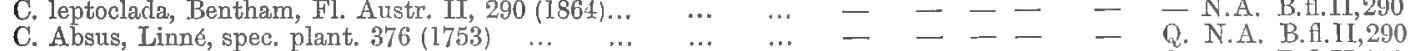

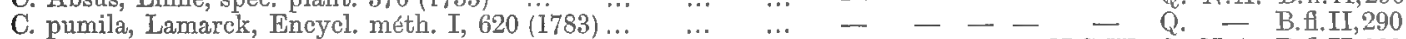

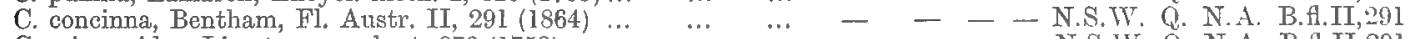

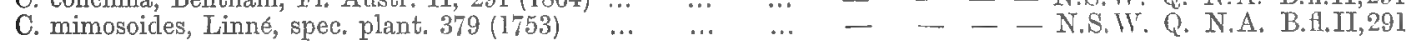
PETALOSTYLIS, R. Brown, App. to Sturt's Centr. Austr. 79 (1849).

P. labichoides, R. Brown, App. to Sturt's Centr. Austr. 17 (1849) W.A. S.A. - - N.S.W. Q. N.A. B.A.II,292 LABICHEA, Gaudichaud in De Cand. prodr. II, 507 (1825).

L. cassioides, Gaudichaud in De Cand. prodr. II, 507 (1825) ... W.A. - $-{ }_{-}$- B.ff.II, 292

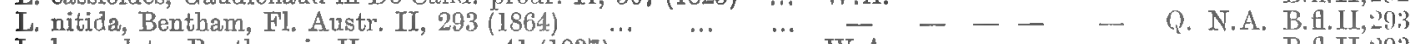

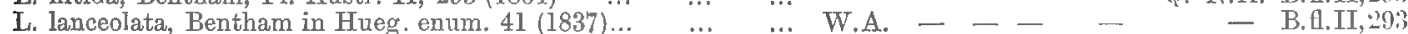

L. rupestris, Bentham in Mitch. Trop. Austr. $342(1848) \quad \ldots$...

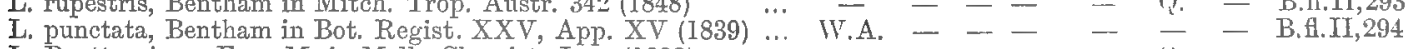

L. Buettneriana, F. v. M. in Melb. Chemist, June (1882) … - - _ TAMARINDUS, Tournefort, inst. 660, t. 445. App. (1700) from C. Bauhin (1623).

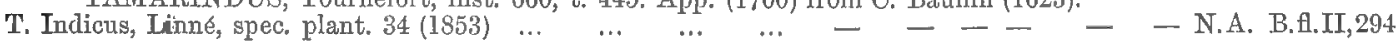
BAUHINIA, Plumier, nov, gen. pl. Amer. 22, t. 13 (1703).

B. Leichhardtii, E. v. M. in Transact. Vict. Inst. III, $50(1858)$ - S.A. - - - - N.A. B.Al.II,295

B. Carronii, F. v. M. in Transact. Vict. Inst. III, $49(1858) \ldots-\ldots-1$ - N.S.T. Q. - B.f.II, 29.

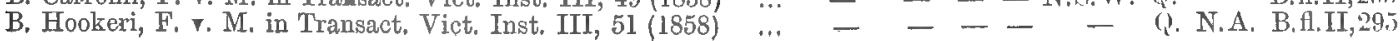

II.fr.X, 7.

M.fr.X, 8.

M.fr.IV, $14 ; \mathrm{X}, 8$.

M.fr.IV, $14 ; \mathrm{X}, 9$.

11 .fr. I, $166 ; X, 9$.

M.fr.I, 165;X, 9 .

II.fr. III, 28;X, 9 .

M.fr.I, 223;X, 9 .

M.fr.IV, $13 ; \mathbf{X}, 9$.

M.fr.X, 9 .

M.fr.III,48.

M.fr.I, 165 .

MI. fr. III, 49 .

Mi.fr. X, 50 .

MI.fr.III, 49;X, 9 .

II.fr. $X, S$.

MI.fr.III, $50 ; \mathrm{X}, 9$.

II.fr.III, $47 ; \mathrm{X}, 10$.

M.fr.X, 10 .

M.fr.III, $48 ; X, 10$.

M.fr.X, 7 .

M.fr. $x, 7$.

MI. fi. $X, 7$.

M.fr. X, 7 .

MI.fr, X,8.

M.fr.X,8.

M.fr.X.8.

M.fr.X,, , 
B. Gilesii, F. v. M. and Bailey in Wing's S. Sc. Record, Jul. (1882) - $-\quad-\quad-$ N.A. AFZELIA, Smith in Transact. Limn. Soc. IV, 221 (1798).

A. bijuga, A. Gray, Bot. Wilk. Expl. Exped. 467, t. 51 (1854) ... CYNOMETRA, Linné in Act. Soc. Ups. 78 (1741).

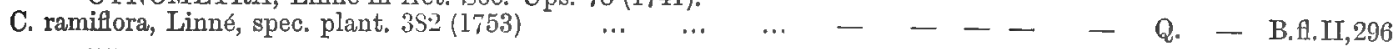
ERYTHROPHLAEUM, Afzelius in Tuckey's Congo 430 (1818). (Laboucheria,)

E. Laboucherii, F. v. M. in Benth. Fl. Austr. II, 297 (1864) ... - - - - Q. N.A. B.H.II,297 ENTADA, Adanson, Familles des plantes II, 318 (1763).

E. Pursaetha, De Cand, mémoir. sur la fam. des Légum. 12 (1825) - - - - - Q. - B.t.II,298 ADENANTHERA, Royen in Linné, coroll. 7 (1737).

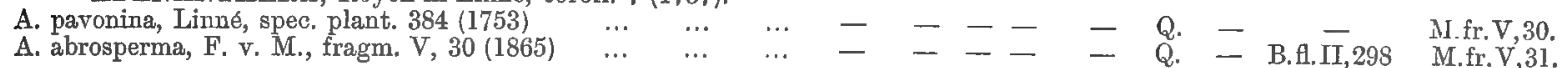
NEPTUNIA, Loureiro, Fl. Cochinch. II, 653 (1790). (Dichrostachys.)

N. cinerea, F.v. M.; Dichrostachys, Wight\&Arnott, prodr.271(1834) - - - - - N.A. B.A.II.299

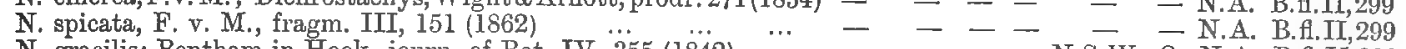

N. gracilis; Bentham in Hook, journ. of Bot. IV, 355 (1842) $\cdots,-\ldots=-1 . \overline{-}=$ N.S.W. Q. N.A. B.A.II,300

N. monosperma, F. v. M. in Benth. Fl. Austr. II, 299 (1864) .. - - - - Q. N.A. B. B.II,300

M.fr.III, 151. ACACIA, Tournefort, inst. 605, t. 370 (1700) from Dioscorides \& Plinius. (Vachellia, Chithonanthus, Tetracheilus, Mimosa partly.)

\section{Alatae.}

A. glancoptera, Bentham in Schlecht. Linnaea XXVI, 604 (1853) W.A. - - - - - - B.fl.II,320

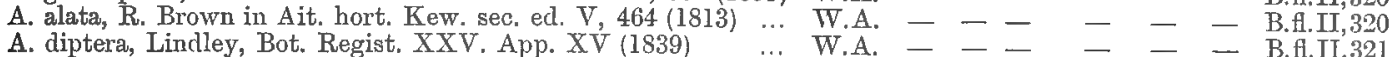

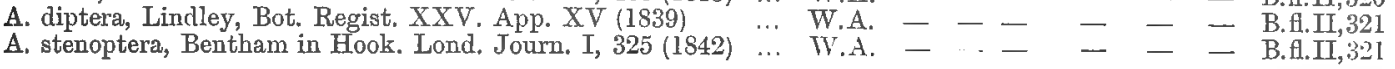
II. Contindae.

A. incurva, Bentham in Hook. Lond. Journ. I, 325 (1842) $\ldots$ W.A. - - - - - - B.fl.II,32:2

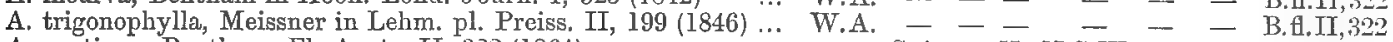

A. continua, Bentham, Fl. Austr. II, 322 (1864) ... $\ldots \ldots \ldots$ - . S.A. - V. N.S.W. -

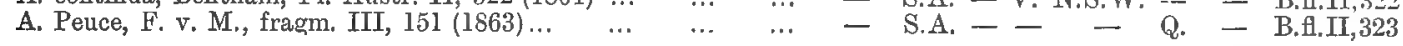
III. Pongentents.

A. spinescens, Bentham in Hook. Lond. Journ. I, $323(1842)$... - S.A. - V. N.S.W. - - B.H.II,323

A. cochlearis, H. L. Wendland, comm. de Acac. aphyll. I5 (1820) W.A. S.A. - - - - - B.A.II,324

A. lanigera, Cunningham in Field's N.S. Wales, 345 (1825) … - - - V. N.S.W. - - B.A.II,324

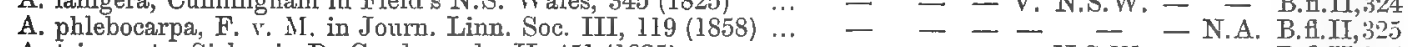

A. trinervata, Sieber in De Cand. prodr. II, 451 (1825) ... ... - - - N.S.W. - - B.f.,I, 325

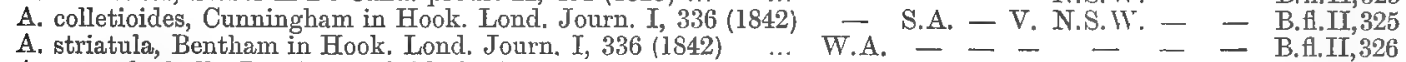

A. campylophylla, Benthañ in Schlecht. Linnaea XXVI, 605(1853) W.A. - - - - - - B.H.II,326

A. teretifolia, Bentham in Hook. Lond. Journ. I, 326 (1842) ... W.A. - - - - - B.fl.II,326

A. sulcata, R. Brown in Ait. hort. Kew. sec. ed. V, 460 (1813) W.A. - - - - - - B.fl.II,3:7

A. costata, Bentham in Hook. Lond, Journ. I, 339 (1842) … W.A. - - - - - - B.H.II,327

A. barbinervis, Bentham in Hook. Lond. Journ. I, 326 (1842) ... W.A. - - - - - - B.H.I,327

A. ataxiphylla, Bentham in Schlecht. Linneea XXVI, 605 (1853) W.A. - - - - - - B.A.II,328

A. Baxteri, Bentham in Hook. Lond. Journ. I, 327 (1842) ‥ W.A. - - - - - - B.H.II,328

A. aureonitens, Lindley, Bot. Regist. XXV, App. XV (1839) .... W.A. $-二-Z-二=$ B.A.II,328

A. quadrisulcata, F. v. M., fragm. IIx, 127 (1863) $\ldots$... W.A. - - - - - - B.fl.II,3ะS

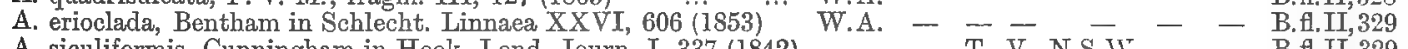

A. siculiformis, Cunningham in Hook. Lond. Journ. I, 337 (1842) - - T. V. N.S.W. - - B.Al.II,329

A, patens, F. v. M. in Journ. Linu. Soc. III, 120 (1858)... ‥ - - - - - - N.A. B.fl.II, 329

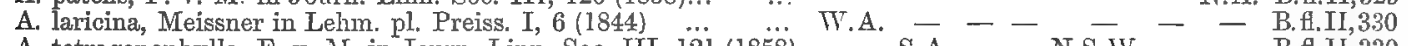

A. tetragonophylla, F. v. M. in Journ. Linn. Soc. III, I201 (1858) - S.A. - N.S.IV. - - B.fl.II,330

A. genistoides, Cunningham in Benth. Fl. Austr. II, 330 (1864) W.A. - - - - - B.fl.II,330

A. spacelata, Bentham in Hook. Lond. Journ. I, 338 (1842) ... W.A. - - - - - - B.H.II,331

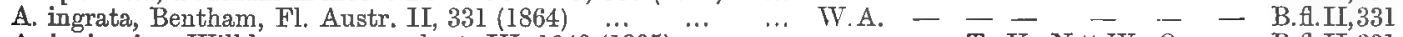

A. juniperina, Willdenow, spec. plant, IV, 1049 (1805) ..... - - T. V. N.S.W. Q. - B.H.II,331

A. asparagoides, Cunningham in Field's N.S. Wales 343 (1825)... - - - - N.S.W. - - B.H.II,332

A. tenuifolia, F. v. M. in Transact. phil. Soc. Vict. I, 37 (1554) - - - V. -- B.fl.II,332

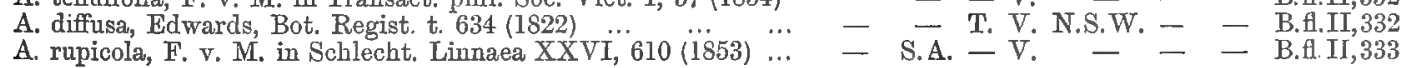
IV. Calamiformes.

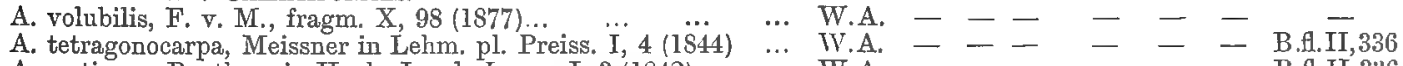

A. restiacea, Bentham in Hook. Lond. Journ. I, 3 (1812) … W.A. - _ - - - - B.A.II,336

A. squamata, Lindley, Bot. Regist. XXV, App. XV (1839) … W.A. - - - - - - B.fl.II,336

A. brachyphylla, Bentham in Schlecht. Linnaea XXVI, 615 (1853) W.A. - - - - - - B.fl.II,337

A. Bynoeana, Bentham in Schlecht. Limnaea XXVI, 614 (1853) - _ - - - - N.A. B.A.II,337

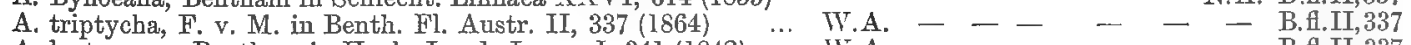

A. leptoneura, Bentham in Hook. Lond. Journ. I, 341 (1842) ... W.A. - - - - - - B.fl.II,337

A. rigens, Cunningham in Loudon, hort. Brit. II, 406 (1830) $\ldots .$. - S.A. - V. N.S.W. - - B.fl.II,337

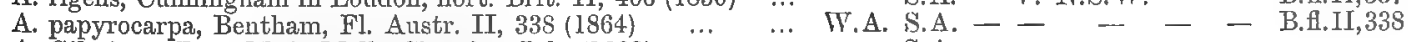

A. Gilesiona F. $\mathrm{M}$ in Melb. C'hemist July (1882)

A. sessiliceps, F. $\checkmark$. M. in Mlelb. Chemist, July (1882)

A. pugioniformis, Wendl., comm. de Acac. aphyll. 38, t. 9 (IS20)

A. juncifolia, Bentham in Hook. Lond. Journ. I, 341 (1842)

A. calamifolia, Sweet in Bot. Regist. t. 839 (1824)

A. scirpifolia, Meissner in Bot. Zeitung 10 (1855)...

A, extensa, Lindley, Bot. Regist. XXV, App. XV (1839)
- S.A. - - - -

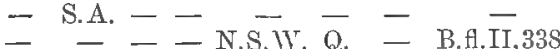

- - - - N.S.W. Q. N.A. B.H.II,339

- S.A. $=\overline{\text { V. N.S.W. }}$ - B.H.I, 339

$\therefore$ W.A. - - - - - B.AlII, 339

… W.A. - - - - - B.E.II,340
M.fr.IV,3.

M.fr.III.151.

M.fr.IV,4.

M.f.III, 127.

M.fr.III, 46 .

II.fr.IV,3.

II.fr.X, 98 .

M.fr.IV, 9. 
A. gonophylla, Bentham in Schlecht. Linnaea XXVI, 613 (1853) A. ericifolia, Bentham in Hook. Lond. Journ. I, 345 (1842)

A. uncinella, Bentham in Schlecht. Linnaea XXVI, $613(1853) \ldots$ A. oxyclada, F, v. M. in Benth. Fl. Austr. II, 341 (1864) V. BRUNIDIDAE.

A. cedroides, Bentham in Schlecht. Linnaea XXVI, 615 (1853)

A. lycopodifolia, Cunningham in Hook. icon. plant. t. 172 (1837)

A. spondylophylla, F. v. M., fragm. VIII, 243 (1874)

A. galioides, Bentham in Hook. Lond. Journ. I, 344 (1842)

A. Baueri, Bentham in Hook. Lond. Journ. I, 344 (1842)

A. subternata, F. v. M. in Journ. Linn. Soc. III, 124 (1858) $\quad \ldots$

A. minutifolia, F. v. MI., fragm. VIII, 243 (1874)

A. brunioides, Cunningham in G. Don, gen. syst. II, $404(1832)$

A. conferta, Cunningham in Hook. Lond, Journ. I, 345 (1842)... VI. UNINERYES.

A. scabra, Bentham in Schlecht. Linnaea XXVI, 605 (1853) ...

A. nodiflora, Bentham in Schlecht. Linnaea XXVI, 621 (1853) ..

A. spinosissima, Bentham in Schlecht. Linnaea XXVI, 621 (1853)

A. ulicina, Meissner in Lehm. pl. Preiss. II, 202 (1846) ...

A. erinacea, Bentham in Hook. Lond. Journ. I, 360 (1842)

A. Huegelii, Bentham in Hueg. enum. 42 (1837) ...

A. nervosa, De Candolle, Mem. sur la fam. des Légum. $\ddot{4} 44$ (1820)

A. obovata, Bentham in Hook. Lond. Journ. I, 329 (1842)

A. congesta, Bentham in Hook. Lond. Journ. I, 327 (1842)

A. dermatophylla, Bentham, Fl. Austr. II, 346 (1864)

A. aspera, Lindley in Mitch. Three Exped. II, 139 (1838)

A. armata, R. Brown in Ait. hort. Kew. sec, ed. V, 463 (1813)

A. idiomorpha, Cunningham in Hook, Lond. Journ. I, 329 (1842)

A. Shuttleworthii, Meissner in Lehm. pl. Preiss. I, 7 (1844)

A. Gregorii, F. v. M., fragm. III, 47 (1862)

A. pilosa, Bentham in Schlecht. Linnaea XXVI, 607 (1853) $\quad \ldots$

A. crispula, Bentham in Schlecht. Linnaea XXVI, 607 (1853)

A. crassistipulea, Bentham in Hook. Lond. Jourm, I, 326 (1842)

A. hastulata, Smith in Rees' Cyclopaed. XXXIX, Suppl. (1819)

A. horridula, Meissner in Lehm. pl. Preiss, I, 9 (1844).

A. divergens, Bentham in Hook. Lond, Journ. I, 331 (1842)

A. vomeriformis, Cunningham in Hook. Lond. Journ. I, $332(18+2)$ A. plagiophylla, F. v. M. in journ. Linn. Soc. III, 131 (1858)

A. biflora, $\boldsymbol{R}$, Brown in Ait. hort. Kew. sec. ed. V, 463 (1813)

A. decipiens, R. Brown in Ait. hort. Kew. sec. ed.' V, 463 (1813) A. cuneata, Bentham in Hneg. enumer. 42 (1837).

A. dilatata, Bentham in Schlecht. Linnaea XXVI, 608 (1853)

A. bidentata, Bentham in Hook. Lond. Journ. I, 333 (1842)

A. acanthoclada, F. จ. M., fragm. III, 127 (1863)

A. obliqua, Cunningham in Hook. Lond. Journ. I, 334 (1842) ...

A. acinacea, Lindley in Mitch. Three Exped. IJ, 267 (1838) ...

A. lineata, Cunningham in G. Don, gen. syst. II, 403 (1832)

A. lachnophylla, F. v. M. in Wing's S. Sc. Record, July (1882)

A. triquetra, Bentham in Hook. Lond. Journ. I, 358 (1842)

A. ligustrina, Meissner in Lehm. pl. Preiss. II, 203 (1846)

A. Meissneri, Lehmann, delect. sem. hort. bot. Hamb. (1842) ...

A. anceps, De Candolle, Mém. sur la fam. des Légum. 446 (1825)

A. hispidula, Willdenow, spec. plant. IV, 1054 (1805)

A. undulifolia, Fraser in Loddiges, Bot. Cabinet XVI, 1544 (1829)

A. flexifolia, Cunningham in Hook. Lond. Journ. I, 359 (1842)...

A. dura, Bentham in Schlecht. Linnaea XXVI, 622 (1853)

A. spathulata, F. V. M. in Benth. Fl. Austr. II, 356 (1864)

A. microcarpa, F. v. M., second gen. Report 7 (1854)

A. montana, Bentham in Hook. Lond. Journ. I, 360 (1842) $\ldots$

A. verniciflua, Cunningham in Field's N.S. Wales 344 (1825) ...

A. leprosa, Sieber in De Cand. prodr. II, 450 (1825)

A. stricta, Willdenow, spec. plant. IV, 1052 (1805)

A. dodonaeifolia, Willdenow, enum. pl, hort. Berol, suppl. 68 (18̈is)

A. Gnidium, Bentham, Fl. Austr. II, 359 (1864) ...
A. ramosissima, Bentham in Hook. Lond. Journ, I, 356 (1842)...

A. Dempsteri, F. v. M., fragm. XI, 65 (1879)

A. Sentis, F. v. M., second gen. Report ll (1854) $\cdots$

A. dentifera, Bentham in Maund, Botanist IV, t. 179 (1839)

A. sclerosperma, F. v. M. in Wing's South. Sc. Rec., July (1882) TI

A. fasciculifera, F. v. M. in Benth. Fl. Austr. II, 361 (1864)

A. falcata, Willdenow, spec. plant. IV, 1053 (1805)

A. macradenia, Bentham in Mitch. Trop. Austr. 360 (1848)

A. penninervis, Sieber in De Cand. prodr. II, 452 (1825)

A. amblyphylla, F. v. M. in Wing's S. Sc. Record, July (I882)

A. retinodes, Schlechtendal, Linnaea XX, 664 (1847)

A. nerifolia, Cunningham in Hook. Lond. Journ. 1, 357 (1842)

A. microbotrya, Bentham in Hook. Lond. Journ, I, 353 (1842)...

A. leiophylla, Bentham in Hook. Lond. Journ. I, 351 (1842) ...

A. cyanophylla, Lindley, Bot. Regist. Misc. 49 (1835)

A. pycnantha, Bentham in Hook. Lond. Journ. I, 3551 (I 1442 ) ...
W.A.

.A. - - - - - B.fl.II, 340

W.A. - - - - - - - B.fI.II,341
W.A. - -

IV.A. - - - - - - B.H.II, 34l

- S.A. - - - Q Q. N.A. B.f.II, 342

- S.A. - - - Q N. N.A.A. B.fl. $\overline{\text { II, 34? }}$

- - - - N.S.W. Q. - B.f.II, 342

- Z - - N.S.W. N. N.A. B.f.II, 343

- S.A. - - $\overline{\text { Q. }}$ - B.f. $\overline{\mathrm{II}}, 343$

- - - - N.S.W. Q. - B.A.I,343

W.A. - - - - - B.fl.II,344

W.A. - - - $=-$ B.f.II 344

W.A. - - - - - B.fl.II,344

TI.A. - - - - - - B.fl.II,345

W.A. - - - - - - B.fl.II,345

W.A. - - - - - B.H.II,345

W.A. - - . - - - B.fl.II, 346

W.A. - - - - - - B.fl.II,346

W.A. - - - - - - B.fl.II,346

W.A. - - - - - B.fl.II,346

- S.A. - V. N.S.W. - - B.H.II,347

W.A. S.A. - V. N.S.W. Q. - B.H.II,347

IV.A. - - - - - - B.A.II,348

WV.A. - - - - - - B.fl.II.348

- - - - - N.A. B.fl.II,34S

IV.A. - - - - - B.fl.II,348

W.A. - - - - - - B.fl.II,349

W.A. - - - - - - B.fl.II,349

W.A. - - - - - - B.fl.II, 349

W.A. - - - - - - B.fI.II,350

IV.A. - - - - - B.fl.II,350

S.A. T. V. N.S.W. - $=$ B.H.II, 350

- - - N.S.W. Q.

W.A. - - - - - B.fl. II.351

W.A. - - - - - - B.H.II,351

TV.A. - - - - - - B.fl.II,35I

W.A. - - - - - - B.fl.II,352

W.A. - - - - - - B.fl.II,352

W.A.S.A. - V. N.S.T - - B.A.II,352

- S.A. - V. N.S.W. - - B.fl.II,353

- S.A. - V. N.S.W. - B.H.II,353

- S.A. - V. N.S.W. - - B.fl.II,353

W.A. - - - - - - B.fl. II, 354

W.A. - - - - - - B fl.11,354

W.A. - - - - - - B.H.II,354

W.A.S.A. - - - - - B.H.II,35.5

- - - - N.S.T. Q. - B.fl.II,350

- - - N.S.II.Q. - B.f.II,355

- - - - N.S.W. - - B.A.II,356

W.A. - - - - - - B.A.II, 356

W.A. - - - - - N.A. B.H.II,356

- S.A. - V. N.S.W. - - B.fl.II.337

- S.A. - V. N.S.W. - - B.H.II,357

- S.A. T. T. N.S.IY - - B.H.II.3JS

- - - V.N.S.W. - - B.fl.II,35S

- - T. V. N.S.W. - - B.fl.II,358

- S.A. - - - - - B.fl.II,359

- - - - Q. - B.f.II,359

W.A. - - - - - - B.H.II,360

W.A. $-\overline{\text { W.A. }}$ - V. N.S.T. $\overline{\text { Q. N.A. B. B.A.II, } 360}$

IT.A. - - - - - B.fl.II,361

- - - N.S.IV. Q. N.A.

- - N.S.IV. (

- T. V. N.S.II. (

W.A. $-\quad--\quad-$

- S.A. - V.

- D. - N.S.W. (? - B H.II,;3:3

II.A. - - - - - - B.fl.II,363

W.A. - - - - - - B.fl.II,364

W.A. - - - - - B.fl.II,364

W.A. S.A. - T. N.S.W. - -- B.fl.II,36.)
M.fr.243.

M.fr.XI, 33 .

M.fr.X, 243 .

M.fr.III, 47 .

M.fr.III, 127.

M.fr.I, $5 ; \mathrm{II}, 177$.

M.fr.I, 6.

II.fr. XI, 65 .

M.fr.XI,65. 
A. gladiiformis, Cunningham in Hook. Lond. Journ. I, $\dddot{3} \bar{y} 4(18 \ddot{4} 2)$

A. obtusata, Sieber in De Cand. prodr. II, $453(1825)$.

S.A. - - NSIT

A. rubida, Cunningham in Field's N.S.Wales, 344 (1825) …

A. amoena, H. L. Wendland, comm. de Acac, aphyll. 10, t. $4(1820)-$

- - - N.S.W. - - B.fl.II,363

- - - N.S.W - - B.A.II,366

- - N.S.M. - - B.Hl.II,360

- - V. N.S.II. - - B.f.II,366

A. hakeoides, Cunningham in Hook. Lond. Journ. I, 35t (1842) - S.A. - V. N.S. II - - B.

A. salicina, Lindley in Mitch. 'Three Exped. II, 20 (1838)

A. rostellifera, Bentham in Hook. Lond. Journ. I, 356 (1842) $\ldots$

A. pycnophylla, Bentham, Fl. Austr. II, 368 (1864)

W.A. S.A. - V. N.S.W. Q. N.A. B.fl.II,367

W.A. - - - - - - B.H.II,368

A. iteaphylla, F. v. M. in Schlecht. Linnaea XXV̈I, 617 (1853)

A. suaveolens, Willdenow, spec, plant. IV, 1050 (1805)... ...

A. subcoerulea, Lindley, Bot. Regist. t. 1075 (1827)

A. Lindleyi, Meissner in Lehm. pl. Preiss. I, 14 (184t)

A. leptopetala, Bentham in Schlecht. Limnaea XXVI, 619 (1853)

A. Dietrichiana, F. v. M. in Melb. Chemist, July (1882)

A. Murrayana, F. v, M. in Benth. FI. Austr. II, 370 (1864) …

A. subulata, Bonpland, pl, rar, cult. a, Malm. 110 t. 45 (1813) ...

A. linifolia, Willdenow, spec. plant. IV. 1051 (1.805)

A. Leichhardtii, Bentham, Fl. Austr. II, 372 (1864)

A. crassiuscula, H. L. Wendland, comm. de Acac. 31, t." 8 (1820)

A. buxifolia, Cunningham in Field's N.S. Wales, 344 (1825)

A. lunata, Sieber in De Cand, prodr. II, 452 (1825)

A. brachybotrya, Bentham in Hook. Lond. Journ. I, $347^{\prime \prime}(1842)$

A. Wattsiana, F. v. M. in Benth. Fl. Austr. II, 374 (1864)

A. podalyrifolia, Cunningham in Loudon, hort. Brit. 707 (1830)

A. uncifera, Bentham in Mitch. Trop. Austr. 341 (1848)

A. vestita, Ker in Bot. Regist. t. 698 (1823)

A. cultriformis, Cunningham in G. Dow, gen. syst. II, 406 (I832)

A. pravissima, F. v. M., first gen. report 12 (1853)

- S.A. T. V. N.S.T. $\bar{Q}$ Q.

Y.A. - - - - B.flII,360

W.A. - - - - - B.H.II,370

V.A. - - - - - B.H.II,370

- S.A - - $-\overline{\mathrm{Q}}-\mathrm{B} \overline{\mathrm{II}} 3 \mathrm{0}$

- - - N.S.W. O

- T. - N.S.W Q.

- - N.S.W.Q

- - V. NS.TY.Q

N.S.W. - B.fl.II,373

- S.A. - - - N.S.W. Q. - B.f.II,3,74

- - - - Q - B.H.H,374

- - - - N.S.W. - - B.fl.II,375

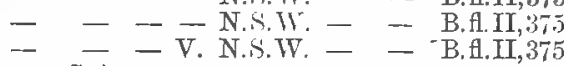

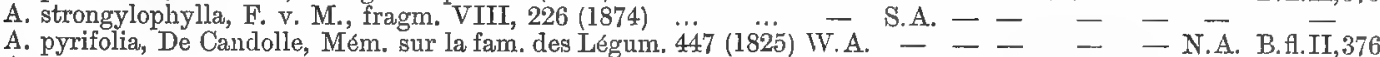

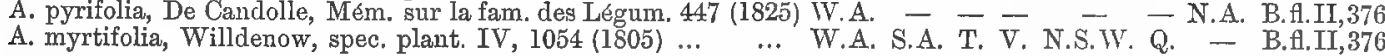
VII. Plurinerves.

A. scalpelliformis, Meissner in Lehm. pl. Preiss. II, 200 (1847)...

A. urophylla, Bentham in Bot. Reg. Misc. 24 (1841) ... ...

A. Luehmanni, F. v. M., fragm. XI, $116(1881) \quad \ldots \quad \ldots \quad \ldots$

A. sublanata, Bentham in Hueg. enum. $42(1837) \ldots . \quad \ldots . \quad \ldots$

A. pravifolia, E. v. M., fragm. I, 4 (1858) ...

A. amblygona, Cunningham in Hook. Lond. Journ. I, $332(1842)$

A. adnata, F. v. M. in Melb. Chemist, July (1882)

A. deltoidea, Cunningham in G. Don. gen. syst. II, 401 (1832)...

A. stipulosa, F. v. M. in Journ. Linn. Soc. III, 119 (1858)

A. loxophylla, Bentham in Schlecht. Linnaea XXVI, 625 (1853)

A. setulifera, Bentham in Schlecht. Linnaea XXVI, 625 (1853)

A. translucens, Cunningham in Hook. icon. plant. t. 160 (1837)

A. impressa, F. v. M. in Journ. Linn. Soc. III, 133 (1858)

A. bivenosa, De Candolle, prodr. II, 452 (1825) ... ...

A. trinenra, F. v. M., pl. of Vict. II, 25 (1860) $\ldots . . .$.

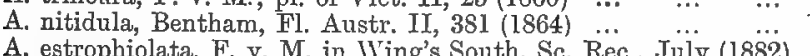

A. heteroclita, Meissner in Lehm. pl. Preiss. I, 318 (1845)

A. elongata, Sieber in De Cand. prodr. II, 451 (1825) ... $\ldots$

A. subporosa, F. v. M., pl. of Vict. II, 24 (1860) ... …

A. Simsii, Cunningham in Hook. Lond. Journ. I, $368(1842) \ldots$

W.A.

- - - - - B.fl.II,377

- S.A. - - - - N.A. B.f. $\overline{I I}_{378}$

- S.A. - - N.S.IV. Q. -

- - - - N.S.W. Q. - B.t.II, 378

W.A. - - - - - N.A. B.fi.II, 378

TV.A. - - - - - N.A. B.H.II,379

W.A. - - - - - - B.Al.II,379

- - - - - N.A. B.fl.II, 3 -

- - - - - N.A. B.fl.II, 379

W.A. - - - - N.A. B.Al.II, 380

- S.A. - V. - - N.A. B.f.II,381

W.A. S.A. - - - - - B.A. B.fl.II,381

W.A. - - - - - - B.fl.II,381

- - - T. N.S.T. $\bar{Q}$ - B.fl.II,381

- - - V. N.S.W. - - B.f.II,382

- - - — Q. N.A. B.f.II,382

A. leptospermoides, Bentham in Schlecht. Limnaea XXVI,626(18̈53) W.A. - - - - - - B.Al.II,383

A. homalophylla, Cunningham in Hook. Lond. Journ. I, $365(1842)-$ S.A. - V. N.S.T. - - B.f.II,383

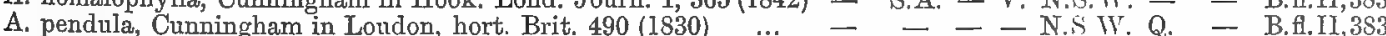

A. Osswaldi, F. v. M., pl. of Vict. II, 27 (1860) $\ldots \ldots \ldots . \quad \ldots$. W.A. S.A. - V. N.S.W. Q. $\quad$ - B.fl.II,384

A. lineolata, Bentham in Schlecht. Linnaea XXVI, 626 (1853) ... W.A. - - - - - - B.fl.I,384

A. quadrimarginea, F. v. M., fragm. X, 31 (1876) $\quad$.. … W.A. - - - $\quad-\quad-\quad-$

A. coriacea, De Candolle, Mém. sur la fam. des Légum. 446 (1825) V.A. - - - - - N.A. B.fl.II,385

A. stenophylla, Cunningham in Hook. Lond. Journ. I, $366(1842)-$ S.A. - V. N.S. W. Q. N.A. B. H.II,3Sã

A. hemignosta, F. v. M. in Journ. Linn. Soc. III, 134 (1858) ... - - - - - - N.A. B.H.II,385

A. sclerophylla, Lindley in Mitch. Three Exped. II, $138(1838)$ - S.A. - V. N.S.W. - - B.A.IL,386

A. farinosa, Lindley in Mitch. Three. Exped. II, 145 (1838) ‥ - S.A. - V. N.S.W. - - B.fl.II,386

A. Whanii, F. v. M. in Benth. Fl. Austr. II, 386 (1864) $\ldots-$ - $-\quad$ - T. - - - B.fl.II,386

A. heteroneura, Bentham in Schlecht. Limnaea XXVI, 624 (1853) W.A. - - - - - - B.f.II,387

A. viscidula, Cunningham in Hook. Lond. Journ. I, 366 (1842) - - - N.S.W. Q. - B.H.II, 387

A. ixiophylla, Bentham in Hook. Lond. Journ. I, $364(1842)$... W.A. - - - N.S.W. Q. - B.fl.II,387

A. dictyophleba, F. v. M., fragm. III, I28 (1863)... $\ldots . . . \quad-$ S.A. - - - Q. N.A. B.A.II,388

A. venulosa Bentham in Hook. Lond. Journ, I, $366(1842)$

A. cyclops, Cunningham in Loudon, hort. Brit. 407 (1830)

A. melanoxylon, R. Brown in Ait. hort. Kew. sec. ed. V, $462(1813)$

A. oraria, F. v. M., fragm. XI, 66 (1879) .

A. implexa, Bentham in Hook. Lond. Journ. I, 363 (1842)

A. harpophylla, F. v. M. in Benth. Fl. Austr. II, 389 (1864) ..

A. excelsa, Bentham in Mitch. Trop. Austr. 225 (1848) ... $\quad \ldots$

A. homaloclada, F, . M., fragm. XI, 34 (1878) ... ... $-\frac{-}{-1}-$ N.S.TV. Q V.A. S.A. - - T. N.IT.
S.A. T. T.

- - V. N.S.W. Q

$=- \pm-$
B. A.II, 370

B.fl. II, 371

B. fl. II, 372

B.H.II, 372

B.fl. II, 372

M.fr.I, 6.

M. fr.XI, 123.

M.fr.I. 5 .

M.fr. VIII, 226

M. fr. III, 17 .

M.fr.XI, 116.

M.fr.XI, 117.

M.fr.I, 4

M.fr.IY, 3 .

M.fr.XI, 11\%.

M.fr. $\Gamma, 5$.

M.fr.IV,6.

M.fr.IT, 5 .

M.fr.IV,5.

M.fr.X, 31 .

M.fr.XI, 67

M. fr. TY 6

M.fr.III,128

B.fl.I1,388

- B.fl.II,388

- B.fi.II, 389

B. fl.1I,389

B. A.II, 390

M. fr. XI, 66 ,

M.fr.IV, 6.

M.fr.XI, 34 . 
A. complanata, Cunningham in Hook. Lond. Journ. I, 369 (1842) A. dineura, F. Y. M. in Journ. Linn. Soc. III, 130 (1858)

A. binervata, De Candolle, prodr. II, 452 (1825) ... ... ... A. latescens, Bentham in Hook. Lond. Journ. I, 380 (1842) $\quad \ldots$ A. platycarpa, F. v. M. in Journ. Linn. Soc. III, 145 (1858) A. flavescens, Cunningham in Hook. Lond. Journ. I, 381 (1842) A. retivenea, F. v. M., fragm. III, I2S (1863)

A. triptera, Bentham in Hook. Lond. Journ. I, $3 \ddot{2} 5$ (1842)

A. rhigiophylla, F. v. M. in Schlecht. Linnaea XXVI, 611 (1853)

A. Oxycedrus, Sieber in De Cand, prodr. II, 453 (1825) ...

A. verticillata, Willdenow, spec. plant. IV, 1049 (1805)...

A. Riceana, Henslow in Maund, Botanist III, t. 135 (1839) VIII. JULIFERAT.

A. amentifera, F, v. MI, in Journ. Linn. Soc. IXI, 141 (1858) ... A. Wickhami, Bentham in Hook, Lond. Journ. I, 379 (1842) ... A. lysiphloia, F. v. M. in Journ, Linn. Soc. III, 137 (1858) ‥ A. limarioides, Bentham in Hook. Lond. Journ. I, 371 (1842) ... A. stipuligera, F. v. M. in Journ. Limn. Soc. III, 144 (1858) ... A. stigmatophylla, Cumningham in Hook. Lond. Journ, I, 377 (1842) A. umbellata, Crunimgham in Hook. Lond. Journ. I, 378 (1842) A. leptophleba, F. v. M. in Journ. Linn. Soc. III, 143 (1858) ... A. limbata, F. v. M. in Journ. Linn. Soc. III, 145 (1858)

A. brevifolia, Bentham, Fl. Austr. II, 395 (I864) ...

A. megalantha, F. v. M. in Journ, Linn. Soc. III, 143 (1858) “'

A. gonoclada, F. v. M. in Journ. Linn. Soc. III, 140 (1858) ...

A. pycnostachya, F. จ. M., pl. of Vict. II, 33 (1860)

A. subtilinervis, F. v. M., pl. of Vict. II, 32 (1860)

A. cochliocarpa, Meissner in Bot. Zeiting 10 (1855)

A. Dallachiaula, F. v. M. fragm. I, 7 (1858)

A. alpina, F. v. M., fragm. III, 129 (1863)...

A. longifolia, Willdenow, spec. pl. IV, 1052 (1805)

A. linearis, Sims, Bot. Mag, t. 2156 (1820)...

A. aciphylla, Bentham in Schlecht. Linnaea, XXVI, $6277^{\prime 1853)}$

A. ephedroides, Bentham in Hook. Lond. Journ. I, 370 (1S42) ...

A. Burkittii, F. v. M. in Benth. Fl. Austr. II, 400 (1S64)

A. microneura, Meissner in Lehm. pl. Preiss. I, 19 (1844)

A. cyperophylla, F. Y. II. in Benth. Fl. Austr. II, 400 (1864) ...

A. multispicata, Bentham, Fl. Austr. II, 400 (1864)

A. pityoides, F. v. M. in Journ. Linn. Soc. III, 135 (1858)

A. xylocarpa, Cunningham in Hook. Lond. Journ. I, 370 (1842)

A. gonocarpa, H'. v. M. in Journ. Linn, Soc. III, 136 (1858)

A. oncinophylla, Lindley, Bot. Regist. XXV, App. XV (1839)...

A, conjunctifolia, F. v. M., fragm. XI, 68 (1879) ...

A. drepanocarpa, F. v. M. in Journ. Limn. Soc. III, 137 (1858)...

A. arida, Bentham in Hook. Lond. Journ. I, 370 (1842)

A. aneura, F. v, II. in Schlecht, Linnaea, XXVI, 627 (1853) '..

A. cibaria, F. v. M. in Melb. Chemist, July (1882)

A. Kempeana, F. v. M. in Melb. Chemist, July (1882) ...

A. conspersa, F. v. M. in Journ, Linn. Soc. III, 140 (1858)

A. Doratoxylon, Cumingham in Field's N.S. Wales, 345 (1825)

A. acuminata, Bentham in Hook, Lond. Journ. I, 373 (1862) ..

A. stereophylla, Meissner in Lehm, pl. Preiss. II, 203 (1847) ...

A. signata, F. v. M., fragm. IV, 7 (1864) ‥

A. delibrata, Cunningham in Hook. Lond. Journ, $\dddot{1}, 374$ (1842)

A. oligoneura, F. v. M. in Journ. Linn. Soc. III, 139 (1858) ...

A. torulosa, Bentham in Journ. Linn. Soc. III, 139 (1858)

A. julifera, Bentham in Hook. Lond. Journ. I, 374 (1842)

A. Solandri, Bentham, Fl. Austr. II, 406 (1864) ...

A. leptostachya, Bentham, Fl. Austr. II, 406 (1864)

A claucosens, Willdenow, spec. plant. IV 1059 (1805). '

A. Cunninghamii, Hooker, icon. plant. t. 165 (1837)

A. leptocarpa, Cunningham in Hook. Lond. Journ. I, 376 (1842)

A. polystachya, Cunningham in Hook. Lond. Journ. I, 376 (1842)

A. holcocarpa, Bentham, FI. Austr. II, 408 (1864)

A. plectocarpa, Cunningham in Hook. Lond. Journ. I, 375 (1842)

A. pachycarpa, F. v. M. in Journ. Limn. Soc. III, 139 (1858) ...

A. tumida, F. v. M. in Journ. Linn. Soc. III, 144 (1858)

A. loxocarpa, Bentham in Hook. Lond. Journ. I, 377 (1842) $\ldots$

A. oncinocarpa, Bentham in Hook. Lond. Journ. I, 378 (1842) ...

A. retinervis, Bentham in Hook. Lond. Jonrn. I, 379 (1 1842$)$

A. aulacocarpa, Cumningham in Hook. Lond. Journ. I, 378 (1842)

A. calyculata, Cunningham in Hook, Lond. Journ. I, 379 (1 84 ㄴ)

A. crassocarpa, Cunningham in Hook. Lond. Journ. I, 379 (1842)

A. auriculiformis, Cumningham in ITook. Loud. Journ. I, 377 (1842)

A. latifolia, Bentham in Hook. Lond. Journ. I, 382 (1842)

A. Mangium, TVilldenow, spec. plant. IV, 1053 (1805)

A. holosericea, Cuuningham in $G$. Don, gen. syst. II, 407 (1832)

A. cincinnata, F. v. M., fragm. XI, 35 (1878)

A. dimidiata, Bentham in Hook, Lond. Journ, I, 381 (1842) $\ldots$

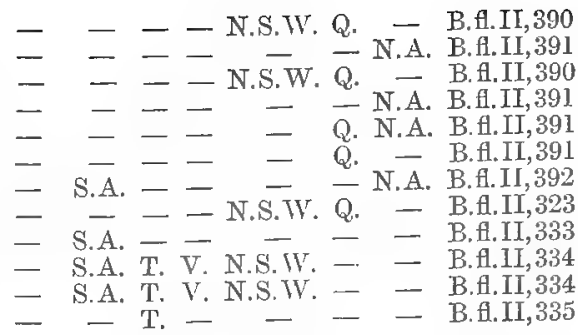

M.fr.XI, 34 .

M.fr.XI, 65 .

M.fr.XI, 66 .

M.fr.XI,67.

MI.fr.XI,68.

MI.fr.III, 128.

M.fr.IV, 8.

M.fr.I,7.

M.fr.III, 129

M.fr.XI,68.

M.fr.IV, 8 .

W.A. $\overline{\text { S.A }}-\overline{\mathrm{V}}$. N.S.W. $\overline{\mathrm{Q}}$ N.A. B.f.II, 402

IV.A. - - - N. S.IV. Q. - -

- - - - - N.A. B.f.JI, 403

- S.A. - V. N.S.W. Q. - - B.f.II,403
W.A. - - - B.fl.II,404

W.A. - - - - - - B.f.II, 404

W.A. - - - - - B.fl.II,404

- - - - - - N.A. B.fl.II, 404

- - - - - - N.A. B.fl.II, 405

- - - - - Q. N.A. B.f.II, 405

- - - - Q. - B.fl.II, 405

- - - - Q. - B.f.II,406

- - - - Q. N.A. B.f.II, 406

- - - - N.S.WV.Q. - B.fl.II,406

- - - - N.N.W.Q. - B.f.II, 407

- - - - N.W.W. Q. - B.f.I, 407

- - - - - Q. N.A. B.fl.II,407

- - - - Q. - B.f.II,408

M.fr.XI, 69.

M.fr. I $V, 7$.

M.fr.IV,7.

- N.A. B.fl.II, $40 \mathrm{~S}$

- N.A. B.fl.II, 408

- N.A. B.fl. II, 409

- N.A. B.fl.II, 409

- N.A. B.fl.II, 409

- N.A. B.fl.II, 410

Q. - B.f.II.410

i. - B.f.II,410

i.) N.A. B.H.1I, 410

(l. N.I. Ii.11.I1, 111

- N.A. B.fl.II,411

- @. N.A. B.A.II, 411

- $\quad$ N.A. B.fl.II, $4 \mathrm{l}_{2}$

M. fr. XI, 69.

M.fr. XI, 36.

M. fr. XI, 35. 
4. humifusa, A. Cumningham in Lond. Journ. I, 382 (1842)

‥ -- Q. N.A. B.fl.II,412

A. denticulosa, F. v. M., fragm. $\mathrm{X}, 32$ (1876)

V.A. - - -

$-$

$-$

M.fr.X, 3:

IX. BtPINNATAR.

A. Drummondii, Lindley, Bot. Regist. XXV, App. XV (1839).. W. A. - - - - - - B.fl.II.419

A. pulchella, R. Brown in Ait. hort. Kew. sec. ed, V, 464(1813) W.A. - - - - - - B.fl.II,416

A. Mitchelli, Bentham in Hook. Lond. Journ. I, 387 (1842) ‥ - S.A. - V. - - - B.t.II,417

A. pentadenia, Lindley, Bot. Regist. t. 1521 (1832)

A. Gilberti, Meissner in Lehm. pl. Preiss. II, 204 (1847)

A. nigricans, R. Brown in Ait. hort. Kew. sec. ed. $V, 465(1813)$

A. Tayloriana, F. v. M. in Wing's S. Sc. Record, July (1882) ...

A. strigosa, Link, enum. pl. hort. Berol. 444 (1822)

A. elata, Cunningham in Hook. Lond. Journ. I, 383 (1842) $\ldots$

A. pruinosa, Cunningham in Hook. Lond. Journ. I, 383 (1842)...

A. spectabilis, Cunningham in Hook. Lond. Journ. I, 383 (1842)

A. polybotrya, Bentham in Hook. Lond. Journ. I, 384 (1842) ...

A. discolor, Willdenow, spec. plant. IV, 1068 (1805) $\ldots$...

A. decurrens, Willdenow, spec. plant. IV, $1872(1805) \ldots . \quad \ldots$

A. cardiophylla, Cunningham in Hook. Lond. Journ. I, 385 (1842)

A. leptoclada, Cunningham in Hook. Lond. Journ. I, 385 (1842)

A. pubescens, R. Brown in Ait. hort. Kew. sec. ed. V, 464 (1813)

A. Farnesiana, Willdenow, spec, plant. IV, 1083 (1805)...

A. suberosa, Cunningham in Hook. Lond. Jourm. I, 499 (1842)...

A. Bidwilli, Bentham in Schlecht. Limnaea XXVI, 629 (1853) ...

W.A. - - - - - - B.fI.II,417

W.A. - - - - - B.AlII,417

W.A. - - - - - B.fl.II,418

W.A. - - - - - - B.f. $\overline{\text { II }}, 418$

- - - - N.S.W. - - B.f.II,413

- - - - N.S.W. - - B.fl.II,413

- $\quad-\quad-$ N.S.W. Q. - B.f.T, 413

- - - - N.S.W. Q. - B.fl.II,414

- - T. V. N.S.W. - - B.H.II,414

- S.A. T. V. N.S.W. Q. - B.f.II,414

- S.A. T. V. N.S.W. - - B.fl.II,415

- - - - N.S.WV. - - B.f.II,415

- - - - N.S.W. Q. - B.f.II,416

- - - - N.S.W. - - B.fl.II,416

- S.A. - - N.S.W. Q. N.A. B.fl.II,419

- - - - - N.A. B.fl.II, 420

- - - - - Q. N.A. B.fl.II,420

M fr. XI, 30 .

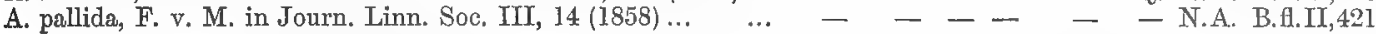

ALBIZZTA, Durazzini in Magazzino Toscano III, part IV, 13 (1772). (Zygia 1750, Pithecolobium, Cathormion, Calliandra Enterolobium, Serianthes.)

A. lophantha, Bentham in Hook. Lond. Journ. III, 86 (1844) ... W.A. - - - - - - B. I.II,421

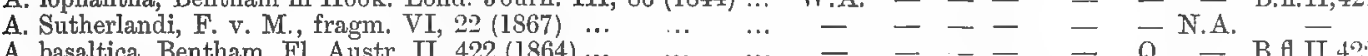

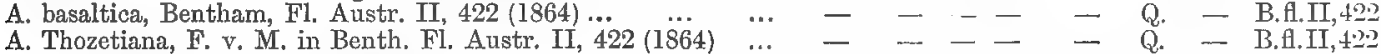

A. amoenissima, F. v. M., fragm. VIII, $165(1874) \quad \ldots \quad \ldots-\ldots-$ - - V. N.S.W. Q. - -

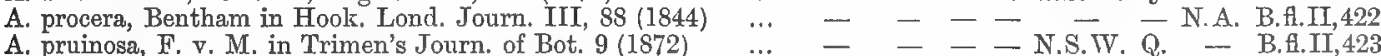

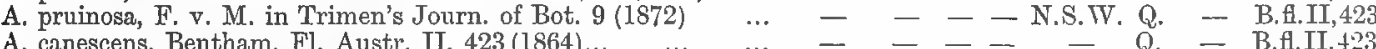

A. Tozeri, F, v. M. in Trimen's Journ, of Bot. $10(1872) \quad \cdots,-\ldots-$ - - N.S.W. Q. - B.,1.II, 424

A. Hendersoni, F. v. M. in Trimen's Joum. of Bot. 10 (1872) ... - - - - N.S.W. Q. - A.

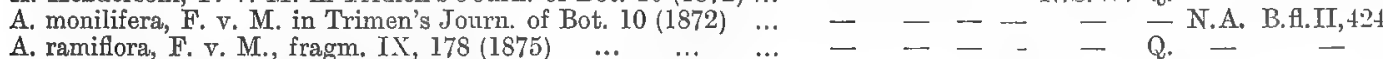

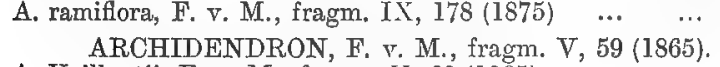

A. Vaillantii, F, v. M., fragm. V, 60 (1S65) … ...

A. Lucyi, F. v. M., fragm. VI, 201 (1868)...

$\ldots$

$\begin{array}{llllllll}\ldots & - & - & - & - & \mathrm{Q} . & - & - \\ \cdots & - & - & - & \mathrm{Q} . & - & -\end{array}$

M.fr.IX, 179 .

MI.fr.IV, $9 \& 176$

M.fr.IX, 179 .

M.fr.IX, 179 .

M.fr.IX, 179 .

M.fr.IX, 179 .

M.fr. X, 178 .

M.fr. V, 10;IX, 178 .

M.fr. $\mathrm{V}, 9 ; \mathrm{IX}, 178$.

M.fr.IX, 178 .

ROSACEAE.

A. L. de Jussieu, gen. pl. 334 (1789), from B. de Jussieu (1759).

PARINARIUM, A. L. de Jussieu, gen. pl. 342 (17S9).

P. Nonda, F. V. M. in Benth. Fl. Austr. II, 426 (1864) .. ... - - - - - Q. N.A. B.fl.II, 426

P. Griffthianum, Bentham in Hook. Niger-Flora 334 (1849) $\ldots-\ldots-\ldots$

STYLOBASIUM, Desfontaines in Mém. du Mus. V, 37, t. 2 (1819). (Macrostigma.)

S. spathulatum, Desfontaines in Mém. du Mus. V, 37, t. 2 (1819) W.A. S.A. - - - - N.A. B.fl.II,427

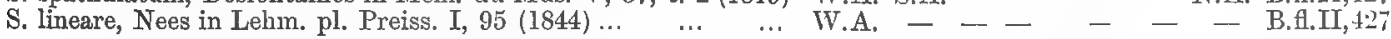

GEUM, Linné, gen. plant. 148 (1737) from Plinius, (Sieversia.)

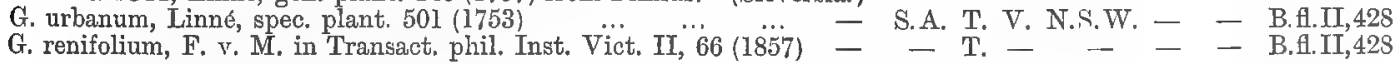

POTENTILLA, Linné, gen. plant. 147 (1737) from Camerarius (1586).

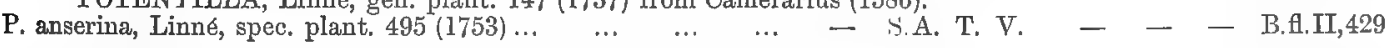
RUBUS, Tournefort, Inst. 614, t. 385 (1700) from Plinius.

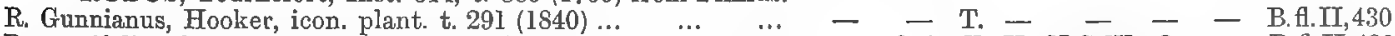

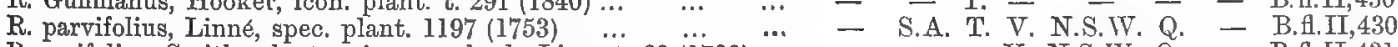

R. rosifolius, Smith, plantar. icon, ex herb. Linn.

R. Moluccanus, Linné, spec. plant. 1197 (1753) $\ldots \ldots \ldots \ldots$-...

R. Moorei, F. v. M. in Transact. phil. Inst. Vict. II, 67 (1857)... - - - - N.S.W. Q. - B.H.II,431 AGRIMONIA, Tournefort, inst. 301, t. 155 (1700) from C. Bauhin (1623).

A. Eupatoria, Linne, spec. plant. 448 (1753) ... ... ... _ _ _ ALCHEMILLA, Linné, gen. pl. 30 (1737) from C. Bauhin (1623). (Alchimilla.)

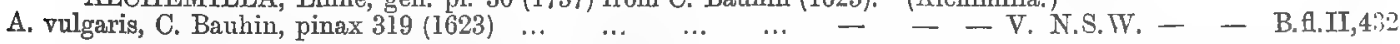
ACAENA, Mutis in Linné, mantiss. II, 200 (1771). (Ancistrum.)

A. ovina, Cunningham in Field's N.S. Wales, 358 (1825) $\quad$... W.A. S.A. T. V. N.S.W. Q. - B.H.II, 433

A. sanguisorbae, Vahl, enum. plant. I, $294(1804) \quad \ldots \quad \ldots . \quad-\quad$ S.A. T. V. N.S.W. Q. - B.fl.II,434

A. montana, J. Hooker in Hook. Lond. Journ. VI, 276 (1847) … - - T. - - - - B.H.II,434

\section{SAXIFRAGEAE.}

Ventenat, Tabl. III, 277 (1799).

ARGOPHYLLUM, R. et G. Forster, charact. gen, 29, t. 15 (1776).

A, Lejourdanii, F. V. M., fragm, IV, 33 (I864)

... 29, t. $15(1776)$.

- - N.S.W. Q. - B.H.II,437

M. fr, TI, 188.

MI.fr.IS, 30.

MI.fr.IV. $3 \pm ;$ TIII, 34.

M.fr.IT,31; YIIT, 34.

M.fr.IV,29\&176;VIII, 34.

MI. fr. TIII, 34 . 
ABROPHYLLUM, J. Hooker in Benth. fl. Austr. II, 437 (1864). (Brachynema.)

A. ornans, J. Hooker in Benth. pl. Austr. II, 437 (1864)

CUTTSIA, F. v. M., fragm. V, 47 (1865).

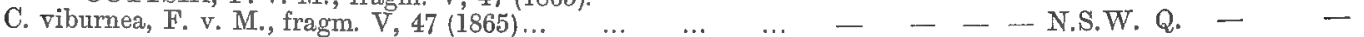
COLMEIROA, F. v. M., fragm. VIr, 149 (1871).

C. carpodetoides, F. V. M., fragm. VII, $149(1871)$ QUINTINIA, A. de Candolle, Mon. Campan. 92 (1830).

Q. Sieberi, A. De Candolle, Monogr. Camp. 92 (1530)

Q. Verdonii, F. v. M., fragm. II, 225 (1861)

Q. Fawkneri, F. v. M., fragm. VI, 92 (1867) POLYOSMA, Blume, Bijdr. 658 (1825).

P. Cunninghamii, J. J. Bemnett, pl. Jav. rar. $196(1840)$

P. alangiacea, F. v. M., fragm. VIII, 8 (1872) ANOPTERUS, Labillardière, Nov, Holl. pl. spec. I, 86, t. 112 (1804.)

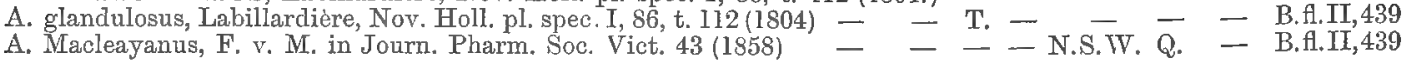
CALLICOMA, Andrews, Bot. Reposit. t. 566 (1809). (Calycomis partly.)

C. serratifolia, Andrews, Bot. Reposit. t. 566 (1809) $\ldots \quad \ldots \quad-\quad-\quad-\quad$ - N.S.W. Q. - B.f.II,440

C. Stutzeri, F. r. M., fragm. V, 34 (1865) ...

ANODOPETALUM, Cunningham in Endl. gen. 818 (1839).

A. biglandulostm, Cunningham in J. Hook. Fl. Tasm. I, $148(1860)$ APHANOPETALUM, Endlicher, nov. stirp. decad. I, 34 (1839). (Platyptelea.)

A, resinosum, Endlicher, nov, stirp. dec. I, 35 (1839) _.. ‥ - - - - V. N.S.W. Q. - B.f.II,44I

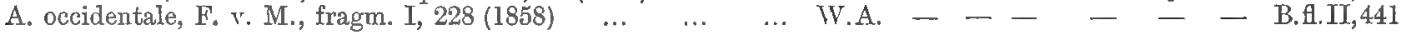
CERATOPETALUM, Smith, specim, of the Bot. of New Holland, 9 (1793).

C. gummiferum, Smith, specim, of the Bot. of New Holl. t. $3(1793)---1$ N.S.W. -- - B.fl.II,442

C. apetalum, D. Don in Edinb. new phil. journ. (1830) $\quad$. $-\quad-\quad-$ N.S. W. $-\quad-$ B.fl.II,442 SCHIZOMERIA, D. Don in Edinb. new phil. journ. IX, 94 (1830).

S. ovata, D. Don in Edinb. new phil. journ. IX, 94 (1830) ‥ - - - - N.S.W. Q. - B.fl.II,443 ACROPHYLLUM, Bentham in Maund \& Henslow, Botanist II, 95 (1840). (Calycomis partly.)

A. venosum, Bentham in Maund \& Henslow, Bot. II, 95 (1840) - - - N.S. W. - - B.fl.II,443 WEINMANNIA, Linné, syst. ed. X, 1005 (1759). (Ackama.)

W. pariculosa, F. v. M., fragm. II, $175(1861) \quad \ldots \quad \ldots \quad \ldots \quad-\quad-\quad-\quad-$ N.S.W. Q. $\quad-\quad$ B.fl.II,444

W. Biagiana, F. V. M., fragm. $T, 16(1865) \quad \ldots \quad \ldots \quad \ldots \quad \ldots \quad \ldots$

- - - - Q

V. lachnocarpa, F. v. M., fragm. VIII, 7 (1872).. $\quad \ldots \quad \ldots \quad-\quad-\quad-\quad-$ N.S. W. Q.

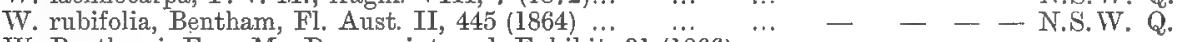
GILLBEEA, F. v. M., fragm. V, 17 (1865).

G. adenopetala, F. v. M., fragm. V, 17 (1865) …

DATIDSONIA, F. v. M., fragm. VI, 4 (1867).

D. pruriens, F. v. M., fragm. VI, 4 (1867).. TETRACARPAEA, J. Hooker, icon. plant, t. 264 (1840).

T. Tasmanica, J. Hooker, icon. plant. t. $264(1840) \quad \ldots \quad \ldots-$ - $\quad$ - T. EUCRYPHIA, Cavanilles, icon. IV, 48, t. 372 (1797). (Carpodontos.)

E. Billardieri, Spach, veg. phan. V, $344(1836) \quad \ldots \quad$.. $\quad \ldots \quad-\quad-\quad$ T. $-\quad-\quad-\quad-$ B.fl.II,446

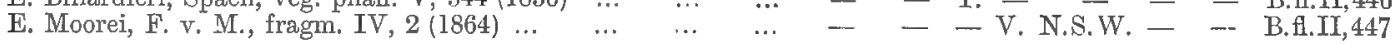
BAUERA, Banks \& Kennedy in Andrews, Bot. Rep. t. 198 (1793).

B. rubioides, Andrews, Bot. Reposit. t. 198 (1793) $\ldots . \ldots \ldots-\ldots$ S.A. T. V. N.S.W. - - B.fl.II,447

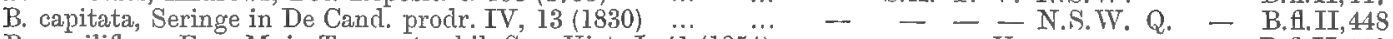

B. sessiliflora, F. . M. in Transact. phil. Soc. Vict. I, 41 (1854) EREMOSYNE, Endlicher in Hueg, enum. pl. Nov. Holl. austr. occ. 53 (1837).

E. pectinata, Endlicher in Hueg. enum. pl.53 (1837) ‥ … W.A. -
CEPHACOTUS, Labillardière, Nov. Holl. pl. spec. II, 7, t. I45 (1806).

C. follicularis, Labillardière, Nov. Holl. pl. spec. II, 7, t. 145 (1806) W.A. - - - — - - B.fl.II,449
M.fr.VI, 189;VII, 150

M.fr. V,47\&189;VI,189

M.fr.VII, 149.

M.fr.III, 126;VI, 189.

M.fr. VI, 189.

M.fr, VII, 150 .

M.fr. VI, 189.

M.fr.VIII, 8.

M.fr. VI, 188;VII, 150.

M.fr. V, 32.

M.fr.VI, 188\&252;VII, 150 .

M.fr.VI, 189.

M.fr. I, 228 .

M.fr.XI, 20.

M.fr.VI, 189; VII, 150.

M.fr. VI, 189.

M.fr. VI, 1\$9;VII, 150 .

M. fr. II, 83; VI, 188; VII, $\mathrm{M}$ fr. VII, 150. [150. M.fr. VIII, 7 .

M.fr.II, 82\&175;VI, 181.

M.fr.V,180;VI, 188.

M.fr. V, 17;VI,188.

M.fr. VI, 249.

M.fr.VI, 189;VII, 150. M.fr.IV,176; VI, 189;VII

M.fr.IV, 23. $[150$

M. fr.IV, 24.

M.fr.IV, 24; TII, 150

M.fr. VI, 189.

M.fr.VI, 189.

\section{CRASSULACEAE.}

De Candolle, Bull. de la soc. philom. n. 49 (1801),

TILLAEA, Micheli, nov. gen. 22, t. 20 (1729). (Bulliarda, Crassulae subgentis).

T. verticillaris, De Caniolle, prodr. III, 382 (1828) … W.A. S.A. T. V. N.S. II. Q.

T. purpurata, J. Hooker in Lond. Journ. of Bot. VI, $472(1847) \quad$ IV.A. S.A. T. V. N.S.W. -
T. macrantha, J. Hooker in Hook. icon. plant. t. $310(1841) \quad \ldots \quad-$ S.A. T. V. N.S. W. -

T. pedicellosa, F. v. M., fragm. XI, 118 (1881)

T. intricata, Nees in Lehm. pl. Preiss. I, 278 (1844)

T. recurva, J. Hooker, Fl. Tasm. I, I46 (1860)

... $\ldots$

$\ldots \quad \cdots \quad$ W.A. $\quad-\quad--\frac{}{-}-$

... W.A. S.A. T. T. N.S.W. Q.
- B. A.II, 451

- B.H.II,451

- B.fi.II, 452

- $=$

- B.f. $\overline{\mathrm{II}}_{, 452}$
M.fr.XI, 118 .

M.fr.XI, 118

M.fr.XI, 117 .

M.fr.XI,118.

M.fr. XI, 117 .

M.fr. XI, ] 18 .

HAMAMELIDEAE.

Genus and species undetermined

R. Brown in Abel, Narr. journ. Chin. 374 (1818). 
ONAGREAE.

Adanson, Familles des plantes II, 81 (1763), from B. de Jussieu (1759).

OENOTHERA, Linné, syst. nat. 8 (1735); Linné, gen. plant. 112 (1737).

0. Tasmanica, J. Hooker, Fl. Tasm. I, $119(1860)$ …

EPILOBIUM, Dillenius in Linné, syst. nat. 8 (1735), from Gesner (1jt2).

E. tetragonum, Linné, spec. plant. $348(1753) \quad \ldots \quad \ldots \ldots$... $\quad$ W.A. S.A. T. V. N.S. W. Q. - B.A.III,305 JUSSIEUA, Linné, gen. plant. 126 (1737). (Jussiaea.)

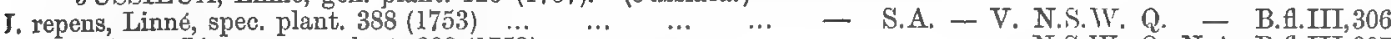

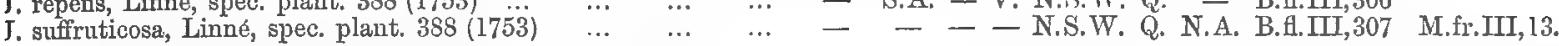

LUDWIGIA, Linné, coroll. $3(1737)$.

L. parviflora, Roxburgh, Fl, Ind. edit. Carey. I, 440 (1820)

SALICARIEAE.

Adanson, Familles des plantes II, 232 (1763), from B. de Jussien (1759).

ROTALA, Linné, mantissa altera 143 (1771).

R. verticillaris, Linné, mantissa altera $143(1771)$
R. Roxburghiana, Wight, Icon. t. $260(1840)$

Roy. Soc. of N.S.Wal 390$)=-$ Q. N.A. B.fl.III,296 M.fr.XI,118.

AMMANNIA, Houston in Linné, gen. plant. 337 (1737).

A. crinipes, F. v. M. in Transact. phil. Soc. Vict. III, 49 (1858)

A. triflora, R. Brown in Benth. Fl. Austr. III, 297 (1866) ‥ - - - - - - N.A. B.f.III,297

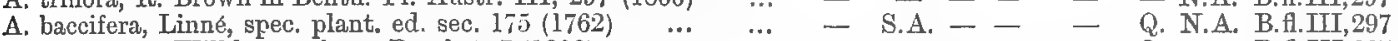

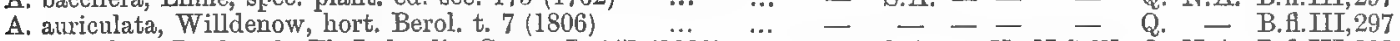

A. multillora, Roxburgh, Fl. Ind. edit. Carey. I, 447 (1820) $\ldots-$ - S.A. - V. N.S.W. Q. N.A. B.A.III,298 LAGERSTROEMIA, Linné, syst. ed. X, 1076 (1759).

I. Indica, Linné, spec. plant. 734 (1753)

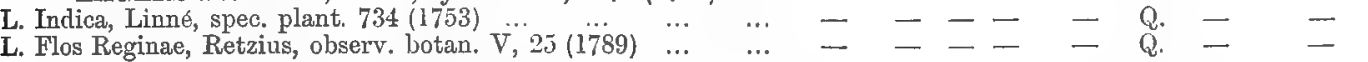
LYTHRUMI, Linné, syst. nat. 8 (1735); Linné, gen. plant, 136 (1737).

L. Salicaria, Linné, spec. plant. 446 (1753) $\quad \ldots \quad \ldots \quad \ldots-\quad-\quad$ S.A. T. V. N.S.W. Q. $\quad-$ B.H.III, 298

L. Hyssopifolia, Linné, spec. plant. 447 (1753) $\ldots . . \quad \ldots . \quad \ldots . \quad-$ S.A. T. V. N.S.W. Q. - B.H.II,299 NESAEA, Commerçon in A. L. de Jussieu, gen. plant. 332 (1799).

N. Robertsii, F. v. M., fragm. VIY, $145(1871)$
N. Arnhemica, F. v. M., fragm. VII, $146(1871) \ldots$
$\ldots$ PEMIPHIS, R. et G. Forster, charact. gen. 67, t. 34 (1776).

P. acidula, R. \& G. Forster, charact. gen. 67 , t. $34(1776)$.. $-\quad-\quad$ - — Q. N.A. B.H.III, 300 LAWSONIA, Linné, gen. plant. 111 (1737).

L. alba, Lamarck, Éncycl, méth. IL, 106 (1789) ...

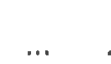

\section{HALORAGEAE.}

R. Brown in Flind. voy. II, 549 (1814).

LOUDONIA, Lindley, Bot. Regist. XXV, App. XLII (1839). (Glischrocaryon.)

L. anrea, Lindley, Bot. Regist. XXV, App. XLII(1839) ‥ W.A. S.A. - - - - - B.fl.II, 472

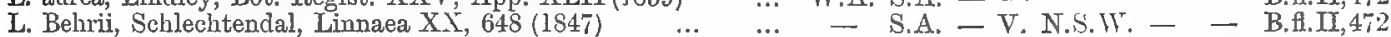

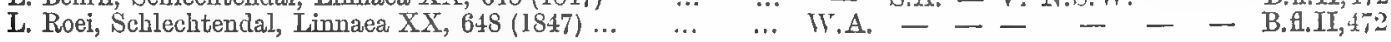
HALORAGIS, R. et G. Forster, charact. gen, 61, t. 31 (1776). (Cercodia, Goniocarpus.)

H. Gossei, F, v. M., fragm. VIII, 161 (1874) ... … ‥ - S.A. - - - -

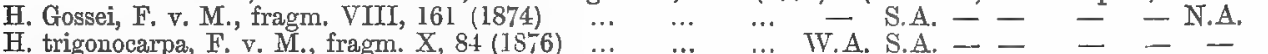

H. digyna, Labillardière, Nov. Holl. pI. spec. I, 10̈1, t. 129 (1904) W.A. - - - - - - B.fl. II, 475

H. mucronata, Bentham, Fl. Austr. II, 475 (1864) $\quad \ldots \quad \ldots$ W.A. S.A. - T. N.S.IV. - - B.f.II,475

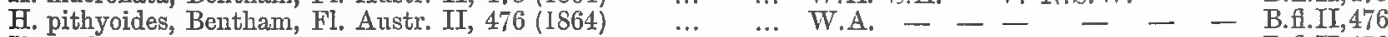

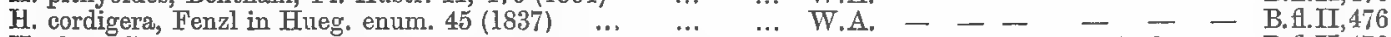

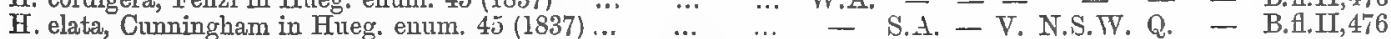

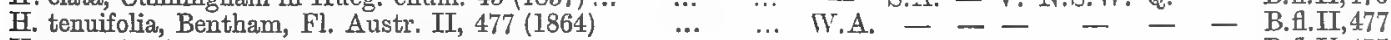

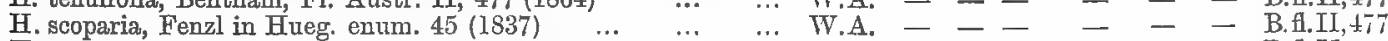

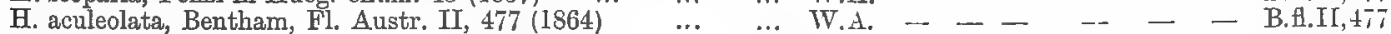

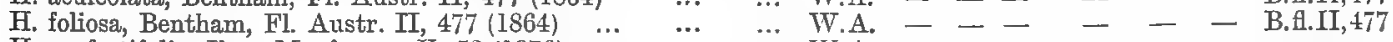

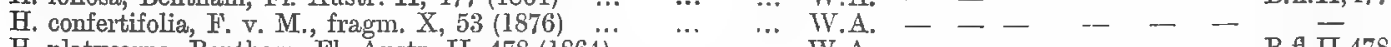

H. platycarpa, Bentham, FI. Austr. II, 478 (1S64)
H. ceratophylla, Zahlbruckner in Endl. Atakta, 16, t. 15 (1833)

H. ceratophylla, Zahlbruckner in Endl. Atakta, 16, t. 15 (1833) - S.A. T. V. N.S.W. Q. N.A. B.fl.II,478

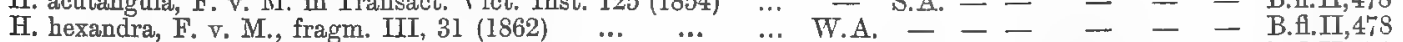

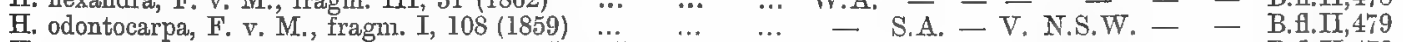

H. serra, Brongniart in Duperr. Toy. sur la Coquill. t. 69 (1829) - S.A. - - N.S.W. - - B.f.II, 479

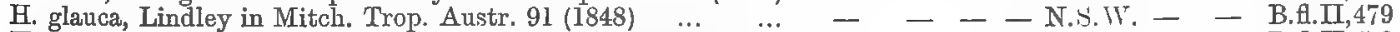

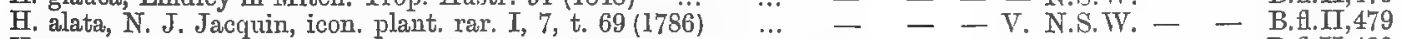

H. racemosa, Labillardière, Nov. Holl. pl. spec. I, 100, t. 128 (1804) W.A. - - - - - - B.H.II,480

H. rotumdifolia, Bentham, Fl. Austr. II, 480 (1864) $\quad$... $\quad$... W.A. $\quad$ W - -

H. rudis, Bentham, Fl, Anstr. II, 480 (1864)

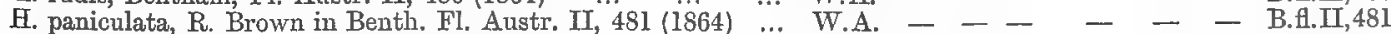

H. pusilla, R. Brown in Benth. Fl. Austr. II, 4Sl (1864) $\quad$.. W.A. - - - - - - - B.fl.II,4SI

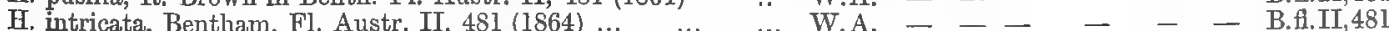

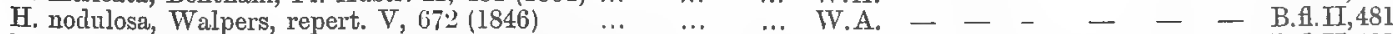

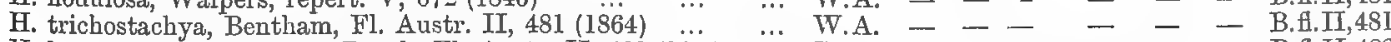

H. lanceolata, R. Brown in Benth. Fl. Austr. II, 481 (IS64) $\ldots$. W.A. $\quad-\quad-\quad-\quad-\quad-\quad-$ B.H.II,482

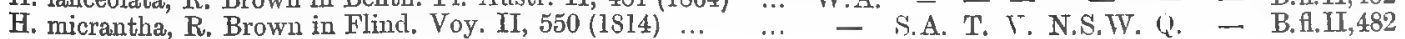

M.fr.VIII, 35.

MI.fr.XII.

M.fr.VII, 147 .

M.fr.VII, 147.

I.fr. VII, 145 .

II.fr.III, 109; VII, 146.

M.fr. VIr, 147.

M.fr. VIII, 162;XI, 20.

M.fr. VIII, 162 .

II. fr. VIII, 162 .

MI.fr.XI, 134.

M.fr.X,84.

MI.fr. VIII, 162.

M.fr. X, 54 .

M.fr.X, 54 .

M.fr. X, 54 .

XI.fr.III, 31.

M.fr.I, 108;X, J4.

MI.fr. VIII, 162.

MI.fr. VIII, 162.

M.fr. VIII, 162;X, 54 . 
H. stricta, R. Brown in Benth. Fl, Austr, II, 482 (1864)

H. heterophylla, Brongniart in Duperr. Voy. t. 68 (1829)

H. pinnatifida, A. Gray, Bot. Wilk. Expl. Exped. I, 627 (1854)

H. leptothoca, F. . . M. fragn. IIT $32(1862)$.

$\mathrm{H}$. tetragyna, $\mathrm{R}$. Brown iu Flind. Voy. II, 550 (18̈14) $\ldots .$.

H. teucrioides, A. Gray, Bot. Wilk. Fxpl. Exped. I, 625 (I854)

H. scordioides, Bentham, Fl. Austr. II, 485 (1864)

H. depressa, Walpers, repertor. II, 99 (1843)

H. salsoloides, Bentham, Fl. Austr. II, 485 (1864) $\cdots \quad \cdots$ $\cdots$

MIEIONECTES, R. Brown in Flind. Voy. II, 550 (1814).

M. Brownii., J. Hooker in Hook. icon. plant. t. 306 (1S41)

MYRTOPHYLLUM, l'Ecluse, rar. stirp, bist. II, 252 (1583) from Dioscorides. (Pelonastes).

M. variifolium, J. Hooker in Hook, icon, plant. t. 289 (1840) ... W. A. S.A. T. V. N.S.W. Q. - B.fl.II,487 M. elatinoides, Gaudichaud in Ann. des sc. nat. V, 105 (1825) ... - - S.A. T. V. N.S.W. - - B.fl.II,487 M. verrucosum, Lindley in Mitch. Trop. Austr. 384 (1848) … W.A. S.A. - V. N.S.W. Q. N.A. B.fl.II,488 M. latifolium, F. v. M., fragm. II, 87, (1860) $\quad \ldots \quad \ldots, \quad \ldots \quad-\quad-\quad-\quad-$ N.S.W. Q. $\quad-\quad$ B.tl.II,488 M. Muelleri, Sonder in Linnaea, XXVIII, 233 (1855) $\ldots . \quad \ldots$. W.A. S.A. - V. $\quad$ - $\quad$ - $\quad$ B.H.II, 488 MT amphibium, Labillardiere, Nov. Holl. pl. spec.II, 70, t.220(1806) - S.A. T. V. - - - B.fl.1I,489 M. pedunculatum, J. Hooker in Lond. Journ. VI, $474(1847)$... W.A. S.A. T. V. - - - B.t.II,489 M. dicoccum, F, v, M. in Transact. phil. Inst. Vict. III, 41 (1858) - - - - - - N.A. B.fl.II, 489 M. trachycarpum, F. v. M., fragm. II, $87(1860) \ldots \ldots$...

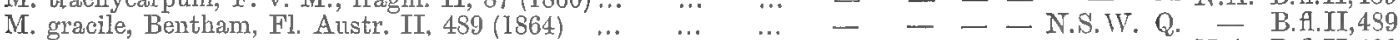

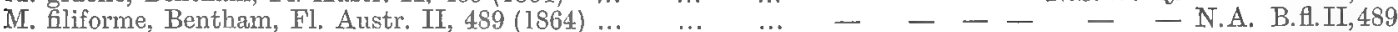
M. integrifolium, J. Hooker, Fl. Tasm. I. 123, t. 23 (1860) … W.A. S.A. T. V. N.S.W. - - B.f.II,490 M. Drummondii, J. Hooker, Fl. Tasm. I, 123, implied (1860) ... W.A. - - - - - - B.A.II,490 GUNNERA, Limne, mantiss. plant. 16 (1767). (Milligania.)

G. cordifolia, J. Hooker, Fl. Tasm. I, 125 (1860) ... $\ldots$... - $\quad$ - T. - - - - B.f.II,491 CERATOPHYLLUM, Linné, syst. nat. 9 (1735); Linné, gen. plant. 290 (1737).

C. demersum, Linné, spec, plant. $992(1753) \quad \ldots \quad \ldots \quad \ldots \quad-\quad$ S.A. - V. N.S. W. Q. $\quad$ - B.fl.II, 491 CALLITRICHE, Linné, syst, nat. ed. sext. 82 (1748).

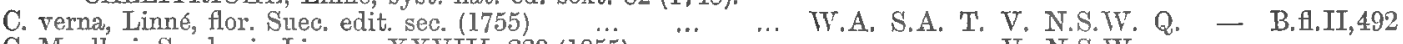

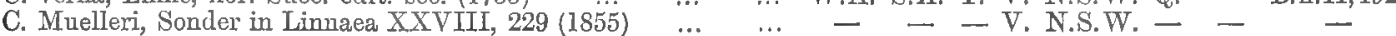

\section{RHIZOPHOREAE.}

R. Brown in Flind. Voy. II, 549 (1814).

RHIZOPHORA, Linne, gen. plant. 137 (1737).

R. mucronata, Lamarck, Encycl. méth. VI, 169 (1804)

CERIOPS, Arnott in Jardine, Ann. of nat. hist. I, 363 (1838).

C. Candolleana, Arnott in Amn. of nat. hist. I, 364 (]838) … BRUGUIERA, Lamarck, Encycl. méth. IV, 696, t. 397 (1796).

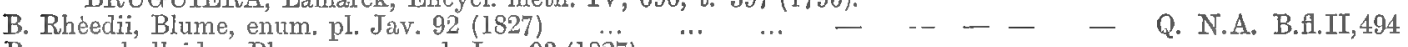

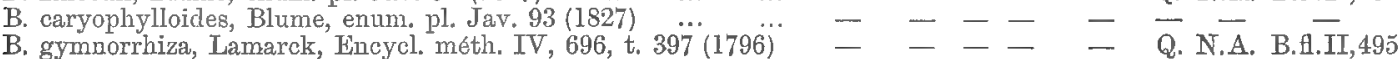
CARALLIA, Roxburgh, in Flind. Voy. II, 549 (1814).

C. integerrima, De Candolle, prodr. III, 33 (1828)

--- N.S.IW. Q. N.A. B.fl.II,493
$---\quad$ Q. N.A. B.H.II,494
$--\quad$ - - Q. N.A. B.H.II, 494
$-\overline{-}-\overline{\text { Q. N.A. B.H.II, } 495}$
$-\quad-\quad-$ Q. N.A. B.H.II, 495

M.fr, VIII, 162.

M. fr.IV, 26 .

M.fr.VIII, 163.

M.fr. VIII, 163.

M.fr.II,87;IV,172;X,54.

M.fr. VIII, 162 .

M.fr.VIII, 163 .

M.fr.VIII, 162 .

M.fi. TIII, 162 .

M.fr.II, $\$$.

M.fr, VIII, 162.

M.fr. VIII, 163.

M.fr. VIII, 163.

M.fr.IX, 159

M.fr.IX, 159.

M.fr.IX, 159

M.fr.IX, 159 .

M.fr.IX, 159.

\section{COMBRETACEAE.}

R. Brown, prodr. 351 (1810).

TERMINALIA, Linné, mantiss, plant. 21 (1767). (Chuncoa.)

T. platyptera, F. v. M., fragm. II, 15l (1861)

T. volneris, R. Brown in Benth. Fl. Austr. II, 498 (1864)

T. oblongata, F. v. M., fragm. II, I52 (186I)

T. bursarina, F, v. M., fragm. II, 149 (1861)

T. circumalata, F. v. M., fragm. III, 91 (1862) ..

T. pterocarya, F. v. M., fragm. II, 152 (1861) ...

T. Thozetii, Bentham, Fl. Austr. II, 500 (1864) ...

T. Catappa, Linné, mantiss. pl. 128 (1767)..

T. melanocarpa, F. v. M., fragm. III, 92 (1862) ...

T. Muelleri, Bentham, Fl, Austr. II, 500 (1864) ...

T. latipes, Bentham, Fl. Austr. II, 501 (1864) ... ...

T. edulis, F, v, M., fragm. II, 151 (1861)

T. discolor, F. v. M., fragm. III, 92 (1862)

T. porphyrocarpa, F, v. M. in Benth. Fl. Austr. II, 501 (1864)

T. platyphylla, F. v. M., fragm. II, 150 (1861)

T. sericocarpa, F. v. M., fragm. IX, 159 (1875) ..

T. microcarpa, Decaisne in Ann. du Mus. III (I834)

T. petiolaris, Cunningham in Benth. Tl. Aust. II, 502 ( 1364 ) ”.

T. erythrocarpa, F. v. . I., fragm. II, 150 (1861) ..

T. grandiflora, Bentham, Fl. Austr. II, 503 (1864)

LUMNITZERA, Willdenow, in den Verh. der Ges. Nat. Freunde zu Burl. IT. 156 (1S03).

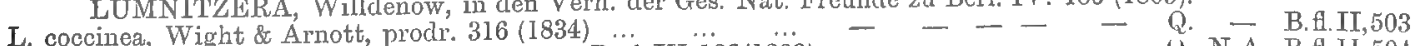

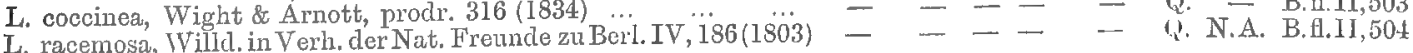

M.fl:II, 151;IX,160.

M. fr. III, $167 ;$ IX, 160

M.fr.II, 149 .

MI.fr.III, 91.

MI.fr.II, 152.

M.fr.IX, 160 .

M.fr.IS, 160

M.fr.III, 92:LX, 160.

M.fr,IX, 160 .

M. fr.II, 151;IX, 160. M.fi.III, 92.

NI.fr.II, 150.

M.fr.IX, 159 .

MI.fr. II, 150 .

M.fr.IX, 160 .

M.fr,IX, 160 .

M.fr.IX, 160. 
MACROPTERANTHES, F. v. M., fragm. III, 91 (1862).

M. montana, F. v. M., fragm. III, $91(1862)$
M. Kekwickii, F. v. M., fragm. III, $151(1863)$

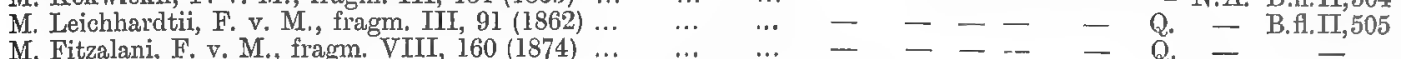

M. Fitzalani, F. v. M., fragm. VIII, $160(1874) \ldots \ldots \quad \ldots \quad \ldots \quad-$
GYROCARPUS, N. Jacquin, select. stirp. Amer, hist. 282 (1763).

G. Americanus, N. Jacquin, select. stirp.Amer, hist. 282, t.178(1763) - - - - — Q. N.A. B.Al.II,50.J

M.fr.II, 149;II, $91 \& 166$.

M.fr.III, 151 .

M.fr.III,9l.

M.fr. VIII, 160 .

\section{MYRTACEAE}

Adanson, Familles des plantes II, 86 (1763), from B. cle Jussieu (1759).

ACTINODIUM, Schauer in Schlecht. Linnaea X, 311 (1835). (Triphelia.)

A. Cunninghamii, Schauer in Lindl. introd. to the nat. syst., sec, ed. 440 (1835)

W.A. - - - - - - B.fl.III,5

DARWINTA, Rudge in Transact. Linn. Soc. XI, 299 (1813). (Genetyllis, Chamaelaucium, Homoranthus, Hedaroma, Polyzone, Francisia, Decalophium, Schuermannia, Cryptostemon.)

D. macrostegia, Bentham in Journ. Linn. Soc. IX, $179(1865)$..

D. Hookeriana, Bentham in Journ. Linn. Soc. IX, 179 (1865) ...

D. fimbriata, Bentham in Joury. Linn. Soc. IX, 179 (1865) $\quad \ldots$

W.A. $\rightarrow---\rightarrow-$ B.fl.III, 8

W.A. - - - - - B.fl.III,9

D. Meissneri, Bentham in Journ. Lim. Soc. IX, 179 (1865) $\quad \ldots$ W.A. - - - - - - B.fl.III,9

D. helichrysoides, Bentham in Journ. Linn. Soc. IX, 179 (1865)

D. Neildiana, F. v. M., fragm. IX, 177 (1875)

D. oederoides, Bentham in Journ. Linn. Soc. IX, 179 (1865) $\cdots$

D. virescens, Bentham in Journ. Linn. Soc. IX, 179 (1865)

D. Oldfieldii, Bentham in Journ. Linn. Soc. IX, 180 (1865)

D. purpurea, Bentham in Journ. Linn. Soc. IX, 180 (1865)

D. citriodora, Bentham in Journ. Linn. Soc. IX, 180 (1865)

D. thymoides, Bentham in Journ. Linn. Soc. IX, 180 (1865)

D. taxifolia, Cumningham in Field's N.S. Wales 352 (1825)

D. vestita, Bentham in Journ. Linn. Soc. IX, 180 (1865)

D. pauciflora, Bentham in Journ. Linm. Soc. IX, 180 (1865)

D. diosmoides, Bentham in Journ. Linn. Soc. IX, 180 (1865)

D. fascicularis, Rudge in Transact. Linn. Soc. XI, 299, t. 22 (1813)

D. pinifolia, Bentham in Journ. Linn. Soc. IX, 181 (1865)

D. rhadinaphylla, F. v. M., fragm. IX, 175 (1875)

D. sanguinea, Bentham in Journ. Limn. Soc. IX, 181 (1865)

D. micropetala, Bentham in Journ. Linn. Soc. IX, 181 (1865) ..

D. Schuermanni, Bentham in Journ. Linn. Soc. IX, 181 (1865) ...

D. verticordina, Bentham in Journ. Linn. Soc. IX, 181 (1865) ...

D. Thomasii, Bentham in Journ. Linn. Soc. IX, 181 (1865)

D. virgata, F. v. M., fragm. IX, 176 (1875) Cha ... $\dddot{\text { Mém. }}$... Mus, Par. V, 40 , t. 3 (1821)

D. gracilis; Chamaelaucium, F. v. M., fragm. IV, 62 (1864) $\ldots$

D. Forrestii, F. v. M., fragm. XI, 9 (1878)... … … ...

D. heterandra, F. v. M.; Cham., Benth., Fl. Austr. III, 36 (1866)... Linn, Soc. I, 44 (1857)

D. Endlicheri, F. v. M.; Chamaelaucium virgatum, Endicher in Ann. Wien. Mus, II, $193(1838)$... $\quad \ldots \quad \ldots \quad$...

D. brevifolia, F.v. M. ; Cham, Benth., Fl. Äustr. III, 37 (1866)...

W.A. - - -

W.A. - - -

W.A. - - -

$-$

-

-

B. fi.III, 10

W.A.

W.A.

IV.A.

IV.A.

IV.A.

W.A.

W.A.

W.A.

W.A.

W.A

W.A.

- S.A.

S.A. - -

- S.A. -

W.A.

- -

二

W.A.

W.A. - -

IV.A. - -

W.A

W.A.

- - -

W.A

D. uncinata, F. v. M.; Cham., Schauer in Pl. Preiss. I, 97 (1844) W.A.

D. megalopetala; Cham., F. v. M. in Benth. Fl. Austr. III, 38 (1866) W. A.

D. Turczaninowii, F. v. M.; Chamaelaucium pauciflorum, Benth., Fl. Austr. III, 38 (1866)

W.A.

D. axillaris, Cham., F. v. M. in Benth. Fl. Austr. III, $\dddot{38}$ (1866)

A. - - -

VERTICORDIA, De Candolle in Dict. class. XI, 400 (1826). (Chrysorrhoea.)

V. Wilhelmii, F. v. M. in Transact. Vict. Inst. I22 (1535)

V. densiflora, Lindley in Bot. Regist. XXV, App. VI (1839) $\ldots$

V. stelluligera, Meissner in Journ. Linn. Soc. I, 38 (1857)

V. minutiflora, F. v. M., fragm. IV, 58 (1864)

TV.A.

S.A. - -

V. Fontainesii, De Candolle, prodr. III, 209 (1828)

V. helichrysantha, F. v. M. in Benth. Fl. Austr.

V. Brownii, De Candolle, prodr. III, 209 (1828) ... … …

V. Harveyi, Bentham, Fl. Austr. III, $22(1864) \ldots \ldots$... $2 . . . \quad$ W.A.

V. fimbrilepis, Turczaninow in Bull. Mosc. XX, 158 (1847) … W.A.

V. serrata, Schauer in nov, act. acad. Caes. XIX, suppl. II,70 (1841) W.A.

V. nitens, Schauer innov, act. acad. Caes. XIX, suppl.II, 71, t.4 (1841) W.A.

V. grandifora, Fndlicher in Ann. Wien. Mus. II, 195 (1838) ... W.A.

V. chrysantha, Endlicher in Ann. Wien. Mus. II, 195 (1838) .. W.A.

V. Preissii, Schauer in Lehm. pl. Preiss. I, 101 (1844) ... ... TV.A.

V. acerosa, Lindley in Bot. Regist. XXV, App. VI (1839) … W.A.

V. polytricha, Bentham, Fl. Austr. III, 25 (1866) $\quad . . \quad \ldots$ W.A.

V. oxylepis, Turcz. in Bullet. de l'Ac. de St. Petersb. 402 (1852)

V. humilis, Bentham Fl Austr. III, 26 (1866)

W.A.

V. penicillaris, F. v. M., fragm. I, 226 (1859) 7 .

V. Huegelii, Endlicher in Hueg. enum. 16 (1837)...

... W.A.

W.A -- -

$-1-$

B.H.III, 10

B. fi.III, 10

B. fl.III, 10

- B.fl.III, 11

B.fl.III, 11

B.fl.III, 11

- B.fl.III,12

B.fl.III, 12

B.fl.III, 12

- B.f.III, 13

- B.Hl.III, 13

B.A.III, 14

B.ㅂ. III, 14

- B.fl.III, 14

- B.fl.III, 14

- B.H.III, 15

- B.fl.III, 15

B. Al.III, 16

M.fr.IX, 160.

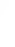


V. insignis, Endlicher in Hueg, enum. 47 (1S37) ... V. habrantha, Schauer in Lehm. pl. Preiss. I, $100(1844) \quad \ldots$. W.A. - - V. monadelpha, Turczaninow in Bull. Mosc. XX, 158 (1847) ... TV.A. - - V. Lehmanni, Schauer in Lehm. pl. Preiss. I, 99 (1844)... $55(1841)$

V. picta, Endlicher in Än. des Wien. Mus. II, 194 (183̈) $\quad \ldots$

V. pennigera, Endlicher in Hueg. enum. 46 (1S37) XIX, suppl. II, 56 (1841) ...

V. Hughani, F. v, M., fragm. XI, $10(1878) \quad \cdots \cdots \quad \cdots \quad \cdots$

V. pholidophylla, F. Y. M. fragm. I, 227 (1859) ...

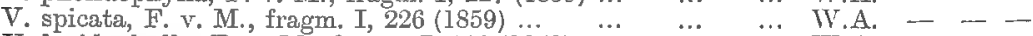

V. lepidophylla, F. V. M., fragm. I, 228 (1859) … W. A. - - -

V. ovalifolia, Meissner in. Journ. Linn. Soc. I, $40(1857)$... ... W.A. - - -

V. chrysostachya, Meissner in Journ. Limn, Soc. I, 41 (1857)
V. oculata, Meissner in Journ. Linn. Soc. I, 41 (1857) $\ldots$.
...

V. grandis, Drummond in Hook, Kew Mise. V, 119 (1853)

PILEANTHUS, Labillardière, Nov. Holl. pl. specim. II, 11 (1806).

P peduncularis Endl in Ann des Wien. Mus. II 196 (1838) W A

P. Límacis, Labillardière, Nov. Holl.pl. specim. II, 11, t.149(1806) W.A. - - - _ - - B.fl.III,34

P. filifolius, Meissmer in Journ. Linn. Soc. I, $45(1857) \ldots \ldots$... W.A. - - - - - - B.A.III,35

CAL ICOTHRIX, Labillardière, Nov. Holl, pl, specim. II, 8, t. I46 (1806). (Calytrix, Calythrix.)

C. aurea, Lindley, Bot. Regist. XXV, App. V, t. 3 (1839)

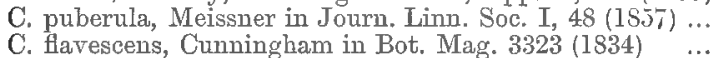

C. asperula, Schaner in Lehm. pl. Preiss. I, 106 (1844) ...

C. sapphirina, Lindley, Bot. Regist. XXV, App. V (1839)

C. breviseta, Lindley, Bot. Regist. XXV, App. V (1839)

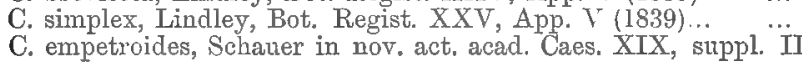
$102(1841)$

C. variabilis, Lindley, Bot. Regist. XXX, App. $\dddot{\mathrm{V}}(1839)$

C. muricata, F. V. M. , fragm. I, 224 (1859)

C. gracilis, Bentham, Fl. Austr. III 45 (1866)

C. brevifolia, Meissner in Journ. Linn. Soc. I, 46 (1857)

C. brachyphylla, Turezaninow in Bull. Mosc. XX, 161 (1847) ...

C. Leschenaultii, Schaner in Lehm. pl. Preiss. I, 104 (1844) $\ldots$

C. Oldfieldii, Bentham, Fl. Austr. III, $46(1866) \ldots \ldots$

C. glutinosa, Lindley, Bot. Regist. XXV, App. V (1839) $\quad \ldots$
C. angulata, Lindley, Bot. Regist. XXV, App. VI (1839) $\quad \ldots$

C. angulata, Lindley, Bot. Regist. XXV, App. VI (1839)
C. depressa, Turczaninow in Bull. Soc. Mosc. XX, 162 (1847) ...

C. tenuifolia, Meissner in Journ. Timn. Soc. I, 46 (1S57)...

C. strigosa, Cunningliam in Bot. Mag. 3323 (1834)

C. plumulosa, F. r. M. fragm. X, 27 (1876)

C. decandra, R. Brown in De Cand. prodr. III, 208 (1828)

C. Birdii, F. v. M., fragm. X, 26 (IS76)

C. tenuiramea, Turczaninow in Bull. Mosc. XXII, 20 (1849) ...

C. Fraseri, Cunningham in Bot. Mag. 3323 (1834)

C. granulosa, Bentham, Fl. Austr. III, 49 (1866)

C. Creswelli, F. r. MI., fragm. X, 27 (1S76)

C. microphylla, Cunningham in Bot. Mag. 3323 (1834) ...

C. longifiora, F. v. M., fragm. I, $12(1858)$

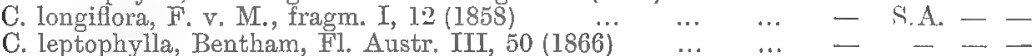

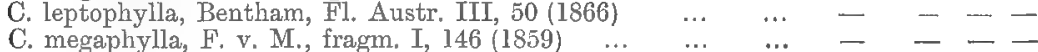

C. tetragona, Labillardière, Nov. Holl. pl. specim. II, 8, t. I46(1806) W.A. S.A. T. V.

C. Sullivani, F. v. M., fragm. IX, 1 (1875)...

C. conferta, Cunningbam in Bot. Mag. 3323 (1834)

C. arborescens, F. v. MI. in Transact. phil. Inst. Vict. III, 42 (1858)

C. brachychaeta, F. v. Ir. in Transact. phil. Inst. Vict. III, 43(1858) -

C. achaeta, F. v. M. in Transact. phil. Inst. Vict. III, 43 (1858)

C. laricina, R. Brown in Benth. Fl. Austr. III, 52 (1866)

LHOTZKYA, Schauer in Linnaea X, 309 (1835).

L. glaberrima, F. v. M., fragm. I, 13 (1858)

L. genetylloides, F. v. M. in Transact phil Soc. Vict. I $16(1854)-S \cdot A_{1}-$ -

L. violacea, Lindley, Bot. Regist. XXV, App. VII (1839)

L. ciliata, F. v. M. in Benth. Fl. Austr. III, 54 (1866) ...

L. brevifolia, Schauer in Lehm. pl. Preiss. I, 103 (1844)...

L. purpurea, F. v. M., fragm. I, 224 (1859)

L. ericoides, Schater in Lindl. Introd. sec. ed. 439 (IS35)

L. acutifolia, Lindley, Bot. Regist. XXV, App. VII (1839)

L. Harvestiana, F. V. M., fragm. XI, 8 (1878)

THRYPTOMENE, Endlicher in den Ann, des Wien. Mus. II, 192 (1838). Micromyrtus.)

T austrolis, Enllicher in

(1838) W.A.

T. tenella, Bextham, Fl. Austr. ITI, 59 (1866)

T. prolifera, Turezaninow in Bull, Mose. XXXV, $324(1862) \quad \ldots, \quad W . A$.

$--\mathrm{V}$.

- -

$--$

A. - -

... W.A. - -

W.A. - - -

W. W.A.

W.A.

W.A.

.. W.A.

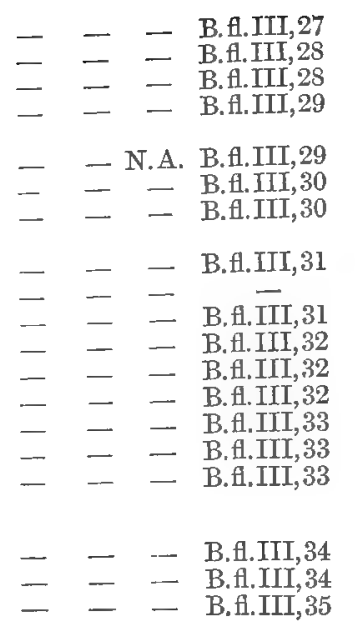

M.fr. X,28.

M.fr. I, 164 .

M.fr.X, 28.

M.fr. X, 28

M.fr. $X, 28$.

M.fr. X, 28 .

M.fr.XI, 10

M.fr.I,227.

M.fr.I,226.

M.fr. I, 228.

M.fr.X,28.

M.fr.X,28.

M.fr.X,28;XI, 10.

M.fr.I,225.

- - - B.f.III,4I

- - - B.f.III,42

- - - B.fl.III,42

- - - B.fl.III,42

- - - B.fl.III,43

- - B.AlIII,43

- - B f III 44

- - - - B.H.III,44

- - - B.fl.III,44

- _ - B.fl.III,45

- - - B.H.III,45

-_ - - B.H.III,45

- - - B.fl.TII,46

- - - B.t.III, 46

-. - B.fl.III,46

- - - B.fl.III,47

- - - B.fl.III,47

- - - B.fi.III,47

- - - B.fl.III,47
- - - B.f., III,48

- $-\cdots$

$-\quad-$

B.fl. III, 48

B. fl. III, 48

-. $\quad$ - B.fl.III, 49

- N.A. B.fl. $\overline{I I I}, 49$

Q. - B.fl.III,49

Q. - B.f.III, 50 - N.A. B.fl.III, 50 Q. - B.fl.III,50 - N.A. B.fi.III, 51

- - N.A. B.f.III,51

- - N.A. B.f.III,52

- - NA B.H J

- N.A. B.fl.III,52

$\begin{array}{llll}- & - & - & \text { B.fl.III,53 } \\ - & - & - & \text { B.fl.III,54 } \\ - & - & - & \text { B.fl.II,,54 } \\ - & - & - & \text { B.fl.III,54 } \\ -\quad- & - & \text { B.H.III,54 } \\ -\quad & - & \text { B.fl.III,55 } \\ -\quad & - & \text { B.Hl.III,55 } \\ -\quad-\quad- & \text { B.H.III,55 }\end{array}$

M.fr. I, 13 .

MI.fr.XI, S.

M.fr. T, 224

M.fr.XI,9.

M.fr.XI,

M.fr.XI, 8 .

M.fr. X, 27.

M.fr. I, 146.
M.fr.X, 26.

M.fr. X, 27.

M.fr.I, 12.

M.fr. I, 146 .

I.fr.IV, 36 ;VIII, 183

II.fr.IX, I.

(Homalocalyx, Paryphantha, Astraea, Eremopyxis,

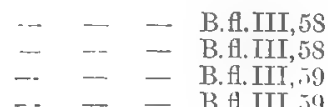

MI.fr, VIII, $182 ; \mathrm{X}, 24$ 
T. saxicola, Schauer in Lehm. pl. Preiss. I, 102 (1844) ..

T. Johnsonii, F. F. M., fragm. IV, 77 (1864)

T. racemulosa, Turczaninow in Bull. Mosc. XX, $\ddot{1} 6$ (1847)

T. denticulata, Bentham in Fl. Austr. III, 60 (1866)

T. baeckeacea, F. v. M., fragm. IV, 65 (1864)

T. strongylophylia, F. v. M. in Benth. Fl. Austr. III, 61 (1866)

T. hyporhytis, Turczaninow in Bull. Mosc. XXXV, 324 (1862)

T. Maisonneuvii, F. v. M., fragm. IV, $6 \pm$ (1864) ...

T. urceolaris, F. v. M., fragtm. X, 25 (1876)

T. Mitohelliana, F. F. M., fragm. I, 11 (1858)

T. micrantha, J. Hooker in Hook. Kew Misc. V, 299, t. 8 (185̈3)

T. stenocalyx, F. v. M., fragm. X, $23(1876)$

T. ericaea, F. v. M., fragm, I, 12 (1858)

T. oligandra, F. v. M., fragm. I, 11 (1858)...

T. elobata, F. v. M., fragm. IV, 63 (1864)..

T. racemosa, F. V. M. in Benth. Fl. Austr. III, 64 (1866)

T. trachycalyx, F. v. M., fragm. X, 25 (1876) ‥ … …

T. flaviflora, F, v. M., fragm. VIII, 13 (1873)

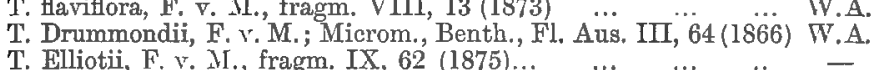

T. auriculata, F. v. N., fragm. X, 24 (1876)

T. hymenonema, F. v. II., fragm. X, 26 (1876)

T. ciliata, F, v. M. in Woolls, pl. of the neighb. of Sydney $23(1880)$

T. minutiflora, F.r.M. in Woolls, pl. of the neighb. of Sycn. 23 (1880)

T. leptocalyx, F. v. M.; Microm., Benth., Fl. Austr. III, 65 (1866)

T. homalocalyx, F. v. II., fragm. IV, 63 (1864) ..

T. polyandra, F. v. M., fragm. IV, 77 (1864)

WEHLIA, F. v. M., fragm. X, 22 (1876).

W. thryptomenoides, F. $\vee$. M., fragm. X, 22 (1876)

W. coarctata, F. v. M., fragm. X, 23 (1876)

BAECKEA, Linné, spec plant 358 (1753) (Jungia Tetrapora, Harmogia, Oxymyrrhine, Babingtol

B. platystemonea, Bentham, FI. Austr. III, 74 (1866)

B. Fumana, F. v. M., fragm. IV, 68 (1864)

B. dimorphandra, F, v. M. in Benth. Fl. Austr, III, 74 (1866) ...

B. schollerifolia, Lehmann in Lehm. pl. Preiss. II, 369 (1847) ...

B. oxycoccoides, Bentham, Fl. Austr. III 75 (1866)

B. Drummondii, Bentham, Fl. Austr. III, 75 (1866)

B. diffusa, Sieber in De Cand. prodr. III, 230 (1S28)

B. crassifolia, Lindley in Mitch. Three Exped. II, 115 (IS3S) ...

B. cryptandroides, F. v. M., fragm. X, 29 (1876)...

B. tetragona, F. v. IT. in Benth. Fl. Austr. III, 77 (1866)

B. ericaea, F. v. MI., fragm. I, 31 (1858)

B. polystemonea, F. r. M., fragta. II, 124 (1861)..

B. crenulata, R. Brown in Flind. voy. 548 (1814)...

B. brevifolia, De Candolle, prodr. III, 230 (1828)...

B. Gunniana, Schauer in Walp. Rep. II, 920 (1843)

B. diosmifolia, Rudgre in Transact Linn. Soc VITI, 298 $\ldots 13$ (1807)

B. leptocaulis, J. Hooker, icon. pl. t. $298(1840)$

B, arbuscula, R. Brown in Benth. Fl. Austr. III, 79 (1866)

B. astarteoides, Bentham, Fl. Austr. ITI, 80 (1866)

B. linifolia, Rudge in Transact. Linn. Soc. VII, 297, t. 12 (1807)

B. stenophylla, F. v. MI., fragm. I, 13 (1858)

B. camphorata, R. Brown in Bot. Mag. t. 2694 (1826)

B. virgata, Andrews, Bot. Reposit. t. 598 (1810) ...

B. crenatifolia, F. v. M., fragm. IV, 70 (1864)

B. Cunninghamii, Bentham, Fl. Austr. III, 82 (1866)

B. densifolia, Smith in Transact. Linn. Soc. III, 260 (1797)

B. Behrii, F. v. M., fragm. IV, 68 (1864) ...

B. uncinella, Bentham, Fl. Austr. III, 84 (1866) $\ldots$

B. polyandra, F. v. M., fragm. IV, 12 (1864)

B. corynophylla, F. v. M., fragm. IV, 72 (1864) ...

B. pachyphylla, Bentham, Fl. Austr. III, 85 (1866)

B. crispiflora, F. v. MI., fragm. IV, 72 (1864)

B. Blackettii, F. จ. M., fragm. VIII, 181 (1874) .

B, camphorosmae, Endlicher in Hueg. enum. 51 (1837) ...

B. pulchella, De Candolle, prodr. III, 230 (1828) ...

B. ochropetaIa, F. จ. M., fragm. X, 29 (1876)

B. pygmaea, R. Brown in Benth. Fl. Austr. III, $\ddot{86}$ (1866)

B. corymbulosa, Bentham, Fl. Austr. III, 87 (1866)

B. floribunda, Bentham, Fl. Austr. III, 87 (1866)

B. pentandra, F. v. M., fragm. II, 31; IV, 72 (1864)

B. pentagonantha, F. v. M., fragm. IV, 73 (1864)

B. robusta, F. v. M., fragm. IV, T2 (1864) ..

B. ovalifolia, F. v. M., fragm. IV, 72 (1864)

B. subcuneata, F. v. M., fragm. IV, 73 (1864)

B. grandiflora, Bentham, Fl. Austr. III, 89 (1866)

B. uberiflora; Scholtzia, F. v. II., fragm. IV, 74 (1864) ...
W.A. - - -

W.A. - - -
W.A. $-2-$

W.A. - - -

W.A. --

W.A. - - -

W.A. $\overline{\text { A }}$ - -

W.A. S.A. - -

S.A. - V

- - - B.fi.III, 61

- N.S.W. - - B.fl.II,62

- - T. - - - - B.fl.II, 62

D.A. S.A. - - - - - B.f. III,62

W.A. - - -

W.A. - - -

IV.A. - - -

W.A. - - -

W.A.A. S.A. - -

W.A. S.A. - -

W.A. S.A. -

$\bar{A}-\overline{\mathrm{V}} \mathrm{N} \overline{\mathrm{S}} \mathrm{V}$

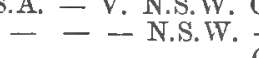

- - N.S.W. $\frac{\text { Q }}{2}$

- - - -

W.A.

W.A.

$-\ldots$

-

holtzia, Schidiomyrtus, Rinzia,
yrtus, Piptandra, Anticoryne.)

W.A. - - P - - - B.fl.III, ,4

W.A. - - - - - - B.H.III, 7

W.A. - - - - - - B.H.III, 74

W.A. - - - - - B.flIII,75

W.A. - - - - - B.fl.III,7.J

W.A. - $-\overline{\text { T. }}$ V. N.S.T. - - B.fl.III, 50

W.A. S.A. - V. N.S.W. - - B.A.II, 6

W.A. - - - - -

TV.A.

S.A. - V. N.S.W. -

B.f. IIT, 77

- S.A. - - $\overline{\mathrm{SW}}$

- - - N.S.W. Q.

- T. V. N.S.W. -

- - - N.S.W. -

W.A.

W.A.

- -

- - - V. N.S.W. -

- - - N.S.W. Q

B.t.IUI, 77

B.fl.III, 77

B.fl.III, 78

B.f.III, 78

- B.H.III, 78

B.A.III, 79

B.fl.III, 79

- B.fl.III,79

- B.fl.III,80

- - B.fl.III,80

- - - N.S.W. - - B.fl.III, 8I

- - V. N.S.W. Q. N.A. B.f.III,81

$-\quad \mathrm{V}$$$
\mathrm{N}
$$$$
\text { N.S.W. - }
$$

B.H III,82

- - - - N.S.W. - - - B.S.W.III,82

iv.A. S.A. - V. N.S.W. - - B.H.III,83

W.A. - - - - - - B.fl.III, 84

W.A. - - - - - B.fl.III,84

W.A. - - - - - - B.H.III,84

W.A. - - - - - B.fl.III,85

W.A. - - - - - - B.A.III,85

W.

W.A. - - -

W.A. - - -

W.A. - - -

W.A. - - -

W.A. - - -

W.A. - - -

W.A. - - -

W.A. - - -

W.A. - - -

W.A. - - -

W.A. - - -

W.A. - - -

W.A. - - -
- - -

- - - B.fl.III, 86

- - - B.fl.̄II, 86

- _ - B.fl.III,87

- - - B.f.III,87

- $\quad$ - B.fl.III, 87

- - - B.fl.III, S7

- _ - B.ff.III,8s

- - - B.fl.III,88

- - - B.fl.III,88
- - - - B.fi.III,89
M.fr.IT, 75.

M.fr.IV, 7 .

MI.fr.IV, 75.

M.fr.IV, 65 .

M.fr. $\Pi, 61$.

M.fr.X, 25 .

M. fr. I, 11 ;IX, 62 .

M.fr.I, 11 .

M.fr.X,23.

MI.fr.I, 12 .

M.fr.I,Il;IV, 169 .

M.fr.IV,63.

II.fr. X, 25.

M.fr. $X, 25$.

M. fr. VIII, 183 .

MI.fr.IX, 62

M.fr.X,24.

I.fr. $X, 26$.

M.fr.I,30\&242;IV, 63 .

M.fr.I, 30 .

M.fr.IV, 63 .

II.fr.IV, 7 .

M.fr.X,22.

II.fr. $X, 23$.

tus, Camphoromyrtus,

M.fr. TIII, 183.

M.fr. VIII, 183.

M.fr.VIII, 183

M.fr. VIII, 183.

MI.fr. $T \backslash, 67$.

M.fr.TV,66.

M.fr. X, 29.

M.fr. II, 124

M.fr.TV, 65 .

M.fr.IV, 66.

M.fr.I,29.

M.fr.IV,71.

M.fr.I, 13 .

M.fr.IV,70;IV, 177 .

M.fr.I, 69 .

M.fr.IV, 70 .

M.fr.IV, 71 .

M.fr.IV,68.

M.fr.II,30;IV,72.

M.fr.II,31;IV,72.

II.fr. VIII, 181.

M.fr.X,29.

M.fr.VIII, 183.

XI.fr. II, $31 ;$ IV , 72;V III,

M.fr.IV, 73.

M.fr.IV, 72 .

II fr II 32;IV 72 ;VIII

M.fr.IV, 73 .

M.fr.IV,74. 
B. involucrata, Endlicher in Hueg. enum. 51 (1837)

W.A.

B. spathulata, F. r. M.; Scholtzia, Benth., Fl. Austr. III, 68 (1866) IV.A.

B. ciliata; Scholtzia, F. v. M., fragm, IV, 76 (1864)

B. capitata; Scholtzia, F. v. M. in Benth. Fl. Austr. III, 69 (1866) W.A.

B. umbellifera; Scholtzia, F. v. M., fragm. IV, 75 (1864) ... W.A.

B. laxiflora, F. v. AI.; Scholtzia, Benth., Fl. Austr. III, 69 (IS66) W.A.

B. leptantha, F. v. M.; Scholtzia, Benth., FI. Austr. III, 69 (1866) W.A.

B. parviflora; Scholtzia, F. v. M., fragm. IV, 76 (I864) ... ... W.A.

B. oligandra; Scholtzia, F. v. M. in Benth. Fl. Austr. III, 70 (1866) W.A.

B. serpillifolia, F. v. M., fragm. X, 30 (1876) … … … A.

$--$

ASTARTEA, De Candolle in Dict. class. XI, 400 (IS26).

A. ambigua, F. v. M., fragm. II, 32 (1860)

A. fascicularis, De Candolle, prodr. III, $210(1828)$... $\quad \ldots .$. W.A.

A. intratropica, F. Y. M., fragm. I, 83 (1858)

HYPOCALYMMA, Endlicher in Hueg. enum. pl. Nov. Holl.

H. xanthopetalum, F. v. .I., fragm. II, 29 (1860)

H. robustum, Endlicher in Hueg. enum. 50 (1837)

H. longifolinm, F. จ. M., fragm. II, 28 (1860)

H. strictum, Schauer in Lehm, pl Preiss, I, 111 (1844) ...

H. tetrapterum, Turczaninow in Bull. Mosc. XXXV, $32 \ddot{5}$ (1862)

H. linifolium, Turczaninow in Bull. Mose. XXXV, 325 (1862) ...

H. angustifolium, Endlicher in Hueg. enum. 50 (1837) ..

H. ericifolium, Bentham, FI. Austr. III, 94 (1866)

H. cordifolium Lehman plant Preiss. I 112 (1844)

H. speciosum, Turcz. in Bull, de l'Acad. de St. Pet. 409 (1852)

H. Phillipsii, Harvey in nat, hist. Rev. V, 296, t. 22 (1865)

H. myrtifolium, Turcz, in Bullet. de St. Petersb. 410 (1852)

BALAUSTION, Hooker, icones plantarum 852 (1852).
B. pulcherrimum, Hooker, icon, plant. $852(1852)$

AGONIS, Lindley, Bot. Regist. XXV, p. X (1839).

A. spathulata, Schaner in Lehm. pl. Preiss. I, 117 (1844)

A. floribunda, Turczanizow in Bull, Mosc. II, 20 (1849)

A. marginata, Schaner in Lehm. pl. Preiss. I, 117 (I844)

A. linearifolia, Schaner in Lehm. pI. Preiss. I, 118 (1844)

A. juperina, Schauer in Lehm. pl. Preiss. I, 118 (1844) ...

A. parviceps, Schauer in Lehm. pl. Preiss. I, 119 (1844)

A. obtusissima, F. v. M., fragm. XI, 119 (1881)

A. flexuosa, Schauer in Lehm. pl. Preiss. I, 116 (1814) ...

A. undulata, Bentham, Fl. Austr. III, 100 (I866)

A. hypericifolia, Schauer in Lehm. pl. Preiss. I, 117 (1814)

A. Scortechiniana, F'. v. M., fragm. XI, 118 (1881)

A. grandiflora, Bentham, Fl. Austr. III, 100 (1866) IEPTOSPERMUM, R. et G. Forster, char.

L. Fabricia, Bentham, Fl. Austr. III, 102 (1866) ...
L. laevigatum, F. v. M., Annual Report 22 (1858)

L. frmum, Bentham, FI. Austr. III, 104 (1866) ...

L. flavescens, Smith in Transact. Linn. Soc. III, 262 (1797)

L. scoparium, R. \& G. Forster, char. gen. 48 (1776)

L. arachnoideum, Smith in Transact. Linn. Soc. III, 263 (1797)

L. spinescens, Endlicher in Hueg, enum. 51 (1837)

L. lanigerum, Smith in Transact. Linn. Soc. III, 263 (1797) ...

L. parvifolium, Smith in Transact. Linn. Soc. III, 263 (1797) ...

L. stellatum, Caranilles, icon. pl. IV, 16, t. 330

L. attenuatum, Smith in Transact. Linn. Soc. III, 262 (1797) ...

L. myrtifolium, Sieber in De Cand., prodr. III, 238 (182S)

L. rupestre, J. Hooker in Hook. icon. pl. t. 308 (1841)

L. myrsinoides, Schlechtendal in Linnaea XX, 653 (1847)

L. erubescens, Schauer in Lehm. pl. Preiss. I, 12 (1844)

L. abnorme, F. v. M. in Benth. F'. Anstr. III, 109 (I866)

L. Roei, Bentham, Fl. Austr. III, 110 (1866)

L. foridum, Bentham, Fl. Austr. III, 110 (1866)... $\quad \ldots$

L. ellipticum, Endlicher in Hueg. enum. 51 (I837) ...

L. crassipes, Lehmann, ind. sem. hort. Hamb. (1842) ... KUNZEA, Reichenbach, conspect. regn, veget. 175 (1828)

K. micrantha, Schauer in Lehm, pl. Preiss. 1, 125 (1844)

K. eriocalyx, F. v. M., fragm. II, 28 (1860)

K. Muelleri, Bentham, Fl, Austr. III, $113(1866)$ ” ...

K. ericifolia, Reichenbach, consp. 175 (1828)

K. Preissiana, Schauer in Lehm. pl. Preiss. I, $12 \breve{5}(1844)$

K. recurva, Schauter in Lehm. pl. Preiss. I, 12.5 (I844)

K. micromera, Schauer in Lehm. pl. Preiss. III, 223 (1847)

K. pauciflora, Schauer in Lehm. pl. Preiss. I, 124 (1844)

K. calida, F. v. M., fragm. VI, 23 (1867)

K. parvifolia, Schauer in Lehm. pl. Preiss. I, I 24 "(1844)"

K. peduncularis, F. v. M. in Transact. Vict. Inst. 144 (1855) ...

$\mathrm{K}$. corifolia, Reichenbach, consp. 175 (1828)

K. capitata, Reichenbach, consp. 175 (1828

K, pomifera, F, v. M, in Transact. Vict. Inst. 124 (185E)

YVA. - -
Holl. Austr. occ. 51 (1837)

TV.A. -

IV.A. - - - - - - B.fl.III,92

W.A. - - - - - B.f.III,92

W.A. - - - - - - B.f.III,93

W.A. - - - - - - B.f.III, 93

W.A. - - - - - - B.f.IIT,93

W.A. - - - - - - B fl.IJI,94

W.A. - - - - - B.fl.III94

W.A. - - - - - - B.fl.III,94

W.A. - - - - - - B.fl.III,95

IV.A. - - - - - - B.fl.III 95

W.A. - - - - - - B.fl.III,95

(Punicella, Cheynia.)

... W.A. - -

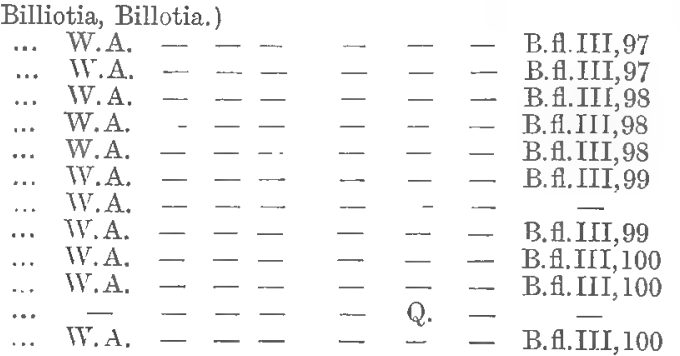

-

(1776). (Fabricia, Homalospermum, Pericalymma.)

- - $-\overline{-}$ Q. - B.f.III, 102

T. S.A. T. V. N.S.W. - - B.f.III, 103

- - - $\overline{\text { T. }}-$ - B.fl.III,104

- - T. V. N.S.W. Q. - B.fl.III,104

- S.A. T. V. N.S.W. Q. - B.f.III, 105

- - - - N.S.W. - - B.fl.III,105

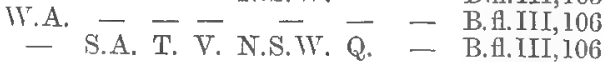

- - - - N.S.W. - - B.f.III,107

- - - - N.S.W. Q. - B.f.III, 107

- - - V. N.S.W. Q. - B.f.III,108

- - T. V. N.S.W. Q. - B.f.III,108

- - T. - - - - B.f.III, 108

- S.A. - V. N.S.W - - B. -

.. W.A. - - - - - - B.f.III, 109

- - - - N.S.W. Q. N.A. B.f.III, 109

W.A. - - - - - - B.f.III,110

W.A. - - - - - - B.fl.III,110

W.A. - - - - - - B.H.III,110

W.A. - - - - - - B.fi.III,110

(Salisia, Pentagonaster.)

... W.A. — - - -

W.A. - - V. N.S.TH -

V.A. - - V. N.S.W. -

W.A.

W.A. - -

$-\quad-$

- - - - $\mathrm{Q}$.

- $\quad-$ - T. N.S.W. -

- $\quad$ - T. V. N.S.W.

- - - - N.S.W. -

$\cdots$
B.fl.III, 112

- B.fl.III, 11:

B.f.III, 113

B. fl, III, 113

- B.A.III,113

- B. Al. [II, 114

- B.f.III,114

- B.fl.III, I14

- B.fl.III 115

B.A.III, 115

B. fl. III, 1 I

B.fl.III,116

- B.f.III,116
M.fr.IV, T4.

M.fr.IV, 76 .

M.fr.IV,75.

M.fr.IV,76.

M.fr.VIII, 183.

M.fr.II, 32.

M.fr. VIII, 183.

M.fr.I,83.

M.fr.II, 29.

M.fr.II,28.

M.fr.VIII, 183.

M.fr.VIII, 183 .

M.fr. VIII, 183.

M.fr.XI, 120.

MI.fr.XI, 120.

M.fr.VIII, 183;XI, 120.

II. fr.XI, 120 .

M.fr.XI, 120.

II.fr.XI,119.

H.fr.XI, 120.

I.fr.XI, 120.

M.fr. XI, 118.

I.fr.XI, $1 \geq 0$.

M.fr. IV, 60 .

M.fr.VIII, 183.

M.fr. II, 27.

II.fr. VIII, 183.

M.fr. II, 28 .

MI. fl. VIII, I83.

MI.fr. VIII, 183.

M.fr. YII, 23 .

II.fr. Y III, 13.

I.fr. $X, 55$, 
K. sericea, Turczaninow in Bull. Mosc. XX, $162(18+7)$...

K. Baxteri, Schauer in Lehm. pl. Preiss. I, 123 (1844) ...

K. opposita, F. v. M., fragm. VI, 24 (1867)

CALLISTEMON, R. Brown in Flind. voy. II, 547 (1814).

C. speciosus, De Candolle, prodr. III, 224 (1828).

C. phoenicus, Lindley, Bot. Regist. XXV, App. X (1839)

C. lanceolatus, De Candolle, prodr. III, 224 (I828)

C. coccineus, F. v. M., fragm. I, $13(1858)$...

C. salignus, De Candolle, prodr. III, $22 \%$ (1828) ...

C. paludosus, F. v. M., fragm. I, 14 (1858)

C. rigidus, R. Brown in Bot. Regist. t. 393 (1819)

C. linearis, De Candolle, prodr. III, 22, (1828)

C. pinifolius, De Candolle, prodr. III, 223 (1828)

C. teretifolius, F. r. M. in Schlecht. Linnaea XXV, 387 (1852)

C. brachyandrus, Lindley in Journ. Hort. Soc. IV, 112 (1849).

MELALEUCA, Linné, mantiss. plant. 14 (1767). (Gymnag

II. macronychia, Turcz. in Bullet. de St. Petersb. 420 (1852) ...

M. lateritia, Otto in allgem. Gart. Zeit. II, 257 (1834) ..

M. calothamnoides, F. V. M., fragm. III, 114 (1862)

I. blaeriaefolia, Turczaninow in Bull. Mosc. XX, 165 (1847) ...

M. diosmifolia, Andrews, Bot. Reposit. t. 476 (1808)

M. elliptica, Labillardière, Nor. Holl, pl. spec. II, 31, t. 1-3 (I806)

M. hypericifolia, Smith in Transact. Linn. Soc. III, 279 (1797)...

M. fulgens, R. Brown in Ait. hort. Kew. sec, ed. IV, 41J (1812)

M. acuminata, F. v. 2l., fragm. I, 15 (1858)

M. leptoclada, Bentham, Fl. Austr. III, $132(1866) \quad \ldots$.

M. basicephala, Bentham, Fl. Austr. III, 133 (1866)

M. gibbosa, Labillardiere, Nov. Holl. pl. spec. II, 30, t. 172 (I806)

M. decussata, R. Brown in Ait, hort. Kew. sec. ed. IV, 415 (1SI2)

M. Wilsonii, F, v, M., fragm. II, 124, t. 15 (1861)

M. thymifolia, Smith in Transact. Linn. Soc. III, 278 (1797) $\quad \ldots$
M. violacea, Lindley, Bot. Regist. XXV, App. VIII (1839) $\quad \ldots$

M. cardiophylla, F. V. M, fragm. I, 2.25 (1859)

M. undulata, Bentham, F1. Austr. III, I35 (1866) $\ldots$.

M. lateriflora, Bentham, Fl. Austr. III, 136 (1866)

M. exarata, F. ฬ. M., fragm. III, I14 (1862)
M. fasciculiflora, Bentham, Fl. Austr, III, 137 (1866)

M. teretifolia, Endlicher in Hueg, enum. 49 (1837)

M. alsophila, Cunningham in Benth. Fl. Austr. III, 137 (1866)...

M. acacioides, F. v. M., fragm. III, 116 (1862)

M. symphyocarpa, F. v. M. in Transact. phil. Inst. Vict. III, 44(18̈8)

M. angustifolia, Gaertner de fruct. I, 172, t. 35 (1788)

M. pauciflora, Turczaninow in Bull. Mosc. XX, 166 (1847)

i. squarrosa, Donn, Hort. Cantabr. ed. sec. 101 (1800)...

M. adnata, Turcz, in Bullet. de St. Petersb. 425 (1852) ..

M. linariifolia, Smith in Transact. Linn. Soc. III, 278 (1797)

M. trichostachya, Lindley in Mitch. Trop. Austr. 277 (1848) ...

M. radula, Lindley, Bot. Regist. XXV, App. VLII (1839)

M. pulchella, $R$. Brown in Ait. hort. Kew. sec. ed. IV, 414 ...

M. conferta, Bentham, FI. Austr. III, 142 (1866)...

M. Leucadendra, Linné, mantiss. $105(1767)$

... $\quad \cdots \quad$ VV.A

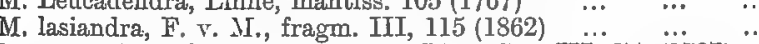

M. genistifolia, Smith in Transact. Linn. Soc. III, 277 (ï797) .”.

M. styphelioides, Smith in Transact. Linn. Soc. III, 275 (1797)

M. Huegelii, Endlicher in Hueg, enum, 4S (1837)

M. dissititlora, F. v. M., fragm. III, $153(1863) \quad \ldots \quad \ldots . \quad \ldots$

I. linophylla, F. v. M., fragm. III, 115 (1863)

M. parviflora, Lindley, Bot, Regist. XXV, p. VIII (1839)

M. laxiflora, Turczaninow in Bullet. de St. Petersb. 421 (1852)

M. armillaris, Smith in Transact. Linn. Soc. III, 277 (1797) ...

M. hamulosa, Turczaninow in Bull. Mosc. XX, 165 (1847)

M. subfalcata, 'Turczaninow in Bullet, de St. Petersb. 422 (1852)

M. glaberrima, F. v. M., fragm. III, 119 (1862)

M. rhaphiophylla, Schaner in Lehm. pl. Preiss. I, 143 (I 844$)$..

M. cymbifolia, Bentham, Fl. Austr. IIT, 148 (1866)

M. cuticularis, Labillard., Nov. Holl. pl. spec. II, 30, t. 17 i (1806)

M. sparsiflora, Turezaninow in Bull. Mosc. XX, 167 (1847)

M. sarsilora, Turczaninow in bul. Mosc. XX, IV 416 (18I2)

M. cordata, Turcz, in Bullet. de St. Petersb. 418 (1852)..

M. globifera, R. Brown in Ait. hort. Kew. sec, ed. IV, 411 (1812)

M. megacephala, F. v. M., fragm. III, 117 (186:)

M. nesophila, F. v. M., fragm. III, 113 (1862)

M. Oldfieldii, F. v, M., fragm. II, 118 (1862)

… W.A.

W.A. - - - - - - B.f.III,117
W.A. - - B.f.II, 117

W.A. - - - N.S.W. - - B.H.III,117

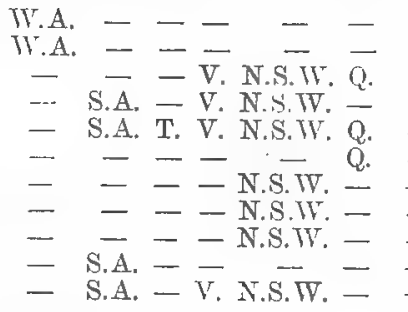

B.f.III, 119

B.fl.III, 119

- B.H.III, 120

- B.f.III,I20

B.fl.III, I20

- B.f.III, l2l

B.fi.III. 122

B.f.III, 122

- B.H.III, 122

gathis, Asteromyrtus.)

IV.A.

- - - - - B.fl.III,129

TV.A. - - - - B.H.III, 130

W.A. - - - - - B.fl.III, 130

A. - - - - - B.A.III, 130

IT.A. - - - - - B.H.III, 130

W.A. - - N.S.W. -

IV.A. $-\overline{\text { IV. }}-\overline{\text { V. N.S.T. }}=$

W.A. S.A. - V. N.S.W. -

B.fi.III 131

- B.fl.III, 131

- B.H.III,131

- B.fl.III, 132

B.f.III, 132

B.H.III, 132

- S.A. T. V. - - - B.fl.III, 133

- S.A. - V. - - - B.fl.III,133

- S.A. - T. - - - B.H.III, 134

- - - N.S.W. Q. - B.fl.III,134

W.A. - - - - - - B.fl.III,135

W.A. - - - - - - B.H.III,135

W.A. - - - - - - B.f.III,135

TI.A. - - - - - - B.fl.III, 136

W.A. - - - - - - B.fl.III,I36

W.A. - - - - - - B.f.III,136

TV.A.

W.A.

-

T.A.

-

-

W.A.

- - - - N.S.T. Q

- S.A. - - N.S.T. Q. N. B. B.III, 140

W.A. - - - - - B.f.III,141

V.A. - - - - - - B.H.III,142

W.A. - - - N.S.W. Q. N.A. B.H.III, 142

- - - - - - N.A. B.fl.III, 143

- - - - N.S.W. Q. N.A. B.fl.III,143

TT.A. - - N.S.W. Q. - B.f.III, 144

- - - - - - N.A. B.fl.III,144

$-\quad---$ - N.A. B.fl.III, 145

TV.A. E.A. - V. N.S.W. Q. - B.fl.II, 145

W.A. - - - - - - B.HIIII, 145

- - - V. N.S.W. - - B.fl.III, 146

W.A. - - - - - - B fl.III, I46

W.A. - - - - - B fi.III,146

W.A. - - - - - B.fl.III, 147

W.A. - - - - - - B.fl.III, 147

W.A. - - - - - - B.fl.III,148

W.A. - - - - - B.fl.III, 148

W.A. - - - - - - B.fl.III, 148

W.A. - - - - - B.fl.III, 14S

W.A. - - - - - - B.fl.III, 149

W.A. - - - - - B.fl.II, 149

W.A. - - - - - - B.f.III, 149

IV.A. - - - - - - - B.H.III,150
WV.A. - - B.III, 150

M. uncinata, R. Brown in Ait. hort. Kew. sec. ed. IV, 414 (18i2) W.A. S.A. - T. N.S.W. -- - B.fl.III, 150

M. concreta, F. v. M., fragm. III, 118 (1862)

M. filifolia, F. $\checkmark$. MI, fragm. III, 119 (1862)

M. hakeoides, F. v. MI. in Benth. Fl. Austr. III, I5l (1866) ,

M. glomerata, F. v. M., Rep. Babb. Exped. S (1858)

IV.A.

B.

B.fl.III, 151

W.A. S.A. - - N.S.W. - N.A. B.f.III, 151
B.HI,
MI.fr, III, 153 .

MI.fr. VI, 24.

M.fr.I, 14

M.fr.IV, 53.

M.fr.IV, 53.

M.fr.I,13.

M.fr.I ${ }^{\top}, 54$.

M.fr.I, It.

M.fr. TV, $5 ?$

M.fr.VIII, 183.

II. fr. III, 114 .

M.f. VIII, 183.

M.fr.I, IJ.

MI,fr.II, 1:4.

M.fr.X, 55 .

MI. fr. I, 225;III, 164.

M.fr.III, 120; VIII,183.

M.fr.III, 114; VII, 184.

M.fr. VIII, 184.

M.fr.III,117.

M.fr.III, 116.

M.fr.IIT, 115.

M.fr.I, $15 ; X, 55$.

M.fr.III, 153.

M. fr.III, 115 .

M.fr. VIII, 184;X,55.

M.fr.III, 119;VIII, 184.

II.fr.III, 119 .

M.fr. VIII, 184.

M.fr. VIII, 184.

M.fr.III, 117.

M.fr.III, 113.

M.fr.II, 118.

M.fr.HI, 119. 
M. pentagona, Labillardière, Nov. Holl. pl. spec. II, 27, t. 166 (1806)W. A M. ciliosa, Turczaninow in Bull. Soc. Mose. XXXV, 326 (1862)

M. polycephala, Bentham, Fl. Austr. III, 152 (1866)

M. spathulata, Schauer in Lehm. pl. Preiss. I, 134 (1844)

M. eriantha, Bentham, Fl. Austr. III, 153 (1866)

M. subtrigona, Schauer in Lehm. pl. Preiss. I, 139 (1S44)

M. seriata, Lindley, Bot. Regist. XXV, App. VIII (1S39)

M. scabra, R. Brown in Ait. hort. Kew, sec. ed. IV, 414 (1812)

M. urceolaris, F. Y. .I. in Benth. Fl. Austr. III, 154 (1866)

M. trichophylla, Lindley, Bot. Regist. XXT, App. VIII (1839)

11. holosericea, Schauer in Lehm. pl. Preiss. I, 139 (184t)

M. squamea, Lillardierre Nov. Hoil

M. densa, R. Brown in Ait. hort. Kew. sec, ed. IV, 416 (1812)... W.A.

II. thymoides, Labillardière, Nov. Holl. pl. spec. II, 27, t. 167 (1S06) W.A.

II. striata, Labillardiere, Nov. Holl. pl. spec. II, 26, t. 165 (1S06) W.A.

M. polygaloides, Schauer in Lehm. pl. Preiss. I, 142 (1844)

M. incana, R. Brown in Bot. Regist. t. 410 (1819)

M. nodosa, Smith in Transact. Linn. Soc. III, 276 (1797)

M. pungens, Schaner in Lehm pl. Preiss. I, 138 (1844)

M. ericifolia, Smith in Transact. Linn. Soc. III, 276 (1797)

M. pustulata, J. Hooker in Lond. Jonrn. VI, 476 (1844)

M. viminea, Lindley, Bot. Regist. XXV, App. VIII (1839)

M. microphylla, Smith in Rees, Cyclop. XXIII (1812)

M. tenella, Bentham, Fl. Austr. III, 160 (1866) ...

M. leiocarpa, F. v. M., fragm. X, 55 (1876)

M. leiopyxis, F. v. M. in Bentham Fl. Austr. III, 160 (1866)

iI, acerosa, Schauer in Lehm. pl. Preiss. I, 137 (1844) ..

M. pauperiflora, F. v. HI, fragm. III, 116 (1862)

M. aspalathoides, Schaver in Lehm. pl. Preiss, I, 140 ("̈844)

M. cucullata, Turczaninow in Bullet. de St. Petersb. 424 (1S.2)

M. minutifolia, F. v. M. in Transact. phil. Soc. Vict. III, 45 (1858)

M. foliolosa, Cunningham in Benth. Fl. Austr. III, 162 (1866)..

M. micromera, Schauer in Lehm. pl. Preiss. I, 146 (1844)

M. thyoides, Turczaninow in Bull. Soc. Mosc. XX, 167 (1847),...

CONOTHAMNUS, Lindley, Bot. Regist. XXV, App. IX (1839).

C. trinervis, Lindley, Bot, Regist. XXV, App. IX (1839) ‥ W. A.

C. divaricatus, Bentham, FI. Austr. III, $164(1566) \quad \ldots \quad \ldots$ IV.A.

BEAUFORTIA, R. Brown in Ait. hort. Kew. sec. ed. IV, 418 (1812). (Schizopleura.)

B. sparsa, R. Brown in Ait. hort. Kew. sec. ed. IV, 419 (1812) W.A.

B. decussata, $R$. Brown in Ait. hort. Kew. sec. ed. IV, 418 (1812) W.A.

B. squarrosa, Schauer in nov. act. acad. Caes. XXI, 11 (1844)... WV.A.

B. orbifolia, F. v. M., fragm. III, 110 (1863)

B. anisandra, Schauer in nov. act. acad. Caes. XXI, $13, \ldots$ t. I, (1844) W.A.

B. macrostemon, Lindley, Bot. Regist. XXV, App. X (1839) … W.A.

B. heterophylla, Turczaninow in Bullet. de St. Petersb. 427 (1852) W.A.

B. Schaueri, Preiss in nov. act. acad. Caes. XXI, $18(1844) \ldots$
B. purpurea, Lindley, Bot. Regist. XXV, App. X, t. 3 (1839) ... W.A.

B. Dampieri, Cunningham in Bot. Mag. t. 3272 (1833) ... ...

B. elegans, Schauer in nov. act, acad. Caes, XXI, $20(1844) \quad \ldots$
B. interstans, F. v. M., fragm. X, $30(1876) \quad \ldots$

W.A. - -

W.A. - - -

W.A. - - -

B. micrantha, Schaner in nov. act, acad. Caes. XXI, 22 (1814)... REGELIA, Schauer in Linnaea XVII, 243 (1843).

R. grandiflora, Bentham, Fl. Austr. III, $170(1866)$...

R. inops, Schaner in Lehm. pl. Preiss. II, $224(1847) \quad \ldots$

PHYMATOCARPUS, F, v, Mueller, fragm. III, 120 (1862)

P. porphyrocephalus, F, v. M, fragm. III, 121 (1862) ... ... W.A.

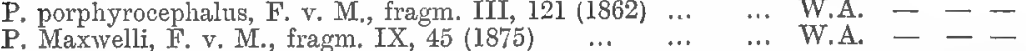
CALOTHAMNUS, Labillardière, Nov, Holl. pl. specim. II, 25 (1806). (Billotia.)

C. pachystachyus, Bentham, Fl. Austr. III. 173 (1866) ... … W.A. - - -

C. longissimus, F. v. M., fragm. III, $112(1862) \ldots \ldots$ … $\quad \cdots \quad$ W.A. - - -

C. blepharantherns, F. v. M., fragm. III, $112(1862)$... $\ldots$

C. sanguineus, Labillardière, Nov. Holl, pl. spec. II, 25, t. 164(1 (1806)
C. torulosus, Schaner in nov act. acad. Caes. XXI, 25 (1844) ...

C. torulosus, Schaner in nov. act. acad. Caes. XXI, $25(1844) \ldots$
C. gibbosus, Bentham, Fl. Austr. III, $175(1866) \ldots$

C. gracilis, R. Brown in Ait. hort. Kew. sec. ed. IV, 418 (1812)

C. blepharospermus, F. v. M., fragm. III, 111 (1862) ... ...

C. Gilesif, F. v, M., fragm. X, 31 (1876) ... ... ... ... IV.A.

C. chrysantherus, F. v. M., fragm. III, $112(1862) \quad \ldots \quad \ldots \quad$ W.A.

C. Oldfieldii, F. v. M., fragm. III, $113(1862)$ … $\dddot{7}$ (1842)

C. planifolius, Lehmann, delect. sem, hort. bot. Hamb.

C. affinis, Turczøninow in Bullet. de St. Petersb. 428 (1852) $\ldots$

C. Preissii, Schauer in nov, act. acad. Caes. XXI, 31 (1844) .

C. Schaueri, Lehmann, delect. sem. hort. bot. Hamb. 7 (1842)...

C. Lehmanni, Schauer in nov, act. acad. Caes. XXI, 31 (1844)...

C. villosus, R. Brown in Ait. hort. Kew, sec. ed. IV, 418 (1812)

C. pinifolius, F. v. M., fragm. III, 153 (1863)

$=$
$=$
-
-
-
-
-
-

$-$

-

-

-

-

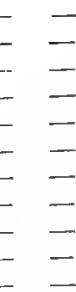

B. Al.III, 152

B.A.III, 152

B. A. III, 152

B.fi.III, 153

B. fl.III, 153

B. fl.III, 153

B.fl.III, 154

B.f.III, 154

B.H.III, 154

B.fl.III, 155

B.fl.III, 155

B.fl.1II, 155

B. Al.III, 156

B.A.III, 156

B.fl.III, 157

B. fl.III, 157

B.H.III, 157

B.f.III, 158

B. f.III, 158

B.H.IIT, 159

B.fl.III, 160

B.fl.III, 159

B.fl.III, 159

B.fl.III, 160

-

B.fl.III, 160

B.fl.III, 160

B. fl.III, 161

B. ff.III, 161

- B.fl.III, 161

Q. N.A. B.fl.III, 162

Q. - B.fl.III, 162

- Q. - B.f.III,162

B.fl.III, 162

B. II.III, 163

M.fr. III, 114.

B. Al.III, 163

B.fl.III, 164

M.fr. VIII, I84.

M.fr. $\mathrm{X}, 55$.

M.fr.III, 118 .

M.fr.III, 116.

M.fr. VIII, 184.

B. Al.III, 165

- - - B.H.III,166

- - - B.fl.III,166

- - - B.fl.III, 166

- - - B.A.III,167

- - - B.fl.III,167 
C. rupestris, Schauer in nov, act. acad. Caes. XXI, 26 (1844) ... W. A

C. quadrifidus, R. Brown in Ait. hort. Kew. sec. ed. IV, 418(1812) W.A.

C. asper, Turczaninow in Bull. Mosc. XXII, 25 (1849) ... ... W.A.

C. homalophyllus, F. v. M., fragm. III, 111 (1862) $\ldots . \quad \ldots \quad$ W.A.

LAMARCHEA, Gaudichaud in Freyc. Voy. Bot. 483, t. 110 (1826)

L. hakeaefolia, Gaudichaud in Freyc. Voy. 484, t. 110 (1826) ... W.A. EREMAEA, Lindley, Bot. Regist. XXV, App. XI (1839).

E. fimbriata, Lindley, Bot. Regist. XXV, App. XI (1839) ...

E. acutifolia, F. v. M., fragm. II, 30 (I860)

E. violacea, F. v. M., fragm. XI, 10 (1878)

E. pilosa, Lindley, Bot. Regist. XXV, App. XI (1839) ..'

E. ebracteata, F. v. M., fragm. II, 29 (1860)

E. beaufortioides, Bentham, Fl. Austr. III, I82 (1866) ... ANGOPHORA, Cavanilles, icon. IV, 21, t. 331 (1797).

A. cordifolia, Cavanilles, icon. IV, 2l, t. 338 (1797)

A. subvelutina, F. v. M., fragm. I, 31 (1858)

A. intermedia, De Candolle, prodr. III, 222 (1828)

A. lanceolata, Cavanilles, icon. IV, 22, t. 339 (1797) EUCALYPTUS, L'Heritier, sertum Anglicum 18 (1788). I. RENANTHERAE,

E. stellulata, Sieber in De Cand., prodr. III, 217 (1828)...

E. pauciflora, Sieber in Sprengel, cur. poster. 195 (1827)

E. regnans, F. v. M. in Report Acclim. Soc. Vict. 20 (1870)

E. amygdalina, Labill., Nov. Holl. pl. spec. II, 14, t. 154 (1806)

E. coccifera, J. Hooker in Lond. Journ. of Bot, VI, 477 (1847)...

E. obliqua, L'Heritier, sertum Anglicum ] 8, t. 20 (1788)

E. stricta, Sieber in De Candolle, prodr. III, 218 (1828)...

E. Planchoniana, F. v. M., fragm. XI, 43 (1878) ...

E. Baileyana, F. v. M., fragm. XI, 37 (1878)

E. sepulcralis, F. v. M., Eucalyptographia eight dec. (1 1882 ) ...

E. buprestium, F. v. M., fragm. III, 57 (1862)

E. marginata, Smith in Transact. Linn. Soc. VI, $302(1002)$

E. santalifolia, F. v. M. in Transact. Vict. Inst. I, 35 (1854)

E. macrorrhyncha, F. v. M., First general Report, I2 (1853)

E. capitellata, Smith in White, Journ. Voy. N.S.W. $216(1790)$

E. eugenioides, Sieber in Spreng. cur. poster. $195(1827)$
E. piperita, Smith in White, Journ. Voy. N.S. Wales $226(1790)$

E. pilularis, Smith in Transact. Linn. Soc. IIT, 284 (1797)

E. triantha, Link, enum. pl. hort. bot. Berol. II, 20 (1822)

E. haemastoma, Smith in Transact. Linn. Soc. III, 285 (1797) ...

E. Sieberiana, F. v. M., Eucalyptogr. sec. decade (I879)

E. microcorys, F. v. M., fragm. II, 50 (1860)

II. Porantherae.

E. paniculata, Smith in Transact. Linn. Soc. III, 287 (1797)

I. Leucoxylon, F. v. M. in Transact. Vict. Inst. I, 33 (1854) …

E. melliodora, Cumningham, in Walp. rep. bot. syst. II, 924 (184.3)

E. polyanthema, Schauer in Walpers, rep. bot. syst. II, 924 (1843)

E. populifolia, Hooker, icon. plant. 279 (1852)

E. ochrophloia, F. v. M., fragm. XI, 36 (1878)

E. gracilis, F, v. M. in Transact. Vict. Inst. I, 35 (1854)

E. uncinata, Turczaninow in Bull. de Mosc. XXII, 23 (1849)

E, odorata, Behr in Schlecht. Linnaea XX, 657 (1847)

E. largiflorens, F. v. M. in Transact. Vict. Inst. I, 34 (1854) ...

E. Behriana, F. v. M. in Transact. Vict. Inst. I, 34 (1854)

E. hemiphloia, F. v. M., fragm. II, $62(1860)$

E. pruinosa, Schauer in Walp. rep. bot. syst. II, 926 (1843)

E. melanophloia, F. v. M. in Journ. Linn. Soc. III, 93 (1858) ...

III. Paralletantherate.

E. Cloeziana, F. v. M., fragm. XI, 44 (1878)

E. Howittiana, F. v. M. in Wing's S. Sc. Record “̈ug. (1882) ..

E. drepanophylla, F. v. M. in Benth. Fl. Atustr. III, 221 (1866)

E. crebra, F, v. M. in Journ. Linn. Soc. III, 87 (1858)

E decipiens, Endlicher in

E. concolor, Schauer in Lehm. pl. Preiss. I, 129 (1844) ...

E. siderophloia, Bentham, Fl. Äustr. III, 220 (1866)

E. microtheca, F. v. M. in Journ. Linn. Soc. III, 87 (1858)

E. Raveretiana, F. v. M., fragm. X, 99 (1877)

E. brachyandra, F. v. M. in Journ. Linn. Soc. III, 97 (1858) '.'

W. pachyphylla, F. v. M. in Journ. Linn. Soc. III, 98 (1858) $\ldots$

E. pyriformis, Turczaninow in Bull. Mosc. XXII, 22 (1849)

E. macrocarpa, Hooker, icones plant. t. 405-407 (1842)

E. alpina, Lindley in Mitch. Three Exped. II, 175 (1838)

E. Globulus, Labillardière, Voy. I, 153, t. 13 (1799)

E. megacarpa, F. v. M., fragm, II, 70 (1860)

E. cosmophylla, F. v. M. in Transact. Vict. Inst. "32 (1854)

F. cordata, Labillardière, Nov. Holl. pl, spec. II, 13, t. 152 (1806)

E. urnigera, J. Hooker in Lond. Journ. of Bot. VI, 477 (1847) ...

W.A.

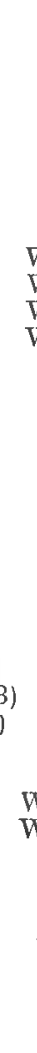

W.

W.A.

W.A.

$-\ldots$

W.A - - -

W.

- $\quad-\quad-$ N.S.W.

- $\quad-\quad-\quad$ - N. N.S.W. Q. Q.

N.S.W. Q.
N.S.W. Q.

(Eudesmia, Symphyomyrtus.)

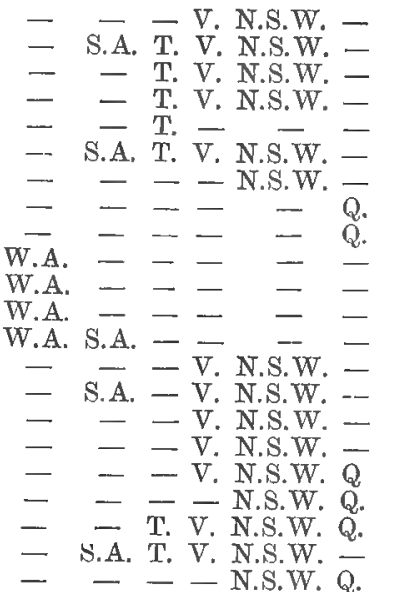

V. N.S.W. S.A. - V. N.S.W. Q. - - V. N.S.W. - - V. N.S.W. $\overline{\text { N.S.W. }}$

W.A. S.A. - V. N.S.W.W. Q. W.A. S.A. - V. N.S.W. -

- S.A. - - - -

- S.A. - V. N.S.W. Q.

- S.A. - V. - -

- S.A. - V. N.S.W. Q.

- $--\overline{\text { N.S.W. Q. }}$

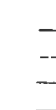

- B flIII 184

B.fl.III, 184

M.fr.I,31.

M.fr. XI, 11

M.fr.II, 30 .

II.fr.XI, 10 .

M.fr.XI,11.

M.fr.XI, 11 .

M.fr.XI, 11 .

(n)

M.fr. III, 52.

M.fr.XI, 38 .

M.fr.XI, 43 .

M.fr.XI,37.

M.fr.III, 57.

M.fr. XI, 45 .

M.fr.II, 50 .

M.fr.VII, 43 .

M.fr.VII, 44.

M.fr.II, 66.

M.fr. VII, 43.

M.fr.II,62.

M.fr.III, 132.

M.fr, XI, 44 .

- - - - - - Q. - $\quad$ Q. B.f.IIII,221
M.fr.X, 31.
M.fr.X, 31.

M.fr.X, 31 .

M.fr.II, 45;IX, I72.

M.fr. $\amalg, 53 ; \mathrm{VII}, 42 \& 44$.

M.fr.II,44\&171;XI,33. M.fr.II,40\&174;VII,43;

M.fr.II, $173 ; \mathrm{XI}, 38$.

M.fr.II, 173;VII,44;XI, 38

M.fr.II, $61 \& 172$; VII, 44 ;

[XI,38.

II, 43 ;

M.fr.XI,37. [XI,38.

M.fr.II, $174 ; \mathrm{VII}, 43$.

M.fr.II, $60 \& 175$.

M.fr.XI,36. [XI, 36.

M.fr. VII, 43; VIII, 184;

- - - - N.S.W. Q. N.A. B.fl.III,221

W.A. - - - - - - B.f.MI, 218

W.A. - - - - - - B.fl.III,247

- - - N.S.W.Q. - B.H.III,220

- S.A. - - N.S.W. Q. - B.f.III,223

- - - - Z Q. N.A. B.fl.III, 223

- S.A. - - - - N.A. B.f.III,237

W.A. S.A. - - - - - B.H.III,226

W.A. - - - - - B.f.III,224

W.A. - - - - - - B.fl.III,232

B.tl.III,225

B.fl.III, 225

B.f.III, 232

B.fl.III, 225

B. fl.TI, 224

B.fl.III, 227
M.fr.II, $61 ;$ VII, 44.

M.fr.X, 99 .

M.fr.II, $32 ; \mathrm{X}, 5 \& 84$.

M.fr.II, $41 ; X I, 38$.

M.fr.II, 38; VII, 42.

M.fr.II, $68 ; \mathrm{VII}, 42$.

M.fr.II, 68 .

M.fr. VII, 43.
M. fr. VII, 43 . 
E. longifolia, Link, enum, pl. hort. bot. Berol. II, 29 (1822)

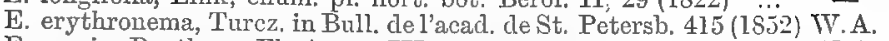

E. cæesia, Bentham, Fl. Austr. III, 227 (1866)

E. tetraptera, Turczaninow in Bull, cle Mosc. XXII, 22 (1849)... W. $\mathrm{W}^{\mathrm{A}}$.

E. miniata, A. Cunningham in Walp. rep. bot. syst. II, 925(1843)

E. phoenicea, F. v. M. in Journ. Linn. Soc. III, 91 (1858)

F. robusta, Smith, Specim. Bot. New Holl. 40, t. 13 (1793)

E. botryoides, Smith in Transact. Limn. Soc. III, 286 (1797)

E. goniocalyx, F. r. M., fragm. II, 48 (1860)

E. pallidifolia, F. v. M., fragm. III, 131 (1863)

E. incrassata, Labillard., Nov. Holl. pl. spec. II, 12 , t. $\dddot{150}$ (1so6)

E. oleosa, F. v. MI. in Nederl. Kruidk. Arch. IV, I27 (1859)

E. goniantha, Turczaninow in Bullet. de Mosc. XX, 163 (1847)

E. falcata, Turczaninow in Bullet. de Mosc. XX, 163 (1847)

E. salmonophloia, F. v. M., fragm. XI, 11 (1878)...

E. Ieptopoda, Bentham, Fl. Austr. III, 238 (1866)

E. angustissima, F. v. M., fragm. IV, 25 (1863) $\ldots$

E. Doratoxylon, F. Y. M., fragm. II, 55 (1860)

E. Cooperiana, F. v. M., fragm. XI, 83 (1880)

F. corynocalyx, F. v. M., fragm. II, 43 (1860)

E. gomphocephala, De Candolle, prodr. III, 220 (IS2S) $\ldots$

E. Oldfieldii, F. v. M., fragm. II, 37 (1860)

E. orbifolia, F. v. M., fragm. V, 50 (1865)...

E. diversicolor, F. v. M., fragm. III, 131 (1863) .

E. patellaris, F. v. M. in Journ. Linn. Soc. III, 84 (1858)

E. tessellaris, F. v. M. in Journ. Lim. Soc. III, 88 (1858)

E. clavigera, A. Cumningham in Walp. rep. bot. syst. II, 926 (1843)

E. ferruginea, Schauer in Walp. repert. II, 926 (IS43) „
E. grandifolia, R. Brown in Benth. Fl. Austr. IIT, 250 (1866) $\ldots$

E. aspera, F. v. M. in Journ. Linn. Soc. III, 95 (1858) ...

E. patens, Bentham, Fl. Austr. III, 247 (1S66)

E. Todtiana, F. v. M., in Wing's S. Sc. Record Aing. (1 1882 )

E. vernicosa, J. Hooker in Lond. Journ. of Bot. VI, $478(18+7) \ldots$

E. Gunnii, J. Hooker in Lond. Journ. Bot. III, 499 (1844)

E. resinifera, Smith in White's Journ. Voy. N.S. Wales 231 (1790)

E. saligna, Smith in Transact. Limn. Soc. III, 285 (1797)
F. punctata, De Candolle, prodr. III, 217 (1828) ...

E. oligantha, Schauer in Walp. rep. II, 926 (1843)

E. alba, Reinwardt in Blume, Bijdr. 1101 (1826) ...

E. pulverulenta, Sims, Bot. Mag. t. 2089 (1819) ...

E. Stuartiana, F. v. M. in Benth. Fl. Austr. III, 243 (1 1366$)$

E. viminalis, Labillardiere, Nov. Holl. pI. spec. II, I2, t. 151 (1S06)

E. rostrata, Schlechtendal, Liniaea XX, 655 (1847)

E. tereticornis, Smith, Spec. Bot. New Holl. 41 (1793) ...

E. rudis, Endlicher in Hueg. enum. 49 (1837)

E. foecuncla, Schauer in Lehm. pl. Preiss. I, 130 (1844) ...

E. redunca, Schaner in Lehm, pl. Preiss. I, 127 (1844)

E. obcordata, Turczaninow in Bull. Acad. Petersb. 416 (1852) ...

E. occidentalis, Endlicher in Hueg. enum. 49 (I837)

E. cornuta, Labillardière, Voy. I, 403, t. 20 (1799)

E. gamophylla, F. r. M. fragm. XI, 40 (1878)

E. perfoliata, R. Brown in Benth. El. Austr. III, 253 (1866)

E. setosa, Schauer in Walpers, rep. bot. syst. II, 926 (1843)

E. Torelliana, F. V. M., fragm. X, 106 (1877)

E. peltata, Bentham, Fl. Austr. III, 251 (1866)

E. latifolia, F. v. M. in Journ. Limm. Soc. III, 94 (1858)...

E. ptychocarpa, F. v. M. in Journ. Linn. Soc. III, 90 (1858)

E. Abergiana, F. v. M., fragm. XI, 41 (1878)

E. calophylla, R. Brown in Journ. Geogr. Soc, 20 (1831)

E. ficifolia, F. v. M., fragm. II, 85 (1860)

E. corymbosa, Smith. Specim. Bot. New Holl. 43 (1793)...

E. terminalis, F. v. M. in Journ. Linn. Soc. III, 89 (1858)

E. dichromophloia, F. v. M. in Journ. Linn. Soo. III, 89 (1S58)

E. trachyphloia, F. v. M. in Journ. Limn. Soc. III, 90 (1858)

E. eximia, Schauer in Walpers, rep. bot. syst. II, 925 (1843)

E. maculata, Hooker, icones plant. t. 619 (184t) ...

E. Watsoniana, F, r. M., fragm. X, 98 (1877)

E. odontocarpa, F. v. M. in Journ. Linn. Soc. III, 98 (1858)

E. tetrodonta, F. v. M. in Journ. Limn. Soc. III, 97 (1858)

E. erythrocorys, F. v, M., fragm. II, $33(\mathbf{1 8 6 0 )}$

E. tetragona, F. v. M., fragm, VI, 51 (1864)

E. eudesmioides, F. v. M., fragm. II, 35 (1860)
TRISTANIA, R. Brown in Ait. hort. Kew. sec. ed. IV, 417

T. nerififolia, R. Brown in Ait. hort. Kew. sec. el. IV, 417 (1812)

T. suaveolens, Smith in Rees, Cycl. XXXVI (1817)

T. conferta, R. Brown in Ait. hort. Kew. sec. cd. IV, 417 (1812)

T. lactiflora, F. v. M., fragm, I, s: (1858)...

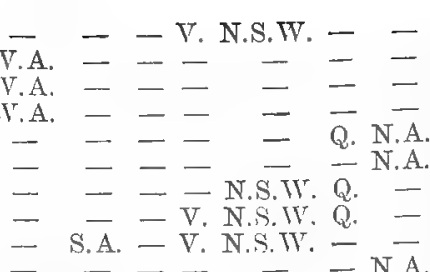

B. Al. III, 226 B.fl.III, 227

B. fl.III, 227

B.fl.III, 228

$-$

-

W.A. S.A. - V. N.S.IV.
W.A. S.A. - V. N.S.W.
W.A. - - -

W.A. - - - -
W.A. -

W.A. - - -
W.A. -

W.A. - - -
W.A. -

W.A. S.A. - -

W.A. - - -

W.A. - - -

WT.A. S.A. $=$ - -

W.A. - - -

W.A. - - -

W.A. - - -

-

$-$

-

W.A.

W.A.

-

-

-

- T. - - - - B.fl.III, 232

S.A. T. V. N.S.IT. - - B.fl.III, 246

- - - N.S.W. Q. - B.fI.III, 245

- - N.S.W. - - B.H.IH, 245

- - - N.S.W.

- - - - - N.A. B. H.III,213

- - - - Q. N.A. B.f.III, 213

- - V. N.S.W. - - B.fi.IT, 224

S.A. T. V. N.S.IV. - - B.fl.IJI, 243

S.A. T. V. N.S.W. - - B.fl.III, 239

W.A. S.A. - V. N.S.WV. Q. N.A. B.A.III, 240

- - - V. N.S.W. Q. - B.fl.III,24I

W.A. - - - - - - B.fl.III,244

WV.A. - - - - B.A.III, 25:

IV.A. - - - - - - B.f.III,253

W.A. -. - - - - B.fl.III,232

W.A. - - - - - - B.H.ITI,234

WV.A. - - - - - - B.H.III,235

IV.A. - - - - - - B.fl.III, 234

- S.A. - - - - N.A.

- N.A. B.A.III, 253

Q. N.A. B.fl.TI, $25 t$

Q. - B.fl. III, 254

- N.A. B.f.TII, 25. .

Q. N.A. B.H.II, 255

W.A. - - - - - - B.fl.III, 25.

IV.A. - - - N.S.W. Q. N.A. B.fl.III, 256

$\begin{array}{lll}\text { S.A. - - N.S.W. Q. N.A. B.H.MT,256 } & \text { Q. N.A. B.A.TI, } 257\end{array}$

$-\quad-A$
$-\quad-\quad-\quad-\quad-$
Q N.A. B.AlIII, 251

- - - - - N.S.WV. - N.S.W. 1 - B.H.III,2.58

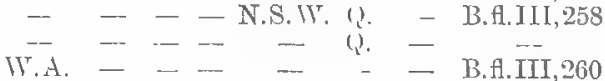

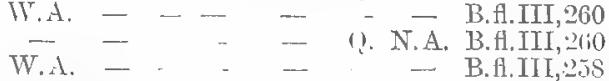

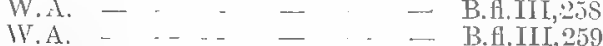

IV.A. - - - - - B.fl.III,260

is.)

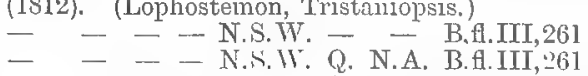

_ - N.S.TV. Q. N.A. B.H.III,263

- - - - - N.A. B.f.III, 263
M. fr.II, $50 ; \mathrm{VII}, 43$.

M.fr. VIII, 184 .

M.fr. XI, 40.
M.fr.II, 34;IV, 172; VIII,

M.fr.XI, $38 \& 42$.

M.fr. II, $43 \& 171 ;$ VII, 44 .

M.fr.II, 48\&175; VII, 43 .

M.fr.IV, 52 .

M.fr.III, 131 .

M. fr.II, $56 ; \mathrm{XI}, 14$.

M.fr.XI, 11 .

M.fr. XI, $11 \& 13$.

M.fr.XI,12.

M.fr.IV,25.

M.fr.II, 55.

M.fr.III, 130.

M.fr.XI, 83.

M.fr.VIL, 43 .

M.fr.II, 36.

M.fr.II, 37.

M.fr.V,50.

M.fr.III, 131.

M.fr.II, 62; Y II, $4: ; \times \mathrm{XI}, 8 \mathrm{~S}$

MI.fr.II, 1;2:VII, 43 .

II.fr. II, 173; VII, 41.

II. fr. TII, 4 .

M.fr.IT, 70 .

II. fr. II, 6?

M.fi.II, $64 ;$ VII 44

I.fr. VII, 44.

M. fl: XI, 14 .

MI.fr...II, 15 .

MI.fr. VII,41,

II.fr. TII, 44; YIII, 184.

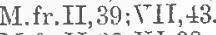

M.fr.II,39; II,38.

II.fr, XI, 40.

M. fr. III, $123 ; \mathrm{X}, 106$.

M.fr. X, 106.

MI.fr. YII, 41 .

M. fr. XI, 41.

M.fr.II,33171. [XI,15.

M.fr.II, S5\&176;VI, 25

II.fr, II, $46 \notin 174$.

M.fr.II, $4:$ VIII, 43 .

M.fr. X, 95 .

MI.fr.II, 33 .

II.fr. II, 37 .

M.fr.II, 35.

M.fr. IT, 56.

M.fr.I, 81 .

M.fr. T, s'.

M. fr, 1,82 . 
T. exiliflora, F. v. M., fragm. $T, 11$ (1S65)..

T. laurina, R. Brown in Ait. hort. Kew. sec. ed. IV $41 \ddot{7}$ (1812̈)

T. psidioides, Cunningham in Bot. Regist. XXII, n. 1839 (1837)

T. umbrosa, Cunningham in Bot. Regist. XXII, n. 1839 (1837)... METROSIDEROS, Banks in Gaertner, de fruct. I, 170, t. 34 (178S) Fremya, Cloezia, Spermalepis.

M. glomulifera, Smith in Transact. Linn. Soc. III, 269 (1797) ... M. leptopetala, F. r. MI., Docum. Intercol. Exhib. 30 (1867) …

M. ternifolia, F. r. M., Docum. Intercol. Exhib. 30 (1867)

MI. eucalyptoides, F. v. MI., fra!n. I, $2 \pm 3(1558)$..

M. polymorpha, Gaudichaud, Freye, Voy. Bot. 99 t. 108 (1826)

M. nervulosa, C. Moore \& F. Ү. גI., fragm. VIII, 15 (1873)

M. chrysantha, F. v. Al., frarm. IV, 159 (1864) ...

M. paradoxa, F. v. M., fragm. I, 243 (1859)

BACKHOUSIA, Hooker and Harvey in Bot. Mag. t. 4133 (1845).

B. myrtifolia, Hooker \& Harvey in Bot. Mag. t. 4133 (1845) ... -

B. angustifolia, F. v. M., fragm. I, 79 (1858)

B. sciadophora, F. T. II., fragm. II, 26 (1860)

B. citriodora, F. V. M., fragm. I, 78 (1858)

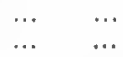
OSBORNIA, F. v. Mueller, fragm. III, 30 (1862). 0. octodonta, F. r. MI., fragm. III, 31 (1862) ... … ..
RHODOMYRTUS, De Candolle, prodr. III, 240 (182S).

R. psidioides, Bentham, Fl. Austr. III, 27? (1S66)

R. trineura, F, V. II il Benth. Fi. Austr. III, 272 (1866)

R. cymiflora, F. v. M. in Benth. Fl. Anstr. III, 273 (1866)

R. macrocarpa, Bentham, Fl. Austr. III, 273 (1866) MYRTUS, Tournefort, inst. 640, t. 409 (1700), from Plinius.

M. rbytisperma, F. v. M., fragm. I, 77 (1858)

M. tenuifolia, Smith in Transact. Linn. Soc. III, 280 (1797)

M. gonoclada, F. r. II. in Benth. Fl. Austr. III, 275 (1866)

M. lasioclada, F. v. M., fragm. IX, 148 (1875)

M. Hillii, Benthan, Fl, Austr. III, 275 (I866)

M. Becklerii, F. v. MI., fragm. II, 85 (1861)

M. Bidwillii, Bentham, Fl, Austr. III, $27 j$ (1866)

M. racemulosa, Bentham, Fl. Austr. III, $276(1866)$

M. acmenioides, F. v. M., fragm. I, 77 (1858)

M. fragrantissima, F. v. M. in Benth. Fl. Austr. III, 277 (1866)

II. Shepherdi, F. v. II, fragm. IX, 148 (1875) RHODAMNIA, Jack in Hook. Comp. to the Bot. Mag. I, 153 (1835)

R. sessiliflora, Bentham, El. Austr. III, 275 (1866)

R. trinervia, Blume, Mus. Bot. Lngd. I, 79 (1849)

$\mathrm{R}$. argentea, Bentham, Fl. Austr. III, 276 (1866)...

R. Blairiana, F. v. M., fragm. IX, I4l (1875) FENZLIA, Endlicher, Atacta 10, t. 17 \& 18 (1933).

F. olstusa, Endlicher, Atacta 19, t. 17 (1833)

F. retusa, Endlicher, Atacta 20, t. 18 (1833) DECASPERMUM, R. \& (4. Forster, char. gen. 73, t. 37 (17j6).

D. paniculatum, Baillon, hist. des plant. 350 (1871) EUGENIA, Micheli, nov. pl. gen. 226, t. 108 (1729).

E. rariflora, Bentham in Hook. Lond. Jonrn. II, 22l (1843)

E. Smithii, Poiret, Encycl. móthod. SuppI. III, I26 (1813)

E, hemilampra, F. v. M., fragm. IX, $145(1875) \ldots$

E. Ventenatii, Bentham, Fl. Austr. III, 283 (1866)

E. leptantha, Wight, Illustr. II, I5 (1850) ..

E. Moorei, F. v. II., fragm. T, 33 (1865)

E. corynantha, F. v. MI., fragm. IX, 144 (1875) ...

E. cormitlora, F. v. M., fragm. V, 32 (1865)

E. Hodgkinsoniae, F. r. M., fragm. IX, 145 (15,5)

E. Tierneyana, F. v. M., fragm. V, $1+(1865)$

E. grandis, Wight, Illistr. II, 17 (1850)

E. suborbicularis, Bentham, Fl. Austr. III, 285 (1866) ...

E. Wilsonii, F. v. MI., fragm. T, $12(1865) \ldots \ldots$

E. myrtifolia, Sims, Bot. Mag. t. 2230 (1821)

E. angophoroides, F. r. ir., fragm. T, $33(1865) \ldots$

E. Armstrongii Bentham FI. Anstr. III, 286 (1866)

- ...

E. oleosa, F. v. M., fragm. V, 15 (1865) ... ... ...

E. Dallachiana, F. r. M. in Benth. Fl. Austr. IIT, 287 (1866)

E. cryptophlebia, F. v. M., fragm. IX, 144 (1875)

ACICALyPTUS, A Gray, New Gen. Un St Trped 9(1853).

A. Fullageri, F, v. M fragn. VII

BARRINGTONIA, R. \& G. Forster, char. gen. 75, t. 39 (1776).

B. speciosa, R. \& G. Forster, char, gen. 76, t. 38 (1776)

B. racemosa, Gaudichand in Freyc. Voy. Bot. 483, t. 107 (1826)

B. acutangula, Gaertner, de fruct. II, $97, \mathrm{t}$; 101 (1791) ...

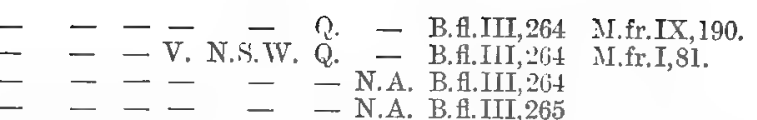

(Syncarpia, Kamptzia, Lysicarpus, Xanthostemon, Nania,

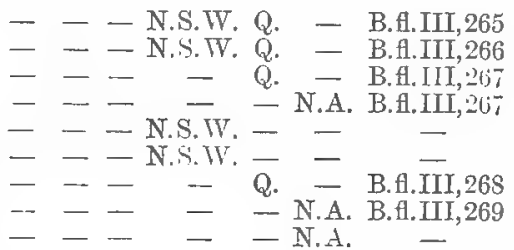

- $\quad--$ N.S.W. Q.

- - - N.S.W.

\section{Q.}

B.H.III,269

B.HI.III, 270

B.fl,III, 270

B. fl. III, 270

Q. N.A. B.fl.III,271

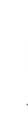$$
\begin{gathered}
-\quad-\text { N.S.W. } \\
-\quad-\quad- \\
-\quad-\quad-
\end{gathered}
$$$$
\begin{aligned}
& \text { Q. - } \\
& \text { Q. - }
\end{aligned}
$$

B. 1. III, $2 \div 2$

B. I. III, 2,2

- B.H.III, 273

Q. - B.fl.III, 273

$-\quad-$ N.S.W.

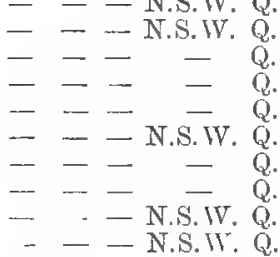

$$
\begin{aligned}
& - \\
& - \\
& - \\
& -
\end{aligned}
$$

B.fl.IIr, $2-4$

B. A.III, 274

B. A.III, 275

B.fl. III, $27 \overline{\text {; }}$

B. fl.III, 275

B.fl.III, 27.5

B. fl. III, 276

B. fl. III, 276

B.f.III, 277

$$
\text { - }
$$

- - - - Q. - B.fl.III,2;7

- - N.S.W.Q

- - - N.S.W. Q

B.fl.III, 275

B. Al.II, $27 \mathrm{~S}$

$\longrightarrow$

- - - - Q. - N.A. B.f.III, 279

(Nelitris.)

(Plinia, Plumier 1703, Acmene, Jambosa, Syzygium.)

- O - B.flII, 282 - - V. N.S.T. Q. N.A. B.fl.III,282

- - - N.S.W. Q

- - - N.S.IV.Q.

- - - N.S.W. Q

$=--$ N.S.W.Q.

- - - - Q

- - - N.S.T. O

- - - ? -

- - - - -

- - - -

$-\quad-$ N.S.TV.

B.fl. $\overline{\text { III }}, 283$

B.fl.III,283

- B.fl.III,283

-

B. fl. III, 284

- T.

B. Al. III, $2 S 4$

B fl.III, 27.,

B.fi.III, 285

- B.H.III,2SJ

N.A. B.f.III, 285

- - - N.S.IV. Q. - B.fl.III,2S6

- - - Q. B.f.III,2S6

- - - - $\overline{\text { Q. N.A. B.H.III,2S7 }}$

- - - - Q. - B.fl.III,2S7

Calyptranthes partly.)

--1 -
(Straradium.)

- - - - Q. - B.fl.III,2SS

- - - - U N.A. B.fl.III, 288
M.fr. I, 79 .

II.fr.I, T.

M.fr.I.S1\&243.

M.fr.IX, 14.

I.f. I, 80 .

M.fr.IV, 159 .

M. fr. I, 80 .

II. fr. VII, 41.

II.fr.I, $78 ; \mathrm{IX}, 43$

M.fr. I, $79 ; \mathrm{IX}, 43$.

M.fr.II, 171 .

M.fr.I,7s.

\section{M.fr.IX, 143.}

M.fr.II, $86 ; \mathrm{IX}, 142$.

I.fr. IT,117;IX, $1 \pm 2$

MI.fr.T.12;IX, 149 .

M.fr.IX, 142.

II. fr.IX, 147 .

II.fr.IX, 147.

II.fr.IX, 149 .

I.fr.IX, I48.

M. fr. IX, 149 .

M.fr. IX, 147.

AI.fr.IX, 147.

I.fr.IX, 147.

M.fr.IX, 148.

II. fr. IX, $1+2$.

II.fr. I, $76 ; \mathrm{IX}, 142$.

II.fr.IX, 142.

II.fr.IX, I4l.

M.fr.IX, 142.

X.ff.:TV, $56 ; \mathrm{IX}, 149$.

II. fr.III, $130 ; \mathrm{V}, 15 ; \mathrm{IS}, 143$

M.fr.IT, 59;IX, 145.

II. fr.IX, 145 .

II.fr. IV, $58 ;$ IX, 145.

M.fr.IX, 143.

II. fr. IX, 143.

M.fr. IX, 144.

M.fr. T,; ;

II.fr.IX, 145.

M.fr.IX, 143.

II.fr. I, 13; IX, 143.

M. fr. IX, 143 .

M.fr.IX, 143 .

NI.fr. [X, 143.

M.fr.I, 225;IX, 143.

M.fr.IX, 14J.

M.fr.IX, 146.

M.fr. IX, 145 .

I. fr.IX, 144 .

M.fr.VIII, 15.

Mi. fr. TX, $11 \mathrm{~S}$.

M.fr.IX, 118 .

M.fr.IX, 119 . 
CAREYA, Roxburgh, pl. Corom, III, 13, t. 217 (1816).

C. australis, F. v. M., fragm. V, 183 (1866) … ... SONNERATIA, Linné fil., stuppl. plant. 38 (1781).

S. acida, Linné fil., suppl. plant. 38 (1781)... ... ...

\section{MELASTOMACEAE.}

MEMECYLON, Linné, Fl. Zeyl. app. 9 (1747).

A. L. de Jussieu, gen. plant. 328 (1789).

II. umbellatum, N. L. Burmann, Fl. Tnd. 87 (1768)

OSBECKIA, Linné, spec. plant. I, 345 (1753).

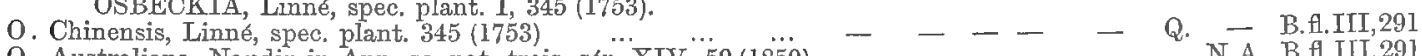

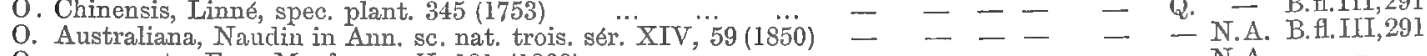

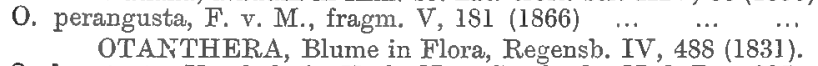

0 . bracteata, Korthals in Verh. Nat. Gesch. der Ned. Bes. 235, t. 51 (1841)

MELASTOMA, J. Burmann, thesaur. Zeyl. 72 (1737).

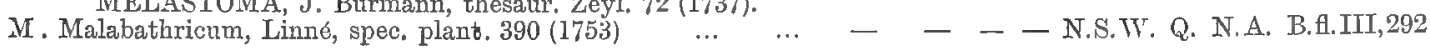

Q. N.A. B.Al.III,289 MI.f1: T,1S3.

- N.A. B.f.III, 301

RHAMNACEAE.

A. L. de Jussieu, gen. plant. 376 (1789), from B. de Jussieu (1759).

VENTILAGO, Gaertner, de fruct. I, 223, t. 49 (1788).

V. viminalis, Hooker in Mitch. Trop. Austr. $369(1848)$.. - S.A. - - N.S.W. Q. N.A. B.f.I,411

M.fr.IX, 140.

ZIZYPHUS, Tournefort, inst. 627, t. 403 (1700), from Plinius (Ziziphus).

. Oenoplia, Miller, Gardeners Dictionary n. $3(1731)$.. $\quad \ldots \quad-\quad-\quad-\quad$ - - N.A. B.H.I,412

. Jujuba, Lamarck, Encycl. méth. III, 318 (1789) $\quad . . \quad \ldots \quad-\quad$ - $\quad$ - $\quad$ - $\quad$ Q. - B.fl.I,412

. quadrilocularis, F. v. M., fragm. III, $57(1862) \quad \cdots . \quad \ldots \quad-\quad-\quad-\quad-\quad-\quad-$ N.A. B.Al.I,4l2

DALLACHYA, F. v. M., fragm. IX, 140 (1875). (Rhamuus partly.)

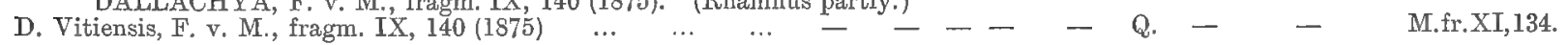

BERCHEMIA, Necker, elem. bot. II, 122 (1790).

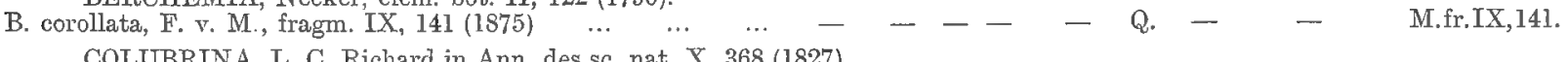

$\begin{aligned} & \text { COLUBRINA, L. C. Richard in Ann. des sc. nat. X, } 368 \text { (1827). } \\ & \text { iatica, Brogniart in Ann. des sc. nat. X, } 369(1827)\end{aligned}-\ldots--\quad-\quad$ Q. N.A. B.fl.I,413 M.fr.IX,139.

C. Asiatica, Brogniart in Ann. des sc, nat. X, 369 (1827) $\ldots$
ALPHITONIA, Reisseck in Endl. gen. plant. 1098 (1540).

A. excelsa, Reisseck in Endl. gen. plant. 1098 (1840) $\ldots \quad \ldots \quad-\quad-\quad-\quad$ N.S.TT. Q. N.A. B.A.I,4l4 ELIMENOSPERMUM, F. v. M., fragm. III, 63 (1862).

E. alphitonioides, F. v. M., fragm. III, $63(1862) \ldots \quad \ldots \quad \ldots \quad-. \quad-\quad-\quad-$ N.S.W. Q. $\quad-\quad$ B.fl.I,415

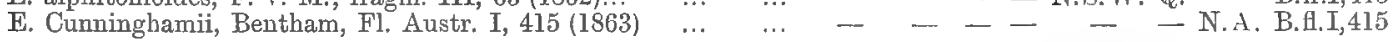
POMADERRIS, Labillardière, Nov. Holl. pl. specim. I, 61, t. 86 (1804).

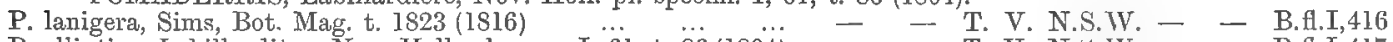

P. elliptica, Labillardière, Nov. Holl. pl. spec. I, $\ddot{6} 1$, t. $36(1804)-$ - T. V. N.S.IV. - - B.f.I.417

P. phillyroides, Sieber in De Cand, prodr. II, $33(1825) \ldots \ldots \ldots-\ldots-\ldots$ N.S.W. Q. - B.H.I,418

P. vacciniffolia, Reisseck \& F. v. M. in Linnaea XXIX, $266(1856)--$ - V. N.S. W. - - B.fl. I.418

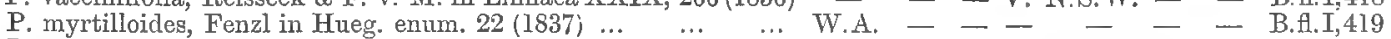

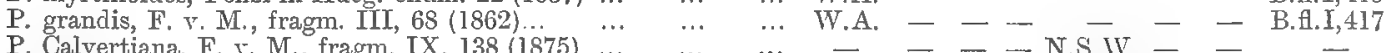

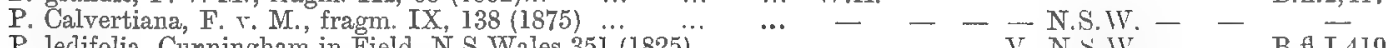

P. ledifolia, Cunningham in Field, N.S. Wales 351 (1825) $\ldots-$ - - - T. V. N.S.W. - - B.fl.I,419
P. apetala, Labillardiere, Nov. Holl. pl, spec. I, 62, t. $87(1804)-$ S.A. T. V. N.S.W. - - B.f.I,419

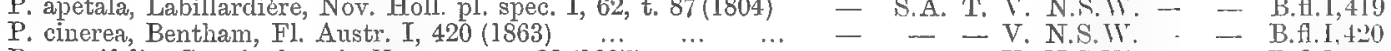

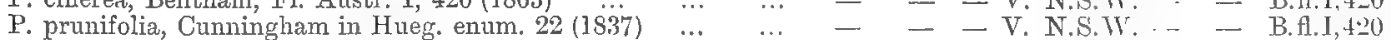

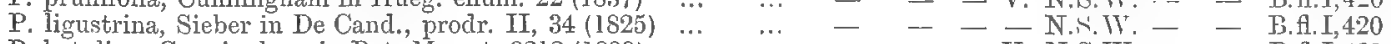

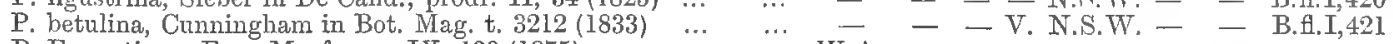

P. Forrestiana, F. v. M., fragm. IX, 139 (1875) $\ldots . \quad \ldots \quad$... W. A. $\quad-\quad-\quad-\quad-\quad-$

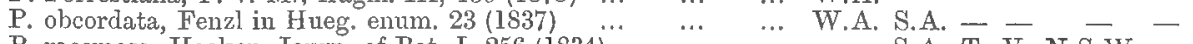

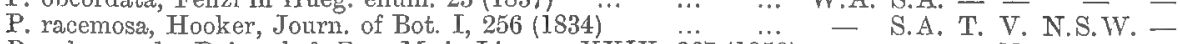

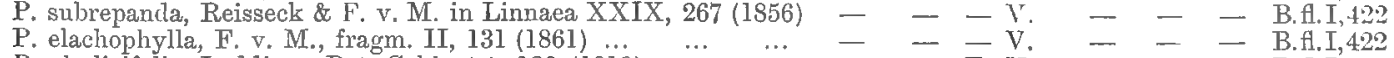

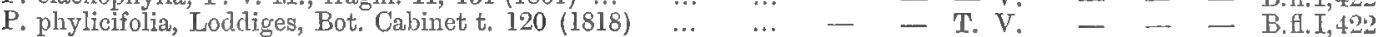

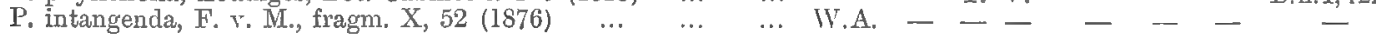

II.fr.IX, 140.

M.fr.IX,140.

MI.fr.IX, 139 .

M.fr.III, 71.

M.fr.IX, 138 .

M.fr.IX, 139 .

M.fr.IX,13S.

M.fr.III, 69.

M. fr.III, 69\&:3;IX, 139.

II.fr.IIT,75;IX, 139.

M.fr. III, 71 ; IX, 139.

M. fr.III, $76 ; \mathrm{IX}, 139$.

M.fr.IX, 139 .

M.fr.III, 73.

I. fr.III, $75 \& 168 ;$ IX, 139 .

M.fr.III, 74 .

M.fr.II, 166;IX, 139 .

M.fr. III, 73 .

I. fr. X, 53 .

CRXPTANDRA, Smith in Transact. Linn. Soc. IV, 217 (1798). (Trymalium, Spyridium, Stenanthemum, Wichurea, Stenodiscus.)

C. albicans, F. v. M.; Trym., Reisseck in pl. Preiss. II, 280 (1847) W.A. - - - - - - B.fl. I,423

C. Billardieri, F. v. M. Trym., Fenzl in Hueg. enum. 25 (1837) W.A. - - - - - - B.H.I,

C. ledifolia, F. V. II.; Trymalium, Fenzl in Hueg. enum. 24(1837) W.A. $--\frac{-}{-}-\quad-$ B.fl.I,424

C. Daltoni ; 'Trymalium, F. v. M., fragm. IX, 135 (1875) … - - - V. $-\overline{-}$

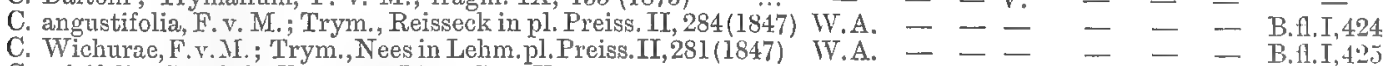

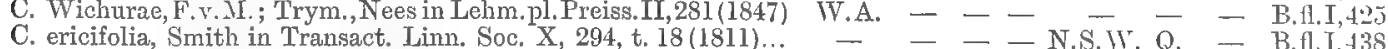

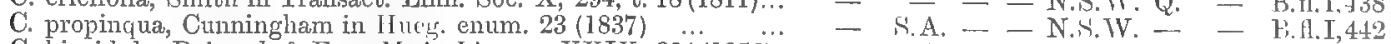

C. hispidula, Reisseck \& F. v. I. in Linnaea XXIX, $294(1856)$ - S.A. - - N.H. - - - - B.A.I,442

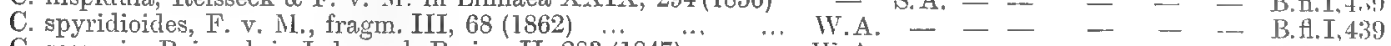

C. scoparia, Reisseck in Lehm. pl. Preiss. II, 283 (1847) $\cdots \quad$ W.A. - - - - - - - - B.H.I.439

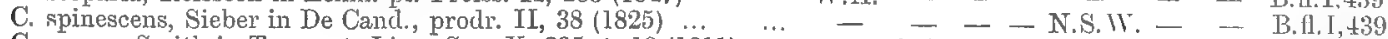

C. amara, Smith in Transact. Linn. Soc. X, 295, t. $18(1811) \ldots-\ldots-$ S.A. T. V. N.S.W. Q. - - B.H.I,440

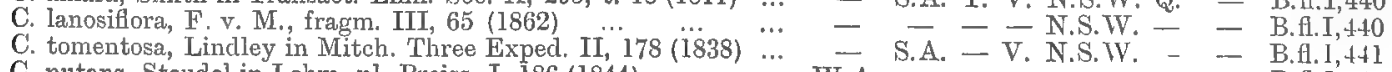

C. nutans, Steudel in Lehm. pl. Preiss. I, $186(1844) \quad \ldots \quad$ … W.A.

M.fr. IX, 135.

M.fr.IX, 135.

M.fr.IX,135.

M.fl. IX, 137.

M.fr.IX, 137 .

M.fi. IX, 138.

M. fr. III, $66 \& 67$;IX, 137 .

M. fr. III, 6.5 .

M.fr.III, $67 ; I X, 138$.

M. fr.IX, 133 . 
C. glabriflora, Bentham, Fl. Austr. I, 441 (1863) ...

C. alpina, J. Hooker, Fl. Tasm. I, 75, t. $12(1860)$

C. Leissner in Lehm. pl. Preiss. II, 287 (184)

C. buxifolia, Fenzl in Hueg. enum. 23 (1837)

C. pungens, Steudel in Lehm. pl. Preiss. I, $187\left(17_{444}{ }^{\cdots}\right.$

C. mutila, Nees in Lehm. pl. Preiss. II, 289 (1847)

C. longistaminea, F. v. M., fragm. III, 64 (1862)..

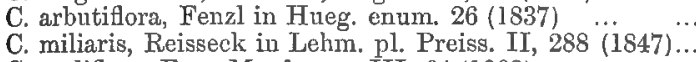

C. nudifiora, F, v. M., fragm. III, 64 (1862)

C. ulicina, Hooker, Journ. of Bot. I, 257 (1834) $\ldots . \quad \ldots$

C. pomaderroides, Reisseck in Endl. nov. stirp. dec. 29 (1839)."

C. pumila; Spyridium, F. T. M., fragm. IX, 137 (1875)..

C. leucophracta, Schlechtendal, Linnaea XX, 640 (1847)

C. coronata, Reisseck in Lehm. pl. Preiss. II, 288 (1847)

C. humilis, F. v. II.; Stenanthemum, Benth., Fl. Austr. I, 436(1863)

C. Waterhousii; Spyridium, F. v. M., fragm. III, 83 (1862)

C. tridentata, Stendel in Lehm. pl. Preiss, I, 186 (1S44)...

C. obcordate Hor Tasm I 71 (1860)

C. Lawrencii, J. Hooker, Fl. Tasm. I, 72 (1860) $\ldots$ Mookeri, F. v. M.; C. parvifolia, J. Hooker, Fl. Tasm. I, $73(1860)-$

C. spadicea, F. r. M. ; Trymalium, Fenzl in Hueg. enum. 26 (1837) W.A.

C. globulosa, F. v. M.; Pomad, G. Don in Loud. hort. Brit. 84(1830) W.

C. obovata, J. Hooker, Fl. Tasm. I, 74 (1860)

C. spathulata; Trymalium, F. v. M. in Trans. Viet. Inst. I, $122(1855)$

C. cordata, Turczaninow in Bull, de Mosc. XXXI, 459 (1858) ... W.

C. rotundifolia; Spyridium, F. v. M., fragm. IV, 25 (1863) ‥ W. A.

C. coactilifolia, F, v. MI.; Spyr., Reiss. in Linnaea XXIX, 291 (1856) -

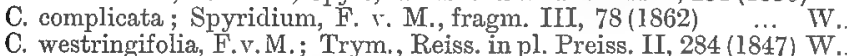

C. villosa, Turczaninow in Bull. de Mosc. XXXI, 458 (1858) ... W.A. - - -

C. paucifiora, Turczaninow in Bull. de Mosc. XXXI, 458 (1858)

C. halmaturina; Trym., F. v. M. in Linnaea XXIX, 283 (1856)

TI.A.

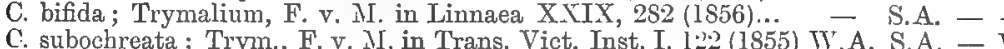

C. vexillifera, Hooker, Jouru. Bot. I, 257 (1834)

COLLETIA Commercon in A. I de Jussieu, on 380 (1789) (Tiscori Tetrapasm.

C. pubescens, Brogniart in Annal. des sc. nat. X, 366 (1827) .. - - T. V. N.S.W. GOUANIA, N. J. Jacquin, stirp. Amer. hist. 263, t. 179 (1763).

(t. Hillii, F. v. M., fragm. IX, $163(1875) \ldots \ldots \ldots$....

G. Australiana, F. v. M., fragm. IV, $144(1864)$ ․

\section{ARALIACEAE}

Ventenat, Tabl. III (1799). (Umbelliferarum_subordo.) ASTROTRICHA, De Candolle, Mémoire sur la fam. des Ombellifères 25 (1829).

A. pterocarpa, Bentham, Fl. Austr. III, 379 (1866)

A. Hoccosa, De Candolle, Mém. des Ombell. 30, t. 5 (1829)

A. longifolia, Bentham in Hreg. exum. 55 (1837) ...

A. ledifolia, De Candolle, Mém. des Ombell. 30, t. 6 (1829)

A. Hamptoni, F. v. M., fragm. VI, 125 (1868)

POROSPERMUM, F. v. M., fragm. VII, 94 (1870).

P. Michieanum, F. v. M., fragm. III, 95 (1870) .. PANAX, Limné, spec, plant, edit. sec. 1513 (1763). (Nothopanax.)

P. Gunnii, J. Hooker in Lond. Journ. of Bot. VI, 466 (1847) ... -

P. Murrayi, F. v. M., fragm. II, 106 (1861)

P. mollis, Bentham, Fl. Austr. III, 382 (1S66)

P. Macgillivraei, Bentham, Fl. Anstr. III, 382 (1866)

P. sambucifolius, Sieber in De Cand. prodr. III, 255 (1820̈)

P. cephalobotrys, F. v. M., fragm. II, 83 (1861)

P. elegans, C. Moore \& F. v. M. in Trans. phil. Inst. $\dddot{V}$ ict. II, $68(1858)$ -

P. Cissodendron, C. Moore \& F. v. M., fragm. VII, 96 (1.870) ...

MOTHERWELLIA, F. v. M., fragm. VII, 107 (1871).

M. haplosciadea, F. v. M., fragm. VII, 107 (1871)

CISSODENDRON, Seemann, Journ. of Bot. II, 303 (1864). (Hedera partly, Irvingia, Kissodendron.)

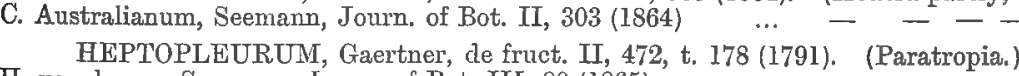

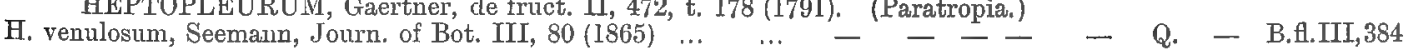
BRASSAIA, Endlicher, nov. stirp. decad. I, 89 (1839).

B. actimophylla, Endlicher, nov. stirp. dec. I, S9 (I839) ...

MERYTA, R. \& (. Forster, char. gen. 119, t. 60 (1776). (Bothryodendron.)

M. latifolia, Seemann in Bonplandia, 295 (1862)

M. angustifolia, Seemann in Bonplandia, 296 (1862)

MACKINLAYA, F. v. M., fragm. IV, 120 (1864).

M. macrosciadea, F, v. MI, fragm, IV, 120 (1864) ...

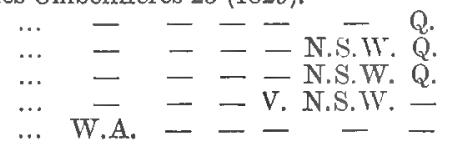

B.fl.III, 379

B. fl. III, 380

B.f..III, 380

B.fl.III, 350

-

M.fr. VII, 148.

M.fr. VI, $125 \& 250$

MI. fr. TII,95.

B. fl.III, 381

B. H.III, 381

B. Al.III, 382

B. fl. III, 35:2

B.fl.III, 382

B. fl. III, 382

B.H.III, 383

M.fr. VII, 96.

M.fr.VII, 96.

M.fr. TII, 95 .

M.fr. UI, 83; \II, 95 .

M.fr.IX, 96 .

M.fr.VII, 107.

M.fr.IT, $120 ; \Gamma, 18$.

Q. - B.fl.III,3S5

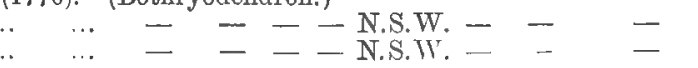

ALfr. IX, 169.

M.fr.IX, 169 . 


\section{UMBELLIFERAE:}

Morison, hist. pl, II, lib. 3, sects 9 (1680).

HYDROCOTYLE, Toumefort, inst. 328, t. 173 (1700).

H. vulgaris, Linné, spec. plant. $234(1-53) \ldots$... .. ... ... - S.A. - V. N.s.IV. Q H. hirta, R. Brown in annal. des sc. phys. VI, $6+(1820) \ldots \ldots$ W.A. S.A. T. V. N.S. IV. Q. H laxifora, De Candolle, pros.

... $\quad \cdots \quad-$ S.A. - V. N.S.W. Q.

N- - - - - N.S.W. -

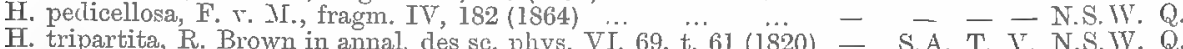

H. plebeia, R. Brown in annal. des sc. phys. VI, 46, t. $60(1820)$ W. A. - - - - -

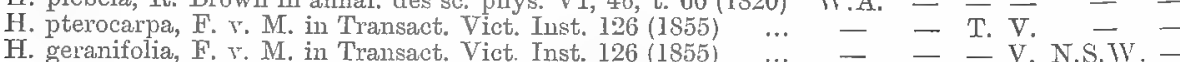

H. medicaginoides, Turczaninow in Bull. Mosc. XXII, 27 (1849)

H. muriculata, Turczaninow in Bull. Mosc. 28 (1849)

H. callicarpa, Bunge in Lehm. pl. Preiss. I, 2S:3 (1844) $\cdots$

H. scutellifera, Bentham, Fl. Austr. III, 343 (1866)

H. hispidula, Bunge in Lehm. pl. Preiss. I, 283 (1844)

F. trachycarpa, F. v. II. in Schlecht. Linnaea XXV, 394 (1852)

H. pilifera, Turczaninow in Bull. Mosc. XXII, 26 (1849)

H. capillaris, F. r. M., fragm. IV, ITS (1S64)

H. xugulosa, Turczaninow in Bull. Soc. Mose. XẌIT, 27 (1849)...

H. grammatocarpa, F. r. M., fragm. II, I 198 (1861)

H. diantha, De Candolle, prodr. IT , 63 (1830)

W.A. S.A. - V. N.S.TV. -

W.A. S.A. T. V. N.S.W. -

IV.A. - - - - -

S.A. - N.S.W. -

W.A. $\overline{\text { IV.A.A }}-\overline{\mathrm{V}} \mathrm{N}$. $\overline{\mathrm{S}} \mathrm{W}$. -

B. A.III, 339

B.fl.III, 340

B.fl.III,341

M.fr.IV, $179 \& 180$

M.fr.VII, 147.

M.fr.VII, 147.

B.fl.III, 341 M.fr. VII, 147.

B.f.IIT, 341

B.fl. III, 342

- B.fl.III, 342

B.f1.III, 342

- B.Hl.III,342

- B.fl.III,343

- B.f.III, 343

- B.fl.III,343

- B.H.III, 343

- B.fl. ITI, 344

- B.f.III.344

W.A. - - - - - B.fl.III, 344

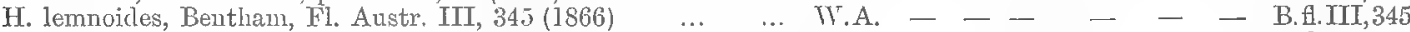

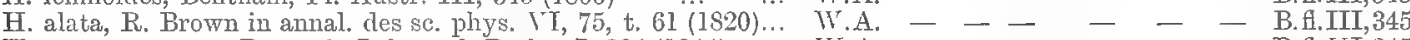

H. tetragonocarpa, Bunge in Lehm. pl. Preiss. I, 284 (1844) ... W.A. - - - _ - - B.fl.III,345

H. glochidiata, Bentham, Fl. Austr. III, $346(1866) \quad \ldots \quad$... TV.A. $\quad-\quad-\quad-\quad-\quad$ - $\quad$ - B.Al.III,346

H. verticillata, Turczaninow in Bull. Mosc. XXII, 28 (1849) $\ldots$ W.A. $-\quad-\quad-\quad-\quad-\quad-$ B.H.III,346

H. Asiatica, Limné, spec. plant, 234 (1753) ...

W.A. S.A. T. V. N.S.TY. Q. - B.f.III, 346

M.fr.II, 129;:II, 147.

DIDISCUS, De Candolle in Bot. Mag. t. 2857 (1828). (Dimetopia, Pritzelia, Hueyelia, Cesatia, Hemicarpus.)

D. pusillus, F. v. M., fragm. IX, $47(1875) \quad \ldots \quad \ldots . \quad \ldots \quad$ W.A. S.A. - V. N.S.T. - - B.Al.III,348

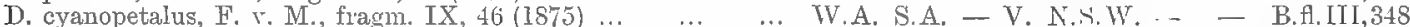

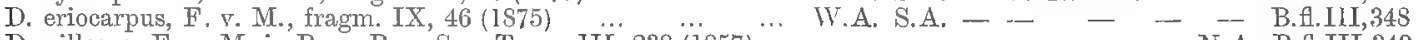

D. villosus, F. v. M. in Proc. Roy. Soc. Tasm. IIr, 238 (1857) ‥ - - - - - - N.A. B.A.III,349

D. coeruleus, De Candolle in Hook. Bot. Mag. 287J(1828) ‥ W.A. - - - _ - - B.fl.III,349

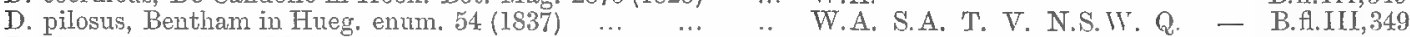

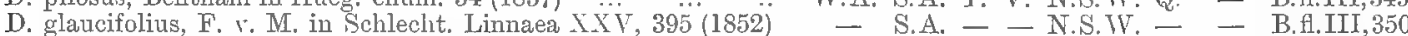

D. glandulosus, F. v. M. in Proc. Roy. Soc. Tasm. III, 238 (1857) - - - - - N.A. B.Al.III,350

D. incisus, Hooker, Bot. Magaz. 2875 (1828) $\ldots . \quad \ldots \quad \ldots \quad-\quad-\quad-$ N.S.TV. Q. $\quad$ - B. B.IL.350

D. procumbens, F. v. M. in I'roc. Roy. Soc. Tasm. III, 237 (1857) - - - - N.S.IV. Q. - B.A.III, 350

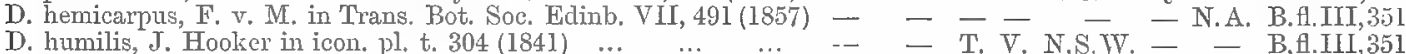

M.fr.IX, 47.

M.fr.I,231.

M.fr.IX,46.

M.fr.IX, 47.

M.fr.IX, 47 .

M.fr.IX, 47.

M.fr. IX, 47 .

TRACHYMENE, Ruclge in Transact. Linn. Soc. X, 300 (1810). (Siebera, Fischera, Platysace, Platycarpidium.)

T. compressa, Rudge in Transact. Limn. Soc. $\mathrm{X}, 300(1810) \quad \ldots$ W.A. $-\quad-\quad-\quad-$ B. 11.III,352

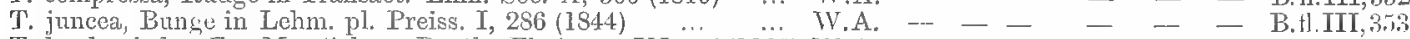

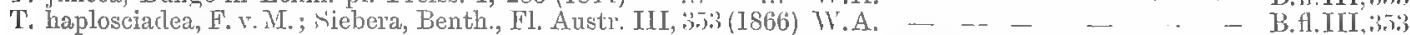

T. cirrosa, F. Y. M.; Platysace, Bunge in pl. Preiss. I, 285 (1844) - S.A. - V. - - - B.ll.III,3i.

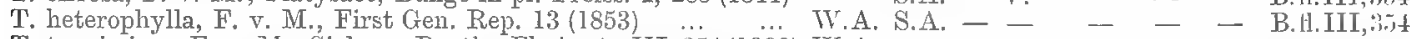

T. tenuissima, F. T. MI.; Siebera, Benth., Fl. Austr. III, 354 (1866) W.A. - - _ _ _ _ - B.H.III,3it

T. dissecta, F. v. M.; Sicbera, Bentham, Fl. Austr. III, 3int (1866) W.A. - - . - - - B.H.III,354

T. commutata, Turczaninow in Bull. Mose. XXII, 30 (1849) ... W.A. - _ - _ _ _ - B.fl.III, מJ.

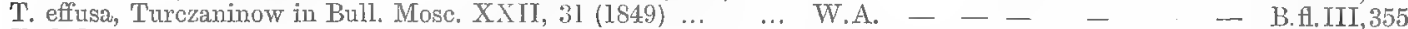

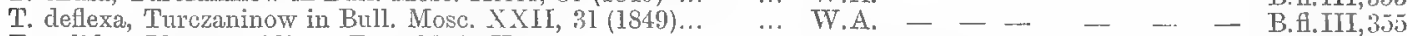

T. valida; Platycarpidium, F. v. II. in Kew. Misc. IX, 310(1857) - - - - - O. - B.fl.III,355

T. ericoides, Sieber in De Candolle, prodr. IV, $73(1830) \quad \ldots \quad-\quad-\quad-$ V. N.S.W. Q. - B.fl.III,356

T. linearis, Sprengel, spec. umbellif.7(1818) $\quad \ldots \quad \ldots \quad \ldots-\quad-\quad-\quad--$ N.S.IV. $-\quad-$ B.f.III.356

T. Billardieri, F. v. M.; Siebera, Benth, Fl. Austr. III, 356 (1866) - - - V. N.S. IV. - - B.f.III,350

T. Stephensonii, Turezaninow in Bull. de Mosc. XX, $170(1847) \quad-\quad-\quad$ - N.S.W. - - B. A.III,357

XANTHOSIA, Rudge in Transact. Limn. Soc. $\mathrm{X}, 361$ (1810). (Leucolaena, Schoenolaena, Pentapeltis.)

X. juncea, Bentham, Fl. Austr. III, 359 (1866) ... $\ldots$... W.A. - $\quad$ -

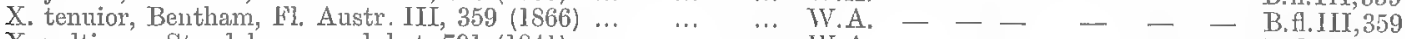

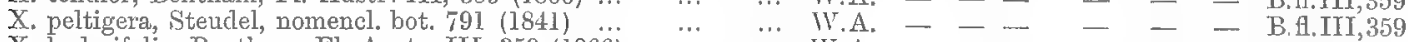

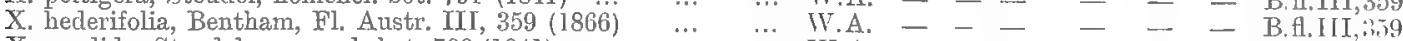

X. candida, Steudel, nomencl, bot. 790 (184I)

X. tridentata, De Candolle, prodr. IV, $75(1830)$...

X. singulillora, F. v. M, fragm. IV, 184 (1864) ...

... W. W.A. - - - - $\quad$ - B.f.III,259

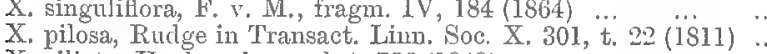

X. ciliata, Hooker, icon. pl. t. 726 (1848)

X. pusilla, Bunge in Lehm. pl. Preiss. I, 291 (1844)

X. fruticulosa, Bentham, Fl. Austr. III, 361 (1866)

X. Huegelii, Steudel, nomenclat. bot. 791 (1841)

X. dissecta, J. Hooker in icon. plant. t. 302 (184 I)

X. peduncularis, Denthan, Fl. Austr. III, :6:- (1866)

$\mathrm{X}$. vestita, Bentham, Fl. Austr. III, 363 (1866)

X. Atkinsoniana, F. v. M., fragm. II, I:27 (1861)...

X. rotundifolia, De Candolle, prodr. IV, F., (1S30)

W.A. - - V. N.S.T. -

B. A. III $\because 60$

W.A. - - - - - B.fI.III,260

W- - T. V. N.S.W. Q. - B.fl.III, 260

M.fr.IV, 184 .

IV.A. - - - - - B.fl.III,261

W.A. S.A. T. V. -- - B.fl.IIT,261

II.A. - - - - - - B.A1,111,261

... W.A. - - - - - - B.I.III,362

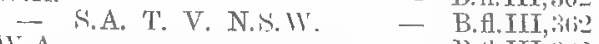

II.A. - - - _ _ B.11.III,;302

W.A. - - - N.S.W. - N.S.W. - - B.Al.III,363
W. B.III,363

W.A. - - - - -. - B.H.III,;60.)

M.fr.II, 127. 
AZoRELlA, Lamarck, Encycl. methodiq. I, 341, t. 189 (1783). (Fragosa, Pozoa, Microsciadium, Schizeilema, Oschatzia, Centclla partly, Dichopetalum.)

A. Muelleri, Bentham, Fl. Austr. III, $364(1866) \ldots \ldots \ldots \ldots$.... - - V. - - - B.A.III,364

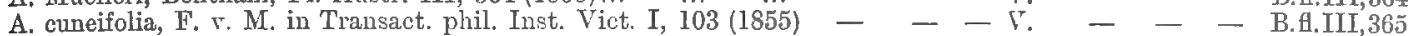

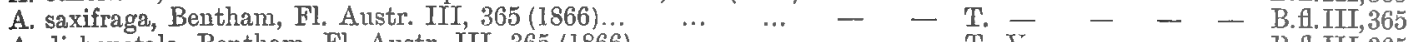

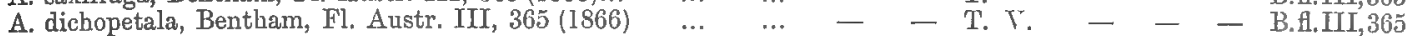

HUANACA, Caranilles, icon. TI, 1S, t. 528 (1801). (Diplaspis, Pozoopsis.)

H. hydrocotylea, Bentham \& J. Hooker, gen, pl. I, si: (1867).. - - T. V. - - - B.f.III,366

H. cordifolia, Bentham \& J. Hooker, gen. pl. I, Sit (1867) .. - - T. - - - - B.A.II,366

ACTINOTUS, Labillardière, Nov. Holl, pl, spec. I, 67, t. 92 (1804). (Hemiphues, Eriocalia, Holotome.)

A. Helianthi, Labillardière, Nov. Holl, pl. spec, I, 67, t. 92 (I804) - - - V. N.S. Ir. Q. - B.f.III,367

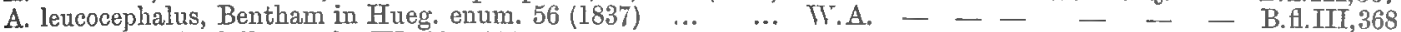

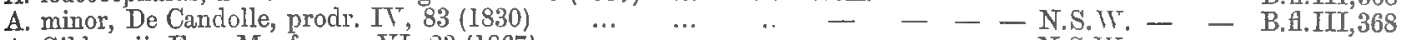

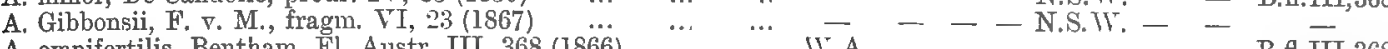

A. omnifertilis, Bentham, Fl. Austr. III, 368 (1866) $\quad$.. .. W.A. - $\quad$ W - - - - B.fl.III,368

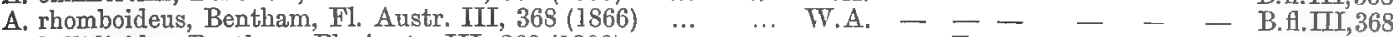

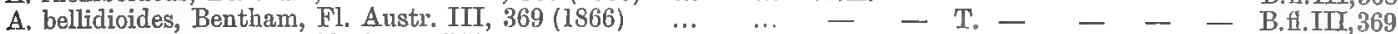

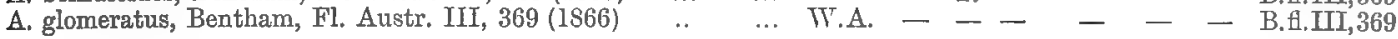

ERYNGIUM, Tournefort, inst. 327, t. 173 (1700) from Theophrastos and Dioscorides).

E. rostratum, Cavanilles, icon. pl. VI, 35, t. 552 (1801) ... … W.A. S.A. - T. N.S.W. Q. - B.f.III,370

E. vesiculosum, Labillardière, Nov. Holl. pl, spec. I, 73, t.98(1804) - S.A. T. Y. N.S.M. Q. - B.fl.III,370 M.fr.VII,148.

E. plantagineum, F. v. M. in Pap. Roy. Soc. Tasm. III, 235 (1857) - S.A. - - N.S.W. Q. - B.fl.III,371 MI.fr.VII, 148.

E. expansum, F. v. M. in Pap. Roy. Soc. Tasm. III, 236 (1857) - - - - N.S.W. Q. - B.fl.III,37I M.fr.VII, 148. APIUM, Tournefort, inst. 305, t. 160 (1700).

A. prostratum, Labillardière, relat, du voy. I, 141 (1799) … TT.A. S.A. T. Y. N.S.W. Q. - B.fl.III.372 MI.fr. VII,148. A. leptophyllum, F. v. M. in Benth. Fl. Austr. II, 372 (1866)... - - - V. N.S.W. Q. - B.t.III,372 I.fr.VII,148. SIUL, Tournefort, inst. 308, t. 162 (1700), from C. Bauhin (1623).

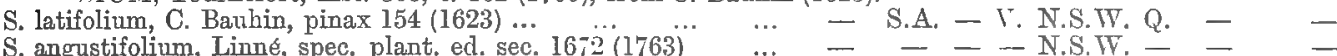
SESELI, Rivinus in Rupp. FI. Jen. 267 (1718).

S. Harveyanm, F. v. AL. in Transact. phil. Soc. Yict. I, 104 (1855) - - - V. - - - B.fl.III,373

S. algens, F. v. M. in Transact. phil. Soc. Vict. I, 104 (1855) ... - - - V. $-\quad$ - - B.H.III,373

CRANTZIA, Nuttall, Gen. of North Amer. plants I, 177 (1818). (Non Scopoli, 1777, Crantziola, F. r. M.)

C. lineata, Nuttall, Gen, of North Amer. pl. I, $178(1818)$.. - S.A. T. V. N.S.W. Q. - B.fl.III,374 OENANTHE, Tournefort, inst. 312, t. $166(1700)$,

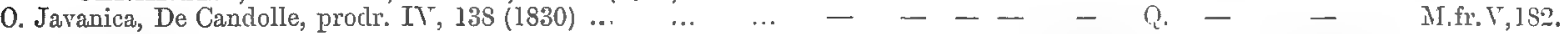

ACIPHYLLA, R. \& G. Forster, char. gen. 135, t. 68 (1776). (Anisotome, Calosciadium, Gingidium partly, Ligustici subgenus.)

A. simplicifolia, F. v. M. in Benth. Fl. Austr. III, 375 (1866) ... - - - T. N.S.W. - - B. H.III,375

A. glacialis, F. r. M. in Benth. Fl. Austr. III, 375 (1866) $\ldots-\ldots-$ - T. N.S.W. - - B.fl.II,375

A. procumbens, F. v. M. in Benth. Fl. Austr. III, 375 (1866) .. - - T. - - - - B.fl.III,375

DAUCUS, Tournefort, inst. 307, t. $161(1 ; 00)$, from l'Ecluse $(1,50)$.

D. brachiatus, Sieber in De Cand., prodr. IV, $214(1830) \quad$... TV.A. S.A. T. T. N.S.W. Q. - B.fl.III, 370 OREOMYRRHIS, Endlicher, gen, plantar, is7 (1839).

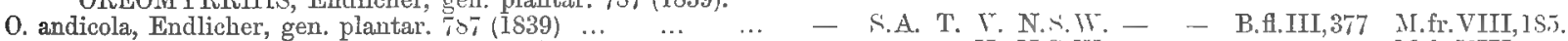

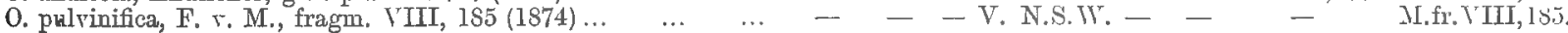

\section{SYNPETALEAE PERIGYNAE.}

F. v, M. in Woolls's plants of the neighb. of Sydney, 27 (1880).

\section{AQUIFOLIACEAE.}

De Candolle, Théor. élém. I, 217 (1813).

BYRONIA, Endlicher in den Ann, des Wien. Mus. I, I84 (1836).

B. Arnhemensis, F. v. M., fragm. II, 119 (1861) ... … .. - - - - - - N.A. B.A.I,397

ILEX, Linné, gen. plant. 33 (1737), from C. Bauhin (1623).

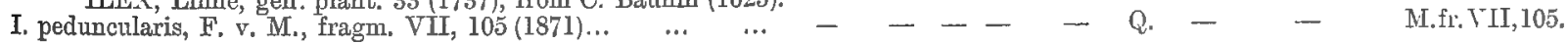

OLACINEAE.

Mirbel in Bull. de la Soc. philomat. 377 (1813).

XIMENLA, PIumier, nora plant. Amer. genera 6, t. 21 (1703).

X. Americana, Linné, spec. plant. $1193(1753) \quad \ldots \quad \ldots \quad \ldots \quad \ldots \quad-\quad \ldots \quad-\quad$ Q. N.A. B.Al.I,391

OLAX, Linné, amoen, acad. 387 (1717). (Spermaxyrum, Lopadocalyx.)

0. phyllanthi, R. Brown, prodr. 358 (1810) -...W.A. - - - - - - B.fl.I.392

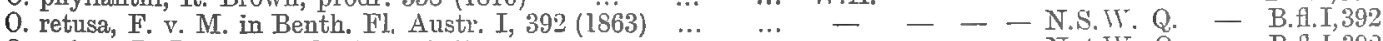

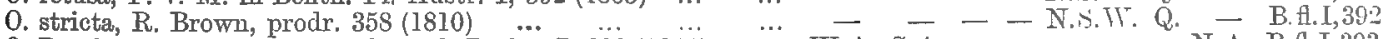

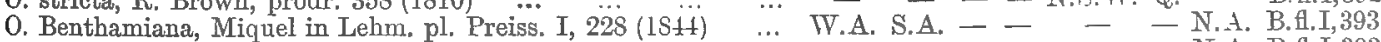

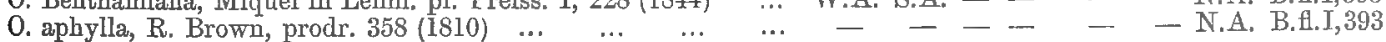

CANSJERA, A. L. de Jussieu, gen. pl. 448 (1789).

C. leptostachya, Benth. in Hook. Lond. Journ, of Bot. II, 231 (1843) - - - - — Q. - B.fl.I,394

OPILIA, Roxburgh, pl. Corom. II, 31, t. 158 (1799).

O. amentacea, Roxburgh, pl. Corom. II, 31, t. $158(1799) \quad \ldots \quad-\quad-\quad$ - $\quad$ - Q. N.A. B.fl.I,394

PHLEBOCALTIIIA, Griffith in Benth. \& J. Hook. gen. pl. I, 353 (1862). (Phlebocalymna).

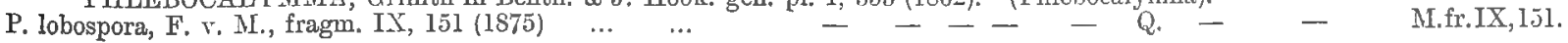

MI.fr. TI,3;TX, 150.

MI.fr. IX, Iอ̃ิl.

M.fr. IX, 151

M.fr.IX, 151 .

II.fr.IX, 151 .

M.fr.LX, 15I.

MI.fr. VI, 3.

M.fr. VI, $4 ; I X, 150$. 
PENNANTIA, R. \& G. Forster, char. gen. 133, t. 67 (1776).

P. Cunninghamii, Miers in Ann. of nat. hist. sec. ser. IX, 491 (1852)

P. Endlicheri, Reisseck in Sohlecht. Linnaea XVI, 341, t. 13 (1843) -

APODYTES, E. Meyer in Hook. Journ. of Bot. III, 155 (1841).

A. brachystylis, F. V. II, fragm. IX, $149(1875) \ldots \ldots$... $\ldots$ VILLARESIA, Ruiz \& Pavon, flor. Peruv. et Chil. prodr. 35 (1794).

V. Moorei, F. v. M. in Benth. Fl. Austr. I, 396 (IS63) ... ‥ - - - - N.S.T. - - B.H.I,395

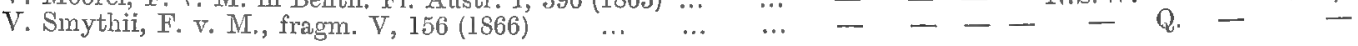
GOMPHANDRA, Wallich, Catal. n. 3718 \& 7204 (1832).

G. Australiana, F. r. M., fragm. VI, 3 (1877)
$\mathrm{M}$ fr. VI, $4 ; \mathrm{IX}, 150$.

I.fr.IX, 150 .

M.fr.IX, 149 .

M.fr.IX, 150 .

M.fr. VI, 3\&253;IX, 150 .

\section{ELAEAGNEAE.}

R. Brown, prodr. I, 350 (1810.)

ELAEAGNUS, Tournefort, coroll. 53, t. 459 (1703) from Camerarius (1586).

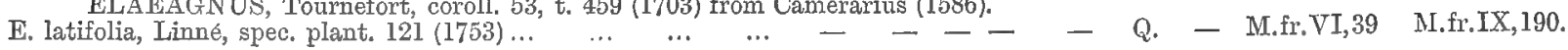

SANTALACEAE.

R. Brown, prodr. I, 350 (1810).

THESIUM, Linné, gen, pl. 60 (1737).

T. australe, R. Brown, prodr. 353 (1810) ...

SANTALUM, Linné, gen. ed. sec., 165 (1742), from C. Bauhin (1623). (Fusanus, Eucarya),

S. lanceolatum, R. Brown, prodr. $356(1810)$

S. oratum, R. Brown, prodr. 355 (1810)

S. obtusifolium, R. Brown, prodr, 356 (1810)

S. acuminatum, A. de Candolle, prodr. XIV, 684 (1856)...”

S. persicarium, F. r. M. in Transact. Vict. Inst. 41 (1854)

S. cygnorum, Miquel in Lehm. pl. Preiss. $X$, 615 (1845) ...

S. crassifolium, A de Candolle, prodr. XIV, 685 (1856)...

CHORETRUM, R. Brown, prodx. 354 (1810).

C. glomeratum, R. Brown, prodr. 354 (1810)

C. spicatum, F, v II, fragm. I 21 (1858)

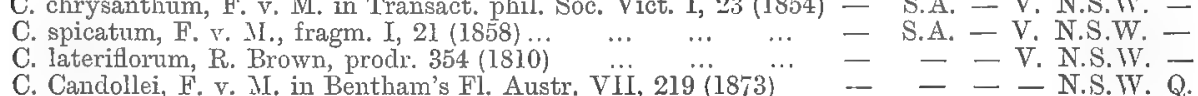
LEPTOMERIA, R. Brown, prodr. 353 (1810).

L. spinosa, A. de Candolle, prodr. XIV, 67 S (1856)

L. Preissiana, A. de Candolle, prodr. XIV, 678 (1856) ...

L. pauciflora, R. Brown, prodr. 354 (1810)...

L. scrobiculata, R. Brown, prodr. 354 (1810)

L. acida, R. Brown, prodr. 353 (1810)

L. Billardieri, R. Brown, prodr. 354 (1810)

L. aphylla, R. Brown, prodr. 354 (1810) ...

L. glomerata, F. v. .I. in J. Hook. Tasm. II, 370 (1860)...

L. squarrulosa, R. Brown, prodr. 354 (1810)

L. Cunninghamii, Miquel in Lehm. pl. Preiss. I, 611 (1845)

L. empetriformis, Miquel in Lehm. pl. Preiss. I, 610 (1845)

L. axillaris, $R$. Brown, prodr. 354 (1S10)

L. laxa, Míquel in Lehm. pl. Preiss. I, 612 (1845)... $\quad \ldots$

L. obovata, Miquel in Lehm. pl. Preiss. I, 613 (1845) $\ldots$

OMPHACOMERIA, Endlicher, gen. pl. 326 (1838).

O. acerba, A. de Candolle, prodr. XIV, 681 ((1856) ANTHOBOLUS, R. Brown, prodr. 357 (1810).

A. filifolius, $R$. Brown, prodr. 357 (1810) ...

A. triqueter, $R$. Brown, prodr. $357(1810) \cdots \cdots$

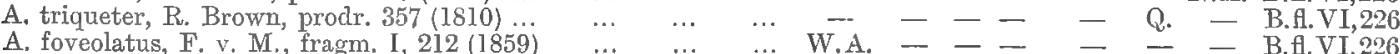

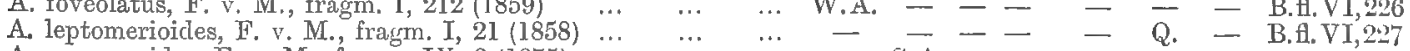

A. exocarpoides, F. . M. fragm. IX, 3 (1875) …

EXOCARPOS, Labillardiere, Relat. du voy, d la rech.

E. latifolia, R. Brown, prodr. 356 (1810)

E. odorata, A. de Candolle, prodr. XIV, 689 (1856)

H. cupressiformis, Labillardiere, voy. I, 155, t. $14(1798)$

F. spartea, R. Brown, prodr. 356 (1810)

E. aphylla, R. Brown, prodr. 357 (1810) ... . . . 9 (1871)

i. phyllanthoides, Endlicher, prodr. pl, ins. Norf. 46 (1833)

F. stricta, R. Brown, prodr. 357 (1810)

E. humifusa, R. Brown, prodr. 356 (1810).'

E. nana, J. Hooker in Lond. Journ, of Eot. VI, 281 (1847)

... W.A. S.A. - - N.S.T. Q. N.A. B.fl.VI, 214

-. - - - - - N.A. B.A.VI,214

․ - - - V. N.S.V. - - B.fl.VI,215

... W.A. S.A. - V. N.S.W. - - B.fl.VI,2l6

... W.A. S.A. - V. N.S.W. - - B.fl.VI,2l6

... W.A. - - - _ - B.fl.VT,217

... - - - - - N.S.W. - - B.f.VI,217

... W.A. S.A. - V. N.S.W. - - B.f.VI,218

- B.fl. $\overline{\mathrm{VI}}, 218$

- B.fl. VI,219

— B.fl.VI, 219

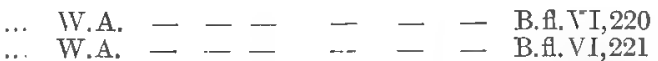

... W.A. - - - - - - B.甘.VI,201

.. W.A. - - - - - - B.H.VI,221

... - - - V. N.S.W.Q. - B.H.VI,222

.. - - T. - N.S.W. Q. - B.fl.VI, 2.2

.. - S.A. - V. N.S.W. - - B.fl.VI,222

․ - - T. - - - - B.f.VI, 2.3

... W.A. - - - - - - B.fl.VI, 2.,

‥ W.A. - - - - - - B.fl.VI,223

W.A. - - - - - B.fl.VI,

... W.A. - - — - - - B.fl.VI, 2.24

... W.A. - - - - - - B.H.VI,224

.. - - - V. N.S.W. - - B.f.VI,224

… - $\mathrm{SAA}=-$ - $-\mathrm{Q}$.

de La Pérouse I, 155 (1798). (Exocarpus.)

... - - - - N.S.IT. Q. N.A. B.fl.T, 2.28

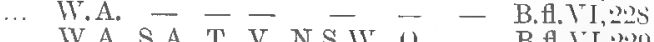
‥ W.A. S.A. T. V. N.S.IT. Q. - B.fl.VI. ... W.A. S.A. - V. N.S.W. (1. - B.fl.VI, 2.29 ‥ W.A. S.A. - V. N.S.W. Q. - B.f.YI.2:30 ... - - - - N.S.IW. - - B.H.VI,230 .. $\quad-\quad-\quad-$ N.S.W. ‥ - S.A. T. V. N.S.W. -. - - T. - - -

\section{LORANTHACEAE.}

A. L. de Jussieu, in Ann, du Mus, XII, 292 (1808).

VISCUM, Tournefort, inst. 609, t. 380 (1700), from Camerarius (1586).

V. orientale, Willdenow, spec. plant. IV, 737 (1805)

V. angulatum, Heyne in De Cand., prodr. IV, 283 (1830)

V. articulatum, N. L. Burmann, Fl. Ind. 311 (1768)
.. - - - - - - Q. - B.f.III,396
$\ldots-$ Q.

$\cdots-$-.. - - - N.S.W. Q. - B.H.III,390
B.A.III,390
B.fl. YI, 230

B. A. YI, $2: 31$

B. II. I I, 230
M.fr.I, 85;VIII, 11;IX,3.

MI.fr.VIII, 11.

M. fr. IX, 169;XI, 20 .

M.fr.I,86;IX, 3;XI, 20.

M.fr. VII, 11 .

M.fr. VIII,11;XI, 135.

M.fr. VIII, 11 .

M. fr. VIII, 10.

M.fr.VIII, 10

MI.fr. VIII, 10 .

MI.fr. VIII, 10.

M.fr. TIII, 10 .

M.fr. VIII, 10 .

M.fr. VIII, 10.

II.fr. VIII, 10.

M.fr. VIII, 11.

MI.fr. YIII, 10 .

M.fr.IX, 3.

M.fr.II, 181;IX, 3;XI, 20.

M. fr.IX, 3.

MI.fr.IX, 3.

M,fr, VIII, 10.

M.fr.VIII, 10.

M. fr. VIII, $10 ; X I, 20$. [20.

M.fr. VIIT, 10;IX, 169;XI,

Ml.fr. VIII, 9.

M.fr. 1 169.

Ml.fr. IX, 169. 
NOTOTHIXOS, Oliver in Journ. Linn. Soc. VII, 104 (1865).

$\mathrm{N}$. incanus, Oliver in Journ. Linn. Soc. VII, 104 (1865)...

LORANTHUS, Linné, syst. nat. ed. sec. $22(1740)$.

L. celastroides, Sieber in Roem. \& Schult. syst. veg. VII, 163 (1829)

L. Bidwillii, Bentham, Fl. Austr. III, 390 (1866).

- - - Q Q B.f.III, 390

L. longiflorus, Desrousseaux in Lam. Encjcl. méth. IV, $598(1796)--\quad-$ - N.S.W. Q. N.A. B.f1.III,390

L. angustifolius, R. Brown in Benth. Fl. Austr. III, 390 (1866) - S.A. - - - - - B.fl.IT, 390

L. dictyophlebus, F. v. M., Rep. Burdek. Exped. 14 (1858) ‥ - - - - N.S.W. Q. - B.f.III,391

L. alyxifolius, F. V. M. in Bentham Fl. Austr. III, 391 (1866) .. - - - - N.S.W. Q. - B.H.III,391

L. odontocalyx, F. v. M. in Bentham Fl. Austr. III, 391 (1866) - - - - - Q. N.A. B.Al.XII,391

L. linearifolius, Hooker in Mitchell, Trop. Austr. 102 (1848) ‥ W.A. S.A. - - - Q Q. - B.f.III,39l

L. Exocarpi, Behr in Schlecht. Linnaea XX, 624 (1847) ... … W.A. S.A. - V. N.S.W. Q. N.A. B.A.MI,392

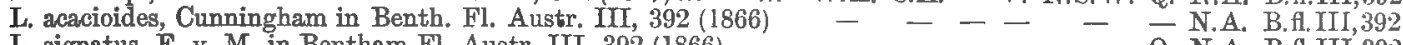

L. signatus, F. v. M. in Bentham Fl. Austr. III, $392(1866) \ldots-1-z_{-}-$Q. N.A. B.fl.III,39:

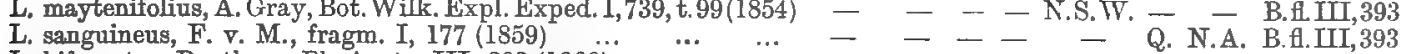

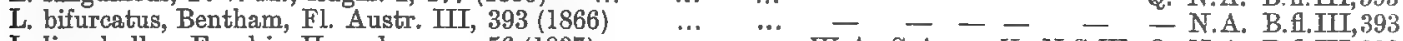

L. linophyllus, Fenzl in Huegel, enum. 56 (1837) ... ‥ … W.A. S.A. - V. N.S.WV. Q. N.A. B.f. II,393

L. pendulus, Sieber in De Candolle, prodr. IV, $294(1830) \quad \ldots$ T.A. S.A. - V. N.S. W. Q. N.A. B.A.III,394

L. Quandang, Lindley in Mitch. Three Exped. II, 69 (1838) … W.A. S.A. - V. N.S.W. Q. N.A. B.f.II,395

L. grandibracteus, F. v. M., Rep. Burdek. Exped. 14 (1858) ‥ - S.A. - — - Q. N.A. B.fl.III,395 ATKINSONIA, F. v. Mueller, fragm. V, 34 (1865).

A. ligustrina, F. v. M., fragm. V, $34(1865) \quad \ldots \quad \ldots$.
NUYTSLA, R. Brown in Journ, of the geogr. Soc. I, $17(1831)$

N. floribunda, R. Brown in Journ. geogr. Soc. I, 17 (1831) … W.A. - - - _ - - B.fl.III,387 M.fr.VI,252.

\section{PROTEACEAE.}

A. L. de Jussieu, gen. pl. 78 (1789).

PETROPHILA, R. Brown in Transact. Linn. Soc. X, 67 (1809).

P. teretifolia, R. Brown in Transact. Linn. Soc. X, 68 (1809) ... W. A

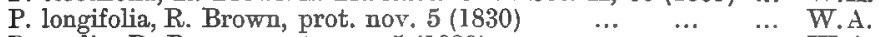

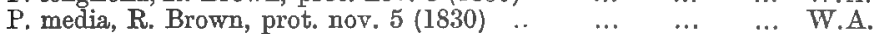

P. acicularis, $R$. Brown in Transact. Linn. Soc. X, 69 (1809) $\cdots$. W.A.

$\begin{array}{llllll}\text { P. megalostegia, F. } \nabla . \text { M., fragm. X, 61 (1876) } & \ldots & \ldots & \ldots & \text { W.A. } \\ \text { P. linearis, R. Brown, prot. nov. } 6(1830) & \ldots & \ldots & \ldots & \ldots & \text { W.A. }\end{array}$

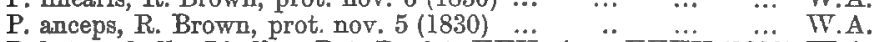

P. heterophylla, Lindley, Bot. Regist. XX

P. biloba, R. Brown, prot. nov. 7 (1830) $\ldots$... ... $\quad \ldots$. W.A.

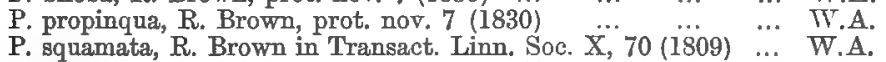

P. colorata, Meissner in Lehm. pl. Preiss. II, 246 (1847) ‥ W.A.

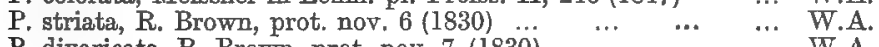

$\begin{array}{lllll}\text { P. divaricata, R. Brown, prot. nov. } 7(1830) & \ldots & \ldots & \ldots & \text { W.A. } \\ \text { P. Serruriae, R. Brown, prot. nov. } 6(1830) & \ldots & \ldots & \ldots & \text { W.A. }\end{array}$

$\begin{array}{lllll}\text { P. Serruriae, R. Brown, prot. nov. } 6(1830) & \ldots & \ldots & \ldots & \text { W.A. } \\ \text { P, inconspicua, Meissner in Hook, Kew IIisc. VII, } 68 \text { (1855) } & \ldots & \text { W.A. }\end{array}$

P. trifida, R. Brown in Transact. Linn. Soc. X, 70 (1809) $\ldots$. W.A.

$P$. carduacea, Meissner in Hook. Kew Misc. IV, 182 (1852) … W.A.

$P$. Shuttleworthiana, Meissner in Lehm. pl. Preiss. II, 246 (1847) W.A.

P. macrostachya, R. Brown, prot. nov. 7 (1830) ... … .. W.A.

P. diversifolia, $\mathbf{R}$. Brown in Transact. Linn. Soc. " $X, 70$ (1809)... W.A.

P. biternata, Meissner in Hook. Kew Misc. VII, 69 (1855) ... W.A.

P. plumosa, Meissner in Hook. Kew Misc. VII, 69 (1855) ‥ W.A.

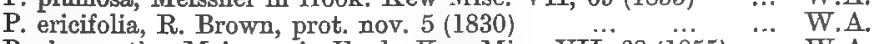

P. chrysantha, Meissner in Hook, Kew Misc. VII, 68 (1855) $\ldots$. W.A.

P. pedunculata, R. Brown in Transact. Linn. Soc. X, 70 (1809)

P. pulchella, R. Brown in 'Iransact. Linn. Soc. X, 69 (1809)

P. sessilis, Sieber in Roem. \& Schult. mant. 262 (1822)

P. fastigiata, R. Brown in Transact. Linn. Soc. X, 70 (1909) $\ldots$.

P. seminuda, Lindley, Bot. Regist. XXV, App. XXXIV (1839)

P. circinata, Kippist in Hook. Kew Misc. VII, 67 (1855)

. Drummondii, Meissner in Lehm. pl. Preiss. I, 496 (1845) ... W.A.

P. crispata, R. Brown, prot. nov. 6 (1830)..

P. rigida, R. Brown in Transact. Linn. Soc. X, 69 (1809)

P. multisecta, F. v. M., fragm. VI, $242(1868)$
P. conifera, Meissner in Hook. Kew Misc. VII, 67 (1855) $\quad \cdots$

P. semifurcata, F. v. M. in Bentham FI. Austr. V, 335 (1870) … W.A. - - ISOPOGON, R. Brown in Transact. Linn. Soc. X, 70 (1809).

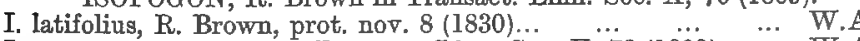

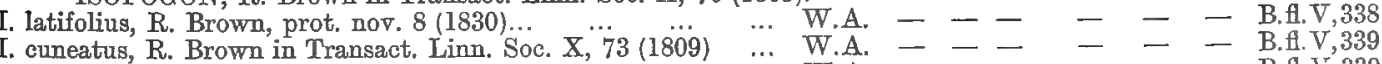

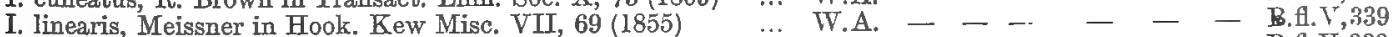

I. polycephalus, R. Brown in Transact. Linn. Soc. X, 73 (1809) W.A. - - - - - - B.甘.V.339

I. attenuatus, R. Brown in Transact. Linn. Soc. X, 73 (1809) T.A - - - - - B.V.

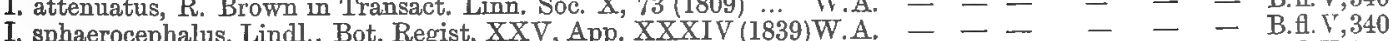

W A

I. buxifolius, R. Brown in Transact. Linn. Soc. $\mathrm{X}, 74$ (18̈09) $\quad \cdots \quad$ W.A.

I. axillaris, $R$. Brown in Transact. Linn. Soc. $X, 74$ (1809) $\cdots$ W.A.

I. tridens, F. v. M., fragm. VI, 239 (1868)... 
I. Baxteri, R. Brown, prot. nov, $9(1830) \ldots$.
I. roseus, Lindley, Bot. Regist. Misc. n. $37(1842)$
I. adenanthoides, Meissner in Hook. Kew Misc. VII, 69 (1855)

I. tripartitus, R. Brown, prot. nov. 8 (1830)

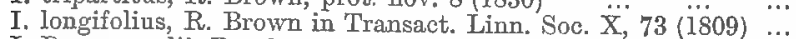

I. Drumpiondii, Bentham Fl Austr. V $344(1870)$

I. heterophyllus, Meissner in Lehm. pl. Preiss. I, 504 (1845) “.

I. villosus, Meissner in De Candolle, prodr. XIV, 277 ( , , ...

I. teretifolius, R. Brown in Transact. Linn. Soc. X, 71 (1809) ...
I. anethifolius, R. Brown in J. Knight, proteac. 94 (1809)

I. petiolaris, Cunningham in R. Br., prot, nov. 8 (1830)... ...

I. anemonifolius, $R$. Brown in J. Knight, prot. 93 (1809)

I. ceratophyllis, R. Brown in Transact. Linn. Soc. X, 72 (1809)

I. asper, $R$. Brown, prot. nov. 8 (1830)

I. formosus, R. Brown in Transact. Limn. Soc. X, 72 (1809)

I. divergens, $R$. Brown, prot. nov, 7 (1830)
I. scabriusculus, Meissner in Hook. Kew Misc. IV, 182 (1852) ...
I. trilobus, R. Brown in Transact. Linn. Soc. X, 72 (1809)

I. crithmifolius, F. v. M., fragm. VI, 239 (1868) $\ldots . .6 \%$

W.A. - - -

- S.A. T. - N.S.W. ADENANTHOS, Labillardière, Nov. Holl. pl. specim. I, 28, t. 36 (1804).

A. Detmoldi, F. v. M., fragm. VIII, 149 (1874) ... … … W.A. - -

A. barbigerus, Lindley, Bot. Regist. XXV, App. XXXVI (1839) W.A. - - -

A. obovatus, Labillardière, Nov. Holl. pl. spec. I, 29, t. 37 (1804) W.A. - - -

A. cuneatus, Labillardière, Nov, Holl. pl. spec. I, 28, t. 36 (1804)

A. Cunninghami, Meissner in Lehm. pl. Preiss, I, 513 (1845) ..

A. pungens, Meissner in Lehm. pl. Preiss. I, 515 (1845) ...

A. venosus, Meissner in Hook. Kew Misc. IV, 183 (1852)

A. Dobsoni, F. v. M., fragm. VI, 204 (1868) $2 \ldots 1$.

A. sericeus, Labillardière, Nov. Holl. pl. specim. I, 29, t. 38 (1804)

A. filifolius, Bentham, pl. Preiss. I, 515 (1845)

A. terminalis, $\mathrm{R}$. Brown in Transact. Linn. Soc. $\mathrm{X}, 152$ (1809)...

A. flavidiflorus, F, v. M., fragm. I, 157 (1859) ... ... ...

A. apiculatus, R. Brown, prot. nov. 9 (1830)

SIMSIA, R. Brown in Transact. Linn. Soc. X, 152 (1809). (Stirlingia.)

S. simplex, F.v.M.; Stirl., Lindl. Bot. Reg, XXV,App. XXX (1839)W.A

S. abrotanoides, F.v.M.; Stirlingia, Meissn. in pl. Preiss. I, 517 (1845)W.A.
S. teretifolia, F. v. M.; Stirlingia, Meissn. in pl. Preiss. 515 (1845)W.A.

S. tenuifolia, R. Brown in Transact. Linn. Soc, X, $152(1809) \ldots$
S. latifolia, R. Brown, prot. nov. $9(1830) \ldots$
...

SYNAPHEA, R. Brown in Transact. Linn. Soc. X, 155 (1809).

S. polymorpha, R. Brown in Transact. Linn. Soc. X, 156 (1809) W.

S. dilatata, R. Brown in Transact. Linn, Soc. X, 156 (1809) … W. W.

S. favosa, R. Brown in Transact. Linn. Soc. X, 156 (1809) ‥ W.

S. acutiloba, Meissner in Lehm. pl. Preiss, I, 528 (1845)...

S. petiolaris, $R$. Brown in Transact. Linn. Soc. X, 156 (1809) …

S. decorticans, Lindley, Bot. Regist. XXV, App. XXXII (1839)

S. pinnata, Lindley, Bot. Regist. XXV, App. XXXII (1839) ... CONOSPERMUM, Smith in Transact. Linn. Soc. IV, 213

C. capitatum, R. Brown in Transact. Limn. Soc. X, 155 (1809)...

C. petiolare, R. Brown, prot. nov. 11 (1830)

C. teretifolium, R. Brown in Transact. Linn. Soc. X, 155 (1809)

C. flexuosum, R. Brown, prot. nov. $11(1830)$
C. acerosum, Lindley, Bot. Regist. XXV, App. XXX (1839) $\quad \ldots$

C. amoenum, Meissner in Lehm. pl. Preiss. I, 522 (1845)

C. nervosum, Meissner in Hook. Kew Misc. VII, 71 (1855)

C. diffusum, Bentham, Fl. Austr. V, 367 (1870),

C. glumaceum, Lindley, Bot. Regist. XXV, App. XXX (1839)...

C. ephedroides, Kippist in Hook. Kew Misc. VII, 70 (1855)

C. Toddii, F. v. M., fragm. X, 20 (1876)

C. polycephalum, Meissner in Lehm. pl. Preiss, II, 249 (1847) ...

C. coeruleum, R. Brown in Transact. Linn. Soc. X, $154(1809) \ldots$

C' debile, Kippist in Hook. Kew Misc. VII, 70 (1855)

C. Scaposum, Bentham, Fl. Austr. V, 369 (1870) ... $\ldots$

C. densiflorum, Lindley, Bot. Regist. XXV, App. XXXII (1839)

C. Brownii, Meissner in Lehm. pl. Preiss, II, 248 (1847)

C. longifolium, Smith, Exot. Bot. II, 45, t. 82 (1805)

C. tenuifolium, R. Brown in Transact. Linn. Soc. $X, 154$ (1809)

C. Mitchellii, Meissner in De Candolle, prodr. XIV, 320 (1856)

C. sphacelatum, Hooker in Mitchell, Trop. Austr. 342 (1848) ...

C. patens, Schlechtendal, Linnaea XX, 587 (1847)

C. taxifolium, Smith in Rees's Cycl. IX (1808)

C. ericifolium, Smith in Rees's Cycl. IX (I80s)

$\begin{array}{llll}\text { C. ellipticum, Smith in Rees's Cycl. IX (1808) } & \ldots & \ldots & \ldots \\ \text { C. distichum, R. Brown in Transact. Linn. Soc. X, } 155 \text { (1809) } & \ldots\end{array}$

C. floribundum, Bentham, Fl. Austr. V, 373 (1870)

- $\quad-$ - N.S.W.
W.A. = - -

W.A. - - -

W.A. - - -

W.A. - - -

W.A. - - -

W.A. - - -

W.A. - - -

W.A. - - -

W.A.

W.A. --- N.S.W.
$-\quad-$ - - N.S.W.
- -

W.A. S.A. T. V.

W.A. - - -

W.A. - - -

W.A. - - -

W.A.

W.A. - -

W.A. - - -

W.A. - - -

W.A. - - -

W.A. - - -
W.A. - - -

W.A. $--\frac{}{V}$

S.A. - V.

A. - - -
$---$

- -

- -

- - -

A.

W.A.

W.A.

W.A.

W.A.

W.A.

W.A

W.A.

W.A.

W.A.

W.A.

W.A.

W.A.

W.A.

W.A.

W.A.

W.A.

W.A.

W.A.

W.A.

W.A.

W.A.

- - - - -

- - - N.S.W. -

- - - V

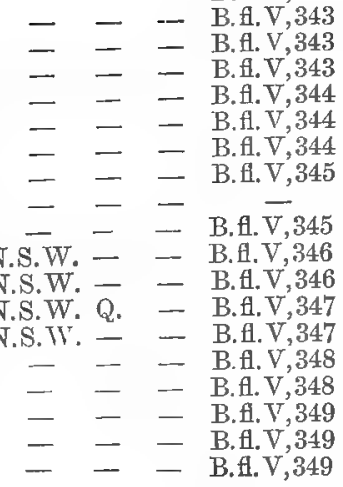

- S.A. - V. N.S.W. Q - T. V. N.S.W. Q

- - - - N.S.T.

- - - - N.S.W. -

W.A.

W.A. - - - - -
M.fr. VI, 240 .

M.fr. VI, 240

M.fr. VI, 241 .

M.fr. VI, 239.

M.fr.VI,237.

M.fr. VI, 241.

M.fr. VI,24l.

M.fr.VI, 241.

M.fr. VI, 241.

M. fr. VI,238.

M.fr.VI,238;X,90.

M.fr.VI, 238.

M.fr. VI, 240.

M.fr. VI,239.

M.fr. VI, 240 .

M.fr. VI, 241.

M.fr. VI, 240.

M.fr. VIII, 149.

M.fr. VI,205; VIII, 149

M.fr.VI,204,

M.fr. I, 157.

M.fr. VI, 224.

M.fr. VI, 224 .

M.fr.I,157;VI, 224.

M.fr. VI,224.

M.fr.X, 20,90.

M.fr. VI,224.

M.fr. VI,223.

M.fr.VI, 223.

M.fi. VI, 223 ,

M.fr. VI, 224 .

M.fr. VI, 224. 
C. incurvum, Lindley, Bot. Regist. XXV, App. XXX (1839) ‥ W.A. - - - - - - - - B.f.V.373 C. brachyphyllum, Lindley, Bot. Regist, XXV, App. XXXI (1839) W.A. - - - - - - - B.f.V.374

C. stoechadis, Endlicher in Ann. Wien. Mus. II, 208 (1838) ‥ W.A. - - - N.S.W. - - B.fl.V.374

C. triplinervium, R. Brown, prot. nov. 11 (1830) ... $\ldots$... W.A. - - - - - - - B.H.V.375

C. bracteosum, Meissner in Lehm. pl. Preiss. I, 518 (1845) $\ldots$. W.A. - - - - - - B.fl.V,375

C. crassinervium, Meissner in Hook. Kew Misc. IV, 184 (1852) W.A. - - - - - - B.f.V,375

FRANKLANDIA, R. Brown in Transact. Linn. Soc. X, 157 (1809).

F. fucifolia, R. Brown in Transact. Linn. Soc. X, 157 (1809) ‥ W.A. - - - - - - B.A.V,376

F. triaristata, Bentham, Fl. Austr. V, $377(1870) \ldots \quad \ldots \quad \ldots$. W.A. - - - - - - - B.f.V,377

SYMPHYONEMA, R. Brown in Transact. Limn. Soc. X, 157 (1809). (Symphionema.)

S. montanum, R. Brown in Transact. Linn. Soc. X, I58 (1809)... - - - - N.S.W. - - B.f.V,377

S. paludosum, R. Brown in Transact. Linn. Soc. X, $158(1809) \ldots-. . \quad--$ - N.S.W. - - B.f.V,378 BELLENDENA, R. Brown in Transact. Linn. Soc. X, 156 (1809).

B. montana, R. Brown in Transact. Linn. Soc. X, $166(1809)$.. - - T. - - - - - B.fl.V,378 AGASTACHYS, R. Brown in Transact. Limn. Soc. X, 158 (1809).

A. odorata, R. Brown in Transact. Linn. Soc. X, $158(1809)$.. - - T. - - - - B.fl.V,379 CENARRHENES, Labillardière, Nov. Holl. pl. spec. I, 36, t. 50 (1804).

C. nitida, Labillardière, Nov. Holl. pl, spec. I, 36, t. $50(1804) \ldots$ - . - T. - - - - B.A.V,380 PERSOONIA, Smith in Transact. Linn. Soc. IV, 215 (1798). (Linkia, 1797, Pentadactylon.)

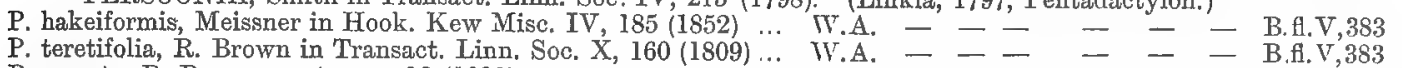

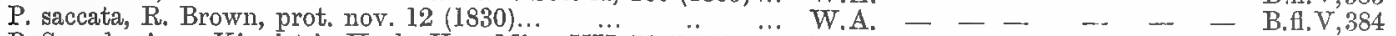

P. Saundersiana, Kippist in Hook. Kew Misc. VIII, 72 (18̈55) $\ldots .$. W.A.
P. diadena, F. v. M., fragm. X, 46 (1876) ...

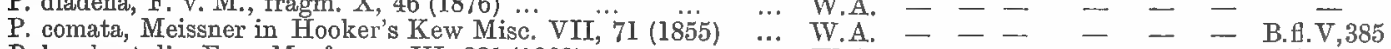

P. brachystylis, F. v. M., fragm. VI, $221(1868) \ldots . \quad \ldots \quad \ldots$. W.A. - - - - - - - - B.f.V,385

P. falcata, R. Brown in Transact. Lim. Soc. X, $1626(1809) \quad \cdots \quad-\quad--\quad-\quad$ Q. N.A. B.A.V,385

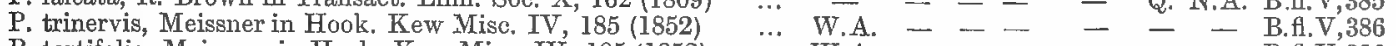

P. tortifolia, Meissner in Hook. Kew Misc. IV, 185 (1852) $\quad \ldots$ W.A. - - - - - - - B.f.T,386

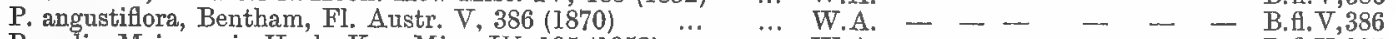

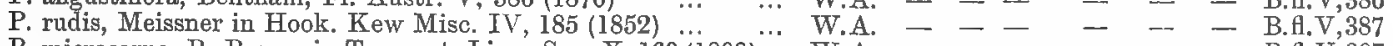

P. microcarpa, R. Brown in Transact. Linn. Soc. X, 160 (1809)... W.A. - - - - - - B.A.V,387

P. sulcata, Meissner in Hook. Kew Misc. IV, 185 (1852) $\quad$.. W.A. $\quad-\quad-\quad-\quad-\quad-\quad$ - B.fl.V,387

P. acicularis, F. v. M., fragm. VI, $220(1868) \quad \ldots \quad \ldots \quad \ldots$, W.A. $\quad$ - $\quad$ - $\quad$ - $\quad$ - $\quad$ - $\quad$ B.f.V.388

P. scabrella, Meissner in Hooker's Kew Misc. VII, 72 (1855) … W.A. - - - - - - - B.f.V,388

P. dillwynioides, Meissner in Hook. Kew Misc. IV, IS5 (1852) W.A. - - - - - - - B.t.V,388

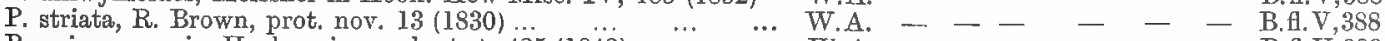

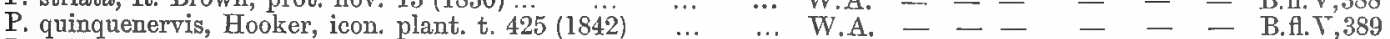

P. rufifiora, Meissner in Hooker's Kew Misc. VII, 72 (1855) $\quad$ … W.A. - - - - - - - B.fl.V,389

P. scabra, R. Brown in Transact. Lim. Soc. X, 162 (1809) … W.A. - - - - - - B.f.V,389

P. graminea, $R$. Brown in Transact. Linn. Soc. X, $164(1809)$... W.A. - - - $-\quad$ - - B.fl.V,390

P. longifolia, R. Brown in Transact. Iinn. Soc. X, 164 (1809) ... W.A. - - - _ - - B.f.V,390

P. articulata, R. Brown in Transact. Linn. Soc. X, 164 (1809) ... W.A. - - - - - - B.A.V.,390

P. elliptica, R. Brown in Transact. Linn. Soc. X, 164 (1809) ... W.A. - - - $-\quad$ - - B.fl.V,391

P. ferruginea, Smith, Exot. Bot. II, 47, t.83(1805) $\ldots$.. - - - - - N.S.T. - - B.f.V,391

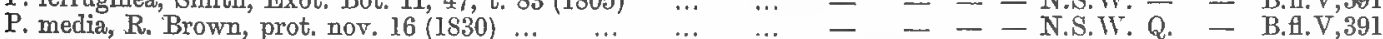

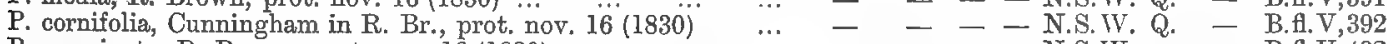

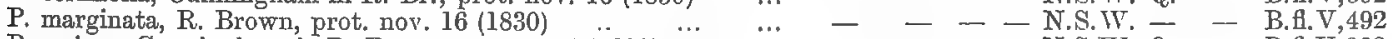

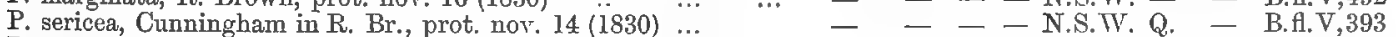

P. Mitchellii, Meissner in Hook. Kew Misc. VII, 73 (1809) ‥ - - - - N.S.IV. Q. - - B.H.V,393

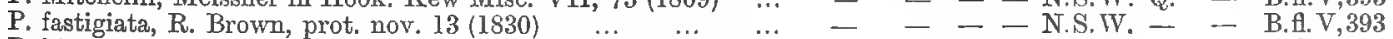

P. hirsuta, Persoon, synops. plant. I, 118 (1805) ... $\ldots \quad \ldots \quad-\quad-\quad-$ - N.S.IV. - - B.f.V,394

P. Chamaepithys, Cunningham in Field, N.S. Wales, 329 (1825) - - - - N.S.W. - - B.f.V,394

P. arborea, F. . M. fram. V, 37 (I865)

P. salicina, Persoon, synops. plant. I, $118(1805) \ldots$
P. prostrata, $\mathrm{R}$. Brown in Transact. Linn. Soc. X, I63 (is09) $\ldots$

P. lanceolata, Andrews, Bot. Reposit. 74 (1800)

P. confertiflora, Bentham, Fl. Austr. V, 396 (1870)

P. lucida, R. Brown in Transact. Linn. Soc. X, 161 (1809)

P. linearis, Andrews, Bot. Reposit. t. 77 (1800) $\cdots$. .

P. Caleyi, R. Brown, prot. nov. 13 (1830) ... ... ... $\ldots$

P. ledifolia, Cunningham in De Cand. prodr. XIV, 339 (I 1856$) \ldots$

P. revoluta, Sieber in Roem. \& Schult. mant. 272 (1822)

P. Gunnii, J. Hooker in Lond. Journ. Bot. VI, 283 (1847)

P. mollis, R. Brown in Transact. Lim. Soc. X, 161 (1809)

P. rigida, R. Brown, prot. nov. 14 (1830) ...

P. curvifolia, R. Brown, prot. nov. 13 (1830)

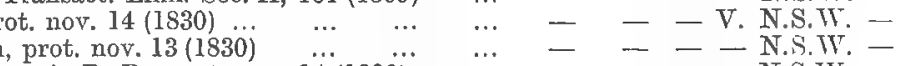

P. oblongata, Cunningham in $\mathrm{R}$. Br. prot. nov. 14 (1830)

P. Cunninghamii, R. Brown, prot. nov. 15 (1830)

P. myrtilloides, Sieber in Roen. \& Schult. mant. 272 (1822)

P. oxycoccoicles, Sieber in Roem. \& Schult. mant. 270 (1822)

P. nutans, R. Brown in Transact. Linn. Soc, X, 162 (1809)

P. angulata, R. Brown, prot. nov. 14 (I830)

P. virgata, $R$. Brown in Transact. Linn. Soc. X, 161 (1809)

P. Chamaepence, Lhotsky in De Cand. prodr. XIV, 336 (1856)...

P. juniperina, Labillardière, Nov. Holl. pl. spec. I, 33, t. 45 (1804)

P. tenuifolia, R. Brown, prot. nov. 12 (1S30)

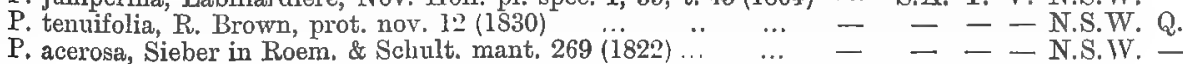

- B.H. $\mathrm{T}, 394$

B.fl.V, 395

- - V.N.S.T. -

$-\quad-$ N.S.W. Q.

- - V. T. -

- - - N.S.W.

- - - N.S.W.

- - - N.S.W. .-

- - - N.S.TW. -

- - - N.S.W. -

- T. - N.S.W. -

- - - N.S.W. -

- - - V. N.S.W. -

- - N.S.TI.

- - - N.S.W -

- - - N.S.T. -

- - - N.S.W. Q.

S.A. T. V. N.S.W. -

B. 1 . $V, 395$

- B. H.V,395

- B.fl.V,396

- B.fl.V,396

- B.fl.V,397

- B.fl.V,397

- B.fi.V,398

- B.H.V,398

- B.fl.V,398

- B.fl. $r, 399$

- B.tl.V,399

- B.fl.V,399

- B.f.V.,400

- B.fl.V, 400

- B.fl.V, 400

- B.A.T.401

- B.fl. T, 401

- B.f. T.40I

- B.fl. V, 402

- B.fi.V,402

- B.t. T,402

- B.fl.V, 403

- B.f.V, 403

M. fr.XI, 20.

M.fr. VI, 224.

MI.fr. VI, 223;VII, 133.

M.fr. VI,223.

M.fr. VI, 223 .

M.fr. X, 90 .

M.fr. VI, 223 .

M.fr. VI, 223.

M.fr. VI, 223 .

M.fr.X, 46 .

M.fr. VI,222,

M.fr. VI,222.

M.fr. VI,220.

M.fr. VI, 222,

M.fr.VI,223.

M.fr. VI, 222.

M.fr. VI, 222 .

M.fr. VI, 222.

M.fr. VI, 222.

M.fr. VI, 223 .

M.fr.V,37;VI, 221 .

M.fr. VI,222.

M.fr.VI, 222.

M.fr. $\mathrm{II}, 222 ; \mathrm{X}, 47$.

M.fr.VI,221.

M.fr.VI,221.

M.fr. VI, 221; VIII, $149, \mathrm{X}$,

NI.fr. VI, 221.

Ml.fr.VI, 221 .

M.fr.VI, 220 .

MI.fr. VIII, 149;X,47.

M.fr. TI,222. 
MACADAMIA, F. v. M. in Transact. phil. Inst. Vict. II, 72 (1857). (Panopsis partly.)

M. ternifolia, F. v. M. in Transact. phil. Inst. Vict. II, $72(1857)---$ N.S.W. Q. HELICIA, Loureiro, Fl. Cochinch. I, 83 (1790).

H. Youngians, C. Moore \& F $\mathrm{T}$ M fragm IV, 84 (1864)

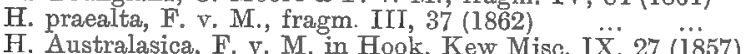

H. glabriflora, F. v. M., fragm. II, 91 (1861) ... ...

H. ferruginea, F. v. M., fragm. III, 37 (1862)

ROUPALA, Aublet, Hist. des pl, de la Guian. I, 83, t. 32 (1775).

R. Bleasdalei, F. v. M. in proceed. Roy. Soc. N.S. Wales, 28 (1881) -

XYLOMELUM, Smith in Transact. Linn. Soc. IV, 214 (1798).

X. pyriforme, Smith in J. Knight, prot. 105 (1809)

X. occidentale, $R$. Brown, prot. nov. 31 (1830)

$\mathrm{X}$. salicinum, Cunningham in $\mathrm{R}$. Br. prot. 31 (1830)

X. angustifolium, Kippist in Hook. Kew Misc. IV., 209 (1852)... W.A. LAMBERTIA, Smith in Transact. Linn. Soc. IV, 214 (1798).

L. uniflora, R. Brown in Transact. Linn. Soc. X, 188 (1809) ‥ W.A.

L. rariflora, Meissner in Lehm. pl. Preiss. II, 263 (1847) ‥ W.A.

L. inermis, R. Brown in Transact. Linn. Soc. X, 188 (1809) ‥ W.A.

L. ericifolia, R. Brown, prot. nov. 30 (1830)

L. multiflora, Lindley, Bot. Regist. XXV, App. “̈XXII (1839)

L. formosa, Smith in Transact. Linn. Soc. IV, 214, t. 20 (1798)

L. echinata, R. Brown in Transact. Linn. Soc. X, 189 (1809) ...

L. ilicifolia, Hooker, icon. plant. t. 553 (1843)

ORITES, R. Brown in Transact, Linn. Soc. X, 189 (1809).

O. excelsa, R. Brown, prot. nov. 32 (1832) ...

O. diversifolia, $R$. Brown in Transact. Linn. Soc. $\dddot{X}, 190$ (1809)

O. Milligani, Meissner in Hook. Kew Misc. IV, 209 (1852)

O. lancifolia, F. จ. M. in Transact. phil. Soc. Vict. I, 108 (1855)

o. revoluta, $R$. Brown in Transact. Linn. Soc. X, $19(1809) \quad \ldots$

o. acicularis, R. Brown, prot. nov. 32 (1830) STRANGEA, Meissner in Hooker's Kew Misc. VII, 66 (1855).

S. linearis, Meissner in Hook. Kew Misc. VII, 66 (1855)

S. cynanchocarpa, F. v. M., fragm. VII, 132 (1871) GREVILLEA, R. Brown in Transact. Linn. Soc. X, 168 (1809).

G. Pinaster, Meissner in Hook. Kew Misc. VII, 76 (1855)

G. obtusifolia, Meissner in Hook. Kew Misc. IV, 187 (1852)

G. sparsiflora, F. v. M., fragm. VI, 206 (1868)

G. macrostylis, F. v. M., fragm. I, 137 (1859)

G. tripartita, Meissner in Hook. Kew Misc. IV, 186 (1852)

G. platypoda, F, v. M., fragm. VI, 205 (1868)

G. patentiloba, F. v. M., fragm. I, 137 (1859)

G. pectinata, R. Brown, prot. nov. 23 (1830)

G. plurijuga, F. v. M., fragm. IV, 84 (1864)

G. nudifiora, Meissner in Hook. Kew Misc. IV, 1066 (1852)

G. stenomera, F. v. M., fragm. IV, 85 (1864)
G. Thelemanniana, Endlicher, nov. stirp. dec. 6 (1839) ...

G. concinna, R. Brown in Transact. Linn. Soc. X, 172 (1809)

G. Hookeriana, Meissner in Lehm. pl. Preiss. I, 546 (1845)

G. Baxteri, R. Brown, prot. nov. 22 (1830)

G. eriobotrya, F. v. M., fragm. X, 44 (1876)
G. pterosperma, F. v. M. in Transact. phil. Soc. $\ddot{V}$ ict. I, $22(1854)$

G. stenobotrya, F. v. M., fragm. IX, 3 (1875)

G. eriostachya, Lindley, Bot. Regist. XXV, App. XXXV̈I (1839)

G. thyrsoides, Meissner in Hooker's Kew Misc. VII, 77 (1855)

G. Chrysodendron, R. Brown in Transact. Linn. Soc. X, 176 (1809)

G. Banksii, R. Brown in Transact. Iinn. Soc. X, 176 (1809)

G. Caleyi, R. Brown, prot. nov. 22 (1830)..

G. asplenifolia, R. Brown in J. Knight, prot. 120 (1869)...

G. cirsiifolia, Meissner in Lehm. pl. Preiss. II, 253 (1847)

G. laurifolia, Sieber in Roem. \& Schult. mant. 279 (1822)

G. Barklyana, F. v. M., gen. report I4 \& 18 (1861)

G. repens, F. v. M. in Schlecht. Linnaea XXVI, 355 (18̈3)

G. Aquifolium, Lindley in Mitchell, Three Exped. IT, 178 (1838)

G. ilicifolia, R. Brown, prot. nov. 21 (1830)

G. Gaudichaudii, R. Brown in Freyc. Voy. Bot. $\dddot{4} 43$, t. $\dddot{4} 6$ (1826)

G. acanthifolia, Cunningham in Field, N.S.Wales, 328 (1825) ...

G. bipinnatifida, $R$. Brown, prot. nov. 23 (1830)

G. armigera, Meissner in Hook. Kew Misc. IV, 186 (1852)

G. asparagoides, Meissner in Hook. Kew Misc. IV, 186 (1852) ...

G. Treueriana, F. r. M., fragm. IX, 123 (1875)

G. cinerea, R. Brown in Transact. Linn. Soc. X, 173 (1809) ...

G. alpina, Lindley in Mitchell, Three Exped. II, 179 (1838) $\ldots$

G. montana, R. Brown in Transaet. Linn. Soc. $\mathbf{X}, 172(1809) \quad \ldots$

G. obtusiflora, R. Brown, prot. nov. 19 (1830)

G. arenaria, R. Brown in Transact. Linn. Soc. X, $172(1809) \quad \ldots$

G. mucronulata, R. Brown in Transact. Linn. Soc. X, 173 (1809)
W.A. - - - - - B.A.V,414

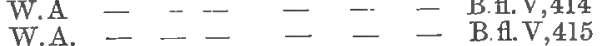

W.A. - - - N.S.W. - - B.fl.V,415

W.A. - - - - - B.fl.V,416

W.A. - - - - - - B.H.V,416

(Oritina.)

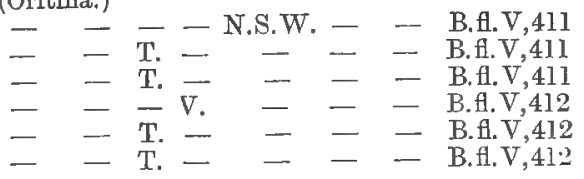

(Molloya.)

- - - N.S.W. Q. - B.f.V, 453

809). (Anadeuia, Lyssanthe, Stylurus, Manglesia.)

W.A. - - - - - - B.H.V,427

W.A. - - - - - B.H.V,427

W.A. - - - - - - B.f.V,428

W.A. - - - - - B.fl.V,428

W.A. - - - _ - - B.fl.V,428

W.A. - - - - - - B.fl.V,428

W.A. - - - - - B.tl.V,429

W.A. - - - - - - B.fl.V,429

W.A. - - - - - B.f.V.430

W.A. - - - - - - - B.H.V,430

W.A. - - - - - B.fl.V,430

W.A. - - - - - B.A.V,431

W.A. - - - - - - B.fl.V,431

W.A. - - - - - - - B.fl.V,432

W.A. - - - - - B.A.V,432

W.A. S.A. - - T. N.S.WV. - - B.f. $\bar{V}, 432$

- S.A. - - - - N.A

- S.A. - - - - N.A. B.A.T, 433

W.A. - - - - - B.f.V, 433

Q. N.A. B.fl. V, 434

- - - - - Q. N.A. B.fl., 434

- - - - N.S.T. - - B.f.V, 435

- - - N.S.W. - - B.fl.V,435

W.A. - - - - B.f.V,436

- - - N.S.W. - - B.fl.V,436

- - - V. - - - B.H.V,436

- - - V. - - - B.fi.T, 437

- - - V. - - - B.fl. $\mathrm{V}^{\mathrm{T}, 437}$

- S.A. - V. - - - B.fl.V,437

- - - - N.S.W. - - B.G.V,438

W.A. - - - N.S.W. - - B.fl. Y,438
- B.f.V,439

W.A. - - - - - - B.A.V,439

W.A. - - - - - - B.fl.V,439

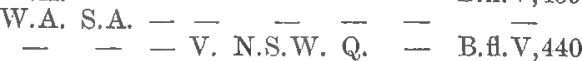

- - - - - N.S.W. - - - B.f.V.V,440

- - - V. - - - B.fl.V,44l

- - - - N.S.W. - - B.H.V,441

- - - - N.S.W. - - B.f.V, 442

- - - - - N.S.W. - - - B.W. B.I. .
[VII,59.

M.fr.II,9I; VI, 191;224,

M.fr. V, 186.

M.fr.IV, $174 ; V_{3}, 224$.

M.fr.II,91.

M.fr. V.38, 186. [224.

M.fr.IV, 174;V, 186;VI,

artly.)

M.fr. V, 90 .

M.fr. VI,220.

M.fr.IV,107,174;VI,220.

M.fr. VI, 220 .

M.fr. VI,248.

M.fr. VI,248.

M.fr.VI,248.

M.fr. VII, 133.

M.fr. VI,248.

M.fr. V,153; VI,223.

M.fr. V,153.

M.fr. V, 153;VI, 223;VII,

M.fr.VI,223;VII, 132.

M. fr. VII, 132 .

M.fr. VI,2I2.

M.fr.VI, 206.

M.fr. VI,211.

M.fr. $V I, 213$.

M.fr. VI, 205.

M.fr.I,137.

M. fr. VI, 212 .

M.fr.VI, 212 .

M.fr.I,35;VI,212.

M.fr.IV, 85.

M.fr. VI,212.

M.fr.VI, 212.

M.fr.VI,212.

B.fl. X, 44 .

M.fr. VI, 210

M.fr. IX, 3.

II.fr.VI,208\&213.

M.fr. VI,2l0.

[46.

M.fr.VI,210;VIII, 150; X,

M.fr. VI, 212.

$[\mathrm{X}, 46$.

II.fr.VII,133;VIII,150;

MI.fr. VI, 212.

MI.fr.VI,212; VIII, 150 .

M.fr. VI, 212 .

M.fr.IX, 123.

M. fr.IX, 123. 
(VIT) G. Baneri, R. Brown in Transact. Linn. Soc. X, 173 (1809)

G. lanigera, Cunningham in $\mathrm{R}$. Br., prot. nov. 20 (1830)..

G. rommarinifolia, Cunningham in Field, N.S. Wales, 328 (1825)

G. Goodii, R. Brown in Transact. Linn. Soc. X, 174 (1809)

In G. venusta, $R$. Brown in Transact. Linn. Soc. $X, 175$ (1809)

G. longistyla, Hooker in Mitchell, Trop. Austr. 343 (1848)

y. Gilsoni, Cunningham in Wilson's Voy. 273 (1835)

G. erectiloba, F, v. M., fragm. X, 44 (1876)

G. lavaudulacea, Schlechtendal, Linnaea XX, 586 (1847)

G. insignis, Kippist in De Cand., prodr. XIV, 379 (1856)

G. Brownii, Meissner in Lehm. pl. Preiss. I, 537 (1845) ...

G. fasciculata, R, Brown, prot. nov. 20 (1830)

G. aspera, R. Brown in Transact. Linn. Soc. X, 172 (1809)

is G. brachystylis, Meissner in Lehm. pl. Preiss. I, 538 (1845)

G. saccata, Bentham, Fl. Austr. V, $450(1870)$

G. Drummondii, Meissner in Lehm. pl. Preiss. I, 536 (1845)

G. disjuncta, F. v. M., fragm. VI, 206 (1868)

G. haplantha, F. v. M. in Bentham Fl. Austr. V, 451 (1870)

G. pinifolia, Meissner in Hook. Kew Misc. 186 (1852) …

G. acuaria, F. v. II. in Bentham Fl. Austr. V, 452 (1870)

G. singuliflora, F. r. M., fragm. VI, 92 (1868)

G. pauciflora, R. Brown in Transact. Lino. Soc. $\dddot{X}, 171$ (1809)...

G. quercifolia, R. Brown, prot. nov, 23 (1830)

G. angulata, R. Brown, prot. nov, 24 (1830)

G. Wickhami, Meissner in Hook. Kew Misc. 187 (1852)

G. agrifolia, Cunningham in R. Br., prot. nov. 24 (1830)

G. Cunninghamii, R. Brown, prot. nov. 23 (1830)..

G. pungens, R. Brown in Transact. Linn. Soc. X, 175 (1809)

G. Huegelii, Meissner in Lehm. pl. Preiss. I, 543 (I845)...

G. dimidiata, F. v. M., fragm. III, 146 (1863)

G. heliosperma, R. Brown in Transact. Linn. Soc. X, 176 (1809)

G. refracta, R. Brown in Transact. Linn. Soc. X, 176 (1809)

G. Dryandri, R. Brown in Transact. Linn. Soc. X, 175 (1809) ...

G. polystachya, R. Brown in Transact. Linn. Soc. X, 177 (1809)

G. robusta Cunningham in $\mathrm{R}$. Br., prot. nov, 24 (1830)...

G. annulifera, F. r. M., fragm. IV, 85 (1864)

G. leucopteris, Meissner in Hook. Kew Misc. VII, 76 (1855) $\ldots$

G. Leucadendron, Cunningham in R. Br., prot. nov, 25 (1830)...

G. pyramidalis, Cunningham in $\mathrm{R}$. Br., prot. nov. 25 (1830) ...

G. striata, R. Brown in Transact. Linn. Soc. X, 177 (1809)

G. mimosoides, R. Brown in Transact. Linn. Soc. X, 177 (1809)

G. Hilliana, F. r. M. in Transact. phil. Inst. Vict. II, 72 (I857)

G. gibbosa, R. Brown in Transact. Linn. Soc. X, 177 (1809) ...

G. buxifolia, R. Brown in Transact. Linn. Soc. X, 174 (1809)

G. phylicoides, R. Brown in Transact. Linn. Soc, X, 174 (1809)

G. sphacelata, R. Brown in Transact. Linn. Soc. X, 174 (1809)..

G. occidentalis, R. Brown in Transact. Linn. Soc. X, 173 (1809)

G. acerosa, F, v. M., fragm. I, 136 (1859) ...

G. umbellulata, Meissner in Lehm. pI. Preiss. II, 252 (18̈7) …

G. oxystigma, Meissner in Lehm. pl. Preiss. I, 540 (1845)

G. Candolleana, Meissner in Lehm. pl. Preiss. I, 541 (1845) ...

G. scabra, Meissner in Lehm. pl. Preiss. I, 541 (1845)

G. Miqueliana, F. v. M. in Transact. Vict. Inst. 132 (1855)

G. Victoriae, F. V. II. in Transact. phil. Soc. Vict. I, 107 (1865)

G. punicea, R. Brown in Transact. Linn. Soc. X, 169 (1809) ...

G. oleoides, Sieber in Roem. \& Schult. mant. 277 (1822)...

G. trinervis, R. Brown, prot. nov. 18 (1830) $\ldots$ ……

G. sericea, R. Brown in Transact. Linn. Soc. X, 170 (1809) ...

G. capitellata, Meissner in Hook. Kew Misc. IV, $187(1852) \quad \ldots$

G. leiophylla, F. V. M. in Bentham, Fl. Austr. V, 471 (1870) ...

G. Inearis, R. Brown in Transact. Linn. Soc. X, 170 (1809) ...

G. parviflora, R. Brown in Transact. Linn. Soc. X, 171 (1809) ...

G. australis, R. Brown in Transact. Linn. Soc. $\mathrm{X}, 171$ (1809) ...

G. commutata, F. v. M., fragm. VI, 207 (1868)

G. pinnatisecta, F. $\nabla . M$. in in Bentham, Fl. Austr. V, 473 (1870)

G. argyrophylla, Meissner in Hook. Kew Misc. VII, 75 (1855)

G. brachystachys, Meissner in Lehm. pl. Preiss. II, 254 (1847) ..

G. Endlicheriana, Meissner in Lehm. pl. Preiss. I, 546 (1S45) ...

G. manglesoides, Meissner in Lehm. pl. Preiss. I, 547 (1845) ...

G. diversifolia, Meissner in Lehm. pl. Preiss. I, 547 (1845)

G. filifolia, Meissner in Lehm. pl. Preiss. I, 547 (1845)

G. hakeoides, Meissner in Lehm. pl. Preiss. II, 252 (1847)

G. teretifolia, Meissner in Lehm. pl. Preiss. II, 255 (1847)

G. eryugioides, Bentham, Fl. Austr. V, 476 (1870)

G. bracteosa, Meissner in Lehm. pl. Preiss. II, 254 (1847)

G. crithmifolia, R. Brown, prot. nov. 23 (1830)

G. trachytheca, F. v. M., fragm. VI, 207 (1868) ..

(t. triternata, R. Brown, prot. nov. 21 (1830)
- - N.S.W.
- - N.S.W.
- - N.S.W.

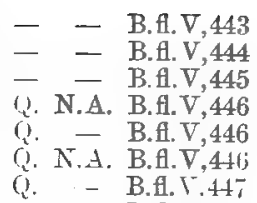

W.A. S.A. - - N.S.W. Q. - B.fl.V.447

W.A. $\overline{\text { W.A. }}$ - T. N.S.T. - - B.f. T. 448

W.A. - - - N.S. - - B.fl.V,448

W.A. - - - - - - B.H.V.449

W.A. - - - - - - B.fl.V,449

W.A.S.A. - - - - - B.H.V,450

W.A. - - - - - - B.A.V,450

W.A. - - - - - B.H.T,450

W.A. - - - - - - B.H.V,451

W.A. - - - - - - B.Al.V.45]

W.A. - - - - - - B.f.T.452

W.A. - - - - - B.fl.V.4.

W.A. - - - - - B.f.V.452

W.A. S.A. - - Q. - B.f.V.,452

W.A. - - - - - B.fl.V,454

- S.A. - - - - N.A. B. B.f.T,4,455

- S.A. - - - - N.A. B.fl.V,455

- - - - - - N.A. B.H.V,455

- - - - - - N.A. B.fl.V,456

IV.A. S.A. - V. N.S.W. - N.A. B.fl. V,456

- - - - - I N.A. B.fl.V,457

- - - - - N.A. B.f.V,457

- - - - - - Q N. N.A. B.ff.V, B.fi.V,458

- - - - - Q. N.A. B.H.V,459

- - - N.S.W. Q. - B.H.V,459

W.A. - - - - - - B.fl.., 460

- - - - - - N.A. B.H. Y,461

- - - - - N.A. B.fi.V,462

- S.A. - - N.S.W. Q. N.A. B.f.V.462

- - - - - - N.A. B.H. T,462

- - - - N.S.T.Q. - B.H.V,463

- $\quad-\quad-$ N.S.W. Q. N.A. B.f.V.,463

- - - - N.S.W. - - B.A.V,4 4

- - - N.S.W. - - B.fl.V,464

W.A. - - - - - - B.H.V,465

IV.A. - - - - - - ${ }_{\text {B.H. } .465}$

W.A. - - - - - B.fl.V,466

W.A. - - - - - - B.f.V,466

W.A. - - - - - - B.f.V,466

- - - V. - - - B.A.V,467

- - - - V.N.S.W. - - - B.f.V.468

- - V. N.S.W. -

- - - N.S.W.

- B.fl.., 468

- B.fl. $\nabla, 468$

- B.A. T,469

- B.fl.V,469

- - - N.S.T. - - B.fl.V,470

- - - N.S.W.

- - - N.S.W. -

S.A. - V. N.S.W. -

- S.A. T. V. N.S.W. -

W.A.

W.A.

IV.A.

IV.A.

W.A.

W.A.

IV.A.

W.A.

IV.A.

W.A.

W.A.

WT.A.

W.A.

-
-
-
-
-
-
-
-
-
-

$-\quad-$

-

B. fl. V, 471

B.A.V, 471

B.fl.V, 471

B.fl.V, 472

B.tl.V, 472

B.fl. $Y, 473$

B.fl. 7,473

B.t.V,474

B.fl. T, 4.4

B.H.V, 4 .t

B. 1 . $T, 475$

B.fl. $\nabla, 475$

B. H. V, 475

B.fl. $Y, 4,6$

B. A. $V, 4,6$

B.tl. V, 476

B.fl.V, 477

B. $f$. V, 477

B. fl. Y, 477

B. $\mathrm{H} . \mathrm{T}, 4-\mathrm{s}$

\section{M.fr. VI,211.}

M.fr. YT, 211.

M.fr. TI, 209 ; I III, 150

M.fr. $Y I, 2 l: 2 ; \mathrm{X}, 45$.

M.fr.X, 41.

M.fr. VIII, I50;X, 45 .

M.fr. VI, 213.

II.fr. VI,211.

MI.fr.VI, 206.

M.fr. TI, 92.

M.fr.I,135;VI,206,208.

M.fr. VI, 213.

MI.fr. VI. $212 ; \mathrm{X}, 46$.

II.fr. III, 146.

M.fr. VI,2IO.

M.fr. YI, 209.

M.fr.TI, 210 .

I.fr.YI.210; VII.133. [XI.20.

.T , 55 ; VI, 212;VII, 13.

M.fr.III, 145;IV, 85, 176;
M.fr.I, 136-7. [XI, 20

MI.fr.VI,210.

M.fr. VI,213.

M.fr.I, 136.

M.fr. VI,213.

M.fr. VIII, 142, 150.

II. fr. XI, 123.

II. fr. VI,211.

M.fr. VI,211.

M.fr.VII, 133 .

M.fr.VII, 207.

M.fr. TI, 411.

M.fr. VI, 211 ,

M.fr. YI, 211 .

N.fr, X, 46 .

M.fr. VI, 20 - 
G. ramosissima, Meissner in De Candolle, prodr. XIV, 388

G. monticola, Meissner in Lehm. pl. Preiss. II, 259 (1847)

G. Muelleri, Bentham, Fl. Austr. V, 479 (1870)

G. trificla, Meissner in Lebm. pl. Preiss. I, 553 (1845) ...

G. Synapheae, R. Brown, prot. nov. 23 (I830)

G. flexuosa, Meissner in Lehm. pl. Preiss. I, 553 (1845) ...

G. Ieptobotrya, Meissner in Lehm. pl. Preiss. II, 256 (1847)

G. brevieuspis, Meissner in Lehm. pI. Preiss. II, 256 (1847)

G. intricata, Meissner in Hook. Kew Misc. VII, 74 (1855)

G. didymobotrya, Meissner in Hook. Kew Misc. IV, 186 (1852)

G. polybotrya, Neissner in Hook. Tiew Mise. IV, 185 (I852)

G. nematophylla, F. v. M., fragm. I, 136 (1859) ...

G. anethifolia, R. Brown, prot. nov. 21 (1830)

G. paradoxa, F. v. I., fragm. VI, 246 (1868)

G. petrophiloides, Meissner in Lehm. pl. Preiss, II 257 (1847)...

G. tenuitlora, Meissner in Lehm. pl. Preiss. I, 554 (1845)

G. pulchella, Meissner in Lehm. pl. Preiss. I, 553 (1845)

G. rudis, Meissner in Hook. Kew Misc. VII, 73 (1855) ..

G. apiciloba, F. v. M., fragm. X, 45 (1876)

G. Shuttleworthiana, Meissuer in Lehm. pl. Preiss. IT, 258 (1847)

G. integrifolia, Meissuer in De Candolle, prodr. XIV, 385 (1856)

G. stenocarpa, F. v. M. in Bentham Fl. Austr. V, 485 (1870)

G. acrobotrya, Meissner in Hooker's Kew Misc. VIII, 74 (1855)

G. glabrata, Meissner in Lehm. pl. Preiss. I, 549 (1845)...

G. ornithopoda, Meissner in Lehm. pl. Preiss. II, 256 (1847) ...

G. paniculata, Meissner in Lehm. pl. Preiss. I, 550 (1845)

G. biternata, Meissner in Lehm. pl. Preiss. I, 549 (1845)

G. triloba, Meissner in Hooker's Kew Misc. VII, 74 (1855)

G. amplexans, F. v. M. in Bentham FI. Austr. V, 488 (1870) ...

G. vestita, Meissner in Lehm. pl. Preiss. I, 548 (1845)

G. tridentifera, Meissner in Lehm. pl. Preiss. I, 547 (1845)

G. erinacea, Meissuer in Hooker's Kew Misc. VII, 74 (1855)

HAKEA, Schrader, sert. Hannov, I, fasc. 3, 27, t. 17 (1797)

H. chorclophylla, F. v. M. in Hooker's Kew Misc. IX, 23 (1857)

H. Cunninghamii, R. Brown, prot. nov. 26 (1830)

H. lorea, R. Brown, prot. nov. 25 (1830) .

H. Fraseri, R. Brown, prot. nov. 26 (1830)

H. macrocarpa, Cunningham in $\mathrm{R}$. Brown, prot. nov. 30 (1830)

H. arborescens, R. Brown in Transact. Linn. Soc. X, 187 (1809)

H. stenophylla, Cunningham in De Cand. prodr. XIV, 417 (1856)

H. trineura, F. v. M., fragm. III, 146 (1863)

H. cyclocarpa, Lindley, Bot. Regist. XXV, App. XXX X

H. crassifolia, Meissner in Lehm. pl. Preiss. I, 570 (1845)

H. pandanocarpa, R. Brown, prot. nov. 29 (1830)

H. Roei, Bentham, Fl. Austr. V, 499 (1870)

H. adnata, R. Brown, prot, nov. 26 (1830)...

H. obliqua, R. Brown in Transact. Linn. Soc. X, iso (1809) $\ldots$

H. Hookeriana, Meissner in Hook. Kew Misc. IV, 208 (1852) ..

$H$. incrassata, R. Brown, prot. nov. 29 (1839)

H. Alabellifolia, Meissner in Hooker's Kew Misc. V̈II, 116 (1855)

H. Brownii, Meissner in Lehm. pl. Preiss. I, 569 (1845)...

H. Baxteri, R. Brown, prot. nov. 28 (1830)

H. ceratophylla, R. Brown in Transact. Linn. Soc. X, $1 \ddot{84}$ (1809)

H. lasiantha, R. Brown, prot. nov. 29 (1830)

H. eriantha, $R$. Brown, prot. nov. 29 (1830)

H. megalosperma, Meissner in Hook, Kew Misc.

H. clavata, Labillardiere, Nov. Holl. pl. spec. I, 31, t. 41 (1804)

H. orthorrhyncha, F. v. M., fragm. V, 214 (1868)

H. Candolleana, Meissner in Lehm. pl. Preiss. II, 262 (1847) ...

H. trifurcata, R. Brown in Transact. Linn. Soc. X, $183(1809) \ldots$

H. erinacea, Meissner in Lehm. pl. Preiss. I, 559 (1845)

H. platysperma, Hooker, icones pl. t. 433 (1842) ...

H. brachyptera, Meissner in Hook. Kew Mise. IV, 208 (1852)

H. Kippistiana, Meissner in Hooker's Kew Misc. VII, 115 (1855)

H. Preissii, Meissner in Lehm. pl. Preiss. I, 557 (1845) ...

H. pugioniformis, Cavanilles, Anmal. hist. nat. I, 213 , t. $11(1800)$

H. Pampliniana, Kippist in Hook. Kew Misc. VII, 115 (1855)

$H$. vittata, $R$. Brown in Transact. Linn. Soc. $X, 182(1809)$..

H. rostrata, F. r. M. in Schlecht. Linnaea XXVI, 259 (1853) ".

H. rugosa, R. Brown in Transact. Linn. Soc. X, 179 (1809)

H. Epiglottis, Labillardiere, Nov. Holl. pl. spec, I, 30, t. 40 (180̈)

H. amplexicaulis, R. Brown in Transact. Linn. Soc. X, 184 (1809)

H. glabella, R. Brown, prot. nov. 28 (1830)

H. auriculata, Meissner in Hook. Kew Misc. VII, 116 (1855) ...

I. cristata, K. Brown, prot. nov. 28 (1830)

$H$. linearis, R. Brown in Transact. Linn. Soc. X, 183 (1809) ...

H. stenocarpoides, F. v. M. in Bentham Fl. V, 511 (1870)

H. ruscifolia, Labillardière, Nov. Holl. pl. spec. I, 30, t. 39 (1804)

$H$. saligna, R. Brown in J. Knirht, prot. 108 (1809)

H. verrucosa, F. v. MI., fragm. V, 25 (1865)
W.A. - - - N.S.W. - - B.f.V.478

W.A. - - - - - - B.H.V,479

W.A. - - - - - - B.fl.V,479

IV A - - - - - BA V 480

W.A. - - - - - - B.fl.V, 480

W.A. - - -- - - - B.f.V.480

W.A. - - - - - B.f.V.481

W.A. - - - - - - B.A.V,481

W.A. - - - - - B.fl.V.481

W.A. - - - - N.A. B.fl.V,482

- S.A. - - N.S.W. - - B.fl.V,482

- - - - N.S.W. - - B.f.V,482

W.A. - - - - - - B.fl.V,483

W.A. - - - - - - B.fl.V,483

W.A. - - - - - - B.fl.V.483

W.A. - - - - - - B.fl. V,484

W.A. - - - - - - B.fl.V,484

IV.A - - -

W.A.

W.A.

W.A.

W.A.

W.A.

W.A.

W.A.

W.A.

W.A.

W.A.

W.A.

W.A.

W.A.

(Conchium.)

- - - - - - -

- N.A. B.fl.V,495

- N.A. B.fl.V, 495

Q. N.A. B. H. V, 495

- - - - N.S.W - - B. V V 490

- - - - - N.A. B.f.V, 496

- - _.. - Q. N.A. B. V.497

- - - - - - N.A. B.f.V.497

W.A - - - Q. - B.f.V,497

- - - B.fl.V,498

W.A. - - - - - - B.fl.V,498

W.A. - - - - - B.H.V,499

W.A. - - - - - - B.fl.V,499

W.A. - - - - - B.fl.V.499

W.A. - - - - - - B.fl.V,500

W.A. - - - - - B.H.T,500

W.A. - - - - - B.fl.V,500

W.A. - - - - - - B.f.V.501

W.A. - - - - - - B.f.V,501

W.A. - - - - - — B.fl.V.501

W.A. - - - - - - B.fl.V,501

W.A. - - - - - - B.fl.V,502

- - - V.N.S.W. - - B.f.V.,502

W.A. - - - - - - B.H.V,503

W.A. - - - - - - B.fl.V,503

IV.A. - - - - - - B.V. V03

W.A. - - - - - B.f.V.504

W.A. - - - - - - B.f.V,504

W.A. - - - - _ - B.fl.V,505

W.A. - - - - - - B.f.V,505

W.A. - - - - - - B.f.V 505

W.A. - - - - - B.H. Y,506

W.A. - - - - - - B.fl.V,506

-

- S.A. T. N. N.S.W. -

S.A. - V. N.S.W. Q

S.A. - -

S.A. - V.

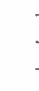

B.fl. V, 506

- B.fl.V,507

-

$-\mathrm{S} . \mathrm{A}$

W.A.

- - -

W.A. - -

IV.A.

W.A.

IV.A.

W.A.

- -

M.fr. VI, 209.

M.fr. VI, 209.

M. fr. I, 136;VI, 208 .

fr, 129;VI, 208

M.fr.I, 136; VI,208.

. 209

M.fr. VI, $209 ; \mathrm{X}, 46$.

M.fr.VI, 209.

M.fr. X, 45 .

M. fr. VI, 213 .

M.fr. VI, 213

M.fr. VI, 213.

M. fr. X, 45

VI, 190.

M.fr.VI, 189;VII, 133 .

M. fr. VI, 190,210.

I, 190 .

M.fr, VI, 216.

M.fr. VI, 218.

M.fr. VI, 215.

M.fr.VI, 217.

I fr. VI, 220

r. VI, 220.

, 217.

M.fr. VI, 219.

M.fr. VI,21S; VII,133. M.fr. VI,218.

M.fr.VI, 217;VII, 133.

M.fr. VI, 217.

B.fl. V, 510

B. fi. V,511

B. fl. V,511

B.f.. V,5I 1

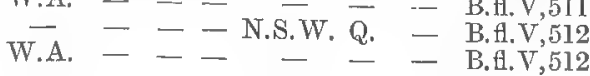

M.fr. VI, 217.

M.fr. V,25;VI,218. 
H. rhombales, F. v. M., fragm. X, 90 (1876)

H. purpurea, Hooker in Mitch. Trop. Austr. 348 (1848)...

H. gibbosa, Cavanilles, Anal. hist. nat. I, 215 (1800)

H. propinqua, Cunningham in Field N.S. Wales, 327 (1825)

H. nodosa, R. Brown in Transact. Linn. Soc. X, 179 (1809)

$H$. acicularis, $R$. THown in Transact. Limn. Soc. X, 181 (1809) ...

H. leucoptera, R. Brown in Transact. Linn. Soc. X, 180 (1809)

H. cycloptera, R. Brown in Transact. Linn, Soc. X, 182 (1809)

H. microcarpa, R. Brown in Transact. Linn. Soc. X, 182 (1809)

H. recurva, Meissner in Hook. Kew Misc. IV, 207 (1852)

H. circumalata, Meissner in Hook. Kew Misc. VII, 114 (1855)...

H. commutata, F. v. M., fragm. V, 26 (1865)

H. strumosa, Meissner in Hook. Kew Misc. IV, 208 (1852)

H. multilineata, Meissner in Lehm. pl. Preiss. II, 261 (1847)

H. laurina, R. Brown, prot. nov. 29 (1830)

H. obtusa, Meissner in Hook. Kew Misc. IV, 209 (1852)

H. cinerea, R. Brown in Transact. Linn. Soc. X, 186 (1809) ...

H. corymbosa, R. Brown, prot. nov. 28 (1830)

H. undulata, R. Brown in Transact. Linn. Soc. X, 185 (1809) .

H. petiolaris, Meissner in Lehm. pl. Preiss. I, 577 (1845)

H. neurophylla, Meissner in Hook. Kew Misc. VII, 117 (1809)

H. loranthifolia, Meissner in Lehm. pl. Preiss. I, 574 (1845)

H. cucullata, R. Brown, prot. nov. 30 (1830)

H. ferruginea, Sweet, Fl. Austral. t. 45 (1828)

H. smilacifolia, Meissner in Lehm. pl. Preiss. I, 567 (1845)

H. elliptica, $R$. Brown in Transact. Linn. Soc. X, 187 (1809) '"

H. ambigua, Meissner in Lehm. pl. Preiss. II, 260 (1847)

H. plurinervia, F. v. M. in Benth. Fl. Austr. V, 523 (1870)

H. dactyloides, Cavanilles, Anal. hist. nat. I, 215, t. $12(1800) \ldots$

H. ulicina, R. Brown, prot. nov. 29 (1830)..

H. falcata, R. Brown, prot. nov. 29 (1830)...

H. pycnoneura, Meissner in Hook. Kew Misc. VII, 117 (1855)

H. stenocarpa, R. Brown, prot. nov. 29 (1830)

H. marginata, R. Brown in Transact. Linn. Soc. X, 185 (1809)...

H. myrtoides, Meissner in Lehm, pl. Preiss. I, 577 (1845)

H. costata, Meissner in Lehm. pl. Preiss. I, 575 (1845) ...

H. oleifolia, R. Brown in Transact. Linn. Soc. X, 185 (1809)

H. florida, R. Brown in Transact. Linn. Soc. X, 183 (1809)

H. varia, R. Brown in Transact. Linn. Soc. X, 183 (1809)

H. sulcata, R. Brown in Transact. Linn. Soc. X, 180 (1809)

H. Meissneriana, Kippist in Hook. Kew Misc. VII, 114 (1809)

H. subsulcata, Meissner in Lehm. pl. Preiss. I, 555 (1845)

H. Lehmanniana, Meissner in Lehm. pl. Preiss. I, 557 (1845) ...

H. flexilis, F. v. M. in Schlecht. Limnaea XXVI, 359 (1853) ...

H. nitida, R. Brown in Transact. Linn. Soc. X, 184 (1809)

H. Oldfieldii, Bentham, Fl. Austr. V, 530 (1870)

H. suaveolens, R. Brown in Transact. Linn. Soc. X, 182 (1809)

H. lissocarpha, R. Brown, prot. nov. 27 (1830) .

H. bipinnatifida, R. Brown, prot. nov. 28 (1830) ... CANARVONIA, F. v. M., fragm. VI, 81 (1867).

C. aralifolia, F. v. M., fragm. VI, t. 55 (1867) ... ... BUCKINGHAMIA, F. v. M., fragm. VI, 248 (1868).

B. celsissima, F. v. M., fragm. VI, 248 (1868) .. DARLINGIA, F. v. M., fragm. V, 152 (1866).

D. spectatissima, F. V. MI., fragm. V, $152(1866) \ldots$ CARDWELLIA, F. v. M., fragm. V, 23 (1865).

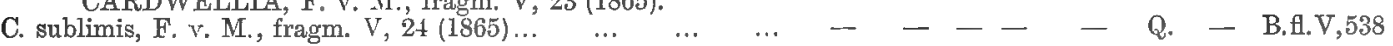
STENOCARPUS, R. Brown in Transact. Limn. Soc. X, 201 (1809). (Cybele, Agnostus.)

S. sinuatus, Endlicher, gen. pl. suppl. IV, 88 (1847) $\ldots \ldots \ldots \ldots-\ldots$

S. salignus, R. Brown in Transact. Linn. Soc. X, 202 (1809) $\ldots-\ldots-$ - - - N.S.W. Q. - B.f.V,539

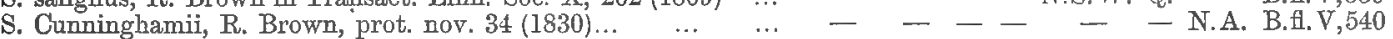
LOMATIA, R. Brown in Transact. Linn. Soc. X, 199 (1809). (Tricondylus.)

L. fraxinifolia, F. v. M. in Benth. FI. Austr. V, $536(1870)$ ‥ - - - - - Q. - B.t.V,536

L. ilicifolia, R. Brown in Transact. Linn. Soe. X, $200(1809) \ldots \ldots--\quad-$ V. N.S.W. - - B.fl.V,536

L. longifolia, R. Brown in Transact. Linn. Soc. X, $200(1809) \ldots \quad-\quad-\quad-$ V. N.S.W. - - B.fl.V.537

L. silaifolia, R. Brown in Transact. Linn. Soc. X, 199 (1809) ... - - - - - N.S.W. Q. - B.fl.V,537

L. tinctoria, R. Brown in Transact. Linn. Soc. X, 199 (1809) .. - - T, - - - - B.f.V,537

L. polymorpha, R. Brown in Transact. Linn. Soc. X, 200 (1809) - - T. - - - - B.fl.V,538 EMBOTHRIUM, R. \& G. Forster, charact. gen. 15, t. 8 (1776).

E. Wickhami, F. v. M., fragm. VIII, 164 (1874)...

TELOPEA, R. Brown in Transact. Linn. Soc. X, 197 (1809).

T. speciosissima, R. Brown in Transact. Linn. Soc. X, 198 (1809)

T. oreades, F. v. M., Annual Report 18 (1861) … …

BANKSIA, Linné, fil. suppl. 15 et 126 (1781).

B. pulchella, R. Brown in Transact. Linn. Soc. X, $202(1809)$.. IV.A. - - - - - - B.fi.V,544

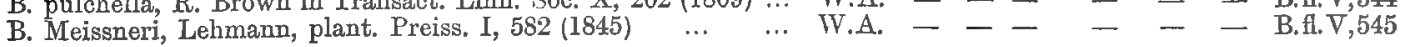

M.fr.X,90.

M.fr.VI, 218.

M.fr.V,26.

M.fr, V, $26 ; \mathrm{VI}, 217$.

M.fr.VI,218.

MI.fr. VI, 219; VII, 133.

II.fr. YI, 217

M.fr.VI,217.

M.fr.VI, 218 .

M.fr. VI,219.

M.fr.VI, 219.

M.fr. VI, 219.

M.fr. VI, 215.

II.fr.IV, $130,216$.

M.fr. VI,216.

M.fr. VI, 216.

M.fr.VI, 216 .

M.fr.VI,216.

M.fr, VI, 217

M.fr. VI,216.

M.fr.VI,215.

M.fr. VI,215.

M.fr.VI,216.

M.fr. VI, 215.

M.fr.VI,219.

N.fr. VI, 219 .

M.fr.VI, 219.

M.fr. VI, 219.

M.fr.VI,218.

II.fr. VI, 216

II.fr. V, $72 ; \mathrm{VI}, 217$.

M.fr. VI, 220.

M.fr.VI,219.

M.fr.VI,81,247;VII,59.

M.fr. VI,248.

M.fr,X,90.

M.fr. V, 24 .

M.fr. VI,224. [VII, 133. M.fr.I, 135, 2 44 ; VI, 224 ;

M.fr.VII, 133 .

M.fr. V,153;VI, 191 .

M.fr. VI, 224 .

M.fr.VIII, 164;IX, 194.

M.fr. X, 90 .

M.fr.II, 170;VII, 133.

M.fr.II, I7I.

M. fr. VII, 54 . 
B. nutans, R. Brown in Transact. Linn. Soc. X, 203 (1809)

B. sphaerocarpa, R. Brown in Transact. Linn. Soc. X, 203 (1809)

B. tricuspis, Meissner in Hook. Kew Misc. VII, 118 (1855)

B. occidentalis, R. Brown in Transact. Linn. Soc. X, 204 (1809)

B. littoralis, R. Brown in Transact. Linn. Soc. X, 204 (1809) .

B. ericifolia, Linné, fil. suppl. 127 (1781)

B. spinulosa, Smith, Specim. Bot. New Holl. 13, t. 4 (1793) ...

B. collina, R. Brown in Transact. Linn. Soc. X, 204 (1809)

B. verticillata, R. Brown in Transact. Linn. Soc. X, 207 (1809)

B. dryandroides, Baxter in Sweet, Fl. Austral. t. 56 (1828)

B. Brownii, Baxter in R. Br. prot. nov. 37 (1830)

B. attenuata, R. Brown in Transact. Linn. Soc. X, 209 (1809)...

B. media, R. Brown, prot. nov. 35 (1830) ...

B. Solandri, R. Brown, prot. nov. 35 (1830)

B. Goodii, R. Brown, prot. nov. 36 (1830)...

B. petiolaris, F. v. M., fragm. IV, 109 (1864)

B. repens, Labillardiere, Voy. I, 411, t. 23 (1798)

B. prostrata, R. Brown, prot. nov. 36 (1830)

B. grandis, Willdenow, spec. plant. I, 535 (1797)

B. quercifolia, $R$. Brown in Transact. Linn. Soc. $X, 210$ (1809)

B. Baueri, R. Brown, prot. nov. 35 (1830) $\ldots \ldots$. $\ldots$

B. marginata, Cavanilles, Anal. hist. nat. I, 227, t. 13 (1800) ...

B. integrifolia, Linné, fil. suppl. 127 (1781)

B. dentata, Linné, fil. suppl. 127 (1781)

B. latifolia, R. Brown in Transact. Linn. Soc. X, 208 (1809) $\ldots$

B. serrata, Linné, fil. suppl. 126 (1781)

B. aemula, R. Brown in Transact. Linn. Soc. X, 210 (1809) $\quad \ldots$

B. ornata, F. v. M. in Suhlecht. Linnaea XXVI, 352 (1853) ...

B. coccinea, $R$. Brown in Transact. Linn. Soc. $X, 207$ (1809) ...

B. Sceptrum, Meissner in Hook. Kew Misc. VII, 120 (1855) ...

B. Menziesii, R. Brown, prot. nov. 36 (1830)

B. laevigata, Meissner in Hook. Kew Misc. IV, 210 (1852)

B. Hookeriana, Meissner in Hook. Kew Misc. VII, $119(1855) \ldots$

B. prionotes, Lindley, Bot. Regist. XXV, App. XXXIV (1839)

B. Victoriae, Meissner in Hook. Kew Misc. VII, 119 (1855)

B. speciosa, R. Brown in Transact. Limn. Soc. X, 210 (1809)

B. Baxteri, R. Brown, prot. nov. 36 (1830)

B. marcescens, R. Brown in Transact. Linn. Soc. ' X, 208 (1809)

B. Lemanniana, Meissner in Hook. Kew Misc. 210 (1852)

B. Caleyi, R. Brown, prot. nov, 35 (1830).

B. Lindleyana, Meissner in Hook. Kew Misc. VII, 120 (1855) ...

B. elegans, Meissuer in Hook. Kew Misc. VII, 119 (1855)

B. Candolleana, Meissner in Hook. Kew Misc. VII, 118 (1855)

B. ilicifolia, R. Brown in Transact. Linn. Soc. X, 211 (1809).

DRYANDRA, R. Brown in Transact. Linn. Soc. X, 211 (1

D. quercifolia, Meissner in Hook. Kew Misc. IV, 210 (1852)

D. praemorsa, Meissner in Lehm. pl. Preiss. II, 265 (1847)

D. cuneata, R. Brown in Transact. Linn. Soc. X, 212 (1809)

D. falcata, R. Brown in Transact. Linn. Soc. X, 213 (1809)

D. armata, R. Brown in Transact. Linn. Soc. X, 212 (1809)

D. longifolia, R. Brown in Transact. Linn. Soc. X, 215 (1809)...

D. Fraseri, R. Brown, prot. nov. 39 (1830)

D. floribunda, R. Brown in Transact. Linn. Soc. X, 212 (1809)

D. carduacea, Lindley, Bot. Regist. XXV, App. XXXIII (1839)

D. carlinoides, Meissner in Lehm. pl. Preiss. II, 267 (1847)

D. polycephala, Bentham, Fl. Austr. V, 570 (1870)

D. Kippistiana, Meissner in Hook. Kew Misc. VII, 122 (1855)

D. squarrosa, R. Brown, prot. nov. 38 (1830)

D. Serra, R. Brown, prot. nov. 38 (1830)

D. concinna, R. Brown, prot. nov. 38 (1830)

D. foliolata, R. Brown, prot. nov. 38 (1830)

D. stupposa, Lindley, Bot. Regist. XXV, App. XXXIII (1839)

D. nobilis, Lindley, Bot. Regist. XXV, App. XXXIII (1839) ...

D. mucronulata, R. Brown in Transact. Linn. Soc. X, 213 (1809)
D. formosa, R. Brown in Transact. Linn. Soc. X, 213, t. 3 (1809)

D. Baxteri, R. Brown, prot. nov. 38 (1830)...

D. nivea, R. Brown in Transact. Linn. Soc. X, 214 (1809)

D. Arctotidis, R. Brown, prot. nov. 39 (1830)

D. nana Meissner in Hook, Kew Misc. VII, 101 (1855) ...

D. Preissii, Meissner in Lehm. pl. Preiss. I, 599 (1845) ...

D. sclerophylla, Meissner in Hook. Kew Misc. VII, 123 (I855) ...

D. pulchella, Meissner in Hook. Kew Misc. IV, 211 (1852)

D. plumosa, R. Brown in Transact. Linn. Soc. X, 214 (1809)

D. senecionifolia, R. Brown, prot. nov, 39 (1830)

D. vestita, Kippist in Hook. Kew Misc. VII, 121 (1855)

D. cirsioides, Meissner in Hook. Kew Misc. IV, 211 (1852)

D. Hewardiana, Meissner in Hook. Kew Misc. IV, 210 (1852) ...

D. patens, Bentham, FI. Austr. V, 578 (1870)

D. conferta, Bentham, Fl. Austr. V, 578 (1870)

D. horrida, Meissner in Hook. Kew Misc. IV, 211 (1852)
W.A

W.A. - - -

W.A. - - -

W.A.

-

W.A.

W.A.

W.A. - - -

W.A.

W.A

W.A. - - -

W.A. - - -

W.A. - - -

W.

W. - -

W.A. S.A. T. V. N.S.W.

- - - V. N.S.W.Q

- $\quad-\quad-$ N.S.W. Q.

- $\quad$ - T. V. N.S.W.

- S.A. - V. N.S.W. Q.

W. A

W.A. - -

W.A.

.A.

W.A.

W.A.

W.A.

W.A.

W.A.

IV.A.

TV.A.

W.A.

W.A.

W.A.

W.A.

W.A.

W.A.

809).

W.A.

IV.A.

W.A.

W.A.

W.

W.A.

W.A.

W.A.

W.A.

IV.A

W.A.

W.A.

W.A.

W.A.

W.A.

W.A.

W.A.

W.A.

W.A.

W.A.

W.A.

W.A.

W.A.

W.A.

W.A.

W.A.

W.A.

W.A.

W.A.

W.A.

W.A.

W.A.

W.A.

W.A.

(Josephia, Hemiclidia.)

- _ _ _ _
B. Al. V, 545

B.fi. V, 546

B.fi.V, 546

B.fl.V,546

B. fl. V, 547

B.fl. V, 547

B.fl. V, 547

- B.fl.V,548

- B.fl.V,548

- B.fl. V,549

- B.fl.V,549

- B.fl.V,550

B.fl. V, 550

- B.fl. V,550

- B.fl.V,55l

- B.fl.V,551

- B.fl.V,55l

- B.fl.V,552

- B.fl. V,552

- B.fl.V,552

- B.fl.V,554

N.A. B.fl. V,555

- B.tl.V,555

- B.Al.V,556

- B.A.V,556

- B. fl.V,557

B.tl. V,557

B.fl.V, 557
- B.fl.V,558

- B.fl.V,558

- B.fl.V,558

- B.fl.V,559

B.fl. V, 559

- B.fl. V.559

- B.fl. V,560

B.fl. V, 560

B.fl. V, 560

- B.H.V,561

- B.fl. V,561

- B.fl.V,561

- B. $1 . V, 561$

- B.Al.V.566

- BAV560

B.tl.V,566

- B.H.V,567

- B.fl. V,567

- B.fl.V,568

- B.H.V,568

- B.fl. V,569

B.H.V,569

B.fl. V, 569

B.fl. V,570

B.fl. V, 570

B.fl.V,571

B. $1 . V, 571$

B. B. V.571

- B.fl.V,572

- B.t.V,572

- B.fl.V,573

- B.fl.V,573

- B.fl.V,573

B.f. V, 574

- B.fl.V.574

- B.fl.V,575

- B.fl.V.575

B.fl.V,576

B.fl. V, 576

- B.f1.V,576

- B.f.V.577

B.A.V,577

B.t. V, 577

- B.fl.V,577

- B.fl.V,578

- B.fl.V,578

B. fl. $Y, 579$

M.fr.IV, 108;VII,54.

M.fr.VII, 54.

M.fr.VII, 54.

M.fr.VII, 54 .

M.fr. VII, 55.

M.fr.VII, 54,

M.fr. VII,54.

M.fr. VII, 58.

M.fr.VII, 58 .

M.fr. VII, 55 .

M.fr. IV 109 .

M.fr.VII, 58 .

M.fr.VII, 58 .

M.fr.TV,109.

M.fr.IV,108,177;VII,58,

M.fr.VII, 57.

M.fr.VII, 57 .

M.fr. VII, 57.

M.fr.IV, 107;VII, 56.

M.fr. VII, 55, 133; X, 90 .

M.fr.VII, 55.

M.fr.VII, 57 .

M.fr.VII, 56 .

M.fr.VII, 56 .

M.fr.VII, 56

M.fr. VII, 56 .

M.fr.VII, 56 .

M.fr. VII, 56.

M.fr. VII, 56.

M.fr. VII, 56 .

M.fr. VII, 58 .

M.fr.VII, 58 .

M.fr. VII, 58 .

M.fr. VII, 56 .

M.fr.VII, $5 \%$.

M.fr. VII, 57.

M.fr. VII, 55 ,

M.fr.VII, 58 .

M.fr. VII, 58.

M.fr.VII, 50.

M.fr.VII, 50 .

M.fr. VII, 50 .

M.fr.VII, 50.

M.fr. VII, 52

M.fr. VI, 92; VII, 50.

M.fr. VII, 51 .

M.fr. VII, 53 .

M.fr. VII, 52.

M.fr.VII, 52 .

M.fr.VII,5l.

M.fr. VII, 52

M. fr.VII, 51 .

M.fr. VII, 51. 
D. serratuloides, Meissner in Hook. Kew Misc. VII, 123 (1855) W.A.

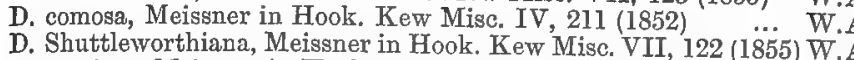

D. Shuttleworthiana, Meissner in Hook. Kew Misc. VII, 122 (1855) W.A.

D. tridentata, Meissner in Hook. Kew Mise. VII, 120 (1855) … W.A.

D. tenuifolia, R. Brown in Transact. Linn. Soc. X, 215 (1809) ... W.A.

D. proteoides, Lindley, Bot. Regist. XXV, App. XXXII (1839) W.A.

D. runcinata, Meissner in Hook. Kew Misc. IV, 210 (1852) ‥ W.A.

D. obtusa, R. Brown in Transact. Linn. Soc. X, 214 (1809) … W.A.

D. bipinnatifida, R. Brown, prot. nov. 39 (1830) ... ... ... W.A.

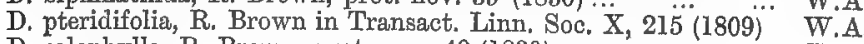

D. calophylla, R. Brown, prot. nov, 40 (1830)

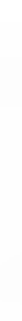

M.fr.VII, 52,

M.fr. VII, 53

M.fr.VII,52,

M.fr.VII, 53.

M.fr.VII,53.

M.fr.V, 185 .

M.fr.VII, 54. M.fr. VII, 54 .

\section{THYMELEAE.}

A. L. de Jussieu, gen, plant. 76 (1789).

PIMELEA, Banks \& Solander in Gaert. de fruct. I, 186 (1788). (Thecanthes, Gymnococca, Heterolaena, Calyptrostegia, Macrostegia;-Banksia, Forst., 1776.)

P. punicea, R. Brown, prodr. 359 (1810) $\cdots \quad \cdots$
P. concreta, F. v. M., fragm. V. 73 (1865) $\cdots$

P. cornucopiae, Vahl, enum. plant. I, 305 (1804)...

P. sanguinea, F. v. M., fragm. I, 84 (1858)...

$\mathrm{P}$. alpina, F. V. M., second general report, 14 (185̈)

P. longifolia, Banks \& Solander in Rees, Cycl. XXVI (1814) ...

P. cinerea, R. Brown, prodr. 361 (1810)

P. Milligani, Meissner in De Candolle, prodr. XI $\dddot{V}, 509$ (1856)...

P. spectabilis, Lindley, Bot. Regist. t. 33 (1841)

P. Lehmanniana, Meissner in Lehm. pl. Preiss, I, 603 (1845) “.

P. hispida, R. Brown, prodr. $360(1810)$

P. rosea, R. Brown, prodr. 360 (1810)

P. ferruginea, Labillardiere, Nov. Holl. pl. spec. I, 10 , † 5 (1

P. brachyphylla, Bentham, Fl, Austr. VI, Il (1873)

P. sylvestris, $R$. Brown, prodr. 361 (1810).

P. brevifolia, R. Brown, prodr. 359 (1810)...

P. Maxwelli, F. v. M. in Benth. Fl. Austr. VI, 12 (1873)

P. angustifolia, $R$. Brown, prodr. 360 (1810)

P. nervosa, Meissner in Lehm, pl Preiss II, 269 (1847)

P. sulphurea, Meissner in Mohl \& Schlecht. Bot. Zeitung, 396 (1848)

P. Horibunda, Meissner in De Candolle, prodr. XIV, 505 (I856)

P. suaveolens, Meissner in Lehm. pl. Preiss. I, 603 (1845)

P. glauca, R. Brown, prodr, 360 (1810)

P. colorans, Cunningham in De Cand. prodr. XIV̈, 499 (i856) ..

P. collina, R. Brown, prodr. 359 (1810)

P. spathulata, Labillardière, Nov. Holl. pl. spec. I, 9, t. 4 (1804)

P. linifolia, Smith, Specim. of Bot. of N. Holl. 31, t. 11 (1793)..

P. Iigustrina, Labillardiere, Nov. Holl. pl. spec. I, 9, t. 3 (1804)

P. humilis, R. Brown, prodr. 361 (1810)

P. sericea, $\mathbf{R}$. Brown, prodr. 361 (1810)

P. nivea, Labillardière, Nov. Holl. pl. spec. I, 10, t. 6 (I804) ...

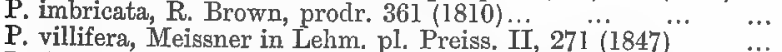

P. drupacea, Labillardiere, Now. Holl. pl. spec. I, 10, t. 7 (1804)

P. haematostachya, F. v. M., fragm. I, 84 (1858)... ...

P. spicata, R. Brown, prodr. $362(1810)$

P. spiculigera, F. v. M. in Bentham Fl. Austr. VI, 23 (1873) ...

P. Forrestiana, F. v. M., fragm. XI, 46 (1879)

P. filiformis, J. Hooker in Lond. Journ. VI, 280 (1847) $\cdots$

P. latifolia, R. Brown, prodr. $362(1810)$

P. simplex, F. v. M. in Linnaea XXV, $443(1852) \cdots \cdots$

P. sericostachya, F. v. M., fragm. IV, 162 (1864)

P. trichostachya, Lindley in Mitch. Trop. Austr. 355 (1S48) ...

P. leptostachya, Bentham, FI. Austr. VI, 24 (1873)

P. argentea, R. Brown, prodr. 362 (1810)

P. clavata, Labillardière, Nov. Holl. pl. spec. I, ï (1804)

P. axiflora, F. v. M., First general Report 17 (1853)

P. leptospermoides, F. v. M., fragm. VII, 2 .(1870)

P. microcephala, R. Brown, prodr. 361 (1810)

P. pauciflora, R. Brown, prodr. 360 (1810)

P. elachantha, F. v. M., First general Report 17 (1853).

P. pygmaea, F. v. M., in Schlecht. Linnaea XXVI, 346 (1853)

P. serpyllifolia, R. Brown, prodr. 360 (1810)

P. flava, R. Brown, prodr. 361 (1810)

P. petrophila, F. v. M. in Schlecht. Linnaea XX $\dddot{V}, 442$ (1852)..”

P. Bowmanni, F. v. M. in Benth. Fl. Austr. VI, 30 (1873)

P. ammocharis, F. v. M. in Hook. Kew Misc. IX, 24 (1857) ...

P. curviflora, R. Brown, prodr. 362 (1810))

P. hirsuta, Meissner in De Cand. prodr. XIV, 513 (1856)

P. altior, F. v, M., fragm. I, 84 (1858)

P. octophylla, R. Brown, prodr. 361 (1810)$$
\text { - }
$$$$
-
$$

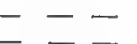

$$
\text { - }
$$$$
\text { - N.A. B.H.VI, } 6
$$$$
\text { Q. N.A. B.H.VI,6 }
$$$$
\text { - - - - V.N.S.W. Q. - B.fl.VI,7 }
$$$$
-\quad \text { - - - N.S.W. - - B.H.VI,7 }
$$$$
\text { - - T. - - - - B.fl.VI,8 }
$$$$
\begin{array}{r}
\text { W.A. - - - - - - - } . \text { B.fl.VI,8 } \\
\text { B.H.VI,9 }
\end{array}
$$$$
\text { W.A. - - - - - - B.fl.VI,9 }
$$$$
\text { W.A. - - - - - - B.A.VI, }
$$$$
\text { W.A. - - - - - B.fl.VI,10 }
$$$$
\text { W.A. - - - - - B.f.VI,11 }
$$$$
\text { W.A. - - - - - B.flVI,11 }
$$$$
\begin{aligned}
& \text { W.A. - - - - - - - B.fl.VI,12 } \\
& \text { W.A. - B.fl.VI,12 }
\end{aligned}
$$$$
\text { W.A. - - - - - B.fl.VI,13 }
$$$$
\text { W.A. - - - - - B.H.VI,13 }
$$$$
\text { W.A. -- - - - - B.A.VI, I4 }
$$$$
\text { W.A. - - - - - - B.fl.VI,14 }
$$$$
\text { W.A. - - - - - - B.H.VI,14 }
$$$$
\text { W.A. - - - - - - B.fl.VI,15 }
$$$$
\text { - S.A. T. V. N.S.W. Q. - B.H.VI,15 }
$$$$
\text { - - - - N.S.W. - - B.fi.VI,16 }
$$$$
\text { - - - V. N.S.W. Q. - B.H.VI, }
$$$$
\text { - S.A. T. V. N.S.W. - }
$$$$
\text { - } \quad \text { - T. V. N.S.W. Q }
$$$$
\text { - S.A.T. V. N.S.WV - }
$$$$
\text { S.A. T. V. N.S.W. - }
$$

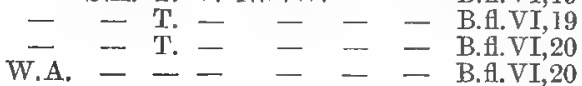$$
\text { W.A. - - - - - - B.f.VI,21 }
$$$$
\text { - - T. V. - - - B.f.VI,21 }
$$$$
\text { - - - - } \text { N.S.W. Q. }_{-} \text {- B.f.VI,22 }
$$$$
\text { W.A. - - - N.S.W. - - B.f.VI,22 }
$$$$
\text { - }-\overline{\mathrm{T}} \text { - }- \text { - N.A. }
$$$$
\text { - } \overline{\text { - B.fl.VI,23 }}
$$$$
\text { - S.A. - V. N.S.W. Q. }
$$$$
\text { - - - - N.S.W.Q. }
$$$$
\text { - } \quad-\quad-\text { N.S.W.Q. }
$$$$
\text { W.A. }
$$$$
\text { - - T. V. N.S.W. - — B.f.VI, } 25
$$$$
\text { - - - - - Q. - B.fl.VI,27 }
$$

W.A. S.A. - V. N.S.W. Q. - - T. V. N.S.W. Q

$$
\text { - }-\frac{\mathrm{T}}{\mathrm{T}} \mathrm{V} \text { - }-
$$

W.A. S.A. T. $\overline{\text { T. }}$ N.S.W. -

W.A. S.A. T. V. N.S.W. Q.

- S.A. - - N.S.W. $\frac{1}{Q}$

$\bar{S} \cdot \bar{A}, \bar{T} \cdot \bar{V}$. N.S.W. $=-$ N.S.W. $\frac{\text { Q.W. }}{-}$

- B.fl.VI,27

- B.fl.VI,27

- B.fl.VI,28

- B.fl.VI,28

- B.fl.VI,29

- B.H.VI,29

- B.H.VI,30

A. B.A.VI,30

- B.H.VI,31

.. - - - - B.fl.VI,32

.. - $-\overline{\mathrm{S}}$ - - - N.S.W. Q. - B.fl.VI,32
M.fr. V,74;VII, 3 .

M.fr.V,73.

M.fr.VII 3 .

M.fr. VII, $3 ; \mathrm{XI}, 47$.

M.fr. VII, 4.
M.fr. VIII, 9.

M.fr.VII, 4 .

M.fr.VII,4.

M.fr.VII, 4.

M.fr.VII,2\&5.

M.fr.VII, 4.

M.fr. VII,3.

M.fr.VII, 3.

M.fr.VII, 5 .

M.fr.VII, 5 .

M.fr.VII, $2,4,5$.

M.fr.VII,4.

M.fr.VII, 4;XI, 123.

M.fr.VII, 3.

M.fr.I,87;IV,163;VII, 5 .

M.fr. VII, 4 ;XI, 47.

M.fr.VII, 5 .

M.fr.VII, 5 .

M.fr.VII, 5.

M.fr. VII 8.

M.fr.I,84;VII, 8;XI,47.

M.fr. VII, 7; VIII, 9 .

M.fr.XI, 46.

M.fr.XI, 46.

M.fr. VII,7.

M.fr.IV, $49 ; \mathrm{VII}, 7$.

M.fr.XI, 47.

M.fr.VII, 7 .

M.fr.VII, 7 .

M.fr.VI, 160; VII, 5.

M.fr.VII, 7.

M.fr.VII, 2.

M.fr. XI,27,47.

M.fr.VII, 8;XI, 47 .

M.fr. VII, 6.

M.fr. VII, 6.

M.fr.VII, 6.

M.fr.VII, 6 .

M.fr. VII,5;XI, 47.

M.fr.VII,6;VIII,9.

M.fr.VII,7.

M.fr.VII, 6 , 
P. petraea, Meissner in Schlecht. Linnaea XXVI, 347 (1853) ... - S.A. - - N.S.W. - - B.fl.VI,33 P. phylicoides, Meissner in Lehm. pl. Preiss. II, 271 (1847) ... - S.A. - V. N.S.W. - - B.H.VI,33

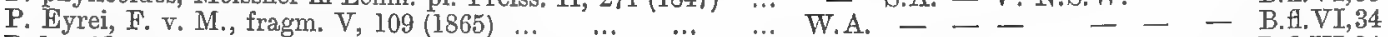

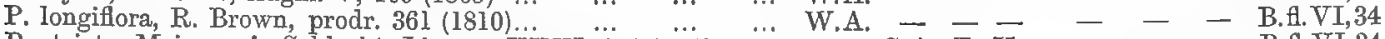

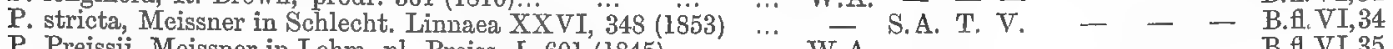

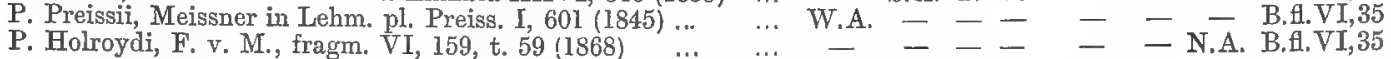
DRAPETES, Lamarck, Journ, d'hist. nat. I, 119, t. 10 (1792). (Kelleria, Daphnobryon).

D. Tasmanica, J. Hooker in Kew Misc. V, 299, t. 7 (1853) ... - - T. V. N.S.W. - - B.f.VI,36 WICKSTROEMIA, Endlicher, prodr. fl. insul, Norfolk. 47 (1833). (Daphne partly).

W. Indica, C. A. Meyer in Bull. de l'Acad. de Petersb. I, 357 (I843) - - - - N.S.W. Q. N.A. B.fl.VI,37 PHALERIA, Jack in Hook. Comp. to Bot. Mag. I, 156 (1835). (Drymispermum).

P. Blumei, Bentham, Fl. Austr. VI, $38(1873)$.. $\quad \ldots \quad$.. $\quad$ -

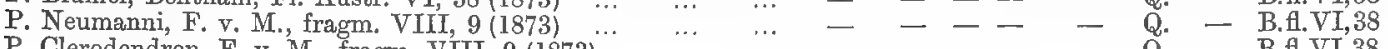

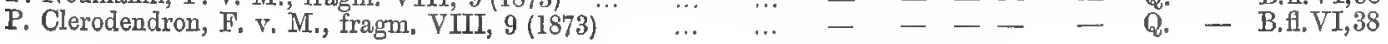

M. fr. VIİ, 6. M.fr. V, 109 . M.fr. VII, 5 . M.fr. VII, 4. M.fr. VIT, 3.

M. fr. 250 .

M.fr. VII, 1 .

M.fr. VII, 1 .

M.fr. V, 26. M.fr.VII, 1.

\section{CORNACEAE.}

Humboldt, Bonpland \& Kunth, nov. gen. Americ. III, 430 (1818).

STYLIDIUM, Loureiro, Fl. Cochinch. I, 220 (1790). (Marlea, Rhytidandra, Pseudalangium).

S. Vitiense, F.v.M.; Rhytid.,AsaGray, Bot.Wilk.Ex.I,303,t.28(1854) - - - - N.S.W. Q. - B.fl.III, 386

\section{RUBIACEAE.}

A. L. de Jussieu, gen. plant. 196 (1789), from B. de Jussieu (1759).

SARCOCEPHALUS, Afzelius in Transact. hort. soc. Lond. V, 422, t. 18 (1824).

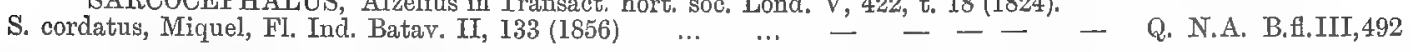
OLDENLANDIA, Plumier, nov. plant. Americ. gen. 42, t. 36 (1703). (Hedyotis, Synaptantha).

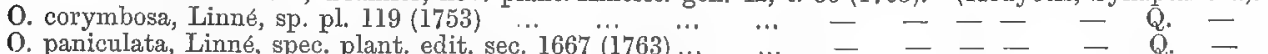

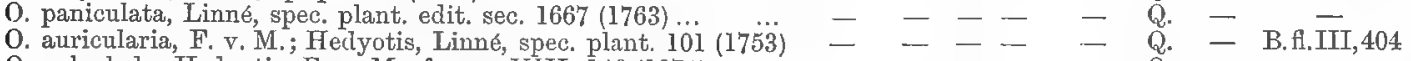

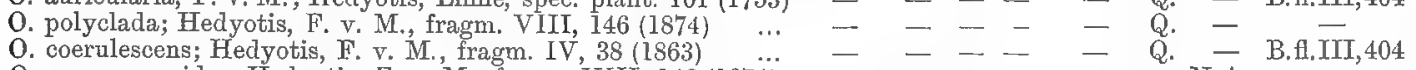

O. spermacocoides; Hedyotis, F. v. M., fragm. VIII, 146 (1874)

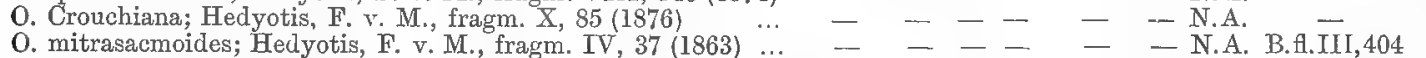

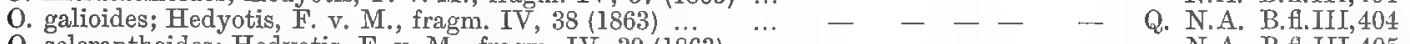

O. scleranthoides; Hedyotis, F. v. M., fragm. IV, $39(1863) \quad \ldots \quad-\quad-\quad-\quad-\quad$ - N.A. B.Al.III,405

O. elatinoides, F. v. M.; Hedyotis, Benth., Fl. Austr. III, 405(1866) W.A. - - - - - - B.fl.III,405

O. tillaeacea; Hedyotis, F. v. M., fragm. IV, 39 (1863) ... ‥ - S.A. - - N.S.W. Q. - B.H.III,405

O. trachymenioides; Hedyotis, F. v. M., fragm. IV, 40 (1863) .. - - - - - Q. - B.f.III,406

O. pterospora; Hedyotis, F. v. M., fragm. IV, $40(1863) \ldots \ldots \ldots \ldots-\ldots \quad-\quad$ - $\quad$ N.A. B.fl.III,407 DENTELLA, R. et G. Forster, char. gen. 25, t. 13 (1776). (Lippaya).

D. repens, R.\& G. Forster, char, gen. 25, t. $13(1776) \ldots \ldots$ … - S.A. - - N.S.IV. Q. N.A. B.A.III,407 OPHIORRHIZA, Linné, fl. Zeylan, 190 et 239 (1747).

O. Australiana, Bentham, Fl. Austr. III, 407 (1866) $\ldots \quad \ldots \quad-\quad-\quad-\quad$ - $\quad$ Q. $\quad$ - B.fl.III,408 ABBOTTIA, F. v. M., fragm. IX, 181 (1875).

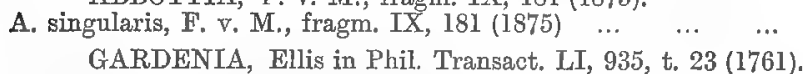
G. edulis, F. v. M., fragm. I, 54 (1858)

G. resinosa, F. v. M., fragm. I, 54 (1858) … $\ldots$

G. megasperma, F. v. M., fragm. I, 54 (1858) $\ldots \ldots \ldots \ldots$

G. Macgillivraei, Bentham, FI. Austr. III, 409 (1866) $\quad \ldots . \quad \ldots$

G. ochreata, F. V. M., fragm. I, 55 (1858) ...

G. suffruticosa, R. Brown in Benth. Fl. Austr. IIT, 410 (1866)...
G. fucata, R. Brown in Benth. Fl. Austr. III, $410(1866)$

G. Jardinei, F. v. M. in Benth. Fl. Austr. III, 410 (1866)

R. hirta, F. v. M., fragm. VII, 46 (1869) ... $\ldots$.. $\ldots$

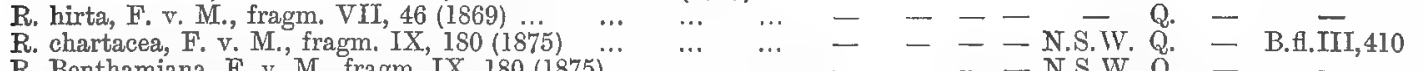

R. Moorei, F. v. M. in Benth. Fl. Austr. III, $411(1866) \quad \cdots \quad-\quad-\quad-$ - N.S.W. Q. - B.H.III, 411

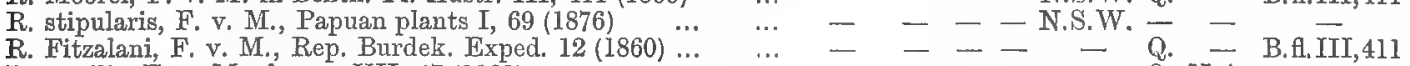

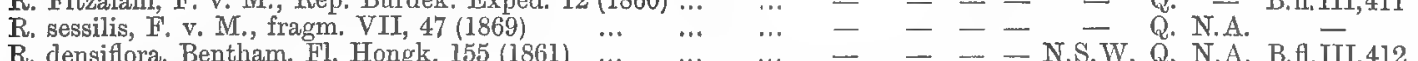

R. densiflora, Bentham, Fl. Hongk. 155 (1861) DIPLOSPORA, De Candolle, prodr. IV, 477 (1830). (Discospermum.)

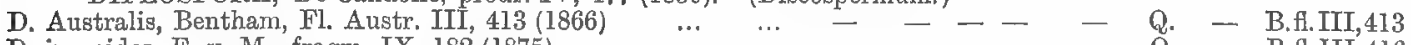

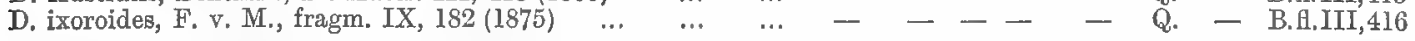
IXORA, Linné, syst, nat. 8 (1735); Linné, gen, pl, 27 (1737). (Pavetta, Webera, Stylocorne.)

I. Paretta, Roxburgh, Fl. Ind. I, 395, ed. Carey (1820)... ... _ - _ - Q. N.A. B.fl.III, 414

I. tomentosa, Roxburgh, Fl. Ind. I, 396, ed, Carey (1820) $\quad \cdots \quad-\quad-\quad-\quad-\quad-\quad$ - N.A. B.fl.III,414

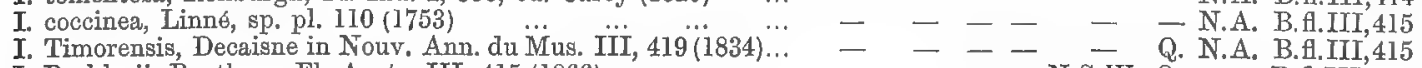

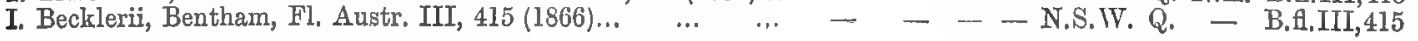

M.fr.VII, 45.

M.fr.VIII, 47 .

M.fr.VIII, 146.

M.fr.IV,38.

MI.fr. VIII, 146.

M.fr. $X, 85$.

M.fr.IV, 37.

M.fr. IV, 38 .

M.fr.IV, 39.

M.fr. VIII, 147.

M.fr.IV,39.

M.fr.IV, 40.

M.fr.IV, $40,177$.

M.fr.IX, 187.

M.fr.IX, 187.

M.fr.IX, 181.

M.fr.I, 54 .

M.fr. I, 54 .

M.fr.I,54.

M.fr. VII, 46.

M.fr. 155 ; VII, 46 .

M.fr. VII, 46.

M.fr.IX, 181.

M.fr.VII, 47 .

M.fr.IX, 180 .

M.fr. VII, 47;IX,70,18I.

M. fr. V, 19;IX, 180 .

M.fr.VII, 47. [180.

M.fr.II, 132; III, 166; IX,

M. fr. IX, 182 .

M.fr.IX, I82.

M.fr. V,18;IX, 183.

M. fr.IX, 183. 


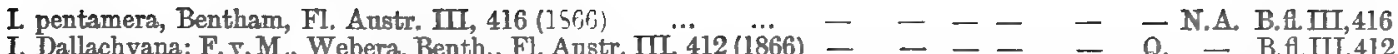

I. Dallachyana; F. . M. M., Webera, Benth., FI. Austr. III, 412 (1866) $-=-二=$ Q. - B.H.III,412 COFFEA, Linnê, syst, nat. 8 (1;35) ); Linné, gen. pl. 55 (1;37), from Ray (1691).

C. Bengalensis, Roxburgh, hort. Bengal. 15 (1814) … … TIMONTCS, Rumphius, herb. Amboin. III, 216, t. 140 (1743). (Polyphragmon,)

T. Rumphii, De Candolle, prodr. IV, $461(1830) \ldots \ldots$ SCTPHIPHORA, K. F. Gaertner, de fruct. III, 91, t. 196 (1805). (Epithinia.)
- _

Q. - - M.fr.VII, 14 .

Q. I.A. B.fl.II, 417 M.fr.II, 134;IX,15i.

S. hydrophylacea, K. F. Gaertner, de fruct. III, 91, t 196 (1805) - - -----
GUETTARDA, Osbeck in Linne, spec. plant. 991 (1533). (Antirrhoea, Guettardella.)

G. speciosa, Linné, spec. plant. $991(1,53)$.

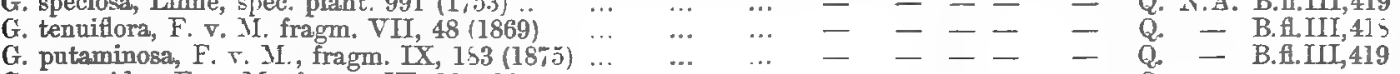

G. myrtoides, F. v. M., fragm. IX, 184 (15,.j) HODGKINSONIA, F, v. MI, fragm. II, 132 (1861).

H. ovatiflora, F. r. MI., fragm. iI, 132 (1861) CANTHICII, Lamarck, Encycl, method. I, 602 (1783). (Psydrax, Plectronia partly.)

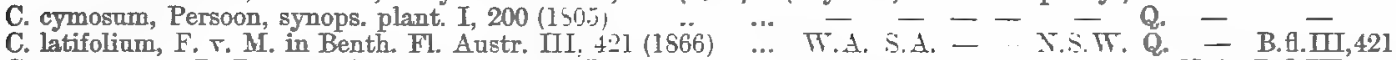

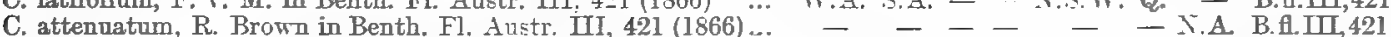

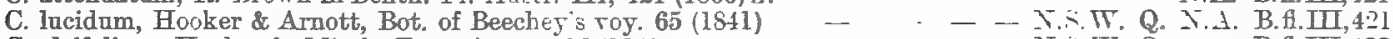

C. oleifolium, Hooker in Mitch. Trop. Austr. 397 (1815) . ․ - - - - N...W. Q - B.fl.II,422

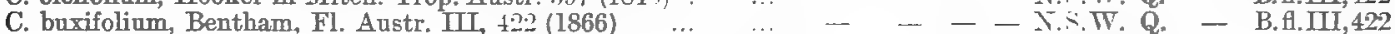

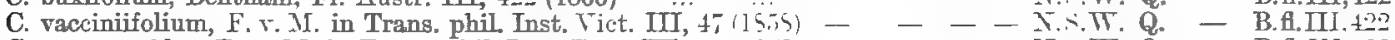

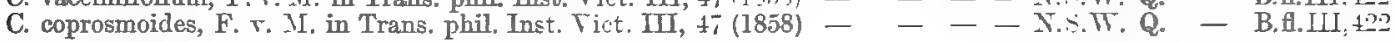
MORLNDA, Vaillant in act. Acad. Par. 202 (1;22).

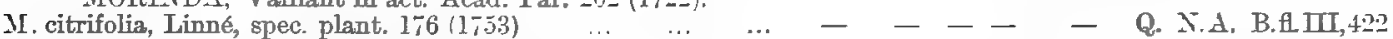

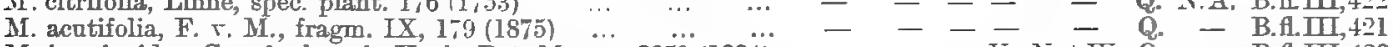

M. jasminoides, Cunningham in Hork. Bot. MLag. t. 33 öl (1834)

M. umbellata, Linné, spec. plant. $176(1753)$... _ _ _ - o - B.fll III 423

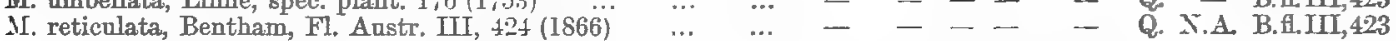
COELOSPERMLM, Blume, Bijdr. 994 (1526). (Pogonolobus.)

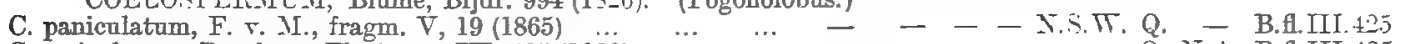

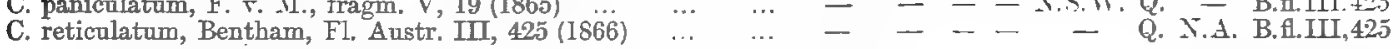
HYDNOPHYTUM, Jack in Transact. Linn. S.c. XIT, 124 (1823).

H. formicarum, Jack in Transact. Linn. Soc. XIV, 124 (1823) ... MYRMECODIA, Jack in Transact. Linn. Soc. XIV, 122 (1523).

M. echinata, Gaudichaud, Bot. roy. Bonite 472, t. 96 (1844) … LASIANTHCS, Jack in Transact. Linn. Soc. XIV, 125 (1823).

I. strigosus, Wight in Cale. Journ. nat. hist. TI, כ12(154fi) .. - - - - Q. - B.fl.II,426 PSTCHOTRIA, Linné, syst. ed. decim. 929 (1759). (Uragoga, 1787, Gromilia.)

P. nesophila, F. จ. M., fragm. II, 135 (1861)

P. Dallachyana, Bentham, F1. Austr. III, 427 (1866)
P. nematopoda, F. r. M., fragm. VII, 15 (1869) ...

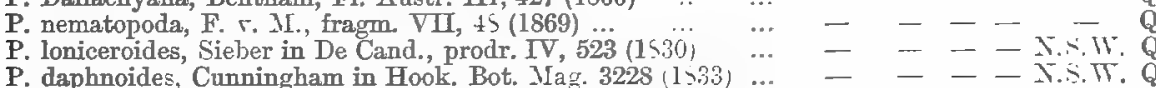

P. poliostemma, Bentham, Fl. Austr. III, 427 (1866)

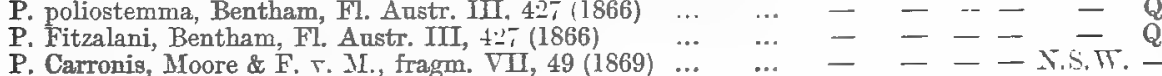
GEOPHILA, D. Don, prodr. Hl, Nepal, 136 (1825). (Cephaelis partly.)

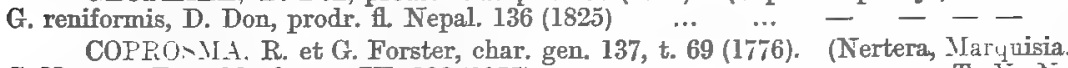

C. Nertera, F. v. M., fragm. IX, 186 (15-5)

C. reptans, F. r. MI., fragm. IX, $186(18,5)$

C. pumila, J. Hooker in Lond. Journ. VI, 465 (1817)

C. nitida, J. Hooker in Lond. Journ. TI, $46 \overline{5}$ (154i)

C. Billardieri, J. Hooker in Lond. Journ. VI, 465 (151;

C. hirtella, Labillardière, Nor. Holl. pL spec. I, 70, t. 9.5 (1801)

C. Baneri, Endlicher, iconogr. gen. pl. XI, t. Ill (15.35)

C. putida C. Moore \& F. T. M. fragm. TTI 45 (1869) …

C. lanceolaris, F. v. MI., fragm. IX, 70 (1875) $\ldots \ldots$ OPERCULARIA, J. Gaertner, de fruct. I, 111, t. $24(1 ; 58)$

0 . vaginata, Labillardière, Nor. Holl, pl. spec. I, 34, t. 46(1804)

0 . spermacocea, Labillard., Nor. Holl. pl. spec. I, 3., t. $4_{i}(1804)$ W.d.

0 . seabrida, Schlechtendal, Linnaea XX, 604 (154-)

O. hirsuta, F. v. M. in Benth. FI. Austr. III, 434 (1866)

0. aspera, Gaertner, de fruct. I, 112, t. $24(1755) \ldots$

0 . hispida, Sprengel, syst. reg. I, $35.5,1525)$

0 . diphylla, Gaertner, de froct. I, $113(1 ; 5,1)$

O. orata, J. Hooker in Lond. Journ. VI, 465 (185-)

0 . varia, J. Hooker in Lond. Journ. TI, 466 (1St)

O. rubioides, A. L. de Jussieu in Annal. du Mus. IV, 428 (1804)

O. volubilis, R. Brown in Benth. Fl. Austr. II, 435 (1866)

O. hispidula, Endlicher in Hueg. enum. 58 (1537)

O. echinocephala, Bentham, Fl. Austr. II, 436 (1866) ...

O. apiciflora, Labillardière, Nov. Holl. pl. spec. I, 35, t. 48 (1804)

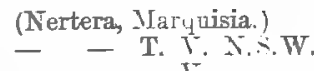

- - - T. T...W. -

- - T. $\because$ - -

- $\quad$ T. T.

- - T. T. N.S. -

- S.A. T. T. T.S.T. -

$-\quad-\quad-1 . S \Pi$ - -

- - - I.S.11.-

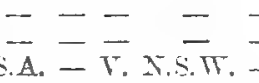

$=-\overline{\mathrm{T}} \cdot \mathrm{x} \cdot \mathrm{\pi} \cdot \overline{\mathrm{Q}}$

$=-\mathrm{r} \cdot \mathrm{N} \cdot \mathrm{a}$

- $\overline{\text { T. }}$. N. N. $\mathrm{N}$.

S. T. Y.S.W.

W.A.

W.A.

TT.A.

T.A.

.

-

$-\quad-$

$-\quad-$

$-1$

$-$

\section{M.fr.IX, 1 ; 9 .}

M.fr.IX, 180.

M.fr. VII, 46;IX, 185.

M.fr.I, $56 ; \mathrm{LX}, 185$.

MI. fr. VII, 45.

MI.fr. TII, 45.

[135.

M.fr.II, 133; LI, 185; II,

M.fr.II, 134;IX, 186.

Q. N.A. B.fliI . 1 -

- B.t.W, 427

B.fi. $\overline{11}, 42-$

B.fl. III, 428

B.fl.III, $4: 25$

B. fl. III, 42 ,$$
\text { - }
$$

M. fr. IX, 70, 185.

M.fr. VII,45;VII, 147.

M.fr.IX, 19R.

M.fr.IX, 186.

M.fr.LX, 156.

E.fl III, 430

B.fl. III, 430

- B.fl.II, 430

- B.f.IU, 429

$\begin{array}{ll}\text { - } & \text { M.fr.IX,69. } \\ -\quad \text { MIfr.IX,69. }\end{array}$

I.fr.IX, 70 .
B.t.III, 433

B.fl.II, 433

B.fl.III,433

B.fl.II, 434

B.fl.II, 434

B.t.III, 434

B.fl.II, 434

B.fl.III, 435

B.H.III, 435

B.fl.II, 435

B.fl.III, 435

B.fl.II, 436

B.fl.II, 436

B.tl.II, 436 
POMAX, Solander in Gaertn., de fruct. I, 112 (1788).

P. umbellata, Solander in Gaertn., de fruct. I, 112 (1788)

ELEUTHRANTHES, F. v. M., fragm. IV, 92 (1864).
E. opercularina, F. v. M., fragm. IV, 92 (1864) ...

KNOXIA, Linné, F1. Zeyl. 189 (1747).

K. corymbosa, Willdenow, spec. pl. I, 582 (1798)

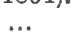

SPERMACOCE, Dillenius, hort. Elth. 369, t. 277 (1732).

S. pogostoma, Bentham, Fl, Austr. III, 440 (1866)

S. marginata, Bentham, Fl. Austr. III, 440 (1866)

S. multicanlis, Bentham, Fl. Austr. III, 440 (1866)
S. exserta, Bentham, Fl. Austr. III, 441 (1866) $\ldots$

S. membranacea, R. Brown in Benth. Fl. Austr. İ̈, 441 (1866)

S. debilis, Bentham, Fl. Austr. III, 441 (1866) $\ldots . \quad \ldots \quad \ldots$

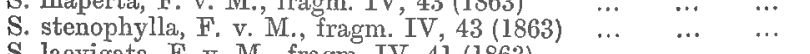

S. laevigata, F. v. M., fragm. IV, 41 (1863)
S. breviflora, F. v. M. in Benth. Fl. Anstr. III, 442 (1866) $\quad \ldots$

ASPERULA, Dodoens, pemptad. 355 (1583).

A. geminifolia, F. F. M., fragm. V, 147 (1865)

GALIUM, Dodoens, pemptad, 335 (1583), from Dioseorides.

G. umbrosum, Solander in G. Forst. prodr. 89 (1786)

G. australe, De Candolle, prodr. IV, 608 (1830)...
S. brachystoma, R. Brown in Benth. Fi. Austr. III, 439 (1866)

S. leptoloba, Bentham, FI. Austr. III, 440 (1866)

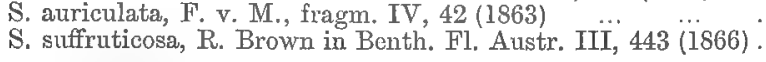

A. oligantha, F. v. M. in Neerl. Kruidk. Arch. IV, 111 et 112 (1859)

\author{
- S.A. - V. N.S.W.Q. - B.H.III,437 \\ W.A. - - - - - B.H.III,437
}

M.f.IX, 187.

M.fr.IV,92. .

- - - - Q. N.A. B.f.III,438 M.fr.IX,187.

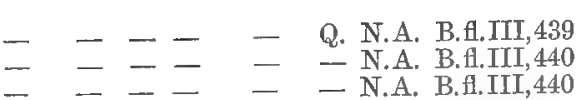

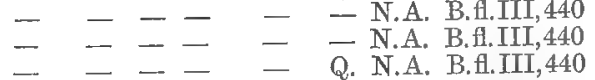

$=\quad=-二$ N.S.W. Q. N. N.A. B.Al.III, 440

- = - Z N.S.W. Q. N.A. B.A.III,441

$=-z=-\overline{\text { N.A. B.f.III,441 }}$

- - - - - Q. - B.H.IM, 441

$-\quad-\quad-\quad-\quad$ - N.A. B.f.III,44I

- -- - - - N.A. B.f.III,442

- $-=-$ - - - N.A. B.A.III,442

- $\quad$ - _ - N.A. B.H.III,443

- S.A. - V. N.S.W. Q. - B.fl.III,443 -- S.A. T. V. N.S.W. Q. - B.flIII,444

- S.A. T. V. N.S.W. - - B.fl.III,446

- S.A.T. V. N.S.W. Q. - B.H.III,446

\section{CAPRIFOLIACEAE.}

Adanson, Familles des plantes II, 133 (1763).

SAMBUCUS, Tournefort, inst. 606, t. 396 (1700), from Dodoens (1583). (Tripetelus.)

S. xanthocarpa, F. v. M. in Transact. phil. Inst. Vict. I, 42 (1855) - - V. N.S.W. Q. - B.fl.III, 398

S. Gaudichaudiana, De Canclolle, prodr. IV, $322(1830) \ldots \ldots$... $\ldots$ S.A. T. V. N.S.W. Q. - B.fl.III,398

\section{PASSIFLOREAE.}

A. L. de Jussieu in Ann, du Mus. VI, 102 (1805).

PASSIFLORA, Plukenet, phytograph. 202 et 282, t. 104, 210, 211, 212 (1692). (Disemma.)

P. Herbertiana, Lindley, Bot. Regist. t. 737 (1823) $\ldots \ldots \ldots$...

P. cinnabarina, Lindley in Gard. Chron. 724 (1855) $\quad \ldots \quad \ldots \quad \ldots \quad-\quad-\quad$ V. N.S.W. $-\quad-$ B.Al.III,311

P. aurantia, G. Forster, fl. ins. Austr. prodr. 62 (1786) $\cdots . . . . . \quad-\quad-\quad--$ N.S.W. $\bar{Q}=$ B.ff.ITI,312

P. brachystephanea, F. v. M. in Benth. Fl, Austr. III, $312(1866)-2-1-$ Q. - B.H.III,312

MODECCA, Lamarck, Encycl. method. IV, 208 (1797).

M. australis, R. Brown in De Cand. prodr. III, 337 (I828) ‥ - $-{ }_{-}-$Q. N.A. B.Al.III,313

\section{CUCURBITACEAE.}

Haller, enum. stirp. Helv. praef. 34 (1742).

TRICHOSANTHES, Linné, gen. plant. 295 (1737).

T. pentaphylla, F. v. M. in Benth. Fl. Austr. III, 314 (1866) ... - $-\quad-\quad-\quad$ Q. - B.fl.III,314

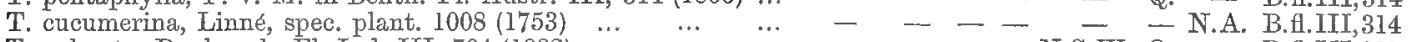

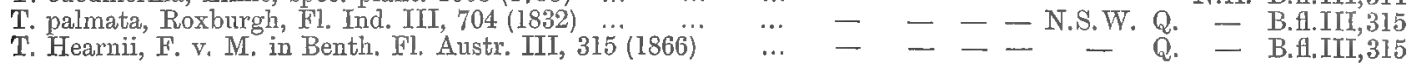

LAGENARIA, Seringe in Miem. Soc. Genèr. III, 25, t. 2 (1825).

L. vulgaris, Seringe in De Cand. prodr. III, 279 (1828) ... … - - - - Q. - B.Al.III,316

LUFFA, Tournefort in act. Acad. Paris. 84, t. 2 (1706), from Vesling (1638).

L. Aegyptiaca, Miller, Gardener's Dictionary (1731)
L. graveolens, Roxburgh, Fl, Ind. III, 716 (1832)
...

L. foetida, Cavanilles, icon. I, 7, t. 9-10 (1791)

ZANONIA, Linné, coroll. 19 (1737). (Alsomitra.)

Z. Capricornica, F. v. M., fragm. VII, $61(1870) \ldots$

Z. Hookeri, F. v. M., fragm. VI, $188(1868)$

$\cdots \quad-\quad--$

-

N.A.

$-$

... $\quad$.. ---

$=8$

Q. $=$ -

M.fr.IV,43.

M.fr.IV,43.

M.fr.IV,4l.

M.fr.IV, 42 .

M.fr.IX,188.

M.fr.IX, 68.

M.fr.IX,68.

M.fr.IX, 68 .

M.fr.I.56.

M. ir.IX, 69.

M.fr.VI,187.

M.fr.IX,188;XI, 27.

M.fr.IX, 187;XI,27.

CUCUMIS, Tournefort, inst. 104, t. 31 (1700), from Plinius,

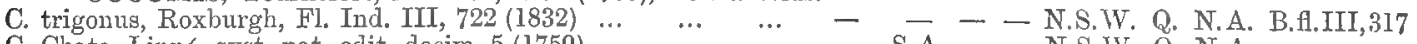

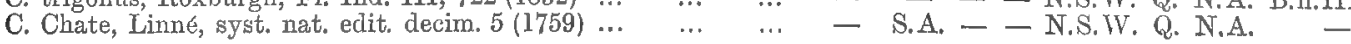

BENINCASA, Savi in Bibl. Ital, IX, 158 (1818).

B. vacua; Cucurbita vacua, F. v. M., fragm. VI, 186 (1868)

MOMORDICA, Tournefort, institut. rei herbar. 103, t. 29 et 30 (1700).

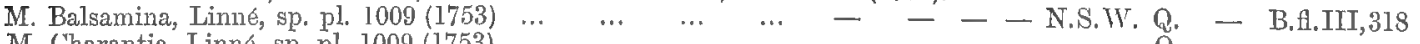

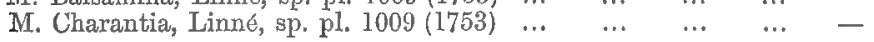

BRYONOPSIS, Arnott in Hook. Journ, of Bot. III, 274 (1841).

B. laciniosa, Nandin in Annal. des sc, nat. sér. cinq. VI, $30(1869)$ - (Bronia partly.)

B. Pancheri, Naudin in Annal. des sc, nat. sér. cinc. V, 30 (1869) _ _ _

(Bryonia partly.) 
MELOTHRIA, Linné, coroll. 1 (1737). (Zehneria, Mukia)

M. Cunninghamii, Bentham, Fl, Austr. III, $320(1866)$.. $\quad \ldots \quad-\quad-\quad-\quad-$ N.S.W. Q. N.A. B.A.III, 320 M. Baueriana, F. v. M., fragm. VI, I88 (1868) ... ... M. Muelleri, Bentham, Fl. Austr. III, $320(1866) \ldots$ ... … $\quad-\overline{\text { SA }}-\overline{\mathrm{V}}$ N.S.W. Q - N.A N.S.W. SICYOS, Linné, syst. nat. 9 (1735); Linné, gen. plant. 297 (1737). S. angulata, Linne, spec. plant. 1013 (1753)

\section{COMPOSITAE.}

Vaillant in act. Acad. Paris. 143 (1718).

CENTRATHERUM, Cassini in Bull, de la Soc. philom. (1817).

C. muticum, Lessing in Schlecht. Linnaea II, 320 (1829) …
PLEUROCARPAEA, Bentham, Fl. Austr. III, 460 (1866).

P. denticulata, Bentham, F1. Austr. III, 460 (1866) ... ...

VERNONIA, Schreber, gen. plant. II, 541 (1791).

V. cinerea, Lessing in Schlecht. Linnaea II, 291 (1829) $\ldots \quad \ldots \quad-\ldots \quad-\quad-\quad-$ N.S.W. Q. N.A. B.fH.III, 459 V. vagans, De Candolle, prodr, V, $32(1843) \quad \ldots \quad \ldots \quad \ldots$
ELEPHANTOPUS, Vaillant in act. Acad, Par. $309(1719)$.

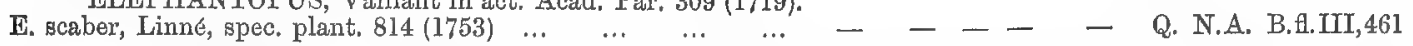
ADENOSTEMMA, R, et G. Forster, char. gen. 89, t. 45 (1776).

A. viscosum, R. \& G. Forster, char. gen. 89, t. $45(1776)$.. - S.A. - V. N.S.W. Q. - B.f.III, 462 AGERATUM, Linné, gen. plant. 247 (1737).

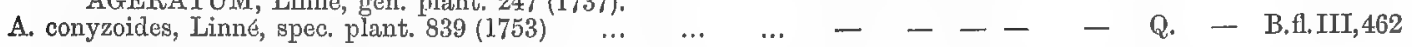
EUPATORIUM, Toumefort, inst. 455, t. 259 (1700), from C. Bauhin (1623).

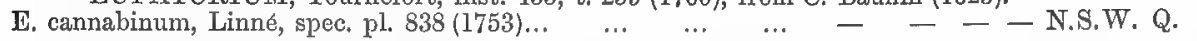
LAGENOPHORA Cassini in Bull de la Soc. philom, 34 (1818). (Ixanchenus, Solenogyne, Tmphysopus.)

L. Billardieri, Cassini in Diction. XXV, 111 (1822) $\ldots . .$. W.A. S.A. T. V. N.S.W. Q. - B.fl.III,507

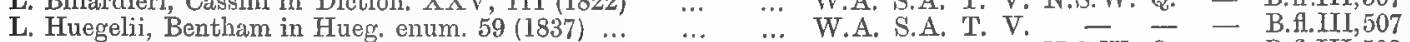

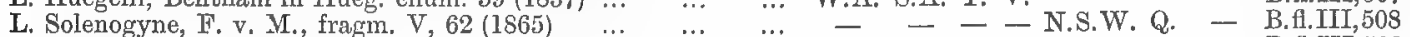

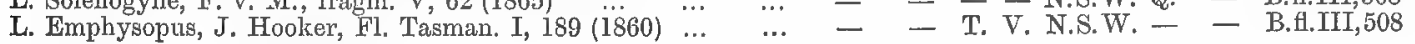
BRACHYCOME, Cassini in Bull. de la Soc. philom. 199 (1816). (Bellidis subgenus, Pacquerina, Brachystephium, Steiroglossa, Silphiospermum.)

B. diversifolia, Fischer \& Meyer, ind. sem. hort. Petrop. 31 (1835) - S.A. T. V. N.S.T. -

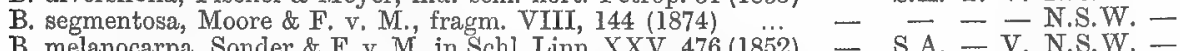

B. melanocarpa, Sonder \& F. v. M. in Schl. Linn. XXV, $476(1852)-$ S.A. - V. N.S.W. -
B. radicans, Steetz in Lehm. pl. Preiss. I, $429(1845) \ldots \ldots$

B. radicans, Steetz in Lehm. pl. I'reiss. I, $429(1845)$
B. goniocarpa, Sonder \& F. v. M. in Schl. Lim. XXV, $474(1852)$ W.A. S.A. - V. N.S.W. -

B. pachyptera, Turczaninow in Bull. de Mosc. XXIV, I75 (1851) W.A. S.A. - V. N.S.W. -

B. iberidifolia, Bentham in Hueg. enum. 59 (1837) $\quad \ldots \quad$... W.A. $\quad$ W. - - - -

B. pusilla, Steetz in Lehm. pl. Preiss. I, 427 (1845)

B. microcarpa, F. v. M., fragm. I, 50 (1858)

B. Stuartii Bentham

B. scapigera, De Candolle, prodr. VII, $277(1838)$..'

B. Muelleri, Sonder in Schlecht. Linnaea XXV, 475 (1852)

B. graminea, F. v. M., fragm. I, 49 (1858)... ‥
B. angustifolia, Cinningham in De Cand., prodr. V, 306 (1836)

B. linearifolia, De Candolle, prodr. V, $306(1836) \ldots \quad \ldots \quad \ldots$

B. basaltica, F. v. M., fragm. I, 50 (1858) ... X $\dddot{2} \ldots, \ldots$
B. trachycarpa, F. v. M. in Schlecht. Linnaea XX $339(1852)$

B. exilis, Sonder in Linnaea XXV, 473 (1852)

B. debilis, Sonder in Linnaea XXV, 477 (1852)

B. decipiens, J. Hooker in Lond. Journ. VI, $114(1847) \ldots$
B. cardiocarpa, F. v. M. in Benth. Fl. Austr. III, 516 (1866) $\ldots$

B. nivalis, F. v. M. in Transact. phil. Soc. Vict. I, 43 (1854) ..

B. scapiformis, De Candolle, prodr. V, 306 (1836)

B. stricta, De Candolle, prodr. V, 305 (1836)

B. heterodonta, De Candolle, prodr. V, 305 (1836) $\quad \ldots$.

B. Billardieri, Bentham, Fl. Austr. III, 518 (1866)

B. cheilocarpa, F. v. M. in Wing's South. Sc. Record, Aüg. (18̈2)

B. ciliaris, Lessing, syn. comp. 172 (1832)..

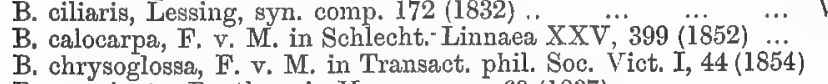

B. marginata, Bentham in Hueg. enum. 60 (1837)

B. Sieberi, De Candolle, prodr. V, 306 (1836)

B. discolor, C. Stuart in Benth. Fl. Austr. III, 520 (1866)

B. multifida, De Candolle, prodr. V, 306 (1836) ...

B. latisquamea, E. v. M., fragm. XI, 16 (1878) ...

B. glandulosa, Bentham, Fl. Austr. III, 520 (1866)

B. collina, Bentham, Fl. Austr. III, 521 (1866) … ERODIOPHYLLUM, F. v. M., fragm. IX, 119 (1875).

E. Eldleri, F. v. M., fragm. IX, 120 (1875).. MINURIA, De Candolle, prodr. V, 298 (1836).

M. Ieptophylla, De Candolle, prodr. V, 298 (1836)

M. Cunninghamii, Bentham, Fl. Austr. III, 498 (1866) ...
W.A. - - - - $-\overline{-}$
W.A. $-\overline{\text { Q. }}$

- - - - - N.S.W. Q

- - - - V. N.S.W. -

- S.A. - - - -

S.A. T. V. N.S.W. -

- - T. V. N.S.W. -

- T. T. T. S.A. - - N.S.W. Q. S.A. - V. N.S.W. Q. S.A. - V. N.S.W. - - V. N.S.W. S.A. - V. N.S.W. S.A. T. V. N.S.W. S.A. T. V. N.S.W. - - V. N.S.W. - T. V. N.S.W. - T. V. N.S.W. - - - N.S.W. - - - N.S.W. $\overline{\mathrm{s}} \overline{\mathrm{T}} \overline{\mathrm{V}} \mathrm{\textrm {S } W}-$ S.A. T. V. N.S.W. -
S.A. - V. N.S.W. S.A. - V. N.S.W. -
S.A. - V. N.S.W. - - - N.S.W. Q. - - - N.S.W. -

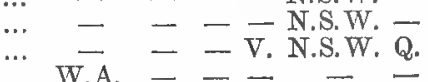

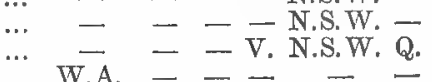
... W.A. - - - - W.A. $-\overrightarrow{\text { W. }}$ - - V. N.S.W. ...

-. - S.A. - - - geron, Elachothamnus, Kippistia.) $\ldots$ W.A. S.A. - V. N.S.W. Q.
$\ldots$ S.A. - V. N.S.W. -
- B.H.III,5I1

- B.fl.III,512

- B.tl.III,512

- B.A.III,512

- B.fl.III, 512

- B.fl.III,513

- B.fl.III,513

B.fl.III, 513

- B.fl.III,513

- B.III 514

B.fl.III, 514

- B.fl.III,514

B.fIII 515

B.A.III,515

B.H.III, 515

B. H.III, 516

B.A.III, 516

- B.t.III,516

B.fl.1II,516

B.fl.IT, 517

B.fl.III, 517

B.fl.III, 517

B.fl.III,518

B. A.III 518

B.,1.III, 518

- B.f. III,518

B.tl.III, 519

- B.f. III,519

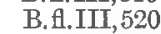

- B.f.III,520

- B.fl.III,520

- B.fl. III, 520

- B.tl.IIr, 521

\section{M.fr.XI, 123.}

M.fr. I, 50 .

M.fr. VIII, 142 .

M.fr. I, 49 .

M.fr.I, 50 .

M.fr.XI, 27.

M.fr. VIII, 145.

M.fr, XI, 16 .

M.fr.IX,119.

- B.fl.IIT, 498 M.fr.IX,119;X,56.
M.fr, VI, 188;VII,62. M.fr. VI, 188.

M.fr.VI, 187.

M.fr.VI, 187;VII, 62.

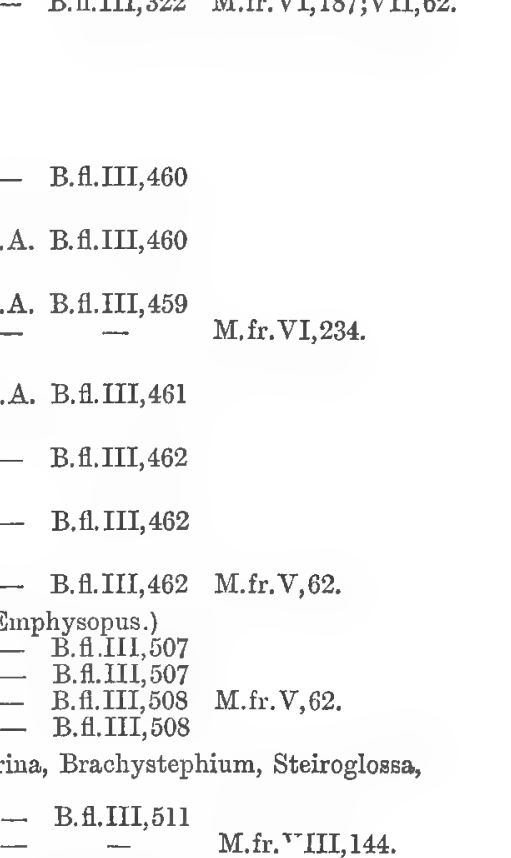

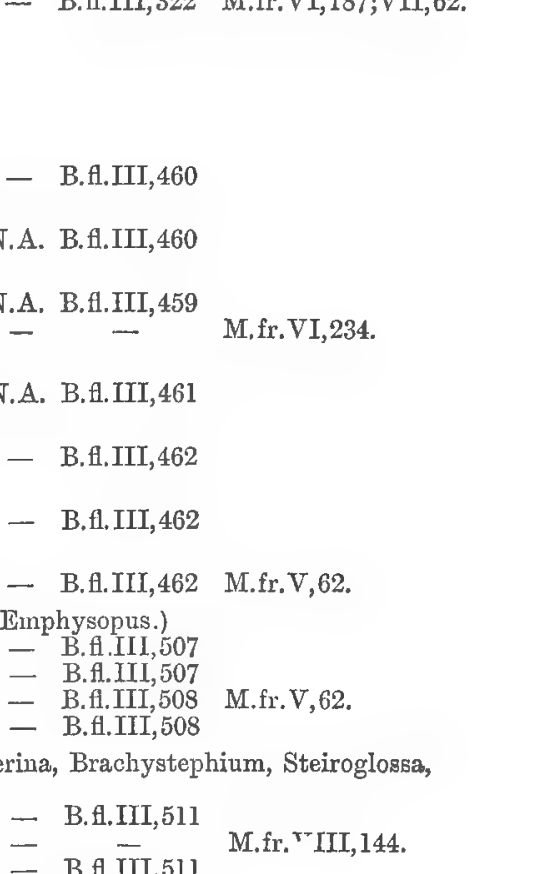

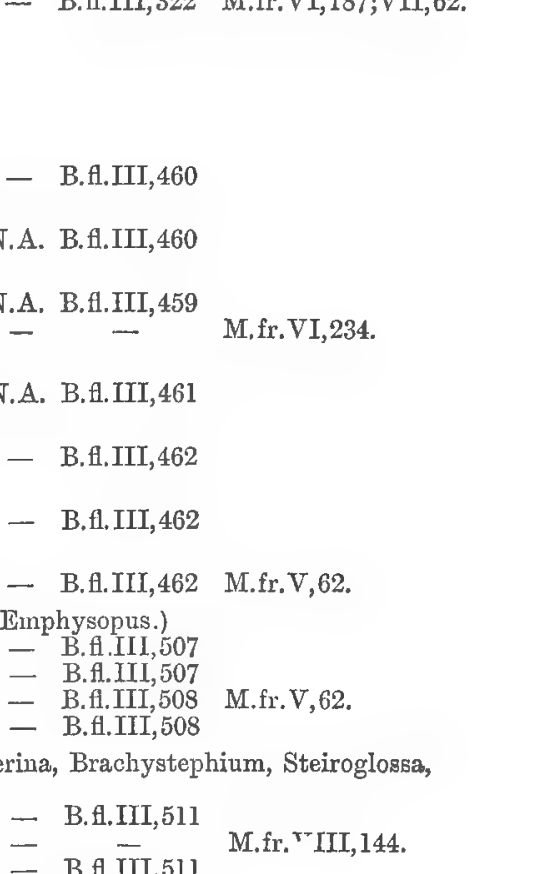

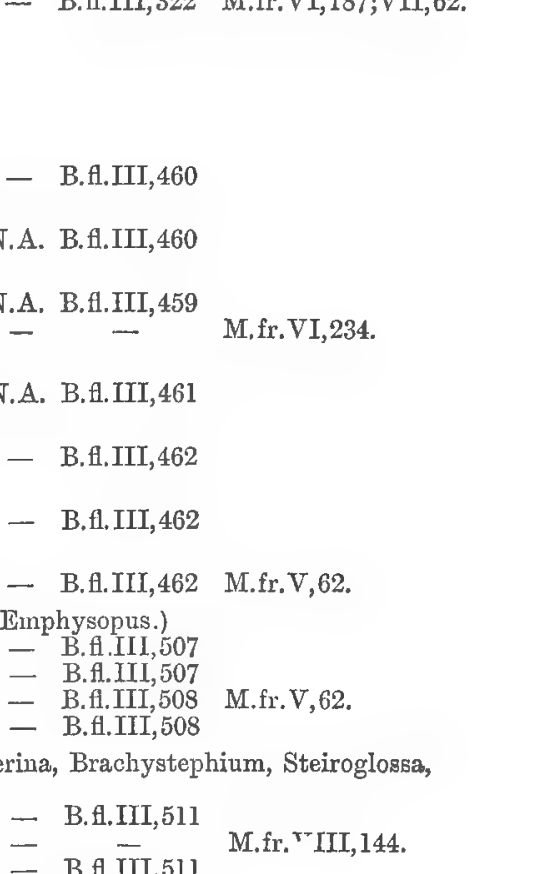


M. Candollei, F. v. M., fragm. IX, $119(1875)$

S.A. - V. N.S.W. Q. N.A. B.f.III, 499

M.fr.X,56.

M. suaedifolia, F. v. M. in Bentham, Fl. Austr. IIII, 499 (1866) M.fr.X, 56.

CALOTIS, R. Brown in Bot. Regist. t. 504 (1820). (Huenefeldia, Goniopogon, Cheiroloma.)

C. dentex, R. Brown in Bot. Regist. 504 (1820) ... … ... - - - N.S.T. Q.

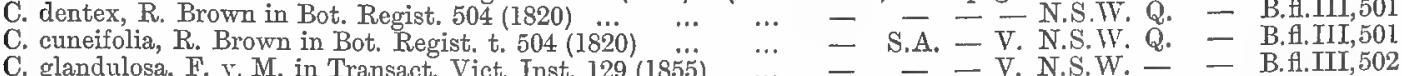

C. glandulosa, F. v. M. in Transact. Vict. Inst. 129 (1855) $\ldots-. .7-$ - V. N.S.W. - - B.fl.III,502

C. erinacea, Steetz in Lehm. pl. Preiss. I, $424(1845) \ldots \ldots \ldots$ W.A. S.A. - V. N.S.W. - - B.fl.III,502

C. scabiosifolia, Sonder \& F. V. M. in Linnaea XXV, 471 (1852) - S.A. - V. N.S.W. - - B.H.XII,503

C. scapigera, Hooker in Mitch. Trop. Austr. 75 (1848) .. ‥ - S.A. - V. N.S.W. Q. N.A. B.fl.III,503

C. anthemoides, F. v. M. in Transact. phil. Soc. Vict. I, 44 (1854) - - - V. $-2-$ B.A.III,504

C. lappulacea, Bentham in Hueg. enum. 60 (1837) 1. ... W.A. S.A. - V. N.S.W. Q. - B.t.III,504

C. microcephâla, Bentham, Fl, Aristr. III, 504 (1866) $\quad \cdots \quad$...

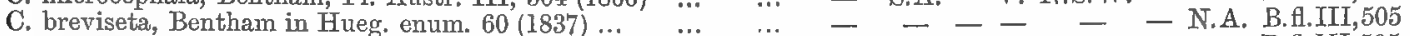

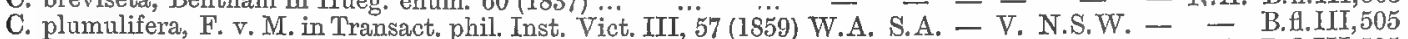

C. porphyroglossa, F. v. M. in Benth. Fl. Austr. III, 505 (1866) - S.A. - - N.S.W. - N.A. B.fl.III,505

C. pterosperma, R. Brown in Benth. Fl. Austr. III, 505 (1866)... - - - - - N.A. B.H.III,505

C. hispidula, F. v. M. in Transact. Vict. Inst. $130(1855) \quad \ldots$ W.A. S.A. - V. N.S.W. - - B.H.III,506

C. Kempei, F. v. M. in Transact. Roy. Soc. S.Austr. IV, 112 (1881) - S.A. - - - - - ASTER, Tournefort, inst. 481, t. 274 (1700), from Dioscorides. (Olearia, Celmisia, Eurybia, Steetzia.)

A. megalophyllus, F. v. M., fragm. V. $70(1865) \ldots$... … - - V. N.S.W. - B.fl.III, 468

A. chrysophyllus, Cunningham in De Cand. prodr. V, $266(1830)=-Z-\mathrm{N}$.S.W. - - B.fl.III,468

A. alpicola, F. v. M., fragm. V, $70(1865) \ldots \ldots, \ldots \ldots$.... $-\ldots-$ V. N.S.W. - - B.H.III,468

A. rosmarinifolius, Cunningham in De Cand. prodr. V, $268(1836)=---$ N.S.W. - - B.A.III,468

A. viscosus, Labillardière, Nov. Holl. pl. spec. II, 53, t. $203(1806)$ - - T. V. - - - B.H.III,468

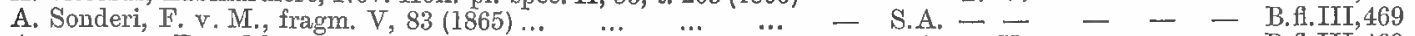

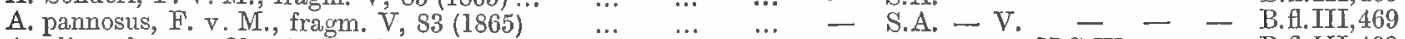

A. oliganthemus; Olearia, F.v. M. in Benth. Fl. Austr. III, 469 (1866) - - - - N.S.W. - - B.fl.III,469

A. argophyllus, Labillard., Nov. Holl. pl. spec. II, 52, t. 201 (1806) - - T. V. N.S.W. - - B.H.III,470

A. cydonifolius, Cunningham in De Cand, prodr. V $267(1836)-{ }_{-}-$N.S.W. - - B.fl.III,470

A. myrsinoides, Labillard., Nov. Holl. pl. spec. IT, 53, t. $202(1806)$ - S.A. T. V. N.S.W. - - B.fl.III,471

A. persoonioides, Cunningham in De Cand. prodr. V, $267(1836)$ -

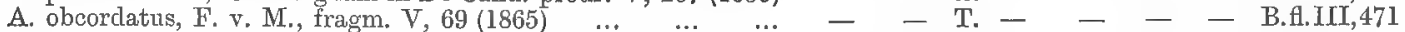

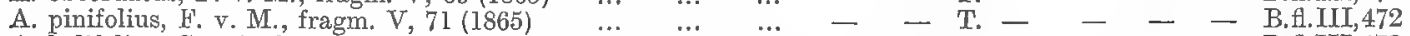

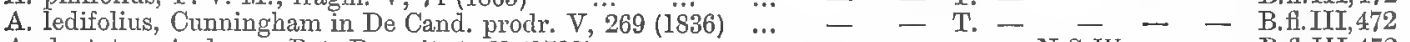

A. dentatus, Andrews, Bot. Reposit. t. 61 (1799)... ... .. - - - N.S.W. - - B.fl.III,472

A. stellulatus, Labillard., Nor. Holl. pl. spec. II, 50, t. $196(1806)$ - S.A. T. V. N.S.W. Q. - B.H.III,473

A. asterotrichus, F. v. M., fragm. V, $79(1865) \ldots \ldots \ldots$... $\ldots$ S.A. - V. N.S.W. - - B.fl.III,473

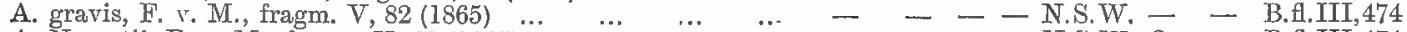

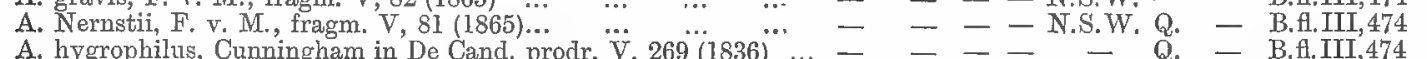

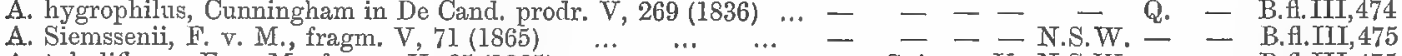

A. tubuliflorus, F. v. M., fragm. V, $65(1865) \quad \ldots . \quad \ldots . \quad \ldots . \quad$ S.A. $-\overline{\text { V. N.S.W. }}$ - - B.Al.III,475

A. axillaris, F. v. M., fragm. V, $64(1865) \ldots \ldots . \ldots . \quad \ldots . .6$ W.A. S.A. T. V. N.S.W. - N.A. B.fl.III, 475

A. revolutus; Olearia, F. v. M. in Benth. Fl.Austr. III, 476 (1866) W.A. - - - - - - B.fl.III,476

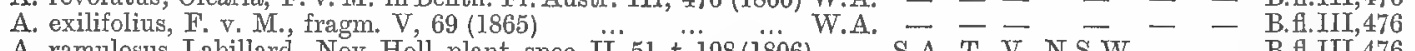

A. ramulosus, Labillard., Nov. Holl. plant. spec. II, 51, t. $198(1806)$ - S.A. T. V. N.S.W. - - B.H.III,476

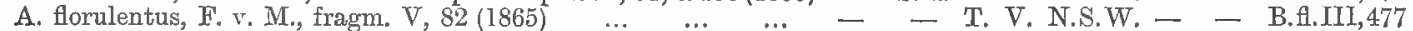

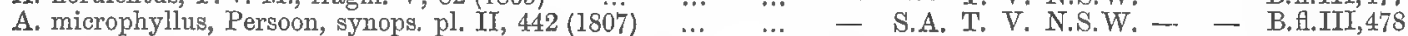

A. Mitchelli, F. v. M., fragm. V, $78(1865) \quad \ldots \quad \ldots \quad \ldots \quad$ - $\quad$ S.A. - V. N.S.W. Q. - B.A.III,478

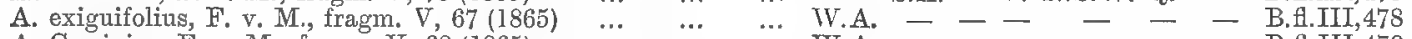

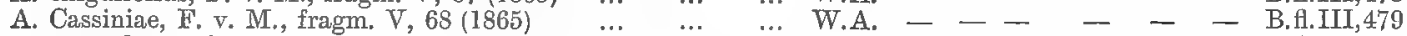

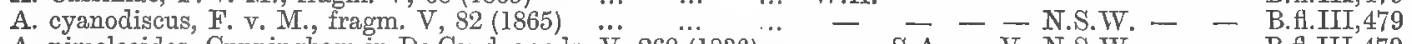

A. pimeleoides, Cunningham in De Cand. prodr. $\dddot{V}, 268(1 S 36) \ldots$ - S.A. - V. N.S.W. - - B.fl.III,479

A. iodochrous, F. v. M., fragm. V, 81 (1865) $\quad \ldots \quad \ldots \quad \ldots \quad \ldots \quad-\quad-\quad-$ V. N.S.W. - $~-~ B . f 1 . I I I, 480$

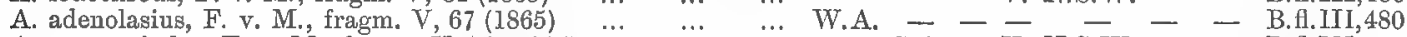

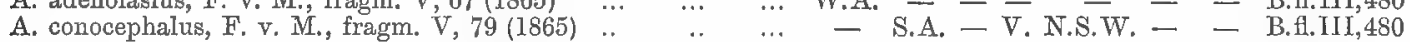

A. calcarens; Olearia, F. v. M. in Benth. Fl. Austr. III, 481 (1866) - S.A. - V. N.S.W. - - B.Al.III,481

A. magniflorus, F. v. M., fragm. V, $80(1865) \quad \ldots \quad \ldots \ldots$ … S.A. - V. N.S.W. -

A. Muelleri; Eurybia, Sonder in Schlecht. Linn. $\dddot{X x V}, 459$ (1852) W.A. S.A. - V. N.S.W. -

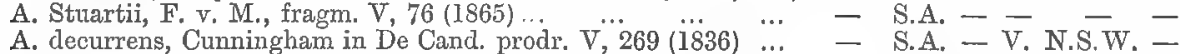

A. decurrens, Cunningham in De Cand. prodr. V, $269(1836) \ldots$.
A. glutescens, F. v. M. fragm. V, 77 (1865)

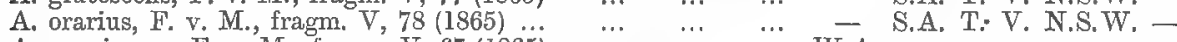

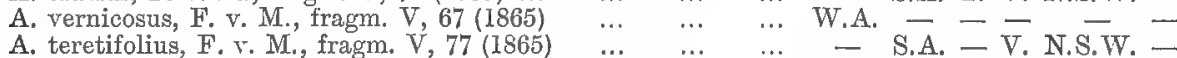

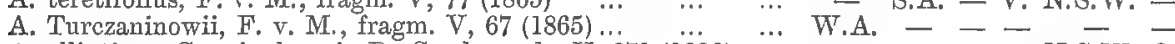

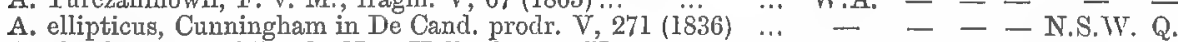

A. glandulosus, Labillard., Nov. Holl. pl. spec. II, 50, t. $197(1806)$ - S.A. T. V. N.S.IV. -

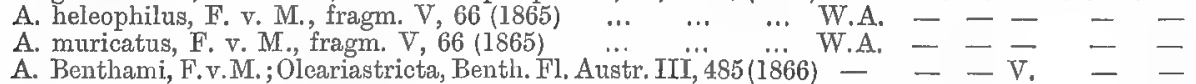

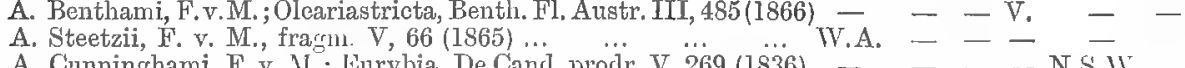

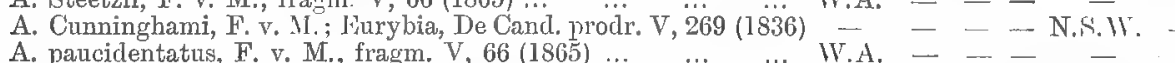

B.fl.III, 481

- B.ff.III, 481

B.Al.III,481

B.f.III,481

- B.H.III, 482

- B.H.III,482

- B.fl.III, 482

B.A.III, 483

B.fl.III,483

B.fl.III, 483

B. fl.III, 484

B.fl.III, 484

B.Al.III, 484

- B.fl.III, 485

- B.fl.III, 4 S.I

B. 11. III, 486

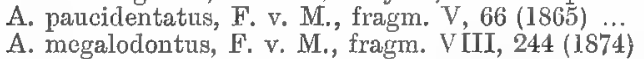

A. adenophorus, F. v. M., fragm. V, 78 (1865) ...

A. homolepis, F. v. M., fragm. V, 65 (1865)

A. xerophilus, F. v. M., fragm. V, 76 (1865)

A. Ferresii, F. v. M., fragm. V, 75 (1865) ...

...

W.A. S.A.

B. H. III, 485

M.fr.XI, 27.

M.fr.XI, 27.

M.fr.XI, 139.

M.fr. V, 70 .

M.fr. V, 70 .

M.fr.V, 71 .

M.fr. V, 83 .

M.fr.V,83.

M.fr. V, 68 .

M.fr.V, 69 .

M.fr. V, 69.

M.fr. V, 69.

M.fr. V,7I.

M.fr.V. 82 .

M.fr.V,81.

M.fr.I,111.

M.fr.V, 82 .

M.fr. V,81.

M.fr.I, 50.

M.fr. V, 65 .

M.fr. V, 64 .

M.fr.V, 69 .

M.fr. $V, 82$.

M.fr.V,70.

M.fr.V,78.

M.fr.V, 67.

M.fr. V, 68 .

M.fr.V,82.

M.fr.II,110.

M.fr.V, 67.

M.fr. $V, 79$.

M.fr. $T, 80$.

M.fr.I, 202 .

M. fr. $V, 77$.

M.fr.V,78.

M.fr.V, 67 .

M.fr. V, 77 .

M.fr. V, 67.

M.fr.I, 16.

M.fr. $\nabla, 66$.

M.fr. V, 66 .

M.fr. $Y, 66$.

MI.fr. $r, 66$.

M.fr. VIII, 24.4 .

B.ff. III, 486

M.fr.I,11l.

M.fr. V, 65 .

M.fr.I,51,76\&86.

M.fr.III, 18. 
A. exul, Lindley, Bot. Regist. XXV, App. XXIV (1839)

A. argutus, R. Brown in Benth. Fl. Austr. III, 488 (1866)

A. Huegelii, F. v. M., fragm. V, 79 (1865)...

A. Balli, F. v. M., fragm. VIII, $143(1874)$..

A. Mooneyi, F. v. M., fragm. VIII, 144 (I874)

A. Celmisia, F. v. M., fragm. V, 84 (1865)...
... W.A. S.A. - V. N.S.W.

- - B.fl.III, 487

․ - - $-\overline{-}-\mathrm{N} . \mathrm{A}$. B.fl.III,488

... W.A. S.A. T. V. N.S.W. - - B.AlIII,488

... - - - - N.S.W. - - -

… - - - T. V. N.S.W.W. - - B.fl. - 489

VITTADINIA, Ach. Richard, Voy. d'Astrol. bot. 250 (1832). (Eurybiopsis, Microgyne.)

V. brachycomoides, F. v. M., fragm. V, 86 (1865)

V. australis, A. Richard, Voy. d'Astrol. 251 (1832)

V. australis, A. Richard, Voy. d'Astrol. 251 (1832)
V. scabra, De Candolle, prodr. V, 281 (1836) $\quad \ldots$.

V. macrorrhiza, A. Gray in Proc. Am. Acad. V, 118 (1863) ‥ - - - - N.S.W Q. - B.fl.TI,491 PODOCOMA, Cassini in Bull. de la Soc. philom. 137 (1817). (Ixiochlamys.)

P. cuneifolia, R. Brown, App. to Sturt, Centr. Austr. 17 (1849) - S.A. - - N.S.W. - N.A B.fl.III, 493 ERIGERON, Linne, hort. Cliffort. 407 (1737). (Haplopappus partly.)

E. pappochromus, Labillard., Nov. Holl. pl, spec. II, 47, t.193(1806) - - T. V. N.S.W. - - B.fl.III, 494

E. ambiguus, F. v. M. in Transact. phil. Inst. Vict. III, $58(1858)--\quad---$ Q. N.A. B.fl.III,494

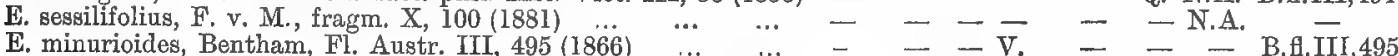

E. conyzoides, F.v. M. in Transact. phil. Soc. Vict. I, $10 \ddot{5}(1855)$ - - - V. N.S.W. - - B.fl.III,495 CONYZA, Lamarck. Encycl. méthod. II, 87, 89 (1786).

C. viscidula, Wallich, numer. list 3006 (1828)

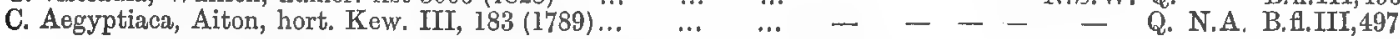
BLUMEA, De Candolle in Guillemin, Arch, de Bot. II, 514 (1833).

B. glandulosa, De Candolle in Wight, contrib. 14 (1834)...

B. amplectens, De Candolle in Wight, contrib. 13 (1834)...

$\mathrm{B}$. integrifolia, De Candolle, prodr. V, 433 (1836)...

B. diffusa, R. Brown in Benth. FI. Austr. III, 525 (1866)

B. hieracifolia, De Candolle in Wight, contrib. 15 (1834)..

B. Cunninghamii, De Candolle, prodr. V, 4.35 (1836)

B. acutata, De Cand in Ann. du Mus, d'hist, nat, Par. III $409\left(1{ }^{3} 34\right)$ -

B. lacera, De Candolle in Wight, contrib. $14(1834) \quad \ldots \quad \ldots \quad-\quad-\quad-$ PLUCHEA, Cassini in Bull, de la Soc. philom. 31 (1817). (Spiropodium, Eyrea.)

P. Indica, Lessing in Schlecht. Linnaea VI, $150(1831) \ldots \ldots \ldots$

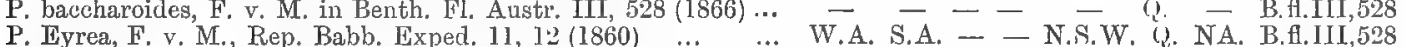

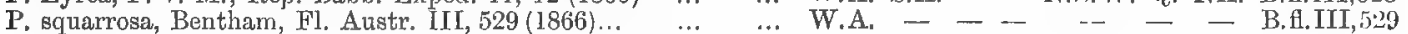

P. dentex, R. Brown in Benth. Fl. Austr. III, $529(1866) \quad \ldots \quad-\quad-\quad-\quad-\quad-\quad$ Q. - B.H.III,529 PTERIGERON, De Candolle, prodr, V, 293 (1836). (Streptoglossa, Oliganthemum.)

P. decurrens, De Candolle, prodr. V, 293 (1836) ... $\quad \ldots \quad$.. $\quad-\quad-\quad-\quad$ -

P. liatroides, Bentham, Fl. Austr. III, $532(1866) \ldots \ldots \ldots \ldots$ W.A. S.A. - $\quad$ - $\quad$ - $\quad$ - - B.fl.III,532

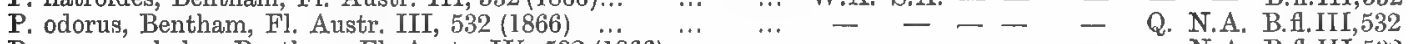

P. macrocephalus, Bentham, Fl. Austr. III, 532 (1866) $\ldots \quad \ldots \quad-\quad-\quad-\quad-\quad-$ N.A. B.fl.III,532

P. microglossus, Bentham, Fl. Austr. III, $532(1866) \quad \ldots \quad \ldots \quad-\quad-\quad-\quad-\quad-\quad$ - N.A. B.fl.III,532

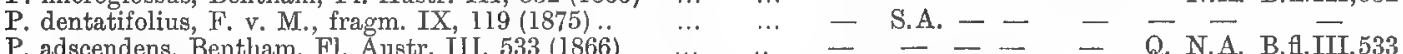

P. adscendens, Bentham, Fl. Austr. II I, 533(1866)
P. filifolius, Bentham, Fl. Austr. III, 533(1866) ...

THESPIDIUM, F, v. M. in Journ. of Landsborough's Exped. app. (1862).

T. basitlorum, F. v. M. in Journ, of Landsb. Exped. App. (1862) - - - - - - N.A. B.fl.III,534 COLEOCOMA, F. v. M. in Hook. Kew Misc. IX, 19 (1857).

C. Centaurea, F. v. M. in Hook. Kew Misc. IX, 19 (1857) EPALTES, Cassini in Bull. de la Soc. philom. 139 (1818). (Sphaeromorphaea partly, Ethuliopsis.)

E. Cunminghamii, Bentham, Fl. Austr. III, 530 (1866) ... ... - S.A. — V. N.S.W. Q. - B.fl.III,530

E. australis, Lessing in Linnaea V, $148(1831) \quad \ldots . \quad \ldots . \quad \ldots .-$ S.A. - V. N.S.W. Q. N.A. B.fl.III,530

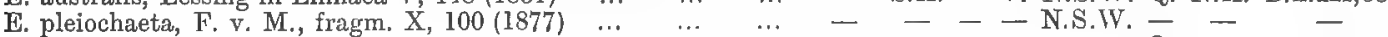

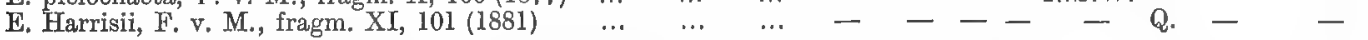
SPHAERANTHOS, Vaillant in act. Acad. Par. 289 (1719). (Sphaeranthus.)

S. hirtus, Willdenow, spec. plant. III, $2395(1800) \quad \ldots \quad \ldots-$-. $-\quad-\quad-\quad$ Q. N.A. B.fl.III, 521

S. microcephalus, Willdenow, spec. plant. III, $2395(1800) \quad \cdots \quad-\quad-\quad-\quad-\quad-$ N.A. B.H.III,522 PTEROCAULON, Elliot, Sketch of the Bot. of S. Carolina and Georgia II, 323 (1824.) (Monenteles.)

P. verbascifolius, Bentham \& J. Hooker, gen. pl. II, 294 (1873)

P. Billardieri, F. v. M., Papman plants, 43 (1S76)...
P. sphacelatus, Bentham \& J. Hooker, gen. plant. II, $94(1873)$

P. glandulosus, Bentham \& J. Hooker, gen. plant. II, 94 (I873)

P. sphaeranthoides, Bentham \& J. Hooker, gen, plant. II, 94 (1873)

STUARTINA, Sonder in Schlechtendal's Linnaea XXV, 521 (1852).

S. Muelleri, Sonder in Schlecht. Linnaea XXV, $522(1852) \quad \ldots-$ S.A. - V. N.S.W. -

- S.A. - - N.S.W. Q. N.A. B. B.fl.III, 523

- S.A. - - N.S.W. Q. N.A. B.H.III, 523
- S.A. - N.S.W. Q. N.A. B.fl.III,523

- - - - N. Q. N.A. B.A.III,523

- _ - _ - - N.A. B.fl.III,524

GNAPHALIUM, Linné, gen, plant. 250 (1737), from J. \& C. Bauhin (1619). (Euchiton.)

G. Iuteo-album, Linné, spec. plant. 851 (1753)

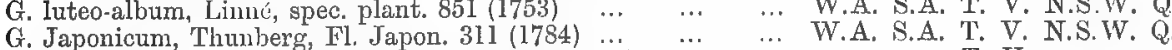

G. alpigenum, F. v. M., second gen. Report 12 (1854)

G. purpureum, Linné, spec. plant. 854 (1753)

G. purpureum, Linne, spec. plant. 854 (17icum, Linné, spec. plant. 852 (1753)..

G. indutum J Hoker in Lond. Journ of Bot. VI, 121 “is47)

G. Traversii, J. Hooker, Handb. N. Zeal. Fl. 154
... - - - T. V. N.S.W. Q

- B.f.III, 654

- - $-\overline{\text { N.S.W. Q. N.A. B.fl.III, } 655}$

W.A. S.A. T. V. N.S.W. $-\quad$ B.H.III,655

- V. N.S.W. - - B.fl.III,656
M. fr.V,75.

M.fr. V, 79.

M.fr. VIII, 143.

M.fr.VII, 144 .

M.fr. V, 84 .

M.fr. $V, 86$.

M.fr. XI, 20 .

M. fr. X, 137 .

M.fr. X, 119 .

M.fr.XI, 101.

M.fr.X, 101.

M.fr.XI, 101. M.fr. $X, 100$.

M.fr.XI,101.

M.fr.III, 138 .

M.fr.III, 138.

M.fr. XI, I01.

M.fr.VIII,145;XI, 27.

M.fr. V, 150

M.fr. V, 150;XI,27.

M.fr. V, 150 .

M.fr. V, 149 .

M.fr. V, 150 . 
ANTENNARIA, Gaertner, de fruct. II, 410, t. 167 (1791). (Raoulia partly.)

A. Planchoni, F.v.M.; Gnaphalium, Hook. FI. Tasm.I,217,t.62(1860) $-\overline{-}-\overline{-}$. N.S.W. $-\overline{-}$ B.fl. $\overline{I I}, 652$ M.fr.VIII, I45.
A. uniceps, F. v. M. in Transact, phil. Soc. Vict. t. $105(1854) .$.

LEONTOPODIUM, R. Brown in Transact. Linn. Soc. XII, 124 (1817). (Raoulia partly).

L. Catipes, F. v. M. in Papers of the Roy. Soc. of Tasm. $44(1882)--$ T. V. N.S.W. - - B.fl.III,651

L. Meredithae; Antennaria, F.v.M. in Pap. Roy.Soc.ofTasm. 15(1870) - - T. - - - - PTERYGOPAPPUS, J. Hooker in Lond. Journ. of Bot. VII, 120 (1847).

P. Lawrencii, J. Hooker in Lond. Journ. VII, 120 (1847) … - - T. - - - - B.A.III,656 PODOTHECA, Cassini in Diction. XXIII, 561 (1822). (Podospermum, Phaenopoda, Lophoclinium.)

P. gnaphalioides, Graham in Bot. Mag. t. 3920 (1842) ...

P. angustifolia, Lessing, synops. gen. compos. 273 (1832)

P. pygmaea, A. Gray in Hook. Kew Misc. IV, 227 (1852)

P. chrysantha, Bentham, Fl. Austr. III, 602 (1866) …

IV.A. - - - - - B.fl.III, 601

IXIOLAENA, Bentham in Hueg. enum. 66 (1837).

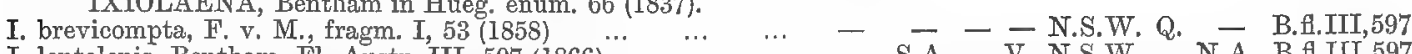

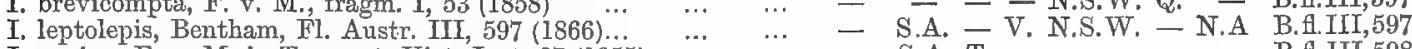

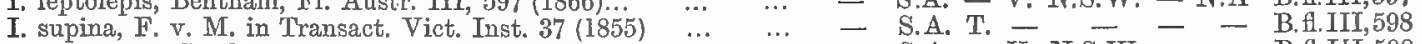

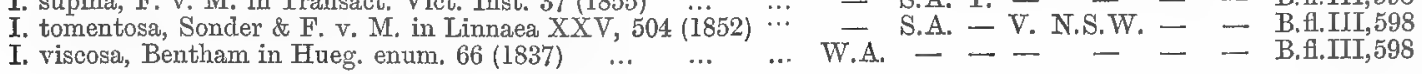

M.fr.XI,20.

I. viscosa, Bentham in Hueg. enum. 66 (1837)

PODOLEPIS, Labillardière, Nov. Holl. pl. specim. II, 57, t. 28 (1806). (Scalia, Panaetia, Scaliopsis, Siemșsenia, Stylolepis, Rutidochlamys.)

P. rhytidochlamys, F. v. M., fragm. IV, 79 (1864)

P. longipedata, Cunningham in De Cand. prodr. VI, $163(1837) \ldots$

P. acuminata, R. Brown in Ait. hort. Kew. ed. sec. V, S2 (1813)
P. canescens, Cunningham in De Cand. prodr. VI, $163(1837) \ldots$

P. aristata, Bentham in Hueg, enum. 64 (1837)

P. pallida, Turczaninow in Bull. de Mose. XXIV, 78 (185i) $\quad \cdots$

P. nutans, Steetz in Lehm. pl. Preiss. I, $464(1 \mathrm{S45})$
P. gracilis, Graham in Edinb. New phil. journ. V, 379 (1828) $\ldots$

P. gracilis, Graham in Edinb. New phil. journ.

P. Lessoni, Bentham, Fl. Austr. III, 606 (1866) $\ldots$
P. Siemssenia, F. v. M. in Benth. Fl. Austr. III, 607 (1866) $\quad \ldots$

P. Siemssenia, F. v. M. in Benth. Fl. Austr. III, $607(1866) \quad \ldots$
P. microcephala, Bentham, Fl. Austr. 607 (1866) ... $\quad \ldots$... ATHRIXIA, Ker in Bot. Regist. VIII, 681 (1823). (Asteridea, Chrysodiscus, Trichostegia.)

A. australis, Steetz in Lehm. pl. Preiss. I, 482 (1845) $\ldots \quad \ldots \quad$ W.A. $\quad-\quad-\quad-\quad-$ - B.H.III, 599

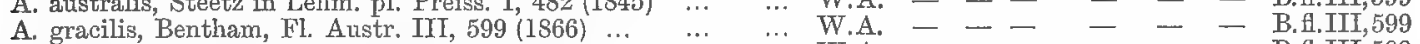

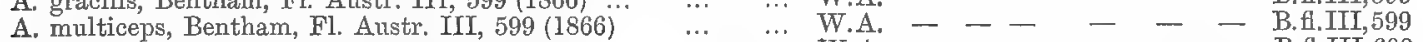

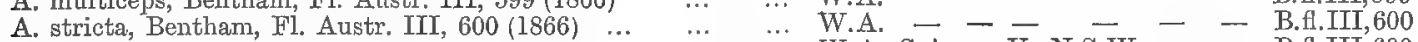

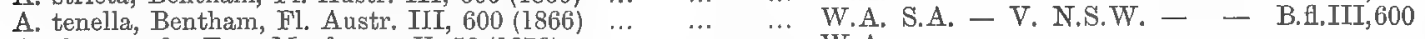

A. chaetopoda, F. v. M., fragm. X, $56(1876) \quad \ldots . \quad \ldots . \quad \ldots$

LEPTORRHYNCHOS, Lessing, syn. composit. 273 (1832).

L. squamatus, Lessing, syn. comp. 273 (1832)

L. panaetioides, Bentham, Fl. Austr. III, 609 (1866)

L. pulchellus, F. v. M. in Linnaea XXV, 500 (1852)

I. Baileyi, F. v. M., fragm. X, 101 (1877) ...

L. elongatus, De Caudolle, prodr. VI, 160 (1837) ...

L. medius, Cunningham in De Cand. prodr. VI, 160 (1837)

L. Waitzia, Sonder in Schlecht. Linnaea XXV, 501 (1852)

L. nitidulus, De Candolle, prodr. VI, 160 (1837) ...

WAITZIA, Wendland, Collect. pl. II, 13, t. 42 (1808).

W. corymbosa, Wendland, Coll. pl. II, 13, t. 42 (I808) ...

W. aurea, Steetz in Lehm. pl. Preiss. I, 452 (1845)

W. nivea, Bentham, Fl. Austr. III, 636 (1866)

W. Steetziana, Lehmann, pl. Preiss. I, 454 (1845)

W. podolepis, Steetz in Lehm. pl. Preiss. I, 450 (1845)

W. paniculata, F. v. M. in Benth. Fl. Austr. III, 637 (1866)

HELIPTERUM, De Candolle, prodr. VI, 211 (1837). (Argyrocome, 1822, Pteropogon, Rodanthe, Xyridanthe, Anisolepis, Hyalosperma, Triptilodiscus, Acroclinium, Monencyanthes, Dimorpholepis, Duttonia, Cassiniola.)

H. Manglesii, F. v. M., fragm. V, 200 (1866)

H. Margaritae, F. v. M., fragm. XI, 48 (1878)

H. roserm, Bentham, Fl. Austr. III, $640(1886)$
H. anthemoides, De Candolle, prodr. VI, $216(1837)$

H. polygalifolium, De Candolle, prodr. VI, 216 (1837) ...

H. rubellum, Bentham, Fl. Austr. III, 641 (1866)

H. chlorocephalum, Bentham, Fl. Austr. III, 641 (1866)

H. floribundum, De Candolle, prodr. VI, 217 (1837)

H. Pyrethrum, Bentham, FI. Austr. III, 642 (1866)

H. heteranthum, Turczan. in Bull. de Mosc. XXIV, 198 (185i)

H. Kendallii, F. v. M., fragm. VIIT, 168 (1874) ..

H. stipitatum, F. v. M. in Bentham, Fl. Austr. MI, 643 (1866)

H. incanum, De Candolle, prodr. VI, 215 (1837) ..

H. Cotula, De Candolle, prodr. VI, 215 (1837)

W.A. - -

- S.A. T. V. N.S.W. - - B.H.III, 608

- S.A. - V. N.S.W. - - B.f.III,609

- S.A. - V. - - - B.fl.III,609

- S.A. - V. N.S.W. - - B.f.III, 610

W.A. S.A. T. V. N.S.W. Q. - B.H. III, 610

W.A. S.A. - V. N.S.WV. - -

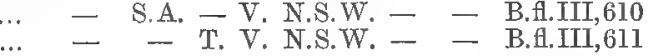

(Viraya, Morna, Pterochaete.)

W.A. S.A. - V. N.S.W. - - B.f.III,635

W.A. - - - - - - B.fl.III, 636

W.A. - - - - - - B.fl.III,636

-

W.A. - - - - - - B.fl.II, 637

M.fr.X, 56.

M.fr.IV.79.

M.fr.XI,27.

M.fr.I, 52 .

M.fr.I, 52 .

M.fr. I,53;XI, 27.

M.fr.X, 101 .

M.fr.XI, 27,28.

M.fr.XI, 85 .

H. hyalospermum, F. v. M. in Benth., Fl. Austr. III, 644 (1866)

H. condensatum, F. v. M., fragm. III, 136 (1863)

H. polyphyllum, F. v. M., fragm. I, 35 (1858)

W.A. - - - - - - B.H.III,640

W.A. S.A. - - - - N.A.

M.fr.X, 108.

M.fr. XI, 48 .

M.fr. $X, 108$.

- S.A. T. V. N.S.W. Q. - B.H.III,641

- S.A. - V. N.S.W. Q. - B.H.III,641

W.A. - - - - - - B.f.III,64I

W.A. - - - - - B.fi.III,641

- S.A. - V. N.S.W. Q. N.A. B.A.III, 642

W.A. - - - - - - - B.fl.III,642
W.A. S.A. - - B.fl.III,642

W.A. $\overline{-1}---$ - - B.f. $\overline{\text { III } 643}$

- S.A. $-\overline{\text { T. }}$ V. N.S.W. $\overline{\text { Q. }}$ - $\quad$ B.A.IIII,643
B.flII,643

W.A. S.A. - V. N.S.W. Q. - B.fl.III, 644

W.A. S.A. - V. N.S.TV. - - B.f.III, 644

B. fl. III, 645

M.fr. X, 108 .

M.fr.X, 107;108.

M.fr.X, 108.

M.fr. X, 108.

M.fr.X, 110 ,

M. fr. I II, 137; X, 108.

M.fr. VIII, 168;X, 107.

M. fr. III, 133;X, 109.

M.fr.II, 157;III, 134;X,

$[110$.

M.fi. X, 109 .

M.fr.III, 136.

M.fr.I, 35 . 
H. Humboldtianum, De Candolle, prodr. VI, 216 (1866)

H. Haigii, F. v. M., fragm. X, 107 (1877) ...

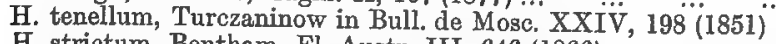

H. strictum, Bentham, Fl. Austr. III, 646 (1866)

H. corymbiflorum, Schlechtendal, Linnaea XXI, 448 (18̈8) $\quad \ldots$

H. pygmaeum, Bentham, Fl. Austr. III, 647 (1866)

H. spicatum, F. v. M. in Bentham, Fl. Austr. III, 647 (1866)...

H. Charsleyae, F. v. M., fragm. VIII, 168 (1874)...

H. moschatum, Bentham, Fl. Austr. III, 648 (I866)
H. Tietkensii, F. v. M., fragm. VIII, 227 (1874) ...

H. pterochaetum, Bentham, Fl. Austr. III, 648 (I866) ...

H. polycephalum, Bentham, Fl. Austr. III, 649 (1866) ...

H. corymbosum, Bentham, Fl. Austr. III, 649 (1866) ...

H. laeve, Bentham, Fl. Austr. III, 649 (1866)

H. exiguum, F. v. M. in Transact. Vict. Inst. 39 (1855)...

H. Dimorpholepis, Bentham, Fl. Austr. III, 650 (1866) ...
W.A. -

W.A. - - -

W.A. S.A. - - - - - -

W.A.S.A. - $-\overline{-}-$ B.fl.III, 646

- B.fl.III,646

- S.A. - V. N.S.W. Q. - B.fl.III,647

W.A. S.A. - V. N.S.W. - - B.fl.III,647

W.A. - - - - - B.H.III,647

W.A. S.A. - $-\overline{\text { S.A. N.S.W. }}$ Q.

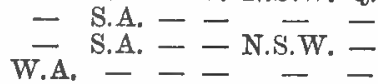

W.A. - - - - -

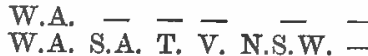

W.A. S.A. - V. N.S.W. -

\section{B.f. III, 648}

B.fi. III, 648

B.fl.III, 649

B.fl.III, 649

B.f.III, 649

B.H.III, 649

B. fl.III, 650
M.fr.III, 135;X, 109

M.fr.X, 107.

$[20$.

M.fr.III, 135;X, 109;XI,

M.fr. X, 109.

M.fr. $X, 109$.

M.fr. X, 109.

M.fr.X, 109

M.fr.VIII, $168 ; X I, 134$.

M.fr. X, 107.

M.fr.VIII, 227.

fr.III, $139 ; \bar{X}, 109$

M.fr. V, 200 .

M.fr.V,200.

M.fr.X, 109

M.fr.X, $109 ; \mathrm{XI}, 27$. Argyroglottis, Acanthocladium, Raoulia partly.)

H. Cassinianum, Gandichaud in Freyc. Voy. Bot. 466, t. 87 (1826)

H. Lawrencella, F. v. M. in Benth. Fi. Austr. III, 616 (1866) ..

H. subulifolium, F. v. M., fragm. III, 134 (1863).

H. filifolium, F. v. M., fragm. III, 134 (1863)

H. semifertile, F. v. M., Rep. Babb. Exped. 14 (1858)

H. Baxteri, Cunningham in De Cand. prodr. VI, 193 (183

H. Ayersii, F. v. M., fragm. VIII, 167 (1874)

H. rutidolepis, De Candolle, prodr. VI, 194 (1837) $\cdots$

W.A. S.A. - - - - - B.fl.III,616

H. oligochaetum, F. v. M., fragm. VI, 235 (1868)... ‥ W.A. - - N.S. Q. B.H.III,618

H. Tepperi, F. v. M. in Wing's South. Science Record, İ̈, 1 (1082) - S.A. - - - - -

H. obtusifolium, Sonder \& F. v. M. in Linnaea XXV, 515 (1852) W.A. S.A. - F. N.S.W. -

H. Calvertianum, F. v. M., fragm. X, $108(1877) \ldots \ldots \ldots \ldots \ldots$... $-\ldots$ - - N.S.W. -

H. Spiceri, F, v. M., fragm. XI, 47 (1878) ...

H. dealbatum, Labillardière, Nov. Holl. pl. spec. II, 45, $\dddot{t} .190(\dddot{1806})$ -

H. pumilum, J. Hooker, Fl. Tasman. I, 213, t. 60 (1860)

H. lucidum, Henckel, adumbr. pl. hort. Hal. 5 (1806)

H. elatum, Cunningham in De Cand, prodr. VI, 193 (1837)

H. adenophorum, F. v. M. in Transact. Vict. Inst. 38 (1855)

H. leucopsidium, De Candolle, prodr. VI, 193 (1837)

H. Blandowskianum, Steetz in Schlecht. Linnaen XXV, $512(1052)-$ W. A. S.A. T. V. N.S.W. -

H. oxylepis, F. v. M., fragm. I, $35(1858) \ldots \ldots \ldots \ldots$... $\quad \ldots \quad$ - $\quad$ - - N.S.W.

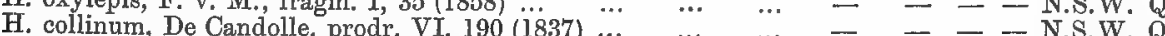

H. rupicola, De Candolle, prodr. VI, 190 (1837) $\ldots$. $\ldots$

H. podolepideum, F. v. M., Rep. Babb.
H. Gilesii, F. v. M., fragm. X, 85 (1876)

H. ambiguum, Turczaninow in Bull. de Mosc. 195 (1851)

H. apiculatum, De Candolle, prodr. VI, 195 (1837)

H. semipapposum, De Candolle, prodr. VI, 195 (1837)

H. Dockerii, F. v. M. in Benth. Fl. Austr. III, 626 (1866)

H. Agyroglottis, Bentham, Fl. Austr. III, 626 (1866) ...

H. ramosum, De Candolle, prodr. VI, 181 (1837) ..

H. cordatum, De Candolle, prodr. VI, 180 (1837)...

H. obovatum, De Candolle, prodr. VI, 180 (1837)...

H. Bidwillii, Bentham, Fl. Austr. III, 627 (1866)..

H. Becklerii, F. v. M. in Benth. FI. Austr. III, 627 (1866)

H. diotophyllum, F. v. M., fragm. V, 150 (1866)...

- - T. - - - - B.fl.III,619

H. Thomsoni, F. v. M., fragm. VIII, 45 (1873) $\ldots . \quad \ldots . \quad \ldots$

H. diosmifolium, Don in Steud. Nom. Bot. ed. sec. 738 (is41) ...

H. retusum, F. v. M., fragm. VIII, 46 (1873)

H. decurrens, F. v. M., fragm. VIII, 46 (1873) $\ldots$

H. reticulatum, Lessing in Steud. Nom. Bot. ed. sec. 740 (1841)

H. cinereum, F. v. M. in Benth. Fl. Austr. III, $629(1866)$
H. bracteolatum, Bentham, Fl. Austr. III, $630(1866) \quad \ldots$

H. Kempei, F. v. M. in Melb. Chemist, Jan. (1882)| $\quad \ldots \quad \ldots$

H. cassinioides, Bentham, Fl. Austr. III, 630 (1866) “.

H. Gunnii, F. v. M. in Benth. Fl. Austr. III, 630 (1866)

H. rosmarinifolium, Lessing, synops. gen. comp. 274 (1832)

H. ledifolium, F. v. M. in Benth. Fl, Austr. III, 630 (1866)

H. ferrugineum, Lessing, synops. gen. comp. 274 (1832)..

H. antennarium, F. v. M. in Benth. Fl. Austr. III, 632 (1866)...

H. obcordatum, F. v. M. in Benth. Fl. Austr. III, 632 (1866) ...

H. Backhousii, F. v. M. in Benth. Fl. Austr. III, 632 (1866) ...

H. cuneifolium, F. v. M. in Benth. Fl. Austr. III, 633 (1866) ...

H. baccharoides, F, v. M. in Benth. Fl. Austr. III, 633 (1866) ...

H. lepidophyllum, F. v. M. in Benth. Fl. Austr. III, 633 (1866)

H. scutellifolium, Bentham, Fl. Austr. III, 633 (1866)

H. pholidotum, F. v. M. in Benth. Fl. Austr. III, 634 (1866) $\ldots$

H. Iycopodioides, Bentham, Fl. Austr. III, 634 (1866)

H. selaginoides, F, v. M. in Benth. Fl. Austr, III, $634(1866) \ldots$

$$
\text { w. }
$$$$
\text { - S.A. - - N.S.W. Q }
$$$$
\text { W.A. - - - - N.A }
$$

W.A. S.A. - - N.S.W. - N.A.

W.A. T. $\bar{V}$ N.S.W. $\overrightarrow{\mathrm{C}}-\mathrm{B}$ B.fl,III,609

W.A. S.A. T. V. N.S.W. Q. - B.fl.III,625

W.A.A. - N.S.W. - - B.f.III,626

W.A. - 二 - $-二-$ - B.H.III, 626
B.H.III, 626

W.A. - - - - - - - B.H.III,626
W.A. - B.III,627

- - - - N.S.W. - - B.H.III,627

- - - - N.S.W. Q. - B.H.III,627

- - - - N.S.W. - - B.H.III,627

- - - - N.S.W. Q.

- S.A. - - N.S.W. Q.

- S.A. - V. N.S.W. Q.

- S.A. - V. V. N.S.W. -

- $\quad-$ - - N.S.W. -

-

-

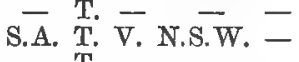

- $\mathrm{T}$. -

S.A. - - - -

二 T. $\overline{\text { T. V. N.S.W. }}$

- T. - -

S.A. T. V. N.S.W. -

- T. $\overrightarrow{\text { V. N.S.W. - }}$

- T.

- T.

二
W.A. $\overline{\text { T. }}$.
- T.

S.A. $\stackrel{\text { T. }}{\text { V. N.S.W. }} \overline{-}$

- T. - -
- T. -
M.fr.III, 134.

M.fr.III, 134 .

M.fr.IX, 195.

M.fr.VIII, 167 .

M.fr.VI, 235.

M.fr.XI, 136.

M.fr.XI, 47 .

M.fr.XI,48.

M.fr. VI, 255 .

M.fr.I,35.

M.fr.X, 86 .

M.fr. II, 150.

M.fr.II, 89.

M.fr.I, 183 .

M.fr.VIII, 46.

M.fr.VIII, 45 .

- B.A.III, 628

二

B.fl.III, 629

B.fl. III, 629

B. H.III, 629

B.fl. III, 630

B.fl.III, 630

B.fl.III, 631

B.fl.III, 630

B.fl.III,631

B.f.III, 632

B.fl.III, 632

B.․․III, 632

B.H.III, 633

B.fl.III, 633

- B.fl.III, 633

- B.H.III,633

B.fl.III, 634

B.t.III, 634

B.tl.III, 634
M.fr. VIIT, 46.

M.fr. VIII, 46.

M.fr.V, 199 .

M.fr. V,199.

M.fr. V, 199.

M.fr. V, 199 .

M.fr.V, 199.

M.fr.V,199.

M.fr.V, 199.

M.fr. V, 199.

M.fr. V, 199.

M.fr.II, 131;V, 199 .

M.fr. V, 199.

M.fr. V, 199. 
CASSINIA, R. Brown in Transact. Linn. Soc. XII, 126 (1817). (Non zoologorum, Apolochlamys, Achromolaena, Chromochiton.)

C. leptocephala, F. v. M., fragm. III, 138 (1863) ..

C. compacta, F. v. M., fragm. I, 18 (1858)... ... $\ldots . . . . \quad=-Z=$ N.S.W. Q. - B.Al.III,585 M.fr.I,18.

C. denticulata, R. Brown in Transact. Linn. Soc. XIII, 127 (1817) - - - N.S.W. - - B.fl.III,586

C. longifolia, R. Brown in Transact. Linn. Soc. XII, $127(1817)$ - - T. V. N.S.W. Q. - B.fl.III,586

C. aurea, R. Brown in Transact. Linn. Soc. XII, $127(1817) \ldots=-\ldots-1-$ N.S.W. - - B.fl.III,586

C. aculeata, R. Brown in Transact. Linn. Soc. XII, $127(1817) \ldots$-... - S.A. T. V. N.S.W. - - B.H.III,586

C. laevis, R. Brown in Transact. Linn. Soc. XII, 128 (I817) ... - S.A. - N.S.W. Q. - B.fl.III,587

C. tenuifolia, Bentham, FI. Austr. III, 585 (1866)

C. quinquefaria, R. Brown in Transact. Linn. Soc. XII, 128 (1817) - - - - N.S.W. Q. - B.fl.III,587

C. arcuata, R. Brown in Transact. Linn. Soc. XII, $128(1817)$... W.A. S.A. - V. N.S.W. - - B.fl.III,587

C. subtropica, F. v. M., fragm. I, 17 (1858) … … … - - - N.S.W. Q. - B.H.III,588

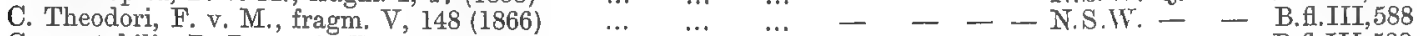

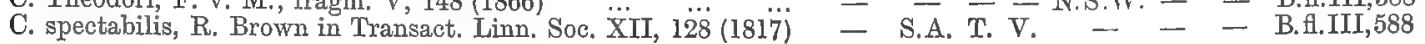

HUMEA, Smith, Exot. bot. I, t. 1 (1804). (Haeckeria, Calomeria.)

H. elegans, Smith, Exot. bot. I, t. l(1804)

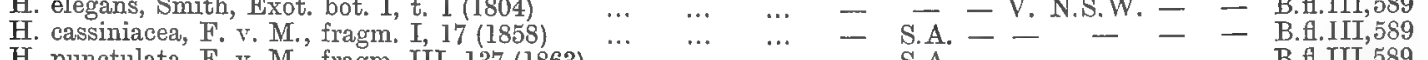

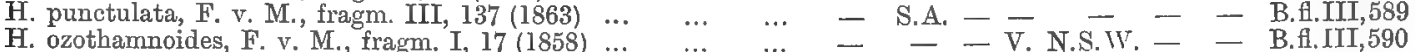

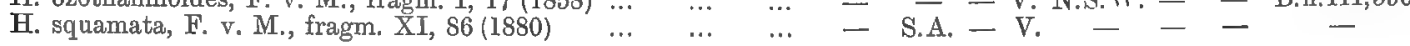
ACOMIS, F. v. M., fragm. II, 89 (1860).

A. Rutidosis, F. v. M., fragm. II, $89(1860) \quad \ldots \quad \ldots \quad \ldots \quad-\quad-\quad-\quad$ - N.S.W. $-\quad$ - B.fi.III,591

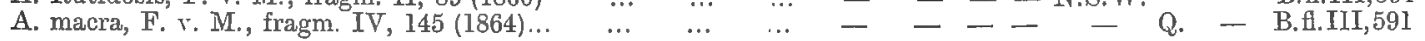
RUTIDOSIS, De Candolle, prodr. VI, 158 (1837). (Pumilo, Actinopappus, Lepidocoma.)

R. leiolepis, F. v. M. in Transact. Vict. Inst. 131 (1855) ‥ - - - V. - - - B.fl.III,593

R. leptorrhynchoides, F. V. M., fragm. V, $148(1866) \quad \ldots \quad \ldots \quad-\quad-\quad-$ V. N.S.W. - - B.H.III,593

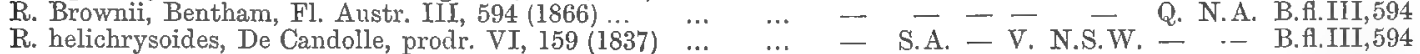

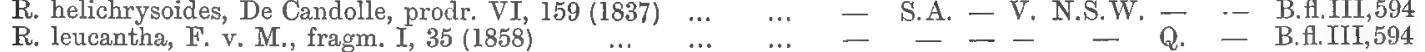

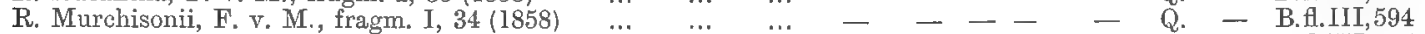

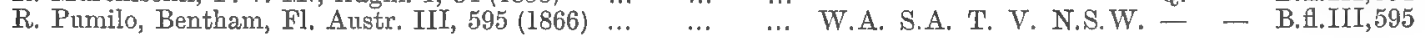
PITHOCARPA, Lindley, Bot. Regist. XXV, App. XXIII (1839).

P. corymbosa, Lindley, Bot. Regist. XXV, App. XXIII (1839) W.A. - - - - - - B.fl.III,590 AMMOBIUM, R. Brown in Bot. Magaz. t. 2459 (1824).

A. alatum, R. Brown in Bot. Magaz. t. $2459(1824)$... .. - - - - N.S.W. Q. - B.fl.III,583

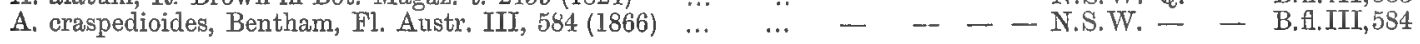
IXODIA, R. Brown in Ait. hort. Kew. sec. ed. IV, 17 (1812).

I. achilleoides, R. Brown in Ait. hort. Kew. sec. ed. IV, $17(1812)$ - S.A. - V. - - - B.tl.III,583 MILlOTIA, Cassini in Ann. sc. nat. XVII, 416 (1829).

M. tenuifolia, Cassini in Amn. sc. nat. XVII, 416 (1829)... ‥ W.A. S.A. T. V. N.S.W. - - B.fl.III,596

M. Greevesii, F. v. M., fragm. III, 18, t. $19(1863) \quad \ldots \quad \ldots-$ S.A. - - N.S.W. - - B.fl.III,596

M. Kempei, F, v. M. in Wing's South. Sc. Record II, I(1882).. - S.A. - - - - - TOXANTHUS, Turczaninow in Bull, de Mosc. XXIV, 177 (1851). (Anthocerastes, Scyphocoronis.)

T. perpusillus, Turczaninow in Bull. Mosc. XXIY, 177 (1851) ... W.A. S.A. - V. N.S.W. - - B.A.III,592

T. Muelleri, Bentham, Fl. Austr. III, 592 (1866) ... ‥ … W.A. S.A. - - - - - - B.fl.1II,592

T. major, Turczaninow in Bull. de Mosc. XXIV, 64 (185i) $\quad \ldots$. W.A. - - - - - - B.H.III,592 QUINETIA, Cassini in Diction. sc. nat. LX, 579 (1830).

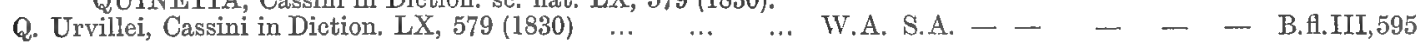
PHACELOTHRIX, F. v. M., fragm. XI, 49 (1878).

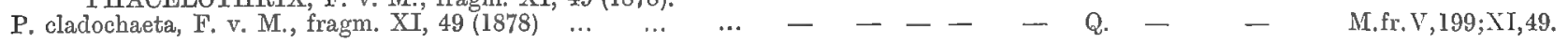
ERIOCHLAMYS, Sonder and F. v. M. in Schlecht. Linnaea XXV, 488 (1852).

E. Behrii, Sonder \& F. v. M. in Linnaea XXV, 488 (1852) ‥ -- S.A. - V. N.S.W. - - B.A.III,591 MYRIOCEPHALUS, Bentham in Hueg. enum. 61 (1837). (Hyalolepis, Antheidosorus, Gilberta, Lamprochlaena, Elachopappus, Polycalymma; some readily to be restituted.)

M. rhizocephalus, Bentham, Fl. Austr. III, 557 (1866) ...

M. nudus, A. Gray in Hook. Kew Misc. III, 174 (1851) ...

M. appendiculatus, Bentham in Hueg. enum. 61 (1837) ...

M. Rudallii, F. v. M., fragm. III, 157 (1863)

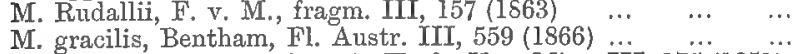

M. helichrysoides, A. Gray in Hook. Kew Misc. III, 173 (1851)

M. suffruticosus, Bentham, Fl. Austr. III, 559 (1866) ..

M. Stuartii, Bentham, Fl. Austr. III, 560 (1866) ...

M. Guerinae, F. v. M' fracm. VIII, 169 (1874) ..........

W.A. S.A. - V. N.S.WT. -

IV.A. - - - - -

W.A. - - - - - - B.fl.III,558

W.A. S.A. - - - - - B.f.III,559

W.A. - - - - - - B.f.III,559

W.A. - - - - - - B.Al.III,559

W.A. - - - - - - B.f.III,559

- S.A. -- V. N.S.TV. - - B.f.III,560
M.fr.I, 17.

M.fr. V, 148 .

M.fr.I, 17 .

M.fr. I, 17.

M.fr.III, 137.

fr. VIII, 14

M.fr.XI, 86 .

M.fr.II, 89 .

M.fr.IV, 145.

M.fr. $V, 148$.

M.fr. I, 35 .

M.fr.I,34.
M.fr.III, 18.

M.fr.XI, 49 .

M.fr.XI, 49 .

M.fr.XI, 49 .

ANGIANTHUS, Wendland, Collect. pl. II, 31 (1809). (Siloxerus, Styloncerus, Ogcerostylus, Cylindrosorus, Phyllocalymma, Skirrophorus, Chrysocoryne, Eriocladium, Pogonolepis, Piptostemma, Epitriche, Gamozygis, Cephalosorus partly, Hyalochlamys, Dithyrostegia, Pleuropappus; some readily to be restituted.)

A. humifusus, Bentham, Fl. Austr. III, 563 (1866)

A. tomentosus, Wendland, Collect. pl. II, 31, t. 48 (1809)

A. pleuropappus, Bentham, Fl. Austr. III, 563 (1866) ...

A. brachypappus, F, V. M. in Trans. phil. Soc. Vict. I, $\dddot{44}$ (1854)

A. myosuroides, Bentham, Fl. Austr. III, 563 (1866)

A. tenellus, Bentham, Fl. Austr. III, 564 (1866) ...

A. pusillus, Bentham, FI. Austr. III, 564 (1866) ...

A. Cunninghamii, Bentham, Fl. Austr. III, 565 (1866) ...

A. phyllocephalus, Bentham, Fl, Austr. III, 565 (1866) ...
... W.A. - - - - - B.fl.III, 563

W.A. S.A. - V. N.S.W. - N.A. B.A.III,562

- S.A. - - - - - B.fl.III, 563

- S.A. - V. N.S.W. - - B.H.III, 563

W.A. - - - - - B.A.III,563

W.A. S.A. - - - - - B.f.III, 564

W.A. S.A. - V. N.S.W. - - B.H.III,564

W.A. - - - - - — B.H.III,564

W.A. - - - - - B.A.III,565

W.A. - - - - - - B.fl.III,565 M.fr.III,159. fr. III, 155 .

I.fr.IIT, $157 ;$ TT, $235 ;{ }^{[145,}$

M.fr.VIII, 169.

M.fr.VIII, 169. 
A. micropoides, Bentham, Fl. Austr. III, 565 (1866)

A. microcephalus, Bentham, F1. Austr. III, 566 (1866) …

A. platycephalus, Bentham, Fl. Austr. III, 566 (1866) $\ldots$

A. Drummendii, Bentham, Fl. Austr. III, 566 (1866) $\ldots$

A. pygmaeus, Bentham, Fl. Austr. III, 567 (1866)

A. globifer, Bentham, Fl. Austr. III, 567 (1866) ...

A. demissus, Bentham, Fl. Austr. III, 567 (1866)...

A. strictus, Bentham, Fl. Austr. III, 568 (1866) ...

A. plumiger, Bentham, Fl. Austr. III, 568 (1866) ...

A. amplexicaulis, Bentham, Fl. Austr. III, 568 (1866) ...

GNEPHOSIS, Cassini in Bull de la Soc philom. Cyathopappus ; some readily to be restituted.)

G. Burkittii, Bentham, Fl. Austr. III, 570 (1866)

G. exiocarpa, Bentham, FI. Austr. III, 570 (1866)

G. macrocephala, Turcz, in Bull. Soc. Mosc. XXIV, 190 (1851) ”.

G. skirrophora, Bentham, FI. Austr. III, 570 (1866) ... ...

G. cyathopappa, Bentham, Fl. Austr. III, 571 (1866)

G. codonopappa, F. v. M. in Giles, geogr. trav. 217 (1875)

G. leptoclada, Bentham, Fl. Austr. III, 571 (1866)

G. arachnoidea, Turez, in Bull. Soc. Mosc, XXIV, 189 (ï851) “.

G. tenuissima, Cassini in Bull. de la Soc. philom. 43 (1820)

G. acicularis, Bentham, Fl. Anstr. III, 572 (1866)

G. pygmaea, Bentham, Fl. Austr. III, 572 (1866)...

G. brevifolia, Bentham, Fl. Austr. III, 572 (1866)

G. eriocephala, Bentham, Fl. Austr. III, 573 (I866)

DECAZFSIA, F. v. M., fragm. XI, 71. (1879).

D. hecatocephala, F. v. M., fragm. XI, 72 (1879)...

… $\quad \cdots$

..

... W.A.
.. W.A. - - - - - - B.f.III,565

... W.A. - - - - - - B.H.III,566 M.fr.III, 158.

.. W.A. - - - - - - B.fl.III,566

.. W.A. - - - - — B.A.III,566

W.A. S.A. T. V. N.S.W. - - B.f.III,566

W.A. - - - - - - B.fl.III,567

W.A. - - - - - - B.fi.III, 567

W.A. - - - - - - B.fl.III,567

W.A. S.A. - V. N.S.W. - - B.H.III,568

W.A. - - - - - - B.f.III,568

W.A. - - - - - - B.fl.III,568

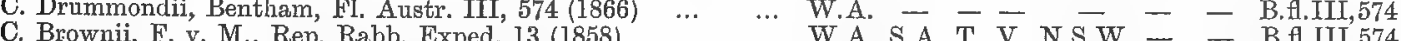

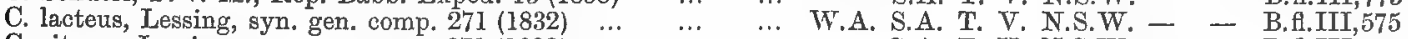

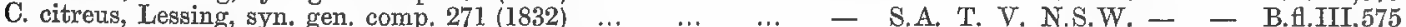

C. angianthoides, Bentham, FI. Austr. III, $575(1866) \quad \ldots . \quad \ldots .6$ W.A. - T. - - - - B.t.III,575

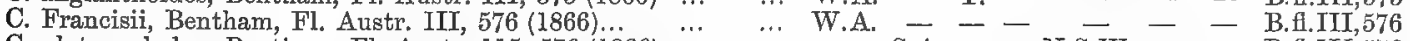

C. platycephalus, Bentham, Fl. Austr, 115, $576(1866)$... … _- S.A. - _ N.S.W. - - B.fl.III,576

C. multiflorus, Bentham, Fl. Austr. III, 576 (1866) $\ldots$ … W.A. - $\quad$ W - - - - B.f1.III,576

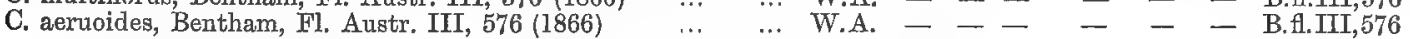

CEPHALIPTERUM, A. Gray in Hook. Kew Misc. IV, 271 (1852).

C. Drummondii, A. Gray in Hook. Kew Misc. IT, 272 (1852) ... W.A. S.A. - - _ - - B.fl.III,577 GNAPHALODES, A. Gray in Hook. Kew Misc. IV, 228 (1852).

G. uliginosum, A. Gray in Hook. Kew Misc. IV, 228 (1852) ‥ W.A. S.A. - V. N.S.W. Q. - B.fl.III,578 M.fr.XI,27.

G. condensatum, A. Gray in Hook. Kew Misc. IV, 228 (1852) ... W.A. - - - - - - B.fl.III,578

G. flifolium, Bentham, Fl. Austr. III, 578 (1866) $\quad \ldots \quad \ldots$ W.A. $\quad-\quad-\quad-\quad$ - - B.H.III,578 CRASPEDTA, G. Forster, florul. insul. Austr. prodr. 306 (1786). (Richea, Pycnosorus.)

C. Richea, Cassini in Diction. XI, $353(1818) \quad \ldots \quad \ldots . \quad \ldots$ W.A. S.A. T. V. N.S.W. Q.

C. pleiocephala, F. v. M. in Schlecht. Linnaea XXV, $404(1852) \quad-\quad$ S.A. - V. N.S.W. - - B.fl.III,580
C. chrysantha, Bentham, Fl. Austr. III, $580(1866)$
C...

C. globosa, Bentham, FI. Austr. III, $580(1866) \ldots \quad \ldots \quad \ldots \quad-\quad$ - S.A. - V. N.S.W. - - B.f.III,580

CHTHONOCEPHALUS, Steetz in Lehm. pl. Preiss. I, 444 (1845). (Chamaesphaerion, Gyrostephium, Lachnothalamus.)

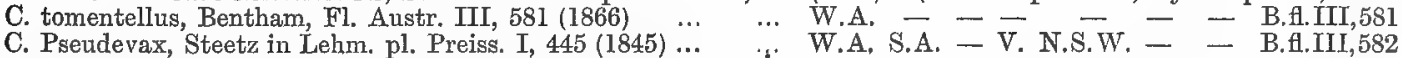

C. pygmaeus, Bentham, Fl. Austr. III, $582(1866) \quad \ldots \quad \ldots$ W.A. $\quad$ - $\quad$ - $\quad$ - $\quad$ B.fl.III,582 NABLONIUM, Cassini in Diction. XXXIV, 101 (1825).

N. calyceroides, Cassini, Dict. XXXIV, $101(1825) \quad \ldots \quad \ldots \quad-\quad-\quad$ T. $\quad-\quad-\quad-\quad-\quad$ B.fl.III,545 CHRYSOGONUM, Linne, hort. Cliffort. 424 (1737). (Moonia, Pentalepis.)

C. trichodesmoides, Bentham \& J. Hooker, gen. pl. II, 350 (1873) - - - - - - N.A. B.fl.III, 540

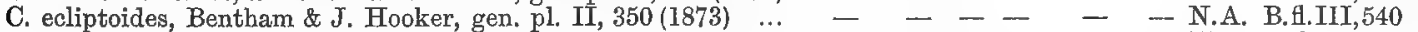

C. procumbens, Bentham \& J. Hooker, gen. pl. II, 350 (1873) ,.. - - - - - - - - N.A. B.1.III,540

M.fr.VIII, 145 .

M.fr.VIII, 145.

SIEGESBECKIA, Linné, hort. Cliffort. 412 (1737).

S. orientalis, Linné, spec. plant. 900 (1753)

W.A. S.A. - V. N.S.W. Q. - B. f.III,535 ENHYDRA, Loureiro, Flor. Cochinch. II, 510 (1790). (Enydra.)

E. paludosa, De Candolle, prodr. V, $637(1836)$... $\quad \ldots \quad \ldots \quad-\ldots \quad-\quad-\quad$ - N.S.W. Q. - B.fl.III,546 M.fr.III, 139. ECLIPTA, Linné, mantissa altera, 157 (1771).

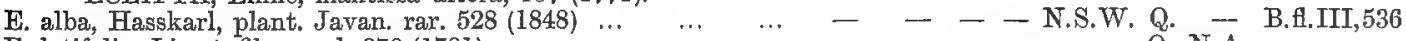

E. latifolia, Linné, fil. suppl. 378 (1781)

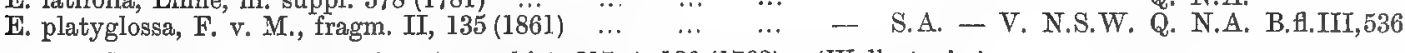

M.fr.VIII, 145 . WEDELIA, Jacquin, stirp. Amer. hist. 217, t. 130 (1763). (Wollastonia.)

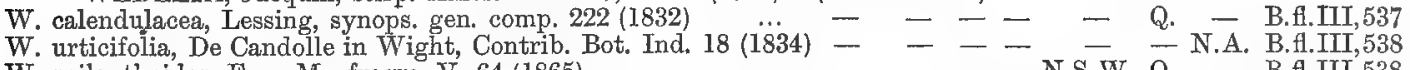

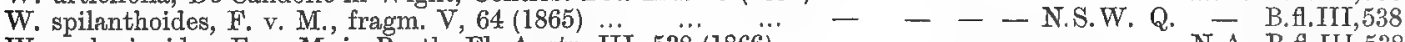

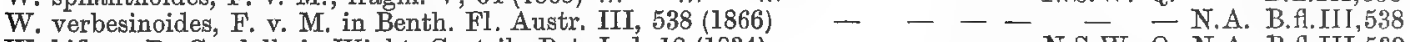

W. biflora, De Candolle in Wight, Contrib. Bot. Ind. 18 (1834) - - - - N.S.W. Q. N.A. B.fl.III,539

W. asperrima, Bentham, FI. Austr. III, 539 (1866)

- N.A. B.fl.III, 539

M.fr.V,64. 
SPILANTHES, Jacquin, stirp. Amer. hist. 214, t. 126 (1763).

S. grandiflora, Turezaninow in Bull. de Mosc. XXIV, 185 (1851) - - - - N.S.W. Q. N.A. B.fl.III,541 M.fr.V,63.

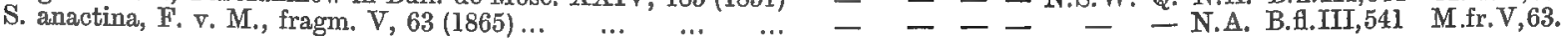

BIDENS, Dillenius, hort. Eltham. 51 and 52, t. 43 et 44 (1732), from Cesalpini (1583). (Possibly immigrated.)

B. tripartitus, Linné, spec. plant. 831 (1753) and 52, t. 43 et 44 (1732), from Cesalpini (1583). (Possibly B.fl.IIl, 543

B. pilosus, Linné, spec, plant. $832(1753) \ldots$
B. bipinnatus, Linné, spec. plant. $832(1753)$
. GLOSSOGYNE, Cassini in Diction. LI, 475 (1827). (Diodontium.)

G. tenuifolia, Cassini in Dict. LI, 475 (1827) $\ldots \ldots \ldots . . . \quad-\quad$ S.A. - - N.S.W. Q. N.A. B.fl.III,544 M.fr.I,51.

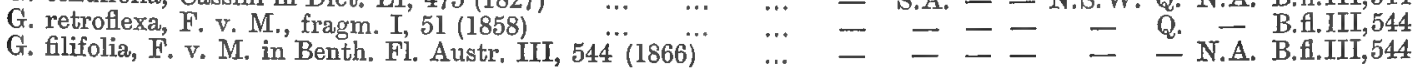

FLAVERIA, A. L. de Jussieu, gen. plant. 186 (1789).

F. Australasica, Hooker in Mitch. Trop. Austr. $118(1848)$.. $--\quad-\quad-\quad$ - Q. N.A. B.fl.III,546 M.fr.I,183.

SOLIVA, Ruiz et Pavon, prodr. 113, t. 24 (1794). (Gymnostyles ; possibly immigrated.)

S. anthemifolia, R. Brown in Transact. Limn. Soc. XII, $102(1817)--1-$ N.S.W. Q. - B.fl.III,552

COTULA, Linné, syst. nat. 9 (1735); Linné, gen. pl. 256 (1737). (Gymnogyne, Strongylospermum, Pleiogyne, Symphyomera, Stenosperma, Leptinella.)

C. filifolia, Thunberg, prodr. pl. Capens. 161 (1800)

C. Gymogyne, F. v. M, in Benth. Fl Austr, III, ${ }_{549}(1866)$ ‥ W.A. S.A. T. V. N.S.W. -

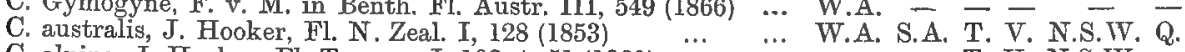

C. alpina, J. Hooker, FI. Tasman. I, 192, t. 51 (1860) $\ldots . \quad \ldots \quad-\ldots$ - $\quad$ - T. V. N.S.W. -

C. Drummondii, Bentham, Fl. Austr. III, 550 (1866) $\ldots$... $\quad$ W.A. $-\frac{1}{-}-1$.

C. reptans, Bentham, Fl. Austr. III, 551(1866) $\ldots \ldots \ldots+\cdots, \ldots-$ S.A. T. V. N.S.W. -

B.H.III, 548

- B.fl.III, 549

- B.H.III,549

- B.fl.III,550

- B.fi.III, 550

- B.f.III,550

- B.fl.III,551

B.A.III,551

CENTIPEDA, Loureiro, Fl. Cochinch. II, 492 (1790). (Myriogyne, Sphaeromorphaea partly.)

C. racemosa, F. v.M.; Myriogyne, Fook. in Mitch.Tr.Austr.353 (1848) - - - N.S.W. Q. N.A. B.fl.III, 553

C. orbicularis, Loureiro, FI. Cochinch. II, $492(1790) \quad \ldots \quad \ldots$. W.A. S.A. T. V. N.S.W. Q. N.A. B.fl.III, 553

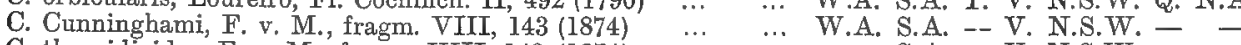

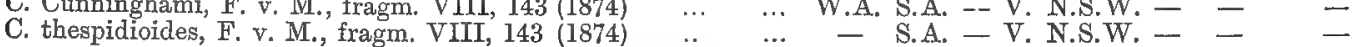

ABROTANELLA, Cassini in Dict. XXXVI, 27 (1825). (Scleroleima, Trineuron.)

A. fosterioides, J. Hooker, Hand. N. Zeal. Fl. $139(1864)$.. - - T. - - - - B.fl.III,554

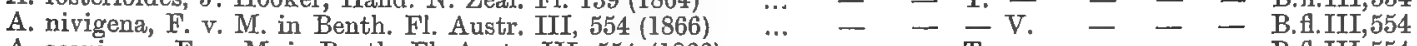

A. scapigera, F. v. M. in Benth. Fl. Austr. III, $554(1866) \quad \cdots \quad-\quad$ T. - - - - B.fl.III,554

ELACHANTHUS, F. v. M. in Schlecht. Linnaea XXV, 410 (1852).

E. pusillus, F. v. M. in Schlecht. Linnaea XXV, $411(1852)$.. - S.A. - V. N.S.W. - - B.H.III,555 CERATOGYNE, Turczaninow in Bull. de Mosc. XXIV, 68 (1851). (Diotosperma.)

C. obionoides, Turezaninow in Bull. de Mosc. XXIV, 68 (1851) W.A. ㄴ - - _ - - B.fl.III,555 ISOETOPSIS, Turczaninow in Bull. de Mosc. XXIV, 175 (1851).

I. graminifolia, Turcz, in Bull, de Mosc. XXIV, 175, t. 3 (1851) W.A. S.A. - V. N.S.W. - - B.fl.III,556 EMILIA, Cassini in Bulletin de la Soc. philomat. 68 (1817).

E. purpurea, Cassini in Diction. des sc. nat. XXXIV, 393 (1825) -
GYNURA, Cassini in Diction. des sc. nat. XXXIV, 391 (1825).

G. Pseudo-China, De Candolle, prodr, VI, $299(1837) \quad \ldots \quad \ldots \quad-\ldots-$ - $\quad$ N.S.W. Q.

SENECIO, Toumefort, inst. 456, t. 260 (1700), from Plinius. (Bedfordia, Centropappus.)

S. Gregorii, F. v. M., pl. of Greg. Search-Exped. for Leichh. $7(\mathbf{1 8 5 9 )}-$ S.A. - V. N.S.W. Q. S. platylepis, De Candolle, prodr. VI, $371(1837) \ldots$

S. papillosus, F. v. M. in Transact. phil. Inst. Vict. Ir, 109 (1857) -

S. primulifolius, F. v. M. in Transact. phil. Inst. Vict. II, $69(1857)-$

S. pectinatus, De Candolle, prodr. VI, 372 (1837)...

S. spathulatus, A. Richard, sert. Astrol. 125 (1833)

S. megaglossus, F. v. M. in Linnaea XXV, 419 (1852)

S. magnificus, F. v. M. in Linnaea XXV, 418 (1852)

S. insularis, Bentham, Fl. Austr. III, 666 (1866) ...

S. Centropappus, F. v. M., annual report 26 (1858)

S. macranthus, A. Richard, sert. Astrol. 126 (1833)

S. Daltoni, F. v. M., fragm. VI, 27 (1867) ... ... - - V. N.S.W. Q.

二 T. - T. - -

- T. V. N.S.W. -

S.A. T. V. N.S.W. -

S.A. - - - -

S. lautus, Solander in G. Forst. prodr. 91 (1786) ...

S. capillifolius, J. Hooker in Lond. Journ VI, $12 \ddot{3}(1847)$

S. vagus, F. v. M. in Transact. phil. Soc. Vict. I, 46 (1854)

S. amygdalifolius, F. v. M., fragm. I, 232 (1859) … 374 (1837) …

S. australis, A. Richard, sert. Astrol. 131, t. 39 (1833)

S. Behrianus, Sonder and F. V. M. in Linnaea XXV, 527 (1852)

S. Ieucoglossus, F. v. M., fragm. II, 15 (I861)

S. brachyglossus, F. v. M, in Schlecht. Linnaea XX X, 325 (1852)

S. Georgianus, De Candolle, prodr. VI, 371 (1837)

S. Gilberti, Turczaninow in Bull. Soc. Mosc. XXIV, 208 (1851)

S. ramosissimus, De Candolle, prodr. VI, 371 (1837)

S. odoratus, Horneman, hort. reg. bot. Hafn. II, 809 (18ї) $\quad \ldots$

S. Cunninghamii, De Candolle, prodr. VI, 371 (1837)

S. anethifolius, Cunningham in De Cand. prodr. VI, 371 (1837)...

S. Bedfordii, F. v. M. annual report 26 (1858)

S. Billardieri, F. v. M. anmual report $26(1858)$
S.A. - V. N.S.W. -

二 T. - N.S.W. -

- T. - N.S.W. -

... -

... -

... -

... -

$\ldots$

$\cdots$

...

W.A. S.A. T. V. N.S.W. Q.

- - - - N.S.W.

- - T. V. N.S.W. -

- S.A. T. V. N.S.W. -

- S.A. - V. N.S.W. -

W.A. $-\overline{\text { W.A. }}$ - V. N.S.W. -

W.A. S.A. T. V. N.S.W. -

W.A. - - - - -

W.A. $\overline{\text { S.A. }}$ T. $\overline{\text { V. N.S.W. }}$

W.A. S.A. - V. N.S.W. -

- S.A. - T. V. N.S.W. Q.

- $\quad$ - T. V. N.S.W. - $\cdots \quad-\quad-$ T. $\overline{\text { V. N.S.W. }}$
M.fr. VI, 114.

M.fr.VI, 114.

M.fr. VIII, 142.

(1)

.


ERECHTITES, Rafinesque, Fl. Ludov. 65 (1817). E. prenanthoides, De Candolle, prodr. VI, 296 (1837) ... E. Atkinsoniae, F. v. M., fragm. V, 88 (1866) $\ldots . \quad \ldots$ E. arguta, De Candolle, prodr. VI, 296 (1837) $\quad$.. $\quad$.. E. mixta, De Candolle, prodr. VI, 297 (1837) .
E. quadridentata, De Candolle, prodr. VI, 295 (1837) E. hispidula, De Candolle, proür. VI, 296 (1837) ... CYMBONOTUS, Cassini in Diction. XXXV, 397 (1825).

C. Lawsonianus, Gaudichaud in Freyc. Voy. Bot. 462, t. $86(1826)$ W.A. S.A. T. V. N.S.W. Q. SAUSSUREA, De Candolle in Ann. du Mus. XVI, 156 (1810). (Aplotaxis, Haplotaxis.)

S. carthamoides, Bentham, Fl. Hongk. $168(1861) \quad \ldots \quad \ldots \quad-\quad-\quad-\quad$ N.S.W. Q. CENTAUREA, Linné, hort. Cliffort. 420 (1737). (Leuzea partly.)

C. Australis, Bentham \& J. Hooker, gen. plant. II, 479 (1873).. - - - V. N.S.W. Q. TRICHOCLINE, Cassini in Bull. de la Soc. philom. 13 (1817). (Amblysperma.)

TRICHOCLINE, Cassini in Bull. de la Soc. philom. 13 (1817). (Amblysperma.)
T. scapigera, Bentham \& J. Hooker, gen. plant. II, 497 (1873)... W.A. - - - B.fl.III,676 MICROSERIS, D. Don in Edinb. phil. Mag. XI, 388 (1832). (Phyllopappus, Scozonera partly.)

M. Forsteri, J. Hooker, FI. N. Zeal. I, 151 (1853) … … W.A. S.A. T. V. N.S.W. - - B.fl.III,676 CREPIS, Linné, gen. pl. 240 (1737). (Youngia.)

C. Japonica, Bentham, Fl. Hongk, 194 (1861)

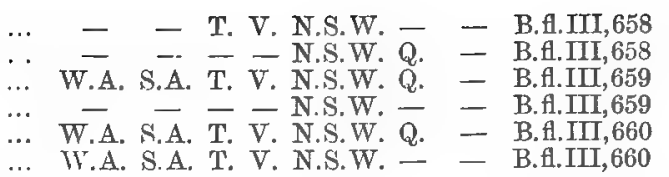

- B.fl.IIr, 456

- B.A.III,457

B. H.III, 679

M.fr. V,88.

- B.fl.III,674 M.fr.XI,27.

B.fl.III,676 M.fr.XI,27.

\section{CAMPANULACEAE.}

A. L. de Jussieu, gen. plant. 163 (1789), from B. de Jussieu (1759).

LOBELIA, Linne, Fl. Lappon. 227 (1737). (Rapuntium, Pratia, Grammatotheca, Holostigma.)

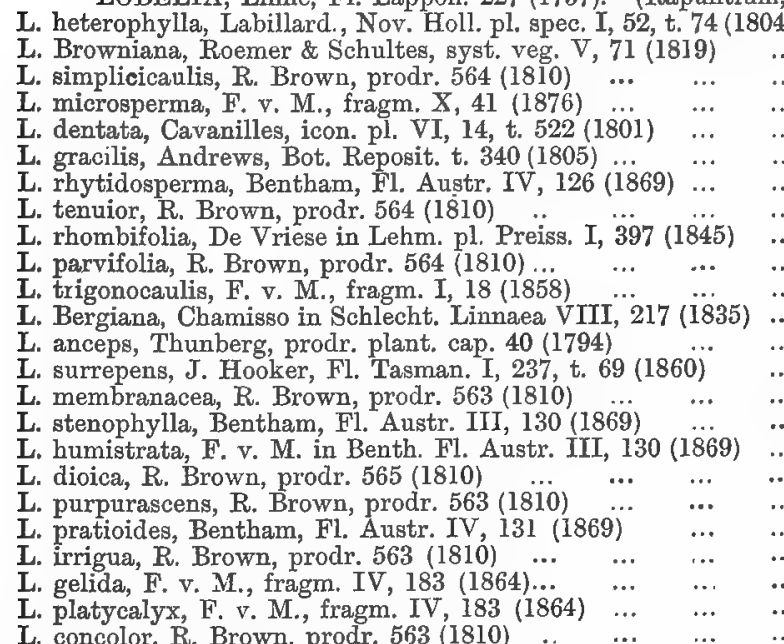

, Pratia, Grammatotheca, Holostigma.
W.A. S.A. - - - S.A. T. V. N.S.W. Q. -

B.fl.IV, 124 - B.fl. $\overline{\mathrm{IV}}, 124$ S.A. T. V. N.S.W. $-\bar{Q}$
W.A. S.A. T. V. N.S.W. Q. W.A. S.A. T. V. N.S.W.Q.
$\cdots \quad-$ - - -
$\cdots$ W.A. - - - - - - B.H.IV,126 W.A. S.A. - V. - - - B.fl.IV,126 W.A. - - - - - B.fl.IV,127 - - - - N.S.W. Q. - B.H.IV,127 W.A. - - - - - B.fl.IV,127 W.A. S.A. T. V. N.S.W. Q. - B.fl.IV,128 ‥ - - T. - - $-\frac{\text { Q. }}{\cdots}$ - B.H.IV,129 ... - - - - - Q. N.A. B.H.IV,130 $\because \quad-\quad-\quad-\quad-$ Q. N.A. B.f.IV,130 .. - - - - - Q. N.A. B.H.IV,130

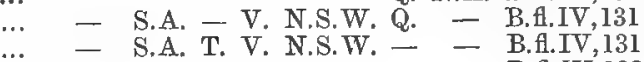
-.. - - T. - - - - B.fl.IV,132 … - - - - V. N.S.W. - - B.H.IV,132 ... - - T. T. $\quad$ - - - - B.fl.IV,133

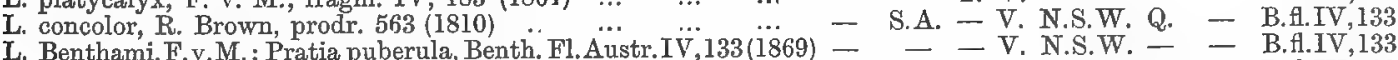
L. pedunculata, R. Brown, prodr. $563(1810) \quad \ldots \quad \ldots . \quad \ldots \quad-\quad$ S.A. T. V. N.S.W. Q. - B.fl.IV,133 ISOTOMA, R. Brown, prodr. 565 (1810). (Lobelia partly, Enchysia partly, Laurencia partly.) I. Brownii, G. Don, gen, syst. III, $716(1834)$
I. axillaris, Lindley, Bot. Regist. t. $964(1826)$
. . I. petraea, F. v. M. in Linnaea XXV, 420 (1852) ... $\ldots \ldots \ldots$... $\ldots$ W.A. S.A. - — N.S.W. Q. - B.A.IV,135

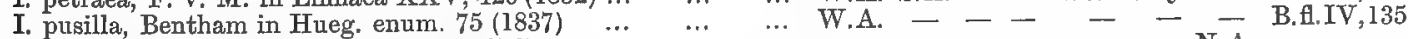

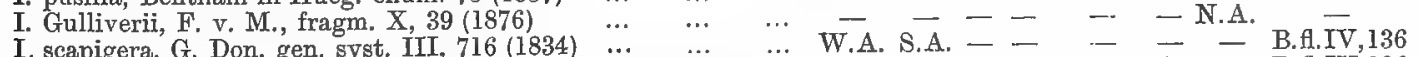

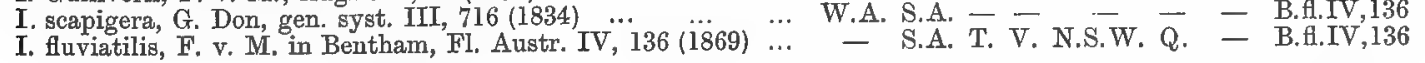
WAHLENBERGIA, Schrader, cat. hort. bot. Goetting. (1814).

W. gracilis, A. De Candolle, Monogr. comp. $142(1830) \ldots \quad$... W.A. S.A. T. V. N.S.W. Q. N.A. B.fl.IV, 137

\section{CANDOLLEACEAE.}

F. v. M., fragm. VIII, 41 (1873). (Stglideae, R. Brown, prodr. 565, 1810.) CANDOLlEA, Labillardière in Ann. du Mus. VI, 451 (1805). (Stylidium, Ventenata, Forsteropsis.)

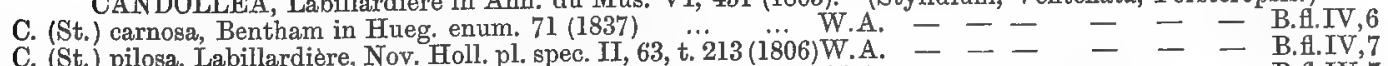

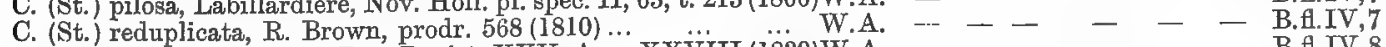
C. (St.) scabrida, Lindley, Bot. Regist. XXV, App XXV̈III (I839)W.A. - - - - - - B.H.IV,8

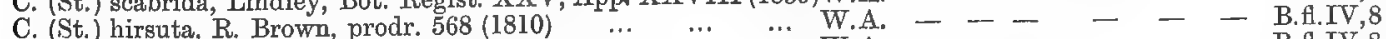
C. (St.) crossocephala, F. v. M., fragm. VI, $5(1866) \quad \ldots \quad \ldots$. W.A. $\quad$ - $\quad$ - $\quad$ - $\quad$ - - B.fl.IV,8

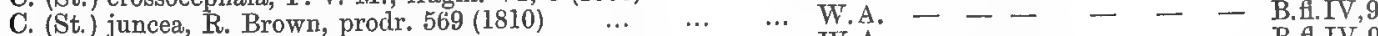

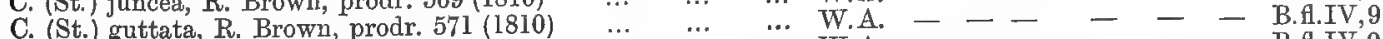
C. (St.) repens, R. Brown, prodr, 571 (1810) $\quad \ldots \quad \ldots \quad \ldots$. W.A. $-\quad--\frac{\ldots}{-}-\quad-$ B.fl.IV,9 C. (St.) repens, R. Brown, prodr. 571 (1810) Ges. Berl. $49(1807)-$ S.A. T. V. N.S.W. Q. - B.A.IV,10 C. (St.) linearis, Swartz, Mag. der nat. Ges. Berl. 50 (1807) C. (St.) elongata, Bentham, Fl. Austr. IV, 11 (1869) - - - N.S.W. - - B.H.IV,11

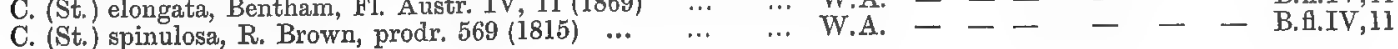

M.fr. VI, 5,254;X,58. M.fr, $X, 58$.

M.fr.X, 58 . M.fr.VI, $6 ; \mathrm{X}, 58$.

M.fr.X, 86 . 
C. (St.) limbata, F. v. M., fragm. X, 57 (1876)

C. (St.) caespitosa, R. Brown, prodr. 569 (I810)

C. (St.) squamellosa, De Candolle, prodr. VII, 782 (1838)

C. (St.) violacea, R. Rrown, prodr. 569 (1810)

C. (St.) lutea, R. Brown, prodr. $570(1810)$.

C. (St.) filifera, R. Brown, prodr. 569 (1810)

C. (St.) sobolifera F.

C. (St.) Floodii, F. v. M., fragm. I, 149 (1859)

C. (St.) disperma, F. v. M., fragm. IV, 93 (1864) ...

C. (St.) calcarata, R. Brown, prodx. 570 (1810)

$\begin{array}{lllll}\text { W.A. } & \text {... } & - \\ \text { V.A. S.A. }\end{array}$

C. (St.) perpusilla, J. Hooker in Lond. Journ. of Bot. VI, $266(18 \ddot{84} 7)$ W.A. S.A. T. V.

C. (St.) eriorrhiza, R. Brown, prodr. $569(1810)$

C. (St.) debilis, F. v. M., fragm. I, 149 (1859)

C. (St.) floribunda, R. Brown, prodr. 569 (1810)

C. (St.) leptorrhiza, F. v. M., fragm. I, 148 (1859)

C. (St.) assimilis, R. Brown, prodr. 569 (1810)

C. (St.) rupestris, Sonder in Lehm, pl. Preiss. I, 375 (1845)

C. (St.) spathulata, R. Brown, prodr. 569 (1810)

C. (St.) Barleei, F. v. M., fragm. VI, 5, t. 69 (1866)

C. (St.) lineata, Sonder in Lehm. pl. Preiss. I, 376 (1845)

C. (St.) glauca, Labillard., Nov. Holl. pl. spec. II, 64, t. 214 (1806)

C. (St.) amoena, R. Brown, prodr. 570 (1810) … XXVIII (18

C. (St.) diversifolia, R. Brown, prodr. 570 (1810)... ... ... W.A

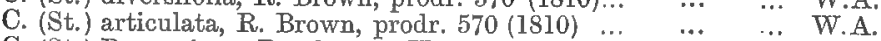

C. (St.) Brunoniana, Bentham in Hueg, enum. 72 (1837)... $\ldots$. W.A.

C. (St.) diuroides, Lindley, Bot. Regist. XXV, App. XXIX (1839) W.A.

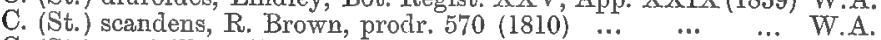

C. (St.) verticillata, F. v. M., fragm. IV, 94 (1864) $\ldots . \quad \ldots$. W.A.

C. (St.) glandulosa, Salisbury, Parad. Lond. t. 77 (1807)

C. (St.) laricifolia, Richard in Pers. syn. II, 210 (1807) ..

C. (St.) Preissii, F. v. M., fragm. III, 122 (1863)..

C. (St.) imbricata, Bentham in Hueg. enum. 73 (1837) $\ldots$

C. (St.) adpressa, Bentham, Fl. Austr. IV, 22 (1869)

C. (St.) despecta, R. Brown, prodr. 571 (1810)

C. (St.) utricularioides, Bentham in Hueg. enum. 73 (183̈)

C. (St.) pygmaea, R. Brown, prodr. 571 (1810)

C. (St.) longitubea, Bentham in Hueg. enum. 73 (1837) ...

C. (St.) diffusa, R. Brown, prodr. 571 (1810)

C. (St.) fissiloba, F. v. M., fragm. I, 154 (1859) ..

C. (St.) trichopoda, F. v. M., fragm. X, 86 (1877)

C. (St.) alsinoides, $R$. Brown, prodr. 572 (1810) ...

C. (St.) tenerrima, F. v. M., fragm. I, 150 (1859)..

C. (St.) brachyphylla, Sonder in Lehm. pl. Preiss. I, 386 (1845)

C. (St.) capillaris, R. Brown, prodr. 570 (1810)

C. (St.) rotundifolia, R. Brown, prodr. 571 (1810)...

C. (St.) schizantha, F. v. M., fragm. I, 152 (1859)

C. (St.) lobulifiora, F, v. M., fragm. I, 153 (1859)...

C. (St.) uliginosa, Swartz in Mag. nat. Fr. Berl. 52 , t. 2 (1807)

C. (St.) pulchella, Sonder in Lehm. pl. Preiss. I, 381 (1845)

C. (St.) petiolaris, Sonder in Lehm. pI. Preiss. I, 382 (1845)

C. (St.) emarginata, Sonder in Lehm. pl. Preiss. I, 383 (1845) ...

C. (St.) corymbosa, R. Brown, prodr. 571 (1810)

C. (St.) lepida, F. v. M. in Benth. Fl, Austr. IV, 27 (1869)

C. (St.) streptocarpa, Sonder in Lehm. pl. Preiss. I, 385 (1845)

C. (St.) uniflora, Sonder in Lehm. pl. Preiss. I, 381 (1845)

C. (St.) pedunculata, R. Brown, prodr. 571 (1810)

C. (St.) pachyrrhiza, F. v. M., fragm. I, $152(1859)$

C. (St.) muscicola, F. v. M., fragm. I, 153 (1859)..

C. (St.) crassifolia, R. Brown, prodr. 571 (1810) ...

C. (St.) pycnostachya, Lindley, Bot. Regist. XXV, App X X

C. (St.) pubigera, Sonder in Lehm. pl. Preiss. 1, 383 (1845)

C. (St.) canaliculata, Lindley, Bot. Regist. XXV,App. XXIX (1839)

C. (St.) leptophylla, De Candolle, prodr. VII, 783 (1838)

C. (St.) dichotoma, De Candolle, prodr. VII, 783 (1838)...

C. (St,) bulbifera, Bentham in Hueg, enum. 73 (1837) ...

C. (St.) breviscapea, R. Brown, prodr. 572 (1810)...

C. (St.) eglandulosa, F. v. M., fragm. I, 150 (1859)

C. (St.) fasciculata, R. Brown, prodr. 572 (1810) ..

C. (St.) falcata, R. Brown, prodr. 572 (1810)

C. (St.) rhynchocarpa, Sonder in Lehm. pl. Preiss. I, 389 (1845)

C. (St.) adnata, R. Brown, prodr. 572 (1810)

LEEWENHOEKIA, R. Brown, prodr. 572 (1810)

L. pusilla, R. Brown, prodr. 573 (1810)

L. dubia, Sonder in Lehm. pl. Preiss. I, 392 (1845)

L. Sonderi, F. v, M., fragm. I, 18 (1858)

L. pauciflora, Bentham in Hueg. enum. 74 (1837)...

L. leptantha, Bentham, Fl. Austr. IV, 35 (1869) ...

L. stipitata, F. v. M., fragm. IV, 94 (1864)

L. Preissii, F. v. M., fragm. IV, 94 (1864)...

-

W.A. - - -
W.A.

$\cdots$

W.A.

W.A.

W.A.

W.A. S.A. T. N $\overline{\mathrm{N}}-$

W.A. - - - N.S.W. - - B.fl.IV,22

W.A. - - - - - - B.fl.IV,23

W.A.

-

(1)

W.A.

-

$-$

W.A. $=-$

W. A.

W.A.

W.A.

W.A. - - -

W.A.

$-\quad--$

$-\quad--$

W.A.

W.A.

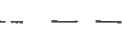

W.A.

$-$

W.A. - - -

W.A.

W.A.

W

A. - - N.S.T.

W.A. -

W.A.

(Levenhookia, Coleostylis.)

W.A. - - - - - - B.fl.IV,34

W.A. S.A. T. V. N.S.W. - - B.f.IV,34

- - - V. - - - B.fl.IV,35

W.A. - - - - - - B.f.IV,35

W.A. - - - - - B.fl.IV,35

... W.A. - - - - - B.f.IV,36

... W.A. - - - - - - B.fIIV,36
M.fr. $X, 57$.

M.fr. $X, 57$.

M.fr.X,56.

M.fr.X, $58,86$.

M.fr, VT, $6 ; \mathrm{X}, 58$.

M.fr.IV,93.

M.fr.VI, 6.

M.fr.VI, 78; X, 58.

M. fr.I, I47;VI, $6 ; \mathrm{X}, 58$.

M.fr. VI, $6 ; \mathrm{X}, 58$.

M.fr.I, $148 ; V I, 6 ; X, 58$.

M.fr.I, 148 .

M.fr.X, 57 .

M.fr.VI,5,249.

M.fr.X, 58.

M.fr. VI, 78 .

M. fr.IV,94.

M.fr. III, 122.

M.fr.VI, 58 .

M.fr.I, L54.

M.fr.X,86.

M.fr.I, $151 ; V I, 6 ; X, 58$.

M.fr. I, 150 .

M.fr.I, 151 .

M.fr.I, 152 .

M.fr.I, 153 .

M.fr.VI, 6.

M.fr. VI, 91 .

M.fr. I, 152 .

M.fr.I, 153.

M.fr. VI, 6;X, 58.

M.fr.X, 58 .

M.fr.X. 58.

M. fr. III, 121; X, 58 .

M.fr. VI, 77.

M.fr.IV, 94.

M.fr.IV, 94 . 
PHYLLACHNE, R. \& G. Forster, char. gen. 115, t. 58 (1776). (Forstera, Helophyllum, Oreostylidium.)

P. bellidifolia, F. v. M., fragm. VIII, $39(1873) \ldots \ldots \ldots \ldots$...

DONATIA, R, et G. Forster, char. gen. 9, t. 5 (1776).

D. Novae Zealandiae, J. Hooker, Fl. N. Zeal. I, 81, t. 20 (1853)

\section{GOODENIACEAE}

R. Brown, prodr. 573 (1810).

BRUNONIA, Smith in Transact. Linn. Soc. X, 366 (1809).

B. australis, Smith in Transact. Linn. Soc. X, 367, t. 28 (1809) DAMPIERA, R. Brown, prodr. 587 (1810). (Linschotenia.)

D. Iuteifolia, F. v. M., fragm. X, 11 (1876)

D. Linschotenii, F. v. M. fragm. VI, 28 (1866)

D. spicigera, Bentham, Fl. Austr. IV, 109 (1869)..

D. candicans, F. v. M., fragm. X, 86 (1876)

D. teres, Lindley, Bot. Regist. XXV, App. XXVIII (1839)

D. trigona, De Vriese in Lehm. pl. Preiss. I, 401 (1845)...

D. prostrata, De Vriese, Goodenov. 83 (1854)

D. alata, Lindley, Bot. Regist. XXV, App. XXVII (1839)

D. coronata, Lindley, Bot. Regist. XXV, App. XXVII (1839)...

D. carinata, Bentham, Fl, Austr. IV, 111 (1869)

D. sacculata, F. v. M. in Benth. Fl, Austr. IV, 111 (1869)

D. incana, R. Brown, prodr. 588 (1810)

D. hederacea, R. Brown, prodr. 588 (1810)...

$D$. ferruginea, $R$. Brown, prodr, 588 (1810)

D. Brownii, F. v. M., fragm. VI, 29 (1866)

D. lanceolata, Cunningham in De Cand., prodr. VII, 503 (1839)

D. altissima, F. v. M. in Benth. Fl. Austr. IV, $113(1869)$

D. marifolia, Bentham, Fl. Austr. IV, 114 (1869)...

D. rosmarinifolia, Schlechtendal, Linnaea XX, 603 (1847)

D. lavandulacea, Lindley, Bot. Regist. XXV, App. XXVII (1

D. juncea, Benthan, Fl. Austr. IV, 115 (1869)

D. oligophylla, Bentham, Fl. Austr. IV, 115 (1869)

D. loranthifolia, F, v. M. in Benth. Fl. Austr. IV, 115 (1869)

D. stricta, R. Brown, prodr. 589 (1810)

D. Scottiana, F. v. M., fragm. XI, 120 (1881) ‥

D. leptoclada, Bentham, FI. Austr. IV, 116 (1869)

D. fasciculata, R. Brown, prodr. 588 (1810)

D. subspicata, Bentham, Fl, Austr. IV, 117 (1869)

D. triloba, Lindley, Bot. Regist. XXV'App. XXVII (10̈39)

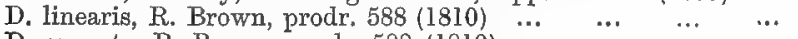

D. cuneata, R. Brown, prodr. 588 (1810) $\ldots$
D. sericantha, F. v. M. in Benth. Fl. Austr. IV, 118 (1869) $\ldots$

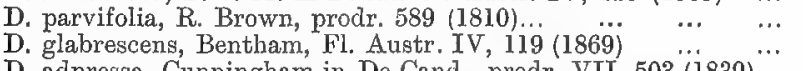

D. adpressa, Cunningham in De Cand., prodr. VII, 503 (1839)....

D. diversifolia, De Vriese in Lehm. pl. Preiss. I, 403 (1845)

D. eriocephala, De Vriese, Goodenov. 118, t. 21 (1854) ...

D. Wellsiana, F. v. M., fragm. X, 12 (1876) DIASPASIS, R. Brown, prodr. 586 (1810).

D. filifolia, R. Brown, prodr. 587 (1810) … ... LESCHENAULTIA $P$ Bro

L. formosa, R. Brown, prodr. 581 (1810) $\cdots$.

L. chlorantha, F. V. M., fragm. II, 20 (1860)

L. linarioides, De Candolle, prodr. VII, 519 (1818)

L. tubiflora, R. Brown, prodr. 581 (1810)

L. superba, F. v. M., fragm. VI, 10 (1866)

L. striata, F, v. M., fragm. VIII, 245 (1874)

L. acutiloba, Bentham, Fl. Austr. IV, 41 (1869)

L. laricina, Lindley, Bot. Regist. XXV, App. XXVII (1839)

L. hirsuta, F. v. M., fragm. VI, 9 (1866)

L. longiloba, F. v. M., fragm. VI, 10 (1866)

L. biloba, Lindley, Bot. Regist. XXV, App. XXVII (1839)

L. expansa, R. Brown, prodr. 581 (1810)

L. floribunda, Bentham in Hueg. enum. 70 (1837)...

L. heteromera, Bentham, Fl. Austr. IV, 43 (1869)

L. divaricata, F. v. M., fragm. III, 33, $167(1863)$

L. filiformis, R. Brown, prodr. 581 (1810) ...

L. agrostophylla, F. v. M., fragm. VI, 8, pl. XLVII (1866) ANTHOTIUM, R. Brown, prodr. 582 (1810).

A. humile, R. Brown, prodr. $582(1810) \ldots \ldots$

A. rubriflorum, F. v. M. in Benth. FI. Austr. IV, 45 (1869) CATOSPERMA, Bentham in Hooker, icon. pl. t. 1028 (1868).

C. Muelleri, Bentham in Hook. icon. pl. t. 1028 (1868) ... SCAEVOLA, Linné, mantiss. II, 145 (1771). (Pogonetes, Crossotoma, Temminckia, Ka

S. Koenigii, Vahl, symb. bot. III, 36 (1794)

S. enantophylla, F. v. M., fragm. VIII, $58(1873) \ldots$

\author{
W.A
}

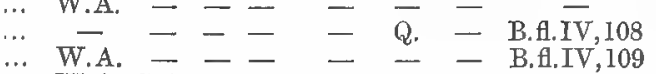

W.A. $\overline{\text { WA }}--$ B.A.IV,109

W..A. - W.A $=-二$ B.fl. $\overline{\mathrm{IV}}, 109$

$\therefore$ W.A. - - - $-二-$ B.A.IV,109

W.A

W A

W.A.

W.A. --

W.A.

W.A.

W.A.

.. W.A.

-

- $-\overline{\text { V. N.S.W. Q }}$

- S.A. - V. N.S.W. -

... W.A. S.A. $-\overline{\text { V. N.S.W. }}$ -

二 S.A. - V. N.S.W. -

W.A.

W.A.

W.A.

A. $--\overline{-}=$

- S.A. T. V. N.S.W.$$
\begin{aligned}
& W \\
& W \\
& W \\
& W \\
& W \\
& W \\
& W \\
& W \\
& W \\
& W \\
& W
\end{aligned}
$$$$
\text { - - - - - B.fl.IV,116 }
$$$$
\text { W.A. - - - - - B.fl.IV,116 }
$$$$
\text { W.A. - 二 二 - - - B.fl.TV,l17 }
$$$$
\text { W.A. - - - - - B.fl.IV,117 }
$$$$
\text { W.A. - - - - - - B.H.IV,117 }
$$$$
\text { W.A. - - - - - - B.H.IV'118 }
$$$$
\text { WA }=- \text { - - - B }
$$$$
\text { W.A. - - - - - B.H.IV'118 }
$$$$
\text { W.A. - - - - - - B.H.IV'119 }
$$$$
\text { W }
$$

W.A.

$--$

W.A. --

(Latourea.)

W.A. - - -

W.A. - - -

W.A.

W.A.

W.A. --

W

W.A.

W.A. - - -

W.A. - - -

W.A. - - -

W.A. - -

W.A. - - -

W. W.A. --

... W.A. - - -

… W.A.

$\cdots \quad$ - S.A. -

- $=$ 二

W.A. - - -

$=$
$=$
$=$
$=$
$=$
$=$
$=$
$=$
$=$
$=$

$=$
$=$
$=$
$=$
$=$
$=$
$=$
$=$
$=$
$=$
$=$
$=$
$\bar{Q} . \mathrm{N} \cdot \mathrm{Z}$

- B.fl.TV, 40

M.fr. VI,226.

M.fr.VI, 26 .

B.A.IV, 40

B.fl. IV, 40

B.fl.TV,41

B.H.IV, 41

B.A.IV, 41

- B.H.IV,41

- B.fl.IV,42

B.fl.IV,42

- B.H.IV, 42

- B.H.IV, 42

- B.fl.IV,43

- B.fl.IV,43

B.fl.IV, 43

N.A. B.fl.IV, 44

M fr, VIII, 246

M.fr.VI, 9,249 .

M.fr.VI, 8;249.

M.fr.VI, 10.

M.fr.VIII, 245.

M.fr.VI, 9.

M.fr.VI, 10 .

- $\quad$ - $\quad$ B.f.IV,44

M.fr.VIII, 58.

— N.A. B.f.IV,83 M.fr.I, 121;VIII,58.

mphusia, Molkenboeria, Merkusia, Verreauxia.) Q. N.A. B fl.IV,86 M.fr.XI, 76 .

M.fr. VI, 225 . 
S. spinescens, R. Brown, prodr. 586 (1810)...

S. Groeneri, F. v. M., fragm. VI, 15 (1866)

S. tomentosa, Gaudichaud in Freyc. Voy. Bot. 460 , t. 81 (1826)

S. atriplicina, F. v. M., fragm. II, 18 (1860)

S. striata, R. Brown, prodr. 586 (1810)

S. phlebopetala, F. v. M., fragm. II, 18 (1860)

S. pilosa, Bentham in Hueg. enum. 69 (1837)

S. hispida, Cavanilles, icon, et descr. pI VI 7, t. 510 ( $\dddot{10} 01)$

S. apterantha, F. v. M., fragm. I, 121 (1859)

S. Hookeri, F. v. M. in Benth. Fl. Austr. IV, 90 (1869).”

S. parvifolia, F. v. M. in Benth. Fl. Austr. IV, 91 (1869)

S. oxyclona, F. v. M., fragm. X, 58 (1876)..

S. restiacea, Bentham, Fl. Austr. IV, 91 (1869)

S. depauperata, R. Brown, App. Sturt's Exped, 20 (1849)

S. tortuosa, Bentham, Fl. Austr. IV, 91 (1869)

S. Cunninghamii, De Candolle, prodr. VII, 508 (1838) ...

S. collaris, F. v. M., Rep. Babb. Exped. 15 (1858)

S. angulata, R. Brown, prodr. 586 (1810)

S. nitida, R. Brown, prodr. 584 (1810)

S. globulifora, Tabilardiere,

S. globuliflora, Labillardière, Nov. Holl. pl.
S. porocarya, F. v. M., fragm. II, 19 (1860)

S. attenuata, R. Brown, prodr. 583 (1810)

S. glandulifera, De Candolle, prodr. VII, 510 (1838)

S. anchusifolia, Bentham in Hueg. enum. 68 (1837)

S. holosericea, De Vriese in Lehm. pl. Preiss. I, 408 (18405)

S. suaveolens, R. Brown, prodr. 585 (1810)

S. revoluta, R. Brown, prodr. 586 (1810)

S. ovalifolia, R. Brown, prodr. 584 (1810) ...

S. crassifolia, Labillardière, Nov. Holl. pl. spec. I, 56, t. 79 (1804)

S. macrostachya, Bentham, Fl. Austr. IV, 97 (1869)

S. longifolia, De Vriese in Lehm. pl. Preiss. I, 410 (1845)

S. lanceolata, Bentham in Hueg. enum. 69 (1837).

S. thesioides, Bentham in Hueg. enum. 68 (1837)...
S. macrophylla, Bentham, Fl. Austr. IV, 98 (1869)

S. platyphylla, Lindley, Bot. Regist. XXV, App. XXVI (1839)

S. auriculata, Bentham, Fl. Austr. IV, 99 (1869)...

S, aemula, R. Brown, prodr. 584 (1810)

S. humilis, R. Brown, prodr. 585 (1810)

S. amblyanthera, F. v. M., fragm. I, 121 (1859)

S. microphylla, Bentham, Fl. Austr. IV, $100(1869)$

S. cuneiformis, Labillard., Nov. Holl. pl. spec. I, 56, t. 30 (1804)

S. microcarpa, Cavanilles, icon. et. descr. pI. VI, 6, t. 509 (1801)

S. linearis, R. Brown, prodr. 586 (1810)

S. Oldfieldii, F. v. M., fragm. II, 19 (1860)

S. paludosa, R. Brown, prodr. 586 (1810)

S. sericophylla, F. v. M. in Benth. Fl. Austr. IV, 102 (1869) ..

S. canescens, Bentham in Hueg. enum. 69 (1837).

S. humifusa, De Vriese in Lehm. pl. Preiss. I, 410 (1845)

S. fasciculata, Bentham in Hueg. enum. 68 (1837)

S. stenophylla, Bentham, Fl. Austr. IV, 104 (1869)

S. Reinwardtii De Vriese in Lehm. pl. Preiss. I, 409 (1845)

S. Verreauxii, F. v. M., Bot. Teachings, 65 (1877) SELLIERA, Cavanilles in Anal. Cienz. Nat. I, 41, t. 5 (1799)

S. radicans, Cavanilles, icon. et descr. pl. V, 49, t. 474 (1799) ...

S. exigua, F. v. M., fragm. III, 142 (1862).. CALOGYNE, R. Brown, prodr. 579 (1810), (Distylis.)

C. pilosa, R. Brown, prodr. 579 (1810)

C. Berardiana, F. v. M., fragm. VI, 7 (1867)

C. heteroptera, F. v. Mueller, fragm. X, $43(1876)$

C. purpurea, F. v. M., fragm. VIII, 57 (1873) GOODENIA, Smith in Transact. Linn. Soc. II, 347 (1794).

G. phylicoides, F. v. M., fragm. I, 206 (1859)

G. viscida, R. Brown, prodr. 578 (1810)

G. xanthotricha, De Vriese, Gooden. 155 (1854) ...

G. scapigera, R. Brown, prodr. 578 (1810) ...

G. quadrilocularis, R. Brown, prodr. 578 (1810) ...

G. Ramelii, F. v. M., fragm. III, 20, t. 17 (1862)...

G. pinifolia, De Vriese, Gooden. 157, t. 30 (1854)...

G. decurrens, R. Brown, prodr. 575 (1810)..

G. racemosa, F. v. M., fragm. I, 114 (1859)

G. bellidifolia, Smith in Transact. Linn. Soc. II, 349 (1794)

G. stelligera, R. Brown, prodr. 575 (1810)

G. ovata, Smith in Transact. Linn. Soc. II, 347 (1794)

G. amplexans, F. v. M. in Transact. phil. Inst. Vict. II, 70 (1859)

G. strophiolata, F. v. M., fragm. I, 119 (1859)

G. varia, R. Brown, prodr. 576 (1810)

G. laevis, Bentham, Fl. Austr. IV, 61 (1869)

G. barbata, R. Brown, prodr. 576 (1810)

G. arthrotricha, F. v. M. in Benth. Fl. Austr. IV, 62 (1869) $\ldots$

G. disperma, F. v. M., fragm. I, 113 (1859)

$\cdots$
W.A. S.A. - V. N.S.W. Q. N.A. B.A.IV,87 W. A A - - - - - B.fl.IV,88

W. - - - - - B.H.IV,88

W.A. - - - - - - B.A.IV,88

W.A. - - - - - B.fl.IV, 89

W.A. - - - - - B.f.IV,89

W.A. - - - - - B.fl.IV,89

- - - V. N.S.W. $\overline{\mathrm{Q} .}-$ B.fH.IV,90

- - - V. - - - B.fl.IV,90

- - T. V. N.S.W. - - B.fl.IV,90

W.A. S.A. - - - Q. N.A. B.fl.IV,9l

W.A. - - - - - - B.fl.IV,91

W.A. S.A. - - - - - B.fl.IV,91

W.A. - - - - - B.Al.IV,91

- - - - - N.A. B.H.IV,92

- S.A. - - - - - B.fl.IV,92

- - - - - - N.A. B.H.IV,92

W.A. - - - - - - B.H.IV,93
W.A. -

W.A. - - - - - - B.fl.IV,94

W.A. - - - - - - B.H.IV,94

W.A. - - - - - - B.H.IV,94

W.A. - - - - - - B.f.IV,94

W.A. - - - - - B.H.IV,95 S.A. - V. N.S.W. Q. - B.H.IV,95

- - - - - - N.A. B.fl.IV, 96

- S.A. - - N.S.W. Q. N.A. B.H.IV,96

W.A. S.A. - V. - - - B.A.IV,96

W.A. - - - - - N.A. B.H.IV,97

W.A. - - - - - -- B.fl.IV,97

W.A. - - - - - - B.fl.IV,98

W.A. - - - - - - B.A.IV,98

W.A. - - - - - - B.fl.IV,98

W.A. - - - - - - B.fl.IV,99

W.A. S.A. T. V. N.S.W. - - B.H.IV,99

- S.A. - - - - - B.fl.IV, 100

W.A. - - - - - N.A. B.f.IV,100

W.A. - - - - - - B.f.IV,101

- S.A. T. V. N.S.W. Q. - B.H.IV,101

W.A. A. - - - - - B.H.IV,102

W.A. - - - - - - B.fl.IV,102

W.A. - - - - - - B.fl.IV,102

W.A. - - - - - - B.fl.IV,103

W.A. - - - - - - B.H.IV,103

W.A. - - - - - - B.f.IV,104

W.A. - - - - - - B.f.IV,104

W.A. - - - - - - B.fl.IV,106

W.A. - - - - - - B.f.IV,105

- S.A. T. V. N.S.W. - - B.f.IV,82 W.A. - - - - - - B.A.IV,82

W.A. - - - - - Q. N.A. B.fl.IV,81
N.A. B.fl.IV,81
- - Q. N.A.

- - - - - - N.A. -

W.A. - - - - - - B.fl.IV,55

W.A. - - - - - - B.f.IV,55

W.A. - - - - - B.H.IV,56

W.A. - - - - - - B.H.IV,56

W.A. - - - - - - B.fi.IV,57

W.A. S.A. - - - - N.A. B.fl.IV,57

... W.A. - - - - - B.fl.IV,58

- - - - N.S.W. - - B.H.IV,58

- - - - - Q. - B.fl.IV,58

- - - - N.S.W.Q. - B.fl.IV,58

- - V. N.S.W. Q.

S.A. T. V. N.S.W. Q.

S.A. - V

M.fr.VIII, 58 .

M.fr.III, 181 .

M. fr.II, VI, 16;XI,76.

M.fr.XI, 76

M.fr.I,121.

M.fr.XI, 76

$58 ; \mathrm{X}, 59 ; \mathrm{XI}, 76$

I.fr, X, 58 .

fr.III, 33; VIII, $58 ;$ XI,

M.fr. VIII, 58;XI, 76.

M.fr.II, 19;XI, 76.

M. fr. VIII, $58 ;$ XI, 76

VII, 58;XI, 76 .

fr.XI, 77.

M.fr.XI, 77 .

I.fr. I, 121

M.fr. VI, 16;XI, 77.

fr. II 19;XI, 77.

M fr. XI 77.

M.fr.XI,77.

M.fr.I, $113 ; X I, 77$.

M.fr.VIII, 58 .

M. fr. VI, 7,28 .

M.fr. VI, 7; VIII,58.

M.fr.X, 43.

a, Aillya.)

M.fr. I, 120;III, 35 .

M.fr. VI, 13.

M.fr.I, 114;III, $163 ; \mathrm{XI}, 50$.

, 13 .

M.fr.I, 114.

M. fr. X, 110

M.fr.VI, 15 .

M.fr.I, 119.

M.fr.I, 205.

M.fr.II,110,176;VI,15.

M.fr.IV,145;VI, 15. 
G. geniculata, R. Brown, prodr. 577 (1810)

G. hederacea, Smith in Transact. Linn. Soc. II, 349 (1794)

G. xanthosperma, F. v. M., fragm. X, 12 (1876) ...

G. hirsuta, F. v. M., fragm. III, 35 (1862)...

G. heterophylla, Smith in Transact. Linn. Soc. II, 349 (1794) ”

G. glabra, R. Brown, prodr. 577 (1810)

G. strongylophylla, F. v. M., fragm. VI, $\ddot{12}(1867)$

G. rotundifolia, R. Brown, prodr. 576 (1810)

G. azurea, F. v. M., fragm. I, 117 (1859)

G. scaevolina, F. v. M., fragm. I, 118 (1859)

G. Stobbsiana, H'. v. M., fragm. XI, 49 (1879)

G. incana, R. Brown, prodr. 578 (1810)

G. leptoclada, Bentham, Fl. Austr. IV, 67 (1869).

G. Eatoniana, F. v. M., fragm. VIII, 186 (1874) ...

G. coerulea, R. Brown, prodr. 578 (1810)

G. trichophylla, De Vriese in Benth. Fl, Austr. I $\dddot{V}, 67$ (I869) ...

G. Hassallii, E. v. M., fragm. VI, 10, t. 51 (1867)

G. pterygosperma, R. Brown, prodr. 578 (1810) ...

G. Vilmoriniae, F. v. M., fragm. III, 19, t. $16(1862) \ldots$

G. Bonneyana, F. v. M. in Benth. Fl. Aust

G. albiflora, Schlechtendal, Linnaea XX, 599 (1847)

G. Nicholsoni, F. v. M., fragm. I, 203, t. 4 (1859)

G. Macmillani, F. v. M., fragm. I, 119, t. 5 (1859)

G. grandiflora, Sims, Bot. Mag. t. 890 (1806)

G. Chambersii, F. v. M., fragm. I, 204 (1859)

G. Strangfordii, F. v. M., fragm. VI, 11, t. 52 (18̈7)

G. Mitchellii, Bentham, Fl. Austr. IV, 71 (1869) ...

G. Mueckeana, F. v. M., fragm. VIII, 56 (1873) ... ...

G. melanoptera, F. v. M., fragm. I, $115(1859) \quad \ldots \quad$...

G. heterochila, F. v. M., fragm. III, $142(1863)$...

G. sepalosa, F. v. M. in Benth. Fl. Austr. IV, 72 (1869)...

G. hispida, $R$. Brown, prodr. 577 (1810)

G. auriculata, Bentham, Fl. Anstr, IV, 72 (1869)...

G. Armstrongiana, De Vriese, Goodenov. 138, t. 24 (1854)

G. corynocarpa, F. v. Mi, fragm. II, 16 (1860)

G. mollissima, F, v. M. in Benth. FI. Austr. IV, 73 (1869)

G. cycloptera, R. Brown in App. Sturt's Exped.'20 (1849)

G. tenella, R. Brown, prodr. 577 (1810)

G. elongata, Labillardière, Nov. Holl. pl. spec. I,

G. pinnatifida, Schlechtendal, Linnaea XXI, $440\{1848\} \ldots$

G. coronopifolia, R. Brown, prodr. 577 (1810)

G. heteromera, F. v. M., fragm. I, 115 (1859)

G. concinna, Bentham, Fl. Austr. IV, 76 (1869) $\cdots$

G. glauca, F. v. M. in Transact. Vict. Inst. 40 (1855) ...

G. filiformis, R. Brown, prodr. 578 (1810) ...

G. Armitiana, F. v. M., fragm. X, 110 (1877)

G. microptera, F. v. M., fragm. III, 34 (1862)

G. bicolor, F. v. M. in Benth. Fl. Austr. IV, 80 (ï69) ...

G. humilis, R. Brown, prodr. 575 (1810)

G. paniculata, Smith in Transact. Linn. Soc. II, 348 (179̈)

G. purpurascens, R. Brown, prodr. 578 (1810)

G. gracilis, R. Brown, prodr. 575 (1810)

G. lamprosperma, F. v. M., fragm. I, 116 (1859) ..

G. minutiflora, F. v. M., fragm. VIII, 244 (1874)

G. claytoniacea, F. v. M. in Benth. Fl. Austr. IV, 79 (1869)

G. Pumilio, R. Brown, prodr. 579 (1810) ...

VELLEYA, Smith in Transact. Linn. Soc. IV, 217 (1798).

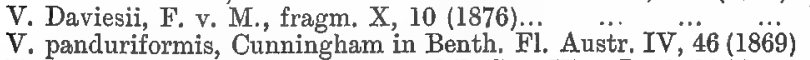

V. connata, F. v. M. in Transact. phil. Soc. Vict. I, $18(1855) \ldots$

V. perfoliata, R. Brown, prodr. 581 (1810)...

V. discophora, F, v. M., fragm. X, 10 (1876)

V. trinervis, Labillard., Nov. Holl. pl. spec. I, $5 \dddot{4}$, t. 77 (1804)

V. macrophylla, Bentham, Fl. Austr. IV, 47 (1869)

V. paradoxa, R. Brown, prodr. 580 (1810)..

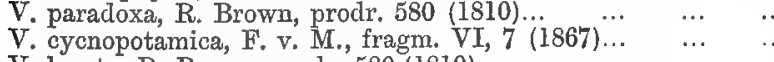

$V$. lyrata, $R$. Brown, prodr. 580 (1810)

V. macrocalyx, De Vriese, Goodenov, 176, t. 34 (1854) ..

V. spathulata, R. Brown, prodr. 580 (1810)

$V$. pubescens, R, Brown, prodr. 581 (1810)

V. montana, J. Hooker in Lond. Journ. of Bot. VI, 265 (1847) ...
W.A. S.A. T. V. N.S.W. Q. - B.fl.IV,62

T. - - V. N.S.W.Q. - B.fl.IV,63

W.A. $-\bar{A}=--\overline{-}$ -

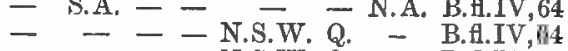

- - - - N.S.W.Q. - B.fl.IV,64

- - - - - Q. - B.fl.IV,65

- - - - N.S.W. Q. - B.fl.IV,65

- - - - - N.A. B.H.IV,66

- - - - - - - N.A. B.fl.IV,66

W.A. - - - - - B. B.H.IV, 66

W.A. - - - - - - B.fl.IV,67

W.A. - - - - - - B.f.IV, 67
W.A. -

W.A. - Z - - W.A. - - B.fl.IV,67
W.A.IV,67

W.A. - - - - - - B.Hl.IV,68

W.A. - - - - - - B.H.IV,68

- S.A. - - - N.A. B.A.IV,68

W.A. $\rightarrow--\overline{-}-$ B.f.IV,69

- S.A. - - N.S.W. -. - B.fl.IV,69

- S.A. - - - - - B.fl.IV,70

- S.A. - - - - - B.H.IV,69

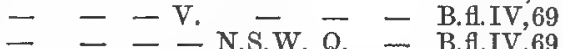

- S.A. - - - - - B.H.IV,70

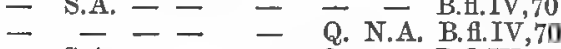

- s.A. - - - Q. - B.fl.IV,71

- S.A. - -

- S.A. -

-

- N.A. $\quad$ -

- N.A. B.fl.IV,71

- - - - - N.A. B.f.IV,72

- - - - - - N.A. B.fl.IV,72

- - - - - - N.A. B.fl.IV,72

W.A - - - - - N.A. B.fl.IV,73

- - - - - $\overline{\mathrm{Q}}$ - $\begin{gathered}\text { B.f.IV,73 } \\ \text { B.fl.IV,73 }\end{gathered}$

- S.A. - V. N.S.W. - - B.f.IV,74

W.A. ---- B.f.IV,74

S.A. T. V. N.S.W. - - B.H.IV,74

- S.A. - V. N.S.W. - - B.fl.IV,75

- - - - - N.A. B.fl,IV,75

W.A. S.A. - V. N.S.W. - - B.fl.IV,76
- - B.fl.IV,76

W.A. S.A. - V. N.S.W. Q. N.A. B.fl.IV,76

W.A. - - - - - B.H.IV,77

- $\overline{\mathrm{S} A \mathrm{~A}}-\mathrm{Z}$ Q. N.A.

- S.A. - - - - N.A. B.H.IV,77

- S.A. T. V. N.S.W. - - B.fl.IV,79

- - - V. N.S.IV. Q. - B.fl.TV,78

- - - - - Q. N.A. B.fl.IV,78

- - - V. N.S.W. Q. N.A. B.H.IV,79

- - - - - N.A. B.fl.IV,79

W.A. - - - - - N.A. ${ }_{\text {B.H.IV,79 }}$

W.A. - - - - Q. N.A.. B.H.IV,79

(Velleia, Euthales.)

W.A. - - - - - N.A. B. B. $\overrightarrow{I V}, 46$

- S.A. - V. N.S.W. - N.A. B.H.IV,46

- - - - N.S.W. - - B.H.IV,46

W.A. - - - - - - - B.H.IV,47
W.A. -

W.A. - - - 二 $=$ - B.H.IV,47

- S.A. T. V. N.S.W. Q. - B.f.IV,48

W.A. - - - - - - B.H.IV,48

- $\quad-$ - N.S.W. - - B.f.T.,49

- - - - N.S.W. Q. - B.H.IV,49

- - - - - N.S.W.Q. - B.H.IV,50

- - T. V. N.S.W. Q - B.f.IV, 59
M. fr. VI, 14

M.fr. VI, 15,110 .

M.fr.X, 12.

M.fr.III, 35.

M.fr.VI, 14 .

M.fr.VI,12.

M.fr.VI, 15.

M.fr.I,117.

M.fr.I,118.

M. fr.XI, 49,137 .

M.fr.I,155;III, 141. [50.

M.fr. VI,227,VIII, 186;XI,

M.fr.XI,51.

M.fr.III, 140;XI, 20 .

M.fr.XI, 50.

M.fr.I, 155;III, 163 .

M.fr.III, 19; VIII, 245 .

M.fr.VI, 226,250.

M.fr.VIII, 245.

M.fr. I, 203.

M.fr. I, 119 .

M.fr.I,204;VI, 14.

M. fr. I, 204.

M.fr,XI,75.

M.fr.IX, 194;XI, 133 .

M.fr.I,115.

M.fr. VIII, 245 .

M.fr. VI, 12 .

M.fr. I, 205;VII, 40 ,

M.fr.II, 16;VI, 15 .

M. fr.VI, 14;XI, 51 .

M. fr. XI, 51 .

$[\mathrm{X}, 110$.

M.fr.VI, 14;VIII, 142,245;

M.fr.VI, 14;XI,5I.

M.fr.VI,14;XI,27.

M.fr.VI,14;XI,27.

M. fr. VI, 14; VIII, 245.

M. fr, X, 110 .

M.fr. VI, 227;XI,75.

$[245 ; \mathrm{X}, 110$

M.fr.VII,227;VIII, 142,

M.fr.I,116.

M.fr. I, 117.

M.fr.XI, 51 .

M.fr.I, 116.

M.fr.VIII, 244 .

M.fr.II, 111 .

M.fr. $X, 111$.

M.fr.X, 10.

M.fr.XI,73.

M.fr. VI, 227; VIII, 246.

M.fr.X, 10 .

M.fr.XI, 75 .

M.fr.XI, 75 .

M.fr.VI, 11;XI, 75.

M.fr.VI,249;X, 10.

M. fr. X, 75 .

M.fr.XI, 75 .

M.fr.VI, 11;XI,75. 
SYNPETALEAE HYPOGYNAE.

F. v. M. in Woolls, pl. of the neighb. of Sydney 34 (1880).

GENTIANEAE.

Necker in Act. Acad. Theod. Pal. II, 477 (1770), from B. de Jussieu (1759).

LIMNANTHEMUM, Gmelin, Nov. Comm. Acad. Petrop. XIV, 257 (1770). (Villarsia, Liparophyllum.)

L. Indicum, Thwaites, enum. pl. Ceyl. 205 (1862) $\quad$.. $\quad \ldots \quad-\ldots$

L. Moonii, Thwaites, enu. plo

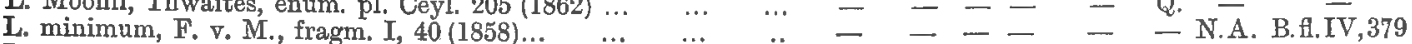

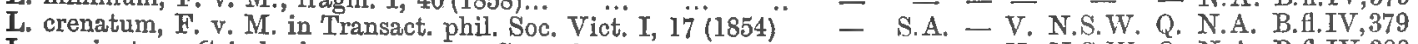

L. geminatum, Grisebach, gen. et spec. Gent. 346 (1839)

L. hydrocharoides, F. v. M. in Benth. Fl. Austr. IV, 380 (1869)

L. exiliflorum, F. v. M., fragm. V, 46 (1865)

L. exiguum, F. v. M., fragm. I, 40 (1858)...

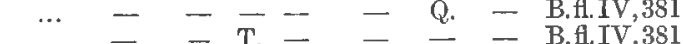

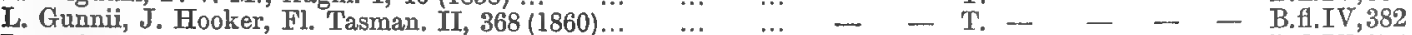

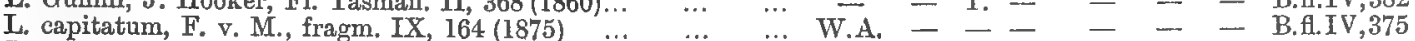

L. calthifolium, F. v. M., fragm. IX, $164(1875) \ldots \quad \ldots \quad \ldots$. W.A. - $\quad$ - $\quad$ - $\quad$ - $\quad$ B.fl.IV,374

L. congestiflorum; Villarsia, F. v. M., fragm. VI, 141 (1868) $\ldots$ W.A. - - - - - - B.H.IV,375

L. latifolium, F. v. M., fragm. IX, $164(1875)$, W.A. - - - - - - B.fl.IV,375

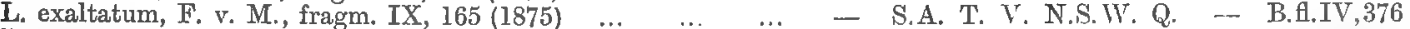

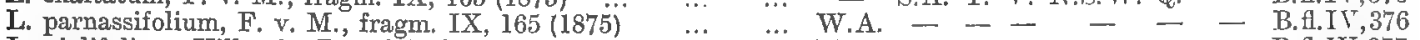

L. violifolium; Villarsia, F. v. M., fragm. VI, 138 (1868) $\quad \cdots$ W.A. - - - - - - - B.fl.IV,377

L. lasiospermum, F. v. M. , fragm. IX, 165 (1875) $\ldots \quad \ldots$. W.A. $\quad$ - $\quad$ - $\quad$ - $\quad$ - $\quad$ B.fl.IV,377

L. albiflorum; Villarsia, F. v. M., fragm. II, 21 (1860) …

W.A. - - - - - - B.H.IV,37 SEBAEA, Solander in R. Brown, prodr. 451 (1810).

S. ovata, R. Brown, prodr. 452 (1810)

S. albidillora, F. v. M. in Transact. phil. Soc. Vict. I, 46 (185

W.A. S.A. T. V. N.S. W. Q. - B.Hl.IV,371 ERYTHRAEA, Reneaulme, specim. hist. plant. 77, t. 76 (1611).

E. australis, R. Brown, prodr. 451 (1810) ... $\ldots . \ldots \ldots . .$. W.A. S.A. T. V. N.S.W. Q. N.A. B.fl.IY,371 CANSCORA, Lamarck, Encycl. method. I, 602 (1783). (Orthostemon.)

C. diffusa, R. Brown, prodr. 451 (1810) GENTIANA, Tournefort, inst. 80, t. 40 (1700), from Dioscorides.

G. saxosa, Forster in Svensk. Kongl. Vet. Ac. Handl. 183, t. 5(1777) - S.A. T. V. N.S.W. - - B.fl.IV,373 M.fr. VI, 136,255;IX,165.

M.fr. VI, 136 .

M.fr.IX, 163 .

M. fr. VI, 137.

M.fr.IV,127;VI,137;IX,

M.fr. VI, 137, 254 .

M.fr.VI, 139;IX., 165.

M.fr. VI, 137.

M.fr.VI, 137.

fr. VI, 137;IX, 164

M.fr.VI, 142 .

M.fr.VI, 140.

M.fr.VI, 141 .

M.fr.VI, 141 .

Mr.II, $21 ; \mathrm{VI}, 139$

M.fr.VI, 140 .

M.fr. VI, 138 .

M.fr.VI, 137.

M.fr.VI, I 38 .

M.fr. VII, 136;IX, 165 . M.fr.VI,136.

M.fr.VI, 136;IX, 165;XI,

M.fr. VI, 136;IX, 165 .

LOGA NIACEAE.

R. Brown in App. Flind, Voy. 564 (1814).

STRYCHNOS, Linné, syst. nat. 8 (1735); Linné, spec. plant. 189 (1753).

S. lucida, R. Brown, prodr, 469 (1810) ‥ $\quad \ldots \quad \ldots \quad-\quad-\quad-\quad$ - Q. N.A. B.fl.IV,369

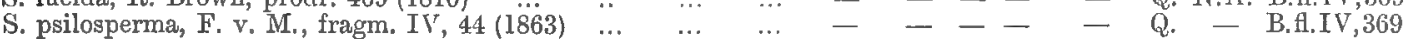

FAGRAFA, Thumberg in Kongl. Svensk. Vetensk. Acad. Handl. 125 (1782).

F. racemosa, Jack in Roxb. Fl. Ind, ed. Carey. II, 35 (1824) - - - - - N.A. B.H.IV,367 M.fr.II, 137.

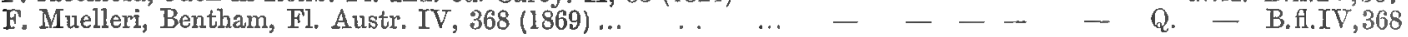
MITREOLA, Linné, hort. Cliffort. 492 (1737).

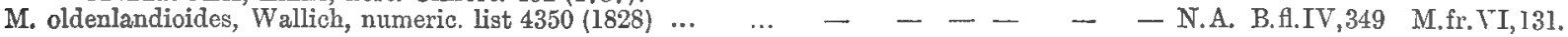
MITRASACME, Labillardière, Nov. Holl. pl. spec. I, 36, t. 49 (1804). (Mitragyne.)

M. Archeri, J. Hooker, Fl. Tasman. II, $368(1860) \quad \ldots \quad \ldots-\ldots-$ - T. - - - - B.fl.IV,351

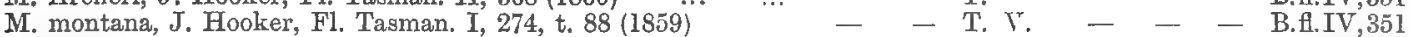

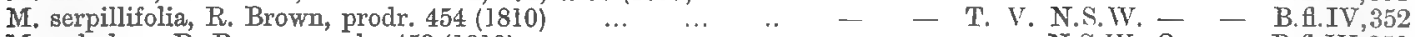

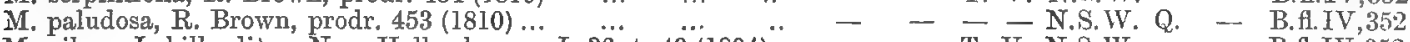

M. pilosa, Labillardière, Nov. Holl. pl. spec. I, 36 , t. $49 \ddot{9}(1804)=\ldots \quad-\quad$ T. V. N.S.W. - - - B.f.IV,352

M. alsinoides, R. Brown, prodr. $453(1810) \ldots \quad$ : $\quad \ldots \quad \ldots \quad-\quad-\quad-\quad-\quad$ N.S.W. Q. $\quad$ - B.fl.IV,353

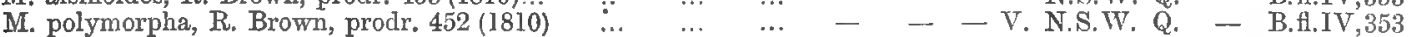

M. longiflora, F. v. M. in Benth. Fl. Austr. IV, 353 (1869)

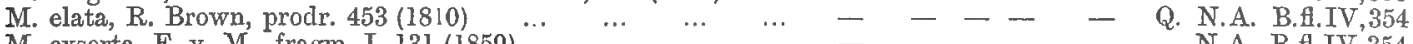

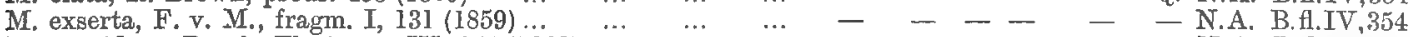

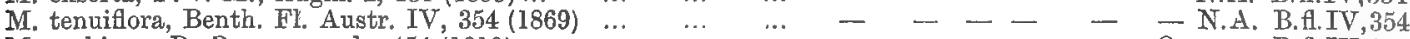

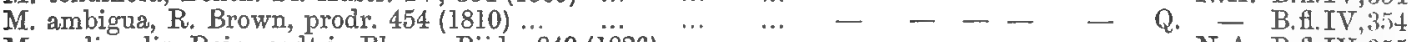

M. nudicaulis, Reinwardt in Blume, Bijdr. 849 (1826) $\ldots \ldots \ldots \ldots-\ldots-\ldots$

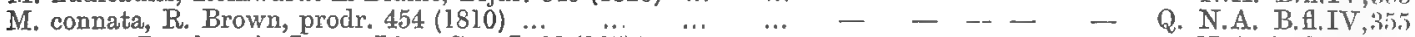

M. laevis, Bentham in Journ. Linn. Soc. I, $03(1857) \quad \ldots \quad \ldots \quad-\quad-\quad-\quad-\quad-\quad-$ N.A. B.f.IV.3..

M. Indica, Wight, icon. pl. Ind. or. IV, t. 1601 (1850) $\ldots . \ldots \ldots-\ldots-$ - $\quad$ - N.S. W. Q. N.A. B.fl.IV,356

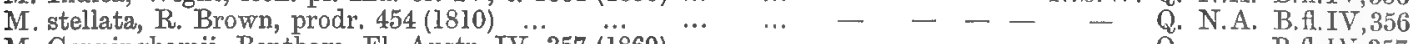

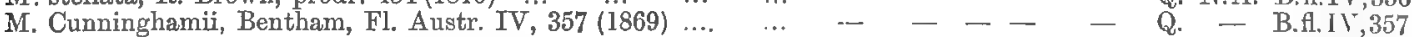

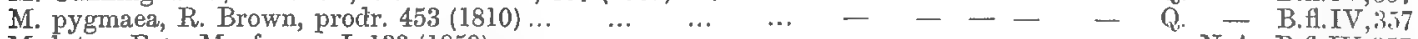

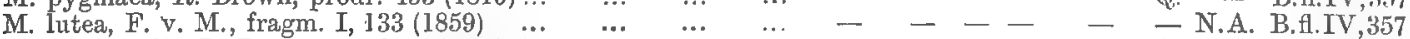

M. multicaulis, R. Brown, prodr. $453(1810) \quad \ldots \quad \ldots \quad \ldots \quad-\quad-\quad-\quad-\quad-\quad$ Q. N.A. B.Al.IV,357

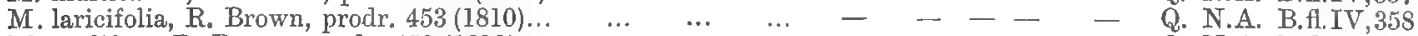

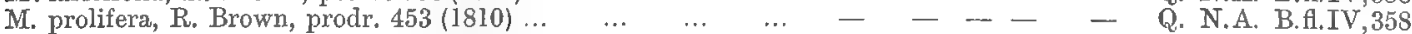

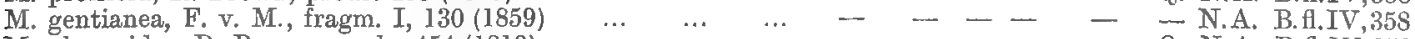

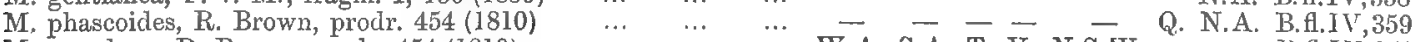

M. paradoxa, R. Brown, prodr. $454(1810) \ldots \quad \ldots \quad \ldots . \quad \ldots \quad$ W.A. S.A. T. V. N.S.W. - $\quad$ - B.fl.IV,359

M. distylis, F. v. M. in Transact. phil. Soc. Vict. I, 20 (1854) ... - - T. V. - - - B.fl.IV,359 GENIOSTOMA, R. \& G. Forster, char. gen. 23, t. 12 (1776).

G. Australianum, F. v. M., fragm. V, 19 (1865)

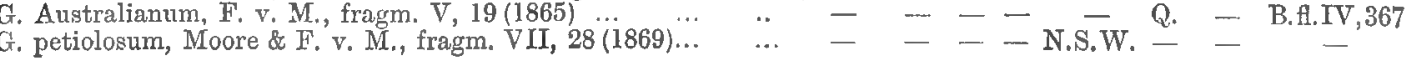

M.fr. VI, 131 .

M.fr.I, 134.

M.fr. VI, 131 .

M.fr. TI, 131 .

M.fr.I, 132.

M.fr.I, 131.

M.fr.I, 131 .

M.fr.I, 133.

M.fr.IV, 170.

M. fr. VI, 131 .

M.fr.I, 130.

M.fr. VI, 131

M.fr. VI, 131 .

M.fr. V, 19 .

M.fr.IX, 193. 
LOGANTA, R. Brown, prodr. 454 (1810). (Euosma 1808, restorable.)

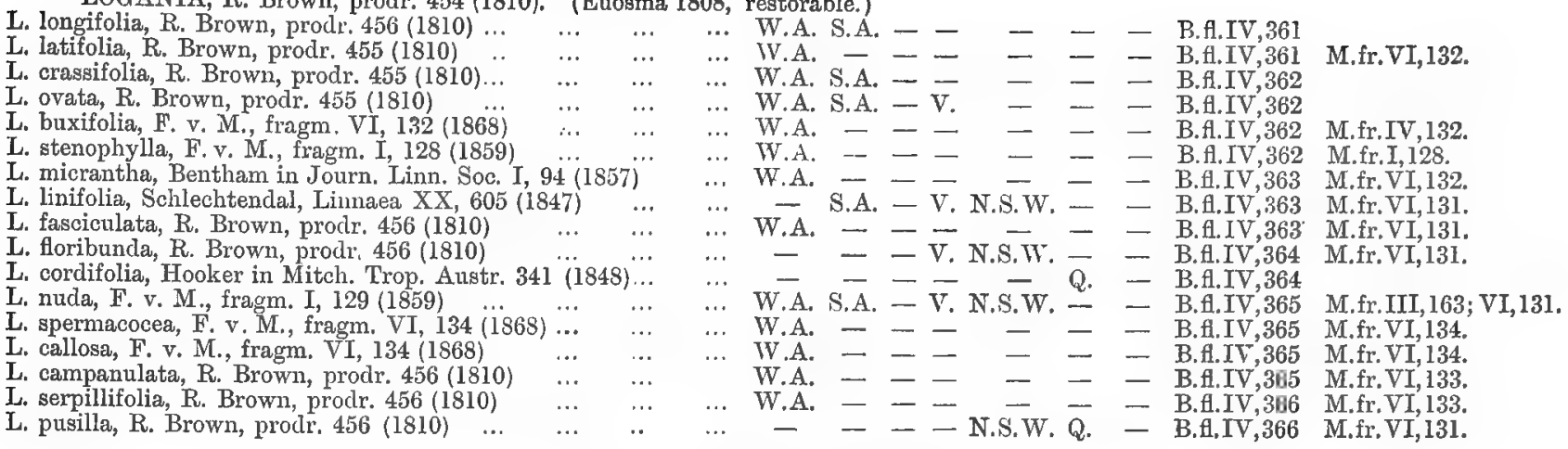

\section{PLANTAGINEAE.}

A. L. de Jussieu, gen. plant. 89 (1789).

PLANTAGO, Tournefort, inst. 126, t. 48 (1700), from l'Ecluse (1576).

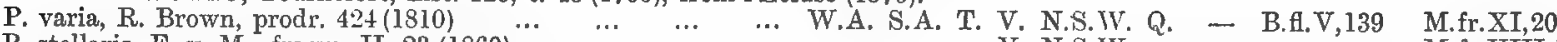

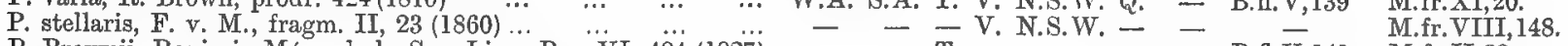

P. Brownii, Rapin in Mém. de la Soc. Linn. Par. VI, $484(1827)-1-$ T. - - - - B.f.V, I41 M.fr.II,23.

P. Gunnit, J. Hooker in Lond, Journ. V, 446, t. $13(1846) \ldots \ldots-$ - - T. V. N.S. IV. - - - B., V.,142 M.fr,VIII,148;XI,134.

\section{PRIMULACEAE.}

Ventenat, Tabl, du régn. végét. II, 285 (1799).

ANAGALLIS, Tournefort, inst. 142, t. $59(1700)$, from Hippocrates and Dioscorides. (Centunculus, Micropyxis.)

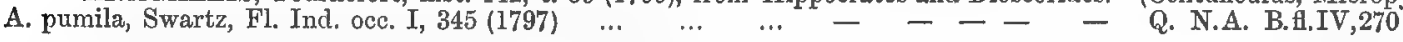

LYSIMACHIA, Tournefort, inst. 141, t. $59(1700)$, from Dioscorides.

L. salicifolia, F. v. M, in Benth. Fl. Austr. IV, 269 (1869) ‥ - - - V. N.S.W. - - B.fl.IV,269

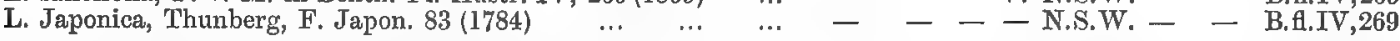

SAMOLUS, Tournefort, inst. 143, t, 60 (1700). (Sheffieldia.)

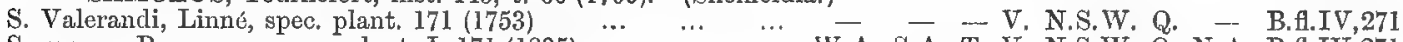

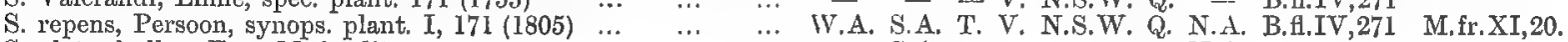

S. platyphyllus, F. T. M. inedit.

$$
\text { … W. S.A. - - - - N.A. - }
$$

\section{MYRSINACEAE.}

R. Brown, prodr. 532 (1810). (Primulacearum subordo.)

MAESA, Forskael, Fl. Aegypt. Arab. 66 (1775). (Baeobotrys.)

M. dependens, F. v. M., fragm. V, $107(1865) \quad \ldots \quad \ldots \quad \ldots \quad \ldots \quad-\quad-\quad-\quad-\quad-\quad$ Q. $\quad-\quad$ B.fl.IV,273 M.fr.V,107.

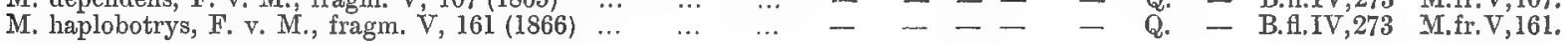

SAMARA, Linne, mantiss. II, 144 (1771). (Choripetalum.)

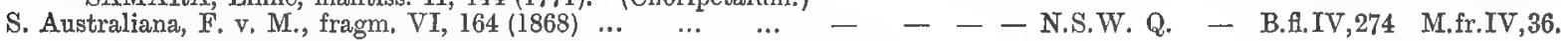

MYRSINE, Linné, syst. nat. 8 (1735); Linné, gen. plant. 54 (1737).

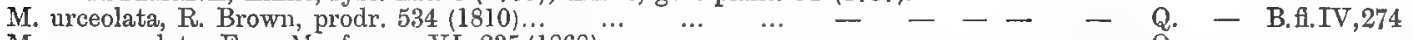

M. campanulata, F. v. MI., fragm. VI, $235(1868) \ldots$
M. crassifolia, R. Brown, prodr. $534(1810)$

M.fr.VI,235. $\quad[48$.

M. platystigma, F. v. M., fragm. VIII, $48(1873)$
M. variabilis, R. Brown, prodr. $534(1810) \ldots$
.

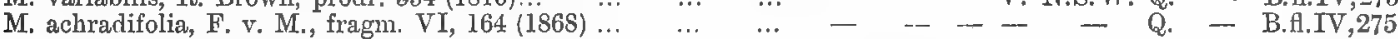

M.fr. VIII, 48 .

ARDISIA, Swartz, nov, gen. et spec. 3 et 48 (1788).

A. Pseudo-Jambosa, F. v. M., fragm. IV, 81 (1864) $\ldots . \quad \ldots \quad-\quad-\quad-\quad-$ N.S.W. Q. $\quad-\quad$ B.f.IV,276

A. breviperata F, M

AEGICERAS, Gaertner, de fruet. I, 216, t. 46 (1788).

A. majus, Gaertner, de fruct. I, 216, t. $46(1788) \ldots \quad \ldots \quad \ldots \quad-\quad-\quad-\quad$ N.S. W. Q. N.A. B.A.IV,277

M.fr.VI, 164 .

\section{SAPOTACEAE.}

A. L. de Jussieu, gen. 151 (1789), from B. de Jussieu (1759).

NIEMEYERA, F. v. M., fragm. VII, 114 (1870). (Chrysophyllum partiy.)

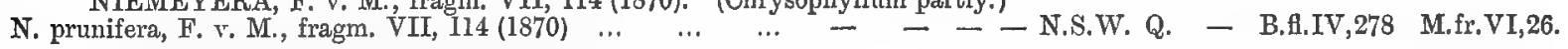

AMORPHOSPERMUM, F. v. M., fragm. VII, 112 (1870).

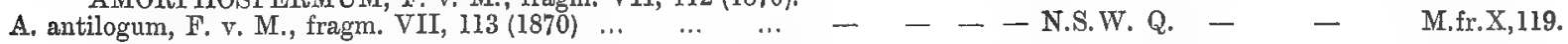

LUCUMA, Molina, Saggio, 186 (1782). (Sersalisia.)
LU - - - - - - Q. N.A. B.f.IV,279 M.fr.VII,112.

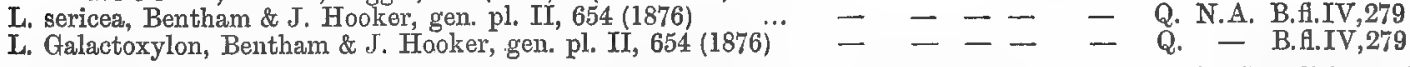

SIDEROXYLON, Dillenius, hort. Eltham. 357, t. 265 (1732). (Achras partly, Sapota partly, Sersalisia partly).

S. Arnhemicum, Bentham \& J. Hooker, gen. pl. II, 655 (1876)... - - - - - - N.A. B.f.IV,280

S. Pohlmanianum, Bentham \& J. Hooker, gen. pl. II, 655 (1876) - - - - - Q. - B.fl.IV,281

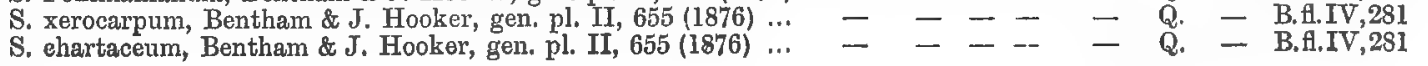

M.fr. V,184. 
S. Richardi, F, r, M.; Sersalisia laurifolia, A. Rich. sert. Astrol. 84 t. 13 (1834)

S. australe, Bentham \& J. Hooker, gen. pl, II, 655 (1876) $\ldots$
S. Brownii, F.v. M. ; Sersalisia obovata, R. Brown, prodr. $530(1010)$

S. myrsinoides, Bentham \& J. Hooker, gen. pl. II, 655 (1876) ...

$\mathrm{S}$ euphlebium; Achras, F. v. M., fragm. VII (1870)

S. Brownlessianum, F. v. M., fragm. VIII, 111 (1870) $\ldots . \quad \ldots$

S. Howeanum; Achras, F. v. M., fragm. IX, $72(1875) \ldots . \ldots$

S. costatum; Achras, Endlicher, prodr, fl. ins. Norf. 49 (1833) ... HORMOGYNE, A. De Candolle, prodr. VIII, 176 (1844).

H. cotinifolia, A. De Candolle, prodr. VIII, 176 (1844) ... MIMUSOPS, Linné, Fl. Zeil. 57 (1747).

M. parvifolia, R. Brown, prodr. 531 (1810)...

M. Browniana, Bentham, Fl. Austr. IV, 285 (1869)

DIOSPYROS, Linné, gen. plant. 143 (1737). (Cargillia.)
D. cordifolia, Roxburgh, pl. Corom. I, 38, t. 50 (1795) ...

D. hebecarpa, Cunningham in Benth. pl. Austr. IV, 286 (1869)

D. maritima, Blume, Bijdrag. 669 (1825)

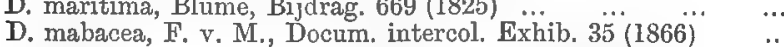

D. pentamera, F. v. M. \& Woolls, Doc. intercol. Exhib. $35(1866)-$ - - - N.S.W. Q. - B.f.IV,288 MABA, R. \& G. Forster, char. gen. 121, t. 61 (1776).

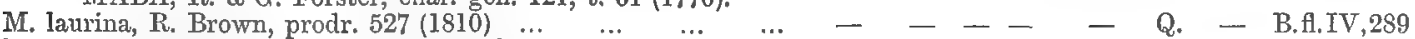

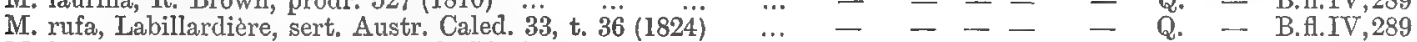
M. hemicycloides, F. v. M. in Benth. Fl. Austr. IV, $290(1869) \quad-\quad-\quad-\quad-\quad$ Q. - B.fl.IV,290 M. fasciculosa, F. v. M., fragm. V, 163 (1866) ... ... .. - - - - N.S.W. Q. - B.fl.IV,290

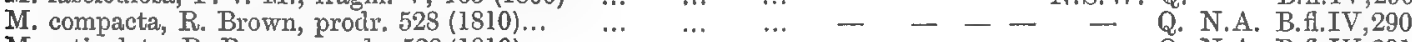

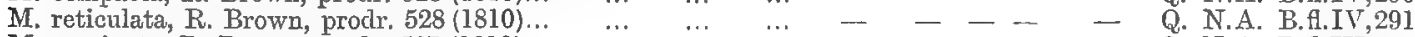

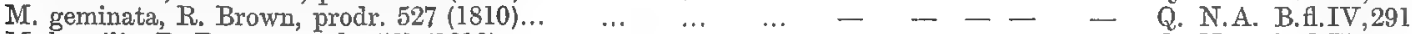

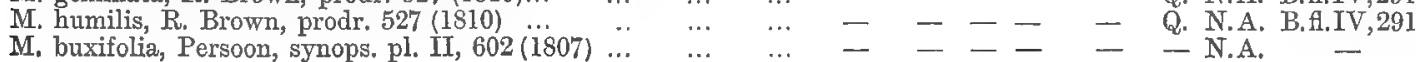

STYRACEAE.

L. C. Richard, Analyse du fruit. 48 (1808).

SYMPLOCOS, N. J. Jacquin, enum. plant. Carib. 24 (1760).

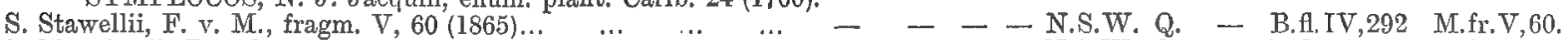

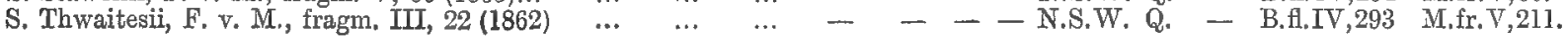

\section{JASMINEAE.}

A. L. de Jussieu, gen, 104 (1789), from Necker (1770).

JASMINUM, Tournefort, inst. 597, t. 368 (1700), from l'Ecluse (1611).

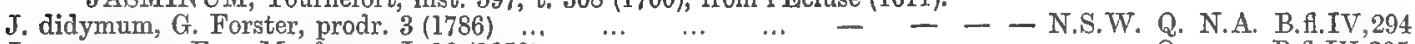
J. racemosum, F. v. M., fragm. I, $19(1858) \quad \ldots . \quad \ldots \quad \ldots . . \quad-\quad-\quad-\quad-\quad-\quad$ Q. - B.fl.IV,295

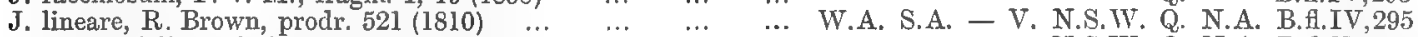

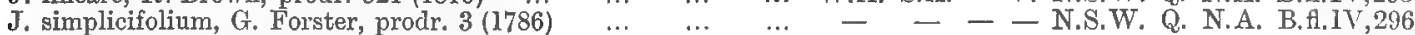

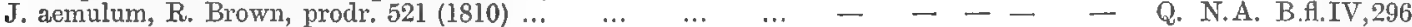

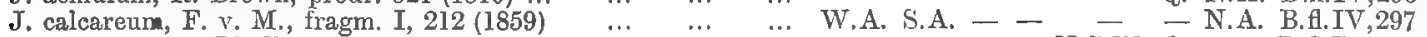

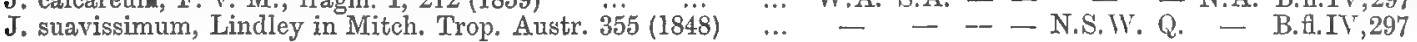

OLEA, Tournefort, inst. 598, t. 370 (1700), from Plinius.

O. paniculata, R. Brown, prodr. 523 (1810)

O. apetala, Vahl, symbol. III, 3 (1794) ...

$$
\cdots \quad \cdots \quad \cdots
$$

-. - - - - N.S.TV. Q. - B.A.IV,297

LIGUSTRUM, Tournefort, inst. 596, t. 367 (1700), from C. Bauhin (1623).

L. Australianum, F. v. M., fragm. V, $20(1865) \quad \ldots \quad \ldots \quad \ldots \quad-\quad-\quad-$

NOTELAEA, Ventenat, Choix des pl, dans le jard. de Cels, t. 25 (1803).

N. ovata, R. Brown, prodr. 524 (1810)

N. longifolia, Ventenat, Choix t. 25 (1803)...

N. punctata, R. Brown, prodr. $524(1810) \ldots$

N. microcarpa, R. Brown, prodr. 524 (1810)

N. ligustrina, Ventenat, Choix t. 25 (1803)

N. linearis, Bentham, Fl. Austr. IV, 300 (1869) .

$\begin{array}{cccc}\ldots & \ldots & \ldots & - \\ \ldots & \ldots & \ldots & - \\ \ldots & \ldots & \ldots & - \\ \ldots & \ldots & \ldots & - \\ \ldots & \ldots & \ldots & - \\ \ldots & \ldots & \ldots & -\end{array}$

$=$

- - N.S.W. Q.

- V. N.S.W. Q.

- - N.S.W. Q.

T. V. N.S.W. -

- - N.S.W. -

MAYEPEA, Aublet, Hist. des pl. de la Guian. I, 81, t. 31 (1775). (Chionanthus partly, Linociera partly, Ceranthes.)

M. ramiflora, F.v.M.; Chion. Roxb. Fl. Ind. I, 106 ed. Carey(1820) - - - - - Q. - B.H.IV,301 M.fr.IV,83.

M. picrophloia; Chionanthus, F. V. M., fragm. III, 139, t.24(1863) - - - - - Q. - B.fl.IV,301 M.fr.IV, 176.

M. axillaris, F. v. M.; Chionanthus, R. Brown, prodr. $523(1810)-$ - - - - Q. N,A. B.fl.IV,301

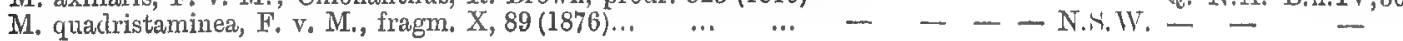

\section{APOCYNEAE.}

A. L. de Jussieu, gen, plant. 143 (1789).

CHILOCARPUS, Blume, catal, hort. Buitenz. (1823).

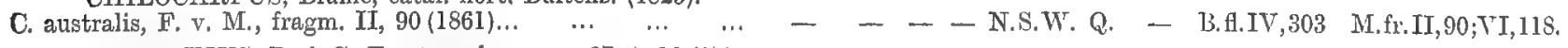

MELODINUS, R. \& G. Forster, char. gen. 37, t. $19(1776)$.

M. acutiflorus, F. v. M. in Transact. phil. Soc. Vict. II, 71 (1857)

M. Guilfoylei, F. v. M., fragm. VI, 118 (1868) .

M. Baueri, Endlicher, prodr. pl. Norfolk. 59 (1833)

$\cdots \quad \cdots \quad-$

- - - Q - B.fl.IV,304

- - - N. Q. Q B.H.IV,304

M.fr. TI,118.

M.fr.IX, 169 .
$[43$.

, 150;VI, 87; VIII

I.fr. VI, 87; VIII, 43 .

M. Ir.VI, $87 ; \mathrm{VIII}, 43$.

M.fr.I, 212; VI, 87, VIII,

M.fr. VIII, 43.

M.fr. TIII, $43 ;$ IX, 169

M. fr. VIII, 41 , 
CARISSA, Linné, mantiss, pl. 7 (1767).

C. Iaxiflora, Bentham, Fl. Austr. IV, 305 (1869) $\ldots \quad \ldots \quad \ldots \quad-\quad-\quad-\quad-\quad-\quad$ Q. $\quad-\quad$ B.fl.IV,305

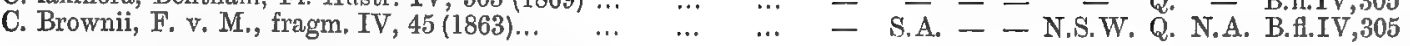

M.fr.IV,45. CERBERA, Linné, gen. plant. 62 (1737).

C. Odollam, Gaertner, de fruct. 193, t. 124 (1791)... ALYXIA, Banks in R. Brown, prodr. 469 (1810). (Gynopogon.)

A. buxifolia, R. Brown, prodr. $470(1810) \ldots \ldots \ldots$........ W. A.

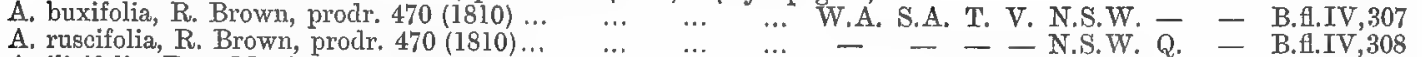

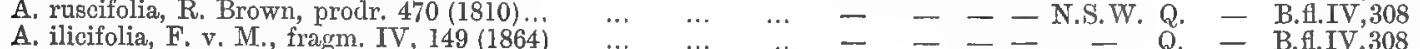

A. squamulosa, C. Moore \& F. v. M., fragm. VIII, 47 (1873) $\ldots$
A. obtusifolia, R. Brown, prodr. 470 (1810)

A. Gynopogon, Roemer \& Schultes, syst. veg. IV, 440 (1819) $\ldots$

A. spicata, R. Brown, prodr, $470(\mathbf{1 8 1 0}) \ldots$
A. Lindii, F, v. M., fragm. VIII, $46(1873)$

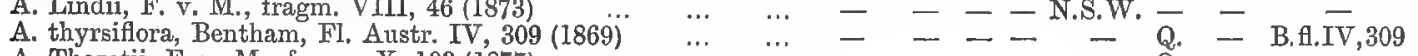

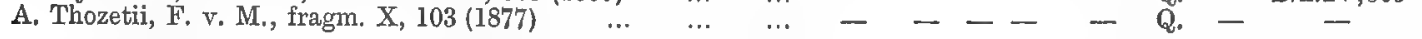
OCHROSIA, A. L. de Jussien, gen. 143 (1789). (Lactaria.)

O. elliptica, Labillardière, sert. Austr. Caled. 27, t. 30(1824) ... - - - - - Q. - B.fl.IV,310

O. Moorei, F. v. M, in Benth. Fl, Austr. IV, $310(1869) \ldots \quad \ldots-$-.. - - - N.S.W. Q. - B.H.IV,310

O. Kilneri, F. v. M., fragm. VII, $129(1871)$... $\ldots \ldots \ldots$ NOTONERIUM, Bentham in B. \& J. H. gen. II, 698 (1876).

N. Gossei, Bentham in B. \& J. H. gen. pl, $698(1876)$... … - S.A. TABERNAEMONTANA, Plumier, nov. pl. Amer. gen, 18, t. 30 (1703).

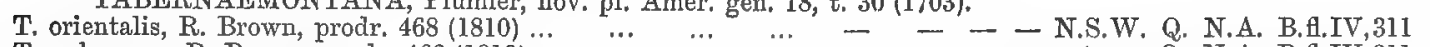

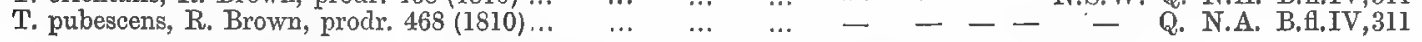
VINCA, Rivinus in Rupp. Fl. Jenens. 27 (1818), from Plinius. (Perhaps immigrated.)

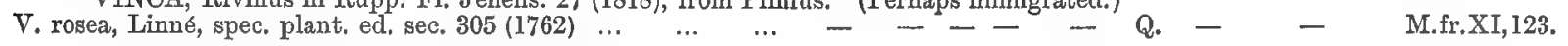
ALSTONIA, R. Brown in Mem. Wern. Soc. I, 75 (1809).

A. scholaris, R. Brown in Mem. Wern. Soc. I, $75(1809) \quad \cdots \quad-\quad-\quad-\quad-\quad$ Q. $\quad$ - B.fl.IV,312 A. verticillosa, F. v. M., fragm. VI, $116(1868) \ldots \quad \ldots \quad \ldots \quad-\ldots \quad-\quad-\quad-\quad-\quad$ Q. N.A. B.fl.IV,313

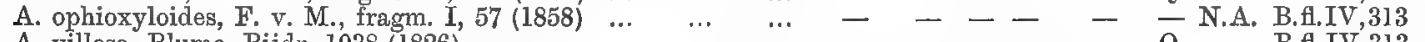

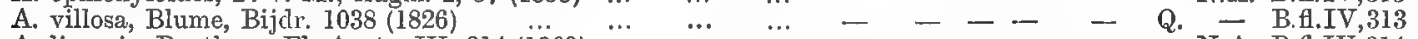

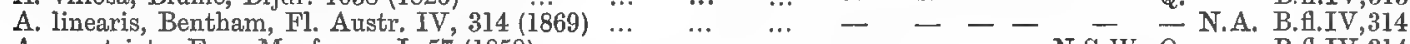

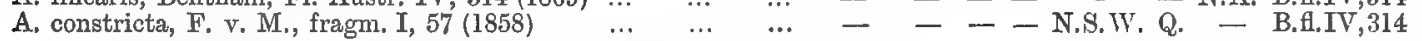
ICHNOCARPUS, R. Brown in Mem. Werm. Soc. I, 75 (1809).

I. frutescens, R. Brown in Mem. Wern. Soc. I, 75 (1809) … - - - - - Q. N.A. B.Hl.IV,315 WRIGHTIA, R. Brown in Mem. Wern. Soc. I, 75 (1809). (Balfouria.)

W. pubescens, R. Brown, prodr. $467(1810)$
W. saligna, F. Y. M. in Benth. Fl. Austr. IV, $316(1869) \quad \cdots \cdots-1-$

W. Cunninghamii, Bentham, Fl. Austr. IV, 317 (1869) ... $\ldots$ W.A. - - - - - - - B.fl.IV,317 PARSONSIA, R. Brown in Mem. Wern. Soc, I, 64 (1809).

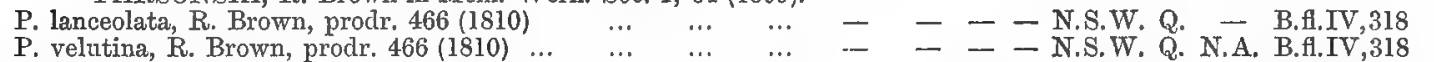

P. Leichhardti, F. v. M., fragm. VI, 128 (1868) … - - - - N.S.W. Q. N.A. B.A.IV,318

P. ventricosa, F. v. M. in Transact. phil. Inst. Vict. II, 71 (1857) - - - - N.S.W. Q. - B.fl.IV,319 LYONSIA, R. Brown in Mem. Wern. Soc. I, 66 (1809).

L. lilacina, F, v. M. in Bentham Fl. Austr. IV, 321 (1869)

L. induplicata, F. v. M. in Benth. Fl. Austr. IV, 321 (1869) $\ldots$

L. stramines $\mathrm{R}$ Brown prodr. 466 (1810).

L. reticulata, F. v. M., Rep. Burdek. Exped. 16 (1860)... $\quad \ldots$

L. diaphanophleba, F. v. M. in Benth. Fl. Austr. IV, 322 (1869)

L. Langiana, F. v. M., fragm. VI, 128 (1868)

L. largiflorens, F. v. M. in Benth. Fl. Austr. IV, 322 (1869) $\quad \ldots$

L. latifolia, Bentham, Fl. Austr. IV, 323 (1869)

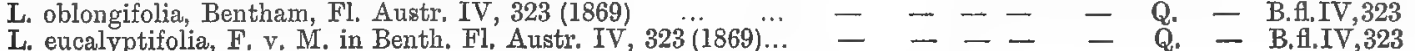

M.fr.VI, 117.

M.fr.VI, 116.

M.fr.VI, 117.

M.fr.VI, 117 .

M.fr.III, $163 ; \mathrm{IV}, 170 ; \mathrm{VI}$,

M.fr.VI, 118.

M.fr, V,161;VI, 118.

M.fr.VI, 126.

M.fr.VI, 130.

M.fr.VI, 128.

M.fr.VI, 130 .

- $\quad-\quad-\quad$ - N.S.W. Q. - - B.fl.TV,321

- - T. T. N.S.W. - - B.f.IV,321

- - - - N.S.W.Q. - B.fl.IV,321

M.fr. VI, 127.

M.fr. VI, 129 .

M.fr.VI, 129 .

M.fr. VI, 129.

M.fr.II,158;VI, 130.

M.fr.VI, 128.

M.fr.II,159;VI,130

\section{ASCLEPIADEAE.}

N. J. Jacquin, Misc. Austr. I, 35 (1778).

GYMNANTHERA, R. Brown in Mem. Wern. Soc. I, 58 (1809).

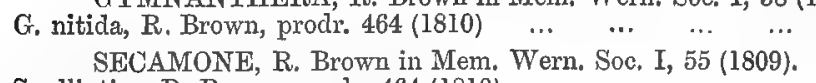

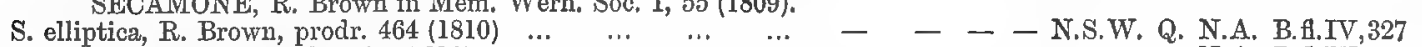

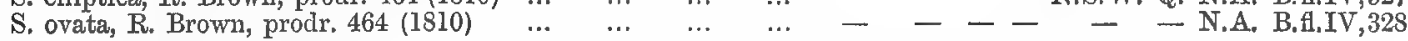

VINCETOXICUM, Dodoens, stirp. hist. pemptades, 704 (1583). (Oxystelma partly, Cynoctonum.)

V. ovatum, Bentham, Fl. Austr. IV, 330 (1869) …

V. elegans, Bentham, Fl. Austr. IV, 330 (1869) …

V. carnosum, Bentham, Fl. Austr. IV, 331 (1869)

V. leptolepis, Bentham, Fl. Austr. IV, 331 (1869)

CYNANCHUM, Linné, gen. plant. 63 (1737).

C. erubescens, R. Browi, prodr. $463(1810)$

C. floribundum, $\mathbf{R}$. Brown, prodr. 463 (1810)

$\begin{array}{lll}\ldots & \ldots & - \\ \cdots & \ldots & -\end{array}$

= - - N.S.W. Q. N.A. B. B.f.IV,330

$\cdots \quad \cdots \quad=-$ - $\quad \cdots$ - N.S.W.Q. N.A. B.A.IV,331

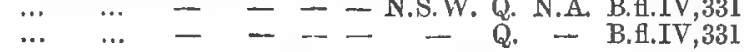

M.fr.V, 160 .

C. pedunculatum, R. Brown, prodr. 463 (1810)

C. puberulum, F. r. M. in Benth. Fl. Austr. IV, 3333 (1869)

... $\quad \cdots \quad-$

S.A. $=-$

- Q. - B.f.IV, 332

.. $=-$ S.A. - - - Q. N.A. B.fl.IV,332

M.fr. $V, 160$

M.fr. V, 160 .

M.fr.V,161.

N.A. B.fl.IV, 333 
SARCOSTEMMA, R. Brown in Mem. Wern. Soc. I, 50 (1809).

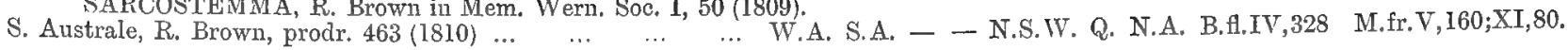

DAEMIA, R. Brown in Mem. Wern. Soc. I, 50 (1809). (Pentatropis, Rhyncharrhena.)

D. linearis, F. v. M.; Pentatropis, Decaisne in De Cand., prodr. VIII, 536 (1833)

D. atropurpuree,

D. Kempeana, F. v. M. in Wing's S. Sc. Record, Aug. (1882) ...

GYMNEMA, R. Brown in Mem. Wern. Soc. I, 33 (1809).

G sylvestre $\mathrm{R}$. Brown in Mem. Wem Soc. I 33 (1809)

... - - - - - N.A. B.f.IV,343

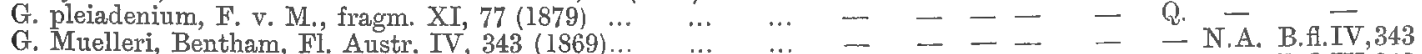

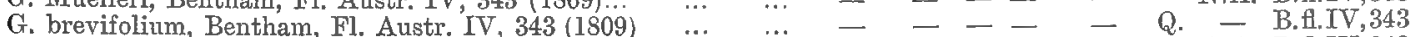

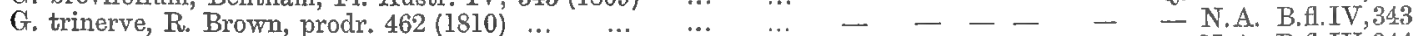

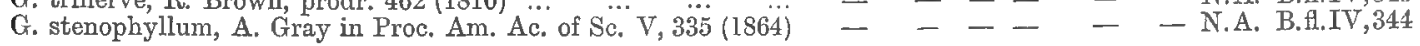
GONGRONEMA, Decaisne in De Candolle, prodr. VIII, 624 (1844).

G. micradenia, Bentham \& J. Hooker, gen. plant. II, 770 (1876) TYLOPHORA, R. Brown in Mem. Wern, Soc, I, 28 (1809).

T. erecta, F. v. M. in Benth. Fl. Austr. IV, 334 (1869) ...

T. macrophylla, Bentham, Fl. Austr. IV, 334 (1869)

T. grandillora, R. Brown, prodr. 460 (1810

T. floribunda, Bentham, Fl. Austr. IV, 335 (1869)

T. calcarata, Bentham, Fl. Austr. IV, 335 (1869)...

T. barbata, R. Brown, prodr. 460 (1810)

T. Woollsii, Bentham, Fl, Austr. IV, 335 (1869) ...

T. paniculata, R. Brown, prodr. 460 (1810)

T, enervis, F. v. M., fragm. IX, 70 (1875) $\quad \cdots \quad \ldots . \quad \ldots$

T. flexuosa, R. Brown, prodr. 460 (1810) ... $\quad \ldots \quad \ldots$

T. biglandulosa, A. Gray in Proc. of Am. acal. sc. vol. $\dddot{V}(1864)$ MARSDENIA, R. Brown in Mem. Wern. Soc. I, 28 (1809).

M. cinerascens, R. Brown, prodr. 461 (1810)

M. rhyncholepis, F. v. M., fragm. XI, 78 (1879) “.

M. flavescens, Cunningham in Bot. Mag., t. 3289 (1833)

M. cymulosa, Bentham, Fl. Austr. IV, 338 (1869)

$M$. velutina, $R$. Brown, prodr. 461 (1810).

M. Hullsii, F. v. M. in Benth. Fl. Austr. IV 333 (1869)

M. araujacea, F. v. M., fragm. VI, 135 (1868) ... ...

M. tubulosa, F. v. M., fragm. IX, 71 (1875)

M. rostrata, R. Brown, prodr. 461 (1810)

M. Fraseri, Bentham, FI. Austr. IV, 339 (1869)

M. Iongiloba, Bentham, FI. Austr. IV, 340 (1869)

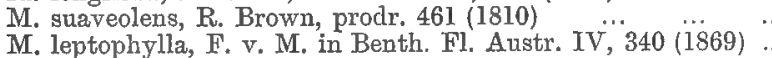

M. Leichhardtiana, F. v. M., fragm. V, 160 (1866)

M. viridiflora, R. Brown, prodr. 461 (1810)

M. coronata, Bentham, Fl. Austr. IV, 341 (1810)...

M. microlepis, Bentham, Fl. Austr. IV, 342 (1869)

(1809).

$$
\text { 三 }
$$

- - - Q Q. - B.fl.IV,334

- - - - T. N.A. B.fl.IV,334

- - - N.S.W. Q. - B.H.IV,334

- - - N.S.W.Q. - Q. Q.IV,335

- - V. N.S.W. - - B.fl.IV,335

- - - N.S.W. - - B.fl.IV,335

- - - N.S.W. - - B.f.IV,336

- - - N.S.IV. - N.A. B.fl.IV, 336

- - - - N.S.W. - N.A. B.fl.IV,336

(Leichhardtia.) - - N.A. B.A.IV,337

- - - - - - Q. N.A. B.fl.IV,337

M.fr.XI,78.

M.fr. V, 160 .

- - - - - Q. N.A. B.fl.IV,338

- _ - - N.A. B.H.IV,338

THOZETIA, F. v. M. in Benth. Fl. Austr. IV, 347 (1869),

T. racemosa, F. v. M. in Benth. Fl. Austr. IV, $347(1869) \quad \cdots \quad-\quad-\quad-\quad-\quad-\quad$ Q. $\quad-\quad$ B.A.IV,347

HOYA, R. Brown in Mem. Wern. Soc. I, 26 (1809).

H. carnosa, R. Brown in Mem. Wern. Soc. I, 26 (1809)...

H. australis, R. Brown in Transact. hort. Soc. VII, 28 (1826) ...

H. Nicholsoniae, F. v. M., fragm. V, $159(1866) \ldots \quad \ldots \quad \ldots$

DISCHIDIA, R. Brown in Mem. Wern. Soc. I, 32 (1809).

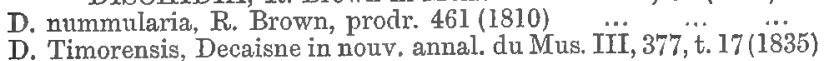
MICROSTEMMA, R. Brown in Mem. Wern. Soc. I, 25 (1809).

M. tuberosum, R. Brown, prodr. $459(1810) \quad \ldots \quad$.. $\quad \ldots \quad$ -

M. glabriflorum, F. v. M., fragm. I, 58 (1858)

CEROPEGIA, Linné, gen, plant. 65 (1737).

C. Cumingiana, Decaisne in De Caand, prodr. VIII, 643 (1844)

\section{CONVOLVULACEAE.}

A. L. de Jussieu, gen. plant. 132 (1789), from B. de Jussieu (1759).

ERYCIBE, Roxburgh, pl. Corom. III, 31, t. 159 (1798).

E. paniculata, Roxburgh, pl. Corom. III, 31, t. 159 (1798)

LEPISTEMON, Blume, Bijdrag. 722 (1825).

L. urceolatus, F. v. M.; L. Fitzalani, F. v. M., fragm. X, $111(1877)-\quad-\quad-\quad-\quad$ Q. - B.fl.IV,427 M.fr.X.111.

IPOMOEA, Linné, syst. nat. 8 (1735); Linne, gen. plant. 47 (]737). (Pharbitis, Batatas, Calonyction, Aniscia, Skimnera.)

I. paniculata, R. Brown, prodr. $486(1810)$

I. Davenporti, F. v. M., fragm. VI, 97 (1868)

I. bona nox, Linné, spec. plant. edit. secund. 228 (1762)

I. palmata, Forskael, Fl, Aegypt. Arabic. 43 (1775)

I. quinata, R. Brown, prodr. 486 (1810)

I. diversifolia, R. Brown, prodr. 487 (1810)
- - - Q. - B.fl.IV,339

- - - V. N.S.W. N. $\bar{Q}$ - - B.fl.IV,339

- - V. N.S.W.Q. - B.fl.IV,339

- - - N.S.W. Q. - B.fl.IV,339

- - - N.S.W. - - B.fl.IV,340

- - - - N.N.W. $\overrightarrow{\text { Q. }}$ - B.H.IV, 340

W.A. S.A. - - V. N.S.W. Q. N.A. B.f.IV, 340

- - - - N.S.W. Q. N.A. B.fl.IV,341

$$
\begin{aligned}
& \text { - - - Q. N.A. B.fl.IV, 4lt iI.fr.VI,9S; X,111. } \\
& \text { S.A. - - - - D. N.A. B.A.IV,H15 M.fr.VIII, 17;X,111. }
\end{aligned}
$$

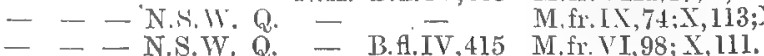

$$
\begin{aligned}
& \text { - - - N.S.W. Q. N.A. B.f.IV,415 M.fr.VI, 98; X,112. } \\
& \begin{array}{l}
-\quad-\quad \text { - Q. N.A. B.A.IV,415 M.fr.VI,98; } \\
-\quad \text { Q. N.A. B.f.IV,416 M.fL.X,112. }
\end{array}
\end{aligned}
$$

M.fr.VI,135.

M.fr. $V, 160 ; V I, 135$.

M.fr, XI, 79 .

M.fr. V, 160.

M.fr. V, 160.

M.fr. XI, so.

.r. $T, 159 ; 1 X, 70,190$.

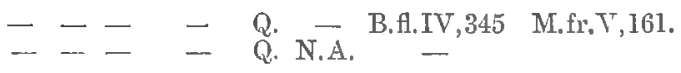

M.fr.I,5s.

M.fr. 1,5 S.

M.fr. V, 159 . 
I. dissecta, Willdenow, phytogr. 5, t. 2 (1794)

I. sinuata, Ortega, decad. nov. pl, hort. Matrit. $\ddot{\text { VII }} 8 \ddot{84}(1799)$

I. hederacea, N. J. Jacquin, Collectan. I, 124 (1786) ...

I. congesta, R. Brown, prodr. 485 (1810) ... ...

I. purpurea, Roth, Catalect. bot. I, 36 (1797)

I. peltata, Choisy, Convolvulac. orient. 70 (1834).

I. Calobra, Hill \& F. v. M., fragm. XI, 73 (1879)

I. alata, $R$. Brown, prodr, 484 (1810)

I. Turpethum, R. Brown, prodr. 485 (1810)

I. longiflora, R. Brown, procir. 484 (1810)

I. costata, F. v. M. in Benth, Fl. Austr. IV, 419 (1869)"

I. Pes Caprae, Roth, nov. spec. plant. 109

I. carnosâ, R. Brown, prodr. 485 (1810)

I. reptans, Poiret, Encycl. méth. suppl. III, 460 (ïs13)."

I. graminea, $\mathrm{R}$. Brown, prodr. 485 (1810) ...

I. velutina, R. Brown, prodr, 485 (1810) ...

I. abrupta, R. Brown, prodr. 485 (1810)

I. denticulata, Choisy in De Cand. prodr. IX $37 \ddot{9}$ (184n̈)

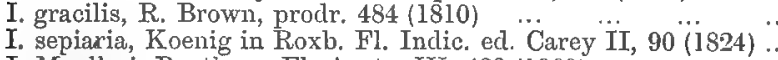

I. Muelleri, Bentham, Fl. Austr. IV, 423 (1869)

I. cymosa, Roemer \& Schultes, syst. veg. IV, 241 (1819)

I. linifolia, Blume, Bijdrag. 721 (1825)

I. chryseides, Ker in Bot. Regist. t. 270 (1818)

I. flava, F. v. M. in Benth. FI. Austr. IV, 424 (1869)

I. obscura, Ker in Bot. Regist. t. 239 (1817)

I. incisa, R. Brown, prodr. 486 (1810)

I. uniflora, Roemer \& Schultes, syst. IV, 247 (1819)

I. angustifolia, N. J. Jacquin, Collect. II, 367 (1788)

I. plebeja, R. Brown, prodr. 484 (1810)

I. eriocarpa, R. Brown, prodr. 484 (1810)

I. heterophylla, $\boldsymbol{R}$. Brown, prodr. 487 (1810)

I. erecta, R. Brown, prodr. 487 (1810)

CONVOLVULUS, Tonrnefort, inst. 82, t. 17 (1700).

C. erubescens, Sims, Bot. Magaz. t. 1067 (1808) ..

C. parviflorus, Vahl, symbol. bot. III, 29 (1794) ...

C. marginatus, Poiret, Encycl. méth. suppl. II (1811)

C. sepium, Linné, spec, plant. 153 (1753) .. POLYMERIA, R. Brown, prodr. 488 (1810).

P. marginata, Bentham, Fl. Austr. IV, 432 (1869)

P. longifolia, Lindley in Mitch. Trop. Austr. 398 (1848)..

P. angusta, F. v. M., fragm. VI, 100 (1868)

P. distigma, Bentham, Fİ. Austr. IV, 433 (1869) ...

P. occidentalis, F. $\checkmark$. M., inedit.

P. calycina, $\mathbf{R}$. Brown, prodr. $488(1810) \quad \ldots$.

P ambigua, R. Brown, prodr. 488 (1810) PORANA, Burmann, Fl. Ind. 51, t. 21 (1768).

P. sericea, F. v. M., fragm. VI, 100 (1868) BREWERIA, R. Brown, prodr. 487 (1810)

B. linearis, R. Brown, prodr. 488 (1810) $\ldots . \quad \ldots$

B. media, R. Brown, prodr. 488 (1810)

B. brevifolia, Benthan, F1. Austr. IV, 436 (1869)

B. rosea, F. v M., fragm. I, 233 (1858)

B. pannosa, R. Brown, prodr. 488 (1810) $\ldots .$. EV OLV ULUS, Linné, spec. plant. ed. sec. 391 (1763). E. linifolius, Linne, spec. plant. ed. sec. $392(1762) \quad \ldots \quad \ldots$
DICHONDRA, R. \& G. Forster, char. gen. 39, t. 20 (1776).

D. repens, R. \& G. Eorster, char. gen. 39, t. 20 (1776) ... CRESSA, Linné, Amoen. Acad. I, 121 (1747).

C. Cretica, Linné, spec. plant. 223 (1753) ... ... WILSONIA, R. Brown, prodr. 490 (1810).

W. humilis, R. Brown, prodr. 490 (1810)

W. rotundifolia. Hooker, icon. plant. t. 410 (1842)

W. Backhousi, J. Hooker in Lond. Journ. VI, 275 (1812) CUSCUTA, Tournefort, inst. app. 652, t. 422 (170

C. Chinensis, Lamarck, Encycl. méth. II, 229 (1786)

C. Chinensis, Lamarck, Encycl. méth. II, $229(1786)$
C. Australis, R Brown, prodr. 491 (1810)

C. Tasmanica, Engelmann in Transact. Acad. St. Louis I, 512(1860) - - T. T.

\section{SOLANACEAE.}

Haller, enum. stirp. Helv. praef. 34 (1742).
Q. N.A. B.f.IV, 416

Q. N.A. B.fl. $\overline{I V}, 416$

Q. N.A. B.A.IV, 416

Q. - B.fl.IV,417

Q. - B.f.IV, 418

Q. N.A. B.fl. $\overline{I V}, 418$

Q. N.A. B.A.IV, 418

Q. N.A. B.f IV, 418

- N.A. B.H.IV,419

Q. N.A. B.H.IV,419

- N.A. B.fl.TV, 420

Q. N.A. B.fl.IV, 420

Q. N.A. B.fl. IV, 421

- N.A. B.fl.TV, 421

Q. N.A. B fl.IV,421

Q. N.A. B.fl.IV,421

Q. N.A. B.fl. IV, 422

Q. N.A. B.fl.IV, 422

- N.A. B.fl.IV, 423

Q. - B.fl.IV, 423

Q. - B.fl.IV,423

Q. - B fl.IV,423

- N.A. B.A.IV, 424

Q. - B.fl.IV, 424

- N.A. B.f.IV,424

Q. - B.fl.IV, 425

Q. N.A. B.fl.IV, 425

Q. - B.fl.IV, 426

Q. N.A. B.H.IV, 426

Q. N.A. B.fl.IV, 426

- N.A. B.A.IV, 427

(Calystegia.)

… - - - N.S.W. Q. N- B. B.H.IV,429

.. - - - V. N.S.W. Q. - B.fl.IV,430 ... W.A. S.A. T. V. N.S.W. - - B.f.IV,430

․ - - - - - Q. - B.fl.IV,432

‥ - S.A. - - N.S.W. Q. - B.H.IV,432

. - - - - - - N.A. B.A.IV,432

W.A. - - - - - - B. B.I.IV,433

... - - - - N.S.T. Q. - B.fl.IV,433

.. - - - - - Q. N.A. B.fl.IV,433

reya.)

- - - - - - - N.A. B.H.IV, 435

- S.A. - - N.S.IV. Q. N.A. B.H.IV,436

- - - - - - N.A. B.fl.IV,436

- - - - Q. N.A. B.f.IV,436

W.A. S.A. - - N.S.W. Q. N.A. B.fl.IV, 437

W.A. S.A. T. V. N.S.W. Q. N.A. B.A.IV, 438

W.A. S.A. - V. N.S.W. Q. N.A. B.fl.IV, 437

W.A. S.A. T, V. N.S.W.

W.A. S.A. - V. N.S.W. -

B. H.TV, 439

B.fl.TV, 440

B. I.IV, 440

B.fl.IV, 441

B.Al IV,441

- B fl. IV,441
M.fr.VI,98; X,112.

M.fr.VIII, $17 ; X, 113$.

M.fr.VI,99;X, 112.

M.fr.VI, $99 ; X, 112$.

M.fr.VI, $99 ; X, 112$.

M.fr.XI,73,137.

M fr.X.112.

M,fr. VI,98.

M.fr.VI, 98;IX, 74; X, 112.

M.fr.X.112

M.fr. VI, $97 ; \mathrm{X}, 112$.

M.fr. VI, 97.

M.fr.X, 112 .

M.fr.I, $185 ; X, 112$.

M.fr. I, 185;X, 112.

M.fr.X, 112.

M.fr. VI,98.

M.fr.I, 186.

M.fr. VI,99.

M.fr. VI, $9^{7} ; \mathrm{X}, 112$

M.fr. VI, $99 ; X, 112$.

M.fr.VI,98;X, 11 .

M.fr. VI,98;X, 112 .

M.fr.VI, 98 .

M.fr.VI, 99;X,113.

M.fr. VI,99;X,113.

M.fr. VI, $99 ; X, 113$.

M.fr.VI, $99 ; \mathrm{X}, 113$.

M.fr. X, 114.

M.fr. VI, 100.

M.fr. VI, 100 .

M.fr.II,22.

II.fr. $\mathrm{X}, 113$.

M.fr.VI, 100;X, 113. M.fr.VI, 100;X, 113 .

M.fr. VI, 100;X,113.

M.fr. VI, 100;X, 113.

M.fr. VI, 100.

M.fr.VI, 101;X, 113.

M.fr.VI, 101 .

M.fr.VI, 101;VIII, 17 .

M.fr.X, 113.

M. fr. I, 100;X, 113.

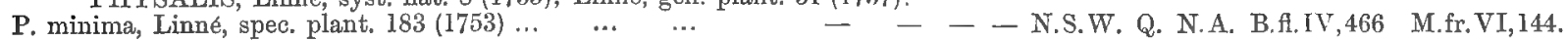
SOLANUM, Tournefort, inst. 148, t. $62(1700)$, from Celsus.

S. nigrum, Linne, spec. plant. 186 (1753) ... … … W.A. S.A. T. V. N.S.W. Q. N.A. B.fl.IV,446 M.fr.VI, 145.

S. vescum, F. v. M. in Transact. Vict. Inst. 69 (I855) $\ldots . . . \quad-\quad-$ T. V. N.S.W. - - 
S. aviculare, G. Forster, prodr. 18 (1786)

S. simile, F. v. M. in Transact. phil. Soc. Vict. I, 19 (10ั3 4 )

S. fasciculatum, F. v. M., fragm. I, 123 (1859)

S. Bauerianum, Endlicher, prodr. fl. Norfolk, 54 (1833)...

S. viride, R. Brown, prodr. 445 (1810)

S. tetrandrum, R. Brown, prodr. $445(1810)$

S. Shanesii, E. v. M., fragm. VI, $144(1868)$

S. verbascifolium, Linné, spec. plant. 184 (1753) ...

S. discolor, R. Brown, prodr. 445 (1810)

S. stelligerum, Smith, Exot. Bot. II, 57, t. 88 (I 1805 )

S. parvifolium, R. Brown, prodr. 446 (1810)

S. ferocissimum, Lindley in Mitch. Three Exped, II, 58 (1838)...

S. sporadotrichum, F. v. M. in Melb. Chemist, Oct. (1882)

S. defensum, F. v. M., fragm. V, 193 (1866)

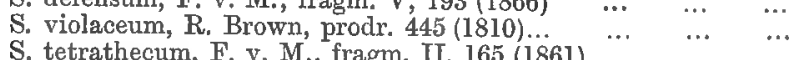

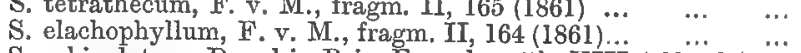

S. orbiculatum, Dunal in Poir. Encycl. méth. XIII, 762 (1817) ...

S. oligacanthum, F. v. M. in Transact. phil. Soc. Vict. I, 19 (1854) -

S. esuriale, Lindley in Mitch. Three Exped. II, 43 (1838)

S. chenopodinum, F. v. M., fragm. II, 165 (1861)..

S. Sturtianum, F. v. M. in Transact. phil. Soc. Vict. I, $\dddot{19}(18 \breve{5} 4)$

S. furfuraceum, R. Brown, prodr. 446 (1810)

S. dianthophorum, Dunal, hist. des Solan. 183 (1813)

S. Dallachyi, Bentham, Fl. Austr. IV, 456 (1869)

S. densivestitum, F. v. M. in Benth. FI. Austr. IV, 456 (1869)

S. Oldfieldii, F. v. M., fragm. II, 161 (1861)

S. semiarmatum, F. v. M., fragm. II, 163 (1861) ...

S, armatum, R. Brown, prodr. 446 (1810)

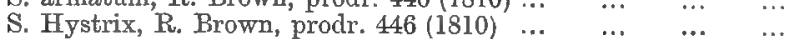

S. cataphractum, Cunningham in Benth. Fi. Austr. IV, $\ddot{459}$ (1869)

S. pungetium, R. Brown, prodr. 446 (1810)

S. eremophilum, F. v. M. in Schlecht. Linnaea X X XV, $4 \ddot{32}$ (1852)

S. campanulatum, R. Brown, prodr. 446 (1810)

S. adenophorum, F. v. M., fragm. II, 162 (1861)...

S. cinereum, R. Brown, prodr. 446 (1810) ...

S. lacunarium, F. v. M. in Transact. phil. Soc. Vict. I, 18 (1854)

S. petrophilum, F. v. M. in Linnaea XXV, 433 (1852) ...

S. diversiflorum, F. $\nabla$. M., fragm. VI, 146 (1868)

S. carduiforme, F. v. M., fragm. II, 163 (1861)

S. melanospermum, F. v. M., fragm. II, 163 (1861)

S. horridum, Dunal, Solan. synops. 28 (1816)

S. echinatum, R. Brown, prodr. 447 (1810)

S. lasiophyllum, Dunal in Poir. Encycl. méth. XIIII, $76 \dddot{4}(1817)$

S. ellipticum, R. Brown, prodr. 446 (1810)

S. quadriloculatum, F. v. M., fragm. II, 161 (1861)

S. phlomoides, Cunningham in Benth. Fl. Austr. IV, 464 (1869)

S. Cunninghamii, Bentham, Fl. Austr. IV, 465 (1869) ..

LYCIUM, Linné, gen. plant. 57 (1737), from Celsus.

L. australe, F. v, M. in Transact. phil. Soe. Vict. I, 20 (1854)... DATURA, Linné, gen. plant. 48 (1737).

D. Leichhardtii, F, v. M. in Transact. phil. Soc. Vict. I, 20 (1854)
S. nemophilum, F. v. M., fragm. II, 161 (1861) ... ...

S.A. T. V. N.S.W. Q. - B.fl.IV, 447 W.A. S.A. - V. N.S.W. - - B.fl.IV,448 W.A. S.A. - - N.S.W. $\frac{-}{-}$

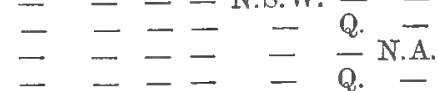
B.fl. $\overline{I V}, 449$ B.fl IV, 449 - - - N.S.W. Q. - B.f.IV,449 - - - N.S.W. Q. N.A. B.fl.IV,450 - - - N.S.W. Q. - B.fl.IV,450 - - - N.S.W. Q. - B.fl.IV,451 S.A. - - N.S.W. Q. - B.fl.IV,451 - - - - Q Q. - B.fl.IV, 451 - - - N.S.W. Q. - B.H.IV,452 - - - N.S.W.Q. - B.fl.IV,453 S.A. - - - Q. - B.fl.IV,453
S.A. - - N.S.IV. - - B.fl.IV,454 S.A. - V. N.S.W. Q. N.A. B.fl.IV,454

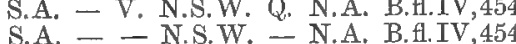
S.A. - - N.S.W. - N.A. B.A.IV,454 - - - - Q. - B.fl.IV,455 - - - - Q. - B.fl.IV,455 - - - N.S.IV. Q. - - B.H.IV,456 - - - - Q. - B.fl.IV,457 -- - N.S.W. $\overline{\mathrm{Q}}$. B.A.IV, 457 - - V. N.S.W. Q B.fl.IV, 458 S.A. - - - - - B.H.IV, 458 - - - - N.A. B.fl.TV,459 - - V. N.S.W. Q. - B.fl.IV,459 S.A. - N.S.W. $\frac{-1}{\text { - N.fl.IV, } 459}$ - - N.S.W.Q. - B.f.IV, 460 - _ - - Q. - B.flIV,460 - - - N.S.IF. Q. - B.fl.IV.460 S.A. - V. N.S.W. - - B.fi.IV,461 S.A. - - N.S.W. - - - - N.A. B.f.IV, 461 - - - - - N.A. B.H.TV,462 - - - - N.A. B.fl.IV,462 - - - - - - N.A. B.fi.IV, 463 W.A. - - - - - - N.A. B.fi.IV, 463
- N.A. B.fI.IV,463 W.A. S.A. - - N.S.W. Q. N.A. B.f.IV,464

- - - - - - N.A. B.fi.IV, 464 - - - - - - - N.A. B.f.IV, 465

W.A. S.A. - V. N.S.W. - - B.fl.IV,467
S.A
Q. N.A. B.A.IV, 468
M.fr. VI,144.

NICOTIANA, Tournefort, inst. 117, t. 41 (1700), from C. Bauhin (1623).

N. suaveolens, Lehmann, gener. Nicot. hist. $43(1818) \quad \ldots \quad$... W.A. S.A. - V. N.S.W. Q. N.A. B.A.IV,469 ANTHOTROCHE, Endlicher, nov, stirp. mus. Vindob. dec. 6 (1839).

A. pannosa, Endlicher, nov, stirp. mus. Vind. dec.7(1839) ... W.A. - - - - - - B.fl.IV,467

A. Blackii, F. v. M., fragm. VIII, $232(1874)$

W...A. S.A. - -

- - - B.fl. $\bar{I}, 468$

ANTHOCERCIS, Labillardière, Nov. Holl. pl. spec. II, 19 (1806). (Cyphanthera, Eadesia.)

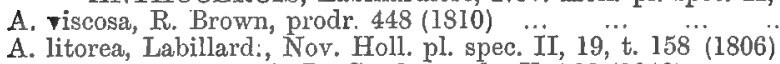

A. gracilis, Bentham in De Cand., prodr. X, 192 (1846).

A. genistoicles, Miers, Illustr. of plants II, App. 27, t. 83 (1857)

A. anisantha, Endlicher in Ann. Wien. Mus. II, 201 (1838) ...

A. intricata, F. v. M., fragm. I, 211 (1859)

A. arborea, F. v. M., fragm. I, 212 (1859).

A. angustifolia, F. v. M. in Transact. phil. Soc. $\dddot{V}_{\text {ict. }} \mathrm{I}, 21$ (1854)

A. fasciculata, F. v. M., fragm. I, 122 (1859)

A. microphylla, F. v. M., fragm. I, 179 (1859)

W.A.

W.A.

- - - - -

B. fl.IV, 475

B.A.IT, 4,6

W.A. - - - - - - B.fl.IV, 476

W.A. - - - - - - B.f.IV,476

W.A. S.A. - - - - - B.fl.IV,477

W.A. - - - - - - B.fl.IV, 477

W.A. - - - - - - B.fl.IV 477

B.f.IV, 477
B.f.IV, 478

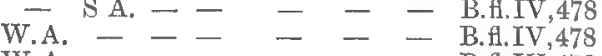

W.A. - - - - - - B.f.IV,478

A. myosotidea, F. v. M. in Transact. phil. Soc. Vict. I, $20(1854)-$ S.A. - V. N.S. W. - - B.fl.IV,478
A. scabrella, Bentham in De Cand., prodr. X, $192(1846) \quad \ldots-$ N.S. IV. - - B.f.IV,47!

A. albicans, Cunningham in Field's N.S.W. 335, t. 2(1825)

A. Odgersii, F. v. M., fragm. X, $19(1876) \ldots$

A. Tasmanica, J. Hooker, Fl. Tasman. I, 289 , t. $\ddot{9} \ddot{2}$ (1860̈)

A. Eadesii, F. r. M., fragm. II, 139 (1861)

A. racemosa, F. v. M., fragm. I, 211 (1859)

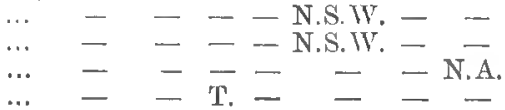

B.H.IV, 479

. - - T. - - - - B.fl. $\overline{I V}, 479$

.. - S.A. - V. N.S.W. - - B.f.IV,480

B. fl. IV, 480
M.fr.I, 83,243;II, 179 .

M.fr.VIII, 232.

I. fr. VI, 144.

1,144.

M.fr.VI, 145.

M.fr. VI, 144.

M.fr. VI, ]45.

.fr.II, 166;VI, 147 .

147.

M.fr. V,193.

M.fr.II, 165 .

M.fr.II, 164 .

M.fr. VI, 146.

M,fr. VI, 146 .

VI, 145.

M.fr.II, 161 .

M.fr. VI, 145 .

M.fr. VI, 146.

M.fr.VI, I46.

M fr. VI, 146 .

M.fr.VI, 146.

M.fr. VI, 146 .

fr. VI, 146.

M.fr. VI, 145.

$V I, 145$.

M.fr.II, 161 .

M.fr. VI, 144. M.fr. I, 123.

MI.fr. VI, ] 43.

I. fr. Y I, 143; XI, 123.

N.fr.I, I22; YI, 143 .

M.fr. YI, 143 .

M.fr.I,211.

M.fr.I,2l:2; YI, 143 .

M.fr, I, 129; VI, 143.

M.fr.I, $179 ; \backslash T, 143$.

M.fr.I, 179; VI, 143.

M.fr. VI, 143.

M. fr.X, 19 .

M.fr. VI, 143 .

MI.fr. VI, 142 .

M.fr.I, 211 .
M. .145.

M.fr.II, 162 .

M.fr.II, 163. 
DUBOISIA, R. Brown, prodr. fl. Nov, Holl, 448 (1810). D. myoporoides, R. Brown, prodr. 448 (1810)

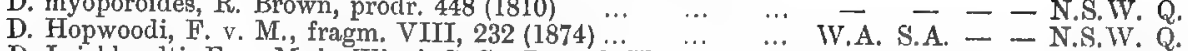
D. Leichhardti, F. v. M. in Wing's S. Sc. Record, II, 2222 (1882)
- - - N.S.W. Q. - B.fl.IV,474 二 B.fi.IV, 480 二 B.fl.IV,480
M.fr. VI, 144

M.fr.II, 138;VI, $143 ; \mathrm{X}, 20$. M.fr.VI, 142 .

\section{SCROPHULARINAE.}

MIMULUS, Linné in Act, Upsal. 82 (1741). (Uvectalia.)

M. Uvedaliae, Bentham in De Cand. X, 369 (1846) $\ldots$.

M. debilis, F. v. M. in Transact. phil. Inst. Vict. I, 62 (1857) ...
M. gracilis, R. Brown, prodr, 439 (1810) ... ... ... ...

M. repens, R. Brown, prodr. 439 (1810) $\ldots$
M. prostratus, Bentham in De Cand. X, 373 (1846) MAZUS, Loureiro, Fl. Cochinch. II, 385 (1790).

M. Pumilio, R. Brown, prodr. 439 (1810) ... ... ... ADENOSMA, R. Brown, prodr, fl. Nov. Holl. 442 (1810).

A. coerulea, R. Brown, prodr. 443 (1810) $\ldots \ldots \ldots \ldots \ldots$

A. Muelleri, Bentham, Fl. Austr. IV, 485 (1869)... STEMODIA, Linné, syst. ed. dec. 1118 (1759). (Morgania, Limnophila.)

S. lythrifolia, F. v. M. in.Benth. Fl. Austr. IV, 486 (1869)

S. grossa, Bentham, Fl. Austr. IV, 486 (1869)

S. viscosa, Roxburgh, pl. of Coromand. II, 33, t. 163 (1798)

S. linophylla, F, v. M., fragm. X, 88 (1876)

S. debilis, Bentham, Fi. Austr. IV, 487 (1869)

S. pedicellaris, F. v. M., fragm. VIII, 231 (1874)...

S. Morgania, F. v. M., fragm. X, 89 (1876)

S. sessiliflora, F. v. M., Limnophila, Blume, Bijdr. 750 (1826) ...

S. gratioloides, F. v. M., fragm. X, 89 (1876)

S. punctata, F. v. M.; Limnophila, Blume, Bijdr. 750 (1̈26) $\ldots$

S. hirsuta, Heyne in Wallich, numer. list. 3930 (1828) ... $\ldots$

S. tenuiflora, Bentham, Scrophul. Indic. 23 (1835) BRAMIA, Lamarck, Encycl. method. I, 459 (1783)

B. floribunda, F. v. M., fragm. IX, 167 (1875) GRATIOLA, Ruppius, Fl. Jenens. 241 (1718), from Docloens (1556).

G. pedunculata, R. Brown, prodr. 435 (1810)

G. Peruviana, Linné, spec. plant. 17 (1753)..

$G$. nana, Bentham in De Cand. prodr. X, 404 (1840) DOPATRIUM, Hamilton in Benth. Scroph. Ind. 4 (1835).

D. junceum, Hamilton in Benth. Scroph. Ind. 4 (1745) ARTANEMA, D. Don in Sweet, Fl. gard. t. 234 (1829).

A. fimbriatum, D. Don in Sweet, Fl. Gard. t. 234 (1829)

L. crustacea, F. v. M. Vandellia Benthel

L. pubescens, F.v.M. ; Vandellia, Benth. in De Cand. pr. X, 415(1846)

L. alsinoides, R. Brown, prodr. 441 (1810)...

L. scapigera, R. Brown, prodr. 441 (1810) ...

L. subulata, R. Brown, prodr. 441 (1810) ...

L. Iobelioides; Vandellia, F. v. M. in Transact. phil. Inst. Vict. III, 61 (1858)

L. clausa, F. v. M., fragm. VI, 102 (1868) ...

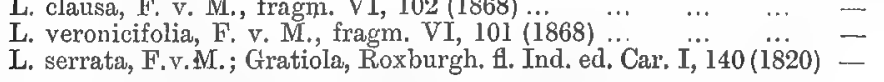
HEMTARRHENA, Bentham, Fl. Austr. IV, 518 (1869).

H. plantaginea, Bentham in Hook, icon. pl. t. 1059 (1869) PEPLIDIUI, Delile, Fl. Aegypt. 148 (1813).

P. humifusum, Delile, Fl. Aegypt. 148 (1813)

P. Muelleri, Bentham, Fl. Austr. IV, 500 (1869) ... MICROCARPAEA, R. Brown, prodr. 435 (I810).

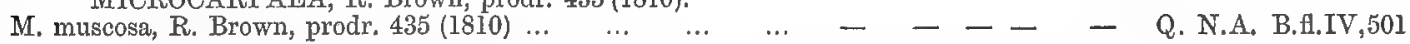
GLOSSOSTIGMA, Arnott in nov, act. acad. Caes. Leop. Carol. XVIII, 355 (1836). (Tricholoma.)

G. spathulatum, Arnott in nov. act. Leop. XVIII, 355 (1836) ... - - - - _ Q. - B.fl.IV,501

G. Drummondii, Bentham in De Cand. prodr. X, 426 (1846) ... W. A. - - - - - - B.fl.IV,502

G. elatimoides, Bentham in J. Hook. Fl. New Zeal. I, 189 (1853) - S.A. T. V. N.S. W. - - B.f.IV,502 LIMOSELLA, Lindern, opusc. plant. Argentorat. 156, t. 5 (1728).

L. aquatica, Linné, spec. plant. 631 (1753)..

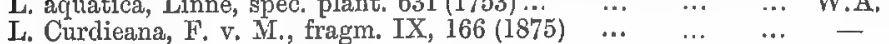
SCOPARIA, Linné, syst. nat. ed. sext. 87 (1748).

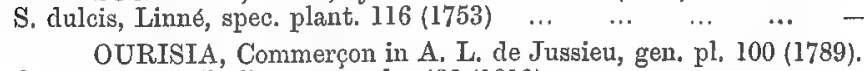

O. integrifolia, R. Brown, prodr. $439(1810)$
VERONICA, Toumefort, inst. 143, t. 60 (1700), from Fuchs (1543).
V. clensifolia, F. v. M., fragm. II, 137, t. $63(1861)$
(Pygmaea.)

V. formosa, R. Brown, prodr. 434 (1810) ..

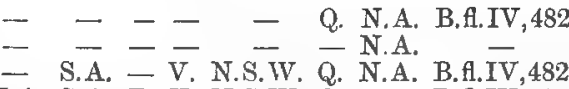
- N.A. B.fl.IV, 486

Q. - B.fl.IV, 490 A. - V. N.S.W. Q. - B.fl.IV,492
.A. S.A. T. V. N.S.W. Q. - B.f.IV, 482

- S.A. T. V. N.S.WV. Q. - B.fl.IV,484 Pterostigma.)

- - - - - Q. N.A. B.fl.IV,484 - N.A. B.fl.IV, 486 - N.A. B.fl.IV, 486 - N.A. B.fl. $\overline{I V}, 487$ Q. N.A. B.fl. $\overline{T V}, 488$ Q N.A. - N.A. B.fl.IV, 490 - N.A. B.H.IV, 490 - - - N.S.W. Q. Q. N.A. B.A.IV, B.A.IV,491 A. T. V. N.S.W. Q. N.A. B.fl.IV,493
T. V. N.S.W. - - B.H.IV, 493 Q. - B.fl.IV, 494 -- - N.S.W. Q. - B.fl.IV,495 M.fr.VI, 104;IX,167. - - - N.S.W. Q. N.A. - - - N.S.W. Q. N.A. B.f.IV, 496 - - - - - Q. N.A. B.Al.IV,497 - - - - - N.A. B.f.IV,497 - - - - N.A. B.fl.IV,498 Q. - B.fl.IV, 498 Q. - - N.A. B.fl.IV,518

M.fr. VI, 102 .

M.fr. VI, 105 .

M.fr.VI, 104.

M.fr.VI, 104. S.A. T. V. N.S.W. - - B.fl.IV,502 M.fr.VI, 105;IX, 165, M.fr.IX, 166. Q. N.A. B.f.IV,504 M.fr.VI,104. M.fr.VI, 105;IX, 168. - V. N.S.W. - - B.Al.IV,505
M.fr.VI, 103. M.fr. VI,102;IX, 167. 
V. decorosa, F. v. M. in Schlechtend., Linnaea XXV, 430 (1852)

V. perfoliata, R. Brown, prodr. 434 (1810).

V. Derwentia, Littlejohn in Andr. Bot. Reposit.

V. arenaria, Cunningham in De Cand. prodr. X, 463 (1846) ..

V. nivea, Lindley, Bot. Regist. Misc. $42(1842) \quad \ldots \quad \ldots . \quad \ldots$

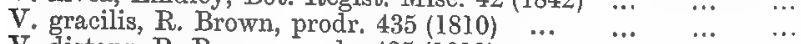

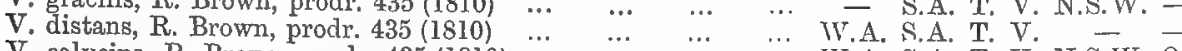

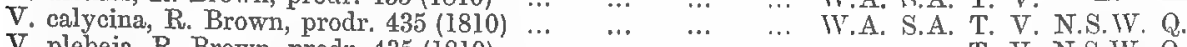

V. plebeja, R. Brown, prodr. 435 (1810) $\ldots$...

V. notabilis, F. v. M. in Benth. Fl. Austr. IV, 5111 (1869)

V. serpillifolia, Linné, spec. plant. 12 (1753)

V. peregrina, Limné, spec. plant. 14 (1753)..

in Hook. Comp. to the Bot. Mag. I, 368 (1835).

CENTRANTHERA, R. Brown, prodr. fl. Nov, Holland, 438 (1810).

C. hispida, R. Brown, prodr. 438 (1810) $\ldots$... $\quad \ldots \quad \ldots \quad \ldots$ SOPUBIA, Hamilton in D. Don, prodrom. flor. Nepalens. 88 (1825).

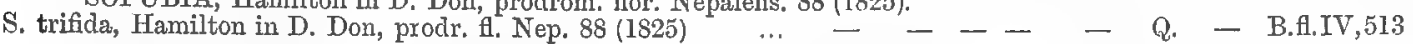
BUECHNERA, Linné, hort. Cliffort. 501 (1737). (Buchnera, Striga.)

B. tetragona, R. Brown, prodr. 437 (1810)...

B. urticifolia, R. Brown, prodr. 437 (1810)...

B. linearis, R. Browu, prodr. 437 (1810) $\ldots$

B. tenella, R. Brown, prodr. 437 (1810)

B. gracilis, R. Brown, prodr. 437 (1810) …

B. ramosissima, $R$. Brown, prodr. 438 (1810)

B. coccinea, Bentham, Scrophul. Ind. 40 (1835)

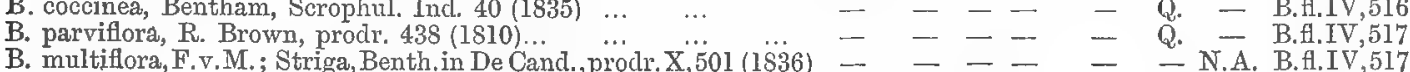

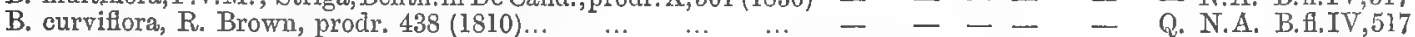
EUPHRASIA, Tournefort, instit. 174, t. 78 (1700), from Matthaeus (1478).

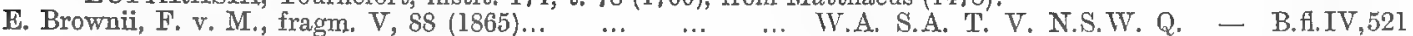

E. scabra, R. Brown, prodr. 437 (1810) ... ... ... ... IV.A. S.A. T. V. N.S.W. Q. - B.fl.IV,521

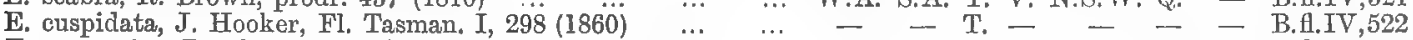

E. antarctica, Bentham in De Cand., prodr. $\mathrm{x}, 555(1846) \quad \cdots \quad-\quad-\quad-$ V. N.S.TV. - - B.H.IV,522

\section{OROBANCHEAE.}

A. L. de Jussieu in Ann. Mus. V, 25 (1804).

OROBANCHE, Tournefort, inst, 175, t. 81 (1700), from l'Ecluse (1583).

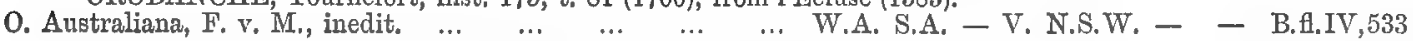

\section{LENTIBULARINAE.}

L. C. Richard in Bulliard, Fl. Paris، ed. sec. I, 26 (1796).

UTRICULARIA, Linné, syst. nat. 8 (1735); Linné, gen. plant. 5 (1737).

U. stellaris, Linne, fil. suppl, plant. $86(1781)$
U. flexuosa, Vahl, enum, plant. I, $198(1804)$
. .

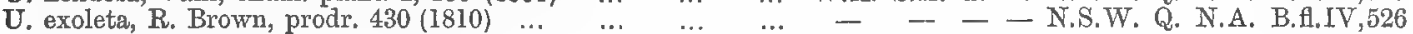

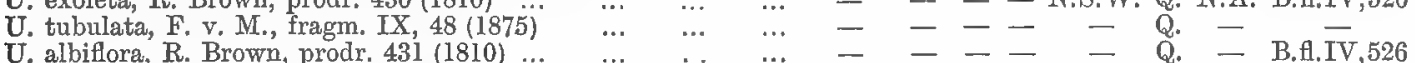

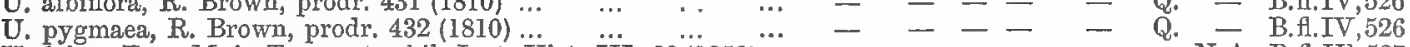

U. fulva, F. v. M. in Transact. phil. Inst. Vict. ÏII, 63 (i858)... - - - - - - N.A. B.f.IV,527

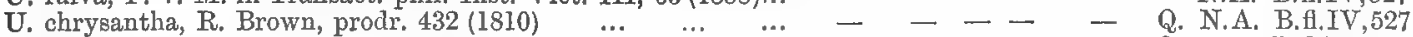

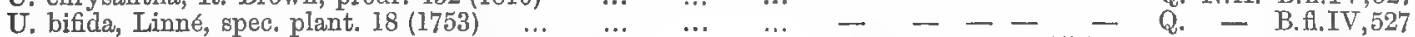

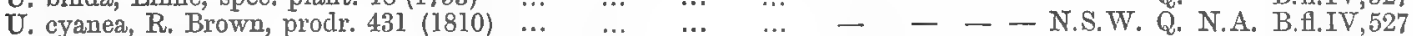

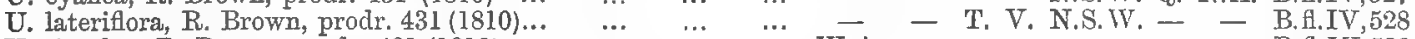

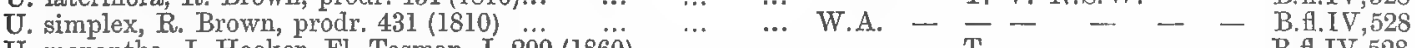

U. monantha, J. Hooker, FI. Tasman, I, $299(1860) \quad \ldots \quad \ldots . \quad-\quad-\quad$ T. $-\quad-\quad-\quad$ B.f.IV,528

U. dichotoma, Labillard, Nov. Holl. pl. spec. I, 11, t. $8(1804)$ - S.A. T. V. N.S.W. Q. - B.f.1V,529

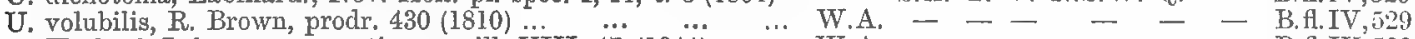

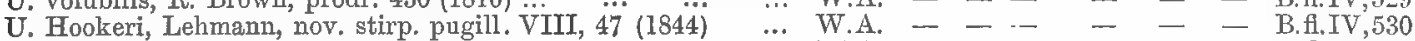

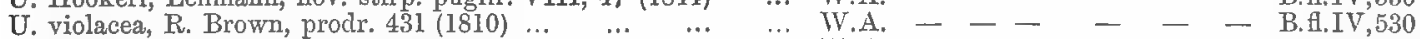

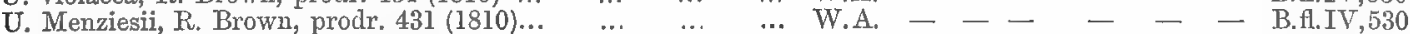

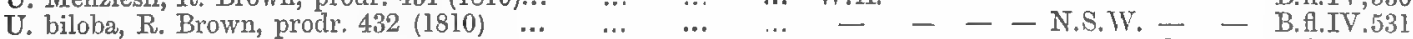

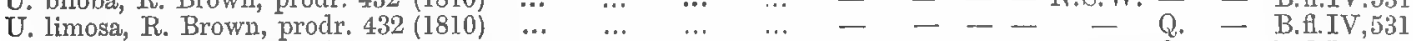

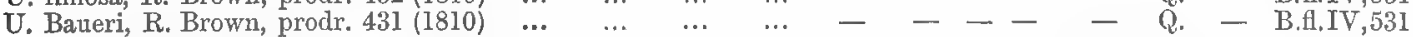

POLYPOMPHOLYX, Lehmann in der Bot. Zeitung, Halle, 109 (1844). (Tetralobus.)

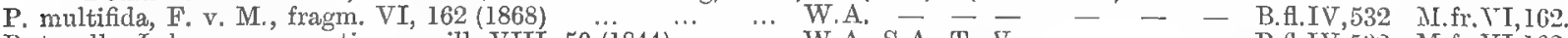

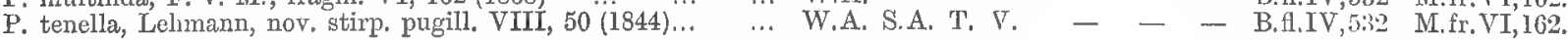

\section{GESNERIACEAE.}

Humboldt, Bonpland \& Kunth, nov. gen. et spec. pl. II, 392 (1817).

FIELDIA, Cunningham in Field's New South Wales 363, t. 2 (1825).

F. australis, Cunningham in Field's N.S.W. 364, t. 2 (1825) $\ldots-\frac{-}{-}-$ V. N.S.W. - - B.fl.IV,535 M.fr.IX,191.

NEGRIA, F. v. M., fragm. VII, 151 (1871). (Rhabdothamnus partly.)

N. rhabdothamnoides, F. v. M., fragm. VII, 152 (1871).. ‥ - - - - N.S.W. - - - - M.fr.VII,152;XI,133.

BAEA, Commercon in Lamarck, Encycl. méth. I, 401 (1783). (Streptocarpus.)

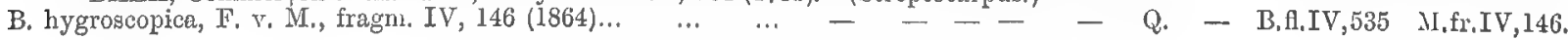




\section{BIGNONIACEAE.}

Ventenat, Tabl. du règn. végét. II, 402 (1799).

TECOMA, A. L. de Jussieu, gen. plant. 139 (1789).

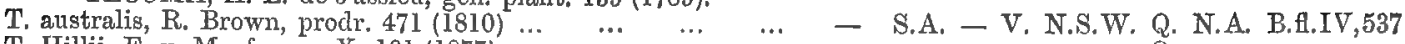

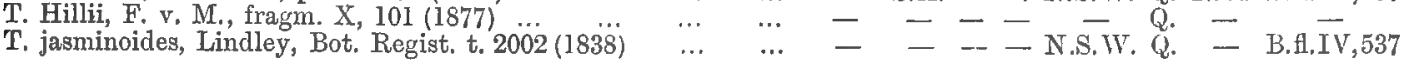

M.fr. X, 101;XI, 136 .

DOLICHANDRONE, Fenzl in Ann, nat. hist, third ser. X, 31 (1862). (Spathodea partly, Dolichandra partly.)

D. heterophylla, F. v. M., fragm. IV, $149(1864)$.. ... ... - - - - - Q. N.A. B.fi.IV, 539 M.fr.IV, 149.

D. filiformis, Seemann, Journ. of Bot. I, $226(1863) \quad \ldots \quad \ldots \quad-\quad-\quad-\quad$ - $\quad$ - N.A. B.fl,IV,539

HAUSSMANNIA, F. v. M., fragm. IV, 148 (1864).

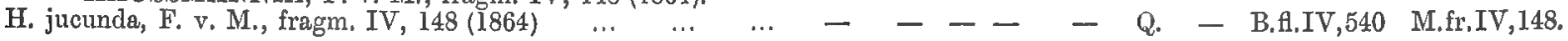

DIPLANTHERA, R. Brown, prodr. Fl. Nov. Holl. 449 (1810). (Deplanchea, Bulweria.)

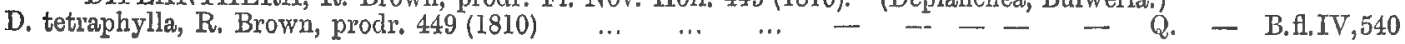

\section{ACANTHACEAE.}

A. L. de Jussieu, gen, plant. 102 (1789), from B. de Jussieu (1759).

THUNBERGIA, Retzius, Physiogr. Saellsk. Handl. I, 163 (1776).

T. Arnhemica, F. v. M., fragm. IX, 73 (1875) $\ldots$.. $\quad$. $\quad-\quad-\quad-\quad-\quad-$ N.A.

T. Powelli, F, v. M. in Wing's South. Sc. Rec. II, 34 (18̈82) … - - - -

NELSONIA, R. Brown, prodr. fl. Nov. Holl. 485 (1810).

N. campestris, R. Brown, prodr. 481 (1810) EBERMAYERA, Nees in Wallich, pl. Asiat. rar. III, 75 (1832).

E. glauca, Nees in De Candolle, prodr. XI, 73 (1847) $\ldots \quad \ldots$

HYGROPHILA, R. Brown, prodr. Fl, Nov, Holl, 479 (1810).

H. angustifolia, R. Brown, prodr. $479(1810) \quad \ldots \quad \ldots$
STROBILANTHES, Blume, Bijdr. tot de $\mathrm{fl}$. van Nederl. Ind. 796 (1826).

S. Tatei, F. v. M. in transact. Roy. Soc. S. Austr. V, Aug. (1882) - - - - - - N.A. RUELLIA, Plumier, nov. gen. 12, t. 2 (1703). (Dipteracanthus.)

R. bracteata, R. Brown, prodr. 479 (1810)

R. primulacea, F. v. M. in Benth. Fl. Austr. IV, $546(1869) \cdots \cdots-$ - $\cdots$ -

R. corynotheca, F. v. M. in Benth. Fl. Austr. IV, 546 (1869) .. - - - - - - Q. - B.fl.IV,546 M.fr.XI,18.

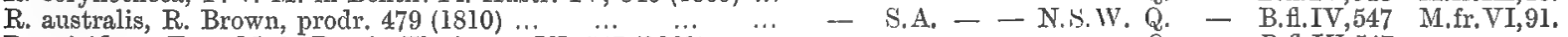

R. spicitlora, F. v. M. in Benth. Fl. Austr. IV, 547 (1869) $\ldots-\ldots--\quad-\quad-\quad-\quad$ Q. - B.A.IV,547

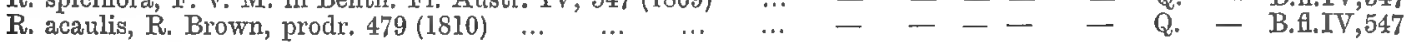
ACANTHUS, Tournefort, inst, 176, t. 81 (1700), from Theophrastos. (Dilivaria.)

A. ilicifolius, Linne, spec. plant. $939(1753) \quad \cdots \quad \ldots \quad \ldots--$

JUSTICIA, Houston in Limé, gen. plant. 4 (1737). (Rostellaria, Rostellularia)
J. procumbens, Linne, spec, plant. 15 (1753) $\ldots$ W. W. S.A. - N. N. W. Q. N.A. B.f.IV,549

Q. N.A. B.A.IV,548 M.fr.X, 18.

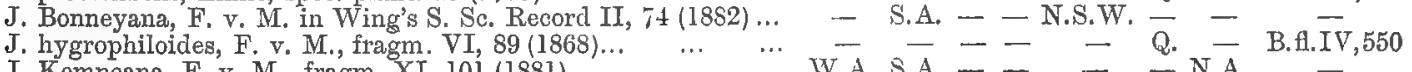

J. Kempeana, F, v. M., fragm. XI, $101(1881) \quad \ldots \quad \ldots \quad \ldots$ W.A. S.A. - $\quad \ldots \quad-\quad$ - N.A.

M.fr. VI, $91 ; X I, 18$. M.fr. X, 73 .

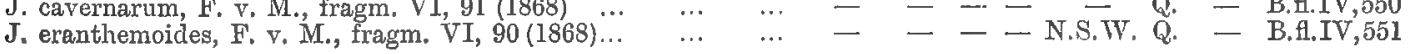
M.fr. VI, 89.

M.fr.XI, 101 . GRAPTOPHYLLUM, Nees in Wall. pl. Asiat, rar. III, 102 (1832). (Earlia, Thyrsacanthus.)

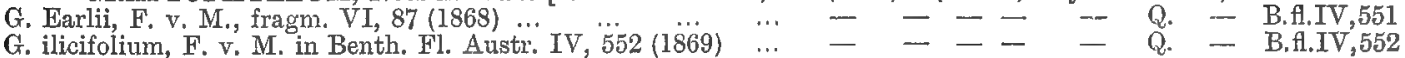

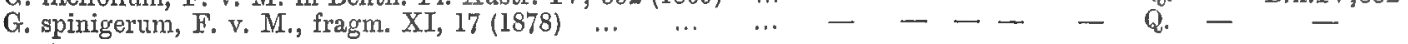
DICLIPTERA, A. L. de Jussieu in Ann. du Mus. IX, 267 (1807). (Brochosiphon.)

D. glabra, Decaisne in Nouv. Annal. du Mus. III, 388 (1834) .. - - - - - - N.A. B.fl.IV,552

D. spicata, Decaisne in Nouv. Annal. du Mus. III, $389(1834) \ldots-\ldots-1-\ldots-$ Q. - - B.f.IV, 553

D. Burmanni, Nees in Wallich, pl, Asiat, rar. III, 112 (1832) . HYPOESTES, Solander in R. Brown, prodr. 474 (1810).

H. floribunda, $R$, Brown, prodr. $474(1810) \quad \ldots \quad \ldots . \quad \ldots \quad-\quad-\quad-\quad-$ N.S.W. Q. N.A. B.A.IV,554 ERANTHEMUM, Linné, Fl. Zeyl. 6 (1747).

E. variabile, R. Brown, prodr. 477 (1810) ... …

E. tenellum, Bentham, Fl, Austr. IV, 555 (1869) ...

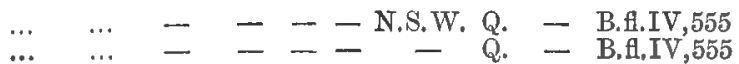

M.ir.

M.fr. VI, 87.

M.fr.XI, 17.

M.fr.VI, 88;XI,18.

M.fr.VI, 89 .

M.fr.VII, 62 .

M.fr.XI, 18.

HYDROPHYLLEAE.

R. Brown in Bot. Regist. t. 242 (1817).

HYDROLEA, Linmé, spec, plant. ed. sec. 328 (1762).

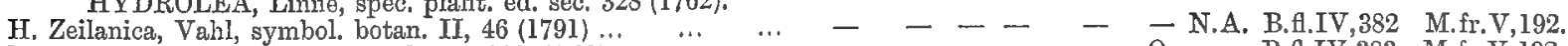

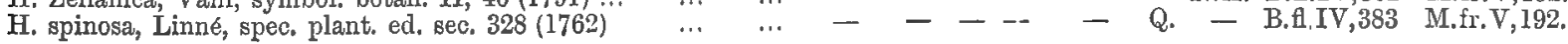

ASPERIFOLIAE.

Haller, enum. stirp. Helv. Praef. 34 (1742).

CORDIA, Plumier, gen. plant. 13, t. 14 (1703).

C. subcordata, Lamarck, Illustr. des genr. I, 421 (1791).. $\ldots-$ - $-\quad-\quad$ - Q. N.A. B.A.IV,385

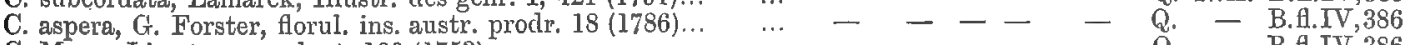

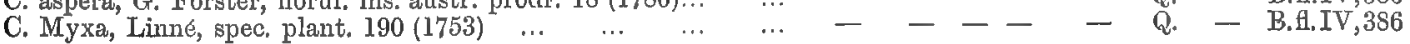

EHRETIA, P. Browne, Civ, and Nat. Hist. of Jamaic. 168, t. 16 (1756.

E. acuminata, R. Brown, prodr. 497 (1810)

E. pilosula, F. v. M., fragm. V, 20 (1865)...

E, saligna, R. Brown, prodr, 497 (1810) ...
,.. $\quad$...

$\begin{array}{ll}\cdots & \cdots \\ \cdots & \cdots \\ \cdots & \cdots\end{array}$ $\ldots$
$\cdots$
$\cdots$
- V. N.S.IV. Q. - B.fl.IV,387

Q. N.A. B.H.IV,388
M.fr. VI,114;IX, 122. [122.

M.fr.V,193; VI, 114;IX,

M.fr.I, $59 ; \mathrm{VI}, 114$.

M.fr. V,21;IX, 122; XI, 124

M.fr. V,20.

M.fr. V,21;IX, 122 . 
E. membranifolia, R. Brown, prodr. 497 (1810)

E. laevis, Roxburgh, Pl. Corom. I, 42, t. 56 (1795)

.. $\quad . \quad$ - $\quad-\quad--\quad$ - Q. $\quad$ - B.fl.IV,388

M.fr.V,2I;VI, 115;IX, 122 TOURNEFORTIA, Linné, syst. nat. 8 (1735); Linné, gen. plant. 55 (1737.)

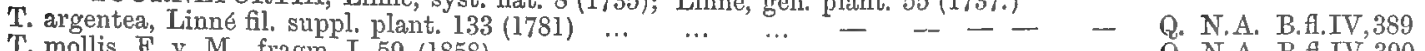

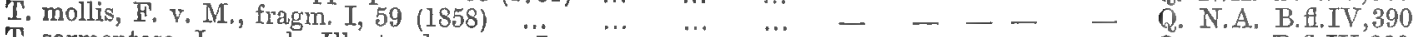

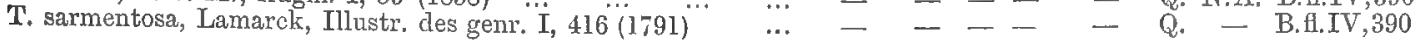

M. fr. TV, 95; VI, 116. COLDENIA, Linné, amoen. acad, ed, prim. 119 (1747). (Lobophyllum.)

C. procumbens, Linné, spec. plant. 125 (1753)

Q. N.A. B.fl.IV,391 M.fr.VI,115.

HELIOTROPIUM, Toumefort, inst. 138, t. 57 (1700), from Theophr, Diosc. \& Plinius. (Tiaridium, Heliophytum.)

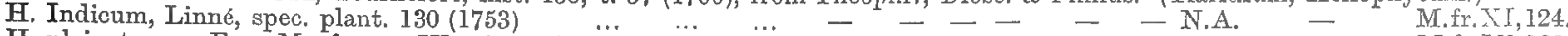

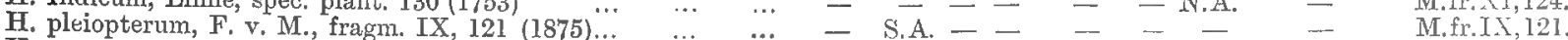

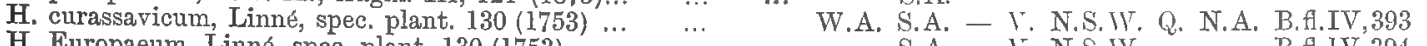

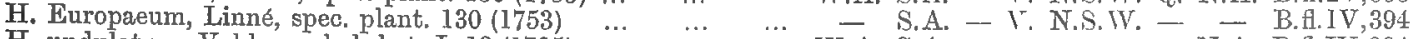

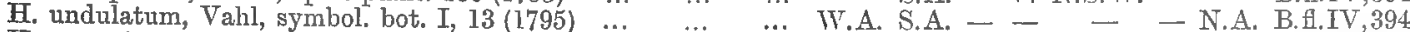

H. asperrimum, R. Brown, prodr. 493 (1810)
H. crispatum, F. v. M. in Benth. Fl. Austr. IV, 395 (1869)

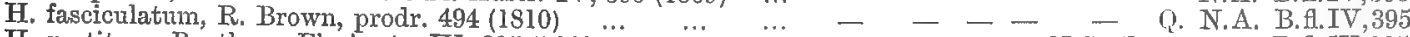

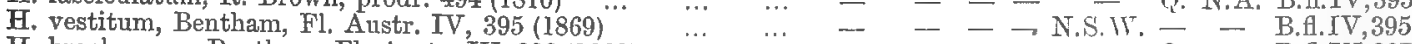

H. brachygyne, Bentham, Fl. Austr. IV, 396 (1869)

H. epacrideum, F. v. M. in Benth. Fl. Austr, IV, 396 (1869) $\ldots \ldots-\ldots-\ldots-\ldots$ N. A. B.A.IV,396

H. ovalifolium, Forskael, Fl. Aegypt. Arab. $47(1775) \ldots \ldots$... . S.A. - - _ - Q. N.A. B.fl.IV,396

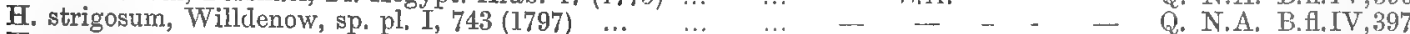

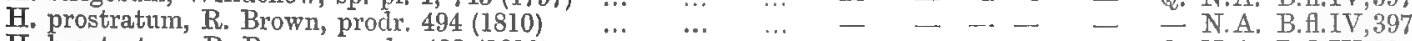

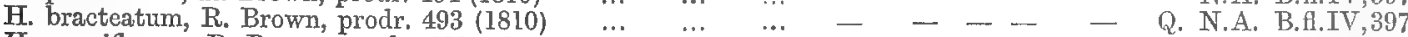

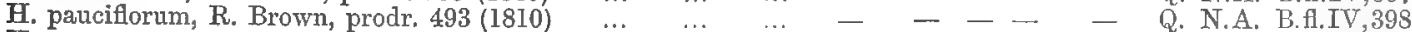

H. conocarpum, F. v. M. in Benth. Fl. Austr. $398(1869) \quad \ldots \quad-\quad-\quad-\quad-\quad--\quad$ - N.A. B.fl.IV, 398

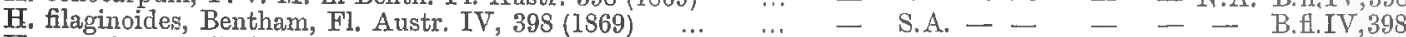

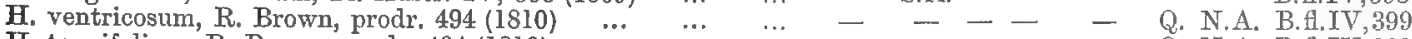

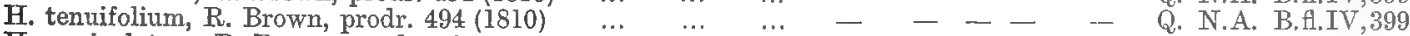

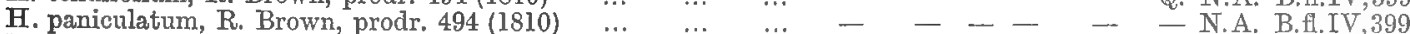

H. Cunninghamii, Bentham, FI. Austr. IV, 400 (IS69) $\cdots \cdots \cdots$

H. diversifolium, F. v. M. in Benth. Fl, Austr. IV, $400(1869) \ldots-\ldots-$ N.A. B.A.IV,400 HALGANIA, Gaudichaud in Freyc. in voy. Botan. 448 (1526).

H. solanacea, F. v. M. in Hook. Kew Misc. IX, 21 (1857)

H. litoralis, Gaudichand in Freyc. voy. Bot. 449, t. 59 (1826) ...

H. corymbosa, Lindley, Bot. Regist. XXV, App. XL (1839) ...

H. sericiflora, Bentham, Fl. Austr. IV, 402 (1869)

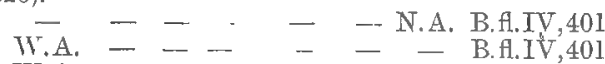

W.A. - - - - - - - B.fl.IV,401
W.A. - B.fl.IV,402

… ... W.A. - - - - - - B.fl.IV,402

H. lavandulacea, Endlicher in Ann. Wien. Mus. II, 205 (1838) W.A. S.A. - V. N.S.W. - - B.fl.IV, 402

H. integerrima, Endlicher in Amn. Wien. Mus. II, 205 (1838) ... W.A. - - - - - - B.H.IV,402 POLLICHIA, Medikus, Botan. Beobach. 247 (1783). (Trichodesma.)

P. Zeylanica, F. v. M.; Trichodesma, R. Brown, prodr, 496 (1810) W.A. S.A. - - N.S.W. Q. N.A. B.A.IV,404

P. latisepalea; Trichodesma, F. v. M., fragm. X, 102 (1577) MYOSOTIS, Ruppius, Fl. Jenens. 9 (1718), from Dioscorides and Plinius. (Exarrheni.)

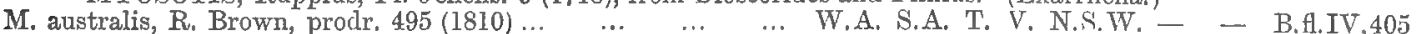

M. suaveolens, Poiret, Encycl. méth. suppl. IV, 44 (18i6) ‥ - - T. V. N.S.TV. - - B.fl.IV,406 ERITRICHUM, Schrader in commentat. Goetting. IV, 186 (1820). (Eritrichium.)

E. Australasicum, A. De Candolle, prodr. X, $134(1846) \quad$ ‥ W. A. S.A. - V. N.S.W. LAPPULA, Rivinus in Rupp. Fl. Jenens. (1718), from Dalechamp. (Echinospermum.)

L. concava; Echinospermum, F. V. M., fragm. II, 139 (1861) ... W.A. S.A. - V. N.S. IV. ROCHELIA, Reichenbach in Fl. Regensb, bot. Zeit. I, 243 (1824).

R. Maccoya, F. v. M. in Benth. Fl. Austr. IV, 408 (1869) ‥ - S.A. -- V. N.S.W. CYNOGLOSSUM, Tournefort, inst. 139, t. 57 (1700), from Dioscorides and Nicandros.

C. latifolium, R. Brown, prodr. 495 (1810)...

C. suaveolens, R. Brown, prodr. 495 (1810)

C. australe, R. Brown, prodr. 495 (1810)

C. Drummondii, Bentham, Fl. Austr. IV, 409 (1869) - T. V. N.S.W. Q. S.A. T. V. N.S.W. - - B.f.IV,409 S.A. T. V. N.S.W. $\bar{Q}-$ B.fl.TV, 409 W.A. S.A. - - - - - B.AlIY,409

M.fr. VI, 116 ;IX, 121. M.fr.VI,116, [124 M.fr.VI,116;IX, 121;XI, M.fr. VI, 116;IX, 121 .

\section{M.fr. VI, 115.}

\section{M.fr. VI, 115.}

M.fr.VI, 115 .

M.fr.VI, $115 ; \mathrm{IX}, 122$.

M.fr. I, 209; VI, 115;XI, 125

M.fr. XI, 125

I.fr. X, 121 .

M.fr.VI, 116;IX, 122 .

M.fr. VI, $115 ; \mathrm{IX}, 122$.

- B.fl.IV,406

M.fr.XI, 125.

B.A.IV, 407

M.fr. VI, 116;IX, 121.

- B.A.IV, 408

M.fr.I,127;IX, 121.

M.fr. VI, 115;IX, 122;XI,

M.fr. VI, $115 ; \mathrm{IX}, 122$.

M.fr. TI, 115;IX, 122.

M.fr. IX, 122 .

\section{LABIATAE.}

Adanson, Famill. cles plant. II, 180 (1763), from B. de Jussieu (1759).

OCIMUM, Tournefort, inst. 203, t. 96 (1700), from Theophrastos and Dioscoricles, (Ocymum.)

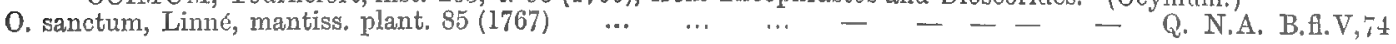
MOSCHOSMA, Reichenbach, conspect. regn. vegetab. 115 (1828). (Basilicum 1802.)

M. polystachya, Bentham in Wall, pl. As. rar. II, 13 (1831) $\ldots-\ldots-\ldots-$ N.S.W. Q. N.A. B.A.T,75 ORTHOSIPHON, Bentham, Labiatar. gen, et spec. 25 (1832).

O. stamineus, Bentham in Wall. pl. As, rar. II, 15 (1831) ...
PLECTRANTHUS, L'Héritier, stirp. nov, I, 85 (1785).

P. longicornis, F. v. M., fragm. V, 51 (1865)

P. parviflorus, Willdenow, hort. Berol. I, t. $65(1806) \ldots$

P. congestus, R. Brown, prodr. 506 (1810)...

$P$. foetidus, Bentham, labiat. 35 (1832)

COLEUS, Loureiro, Fl. Cochinch. II, 372 (1790).

C. scutellarioides, Bentham in Wall. pl. Asiat. rar. II, 16 (1831)

POGOSTEMON, Desfontaines in Mém. du Mus. II, 154, t. 6 (181j). (Dysophyllia.)

P. verticillatus, Hasskarl, catal, hort. Bogor. 131 (1844)

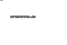

$-\cdot$

- S.A. - V. N.S.W.

$-$

$-\quad-\quad$ - Q. N.A. B.A.T,76

(1) - B.H.Y,77

Q. N.A. B.f.Y,78

(i) - B.fl. T, 7S

Q. N.A. B.fl. T, 79

Q. N.A. B.․ 1,81
M.fr.IV, 46 .

M.fr. TI, 108.

M.fr. VI, 108;IX, 161 .

M.fr. V,5l.

MI.fr. YI, 108;IX, 161 .

M.fr. II, 10s.

M. fr. IX, $161 ; \mathrm{NI}, 135$.

M.fr. VI, 108.

M.fr. V, 200;IX, 161 . 
MENTHA, Tournefort, inst. 188, t. 89 (1700), from Hippocrates and Theophrastos.

M. laxiflora, Bentham in De Cand. XII, $174(1848) \quad \ldots \quad \ldots-\ldots-$ - - V. N.S.W. - - B.fl.V.82

M. grandiflora, Bentham in Mitch. Trop. Austr. 362 (1848) ... - - - - N.S.TV. Q. - B.f.V,B3

M. australis, R. Brown, prodr. $505(1810) \ldots \quad \ldots \quad \ldots \ldots \quad \ldots \quad-\quad$ S.A. T. V. N.S.W. Q. N.A. B.f.V,83

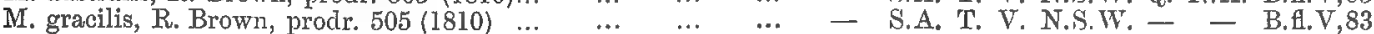

M. saturejoides, R. Brown, prodr. 505 (1810) $\quad \ldots \quad$... $\quad$... W.A. S.A. T. V. N.S.W. Q. - B.fl.V,84 LYCOPUS, Tournefort, inst. 180, t. $83(1700)$, from Plinius.

L. australis, R. Brown, prodr. $500(1810) \ldots \ldots \ldots \ldots$... $\ldots . .$. S.A. T. V. N.S.W. Q. - B.fl.V.85 SALVIA, Linné, gen. plant. 6 (1737), from Plinius.

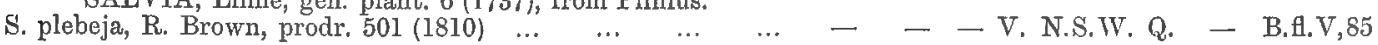
BRUNELLA, L'Ecluse, rar. stirp. hist. II, 42, 43 (1576), from Brunfels (1536). (Prunella.)

B. vulgaris, A. de Candolle, prodr. XII, $410(1848)$.. $\ldots-$ - S.A. T. V. N.S.W. Q. - B.fl.V,87 SCUTELLARIA, Hermann, hort. Lugd. bot. cat. (1687), from Cortusi (1591).

S. mollis, R. Brown, prodr. 507 (1810) $\ldots \begin{array}{llllll}\ldots & \ldots & \ldots & \ldots & \ldots & -\end{array}-$ V. N.S.W. $-\quad-\quad$ B.fl.V,88

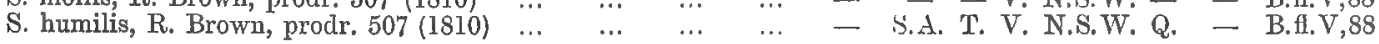
ANISOMELES, R. Brown, prodr. 503 (1810).

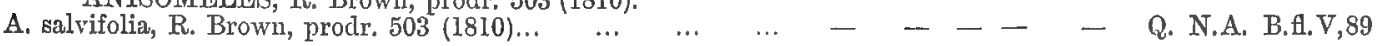
LEUCAS, J. Burmann, thesaur. Zeyl. 140 (1737).

L. flaccida, R. Brown, prodr. $505(\mathbf{1 8 1 0 )} \quad \ldots \quad \ldots \quad \ldots$ DEPREMIESNILIA, F, v. M., fragm. X, 59 (1876).

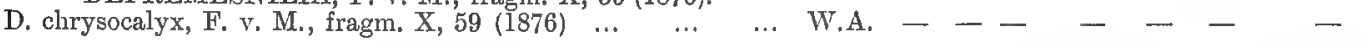
PROSTANTHERA, Labillardière, Nov. Holl. pl. spec. 18, t. 157 (1806). (Chilodia, Cryphia, Klanderia.) P. lasiantha, Labillard., Nov. Holl, pl. spec. II, 18, t. $15(1806)$ - S.A. T. V. N.S.W. Q. - B.H.V,93 P. prunelloides, B. Brown, prodr. 508 (1810)

P. coerulea, R. Brown, prodr. $508(1810) \ldots$
P. ovalifolia, R. Brown, prodr. $509(1810) \ldots$

P. melissifolia, F. v. M., fragm. I, 19 (1858)

P. incisa, R. Brown, prodr. 509 (1810)

P. rotundifolia, R. Brown, prodr. 509 (1810)

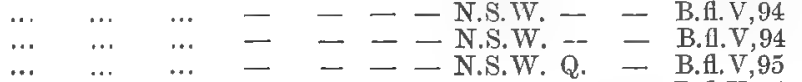

P. violacea, $R$. Brown, prodr. 509 (1810) ...

P. incana, Cunningham in Benth. lab. 455 (1836)..

P. hirtula, F. v. M., first gen. Report 16 (1853) ...

P. denticulata, R. Brown, prodr. 509 (1810)

P. rugosa, Cunningham in Benth. lab. 456 (1836)...

P. marifolia, R. Brown, prodr. 509 (1810) ...

P. marifolia, R. Brown, prodr. 509 (1810) ...
P. rhombea, R. Brown, prodr. 509 (1810) ...
P. spinosa, F. v. M. in Transact. phil. Soc. Vict. I, 48 (1S5̌4) ...

P. cuneata, Bentham in De Cand., prodr. XII, 560 (1848)

P. linearis, R. Brown, prodr. 509 (1810)

P. phylicifolia, F. v. M., fragm. I, 19 (1858)

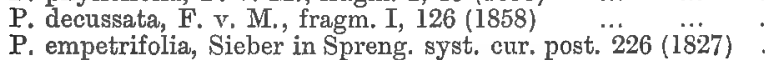

P. lithospermoides, F. v. M., fragm. VI, 107 (1868)

P. Behriana, Schlechtendal, Linnaea XX, $610(1847)$
P. Wilkieana, F. v. M., fragm. VIII, 230 (1874)...

P. Baxteri, Cunningham in Benth. lab. 452 (1836)

P. canaliculata, F. v. M., fragm. VI, 105 (1868) ...
P. nivea, Cunningham in Benth. lab. 452 (1S36) ...

P. striatiflora, F. v. M. in Schlechtend. Linnaea XXV, 425 (1852)

P. saxicola, R. Brown, prodr. 509 (1810) ...

P. debilis, F. v. M., fragm. VIII, $147(1874) \quad \ldots \quad \ldots$

P. Eckersleyana, F. v. M., fragm. X, 17 (1876) ...

P. euphrasioides, Bentham in Mitch. Trop. Austr. 360 (i848) ...

P. staurophylla, E. v. M., fragm. IX, 73 (1875) ...

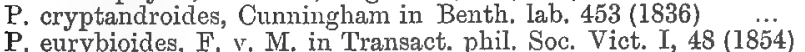

P. ringens, Bentham in Mitch. Trop. Austr. 363 (1848) ... ...

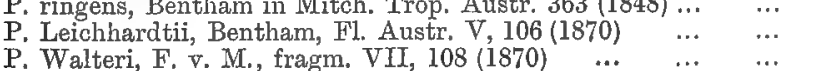

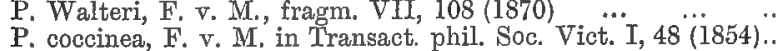

P. chlorantha, F. v. M. in Benth. Fl. Austr. V, 108 (1870)

P. calycina, F. v. M. in Benth. Fl. Austr. V, 107 (1870)

P. Grylloana, F. v. M., fragm. X, 17 (1876) WRIXONIA, F. v. M., fragm. X, 18 (1876).

W. prostantheroides, F. v. M., fragm. X, 18 (1876) HEMIGENIA, R. Brown, prodr. 502 (1810).

(Hemiandra, Colobandra, Atelandra.)

H. macrantha, F. v. M., fragm. I, 210 (1859)

H. rigida, Bentham in De Cand. prodr. XII, 563 (1848)...

H. ramosissima, Bentham in De Cand. prodr. XII, 565 (IS4S) ...

H. microphylla, Bentham in De Cand. prodr. XII, 565 (1848) ...

H. incana, Bentham in De Cand. prodr. XII, 566 (1848)

H. canescens, Bentham in De Cand. prodr. XII, 566 (1848) ...

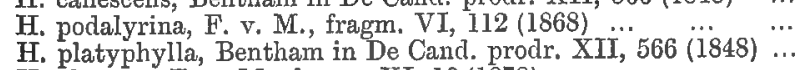

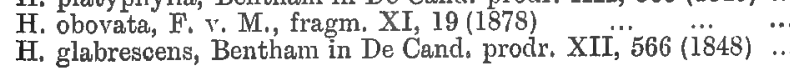

W.A.

W.A.

W.A.

W.A.

W.A.

W.A.

W.A.

W.A.

W.A.
- - V.

- - N - D

S.A. T. V. N.S.W. -

- - - N.S.W. -

- - V. N.S.W. -

- - V. N.S.W. -

- - - N.S.W.

- - - N.S.W. -

- - - N.S.W. -

- T. V. N.S.W. -

- - - V. N.S.W.W. $\overline{\mathrm{Q}}$

- - N.S.W. Q.

- - - N.S.W. -

S.A. - V. N.S.W. -

S.A. - - N.S. -

A. S.A. - - - -

- - - V. N.S.W. $\bar{Q}$.

S.A. - - N.S.W. Q.

- - V. N.S.W. Q.

- 二

$$
\mathrm{s}
$$$$
\text { S.A }
$$$$
\text { A. }
$$

- - N N.S.W. $\bar{Q}$.

S.A.

S.A. - V. N.S.W. Q.

S.A. - V. N.S.IV. -

A. - -

-
B.H.V.95
- B.fl.V.95

- B.f.V,96

- B.H.V,96

- B.fl.V,97

- B.ff.V.97

- B.tl.V,97

- B.fl.V,98

- B.fl.V,98

- B.fl. V,99

- B.H.V,99

- B.fl.V,99

- B.fl. V,100

- B.fl. V,100

- B.fl.V100

- B.fi. V,101

- B.fl.V,101

- B.fl.V,102

- B.fl. V, 102

B.fl. V,102

B.f.V, 103

A. B.fl. V, 103

B. H. V,104

- B.fi. V,104

- B.t. $\overline{\mathrm{V}}, 104$

- B.t. V, 105

- B.fl.V,105

- B.f.V,106

- B.H.V,106

- B.fl. V,106

- B.fl.V,108

- B.H.V,107

$-$

-

B.fl. V, 112

B.fl. V,112

B.fl.V,113

B. fl. V, 113

B.fl.V, 113

- B.fl.V,114

- B.f.V, 114

- B.H.V,115

- B. A, $\overline{\mathrm{V}}, 1] \mathrm{J}$
M.fr. VI, 109;IX, 162.

M.fr.VI, 109;IX, 162 .

M.fr.VI, 109 .

M.fr.VI, 109 .

M.fr.VI, 109 .

M.fr. VI, 109;IX, 161 .

M.fr, VI, 109.

M.fr. VI, 109.

M.fr.VI, 109.

M.fr. VI, 109;IX, 162.

M.fr. VI, 109.

AI.fr. X, 59.

M. fr. VI, 105 .

M.fr. VI, 106.

M.fr. VI, 106.

M.fr. VI, 106 .

M.fr.I, 19,242.

M.fr. VI, 107;IX, 162 .

M.fr. VI, 107; IX, 162 .

M.fr. VI, 107;IX, 162 .

M.fr.IX, 162 .

M.fr. VI, 105.

M.fr.VI, 105.

M.fr. VI, 108;IX, 162.

M. fr. VI, 108 .

M.fr.VI, 106.

M.fr.I, 19;IX, 162.

M.fr.I, 126.

M.fr. VI, 107.

M.fr.VI, 106.

M.fr. VIII, 230.

M.fr. VI, 106.

M.fr.VI, 105.

M.fr.VI, 106 .

M.fr.VI,105;IX, 162.

M.fr.VI, 107;IX, 162.

M.fr. VIII, 147.

M.fr.X, 17 .

M.fr. VI, 108 .

M.fr.IX, 73.

M.fr.VI,105.

M.fr. VII, 108.

M.fr. VI, 108 ;IX, 162 .

M.fr.IX, 162;X, 18 .

M.fr, X, 17 .

M.fr.X, 18.

M. fr.I,210;XI, 19.

M.fr. VI,112;IX, 162 .

M. fr. VI, 112 .

B. $\mathrm{fl}, \mathrm{XI}, 10$ 
H. obtusa, Bentham in De Cand. prodr. XII, 567 (1848)..

H. sericea, Bentham in Hueg. enum. 80 (1837) ... ...

H. barbata, Bartling in Lehm, pl. Preiss. I, 360 (1845) ...

H. curvifolia, F, v. M., fragm. I, 210 (1859)

H. scabra, Bentham, Fl. Austr. V, $117(1870)$ '..

H. humilis, Bentham in De Cand. prodr. V, 117 (1870) ...

H, westringioides, Bentham in De Cand. prodr. XII, 568 (1848)

H. teretiuscula, F. v. M., fragm. VI, 111 (1868) ... ...

H. brachyphylla, F. v. M., fragm. X, 19 (IS76) ..

H. purpurea, R. Brown, prodr. 502 (1810) .

H. cuneifolia, Bentham, Fl. Austr. V, 11 S (1870)...

H. Drummondii, Bentham, Fl. Austr. V, 119 (1870)

H. pimelifolia, F. v. M., fragm. VI, 112 (1868)

H. diplanthera, F. v. M., fragm. VI, 111 (1868) ...

H. pungens, F, v. M., fragm. XI, 20 (1878)

H. leiantha, F. v. M., fragm. XI, 20 (1878)

H. loganiacea, F. v. M., fragm. XI, 19 (1878) MICROCORYS, R. Brown, prodr. 502 (1810). (An

M. longifolia, Benthan in De Cand, prodr. XII, 568 (1848)

M. longiflora, F. v. M., fragm. VI, $113(1868) \ldots$

M. capitata, Bentham in De Cand. prodr. XII, 568 (1848)

M. pimeloides, F. v. M., fragm. I, 156 (1859)

M. subcanescens, Bentham, Fl. Austr. V, 123 (1870) ...

M. ericifolia, Bentham in De Cand. prodx. XII, 569 (1848)

M. glabra, Bentham in De Cand, prodr. XII, 569 (1848)..

M. exserta, Bentham, Fl. Austr. V, 124 (1870)

M. virgata, R. Brown, prodr. 502 (1810) .

M. Macrediana, F. v. M., fragm. VIII, 231 (1874)

M. barbata, R. Brown, prodr. 502 (1810)

M. lenticularis, F. v. M., fragn. VI, 113 (1868) ...

M. obovata, Bentham in De Cand. prodr. XII, 569 (1848)

M. purpurea, R. Brown, prodr. 502 (1810)...

WESTRINGIA, Smith in Kongl. Svenska Vetenskaps Akad. Handlingar 171 (1797).

W. cephalantha, F. v. M., fragm. VI, 110 (1868)... ... ... W.A. - _ - _

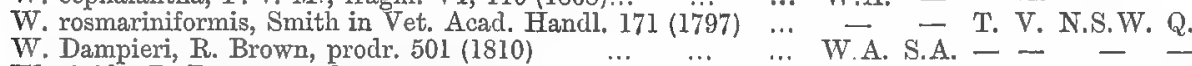

W. rigida, $R$. Brown, prodr. 501 (1810)

W. angustifolia, R. Brown, prodr. 501 (1810) $\ldots . .$.
W. senifolia, F, v. M. in Transact. phil. Soc. Vict. I, $49(1854)$

W. longifolia, R. Brown, prodr. 501 (1810)

W. glabra, R. Brown, prodr. 501 (1810)

AJUGA, Linné, gen. plant. $167(1737)$, from Scribonius (1529).

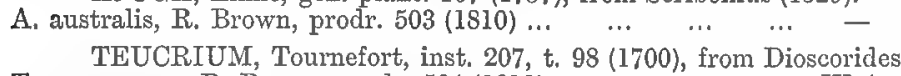

T. racemosum, R. Brown, prodr. 504 (1810) … … … W.A. S.A. - V. N.S. W. Q. N.A. B.fl.V,133 T. integrifolium, F. v. M. in Benth. Fl. Austr, $\dddot{V}, 133$ (1870) ... - S.A. - - - Q. N.A. B.f.V,133

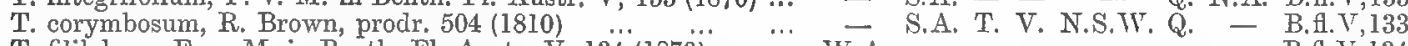

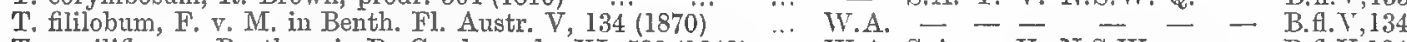

T. sessiliflorum, Bentham in De Cand, prodr. XI, 580 (1848) $\quad$.. $\quad$ W.A. S.A. - V. N.S.W. - - B.fl.V.134 T. argutum, R. Brown, prodr. 504 (1810) ...

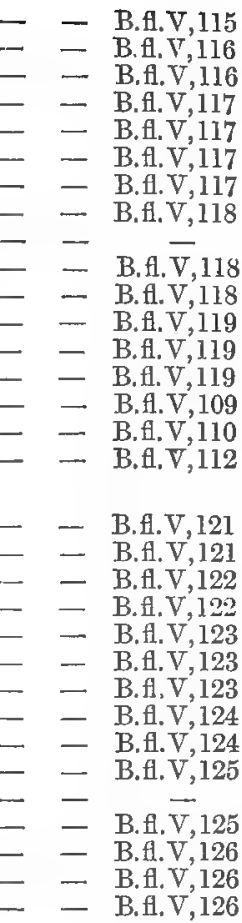

- B.fl.V,127

B. A.V, 128

- B.fl. V, 129

- B.H.V,129

- B.f. $\bar{V}, 130$

B.fl.V,131

- B.fl. V,131

- B.H. V,131

S.A. T. V. N.S.W. Q.
S.A. - V. N.S.W. Q.
S.A. - -
S.A. T. V. N.S.W. Q.
S.A. - - V. N.S.W. ... - - - N.S.W. Q. - B.fl.V,135
M.fr. VI,112.

M.fr.I,2]0.

M.fr.VI, 111 .

M.fr.X, 19 .

M.fr.VI, 112 .

M.fr. VI, 112 .

M.fr. VI, 111 .

M. fr. VI, 113.

M.fr. VI,113.

M.fr.I, 156.

M.fr.VIII,231.

M.fr. VI,113.

M.fr.VI, 110 .

M.fr. VI, 110;IX, 162 .

M.fr.VI, I10;IX, 163 .

M. fr.IX, 163 .

M.fr.VI, 110;IX, 163.

M.fr. VI, 110;IX, 163 .

M.fr.VI,110;IX,163.

M.fr. VI, 110;IX, 163 .

M.fr.VI, 110 .

M.fr. VI, 109.

M.fr. VI, 109;IX,162.

M.fr.IX, 162 .

M.fr. VI, 110 .

M.fr.IX, 162 .

M.fr.VI,110.

I. fr. VI, 110;IX, 162.

\section{VERBENACEAE.}

Adanson, Familles des plantes II, 195 (1763), from B. de Jussieu (1759).

SPARTOTHAMNUS, Cunningham in Loudon's hort. Brit. suppl. (1830).

S. junceus, Cunningham in Loud. hort. Brit. 600 (1830)...

S. teucrifforus, F. V. M. in Wing's S. Sc. Rec. II, 55 (1882) $\ldots-$ - - S.A. - - N.S.W. Q. N.A. B.H.V.55

MI.fr. VI, 153;IX, 5 .

NESOGENES, A. de Candolle, prodr. XI, 703 (1847).

N. enphrasioides, A. de Cand., prodr. XI, 703 (1847)

LIPPIA, Houston in Linné, syst. nat. 8 (1735); Limné, gen. plant. 347 (1737). (Zapania.)

L. nodiflora, Cl. Richard in Mich. A, bor. Amer. II, 15 (1803) … W.A. - - - N.S.W. Q. N.A. B.Al.V,35

L. geminata, Hunboldt, Bonpland et Kunth, nov. gen, et spec. pl.

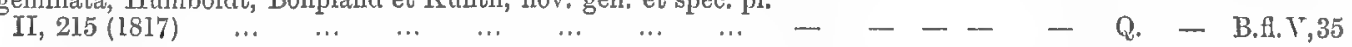

VERBENA, Toumefort, inst. 200, t. 94 (1700), from l'Ecluse (1576).

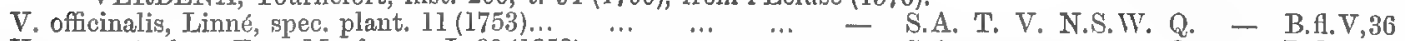

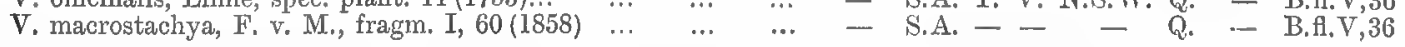

LACHNOSTACHXS, Hooker, icon, plant. t. 414 (1842). (Walcottia, Pycnolachme.)

L. albicans, Hooker, icon. plant. $414(1842)$
L. Cliftoni, F. v. M., fragm. IX, $3(1875) \ldots$
...

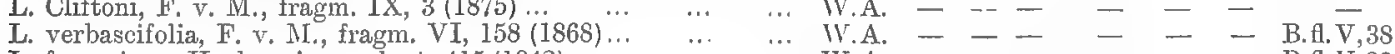

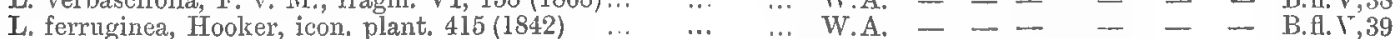

L. Walcottii, F. v. M., fragm. II, $140(1861) \quad \ldots \quad \ldots \ldots \ldots$ W.A. $\quad \ldots \quad-\quad \ldots \quad-\quad$ - B.E.V,39

NEWCASTLIA, F. v. M. in Hook. Kew Misc. IX, 2:2 (1857).

N. cladotricha, F. V. M. in Hook. Kew Misc. IX, $22(1857) \ldots \ldots-$ S.A.
N. bracteosa, F. v. M., fragm. VIII, $49(1873) \ldots \ldots \ldots \ldots$-...

M.fr.VI, $151 ; \mathrm{IX}, 4$.

M.fr. Y I, 151 .

M.fr. VI, $153 ; I X, 5$.

M.fr. I, 60;IX, 5 .

MI.fr. Y 159 .

M.fr.IX, 3 .

M. fr. VI, 15 ; $; \mathrm{IX}, 4$.

M.fr.VI, 159 .

M.fr.II, 140;VI, 159.

M. fr. I, 184;III, 21;VI,155.

M. fr. VIII, 230;IX, 4 . 
N. chrysotricha, F. v. M., fragm. X, $15(1876) \ldots$

N. hexarrhena, F. v. M., fragm. X, $16(1876)$
N. spodiotricha, F. v. M., fragm. III, 21, t. 21 (1862) $\ldots$

N. cephalantha, F. v. M., fragm. IX, 4 (1875) ... ...

PHYSOPSIS, Turczaninow in Bullet. de la Soc. Impe

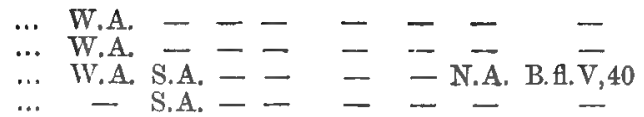

P. spicata, Turczaninow in Bull. de Mosc. XXII, 35 (1849) MALLOPHORA, Endlicher in Annal. des Wien. Mus.

er. des

206

Natur. de Mosc. XXII, 34 (1849).

DICRASTYLIS, Drummond \& Harvey in Hook. Kew Misc. VII, 56 (1855). (Dicrastyles.)

D. Doranii, F. v. M., fragm. VIII, 230 (1874)

D. ochrotricha, F. V. M., fragm. IV, 161 (1864)

D. fulva, Drummond \& Harvey in Kew Misc. VII, 56 (18̈55)

D. reticulata, Drum. \& Harvey in Hook. Kew Misc. VII, 57 (1855)

D. Nicholasii, F. v. M., fragm. X, 15 (1876)

D. parvifolia, F. v. M. fragm. II, 16 (1861)

D. Beveridgei, F, v. M., fragm. VIII, 50 (1873) .

D. Stoechas, Drummond \& Harvey in Kew Misc $\dddot{V}$. $\dddot{7}$

D. Lewellini, F. v. M., fragm. XI, 86 (1880) CHLOANTHES, R. Brown, prodr. Flor. Nov. Holl. 513 (1810)

C. Stoechadis, $\mathrm{R}$ Brown, prodr. 514 (1810)

C. parviflora, Walpers, repertor. bot. syst. IV, 58 (1848)

C. coccinea, Bartling in Lehm. pl. Preiss. I, 352 (1845) ...
C. salvifolia, F. v. M.; Pityrodia, R. Brown, prodr. $513(1810) .$.

C. hemigenioides, F. v. M., fragm. VI, 156 (1868)

C. uncinata, Turczaninow in Bull, de Mosc. XXXVI, 194 (1863)

C. Bartlingii, Lehmann, Ind. sem. hort. Hamb. (1844) ..

C. verbascina, F. v. M., fragm. I, 233 (1859)

C. stachyodes, F. v. M., fragm. V, 50 (1855)

C. loxocarpa, F. v. M., fragm. II, 22 (1861)

C. dilatata, F. v. M., fragm. VI, 157 (1868)

C. cuneata, F.v.M.; Quoya, Gaudich. in Freyc. voy. 454 t. 66 (] 826) W.A.

C. Oldfieldii, F. v. M., fragm. I, 234 (1859)

C. atriplicina, F. v. M., fragm. I, 235 (1859).

C. paniculata, F. v. M., fragm. IV, 80 (I864)

C. loricata, F. v. M., fragm. X, 14 (1876) ...

$\begin{array}{llll}\ldots & \ldots & \ldots & \text { W.A. } \\ \ldots & \ldots & \ldots & \text { W.A. }\end{array}$

$\cdots$

S.A. - -

S.A. - -

-

— N.A. B.fl. V.,42

- - B.f. $\bar{V}, 43$

W.A. - - - - - - - - B.f.V.,43
W.A.V.43

W.A. - - -

W.A. S.A. - -

- - - B.fl., 43

$=--$

- $\quad$ B.fl. $, 4,44$

C. halganiacea, F. v. M., fragm. X, 14 (1876) HEMIPHORA, F. v. M., fragm. X, 13 (1876).

H. Elderi, F. v. M., fragm. X, $13(1876)$... ... DENISONIA, F. v. M., fragm. I, 124 (1859).

D. ternifolia, F. v. M., fragm. I, 124, t. 2 (1859) ... CYANOSTEGIA, Turczaninow in Bull. Soc. Mosc. XXII, 35 (1849). (Bunnya.)

C. Turczaninovii, F. v. M., fragm. VI, 154 (1868)
C. Bunnyana, F. v. M., fragm. V, 36 (1865) CALIICARPA, Linné in act. litter. et scienc. Upsal, 80 (1741).

C. cana, Linné, mantiss, plant. alt. 198 (1771)

C. pedunculata, R. Brown, prodr. 513 (1810)

C. longifolia, Lamarck, Encycl. méth. I, 562 (1783̈) PREMNA, Linné, mantiss. plant. alter. 154 (1771).

P. obtusifolia, R. Brown, prodr, 512 (1810)

P. integrifolia, Linne, mant. plant. alt. 154 (1771)

P. limbata, Bentham, F1. Austr. V, 59 (1870)
P. Dallachyana, Bentham, F1. Austr. V, 59 (1870)

P. acuminata, R. Brown, prodr. 512 (1810) CTERODENDRUTM, Burmann, thesaur Zeylan 66, t 29 (1737)

C. hemiderma, F. v. M. in Benth. FI. Austral. V, 61 (1870)

C. inerme, R. Brown, prodr. 511 (1810)

C. Tracyanum, F. v. M. in Benth. Fl. Austr. V, 62 (1870)

C. tomentosum, R. Brown, prodr. 510 (1810)

C. lanceolatum, F. v. M., fragm. III, 145 (1863) ...

C. floribundum, R. Brown, prodr. 511 (1810)

C. Cunninghamii, Bentham, Fl. Austr. V, 64 (1870)

C. costatum, R. Brown, prodr. 511 (1810) ... GMELINA, Linné, gen. plant. ed. sec. 526 (1742).

G. macrophylla, Bentham, Fl. Austr. V, 65 (1870)

G. fasciculiflora, Bentham, Fl. Austr. V, 65 (1870)

G. Leichhardtii, F. v. M. in Benth. El. Austr. V, 66 (18̈70) VITEX, Tournefort, inst. 603, t. 373 (1700), from Plinius.

V. trifolia, Linné, spec. plant. 938 (1753)

V. Lignum vitae, Cunningham in De Cand. prodr. XI, 692 (1847)

V. acuminata, R. Brown, prodr. 512 (1810)

V. glabrata, R. Brown prodr. 512 (1810) ...

$\cdots \quad \cdots \quad \ldots$$$
\cdots, \quad \cdots \quad \cdots
$$

FARADAYA, F, v. M., fragm. phytogr. Austr. V, 21 (1865).

F. splendida, F. v. M., fragm. V, 21 (1865) AVICENNIA, Linne, syst. nat. $8(1$
(1)
... W.A. - - -

W.A.

... W.A.

(Pithyrodia, Quoya, Dasymalla.)

- - N.S.W. - - B.f.V,45 Q. - B.f.V,46

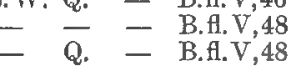
- $\quad$ N.A. B.f.V, 48

- - - B.fl.V,48

- - - B.fl.V,49

- - - B.f.V,50

- - N.A. B.fl.V,51

- - - B.fl.V,5l

- - - B.f.V,5l

- - - B.H.V,52

- - N.A. B.fl.V,52

- - - B.fl.V,53

- - - -

W.A. - - - - - - -

- N.A. B.f.V,54

- - - - N.A. B.f.V, B.fl.V,54
- - - - Q. N.A. B.fl.V,56

- - - - - N.A. B. B.f.V,V,54 - - - N.S.W. Q. N.A. B.fl.V,56 Q. - B.H.V,57 ... - - - - - Q. N.A. B.fl.V,58 ... - $. . \quad-\quad-$ - N.A. B.f.V,59 .. $\quad$.. $-\quad--$ - Q. - B.f.V,59 Q. - B. B. B., 59 N.A. B. H.V, 60 (Clerodendron.)

- - - - - Q. - B.fl.V,61 .. - - - - N.S.W. Q. N.A. B.fl.V,61 - - - - - Q. - B.fl.V,62 W.A. - - - N.S.W. Q. - B. B.V,62 .. - S.A. - - N.S.W. Q. N.A. B.fl.V,63 .. - - - - - Q. N.A. B.fl.V,64

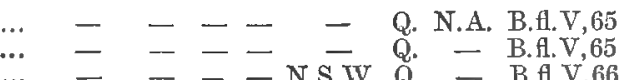
- - - - N.S.W. Q. N.A. B.fl.V,66 - - - - - N.S.W.W.Q. N.A. B.f.V,66 - - - - - Q. Q. N.A. B.H.V,67 - - - N.S.W. Q. N.A. B.t.V,68 - - - — Q. - B.f.V,69 27 (1737).

plant. $27(1737)$.
... W.A. S.A. - V. N.S.W. Q. N.A. B.A. V, 69
- - - B.fl.V,50
M.fr.X, 15 .

M.fr.X, 16 .

M.fr.VIII, $230 ; I X, 4$.

II.fr.IX, 4 .

M.fr.VI, 154 .

M.fr.VI, 154;X,15.

M.fr.VIII, 230.

M.fr.I.60;VI,155;VIII; 230

M.fr.X, 15 .

M.fr.I, 236;VI, 155 .

M.fr.VI, 155;IX, 4 .

M.fr.X, 15 .

M.fr. VI, 155;X, 15.

M.fr. IX, 4 .

M.fr.VI, 155.

M.fr.VIII, 50;XI, 137.

M.fr.VI, 156.

M.fr.IX, 5.

M.fr.VI, 156;IX,5.

M.fr.VI, 155;IX, 5 .

M fr. VI, 156.

M.fr.VI, 156 .

M.fr.VI, 156 .

M.fr.IV, 80;VI, $157 ; \mathrm{IX}, 5$.

M.fr.VI, 158 .

M.fr IV, 80;VI, 156.

M.fr. VI, 157 .

M. fr. IV, 80 .

M.fr. VI, $15 \overline{7} ; \mathrm{IX}, 5$.

M.fr.X,14.

M.fr. X, 14 .

M.fr, X, 13.

M.fr. I, 124 .

M.fr.IX, 5.

M.fr. VI, 153.

M.fr.III, 36.

M.fr.VI, 153.

M.fr.III, 36.

M.fr.VI, $151 ; I X, 5$.

M.fr.VI, 152 .

M.fr. V.61.

M.fr. VI, 152 .

M.fr.III, 145 .

M.fr. III, 145;IX, 5.

M.fr.IV,128.

M.fr.III, 58, 158;VI, 153.

M.fr. III, 59;VI, 152;IX, 5.

M.fr.III, 58;IX, 5 .

M.fr. V, 34; VI, 152, IX, 5.

M.fr.VI, 153 .

M.fr. YI, 153. 


\section{MYOPORINAE.}

R. Brown, prodr. 514 (1810).

MYOPORUM, Banks \& Solander in G. Forster, prodr, 44 (1786). (Bertolonia, Andrewsia, Pogonia, Disoon.) M. tenuifolium, G. Forster, A. ins. Austr. prodr. $44(1786) \quad \ldots$
M. montanum, R. Brown, prodr. 515 (1810) M. deserti, Cunningham in Hueg. enum. 78 (1837) $\quad \ldots . \quad \ldots$ W.A. S.A. - V. N.S.W. Q. - B.A.V,5

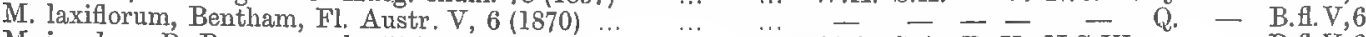

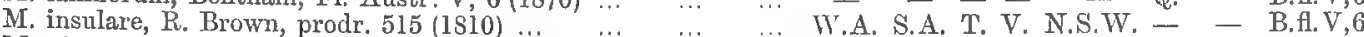

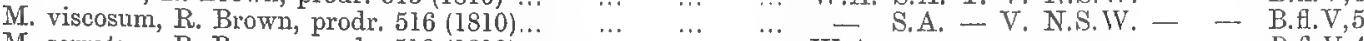

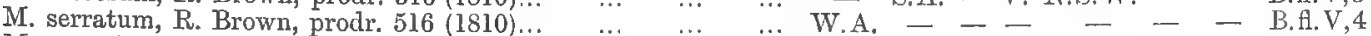

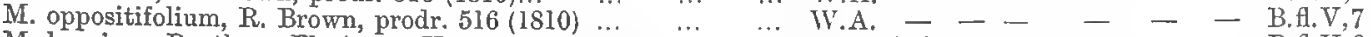

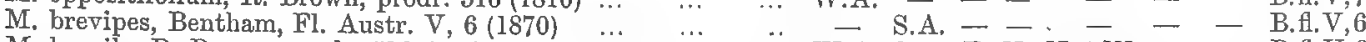

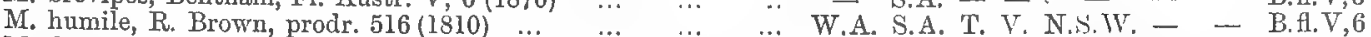

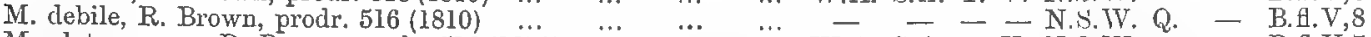

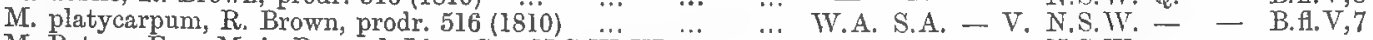
M. Bateae, W. v. M. in Proceed. Lim. Soc. N.S. W.VI, $792(1881)=-1$ - $=-$ N.S.W. $=-$ V. N.S.W. - B.fl. V.8 M. Beckeri, F. v. M. in Benth. Fl. Austr. V, 7 (1870) ... $\quad \ldots$ w.A. $=--{ }_{-}=-$B.fl.V,7

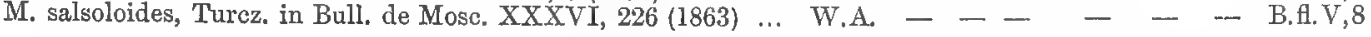

M.fr.VI, 149 . M.fr.VI, 149 . M.fr.VI,148;VII, I10. M.fr.V, 23. M.fr.VI,149;VII,110. M.fr. VII, 109 . M.fr.VI, 149 . M.fr.VI, 149 .

M.fr.VI, 150 . M.fr.VI, 149 . M.fr. VI, 150 . M.fr.XI, 139 .

II.fr.I, 126; VI, 150 . II.fr.I, 126,244;VI, 150 . M.fr.I, 126; VI, 150. EREMOPHILA, R. Brown, prodr. 518 (1810). (Pholidia, Stenochilns, Eremodendron, Pseudopholidia, Sentis, Duttonia, Pholidiopsis,

E. Mackinlayi, F. จ. M., fragm. IV, 80 (1864)

E. Bowmani, F. v. M., fragm. II, 139 (1S63)

E. strongylophylla, F. v. M., fragm. X, $87(1876)$
E. eucophylla, Bentham, Fl. Austr. V, $18(1870)$

E. Turtoni, F. v. M., fragm. X, $87(1876)$...

E. Forrestii, F. v. M., fragm. VII, 49 (1869)

E. eriocalyx, F. v. M., fragm. I, 236 (1859)

E. Maitlandi, F. v, M. in Benth. Fl. Austr. V, $19(1870)$

F. rotundifolia, F. v. M., fragm. I, 207 (1859)

E. Mitchelli, Bentham in Mitch. Trop. Austr. 31 (1848)

E. Paisleyi, F. v. M., Rep. Babb. Exped. 17 (1858)

E. Sturtii, R. Brown, App. to Sturt's Exped. 22 (1849)...

E. exilifolia, F. v. M., fragm. $X, 88$ (1876)

E. Dempsteri, F. v. M., fragm. X, 60 (1876)

E. Gibsoni, F. v. M., fragm. VIII, 227 (1874)

E. Berryi, F. v. M., fragm. VIII, $228(1874)$
E. Clarkei, F. v. M., fragm. I, 208 (1859) $\ldots$..

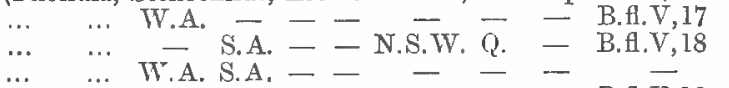

...... W.A. - - - $-二-$ B.H. $\bar{v}, 18$

a. $\quad \cdots$

W.A. S.A --

W.A. $=-二$

-

... W.A.

‥ S.A. $=\overline{\mathrm{V}}$. N.S.W.

$\cdots=-$ - - N.S.W. Q

$\cdots$
$\cdots$ - S.A. $=$ S.A. $=$ N.S.W.

$\cdots$

$$
\text { W }
$$$$
\text { - S.A. - - }
$$$$
\text { 二 }
$$

W.A. S.A. - -

W.A. - =

$=$

$-=$
-
-
-
-

E. Latrobei, F. v. M. in Proceed. Roy, Soc. Tasm. III, 294 (180̈8) W.A. S.A. $=-$ N.S.W. $\overline{\text { Q. N. N.A. }}$

E. gracilitlora, F. v. M., fragm. I, 208 (1858)

E. longifolia, F. v. M. in Proceed. Roy. Soc. Tasm. III, 295 (1858)

F. Drummondii, F. v. M., fragm. VI, 147 (1868)...

W.A.

W.A. S.A. - V. N.S.W. Q. N.A. B.fl.V,23

W.A. - - - - - B.fl.V,24

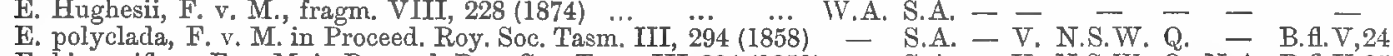

E. bignoniflora, F. v. M. in Proceed. Roy. Soc. Tasm. III, $29 \pm(1858)$ - S.A. - V. N.S.W. Q. N.A. B.fl.V,25

E. Freelingii, F. v. M. in Proceed. Roy. Soc. Tasm. III, 295 (1858) - S.A. - - N.S.W. - - B.fl. V,25

E. Fraseri, F. v. M., fragm. XI, 51 (1878)...

W.A. S.A. - - N.S.W. - N.A.

E. Macdonnelli, F. v. M., Rep. Babb. Exped. 18 (1858)...

E. Goodwinii, F. v. M., Rep. Babb. Exped. 17 (1858) ...

E. Elderi, F. v. M., fragm. VIII, 228 (1874)

E. Willsii, F. v. M., fragm. III, 21 , t. 20 (1862) …

E. platycalyx, F. v. M., fragm. V, 109 (1866)

E. viscida, Endlicher, nov. stirp. dec. 51 (1839) ...

E. Brownii, F. v. M. in Proceed. Roy. Soc. Tasm. III, 297 (1858)

E. subfloceosa, Bentham, Fl. Austr. V, $28(1870) \ldots$

E. Oldfieldii, F. T. M., fragm. I, 208 (1859)

E. Duttonii, F. v. M., Rep. Babb. Exped. 16 (1858)

....

S.A. $=-\overline{-}$ -

-.. S.A. - N.S.W. - - B.H.V,25

$$
\text { ... W W }
$$$$
\text { W.A. S.A. }=-\overline{-}=
$$

W.A. - - - - - - B.fi.V,26

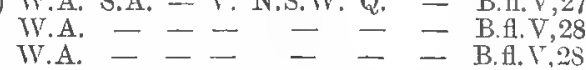

E. maculata, F. v. M. in Proceed. Roy. Soc. Tasm. III, 297 (1858) W.A. S.A. - F. N.S.W. Q. N.A. B.A.V,29

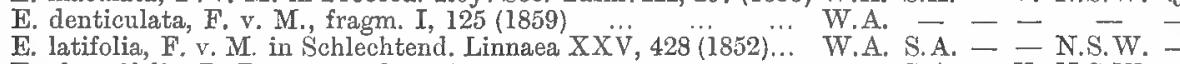

E. alternifolia, R. Brown, prodr. 518 (1810)

E. Dalyana, F. v. M., fragm. V, $22(1865)$

- S.A. - N.S.W. -

E. scoparia, F, v. M. in Proceed. Roy. Soc. Tasm. IIT, $296(1858)$ W. S. - V. N.S.W. -
E. Delisserii, F. v. M., fragm. V, 108, t. $42(1865)$

E. crassifolia, F. v. M. in Proceed. Roy. Soc. Tasm. III, 297 (1858) - S. A. - -

E. resinosa, F. v. M. in Proceed. Roy. Soc. Tasm. III, 296 (1858) W.A. - -

E. Behrii, F. v. M. in Proceed. Roy. Soc. Tasm. III, 296 (1858)

E. Woollsiana, F. v. M., fragm. I, 125, t. 7 (1859)

E. Christophori, F. v. M., fragm. IX, 120 (1875)...

W.A. S.A. $=$

E. brevifolia, F. v. M.; Myoporum, Bartling inpl. Preiss. I, 350(1845) W. A. - - - -
E. Weldii, F. v. M. fragm. VII, 109 (1870)

E. imbricata, F. v. M.; Pholidia, Bentham, Fl. Austr. $\dddot{V}, 13(1870) W . A .-1=-$

E. densifolia, F. v. M., fragm. II, $160(1861)$
E. gibbosifolia, F. v. M., Rep. Babb, Exped. 18 (1858) $\ldots$.

E. divaricata, F. v. M. in Proceed. Roy. Soc. Tasm. III, 293 (1858)

E. microtheca, F. v. M. in Benth. Fl. Austr. V, $14(1870)$

E. adenotricha, F. v. M. in Benth. Fl. Anstr. V, $15(1870)$

W.A.

S.A. - V. N.S.W.

-

$=$

$=$

$-=-$

E. santalina, F, v. M. in Proceed. Roy. Soc. Tasm. III, 295 (1858) - S.A. -

E. Youngii, F. v. M., fragm. X, 16 (1876)...

...

... W.A.

- - -
B.fl. $\bar{V}, 18$

B.fl.V,19

B.H.V, 19

B. H.V, 19

B. $f$. V, 20

B.fl.V,21

B.H.V, 20

B. A.V,, 21

B.fi. $\bar{v}, 22$

B.fl.V,26

B. $A . V, 28$

MI.fr.IV, 80 .

MI.fr.II, 139

M.fr.X,87.

M.fr. X, 87 .

M.fr.I, 236 .

M.fr.I,207.

M.fr.VI, 148 .

M.fr. VI, 148.

M.fr.X,88.

M.fr.X, 60 .

M.fr. VIII, 227.

M.fr.VIII, 228; X,88.

M.fr.I, 208.

M.fr.VIII, 49 .

M.fr.I, 125 .

M.fr.VI, 148.

M.fr.VIII, 228 .

M.fr.VI, 148.

N.fr.VI, 148 .

II.fr.XI,51.

M.fr.VI, 150 .

M. fr.VI, 148.

M.fr.VIII, 228,

M.fr. III, 21

Il.fr. Y, 109.

M.fr, VI, 148.

M.fr.I, 208.

II.fi. IT, 148.

M.fr.IV, 170.

M.fr.VI, 148 .

M.fr.VI, 148 .

MI.fr. $V, 2,2$.

MI. ff. $V, 22 ; Y T$ I, 148.

M.fr. Y, 189.

M..fr. VI, 148.

M.fr. VI, 151

M.fr.IX, 198

M.fr. (I.150)

B. f. $\bar{Y}, 13$

B.f. T, 13

B. fl. $V, 13$

B. f. $\mathrm{V}, \mathrm{I} 4$

B. H. Y, 14

B.fi. V, 14

B. fl. V, 15
M.fr. VII, 49 .

M.fr.VI, 147 .

M.fr. IX, $1 \geq 0$.
M.fr'IT, 160.

M.fr. I I, 150 .

M.fr.IV, $4 \$ ; Y I, 150$.

M.fr. II, 160 .

MI.fr.X, 16 , 
PEDALINAE.

R. Brown, prodr. Fl. Nov. Holl. 519 (1810).

JOSEPHINIA, Ventenat, Jardin de la Malmaison, 103 t. 67 (1804).

J. grandiflora, R. Brown, prodr. 520 (1810)

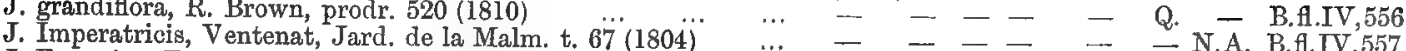

J. Eugeniae, F. v. M. in Hook. Kew Miso. IX, 370, t. 11 (1857) W.A. S.A. $-\overline{-}$ Q. N.A. B.fl.IV,557 M,fr.VI, 163.

\section{ERICACEAE,}

A. L. de Jussieu, gen. plant, 159 (1789).

PERNETTYA, Gaudichaud in Ann. des sc. nat. V, 102 (1825),

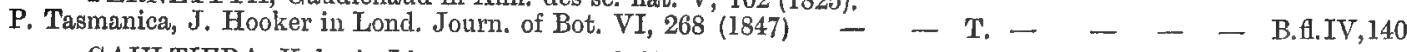

GAULTIERA, Kalm in Linne, amoen. acad. III, 14, fig. 6 (1751). (Gaultheria.)

G. hispida, R. Brown, prodr. 559 (1810) ‥

G. lanceolata, J. Hooker in Lond. Journ. of Bot. VI, 267 (1847) - - T. - - - - B.Al,IV,14L

.. - - T. - - - B.H.IV,142

W. vacciniacea, F. v. M., fragm. II, $136(1861) \ldots \quad \ldots \quad \ldots \quad-\quad-\quad-$ V. $\quad$ - $\quad-\quad$ - B.fl.IV, 139 M.fr.III, 166.

\section{EPACRIDEAE.}

R. Brown, prodr. 535 (1810).

STYPHELIA, Solander in G. Forster, fl. ins. Austr. prodr. 13 (1786). (Epacris, Forst. (1776) partly, Stiphelia, Ardisia, Peroa, Perojoa,

Ventenatia, Cyathodes, Stenanthera, Astroloma, Leucopogon, Melichrus, Acrotriche, Monotoca, Soleniscia, Stomarrhena,

Pentataphrus, Mesotriche, Phanerandra, Froebelia, Pentaptelion, Androstoma, Cyathopsis, Lissanthe partly.)

S. adscendens, R. Brown, prodr. 537 (1810)

S. longifolia, R. Brown, prodr. 537 (1810)...

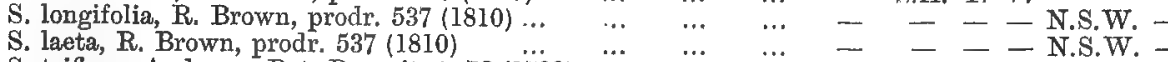

S. triflora, Andrews, Bot. Reposit. t. 72 (1799) $\ldots$... $_{1} \quad \ldots \quad-\quad-\quad-$ - N.S.W. Q.

S. viridis, Andrews, Bot. Reposit. t. 312 (1806) $\ldots$. $\quad \ldots \quad \ldots \quad-\quad-\quad-\quad$ N.S.W. Q

S. Hainesii, F. v. M., fragm. IV, 96, t. 28 (1864)...

S. tubiflora, Smith, Specim. of the Bot. of New Holl. 45,

S. tenuiflora, Lindley, Bot. Regist. XXV, App. XXV (1839)

S. melaleucoides, F. v. M., fragm. IV 97 (18. X

S. melaleucoides, F. V. M., fragm. IV, 97 (1864) ..

S. Leucopogon, F. v. M., fragm. IV, 97 (1864)

S. lasionema, F. v. M., fragm. VI, 40 (1867)

S. macrocalyx, F. v. M., fragm. VI, 37 (1867)

S. xerophylla, F. v. M., fragm. VI, 38 (1867)

S. pentapogonea, F. v. M., fragm. VI, 36 (1867) $\cdots$

S. prostrata, F'. v. M.; Astroloma, R. Brown, prodr. $53 \ddot{8}(1810)$

S. tecta, Sprengel, syst. I, 657 (1825)

S. Candolleana, F. v. M., fragm. VI, 38 (1867) .

S. microdonta; Ástroloma, F.v.M. in Benth. Fl. Austr. IV $\dddot{155}(1869$

S. pallida, Sprengel, syst. I, 658 (1825)

S. compacta, Sprengel, syst. I, 657 (1825) ...

S. humifusa, Persoon, synops. plant. I, $174(1805)$

S. Epacridis, F. v. M., fragm. VI, 38 (1867)

S. Drummondii, F. v. M., fragm. VI, $37(1867)$...

S. microcalyx, F. v. M., fragm. VI, 37 (1867) ...

S. Baxteri, F. v. M. fragm. VI, 37 (1867)...

S. Sonderi, F. v. M., fragm, VI, 36 (1867).

S. longifiora, F. v. M., fragm. VIII, $54(1873)$

S. pinifolia, F. v. M., fragm. VI, $36(1867)$

S. procumbens, Persoon, synops. I, 174 (1805)

S. urceolata, F. v. M., fragm. VI, 38 (1868)

S. Billardieri, F. v. M., fragm. VI, 43 (1867)

S. straminea, Sprengel, syst. I, 656 (1825) ...

S. Hookeri, F. v. M., fragm. VI, 44 (1867)

S. dealbata, Sprengel, syst. I, 659 (1825)

S. abietina, Labillardière, Nov. Holl. pl. spec. I, $\dddot{48}$, t. $\dddot{68}(1804)$

S. Oxycedrus, Labillardiere, Nov. Holl. pl. spec. I, 49, t. 69 (1804)

S. parvifolia, F. v. M. in papers of R. S. Tasm. 86 (1874)

S. sapida, F. v. M., fragm. VI, 42 (1867)

S. strigosa, Smith, Specim. of the Bot. of New Holl. 49 (1793)...

S. verticillata, Sprengel, syst. I, 656 (1825)

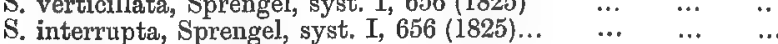

S. amplexicaulis, Rudge in Transact. Linn. Soc. VIII, 292, t. 8. ..

S. alternifolia, Sprengel, syst. I, 655 (1825)

S. lanceolata, Smith, Specim. of the Bot. of New Holl. $\ddot{49}$ (1793)

S. Richei, Labillardière, Nov. Holl. pl, spec. I, 44, t. 60 (1804)...

S. australis, F. v. M., fragm. VI, 43 (1867)

S. capitellata, F. v. M., fragm. VI, 31 (1867) $\quad \ldots .6 \ldots$

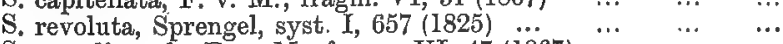

S. grandiuscula, F. v. M., fragm. VI, $47(1867)$
S. reflexa, Sprengel, syst. I, $655(1825)$

S. reflexa, Sprengel, syst. I, 655 (1825) … corifolia, F. v M.; Leucopogon, Endl., nov. stirp. dec. "15 (1839)

S. distans, Sprengel, syst. I, 655 (1825)
-

- - - - -

W.A.

$-1$

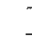

-

W.A. - - -

W.A.

W.A.

W.A.

W.A.

W.A.

W.A.

W.A.

W.A.

W.A.

W.A.

W.A.

W

W.A.

W.A.

$\ldots$

W.A.

W.A. - - T. V. N.S.W. -

- - - N.S.W.Q

- - V. N.S.W. Q.

- T. -

- T. -

- T. -

$-\quad$

$\ldots \mathrm{T}$.

- T.

- T.

-

$-$

T - -

- S.A. T. V. N.S.W. Q.

W.A. - - - - -

W.A. - - - N.S.W.

W.A. - - - N.S.W. -

- T. V. N.S.W. Q

W.A. S.A. T. V. N.S.W. Q

W.A. S.A. T. V. N.S.W. -

W.A.

W.A.

W.A.

W.A.

W.A.

$$
\begin{aligned}
& - \\
& - \\
& - \\
& - \\
& - \\
& - \\
& - \\
& - \\
& - \\
& - \\
& - \\
& - \\
& - \\
& - \\
& - \\
& - \\
& - \\
& - \\
& - \\
& - \\
& -
\end{aligned}
$$
B.H.IV, 146

B.H.IV, 147

B.fl. IV, 147

B.fl.IV,147

B. fl.IV, 148

B.fl.IV, 148

B.fl.IV, 148

B.f.IV,148

B.fl.IV,149

B.fl.IV, 149

B.fl. IV, 149

B.fl.IV, 153

B.fl.IV, 153

B.fl.IV,153

B.H.IV, 153

B.fl.IV, 154

B.fl.IV, 154

B. fl.IV, 154

- B.fl.IV,155

B.fl.IV, 155

B.fl.IV, 155

B. fl. IV, 156

B.f.IV, 156

B.fl.IV, 157

B.fl.IV, 157

B.fl.IV, 158

B.fl. IV, 158

B.fl.IV, 158

B.f.IV, 159

B.f.IV, 162

B. fl.IV, 162

B.fl.IV, 169

B.f.IV, 169

B.fl.IV, 169

B.fl.IV, 170

B.fi.IV, 170

B.f.IV, 170

B.f.IV, 171

B.fl.IV, 175

B.fl.IV, 176

B.fl.IV, 184

B. . IV, 184

B.fl.IV, 185

B.A.IV,185

B.fl.IV,185

B.Al.IV, 186

B.f.IV, 187

B.A.IV, 187

B.Al.TV,187

- B.fl.IV, 188

B.H.IV,188

- B.H.IV, 188

- B.fl.IV,189

M.fr.VI, 36;XI, 122.

M. fr. XI, 122 .

M.fr.VIII, 55.

M.fr. VI,46;VIII,54.

M.fr.VI, 30 .

M.fr, I, 178; VI, 31 .

M.fr.III, 143;VI, 31 .

M.fr.VIII,54.

M.fr. VI, 37.

M.fr.VI,38,

M.fr. VI, 36 .

M.fr.VI, 37.

M.fr.VIII, 54 .

M.fr. VI, 37.

M.fr.VI, 38

M. fr. VI, 37; VIII, 54.

M.fr. VI, 38 .

M. fr.VI, 37

M.fr. VI, 37 .

M.fr.IV 97

$M$ fr VITI 54;IX 48 ;

M.fr.VIII, 54 .

M.fr.VI, 36 .

M.fr.VI, 38;VIII, 55 .

M.fr. VIII, 54.

M.fr.VIII, 54 .

M.fr.VI, 43 .

M.fr.VI, 44; VIII, 54 .

M.fr. VI, 43 ; VIII, 54 .

M.fr.VI, 43 .

M.fr. VI, 43 .

M.fr.VI, 42;VIII, 54 .

M.fr.VI, 42;IX, 48;XI, 122 .

M.fr.VI, 43; VIII, 55.

M.fr. VI, 44 .

M.fr. VI, 43.

M.fr. VI, 42;VIII, 54.

M.fr.IV, 37.

M.fr.VI,31.

M.fr. VI, 31,

M.fr. VI, 47 .

M.fr. VI, 32 .

M.fr.VI, 32 , 
S. gibbosa, F. V. M. ; Leucopogon, Stschegleew in Bull. de Mosc. XXXII, $12(1859) \quad \ldots \quad \ldots \quad \ldots . \quad \ldots \quad \ldots$.

S. thymifolia, F, v. M.; Leucopogon, Lindley in Benth. Fl. Austr. IV, 189 (1869)

S. cordata, F. v. M.; Leucop., Sonder in p̈. Preiss, I, $3 \dddot{1} 3$ (1845)

S. Bossiaea, F. v. M., fragm. VI, $47(1867) \quad \ldots \quad \ldots \ldots \quad \ldots$

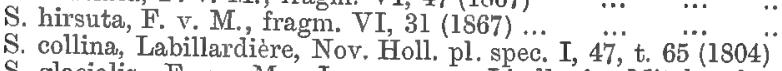

S. glacialis, F. V. M.; Leucopogon, Lindl. in Mitch. three Exped. II, 127 (1838)

S. compacta, F. v. M. ; Leucopogon, Stschegl. in " Bull. đe Mosc. XXXII, 13 (1859)

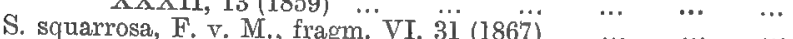

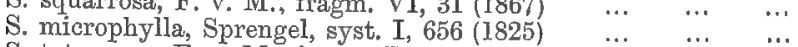

S. tetragona, F. V. M., fragm. VI, 31 (1867)

S. phyllostachys, F. v. M.; Leuc., Benth., f. Austr. IV, 193 (1869)

S. glabella, Sprengel, syst. I, 655 (1825)

S. semiopposita, F. v. M., fragm. VI, 49 (ï867)

S. florulenta, F. v. M. ; Lenc., Bentham, Fl. Austr. IV, 194 (1869)

S. striata, Sprengel, syst. I, 656 (1825)

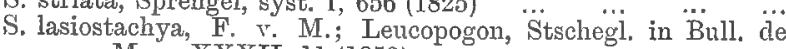

Mosc. XXXII, $11(1859)$
S. carinata, Sprengel, syst. I, 658 (1825)

$\begin{array}{lllll}\text { S. opponens, F. v. M., fragm. VI, } 48(1867) & \ldots & \ldots & \ldots \\ \text { S. oppositifolia, F. v. M. fragm. VI, } 32(1867) & \ldots & \ldots & \ldots\end{array}$

$\begin{array}{lllll}\text { S. oppositifolia, F. v, M. fragm. VI, } 32(1867) & \ldots & \ldots & \ldots \\ \text { S. tamariscina, Sprengel, syst, I, } 656(1825) & \ldots & \ldots & \ldots\end{array}$

S. bracteolaris, F. v. M.; Leuc., Benth., Fl. Austr. IV, $1 \ddot{97}$ (1869)

S. blepharophylla, F. v. M. fragm. VI, 34 (1867)... ... ...

S. gnaphalioides, F. v. M.; Leucopogon, Stschegl, in Bull, de Mose. XXXII, 14 (1859)

S. concurva, F. v. M., fragm. VI, 36 (1867)

S. Gilbertii, F. v. M.; Lencopogon, Stschegl. in Bull. de Mose. XXII, 15 (1859)

S. gracilis, Sprengel, syst. I, 658 (1825)

S. acicularis, H'. v. M.; Leucop., Benth., Fl. Austr. IV, 199 (1869)

S. cryptantha, F. v. M.; Leuc., Bentl., Fl. Austr. IV', 199 (1869)

S. gracillima, F. v. M., fragm. VI, 34 (1867)

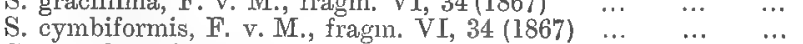

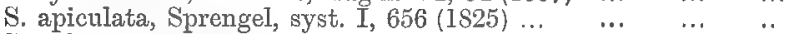

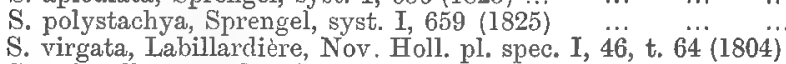

S. pulchella, F. v. M., fragm. VI, 34 (1867)

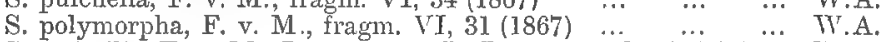

S. assimilis, F. v. M.; Leucopogon, R. Brown, prodr. 545 (1810) W.A.

S. Oldfieldii, F.v.M.; Leucopogon, Bentham, Fl. Austr.IV, 203(1869) W.A.

S. cncullata, Sprengel, syst. I, 656 (1825) ...

S. sprengelioides, F.v. M.; Leuc., Sonder in pl. Preiss. I, 319 (1845)

S. obtusata, F. v. M.; Leuc., Sonder in pl. Preiss. I, 313 (1845)

S. fimbriata, F. v. M.; Lencopogon, Stschegl. in Bull. de Mose. XXXII, 17 (1859)

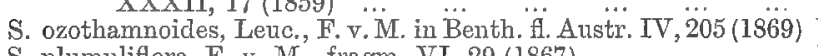

S. plumuliflora, F. v. M., fragm. VI, 29 (1867) ... ... ...

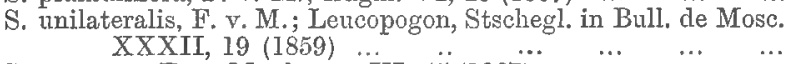

S. montana, F. v. M., fragm. VI, $4 \ddot{5}$ (1867)

S. Macraei, F. v. M., fragm. VI, 46 (1867)

S. pleurandroides, F. v. M., fragm. VI, 32 (1867)

S. linifolia, F. v. M., fragm. VI, 36 (1867)

S. pluriloculata, F. v. M., fragm. VI, 32 (1867) ...

S. pleiosperma, F. v. M., fragm. VI, 41 (1867) ...

S. rubicunda, F. v. M., fragm. VI, 31 (1867)

S. attenuata, F. v. M.; Leucopogon, Cunningham in Field's N.S.W. $341(1825)$

S. conferta, F. v. M.; Leuc., Bentham, Fl. Austr. IV, 208 (1869)

S. mutica, F. v. M., fragm. VI, 45 (1867)..

S. ericoides, Smith, Specim. of the Bot. of New Holl. 48 (1793)

S. brevicuspis, F. v. M. ; Leuc., Benth., Fl. Austr. IV, 210 (1869)

S. propinqua, Sprengel, syst. I, 658 (1825)..

S. subulifolia, F. v. M., fragm. VI, 33 (1867)

S. Allittii, F. v. M., fragm. VI, 34 (1867)

S. racemulosa, F. v. M., fragm. VI, 33 (1867)

S. pendula, Sprengel, syst. I, 657 (1825) ... … . 212 (1869)

S. margarodes, Sprengel, syst. I, 657 (1825)

S. flavescens, F. v. M., fragm. VI, 33 (1867)

S. blepharolepis, F. v. M., fragm. VI, 48 (1867) ...

S. esquamata, Sprengel, syst. I, 658 (1825)

S. rotundifolia, Sprengel, syst. I, 655 (1825)

S. cordifolia, F. v. M., fragm. VIII, 5. (1873)

S. megacarpa, F. $\therefore$.i., fragm. VI, $3:$ (1867)
W.A. - - - - B.f.IV,189

- $--V . \quad-\quad-$ B.fl.IV,I89

W.A. - - - - — - B.f.IV,190 M.fr.VI,47.

W.A. - - - - - B.fl.IV,191 M.fr.VI,31.

- S.A. T. V. N.S.W. - - B.A.IV,191 M.fr.VI,45;VIII, 54 .

- - - V. - - - B.H.IV,191

W.A. -- - - - - - - B.fl.IV,192

W.A. - - - - - - B.f.IV,192

W. - - - N.S.W. -

W.A. - - - - - B.fl.IV,193

W.A. - - - - - - B.H.IV,194

W.A. - - - - - - B.fl.IV,194

WV.A. - - - - - B.fi IV,194

W.A.S.A. - - - - - B.H.IV,194

W.A

- - - - - - B.fl.IV,195

W.A. - - - - - B.H.IV,195

W.A. - - - - - - B.H.IV,196

W.A. - - - - - - B.Al.IV,197

IV.A. - - - - - B.fl.IV,197

IV.A. - - - - - - B.fl.IV,197

W.A. - _ - - _ - B.fl.IV,197

W.A. - - - - - - B.fl.IV,198

- S.A. - - - - - B.fl.IV,198

M.fr.III, 144 ,

W.A. - - - - - B.fl.IV, 198

W.A. - - - - - - B.fl.IV,199 B.fl.IV, 199

- B.fl.IV,199

- B.fl.IV,200

- B.fl.IV,200

- B.fl.IV,201

- B.fl.IV,201

- B.fl.IV,201

- B.fi.IV,202

B.f.IV,202

B.fl.IV,202

B.fl.IV,203

B.f.IV, 203

$\begin{array}{lllll}\text { W.A. - - - - - } & - & - & \text { B.fl.IV,204 } \\ \text { W.A. } & - & - & - & \text { B.fl.IV,204 }\end{array}$

M.fr. VI, 48 .

M.fr. VI, 32 .

II.fr.VI,34.

IV.A. - - - - - - B.fl.IV 204

W.A. - - - - - - B.fl.IV,204

B. $\mathrm{H} . \mathrm{IV}, 205$

M.fr. VI, 155.

M.fr.XI, 122.

W.A. - - - - - B.fl.IV,205

B.f.IV,206

B.H.IV, 206

B.fl.IV, 206

B.fl. 15,207

B.f.IV, 207

B.fl.IV,207

B.fl. IV, 20S

MI.fr. VI 45.

II.fr.VI, $46 ; \mathrm{Y} I I I, 54$

M.fr.III, 143 .

MI.fr. VI, 36.

M.fr.I, 37.

II. fr. VI, 41

IV.A. - - - - -

- $\quad-\quad-$ N.S.IV.

- - - - N.S.W. -

- - - T T. N.S. W.

IT.A.A. T. V. N.S.W. Q

- B.fl.IV,208

- B.fl.IV,208

- B.fl.IV,209

B.f.IY, 209

B.fl.IV, 210

W.A. - - - - - - B.A.IV.210

W.A.

IV.A.

W.A.

B.fl. IV,21 1

B.f.I $Y, 211$

B.fl.IV, 211

W.A.

B.f.IT, 212

B.A.IV, 212

B. A.IV,213

W.A. - - - - - - - B.fl.IV,213
W.A. - - - B.S.W. - - B.f.IV,213
- - B.I.

W.A. - - - - - - B.ff.IV,213
W.A. - - - N.S.W. - - B.f.IV,213
- - B.IV,214

B. A.IV, 214

B. fl.IV, 214

W.A. S.A. - V. N.S.W. -

B. A.IV, 215
M.fr. TT, 4, .

M.fr. VI, 4.5

II.fr. VI, 34

M.fr.IV, 103.

II fr.IV, 103 .

M. fr. VI, 33 .

II.fr.VI,33.

M.fr. VI, 36 .

M.fr.IV, 100 .

MI.fr. VI, 48 .

II.fr. VI, 45

M fr.IV, 102. 
S. ruscifolia, Sprengel, syst. I, 656 (1825) ..

S. imbricata, Sprengel, syst. I, $656(1825)$...

S. cuspidata, Sprengel, syst. I, 657 (1825)...

S. leptospermoides, Sprengel, syst. I, 659 (1825)

S. acuminata, Sprengel, syst. I, 659 (1825)...

S. flexifolia, Sprengel, syst. I, 659 (1825) ...

S. biflora, Sprengel, syst. I, 659 (1825)

S. setigera, Sprengel, syst. I, 659 (1825) ..

S. exolasia, F. v. M., fragm. VI, 34 (1867)... ... ..
S. Fraseri, F. v. M. ; Leucop., Cunn. in Ann. nat. hist. II. 47 (1840) -

S. hirtella, F. v. M.; Leucopogon in Benth. Fl. Austr. IV, 218 (1869)

S. Oldfieldii, F.v.M.; Leuc. ovalifolius, Sond. in pl. Preiss. I, 324(1845)W. A

S. erubescens, F. v. M., fragm. VI, 33 (1867)

S. lissanthoides, F. v. M., fragm. VI, 33 (1867) $\ldots . \quad \ldots . \quad \ldots \quad$ W.A.

S. stricta, F. v. M.; Leucopogon, Bentham, Fl. Austr. IV," 2I9(1869)W.A.

S. Mitchellii, F. v. M.; Leucopogon, Benth. Fl. Austr. IV, $220(1869)$ -

S. juniperina, Sprengel, syst. I, $658(1825)$..

S. rufa, F. v. M., fragm. VI, 46 (1867)

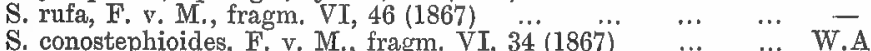

S. deformis, Sprengel, syst. I, 658 (1825).

S. pogonocalyx; Leucop., F.v.M. in Benth. Fil. Austr. IV “222 (1869) W.A

S. breviflora; Leucopogon, F. v. M., fragm. IV, $102(1864)$,.. W. A.

S. dura, F. v. M. ; Leucopogon, Bentham, Fl. Austr. IV, 222 (1869) W.A.

S. multiflora, Sprengel, syst. I, 658 (1825)..

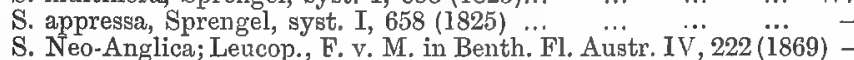

... W.A.

S. obtecta, F. v. M.; Lencopogon, Benth. Fl. Austr. IV, 223 (1869) W.A.

S. crassiflora, F. v. M., fragm. VI, 40 (1867)

S. strongylophylla, F, v. M., fragn. VI, 33 (1867)

,.. W.A.

S. crassifolia, F. v. M., fragm. VI, $33(1867)$ … … … W.A.
S. corynocarpa, F. v. M.; Lencop., Sonder in pl. Preiss. I, 322 (1845)W.A.

S. Woodsii; Leucopogon, F. v. M., fragm. I, $178(1859) \ldots \ldots \ldots$ W.A.

S. leptantha, F. v. M.; Lencop., Bentham, Fl. Austr. IV, 225 (1869)W.A.

S. divaricata, Sprengel, syst. I, $658(1825) \ldots$

S. aggregata, Sprengel, syst. I, 657 (1825) ...

S. serrulata, Labillardière, Nov. Holl. pl. spec, I, 45 , t. 62 (1804)

S. patula, Sprengel, syst. I, 657 (1825)

S. ovalifolia, Sprengel, syst. I, 656 (1825) ...

S. ramiflora, Sprengel, syst. I, 659 (1825) ...

S. depressa, Sprengel, syst. I, 655 (1825) ...

S. fasciculiflora, F. v. M., fragm. VIII, 55 (1873)...

S. elliptica, Smith, Specim. of the Bot. of New Holl. 49 (1793)...

S. glanca Labillardiere Nov Holl pl spec. I 45, t. 61 (1804)

S. scoparia, Smith, Specim, of the Bot. of New Holl. 49 (1793)..

S. ledifolia, F. v. M.; Monotoca, Cunningham in De Cand., prodr. VII, $756(1839)$

S. empetrifolia, F. v. M. in Papers of R. S. Tasm. 86 (1874) $\quad \ldots$

S. oligarrhenoides, F. v. M., fra.gm. IX, $47(1875)$

S. minutiflora, F, v. M., fragm. XI, I22 (1881) OLIGARRHENA, R. Brown, prodr. Fl. Nov. Holl. 549 (1810)

O. micrantha, R. Brown, prodr. $459(1810)$.. ... ... ... W. NEEDHAMIA, R. Brown, prodr. Fl. Nov, Holl, 549 (1810).

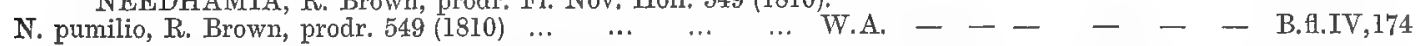
BRACHYLOMA, Sonder in Lehm. pl. Preiss. I, 304 (1845), (Lobopogon, Lissanthe partly.)

B. daphnoides, Bentham, FI. Austr. IV, 173 (1869)

B. ciliatum, Bentham, Fl. Austr. IV, $173(1869)$...

B. depressum, Bentham, Fl. Austr. IV, 173 (1869)

B. Scortechinii, F. v. M., fragm. XI, 121 (1881) ...

B. Preissii, Sonder in Lehm. pl. Preiss. I, 305 (1845)

B. concolor, F. v. M., fragm. VI, 39 (1867)

B. ericoides, Sonder in Schlecht. Linnaea XXVI, 247 (I853) CONOSTEPHIUM, Bentham in Hueg. enum. 76 (1837).

C. pendulum, Bentham in Hueg. enum. 76 (1837)...

C. minus, Lindley, Bot. Regist. XXV, App. XXV (1839)

C. Roei, Bentham, Fl. Austr. IV, 160 (1869)

C. Preissii, Sonder in Lehm. pl. Preiss. I, 304 (1845)

C. planifolium, F. v. M.; fragm. VI, 30 (1867) COLEANTHERA, Stschegleew in Bull. Soc. Mosc. XXXII, 4 (1859). (Michiea.)

C. coelophylla, Bentham, Fl. Austr. IV, 150 (1869)

C.

C. virgata, Stschegleew in Bull. de Mosc. XXXII, 5 (1859) $\ldots$ W.A. - - - $\quad$ - $-\quad$ B.fl.IV,151 TROCHOCARPA, R. Brown, prodr. 548 (1810). (Decaspora, Pentachondra.)

T. laurina, R. Brown, prodr. 548 (1810)

T. disticha, Sprengel, syst. I, 660 (1825)

T. thymifolia, Sprengel, syst. I, 660 (1825)

T. Clarkei, F. v. M., fragm. VI, 57 (1867)...

T. Gunnii, Bentham, fl. Austr. IV, 167 (1869)

T. parviflora, Bentham, Fl. Austr. IV, 167 (1869)

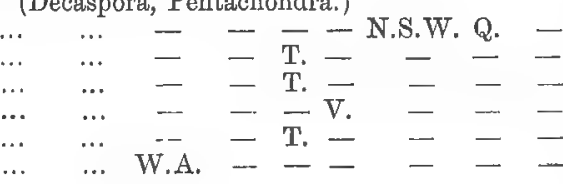

B.fl.IV, 166
W.A. B.fl.IV, 166 B. f.IV, 166 B.fl.IV, 167 B.fi.IV, 167
M.fr.IV, 104.

M.fr. VI, 45 .

M.fr. VI, 34.

M.fr.IV, $105 ; \mathrm{VI}, 46$.

M.fr.IV, 102 .

M.fr.TV, 101 .

M.fr.IV, 104;VI, 46.

M.fr. VIII, $54 ;$ IX, 48.

M.fr.VI, 34 .

M.fr.IV, 102.

M.fr.VI, 32.

M. fr.VI, 40.

M.fr.IV, 101

M.fr.VI, 33 .

M.fr. VI, 33 .

M.fr.VI, 44.

M.fr.VI, $44 ; V I I I, 55$.

M.fr.VI, 44 .

M. fr. VI, $44 ; \mathrm{XI}, 122$.

M.fr.VI, 44 .

M.fr.

M.fr. VI, 44 .

M.fr. VI, 44 .

M.fr. VI, 58 .

M. fr. VI, 58;XI, 122.

M.fr.IX, 47.

M.fr.XI, 122 .

M.fr. VI, $42 ; \mathrm{VIII}, 55$.

M.fr.VI, 42.

M.fr.I,36;VI, 42 .

M.fr.XI,121.

M.fr.VI,39;VIII, 55.

MI.fr.IV, 98 .

M.fr.IV,98;VI, 39;XI, 46.

(Conostephiopsis.)

W.A. - -

W.A.

W.A. - - -

B.tl.IV, 160

M.fr. VI, 40 .

M.fr.VI, 40 .

M.fr.VIII, 55.

M.fr.VI, 40.

M.fr. VI, 30 .

M.fr. VI, 80 .

M.fr. VI, $57 ; \mathrm{IX}, 48$.

M.fr.VI,57;VIII, 55.

M.fr. VI, 57;VIII, 55 .

M.fr.VI, 57.

M.fr.VIII,55. 
T. involucrata, F. v. M., fragm. VI, 57 (1867)

T. pumila, F. r, M., fragm. VI, 57 (1867)...

T. ericifolia, F. v. M. in Papers R. S. Tasm. 86 (1874) ..

T. verticillata, F. v. M. in Papers R. S. Tasm. 86 (1874)

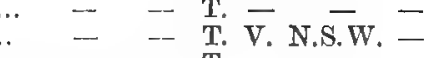

$$
\begin{aligned}
& \begin{array}{l}
. . \\
\cdots-\text { - }- \text { T. } \\
\cdots \text { T. }- \text { - }
\end{array}
\end{aligned}
$$

EPACRIS, Cavanilles, icon. et descr. pl. IV, 25, t. 344 et 345 (1797). (Archeria.)

E. micranthera, F. v. M., fragm. VI, 72 (1867)

E. hirtella, J. Hooker in Lond. Jouxn. of Bot. VI, 271 (I847)

E. longiflora, Cavanilles, icon. et descr. pl. IV, 25, t. 344 (1797)

E. reclinata, Cunningham in Benth. Fl. Austr. IV, 234 (1869) ...

E. impressa, Labillardiere, Nov. Holl. pl. spec. I, 43, t. 58 (1804)

E. sparsa, R. Brown, prodr. 551 (1810)
E. petrophila, J. Hooker, Fl. Tasman. I, 261 (1860)

E. rigida, Sieber in Spreng. cur. poster. 64 (1827)...

E. coriacea, Cunningham in Cand. prodr. VII, 763 (1839)

E. crassifolia, R. Brown, prodr. 551 (1810)...

E. robusta, Bentham, FI. Austr. IV, 237 (1869) ..

E. obtusifolia, Smith, Exot. Bot. I, 77, t. 40 (1804)

E. myrtifolia, Labillardiere, Nov. Holl. pl. spec. I, 41, t. 55 (1804) -

E. exserta, R. Brown, prodr, 551 (1810)

E. mucronulata, R. Brown, prodr. 552 (1810)

E. lanuginosa, Labillardiere, Nov. Holl. pl, spec. $\dddot{I}, 42$, t. 57 (1804) -

E. paludosa, R. Brown, prodr. 551 (1810).

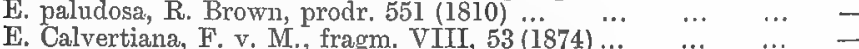

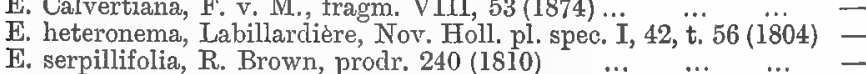

E. microphylla, R. Brown, prodr. 550 (1810)

E. acuminata, Bentham, Fl. Austr. IV, 240 (1869)

E. apiculata, Cumningham in Field, N.'S. Wales, 340 (1825)

E. pulchella, Cavanilles, icon. IV, 26, t. 345 (1797)

E. purpurascens, R. Brown, prodr. 550 (1810) WOOLLSIA, F. v. M., fragm. VIII, 52 (1872). (Lysinema partly.)

W. pungens, F. v. M., fragm. VIII, $52(1872)$

LYSINEMA, R. Brown, prodr. 552 (1810).

L. lasianthkm, R. Brown, prodr. 552 (1810)

L. conspicuum, R. Brown, prodr. 552 (1810)

L. ciliatum, R. Brown, prodr. 552 (1810)

L. fimbriatum, F. v. M., fragm. IV, 125 (1864)

L. elegans, Sonder in Lehm. pl. Preiss. I, 327 (1845) PRIONOTES, R. Brown, prodr. fl. Nov, Holl. 553 (1810).

P. cerinthoides, R. Brown, prodr. $553(1810) \quad \ldots \quad \ldots \quad \ldots$ COSMELIA, R. Brown, prodr. fl. Nov. Holl. 553 (1810).

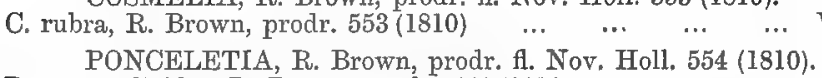

P. sprengelioides, R. Brown, prodr, 554 (1810) $\dddot{2}$. 768 (1843)...
P. monticola, Cunningham in De Cand. prodr. VII, SPRENGELIA, Smith in Svensk. Vetens. Acad. Handling. 260 (1794). (Poiretia.)

S. incarnata, Smith in Vetens. Ac. Handl. 260, t. $8(1794)$... - S.A. T. V. N.S.W. ANDERSONIA, R. Brown, prodr. $553(1810)$. (Atherocephala,
ossea, F. v. M., fragm. VI, 63 (1867) $\ldots$... $\quad$... W.A.

A. colossea, F. v. M., fragm. VI, 63 (1867)
A. patricia, F. v. M., fragm. VI, 79 (1868)

A. grandiflora, Stschegleew in Bull. de Mosc. XẌXII, 201 (1859)

A. setifolia, Bentham, Fl. Austr. IV, 252 (1869) ...

A. involucrata, Sonder in Lehm. pl. Preiss. I, 331 (1845)

A. homalostoma, Bentham, Fl, Austr. IV, 253 (1869) ...

A. sprengelioides, R. Brown, prodr. 554 (1810) ...

A. latillora, F. v. M., fragm. VI, 61 (1867)

A. gracilis, De Candolle, prodr. VII, 767 (1843)

A. aristata, Lincliey, Bot. Regist. XXV, App. XXV (1

A. macronema, F. v. M., fragm. VIII, 51 (1873)..

A. parvifolia, R. Brown, prodr. 554 (1810)

A. depressa, R. Brown, proilr. 554 (1810) ...

A. coerulea, R. Brown, prodr. 554 (1810)

A. subulata, Bentham, Fl. Austr. IV, 256 (1869)...

A. heterophylla, Sonder in Lehm. pl. Preiss. I, 333 (1845)

A. brachyanthera, F. v. M., fragm. VI, 61 (1867)

A. brevifolia, Sonder in Lehm. pl. Preiss, I, 332 (1845)...

A. variegata, Sonder in Lehm. pl. Preiss. I, 334 (1845) ...

A. micrantha, R. Brown, prodr. 554 (1810)

RICHEA, R. Brown, prorli. 555 (1810). (Cystenthe, Pilitis.)

R. sprengelioides, F, v. M., fragm. VI, 68 (1867)

R. procera, F. v. M., fragm. VI, 68 (1867)

R. acerosa, F. v. M., fragm. VI, 69 (1867)...

R. Milligani, F. v. M., fragm. VI, 69 (1867)

R. Gunnii, J. Hooker in Lond. Journ. VI, 273 (1847)

R. scoparia, J. Hooked in Lond. Journ. VI, 273 (1847)
W.A.

A. --
W.A.

W. W.

W... W.A.

... W.A.

... W.A.

... W.A.

... W.A.

... W.A.

W.A.

... W.A.

W... W.

IV.A.

... IV.A.

... W.A.

... W.A.

‥ W.A.

... W.A.

... W.A.

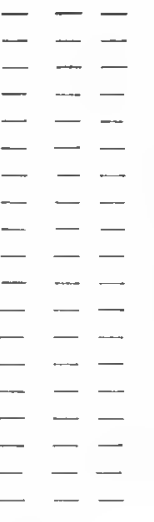

\section{B. fl.IV,I64 M.fr.VIII, 55. B.fl.IV, 164 M.fr.VI,57. \\ B.fl.IV, 164 \\ B.fl.IV, 164}

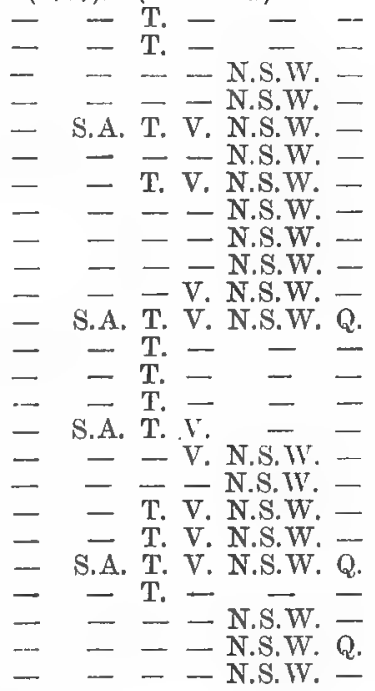

- -
B.fl. VI, 246

B.fl. VI, 246

B.fl.IV, 234

B.fi.IV,234

B.fl.IV, 235

B.fl.IV,235

B.fl.IV, 236

B.fi.IV,236

B.fl.IV, 236

B.fl.IV,237

B.H.IV, 237

B.H.IV, 237

B.fl.IV, 238

B. H.IV, 238

B.fl.IV, 238

B.fl.IV, 238

B.fl.IV, 239

B.fl.IV, 239

B.fl.IV, 240

B.fl.IV, 240

B.fi.IV, 240

B.fl.IV,241

B.fl.IV, 241

B.fi.IV, 241

B.fl.IV, 243

\section{$3 \mathrm{M}$}

.fr.IV, 126;VI,70,88; VIII, 55;IX,48;XI, 122 .

M.fr. VIII, $56 ; \mathrm{XI}, 122$.

M.fr.VIII, 56.

M.tr.VIII, 53.

M.fr.VI,71;VIII, 56.

M.fr. VI, 71;XI,122.

M.fr.IV,127;VIII, 56 .

- B.fl.IV,243 M.fr.III, 142, VI,70; VIII 55.

B.fl.IV, 243

B.H.IV, 243

B.fl.IV 244

B.fl.IV, 244

M.fr.VI, 70;VIII,55;XI,

M.fr.VI, 69,70; VIII, 55 .

M.fr. VI, 70 .

M.fr. VI, 70 .

B.fl.IV, 246

M.fr. VI, 69;XI, 122.

B.fl.IV, 247 M.fr.VI,64.

B.fi.IV,248 M.fr.I, $39 ; V I, 60$.

- B.fl.IV, 249

B. 240

9

[VIII, 56;XI, 122 .

[VIII, $56 ; \mathrm{XI}, 122$
$39 ; \mathrm{VI}, 54 ; \mathrm{IX}, 48 ;$

B.fl.IV,25l M.fr.VI, 63 .

$\begin{array}{ll}\text { B.fl.IV, } & \text { M.fr. } 1,63 . \\ \text { B.fl. } & \text { M.fr.VI,79. }\end{array}$

Homalostoma, Sphincterostoma

$-\quad-\quad-\quad$ B.H.IV,251
$-\quad-\quad$ B.fl.IV,251
$-\quad-$ B.f.IV,252

- - - - B.f.IV,252

- _. - B.fl.IV,252

- B.IV 253

B.H.IV, 253
B.fl.IV, 253

- B.fl.IV,253

- B.fl.IV,254

B.fl.IV, 254

-

B.fl. TI, 254

B.f.IV, 25

B.fl. IV, 255

B.fl. IV, 256

B.fl.IV, 256

B.fl. IV, 256

B.A.IV, 256

B.fl.IV,257

B. fl.IV, 257

M.fr. VI, 62.

M.fr. VIII, 51 .

M.fr. VI, 62.

M.fr. VIII, 56.

M.fr.VI, 64 .

M.fr.VI, 61 .

M.fr, VI, 63 .

M.fr. VIII, 5l,

M fr.IV, 125; I I, 62 .

M. fr. I I, 64 .

M.fr. IV, 124 .

M.fr. VI, 61 .

M. fr. VI, 61 .

M.fr. VI,61.

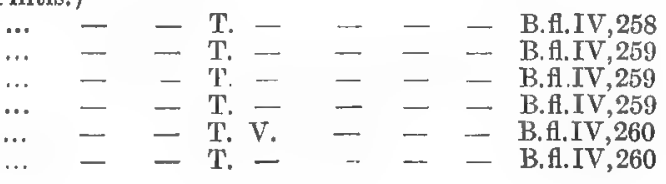

M. fr. I, 38;VI, 68 .

M.fr. I, 38;VI, 68.

M.fr. I, 38;VI, 69.

M.fr.I, 38; VIII, 56.

M.fr. VI,67;XI, 122.

M.fr. VI, 68 . 


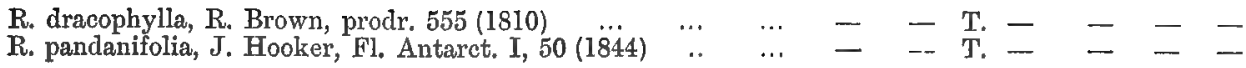

B, fl. IV, 260

B. A.IV, 261

M.fr.VI, 67.

DRACOPHYLLUM, Labillardière, Voy. II, 211, t. 40 (1798). (Epacris, Forst. 1776 partly.)

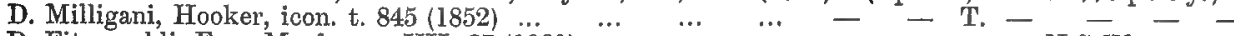

D. Fitzgeraldi, F. v. M., fragm. VII, $27(1869)$
D. secundum, R. Brown, prodr. $556(1810)$
D.

B.fi, IV, 262 M.fr.VI, 65.

- M.fr.X, 119 .

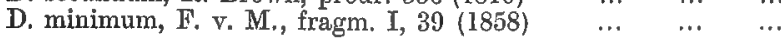

SPHENOTOMA, Sweet, Flora Australasica, t. 44 (1828).

S. squarrosum, G. Don, gen. syst. III, 785 (1834)

S. Drummondii, E, v. M.; Dracophylium, Benth. Fl. Austr. IV, 263 (1869)

S. dracophylloides, Sonder in Lehm. pl. Preiss, I, 33 (1844) ...

S. capitatum, Lindley, Bot. Regist. t. $1515(1832) \quad \ldots . \quad \ldots$

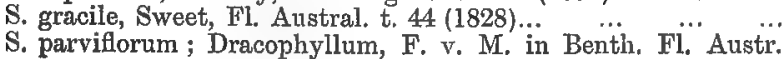
IV $265(1869)$
W.A. - - - - - - B.fl.IV,263 M.fr. VI,66.
W.A. - - - - - -- B.fl.IV,263
W.A. - - - - - - - - B.fl.IV,263
W.A. - - B.fl.IV,264 M.fr.VI,65.
W.A. - - -
W.A. - - - - - - B.fl.IV,264 M.fr, VI,66.
W.A. - - - - - - B.fl.IV,265 M.fr.VI,66.

\section{APETALEAE GYMNOSPERMEAE.}

F, v, M. in Woolls's plants of the neighb. of Sydney 40 (1880).

\section{CONIFERAE.}

Haller, enum, stip. Helv. I, 145 (1742).

DAMMARA, Rumphius, herb. Amboin. II, 174, t. 54 (1741). (Agathis.)

D. robusta, C. Moore in Transact. pharm. Soc. Vict. II, $174(1860)-----$ Q. - B.fl.VI,244 M.fr.XI,104.

ARAUCARIA, A. L. de Jussieu, gen. plant. 413 (1789). (Colymbea, Columbea, Eutassa, Eutacta, Altingia.)

A. Cunninghamii, Aiton in Sweet. hort. Brit. 475 (1827) ‥ - - - N.S.W. Q. - B.H.VI,243

A. Bidwilli, Hooker, Lond. Journ. of Bot. II, 503, t.18(1850).. - - - - - Q. - B.H.VI, 243

A. excelsa, R. Brown in Ait. hort. Kew. sec. ed, V, $412(1813) \ldots-1--$ - N.S.W. -

A. cupressoides, D. Don in Trans. Linn. Soc. XVIII, 173, t. 13(1839) - - T. - - - - B.fl.VI, 242

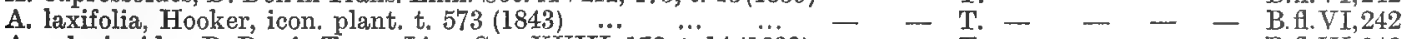

A. selaginoides, D. Don in Trans. Linn. Soc. XVIII, 172, t. $14(1839)-\quad-\quad \mathrm{T} . \quad-\quad--\quad$ - B.f.VI,242 CALLITRIS, Ventenat, decas generum novorum 10 (1808). (Frenela, Actinostrobus, Leichhardtia, Octoclinis.)

C. Macleayana, F. v. M. in Rep. Burdek. Exped. 17 (1860) ‥ - - - - N.S.W. Q. - B.fi.VI, 235

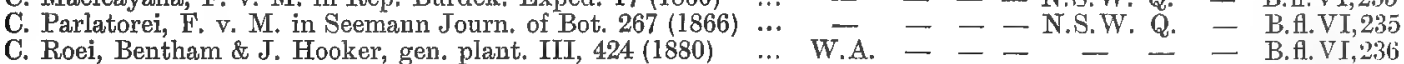

C. Drummondi, Bentham \& J. Hooker, gen. plant. III, 424 (1880) W.A. - - - _ - - B.f.VI,236

C. verrucosa, R. Brown in Mém. du Mus. Par. XIII, 74 (1826) W.A. S.A. - V. N.S. W. Q. N.A. B.tl.VI,236

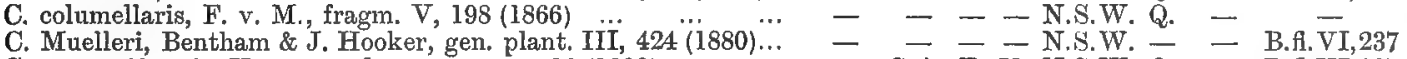

C. cupressiformis, Ventenat, decas gen. nov. $10(1808) \ldots \ldots \ldots-$... $\quad$ S.A. T. V. N.S.W. Q. $\quad-$ B.fl.VI,237

C. calcarata, R. Brown in Mém. du Mus. Par. XIII, 74 (1826)... - - - V. N.S.W. Q. - B.fl.VI,238

C. oblonga, L. Cl. Richard, comment. de conif. 49, t. 18 (1826) - - T. - - - - B fl.VI,238

C. Actinostrobus, F. v. M., Rep. Burdek. Exped, 19 (1860) ‥ W.A. - - - - - - B.f.VI,240

C. acuminata, F. v. M.; Actinostrobus, Parlatore, enum. sem. hort.

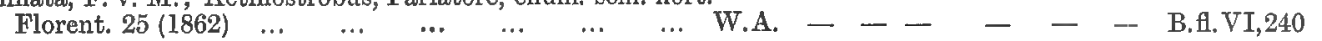

FITZROYA, J. Hooker in Journ. of the hort. Soc. VI, 264 (1851). (Diselma.)

F. Archeri, Bentham \& J. Hooker, gen. pl. III, $426(1880) \quad \ldots-1-$ T. - - - - B.fl.VI,240

PHEROSPHAERA, Archer in Hooker's Kew Misc. II, 52 (1850). (Dacrydium partly.)

P. Hookeriana, Archer in Hooker, Kew Misc. II, 52 (1850) ‥ - - T. - - - -- B.fl.VI,245

P. Fitzgeraldi, F. v. M. in J. Hooker, icon. pl. XIV, 64, t.1383(19382) - - - - N.S.W. - -

MICROCACHRYS, J. Hooker in Lond. Journ. of Bot. IV, 149 (1845). (Dacrydium partly.)

M. tetragona, J. Hooker, Fl. Tasman. I, 358, t. $100(1860) \ldots \ldots-\ldots$ T. - - - - B.f.VI, 24I

DACRYDIUM, Solander in G. Forster, pl, escul. insul. ocean, austr. 80 (1786).

D. Franklinii,.J. Hooker in Lond. Journ. of Bot. IV, 152, t.6 (1845) - - T. - - - - B. I..VI, 245 NAGEIA, Gaertner, de fructib. t. 39 (1788). (Podocarpus.)

N. elata, F. v. M.; Podoc., R. Brown in Mem. de Mus. XIII,75(1826) - - - - N.S.W. Q. - B.A.VI,247

N. spinulosa, F. v. M.; Podocarpus, R. Brown in Mém. du Mus.

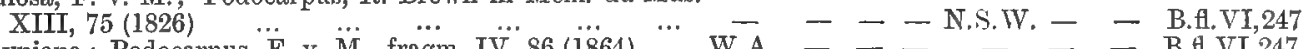

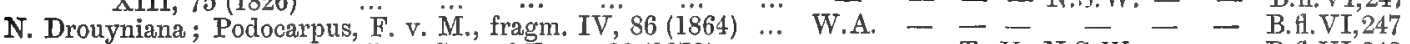

N. D.

THALAMIA, Sprengel, Anleit. zur Kenntn. der Gew. zweite Ausg. II, 218 (1817). (Phyllocladus.)

T. asplenifolia, Sprengel, Anl. zur Kenntn. der Gew. II, 218(1817) - - T. - - - - B.H.VI,246 M.fr.XI, 104.

\section{CYCADEAE.}

L. C. Richard in Persoon, synops. 630 (1807).

CYCAS, Linné, hort. Cliffort. 482 (1737).

C. media, R. Brown, prodr. 348 (1810)

C. Normanbyana, F. v. M., fragm. VIII, 169 (18

C. Kennedyana, F, v. M, in Melb. Chemist, March (1882)

C. Cairnsiana, F. v. M., fragm. X, 63 (1876)

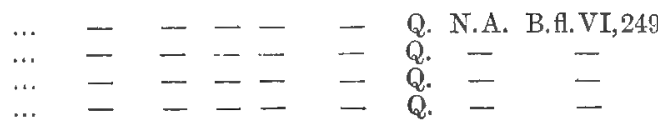

M.fr. VIII, 171 . M.fr.VIII, 169. M.fr.X, 63,121 . 
ENCEPHALARTOS, Lehmann, pugill. VI, 9, t. I, et 3 (1834). (Macrozamia, Lepidozamia, Catakidozamia, Zamia partly.)

E. Pauli Guilielmi, F.v. M. in Trans. Pharm. Soc. Vict. II, $91(1859)-1-0$ - N.S.W. Q. - B.A.VI,25I

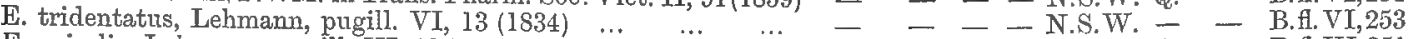

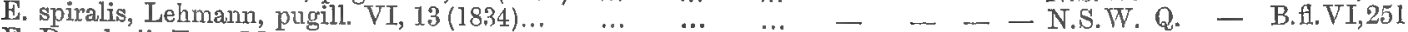

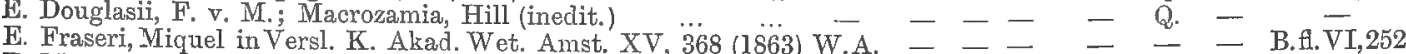

E. Moorei, F. v. M., fragm. XI, 125 (1881). An

E. Macdonnelli, F.v.M. inVersl. K. Akad, Wet. Amst. XV, 376(1863) - s.A - - - Q

E. Denisonii, F. v. M. in Journ. Pharm. Soc. Vict. II, 90 (1859) - - - N.S.W. Q. - B.fl. VI, 253

BOWENIA, Hooker, Bot. Magaz. t. 5398 (1863).

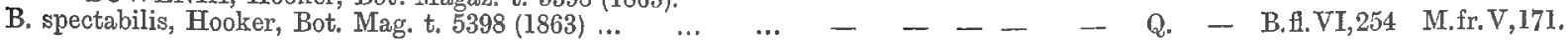

\section{MONOCOTYLEDONEAE.}

Ray, meth. plant. nov. (1682).

CALYCEAE PERIGYNAE.

F. v. M. in Woolls's plants of the neighb. of Sydney $41(1880)$.

\section{ORCHIDEAE.}

Haller, enum. stirp. Helv. praefat. 33 (1742).

PHOLIDOTA, Lindley in Hooker, Exotic Flora II, t. 138 (1825).

P. imbricata, Lindley in Hook. Exot. Fl. II, t. 138 (1825) ‥ - - - - - Q. - B.f.VI,290 M.fr.IV,163;V,105.

STURMIA, Reichembach in Moessler's Handb. II, 1552 (1828). (Liparis, Richard 1818 not of Zoologists 1738.)

S. reflexa, F. v. M., fragm. II, 72 (1861) .. ... .. ‥ - - - N.S.W. Q. - B.f.VI, 273

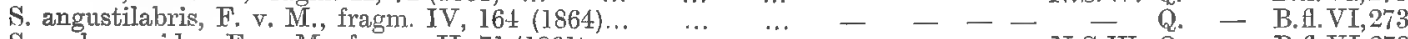

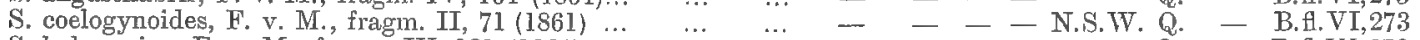

S. habenarina, F. v. M., fragm. IV, 131 (1864) .. $\quad . . \quad \ldots \quad-\quad-\quad-\quad-\quad-\quad$ Q. $\quad-$ B.fl.VI, 273

OBERONIA, Lindley, gen, and spec. of orchid. pl. 15 (1830). (Titania.)

O. iridifolia, Lindley, gen. and spec. of orchid. pl. 15 (1830) $\ldots \ldots-\ldots-$ - N.S.W. Q. $\quad$ - B.fl.VI,274

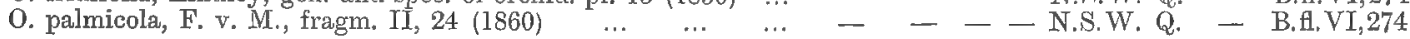
PHREATIA, Lindley, gen. and spec, of orchid. pl. 63 (1830). (Plexanre.)

P. Limenophylax, G, Reichenbach in Seem. Bonpl. 54 (1857) - - - - - Q. - B.fl.VI,290 M.fr.X,64.

DENDROBIUM, Swartz in nov, act. Upsal. VI, 82 (1799). (Thelychiton partly, Pedilonum, Coelandria.)

D. bigibbum, Lindley in Paxt. Flower Gard. III, 25 (1853) ... - - - — Q. N.A. B.A.VI,277

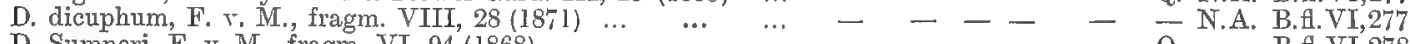

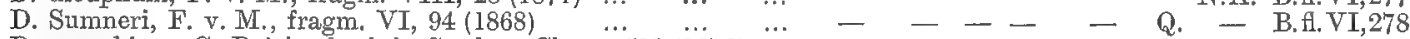

D. superbiens, G. Reichenbach in Garden, Chron. 516 (1876) $\cdots-\ldots$ - - - - - Q. -

D. Phalaenopsis, Fitzgerald in Gardeners' Chronicle, July (1880) - - - - - Q - -

D. Johannis, G. Reichenbach in Garden. Chron, $\ddot{890}(1865) \quad \ldots \quad-\ldots \quad-\quad-\quad-\quad-\quad$ Q. - B.

D. speciosum, Smith, Exot. Botany I, 17, t. $10(1804) \ldots \ldots$

D. falconirostre, Fitzgerald in Sydney Morn. Herald, Nov. 18 (1876) -

D. tetragonum, Cunningham in Bot. Regist. XXV, Misc. $33(1839)-$
D. aemulum, $\mathbf{R}$. Brown, prodr. $333(1810) \ldots \quad \ldots$

- - T S IV. Q

D. brachypus, G. Reichenbach in Garcke, Limmaea XLI, 42 (1876)

D. gracilicaule, F. v. M., fragm. I, 179 (1859)

D. Moorei, F. r. M., fragm. VII, 29 (1870)
D. agrostophyllum, F. v. M., fragm. VIII, 28 (187I)

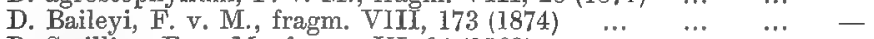

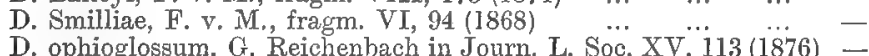

D. canaliculatum, R. Brown, prodr. $333(1810) \ldots \ldots \ldots$...

D. Foelschei, F. v. M. in Wing's S. Sc. Record, Oct. (1882) … -

D. monophyllum, F. v. M., fragm. I, 189 (1859) $\ldots \quad$... $\quad \ldots \quad$ _-

D. hispidum, A. Richard, sert. Astrol. 13, t. $5(1833)$... $\ldots$ ”.

D. cucumerinum, MacLeay in Bot. Reg., new ser. V, Misc. 58 (1842)-

D. rigidum, $\mathrm{R}$. Brown, prodr. $333(1810)$

D. linguiforme, Swartz in Svensk. Vetensk. Ac. Handl. $247(1800)-$

D. teretifolium, R. Brown, prodr. 333 (1810)

D Fairfaxii, Fitzgerald \& F. v. M. in Sydney Mäil 360 , Nov. (1872) -

D. striolatum, G. Reichenbach in Hamburg Gartenz, 313 (1857)

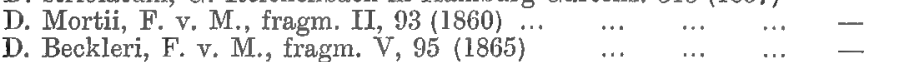

BULBOPHYLLUM, Petit-Thouars, Orchicl. Afric. t, 95 et 108 (182

B. Lichenastrum, F'. V. M., fragn. VII, 60 (1869)

B. Baileyi, F. v. M., fragm. IX, 5 (1875) ...

B. nematopodum, F. v. M., fragm. VIII, 30 (1873)
- - - N.S.W.W. $\overline{\text { Q. }}$

- - N.S.W.Q.

$-\quad-$ N.S.W.Q.

-- - N.S.W. Q.

- - - N.S.W. Q.

- - - N.S.W. $\frac{-}{\mathrm{Q}}$

- - - - Q Q

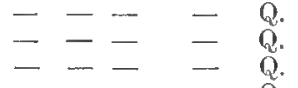

- - - -

- - - $\overline{-} \overline{\mathrm{ST}}$

$-=-$ N.S.W. Q.
$-E-$ N.W. Q.

- - N.S.W. Q.

- - - N.S.T. Q.

- - - N.S.IV. Q.

- T. V. N.S.W. -

$-\quad-$ N.S.W. Q.

- - - N.S.W. Q.

B.f. VI, 279

- B.H. VI, 280

- B.fi.VI, 280

- B.fl.VI,281

- B.fl.VI,281

- B. $1, \mathrm{VI}, 282$

- B.f. VI,2S2

- B.fl. VT,2S2

N.A.

- B.A.TT,2S3

- B.A.VI.2S:

- B.f.VI, 283

- B.fl.VI.

- B.f.VI,:81

- B.fl.VT,284

- B.fl.VI, 285

- B.fl.VI.2S5

- B.f.VI,285

- B.tl.VI,286

(Bolbophyllum, Thelychiton partly.

- - - - Q. - B.fl.VI, 287

M.fr.IX, 5

M. fr, VIII, 130.

M.fr.II, 93 .
[88.

M.fr.VI,119;XI, 87 .

M.fr.VI, 119 .

fr.V1,94.

M.fr.I, 87 .

M. fr. VIII, 249.

M.fr.XI, 87.

..1r. 1,$87 ;, 1,119$

fr. I, 213;XI,87.

M.fr.IV, 171

M. fr.XI, 54 .

fr. VIII, 28.

M.fr. VIII, 173.

II. $\mathrm{f}$ XI 54

M.fr.III, 126.

M.fr. VII, 64

iI. fr.I. 189 .

M.fr. VIII, 248; XI, 87.

M.fr. I,S?.

M.fr. X, 120

M.fri, I, 88;VII, 30. 
B. Shepherdi, F. v. M., fragm. III, 40 (1862)

B. Taylori, F. v. M., fragm. VIII, 150 (1874)

B. aurantiacum, F. v. M., fragm. III, 39 (I862)

- - - - - N.S.IV Q.

B. argyropus, G. Reichenbach in Garcke, Linneae XLI, 42 (1876)

B. exiguum, F. v. M., fragm. II, $72(1861)$...

B. minutissimum, F. v. M., fragm. XI, 53 (1878).

B. Prenticei, F. v. M. in Wing's South. Sc. Record I, 1733 (1881)

B. Elisae, F. v. M., fragm. VI, $120(1868)$.. SARCOCHILUS, R. Brown, prodr. 332 (1810). (Thrixper

S. erectus; Cleisostoma, Fitzcerald, Austr. Orchids part 4 (1877)

S. tridentatus, G. Reichenbach in Walp. Annal. VI, 500 (1861)

S. Armitii, F. v. M., fragm. IX, 49 (1875).

S. Beckleri; Cleisostoma, F. v. M. in Benth. F1. Austr. VI, $296(1870)$

S. brevilabris; Cleisostoma, F. v. M., fragm. XI, 87 (1880) …

S. Macphersoni, F, v. M., fragm. VIII, 248 (1874)

S. Hartmanni, F. v. M., fragm. VIII, 248 (1874)...

S. clivitiflorus, F. v. M. in Benth. Fl. Austr. VI, 292 (18̈70)

S. falcatus, R. Brown, prodr. 332 (1810)

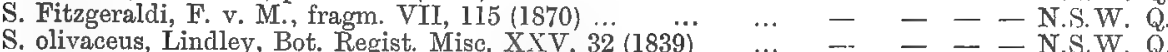

S. parvifloru, Lindley, Bot. Regist. Misc. XXIV, 34 (1838) $\ldots \ldots-\ldots-$ T. V. N.S.W. -

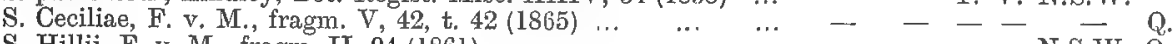

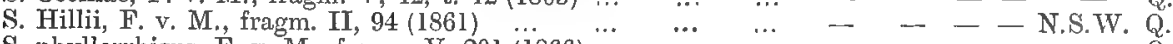

S. phyllorrhizus, F. v. M., fragm. V, $201(1866) \ldots$ TAENIOPHYLLUM, Blume, Bijdr. 355 (1825).

T. Muelleri, Lindley in Benth. Fl. Austr. VI, 291 (1870)

ORNITHOCHILUS, Wallich in Lindley, gen, and spec, of orchid, plants 242 (1833). (Saccolabium partly.)

o. Hilli, Bentham in Journ. Linn. Soc. XVIII, 334 (1880) ‥ - - - N.S.W. Q. - B.fl.TI,298 GEODORUM, Jackson in Andrews, Botan. Reposit. t. 626 (1810).

G. pictum, Lindley, gen. and spec. of orchid. pl. 175 (1833) EULOPHIA, R. Brown in Edwards, Bot. Regist. 686 (1822).

E. venosa, G. Reichenbach in Benth. Fl. Austr. VI, 300 (1870)

E. Fitzalani, F, v. M., fragm. VIII, 30 (1872) DIPODIUM, R. Brown, prodr. 330 (1810).

D. punctatum, R. Brown, prodr. 331 (1810)

D. ensifolium, F. v. M., fragm. V, 42 (1865) CYMBIDIUM, Swartz in nov, act. Upsal, VI, 70 (1799).

C. Hillii, F. v. M. in Regel's Gartenflora, 138 (1879) ... ...

C. canaliculatum, R. Brown, prodr. 331 (1810)

C. albuciflorum, F. v. M., fragm. I, 188 (1859)

C. suave, R. Brown, prodr. 331 (1810) SPATHOGLOTTIS, Blume, Bijdr. 400 (1825).

S. Paulinae, F. v. M., fragm. VI, 95 (1868) PHAJUS, Loureiro, Fl. Cochinch. II, 529 (1790).

P. grandifolius, Loureiro, Fl. Cochinch. II, 529 (1790) .. CALANTHE, R. Brown in Edwards, Bot. Regist. 573 (1821).

C. veratrifolia, $R$. Brown in Bot. Regist. 573 (1821) GALEOLA, Loureiro, Fl. Cochinch. II, 520 (1790). (Erythrorchis, Ledgeria.)

G. cassythoides, G. Reichenbach, Xen. Orchid. II, 77 (1869)

G. foliata, F. v. M., fragm. VIII, 31 (1872) EPIPOGUM, Gmelin, Fl. Sibir. I, 11, t. 2 (1747).

E. nutans, Lindley in Journ. Linn. Soc. I, 177 (1856) GASTRODIA, R. Brown, prodr. 330 (1810).

G. sesamoides, R. Brown, prodr. 330 (1810) ‥ $\ldots$
POGONIA, A. L. de Jussieu, gen. plant. 65 (1789).

P. uniflora, F. v. M., fragm. V, 201 (1866)...

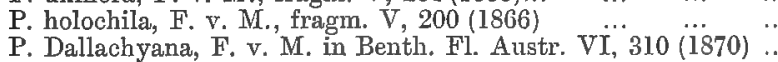

P. pachystomoides, F. v. M., fragm. VIII, 174 (1874) CORYMBORCHIS, Petit-Thouars, hist. des plant. Orchid. re

C. veratrifolia, Blume, Collect. des Orchidées 125 , t. 42 \& 43 (1858) ETAERIA, Blume, Bijdr. 409 (1825). (Hetaeria, Ramphidia.)

E. tenuis, Bentham in Journ. Linn. Soc. XVIII, 345 (1880) ... MICROSTYLIS, Nuttall, Genera of North American plants II, 196 (1818).

M. Bernaysii, F. v. M., fragm. XI, $21(1878) \quad \ldots \quad \ldots \quad \ldots \quad-$
GOODYERA, R. Brown in Aiton. hort. Kew. sec. ed. V, 197 (1813)

G, viridiflora, Blume, Coll. des Orchid. 41, t. 9 (1858) ... ... G. viriditlora, Blume, Coll. des Orchid. $41,4.9(1858)$
G. polygonoides, F. v. M., fragm. VIII, $29(1872)$
SPIRANTHES, L. C. Richard in Mém. du Mus. IV, 40 (1818).

S. australis, Lindley, Bot. Regist. 823 (1824) $\ldots \ldots \ldots \ldots$... $\quad$ - S.A. T. V. N.S.W. Q. THELYMITRA, R. \& G. Forster, char. gen. 97, t. 49 (1776). (Macdonaldia.)

T. ixioides, Swartz in Kong. Svensk. Acad. Handl. 228 , t. $3(1800)$ W.A. S.A. T. V. N.S. W. Q. - B.A. VI,317

T. circumsepta, Fitzgerald, Austral. Orchid, part 4 (1877)

Q. N.A. B. I.VI, 299

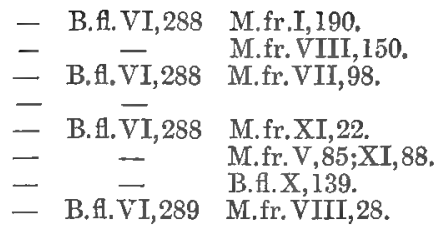

M.fr.XI, 52,139.

M.fr.I, 192;II,181, VII,98.

M.fr.IX, 49.

M.fr.VIII, 248.

M.fr.VIII,248;XI, 139.

M.fr.VII, 96

M.fr. VIII, 248.

B.fi. $\overrightarrow{V I}, 292$

M.fr. IX, 50;XI, 22.

B. H.VI, 293

B.fl.VI, 293

B.fl.VI, 294

B.fl. VI, 294

B.fl.VI,295

B.fl.VI, 295

M.fr. I, 89;VII, 87;X, 50

M.fr.VII, 115 .

M.fr. I,90, 191; VII, 97 .

M.fr.I, 89;VII, 98.

M.fr. VII,98;XI, 22.

M.fr.I, $88 ;$ III, 39 ;VII, 98 .

M.fr. VII, 30 .

\section{M. fr. VII, 97.}

M.fr.III,24;V,102;VIII, [3].

$$
\begin{aligned}
& -\quad--Z-\text { Q. N.A. B.fl.VI,300 M.fr.I,61, } \\
& -\quad \text { Q. }- \text { B.fl.VI, } 300 \text { M.fr.VI, } 30 .
\end{aligned}
$$

M.fr.X, 64 .

$$
\begin{array}{ll}
\text { Q. N.A. B.f.VI,301 M.fr.X,64. } \\
\text { Q. - B. H.VI,30I M.fr.V,42. }
\end{array}
$$

- S.A. - - N.S.W. Q. $\overrightarrow{\text { N.A. B.f. } \overrightarrow{V I}, 302}$ M.fr.XI,88.

- - - N.S.W. Q. - $\begin{array}{ll}\text { B.H.VI,303 M.fr.I,188. } \\ \text { B.H.,303 M.fr.I,187. }\end{array}$

- - - Q. - B.H.VI,304 M.fr.X, 64 .

- N.S.W. Q. - B.f.VI,304 M.fr.I,42;IV,163;X,6 
T. crinita, Lindley, Bot. Regist. XXT, App. XLIX (1839)

T. aristata, Lindley, gen. and spec. of orchid. pl. 521 (1840)

T. longifolia, R. \& G. Forster, char. gen. 98, t. 49 (1776)

T. villosa, Lindley, Bot. Regist. XXV, App. XLIX (1839)

T. tigrina, R. Brown, prodr. 315 (1810)

T. fusco-lutea, $R$. Brown, prodr. 315 (1810)

T. stellata, Lindley, Bot, Regist. XXV, App. X

T. carnea, R. Brown, prodr. 314 (1810)

T. flexusa, Endlicher, nov, stirp. decad. 23 (1839) $\ldots$

T. antennifera, J. Hooker, Fl. Tasman. II, 4, t. 101 (1800)

T. Macmillani, F. v. M., fragm. V, 93 (1865)

T. Mackibbinii, F. v. M. in Melb. Chemist 44 (1881)

T. variegata, Lindley in Benth. Fl. Austr. VI, 323 (1870)

T. venosa, $\mathrm{R}$. Brown, prodr, 314 (1570)

T. cyanea, Lindley in Benth. Fl. Austr. vi, 323 (1870)... EPIBLEMA, R. Brown, prodr. fl. Nov. Holl. 315 (1810)

E. grandiflorum, R. Brown, prodr. $315(1810) \quad \ldots \quad \ldots \quad \ldots$ DIURIS, Smith in Transact. Linn. Soc. IV, 222 (1798).

D. alba, R. Brown, prodr. $316(1810)$
D. punctata, Smith, Exot. Bot. I, 13, t. $8(1804) \ldots$

D. secundiflora, Fitzgerald, Austral. Orchids, part 4 (1877)

D. aurea, Smith, Exot. Bot. 15, t. 9 (1804)

D. palustris, Lindley, gen. and spec. of orchid. pl. $507(1840) \ldots$

D. maculata, Smith, Exot. Bot. I, 57, t. 30 (1804)

D. pedunculata, R. Brown, prodr, $316(1810)$

D. pallens, Bentham, Fl. Austr. VI, 329 (1870) ...

D. abbreviata, F. v. M. in Benth. Fl. Austr. VI, 329 (1870) ..

D. setacea, R. Brown, prodr. 316 (1810)

D. emarginata, R. Brown, prodr. $316(1810)$

D. sulphurea, R. Brown, prodr. 316 (1810)

D. aequalis, F. v. II. in Fitzgerald, Austral. orch. part $\dddot{2}(1876)$

D. dendrobioides, Fitzgerald, Austral. Orchids, part 7 (1882) ...

D. Iongifolia, R. Brown, prodr. 316 (1810)...

D. pauciflora, R. Brown, prodr. $316(1810) \quad \ldots . \quad \ldots . \quad \ldots$ ORTHOCERAS, R. Brown, prodr. fl. Nov. Holl. 317 (1810).

O. strictum, R. Brown, proul. $317(1810) \ldots \ldots \ldots \ldots$ CALOCHILUS, R. Brown, prodr. fl. Nov. Holl. 320 (1810).

C. campestris, R. Brown, prodr. 320 (1810)

C. Robertsoni, Bentlam, Fl, Austr. VI, 315 (1870)

C. paludosus, R. Brown, prodr. 320 (1810)... CRYPTOSPYLIS, R. Brown, prodr. 317 (1810). (Zosterostylis.)

C. longifolia, R. Brown, prodr, $317(1810) \ldots$
C. ovata, R. Brown, prodr, $317(1810)$
...

C. erecta, R. Brown, prodr. 317 (1810) $\quad \ldots$
C. leptochila, F. v. M. in Benth. Fl. Austr. VI, 324 (1870) $\quad \ldots \quad-\ldots \quad-\quad-\quad-$ N.S.W. PRASOPHYLLUM, R. Brown, prodr. 317 (1810). (Genoplesium.)

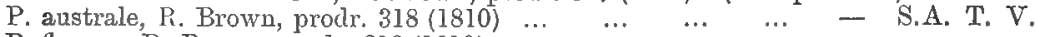

P. flavum, R. Brown, prodr. 318 (1810)

P. elatum, R. Brown, prodr. 318 (1810)

... W.A. S.A. T. V. N.S.W. -

P. hians, G. Reichenbach, Beitr. zur syst. Pflanzenk. 61 (1871)..

P. patens, R. Brown, prodr. 318 (1810)

P. fuscum, R. Brown, prodr. 318 (1810)

P. cyphochilum, Bentham, FI. Austr. VI, 340 (1870)

P. ovale, Lindley, Bot. Regist. XXV, App. LIV (1839)

P. macrostachyum, R. Brown, prodr. $318(1810)$...

P. Fimbria, G. Reichenbach, Beitr. zur syst. Planzenk. 60 (187i) W.A. - - - - $-\overline{-}$ -

P. parvifolium, Lindley, Bot. Regist. XXV., App. LIV (1839) ...

P. - - - - -

P. brachystachyum, Lindley, gen. and spec. of orchid. pl. $513(1840)$

P. despectans, J. Hooker, Fl. Tasman. If, 13, t. $113(1860)$

P. fimbriatum, R. Brown, prodr. 319 (1810)

P. intricatum, C. Stuart in Benth. Fl, Austr. VI, 346 (1870)

P. Woollsii, F. V. M., fragm. V, 100 (1866) MICROTIS, R. Brown, prodr. Al. Nov. Holl. 320 (1810).

M. porrifolia, I. Brown, prodr. 320 (1810)...

M. media, R. Brown, prodr. 32l (1810)

M. alba, R. Brown, prodr. \$21 (1810)

M. pulchella, R. Brown, prodr. 321 (1810)...

i. atrata, Lindley, Bot. Regist. XXV, App. LIV (1839) CORYSANTHES, R. Brown, prodr. 328 (1810),

(Coryhas, Nematoceras.)

C. unguiculata, R. Brown, prodr. 328 (1810) $\quad \ldots \quad \ldots \ldots \ldots-$ N.S. IV. -

C. pruinosa, R. Cunningham in N.S. Wales, Magaz, n, $\dddot{i}(1833)$ W.A. S.A. T. V. N.S.W. -

\author{
B.Hl.VI, 319 M.fr.VI,84. \\ B. A, VI, 319 \\ B.Al.VI,319 \\ B.fl.VI, 320 \\ B.fl.VI, 320 \\ B. $1 . V I, 321$ \\ B.fl.VI, 321 \\ B.fl.VI, 321 \\ B.fl.VI, 322 \\ B.fl.VI, 322 \\ - B.fl.VI,323 \\ B. $\mathrm{A}, \overline{\mathrm{VI}}, 323$ \\ B.fi. VI, 323 \\ M.fr. V,93. \\ M.fr. XI, 139 . \\ M.fr. V, 97,98 . \\ B.ti.VI, 323
}

- B.H.VI,324

M.fr.XI,21.

- B.fl.VI,325

M.fr. V,102.

M.fr. V, 102 .

- B. -

B.fl. $\overline{V I}, 327$

- B.fl.VI,327

- B.fl.VI,327

-- B.fl.VI,327

- B.fl.VI,329

- B.fi.VI,329

- B.fl.VI, 329
- B.fl.VI, 329

- B.ti.VI,330

- B.fl.VI, 330

- B.f., VI, 331

M.fr. $V, 172$.

I.fr. V, 102 .

M.fr. V, 102 .

M. fr. V, 102, 173 .

M.fr. V, 101.

II.fr. V, 102,173.

M.fr. $\mathrm{X}, 65$.

M.fr. V, 101,172.

- B.fl.VI,33I

- B.fl.VI,332

B. fl. VI, 315

- B.fl.VI,315

- B.fi.TI,316

M. fr. VIII, 151.

B.fl. VI, 333

- B.fl.VI,334

- B.f. VI,334

- B.fl.VI,334

M.fr.XI, 21.

- B.f.VI, 337

- B.fl.VI,337

- B.f. YI,337

- B.f.VI,338

- B.f.VI, 338

- B.fl.VI,339

- B.fl.VI,339

- B.fl.VI, 340

- B.Al.VI,341

- B.fl. VI,341

- B.fl. VI,341

- B.H.VI,34:2

- B.f. TY, 342

- B.fl. YI,342

B.f.YI,343
- B.f.VI,343

- B.fl.VI,34t

- B.fl.TI,344

- B. fl. VI,345

- B.fl.YI,345

- B.f. II $^{\top}, 345$

- B. VI,346

- B fi. IT, 346

M.fr. $V, 100$.

il.fr. $V, 101$.

M.fr. V,101.

II.fr. V, 101 .

M.fr. T, 100.

II.fr. $T, 101$.

II fr. $\mathrm{T}, 100$.

M.fr.IX, 50.

II.fr. $V, 100$.

MI.fr. $Y, 100$.

M.fr. T, 100 .

Mi.fr. $Y, 100$.

II.fr, $\mathrm{V}, 100$.

MI.fr. T, 100 . W.A. S.A. T. V. N.S.W. Q.
W.A. - W.A. - - -
W.A. - -

B. fl. VI, 347

M.fr. X,65; XI,21.

B.fl. VI, 348

- B.fl. II, 348

- B.fl. TI,349

B.f. $Y I, 349$

M. fr. I, 90,244;X, 65.

- B.fl. VI,350 II.fr. IX, 49 . 
C. fimbriata, R. Brown, prodr. 328 (1810)

C. bicalcarata, R. Brown, prodr. 328 (1810)

P. concinna, R. Brown, prodr, 326 (1810) ...

P. curta, R. Brown, prodr. 326 (1810)

P. acuminata, $R$. Brown, prodr. 326 (1810)...

P. nutans, R. Brown, prodr. 327 (1810)

P. pedaloglossa, Fitzgerald, Austral. Orchids, part 3 (1877)

P. pedunculata, R. Brown, prodr. 327 (1810)

P. nana, R. Brown, prodr. 327 (1810)

P. cucullata R Brown, prodr 327 (1810) '

P. grandifiora, R. Brown, prodr. 327 (1810)

P. reflexa, R. Brown, prodr. 327 (1810)

P. praecox, Lindley, gen. and spec. of Orchid. pl. $388(1340)$

P. obtusa, R. Brown, procir. 327 (1810)

P. recurva, Bentham, Fl. Austr. VI, 360 (1870) $\ldots$

P. parviflora, R. Brown, prodr. 327 (1810)...

P. barbata, Lindley, Bot. Regist. XXV, App. LIII (1839)

P. turfosa, Endlicher in Lehm. pl. Preiss. II, 5 (1846) ...

P. mutica, R. Brown, prodr. 328 (1810)

P. rufa, R. Brown, prodr. 327 (1810)

P. Daintreyana, F. v. M. in Benth. Fl. Austr. VI, 360 (1870)

P. longifolia, $R$. Brown, prodr. 327 (1810) ...

P. vittata, Lindley, Bot. Regist. XXV, App. LIIÏ (1839) PTERosTyLIS, R. Brown, prodr. ⺆. Nov. Holl, 326 (1810).

P. ophioglossa, R. Brown, prodr. 326 (1810)

P. truncata, Fitzgerald, Austral. Orchids, part 4 (1878) ...

.. - - - N.S.W. - - - N.S.W. Q.

$\cdots \quad$ - S.A. - - N. N.S.W. Q. ... - S.A. T V N W W

... - - - V. N.S.W.

... - S.A. T. V. N.S.W. Q.

.. - - - - N.S.W. -

... - $\quad$ S.A. T. V. N.S.W. -

... W.A. S.A. T. V. - -

.. - S.A. T. V. N.S.W. -

... - - - - N.S.W. -

... W.A. S.A. - V. N.S.W. -

.. - S.A. T. V. N.S.W. -

.. - T. V. N.S.W. -

... W.A. - $\overline{\text { T. }} \overline{\text { V. N.S.W. }} \cdot \overline{\mathrm{Q}}$ W.A. S.A. T. V. N.S.W. W.A. $\overline{\text { TA }} \overline{\mathrm{T}} \overline{\mathrm{T}} \overline{\mathrm{S}} \overline{\mathrm{T}}$ W.A. S.A. T. V. N.S.W. Q. ... W.A. S.A. T. V. N.S.W. Q. $\cdots \quad$ - S.A. T. V. N.S.W. CALEYA, R. Brown in Aiton, Hort. Kew. sec. ed. V, 204 (1813). (Calnana, 1810.)

C. major, R. Brown, prodr. $329(1810) \quad \ldots \quad \ldots . \quad \ldots \quad \ldots .-1-$ T. V. N.S.W. Q.

C. minor, R. Brown, prodr. $329(1810)$ … $\cdots$

C. nigrita, Lindley, Bot. Reg. XXV, App. LIV (1839) $\ldots .$.
C. Sullivani, F. v. M. in Melb. Chemist 44 (1882)
$\ldots$

DRAKAEA, Lindley, Bot, Regist. XXV, App. LV (1839). (Spiculaea, Arthrochilus.)

D. ciliata, G. Reichenbach, Beitr. zur syst. PHanzenk, 68 (187I) W.A. - - - - -

D. irritabilis, G. Reichenbach, Beitr. zur syst. Pflanzenk. $68(1871)---$ - N.S.W. Q.

D. elastica, Lindley, Bot. Regist. XXV, App. LV (1839)

ACIANTHUS, R. Brown, prodr. fl. Nov. Holl. 321 (1810).

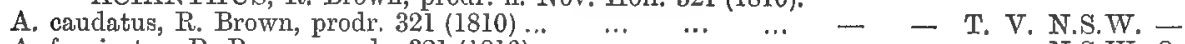

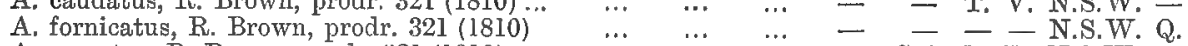

A. exsertus, R. Brown, prodr. $321(1810) \ldots$
A. viridis, J. Hooker, Fl. Tasman, II, $372(1860) \ldots$
$\ldots$

CYRTOSTYLIS, R. Brown, prodr. flor. Nov. Holl, 322 (1810).

C. reniformis, R. Brown, prodr. $322(1810) \quad \ldots . \quad \ldots \quad \ldots$. W.A. S.A. T. V. N.S.W. Q. LYPERANTHUS, R. Brown, prodr. 325 (1810). (Burnettia)

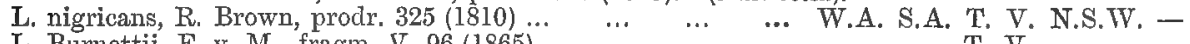

L. Burnettii, F. v. M., fragm. V, $96(1865)$
L. Forrestii, F. v. M. in Wing's S. Sc. Rec. II, $55(1882) \quad \ldots \quad \ldots-1$.

L ellipticus, R. Brown, prodr, 325 (1810)

ERIOCHILUS, R. Brown, prodr. fl. Nov. Holl. 323 (1810).

F. autumnalis, $R$. Brown, prodr. 323 (1810)

E. scaber, Lindley, Bot. Regist. XXV, App. LIII (1839)

E. tenuis, Lindley, Bot. Regist. XXV, App. LIII (1839)

...

I. dilatatus, Lindley, Bot. Regist. XXV, App. LIII (1839)

1. multiflorus, Lindley, Bot. Regist. XXV, App. LIII (1839) ..'

E. fimbriatus, F. v. M. in Wing's S. Sc. Record II, 152 (1882)...

CALADENIA, R. Brown, prodr. 325 (1810). (Leptoceras,

C. Menziesii, R. Brown, prodr. 325 (1810) .. … $\ldots$.
C. Nortoni; Adenochilus, Fitzgerald, Austral. Orch. part $2(1876)$
C. Cairnsiana, F. v. M., fragm. VII, 31 (1869) …

W.A. S.A. T. V. N.S.W. Q.

W.A. - - - - -

W.A. - - -

W.A. - - -

W.A. $-\overline{\text { W.A. }}$ - $-\overline{\mathrm{V}}$

-

Adenochilur.)

C. discoidea, Lindley, Bot. Regist. XXV, App. LII (1839)

C. Patersoni, R. Brown, prodr. 324 (1810) ...

C. Drummondii, Bentham, Fl. Austr. VI, 383 (1873)

C. hirta, Lindley, Bot. Regist. XXV, App. LII (1839) $\ldots$

C. Roei, Bentham, Fl. Austr. VI, 383 (1873)

C. Barbarossae, G. Reichenbach, Beitr. z. syst. Pflanzenk. 64 (1871)

C. flava, R.'Brown, prodr. 324 (1810)

C. latifolia, R. Brown, prodr. 324 (1810)

W.A. S.A. T. V.

W.A. - - - N.S.W. -

1) W.A. - $=--$

… W.A. S.A. T. V. N.S.W. $\overline{\text { W. }}$

W.A. - - - - --

W.A. - - - - -

… $\quad \ldots \quad$... W.A. S.A. T. T. N.S.W.

C. reptans, Lindley, Bot. Regist. XXV, App. LII (1839) _.. W.A. - - - _ -

C. suaveolens, G. Reichenbach, Beitr. zur syst. Pflanzenk. $67(1871)--$ T. V. N.S.W. -

$\begin{array}{lllllll}\text { C. serrata, G. Reichenbach, Beitr. zur syst. Pflanzenk. } 67(1871) & \text { W.A. } & - & - & - \\ \text { C. carnea, R. Brown, prodr. } 324(1810) & \ldots & \ldots & \ldots & \ldots & - & \text { S.A. T. V. N.S.W. Q. }\end{array}$

C. congesta, R. Brown, prodr. $324(1810) \ldots$

C. aphylla, Bentham, Fl. Austr. VI, 387 (1873) ... ...

C. coerulea, R. Brown, prodr. $324(1810) \ldots \ldots$... $\ldots$

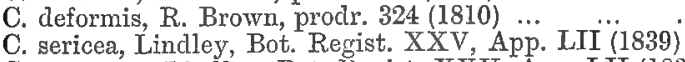

C. gemmata, Lindley, Bot. Regist. XXV, App. LII (1839) $\cdots \quad$ - S.A. T. V. N.S.W. Q.

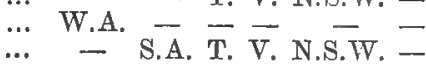

‥ - S.A. T. V. N.S.W. -

... W.A. - - - - -

… W.A. W. - - -
B.fl. VI, 351 M. fr. $X, 65$.

B.fl. VI, 351

B.fl. VI, 354

B.H.VI, 355

B.fl.VI, 355

B.fl.VI, 355

B.fl. VI, 356

B. 田, VI, 356

B.fl. VI, 357

B. 1 .VI, 357

B.fi. VI, 358

B. $\operatorname{fl} \overline{\mathrm{VI}}, 359$

B. fl. VI, 359

B.fl.VI, 360

B.fl.VI, 360

B.fl.VI, 361

B.f.VI, 362

B.t.VI, 362

B.fl. VI, 362

B.丹.VI, 363

B.fl.VI, 360

B.fl.VI, 364

B.fl.VI, 364

$\begin{array}{ll}- & \text { B.fl.VI, } 366 \\ \text { - } & \text { B.fl.VI, } 366 \\ \text { B.fl.VI,366 }\end{array}$

B.A.VI, 367

- B.H.VI,368

- B.fl.VI,368

B.fl. VI, 369

- B.fl.VI,370

- B.fl.VI,370

- B.fi.VI,371

- B.fl.VI, 370

- B.fl.VI,374 B.fl.VI,375

- B.fl. VI,374

B.fl. VI, 372

B.fl.VI, 372

- B.fl.VI,372

- B.fl.VI,372

- B.fl.VI,373

B. 1. VI,377

B.fl.VI,377

B.fl. VI, 378

B.fl.VT, 378

B.fl.VI, 382

B.fi.VI, 382

B.fl.VI,383

B.fl. VI, 383

B.fl.VI, 383

B.fl.VI, 383

B.fl.VI, 384

B.fl.VI, 384

B.fl.VI, 385

B.fl.VI, 385

B.fl.VI, 380

B.fl.VI, 386

B.H.VI, 387

B.fl.VI, 387

B.fl.VI,388

- B.H.VI,388

- B.fl,VI,389

- B.H.VI,389
M.fr. VII,134;X, 65.

M.fr. V,96;VII, 134 .

M.fr.VII, 133.

M.fr. V, $99 ; \mathrm{XI}, 105$.

M.fr. V, 99 .

M.fr. V,99.

M.fr.VIII,249;XI, 105.

I.fr. V,99.

T.fr. V,99.

M.fr. V,99;XI, 105.

M.fr. XI, 105 .

M.fr.IX, $50 ; \overline{X I}, 53$.

M.fr. V, 100 .

M. fr. VIII, 151;XI, 22, 105 .

M.fr. V, 100 .

M.fr. $\nabla, 100$.

M.fr. V, 100.

M.fr.XI, 126.

M,fr,XI, 105.

M.fr. VIII, $151 ; X I, 53$.

M.fr.XI,22, 105 .

M.fr.XI, 105.

M.fr.XI, 104.

M.fr.V,101;XI, 105 .

M.fr.X, 64.

M.fr. VII,31.

M.fr. V, 101.

M.fr.V, 101;VI,83;XI, 105.

M.fr.V,101;XI, 105 .

M. fr. V, 98.

M.fr.V, 99 .

M.fr.V, 101 .

M.fr. V, 101;XI,22.

M.fr. V, $101 ; \mathrm{XI}, 105$.

M.fr. $V, 101$, 
C. ixioides, Lindley, Bot. Regist. XXV, App. III (1839)

C. unita, Fitzgerald in Gardeners' Chronicle 461 (1882) ...

W.A.
.. W.A.
W. - - - $-1-\geq$ B.fl.VI,389

CHILOGLOTTIS, R. Brown, prodr. fl. 322 (1810).

C. diphylla, $R$. Brown, prodr. 323 (1810) ... - ..

C. trapeziformis, Fitzgerald, Austral. Orchids, part 3 (1877)

C. Gunnii, Lindley, gen. and spec. Orchid, 387 (1840) ...

GLOSSODLA, R. Brown, prodr. 325 (1810).

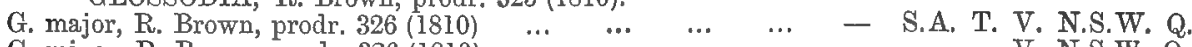

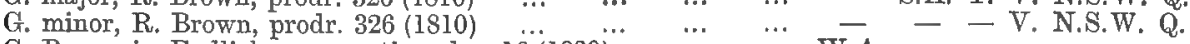

G. Brunonis, Endlicher, nov, stirp. dec. 16 (1839) $\ldots$ … $\quad$ W.A. $\quad$ - $\quad$ - $\quad$ -

G. intermedia, Fitzgerald in Gardeners' Chron. 462 (1882)

G. emarginata, Lindley, gen. and spec. of Orchid. pl. $424(1840)$ HABENARIA, Willdenow, spec. plant. IV, 44 (1805).

H. trinervis, Wight, icon, plant. Ind. or, t. 1701 (1852)...
H. elongata, R. Brown, prodr. 313 (1810) ... ...

H. graminea, Lindley, gen. and spec. of orchid. pl. 318 (1840).

H. ochroleuca, R. Brown, prodr. 313 (1810)

H. xanthantha, F. v. M., fragm. VII, $16(1869) \quad \ldots \quad \ldots$. APOSTASIA, Blume, Bijdr, 423 (1825). (Niemeyera.)

A. stylidioides, G. Reichenbach in Benth. FI. Austr. VI, 396(1873) - - - - - Q. - B.A.VI,396

\section{SCITAMINEAE.}

Iinné, philos, botan. 27 (1751).

MUSA, l'Ecluse, éxoticorum libri decem 229 (1605).

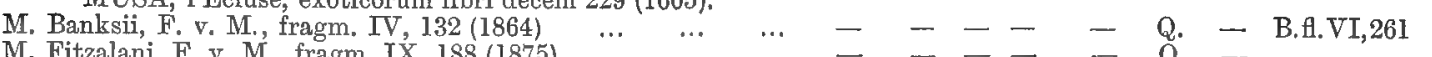

M. Fitzalani, F. v. M., fragm. IX, 188 (1875)

M. Hillii, F. v. M., fragm. IX, 169 (1875)... CURCUMA, Linné, gen. plant. 332 (1737), from Hermann (1687).

C. Australasica, J. Hooker, Bot. Mag. t. 5620 (1867) AMOMUM, Linne, gen. plant. 330 (1737).

A. Dallachyi, F. v. M., fragm. VIII, 25 (1872) ELETTARIA, White and Matton in Transact. Linn. Soc. X, 249 (1809), from Rheede (1692). E. Scottiana, F. v. M., fragm. VIII, 24 (1872) $\ldots . .$.
ALPINIA, Linné, gen. plant. 332 (1737). (Hellenia.)

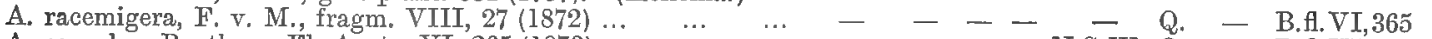

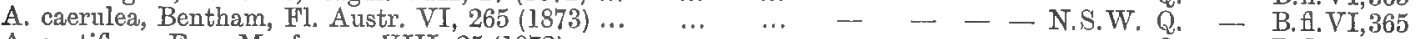

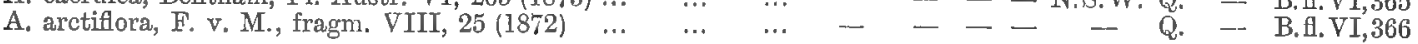
CosTUS, C. Bauhin, pinax, 36 (1623).

C. Potierae, F. v. M., fragm. IV, 164 (1864) TAPEINOCHEILOS, Miquel in annal. mus, bot. Lugdun. IV, 101, t. 4 (1869).

T. pungens, Miquel in ann, mus. Lugd. IV, 101, t. 4(1869) ... _- _ _

\section{IRIDEAE.}

Ventenat, Tableau du règne végétal II, 188 (1799).

DIPLARRHENA, Labillardière, Relation du voy, à la rech. de La Per. I, 275, t. 15 (1799).

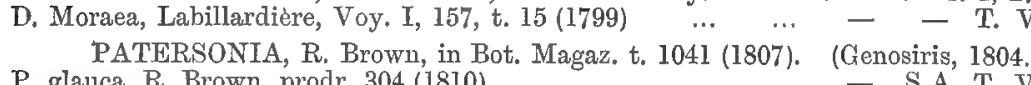

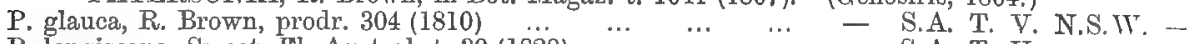
P. longiscapa, Sweet, FI. Austral. t. 39 (1828)

P. occidentalis, R. Brown, prodr. 304 (1810)
P. umbrosa, Endlicher in Lehm. pl. Preiss. II, 31 (1846)
P. xanthina, F. v. M., fragm. I, 214 (1859)

P. limbata, Endlicher in Lehm. pl. Preiss. II, 29 (1846)...

P. juncea, Lindley, Bot. Regist. XXV, App. LVIII (1839)

P. Maxwelli, F. v. M. in Benth. fl. Austr. VI, 405 (1873)

$P$. pygmaea, Lindley, Bot. Regist. XXV, App. LVIII (1839)

P. sericea, R. Brown in Bot. Mag. t. 1041 (1807) ... … ..

P. lanata, R. Brown, prodr. 303 (1810)

P. rudis, Endlicher in Lehm. pl. Preiss. II, 29 (1846)

P. macrantha, Bentham, Fl. Austr. VI, 407 (1873) $\ldots .$.

P. glabrata, R. Brown, prodr. 304 (1810) … $\ldots$.

P. Drummondii, F. v. M. in Benth. F1. Austr. VI, 407 (1873) ...

P. inaequalis, Bentham, Fl. Austr. VI, 408 (1873)

P. graminea, Bentham, Fl. Austr. VI, 408 (1873)...

... W.A.

S.A. T. V.

-.

-

IV.A.

W.A.

VIV. - -

IV.A.

W.A.

W.A.

W.A.

W.A.

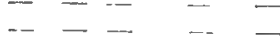

IV.A. - -

W.A. $--\overline{\text { V. N.S.W. }}$ Q.

W.A.

W.A. - - - - -

IRIS, Tournefort, inst. 358, t. 186, $188(1700)$, from Hippocrates, Dioscorides and Pliuius,

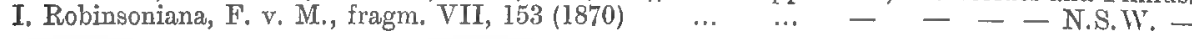
SISYRINCHIUM, Linné, gen. plant. 273 (1737)

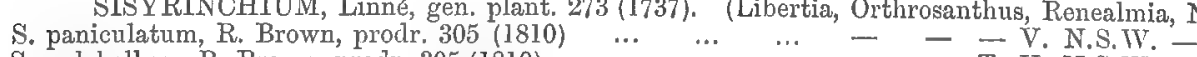
S. pulchellum, R. Brown, prodr. $305(1810) \quad \ldots \quad \ldots \quad \ldots \quad-\quad \ldots \quad-$ T. V. N.S.W. S. cyaneum, Lindley, Bot. Regist. t. 1090 (1827)... $411(1873)$

W.A. S.A. T. V. N.S.IT. -

W.A. - - - - -

\section{$-$} B. fl. VI, 400

$-$ B.fl. TI, 402 B.H. VI, 402 B.fl.VI, 403 B.fl.VI, 404

B.fi.VI,404

- B.fl.VI,404

- B.fl.VI,404

- B.f.VI, 405

- B.f.VI, 405

-- B.A. YI, 406

- B.f.VI, 406

- B.f.VI, 406

- B.t.VI,407

- B.fl.VI, 407

- B.f.TI, 407

- B.fl.VI,408

- B.fl.VI,408

- B.fl.VI,408

(Moraea partly.)

- B.fl.VI, 409 M.fI. YII, 153. atostigma)

- B.f.YI,413 M.fr. VII,91. - B.fl.VI,413 M.fr.VII,92.

- B.fl. TI,410 M.fr. III, 92 . - B.H.VI,411

II.fr, VII, 94.

M.fr. VII, 36 .

M. fr. YII, 36 .

M.fr. VII,31.

M.fr. VII, 32

M. fr. VII, 33.

M. fr.VII, 34 .

M.fr. VII, 33.

M.fr. VII, 35.

M.fr. VII, 35 .

M.fr. VII, 35.

M.fr, XI, 23.
M.fr.X, 116

M.fr.X,116. [116;XI,22

M.fr.VIII, 15l; IX,50; X,

M.fr. VI, 83.

M.fr.XI, 22.

M.fr. VII, 15 .

M.fr.VII, 16 .

M.fr. $V, 102$.

M.fr.VII, 16.

M.fr.IV, 132.

M.fr.IX, 188.

M.fr. IX, 169 .

M.fr.VIII, 26.

M.fr. VIII, 25.

M.fr. VIII,24.

M.fr. VIII, 27

M.fr. VIII, 26.

M.fr. VIII, 25.

M.fr.IV,164;VIII,27.

M.fr.VIII, 26. 
CAMPYNEMA, Labillardiere, Nov, Holl, pl. spec. I, 93, t. 121 (1804).

C. lineare, Labillardière, Nov, Holl. pl, spec. I, 93, t. $121(1804)-{ }^{2}$ T. $-\quad-\quad$ B.f.VI,415 M.fr.TI,96.

\section{BURMANNIACEAE.}

Sprengel, syst. veg. I, 125 (1825).

BURMANNIA, Linné, syst. nat. 8 (1735); Linné, gen. plant. 100 (1737).

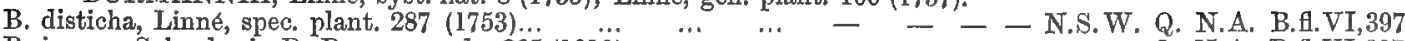

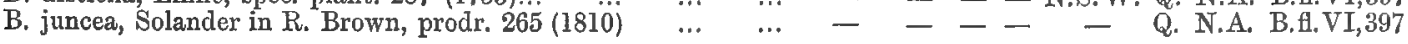

\section{TACCACEAE.}

Presl, reliq. Haenk. I, 149 (1828).

TACCA, R. et G. Forster, char. gen. 65 (1776).

T. pinnatifida, R. \& G. Forster, char. gen. 65 (1776)

\section{DIOSCORIDEAE.}

Du Mortier, Analyse des familles des plantes 57 (1829), from R. Brown (1810).

DIOSCOREA, Plumier, nov. plant. Americ. gen. 9, t. 26 (1703). (Dioscoridea.)

D. hastifolia, Nees in Lehm. pl. Preiss. II, 33 (1846) $\ldots$... W.A.

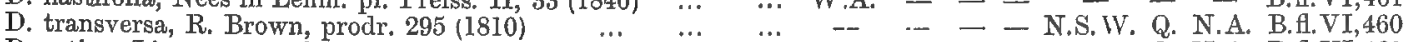

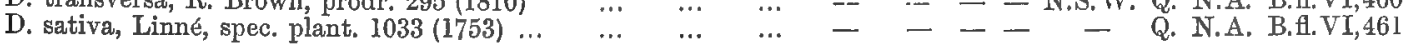

M.fr.VII, $80 ; \mathrm{XI}, 20$.

PETERMANNIA, F. v. M., fragm. II, 92 (1860).

P. cirrosa, F. v. M., fragm. II, $93(1860)$... $\quad \ldots \quad$... $\quad \ldots \quad-\quad-\quad-\quad$ N.S.W. $-\quad$ - B.fl.VI, 462

M.fr. VII, 80 .

M.fr.VII, 81 .

M.fr.II, 176.

\section{HYDROCHARIDEAE.}

Lamarck and De Candolle, Flore Française, III, 265 (1805).

HYDROCHARTS, Linné, syst. nat. 8 (1735); Linné, gen. plant. 308 (1737).

H. morsus ranae, Limné, spec, plant. 1036 (1753)... ... ... -

ENHALUS, L. C. Richard in Mém. de l'Inst. II, 64 (1811).

E. Koenigii, Richard in Mém. de l'Inst. II, 64 (18I1) $\ldots$... -

HALOPHILA, Du Petit-Thouars, gen. nov. Madag. n, 6 (1806).

H. ovata, Gaudichaud, Bot. Voy. Freyc. 470, t. $40(1826)$.

$-\quad-\quad$ Q. $-\quad-$

M.fr. VI, 199.

H. spinulosa, Ascherson in Neumayer, Anleit. zu wiss. Beob. 368(1875)-

S.A. T. V. N.S.W. Q. - B.fl.VII,182 M.fr.VIII,219. OTTELIA, Persoon, synops. plant. I, 400 (1805). (Damasonium partly.)

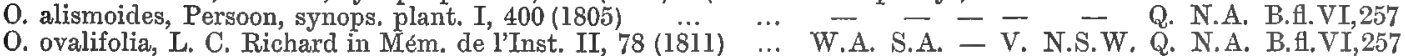
BLYXA, Noronha in Du Petit.Thouars, gen. nov. Madag. n. 14 (1806).

B. Roxburghii, L. C. Richard, Mém. de l'Inst. II, 77, t. 5 (1811) - S.A. - - - Q. N.A. B.A.VI,258

VALLISNERIA, Micheli, nov. plant. gen. 12, t. 10 (1729).

V. spiralis, Linne, spec. plant. $1015(1753) \ldots \quad \ldots \quad \ldots \quad \ldots \quad-\quad$ S.A. T. V. N.S.W. Q. N.A. B.H.VI, 259

HYDRILLA, L. C. Richard in Mém. de l'Inst. II, 61 (1811).

H. verticillata, Caspary in Bot. Zeitung, Dec. (1856) $\ldots$... - S.A. - V. N.S.W. Q. N.A. B.AlvI,259

\section{AMARYLLIDEAE.}

J. St. Hilaire, Expos. des famill. natur. des pl. I, 134, t. 21 (1805).

HAEMODORUM, Smith in Transact. Linn. Soc. 213 (1798).

H. distichophyllum, Hooker, icon. plant. t. 866 (1852)

H. brevicaule, F. v. M., fragm. I, 64 (1858) $\quad \ldots \quad \ldots . \quad \ldots$

H. sparsiflorum, F. v. M., fragm. VII, $117(1870) \quad \cdots \quad \cdots$, W.A. - - -

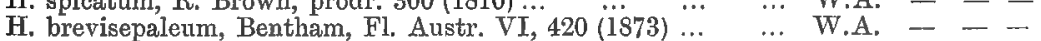

H. paniculatum, Lindley, Bot. Regist. XXV, App. XLIV (1839) W.A. - - -

H. Iaxum, R. Brown, prodr. 300 (1810) … ... ... .

H. simplex, Lindley, Bot. Regist. XXV, App. X $\dddot{\text { LIV }}$ (1839)

H. simulans, F. v. M., fragm. VII, 117 (1870) $\ldots$.

H. planifolium, R. Brown, prodr. $300(1810) \quad \ldots . \quad \ldots$

H. teretifolium, R. Brown, prodr. $300(1810)$
H. coccineum, R. Brown, prodr. $300(1810)$

H. coccineum, R. Brown, prodr. $300(1810)$
H. subvirens, F. v. M., fragm. I, $63(1858)$

H. ensifolium, F. v. M., fragm. I, 64 (1858)
H. parviflorum, Bentham, Fl. Austr. VI, 423 (1873) $\quad \ldots$

H. parviflorum, Bentham, Fl. Austr. VI, 423 (1873)
H. leptostachyum, Bentham, Fl. Austr. VI, 423 (1873) $\ldots$

H. tenuifolium, Cunningham in Benth. Fl. Austr. VI, 425 (1873)

PHLEBOCARYA, R. Brown, prodr. 301 (1810).

P. ciliata, R. Brown, prodr. 301 (1810) … … ‥

P. pilosissima, F. v. M. in Benth. H. Austr. VI, 425 (1873)
P. filifolia, F, V. M. in Benth. fl. Austr. VI, 425 (1873)...
... W.A. - - - - - - B.fl.VI,424

... W.A. - - - - - - B.fl.VI,425

... W.A. - - - - - B.f.VI,425
M.fr.VII, 118;IX,51.

M.fr.VII, 118 .

M.fr.VII, 117 .

M.fr. VII, 118.

- - B.H.VI,420

- - B.f. VI,420

- - B.fl.VI,420

W.A. - - - - - B.A.VI,421

W.A. - - - - - - B.H.VI,42I

... W.A. - - - N.S.W. Q. - $\begin{aligned} & \text { B.fl.VI,421 } \\ & \text { B.f.VI,422 }\end{aligned}$

.. - - - - - N.S.W. - - B.H.VI,422

.. - - - - - Q. N.A. B.A.VI,422

$. \quad-\quad-\quad-$ - N.A. B.H.YI,423

.. - - - - Q. N.A. B.fl.VI,423

- $\quad$ - N.A. B.fl.VI,423

- $\quad$ N.A. B.fl.VI, 423

M.fr. VII, 118.

M.fr. VII, 117.

M.fr.VII, 118.

M.fr. VIJ, 118 .

M.fr. I, 63 .

M.fr, VII, 118 . 
TRIBONANTHES, Endlicher, nov. stirp. dec. I, 27 (1839).

T. brachypetala, Lindley, Bot. Regist. XXV, App. XLIV (1839)

T, uniflora, Lindley, Bot. Regist. XXV, App. XLIV (1839) ...

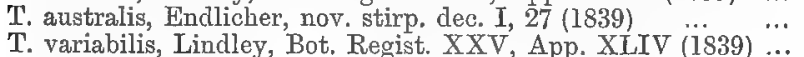

T. longipetala, Lindley, Bot. Regist. XXV, App. XLIV (1839)

CONOSTYLIS, R. Brown, prodr. 300 (1810). (Blancoa, Androstemma.)

C. breviscapa, R. Brown, prodr. 301 (1810)

C. vaginata, Endlicher in Lehm. pl. Preiss. II, $2 \ddot{3}(1846) \ldots$

C. petrophiloides, F. v. M. in Benth. Fl. Austr. VI, 431 (1873)

C. setosa, Lindley, Bot. Regist. XXV, App. XLIV, t. 6 (1839)

C. aurea, Lindley, Bot. Regist. XXV, App. XLIV (1839)

C. melanopogon, Endlicher in Lehm. pl. Preiss. II, IS (1846) $\ldots$

C. setigera, $\mathrm{R}$. Brown, prodr. 300 (1S10)

C. Psyllium, Endlicher in Lehm. pl. Preiss. II, 21 (1846)

C. villosa, Bentham, Fl. Austr. VI, 433 (1873)

C. Drummondii, Bentham, Fl. Austr. VI, 433 (1873)

C. gladiata, Bentliam, Fl. Austr. VI, 434 (1873) 23 (1846)

C. seorsiflora, F. v. M., fragm. I, 158 (1859)

C. teretiuscula, F. v. M., fragm. VIII, is (1872) ...

C. stylidioides, F. v. MI., fragm. VIII, 17 (1872) ...

C. prolifera, Bentham, Fl. Austr. VI, 436 (1839) ...

C. racemosa, Bentham, FI. Austr. VI, 436 (1839)...

C. candicans, Endlicher, nov. stirp. dec. 20 (1839)

C. dealbata, Lindley, Bot. Regist. XXV, App. XLV (1839)

C. Preissii, Endlicher in Lehm. pI. Preiss. II, 18 (1846) ...

C. bracteata, Endlicher in Lehm. pl. Preiss. II, I6 (1846)

C. filifolia, F. v. MI., fragm. VIII, 18 (1872)

C. spimuligera, F. v. M. in Benth. Fl. Austr. VI, 438 (1373)

C. bromelioides, Endlicher in Lehm. pl. Preiss. II, 18 (1846)

C. aculeata, R. Brown, prodr, 300 (1810)

C. laxillora, Bentham, El. Anstr. VI, 439 (1873) $\cdots \cdots$

C. cymosa, F. v. M. in Benth. Fl. Austr. VI, 439 (1873)...

C. serrulata, R. Brown, prodr. 300 (1810) ...

C. caricina, Lindley, Bot. Regist. XXV, App. XLV (1839)

C. Androstemma, F. v. M., fragm. VIII, 19 (1873)

C. Bealiana, F. v. M., fragm. IX, 50 (1875)

C. canescens, F. v. M., fragm. VIII, 19 (1873)

W.A.
W.A. - - -

W.A. - - -

W.A. - - -

W.A. - - -

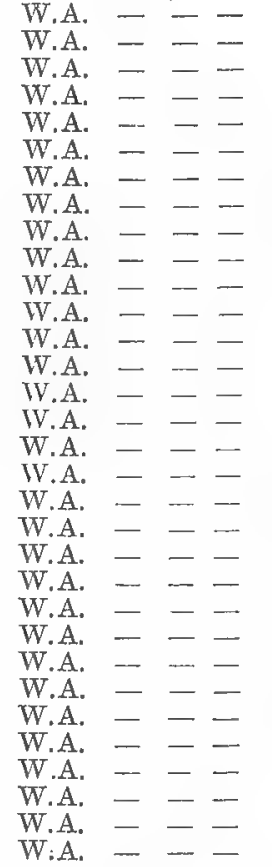

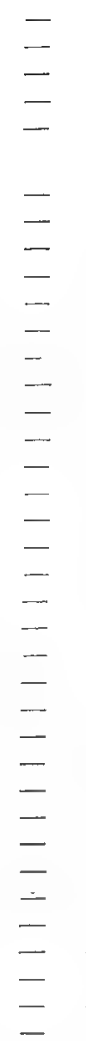

- B.fl.VI, 426

B.f. VI, 427
- B.f. VI, 427
- B.fl.VI, 427

B.fl.VI, 427

B. H. VI, 430

- B.fl.VI,431

B.t.VI, 431

B.fl. VI, 431

B.fl.VI, 432

- B.fl.VI,432

B.fl.VI, 432

B.f.VI, 433

B.fl.VI,433

- B.f.VI, 433

B.fl.VI, 434

- B.fl.VI,434

- B.tl.VI,435

- B.fl.VI,435

B.tl.VI, 435

- B.fl.VI,436

- B.fl. VI, 436

- B.fl. VI, 436

- B.fl. VI, 436

- B.H.VI,437

- B.fl. VI, 437

- B.fl.VI, 438

- Bf VT 438

- B.fl.

- B.H.VI,438

- B.fl. VI, 439

- B.tl.VI,439

- B.fl. VI,440

- B.fl.VI,440

-

B. A. $\overline{V I}, 441$
MI.fr.VIII, 23.

M.fr.VIII, 23 .

M.fr.VIII, 23.

M.fr. VIII, 23.

M.fr. VIII, 19 .

M.fr. VIII, 19 .

M.fr.VIII, 19.

M.fr.VIII, 20

M.fr.VIII, 20 .

M.fr. VIII, 19 .

M.fr.VIII, 18 .

M.fr.VIII, 17 .

M.fr. VI,255.

M.fr. VIII, 20.

M. fr. VIII, 20 .

M.fr. VIII, 18.

M.fr. VIII, 19.

M.fr. VIII, 19 .

M.fr. VIII, 20.

Mi.fr. VIII, 19.

M.fr.IX, 50 .

M.fr. VIII, 19. Schwaegrichenia)

A. rufa, Labillardiere, Voy. I, 41], t, $22(1798)$

A. pulcherrima, Hooker, Bot. Jag. t. 4180 (1845) $\ldots \ldots \ldots$

A. flavida, Redouté et De Candolle, les Liliacées t. 176 (I807) $\cdots$

A. Preissii, Endlicher in Lehm. pl. Preiss. II, 26 (1846)... $\ldots$. W.A. - $\quad$ - - -

A. viridis, Endlicher in Lehm. pl. Preiss. II, 25 (1846)

A. Manglesii, D. Don in Sweet, Brit. Fl. Gard. sec. ser. t. 265 (1836) W.

A. bicolor, Endlicher in Lehm. pl. Preiss. II, 26 (1846) ... ... W.

A. fuliginosus, Hooker, Bot. Mag. t. $4291(1847) \ldots \ldots \ldots$... $\quad \ldots \quad$ W.A.

CURCULIGO, Gaertner, de fruct. I, 63, t. 16 (1788).

C. recurvata, Aiton, hort. Kew. sec. ed. II, 253 (1811) ...

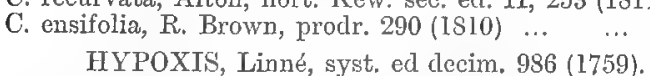

H. hygrometrica, Labillareliere, Nov. Holl. pl, spec. I, 82, t. 108(1804) - S.A. T. V. N.S.W. Q.

H. glabella, R. Brown, prodr. 289 (1810) $\ldots . \ldots . \ldots \ldots$ W.A. S.A. T. V. N.S.W. -

W.A. - - -

W.A. - - - -

W.A. - - -
W.A. - - -
W.A. - -

W.A. - - - -

A. - - -
A. - -

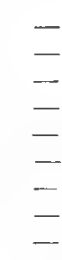

-
-
-
-
-
-

Macropidia, Macropodia,

M.fr. V [II,21.

B.fl. VI, 443 M.fr. VIII, 21 ,

B. fl. VI, 443

B.fl.VI, 444

B.fl. VI, 444

B. $\mathrm{fl}$ VI, 445

B.fl.VI, 445

B.fl.VI, 446

B.fl.VI, 447

M.fr. TIII, 21 .

M.fr. VIII, 23.

M.fr.VIII, 21 .

M.fr. VIII, 22.

M. fr. VIII, 22.

M.fr. YIII, 22.

M.fr.VIII, 20.

M.fr.VIII, 27.

M.fi,VI, 96 .

$\begin{array}{ll}\text { Q. } & \text { B.fl.VI, } 448 \\ \text { Q. N.A. B.fl.VI, } 448\end{array}$

M.fr.VI, 96.

$\begin{array}{ll}\text { B.f.VI, } 449 & \text { M.fr.VI, } 96 . \\ \text { B.fl. VI, } 450 & \text { M.fr.VI, } 96 .\end{array}$

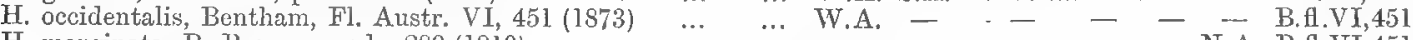

H. marginata, R. Brown, prodr, 289 (1810)

VI, $211(1802)$

DORYANTHES, Correa in Transact. Linn. Soc. VI, 211 (1802).

D. excelsa, Correa in Transact. Linn. Soc. VI, 211, t. 23 (IS02)
D. Palmeri, W. Hill in Benth. FI. Austr. VI, $453(1873)$

- - N.S.W. Q. - B.fl.VI,452

I.fr.IX, 51.

M.fr.IX,5I.

CRINUM, Linné, gen. plant. 97 (1737).

C. venosum, $R$. Brown, prodr. 297 (1810)

C. Asiaticum, Lime, spec. plant. $292(1,5,3)$

C. brachyandlum, Herbert in Bot. Mar. t. 2121 (1820)

C. angustifolium, R. Brown, prodr. 297 (1810)

C. flaccidum, Herbert in Bot. Mag. t. 2133 (1820)...

C. uniflorum, F. v. M., fragin. III, 23 (1862)

C. pedunculatum, R. Brown, prorlr. 297 (1810)

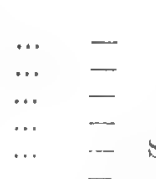

$---\quad-$
--
$\overline{\text { S.A. }}-\overline{-}$
- V. N.S.W.

Q. N.A. B.fl.VI, 453

Q. N.A. B.A.VI, 454

M.fr. III, 22.

M.fr. III, 23.

Ml.fr.XI, ] 39 .

NI. fr. XI, 139.
MI.fi. III, 23.

(3. - B. $\mathrm{A}, \overline{\mathrm{VT}}, 4.54$

Q. N.A. B.A.VI,4.it

Ml.fr. II, 251 .

EURYCLES, Salisbury in Transact. of the Hort. Soc. I, 337 (1812).

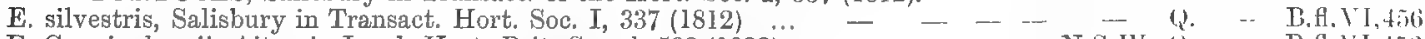

E. Cunninghamii, Aiton in Loud. Hort. Brit. Suppl. 588 (1832) - - - - - N.S.IV. (2. - B.f1.VI,456

CALOSTEMMA, R. Brown, prodr. fl. Nov. Holl. 297 (1810).

C. album, R. Brown, prodr. 298 (1810)

C. purpureum, R. Brown, prodr. $298(1810)$

C. Iuteum, Sims, Bot. Mag. t. 2101 (1819)...

$\begin{array}{ccc}\ldots & \ldots & \ldots \\ \ldots & \ldots & \ldots \\ \ldots & \ldots & \ldots\end{array}$

- - - - - N.A. B.fl.VI,4.is

S.A. - V. N.S.W. - - B.f.VI,4.57

MI.fr. 111,23 . 
CALYCEAE HYPOGYNAE.

F. v. M. in Woolls's plants of the neighb. of Sydney 44 (1880).

\section{ROXBURGHIACEAE.}

Lindley in Wallich, pl. Asiat. rar. III, 50 (1832).

ROXBURGHIA, Jones in Roxburgh, pl. Corom. I, 29, t. 32 (1795). (Stemona 1790.)

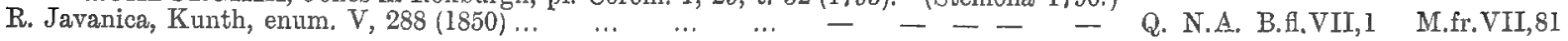

\section{LILIACEAE.}

Haller, enum. stirp. Helv. I, 279 (1742).

PHORMIUM, R. \& G. Forster, charact, gener, 47, t. 24 (1776). (Chlamydia.)

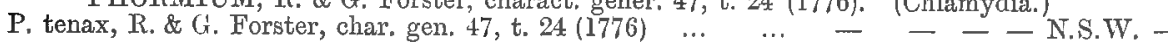

SMILAX, Tournefort, inst. 654, t. 421 (1700), from Theophrastos and Dioscorides.

S. glycyphylla, Smith in White, Voy, to N.S. Wales $230(1790)----$ N.S.W. Q. - B.f.VII,7

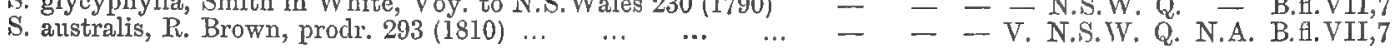
RHIPOGONUM, R. \& G. Forster, char. gen. 49, t. 25 (1776). (Ripogoumm.)

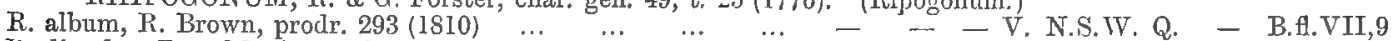

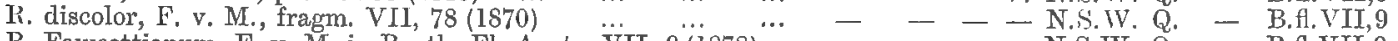

R. Fawcettianum, F. v. M. in Benth. Fl. Austr. VII, 9(1878)... - - - - N.S.W. Q. - B.fl.VII,9

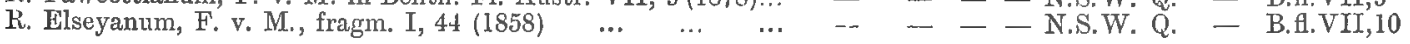
FLAGELLARIA, Linné, amoen. acad. 396 (1747).

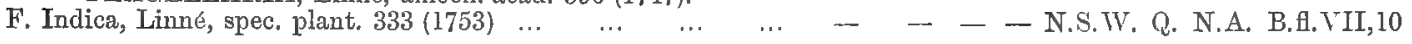
DRYMOPHILA, R. Brown, prodr. fl. Nov. Holl. 292 (1810).

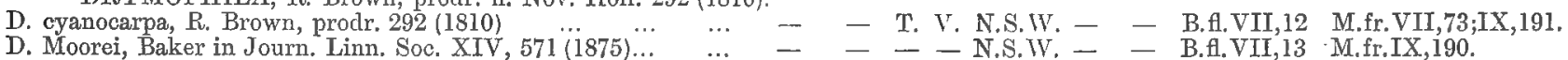
DIANELLA, Lamarck, Encyclop. méthod. II, t. 250 (1786).

D. Tasmanica, J. Hooker, fl. Tasman. II, 57, t. $133(1860) \ldots-$ - - - T. V. N.S.W. - - B.fl.VII,I4 M.fr.VI,121.

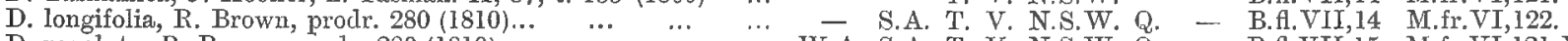

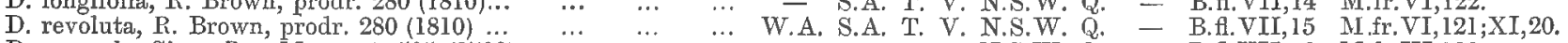

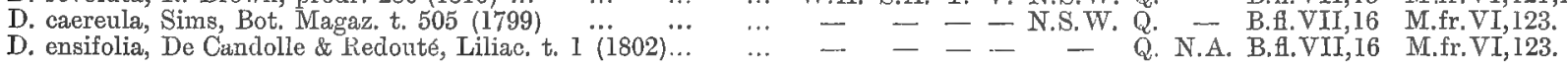
ASPARAGUS, Tournefort, inst. 300, t. 154 (1700), from Theophrastos, Dioscorides and Plinius. (Asparagopsis.)

A. racemosus, Willdenow, spec. plant. II, 152 (1799) $\ldots \ldots \ldots-$ - - - - Q. N.A. B.fl.VII,17 M.fr.VII,73. EUSTREPHUS, R. Brown, prodr. 281 (1810).

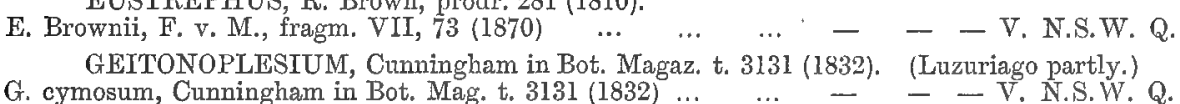

M.fr. TII,77.

M.fr.VII, 79.

M.fr. VII, 75.

M.fr. VII, 80 .

DRACAENA, Vandelli, dissertatio de Dracaena t. 1 (1762).

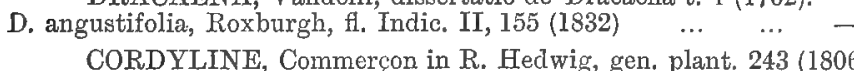
CORDYLINE, Commerçon in R. Hedwig, gen. plant. 243 (1806). (Charlwoodia.)
minalis, Kunth in act. acad. Berol. 30 (1820)

C. terminalis, Kunth in act. acad. Berol. 30 (1820)

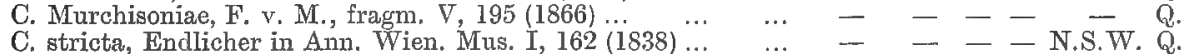

C. Baneri, J. Hooker in Gardeners' Chronicle 792 (1860) BLANDFORDIA, Smith, Exot. Bot. I, 5, t. 4 (1804).

B. marginata, Herbert in Bot. Regist. Misc. $84(1842)$
B. grandiflora, R. Brown, prodr. $296(1810)$
B.

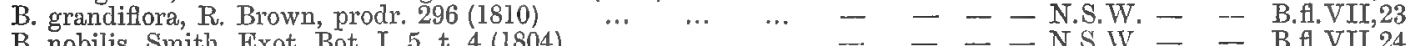

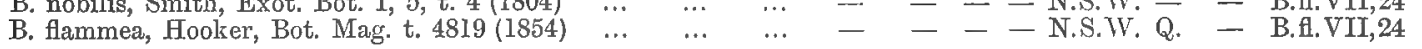
HEWARDIA, Hooker, icon. plant. 858 (1852).

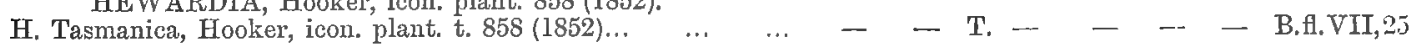
ASTELIA, Banks \& Solander in R. Brown, prodr, 291 (1810). (Hamelinia.)

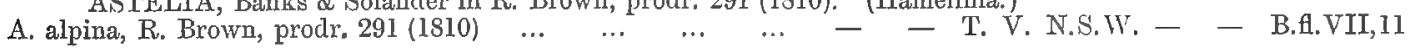
MILLIGANIA, J. Hooker in Kew misc. V, 296, t. IX (1853).

M. densiflora, J. Hooker, Fl. Tasman..II, 62 (1860) $\ldots . . . \quad-\quad-\quad$ T. - $\quad-\quad$ - $\quad$ - B.fl.VII,26

M. Iongifolia, J. Hooker in Kew misc. V, 296, t. IX (1853) $\quad \cdots \quad-\quad-\quad$ T. $-\quad-\quad-\quad-$ B.H.VII,26

M. Johnstoni, F. v. M. in Benth. Fl. Austr. VII, 26 (1878) ․ - - T. - - - - B.H.VII,26

M. stylosa, F. v. M. in papers of the R. S. of Tasm. $11(1876) \cdots--$ T. - $-\quad-$ B.fl.VII,27 WURMBEA, Thunberg, nov. gen. I, 18 (1781). (Anguillaria, Melanthium partly, Pleea partly.)

W. tubulosa, Bentham, Fl. Austr. VII, 28 (1878)... $\quad \ldots \quad$... W.A. $-\quad--\frac{\text { W. }}{-}-$ B.fl.VII,28 M.fr.VII,75.

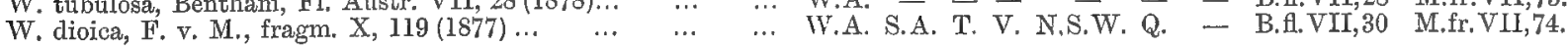
IPHIGENIA, Kunth, enum. plant. omn. huctsq. cognit. IV, 212 (1843).

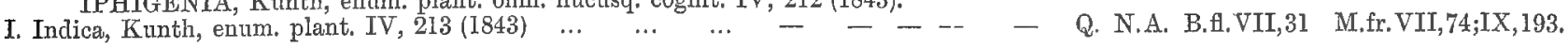
SCHELHAMMERA, R. Brown, prodr. 273 (1810).

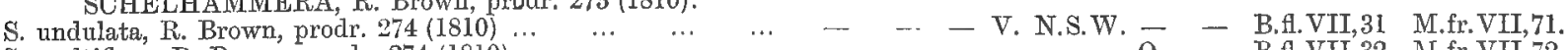

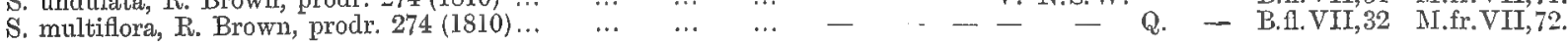
KREYSSIGIA, Reichenbach, iconogr. bot. exotic. III, 11, t. 229 (1829). (Kreysigia, Tripladenia.)

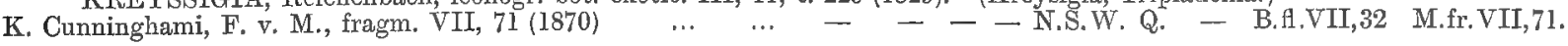
BURCHARDIA, R. Brown, prodtr. $272(1810)$.

B. umbellata, R. Brown, prodr. $272(1810) \ldots$

... W.A. S.A. T. V. N.S.W. Q. - B.fl.VII,33 M.fr.VII,77. 
BULBINE, Linné, hort. Cliffort. 122 (1737). (Anthericum partly, Chrysobactron,)

B. bulbosa, Haworth, revis. pl, succul. 33 (1821)...

B. semibarbata, Haworth, rev. pl. succ. 33 (1821).. Austr. II, 95 (1860).

A. stypandroides, F. v. M., fragm. II, $95(1860)$.. THYSANOTUS, R. Brown, prodr. 282 (1810).

T, multiflorus, $\mathbf{R}$. Brown, prodr. 285 (1810)

T. glancus, Endlicher in Lehm. pl. Preiss. II, 38 (1846)

T. Drummondii, Baker in Journ. Linn. Soc. XV, 341 (1876) ...

T. pauciflorus, R. Brown, prodr. 285 (1810)

T. asper, Lindley, Bot. Regist. XXV, App. LVIIII (1839)

T. chryantherus, F. v. M. in Bentham, flor. Hongk. 372 (1861)

T. exiliflorus, F. v. M. in transact. R. S. of S. Ainstr, IV, $112(1881)$

T. isantherus, R. Brown, prodr. 283 (1810)

T. tenellus, Endlicher in Lehm. pl. Preiss. II, 37 (1846)...

T. scaber, Endlicher in Lehm. pl. Preiss. II, 37 (1846) ...

T. tuberosus, R. Brown, prodr. 282 (1810) ...

T. exasperatus, F. v. M., fragm. I, 21 (1858)

T. thyrsoideus, Baker in Journ. Linn. Soc. XV, $336(1876)$

T. Baueri, R. Brown, prodr. 283 (1810)
T. Patersoni, R. Brown, prodr. $284(1810)$

T. junceus, R. Brown, prodr. 283 (1810)

T. dichotomus, R. Brown, prodr. 284 (1810)

T. arbuscula, Baker in Journ . Linn Soc. XV, 339 (1876)

T. anceps, Lindley, Bot. Regist. XXV, App. LVIII (1839) HODGSONIOLA, F. r. M., fragm. II, 176 (1861)

H. junciformis, F. v. M., fragm. II, 176 (1861) CAESIA, R. Brown, prodr. A. Nov. Holl, 277 (1810).

C. vittata, R. Brown, prodr. 277 (1810)

C. chlorantha, F. v. M., fragm. I, 63 (1858)

C. parviflora, R. Brown, prodr. 277 (1810)

C. rigidifolia, F. v. M., fragm. X, 48 (1876)

C. setifern CHAMAESCILLA, F. v. M., fragm. VII, 68 (1870).

C. corymbosa, F. v. M., fragm. VII, 68 (1870) ... ...

C. spiralis, F. v. M., fragin. VII, 68 (1870) CORYNOTHECA, F, v. M., fragm. VII, $68(1870)$.

C. dichotoma, F, v. M., fragm, VII, $68(1870)$

$68(1870)$.

C. acanthoclada, F. v. M., fragm. VII, 68 (1870).

$\cdots$

... W.A. -
... W.A. S.A. T. V. N.S.W.

W. W.A. S.A. - -

T... A.

(Caesia partly.) (Caesia partly.)
‥ $\quad \cdots \quad-$ S.A. T. V. N.S.W. Q.

C. lateriflora, F. v. M., fragm. VII, 68 (1870)

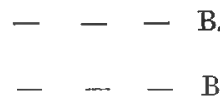

-

$-$

-

$-$

-

$-$

W.A. - - -

... W.A. - - -

$-$

... W.A. S.A. - V.

… - S.A. - -

IV A

... - S.A. - V. N.S.W. -

... - - - N.S.W. -

... W.A.

Hodgsomia.)

- -
B.fl. VII, 34

B. A.VII, 35

B.fl.VII,36

B. fl. VII, 38

- B.fl.VII,39

- B.fl. VII,39

- B.fl.VII,39

- B.fl.VII,39

- B.Hl.VII,39

- B.fl.VII,43

... - S.A. T. V. N.S.W. Q. - B.f.VII, 47

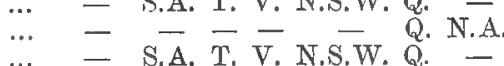

... W.A. - - - - - - B.f.VII,47

... W.A. - - - — - N.A. B.H.VII,47

... W.A. S.A. T. V. N.S.W. - - B.H.VII, 48

.. W.A. - - - - - - B.f.VII,48

... W.A. S.A. - V. N.S.W. Q. N.A. B.fl.VII, 49

... W.A. - - - - - - B.f.VII,50

... W.A. - - - - - - B.fl.VII,50

TRICHORYNE, R. Brown, prodr. f. Nov. Holl. 278 (1810),

T. platyptera, G. Reichenbach, Beitr, zur syst. Pflanzenk.72(1871) - - - - — Q. - B.f.VII,51

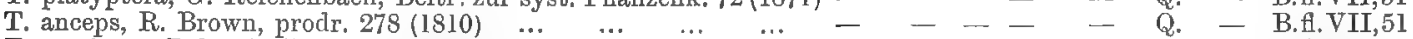

T. muricata, Baker in Journ. Linn. Soc. XV, $363(1876) \quad \cdots \quad-\quad-\quad-\quad-\quad--\quad$ Q. $\quad-$ B.fl.VII,52

T. elatior, R. Brown, prodr. $278(1810) \quad \ldots \quad \ldots . \quad \ldots \quad \ldots$. W.A. S.A. T. V. N.W.W. Q.

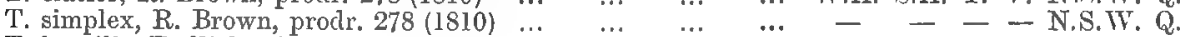

T. humilis, Endlicher in Lehm. pl. Preiss. II, 36 (1846)... $\quad \ldots$ W.A. $\quad-\quad-\quad-\quad-$ STYPANDRA, R. Brown, prodr. flor. Nov. Holl. 278 (1810).

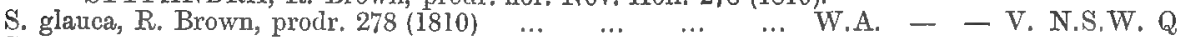

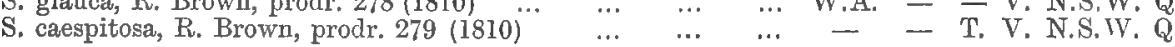

ARTHROPODIUM, R. Brown, prodr. 276 (1810). (Dichopogon.)

A. paniculatum, R. Brown, prodr. $276(1810) \quad \ldots \quad \ldots \ldots \ldots$... $\quad$ S.A. T. V. N.S.W. Q.

A. minus, R. Brown, prodr, 276 (1810) … $\ldots$. 34 (1846)

A. Preissii, Endlicher in Lehm. pl. Preiss. II, 35 (1846)

A. dianellaceum, F. V. M., fragm. X, 65 (1876) ...

A. strictum, R. Brown, prodr. 276 (1810) ...

A. laxum, Sieber in Roemer \& Schultes, syst. VII, 441 (1830)... - S.A. - V. N.S.W. CHLOROPHYTUM, Ker in Bot. Mag. t. 1071 (1808). (Chlorophyton, Phalangium partly.)

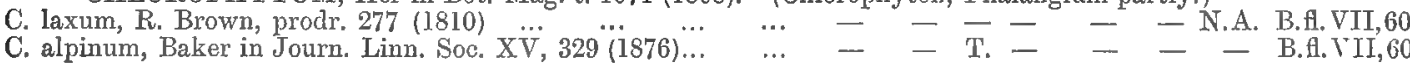
HERPOLIRION, J. Hooker, Fl. of New Zeal. I, 258 (1853).

H. Novae Zelandiae, J. Hooker, FI, of New Zeal. I, 258 (1853) SOWERBAEA, Smith in Transact, Linn. Soc. V, 159 (1800). (Sowerbya.)

S. juncea, Smith in Transact. Linn. Soc. V, 160, t. $6(1800) \ldots \ldots$

S. laxiflora, Lindley, Botan. Regist. t. 10 (1841)

S. alliacea, F: v. M., fragm. VI, 180 (1868) …

ALLANIA, Endlicher, gen. plant. 151 (1837). (Alania.)

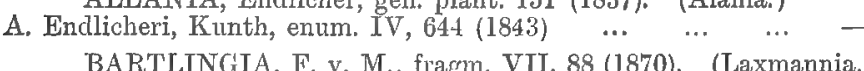
BARTLINGIA, F. v. M., fragm. VII, 88 (1870). (Laxm
ndiflora, F. v. M.; Laxmannia, Lindl. Bot. Reg. XXV, B. grandiflora, F. v. M.; Laxmannia, Lindl. Bot. Reg. XXV,
App. LVI (1839) ... ... ... ...

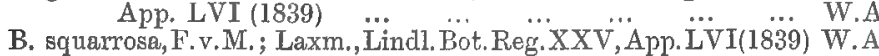

B. minor, F, v, M.; Laxmannia, R, Brown, prodr. 286 (1810)
$-$

- N.A. B.fl.VII, 40

B.fl. VII, 40

B.fl. VII, 40

- B.fl.VII, 41

A. B.fl. VIT,41

B.fl. VII, 42

- B.H.VII,42

- B.fi.VII,43

B.H.VII,44

- B.fl.VII, 45

- B.fl.VII,45

B.f. $\overline{\text { VII }} 47$

B.fl. VII,52

B.f.VII, 52

B.fl.VII, 53

B.fl.VII, 53

B.fl. VII, 54

B.fl.VII, 56

B. fl. VII, 56

B.f. VII, 57

B.Hl.VII, 57

B.Al.VII, 57

B.fl.VII, 5S

B.H. VII, 59

M.fr. V, 202.

M.fr.XI, 139 .

M.fr.VII, 69.

M.fr.I,21.

M.fr.I,22;VII, 69 .

M.fr.VII, $69 ; X I, 20$.

M.fr. VII, 69.

M.fr. VII, 69.

M.fr.II, $95,176$.

M.fr.I, $63 ; \mathrm{VII}, 68$.

M.fr.I, 63.

M.fr.VII, 67.

M.fr, $\mathrm{X}, 48$.

M.fr, VII, 68 .

M.fr.VII, 68 .

M.fr. VII, 68.

M.fr.I,215;XI,20

M.fr.I,215;XI, 20.

MI.fr. VII, 67 .

M.fr.VII,67;XI, 20.

M.fr. VII, 67.

M.fr. VII, 67 .

M.fr. VII, 64.

M.fr. VII, 65.

M.fr.VII, 66.

II.fr.VII, 67;XI, 27.

M.fr. VII, 66.

M.fr. X, 65 .

M. fr. VII, 66 .

M.fr.I,63; YII, 71.

M.fr. VII, 68 .

B.fl.VII, 61 M.fr: YI, 180

B.Al.VII,62 M.fr, VI, 180;VII, 87.

M.fr. VII, 87.

- - N.S.W. - - B.H.VII,63 M.fr.VI, 180.

W.A.

W.A. - - - - - - - B.fi.VII,64
W.A. - B.VII,64

M.fr. I, 159; \II, 88 .

M.fr. VII, $\$ 8,89$. 
B. gracilis, F v. M., fragm, VII, 88 (1870)

B. ramosa, F. v. M., fragm. VII, 88 (1870)

B. ramosa, F. v. M., fragm. VII, 88 (1870) $\ldots ̈$. $\ldots$.
B. sessiliflora, F. v. M. in pap. R. S. Tasm. $116(1877)$
B. brachyphylla; Laxm., F. v. M, in Benth. Fl. VII, 67 (1878)

B. sessilis, F.v.M.; Laxm., Lindl. Bot.Reg. XXV, App.LVI (1839)

STAWELLIA, F. v. M., fragm. phytogr. Austr. VII, 85

S. dimorphantha, F. v. M., fragm. VII, 85 (1870) $\ldots$
JOHNSONIA, R. Brown, prodr. fl. Nov. Holl. 287 (1810).

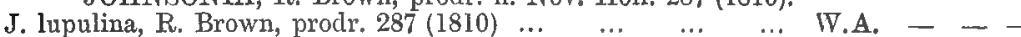

J. pubescens, Lindley, Bot. Regist. XXV, App. ÏVII (1̈339) … W.A. - - ARNOCRINUM, Endlicher \& Lehmann in pl. Preiss. II, 41 (1846).

A. Drummondii, Endlicher in Lehm. pl. Preiss, II, 41 (1846) ... W.A.

A. Preissii, Lehmann, pl. Preiss. II, 42 (1846) ... ... ... W. W.
BORYA, Labillardiere, Nov. Holl. pl. specim. 81, t. 107 (1804).

B. nitida, Labillardiere, Nov. Holl, pl, spec. 81, t. 107 (1804) ... W.A.

B, septentrionalis, F. v. M., fragm. 41 (1856) $\ldots . . . . \quad \ldots$
CALECTASIA, R. Brown, prodr. fl. Nov. Holl. $263(1810)$.

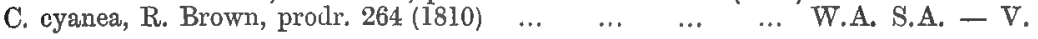

BAXTERIA, R. Brown in Hook. Lond. Journ, of Bot. II, 492, t. 13-15 (1843).

B. australis, R. Brown in Hook. Lond. Journ. II, 492 (1843) ... W. A. - - ACANTHOCARPUS, Lehmann, pl. Preiss. II, 274 (1847). (Chamaexeros).

A. Preissii, Lehmann, pl. Preiss. II, 274 (1847) ... ... … W.A. - -

A. Serra, F. v. M.; Xerotes, Endlicher in pl. Preiss. II, 49 (1847) W.A. - - -

A. fimbriatus ; Xerotes, F, v. M., fragm. VIII, 211 (1874) … W.A.
XEROTES, R. Brown, prodr. 257 (1810). (Lomandra, 1804.)

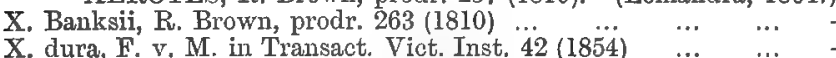

$X$ longifolio $R$ Brown, prodi. $262(1810)$

$\mathrm{X}$. rigida, $\mathrm{R}$. Brown, prodr. 262 (1801)

X. Sonderi, F. ఛ. M., fragm. VIII, 206 (I874) ‥

X. odora, Endicher in Lehm. pl. Preiss. II, 50 (1 $\ddot{846)}$...

X. Brownii, F, v. M., fragm. VIII, 206 (1874)

X. Ordii F. v, M fragm XI, $23(1878)$

$\mathrm{X}$, sororia, F. v. M., first gen, Report 15 (1854) ... $\ldots$

X. Endlicheri, F, v. M., fragm. VIII, 205 (1874) ...

X. sericea, Endlicher in Lehm, pl. Preiss. II, 51 (1846)..

X. purpurea, Endlicher in Lehm. pl. Preiss. II, 49 (1846)

X. Preissii, Eindlicher in Lehm. pl. Preiss, II, 50 (1846)..

X. effusa, Lindley in Mitch. Three Expecl. II, 151 (1838)

X. micrantha, Endl. in Lehm. pl. Preiss. II, 49 (1846) ...

X. Thunbergii, F, v. M., fragm. VIII, 208 (1874)

X. caespitosa, Benth. Fl. Austr. VII, 104 (1878) ...

X. pauciflora, R. Brown, prodr. 261 (1810)..

$\mathrm{X}$ flexifolia R. Brown, prodr 260 (1810)

$\mathrm{X}$. glauca, R. Brown, prodr. $260(1810) \quad \ldots \quad \ldots$.

X. elongata, Bentham, Fl, Austr. VII, 106 (1878) ...

X. rupestris, Endlicher in Lehm. pl. Preiss. II, 50 (1846)

X. collina, $R$, Brown, prodr. 260 (1810)

$\mathrm{X}$. suaveolens, Endlicher in Lehm. pl. Preiss. II, 50 (1846)

X. turbinata, Endlicher in Lehm. pl. Preiss. II, 51 (1846)

X. spartea, Endlicher in Lehm. pl. Preiss. II, 51 (1846)...

X. juncea, F. v. M. in Transact. Vict. Inst. 135 (1855) ...

X. leucocephala, R. Brown, prodr. 260 (1810)

$\mathrm{X}$. hastilis, R. Brown, prodr. 263 (1810)

DASYPOGON, R. Brown, prodr. fl. Nov. Holl. 263 (1810)

D. bromelifolius, $R$. Brown, prodr. 263 (1810)

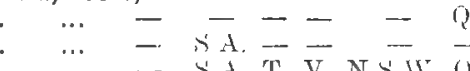

… T.A. T. V. N.S.lF. ?

‥ W.A. - - - - -

W.A. - - - - $=$

- S.A. - T. N.S.W. Q.

W.A. $\overline{\text { S.A. }}$ - V. N.S.W. Q.

W.A.

W.A. - - - - -

W.A. - - - W.A. -

W.A. SA. - V. N.S.W. -

W.A. S.A. - V. N.S.W. -

- S.A. - V. N.S.W. Q.

W.A. - - - -

W.A. - - N S.T.

W.A. S.A. T. V. N.S.W. -

- S.A. - - N.S.W. Q.

W.A. - - - - -

W A

W.A. - - -

W.A. - - -

W.A.

W.A

- S - - - -

. - S.A. - V. N.S.W. Q.

$$
\text { W. }
$$$$
\text { W. }
$$

W.A. -

B. $f$. VII, 66

B.fl.VII, 66 M.fr.I, 158.
B.fl.VII, 67

B.fl.VII,67 M.fr.VII,85.

B.fl.VII, 68 M.fr.VII, 86.

,68 M.fr.VI, 255.

D. Hookeri, Drummond in Hook. Lond. Journ. II, 168 (1843) ... W. W.
XANTHORRHOEA, Smith in Transact. Linn. Soc. IV, 219 (1798).

$\mathrm{X}$ gracilis, Endlicher in Lehm. pl. Preiss. II, 39 (1846)...

X. pumilio, R. Brown, prodr. 288 (1810)

X. macronema, F. v. M., fragm. IV, 112 (1864) $\ldots$

$\mathrm{X}$. hastilis, R. Brown, prodr. 288 (1810) ...

$\mathrm{X}$. arborea, R. Brown, prodr. 288 (1810) $\ldots . .$.

X. semiplana, F. v. M., fragm. IV, 111 (1864)

X. bracteata, R. Brown, prodr. $288(1810) \ldots$

X. minor, R. Brown, prodr. 288 (1810)

X. australis, R. Brown, prodr. 288 (1810) ...

X. quadrangulata, F. v. M., fragm. IV, 111 (1864)

X. Preissii, Endlicher in Lehm. pl. Preiss. II, 39 (IS46)...

... W.A.

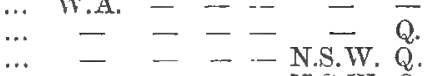

.. - - - - - N.S.W. Q.

.. - - - - N.S.W.Q.

$\cdots$

-

S.A. - -

S.A. T. V. N.S.W. -

$\cdots \quad-$ S.A. T. V.

$\cdots$ - S.A. - -

W.A.

KINGIA, R. Brown in King's Narrat. of survey of Austr. coasts II, 529 (1827).

K, australis, R. Brown in King's Narr, of surv. II, 535 (1827) ... W.A. - - - - - - B.A.VII,119

PALMAE.

Ray, method. pl, emend. 135 (1703).

CALAMUS, Linné, spec. plant. 325 (1753)

C. australis, Martins, hist, nat, palm. III

-
Q. - B.H.VII, 134 M.fr.V,48.

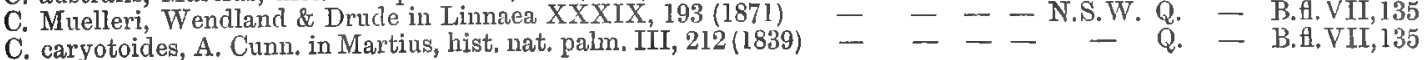


BACULARTA, F. v. M., fragm. VII, 103 (1870). (Linospadix).

B. monostachya, F. v. M., fragm. VII, $103(1870)$

KENTIA, Blume in Bull. Neerl. 64 (1833). (Grisebachia, Hydriastele, Hedyscepe, Kentiopsis, Veitchia partly).

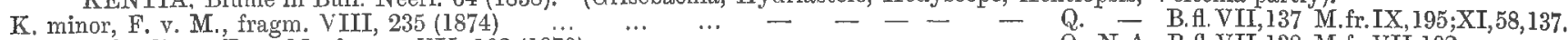

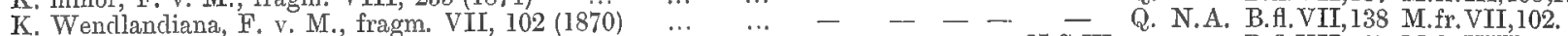

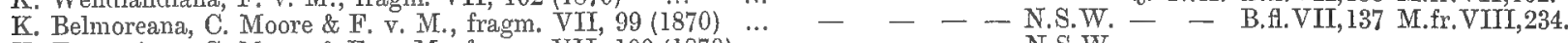

K. Forsteriana, C. Moore \& F. v. M., fragm. VII, $100(1870) \ldots$

T. Bauri Seemann, fl. Vitiens, 269 (1860)

$-\quad-$

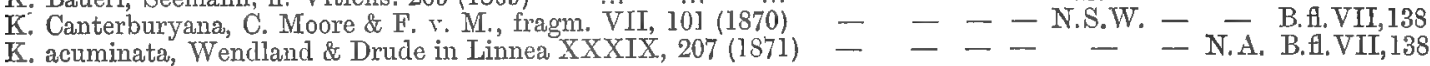

- B.fl.VII, 138 M.fr.VIII, 234;, 119.

CLINOSTIGMA, Herm. Wendland in Seemann's Bomplandia X, 196 (1862). (Cyphokentia). PTYCHOSPERMA, Labillardière in Mém. de l'Inst., ann. 1808, I, 251, pl. X (1809). (Seaforthia, Laccospadix, Archontophoenix).

P. Laccospadix, Bentham, fl. Austr. VII, $140(1878) \quad \ldots \quad \ldots \quad-\quad-\quad-\quad-\quad$ Q. - B.fl.VII,140

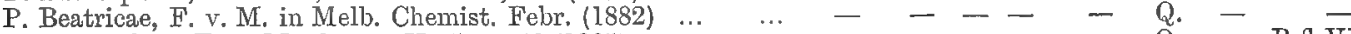

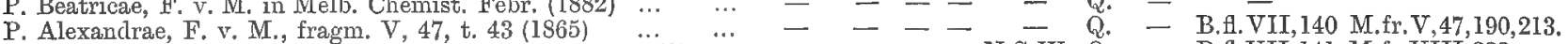

P. Cunninghamii, Herm. Wendland in Bot. Zeit, $346(1858)$.. $-\quad-\quad-\quad-$ N.S.W. Q. $\quad-$ B.fl.VII, I41 M.fr.VIII,222.

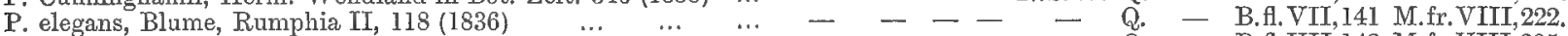

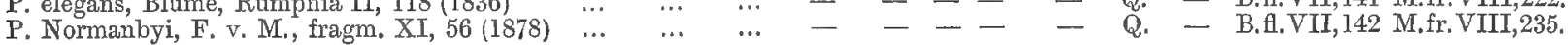
ARECA, Linne, spec. plant. 1189 (1753) from Ray (1688).

A. Alicae, F. v. M. in Regel's Gartenflora, 199-201 (1879) ,... COCOS, Linné, spec. plant. 1188 (1753).

C. nucifera, Linné, spec. plant. 1188 (1753) CARYOTA, Linné, gen. plant. 355 (1737).

C. Rumphiana, Martius, hist. nat. palm. III, 195 (1839) $\quad \ldots \quad-\quad-\quad-\quad$ - $\quad$ Q. $\quad$ - B.fl,VII,144 LICUALA, Rumphius, herb. Amboin. I, 44, t. 9 (1741).

L. Muelleri, Wendland \& Drude in Linnaea XXXIX, 223 (1871) LIVISTONA, R. Brown, prodr. f. Nov. Holl. 267 (1810).

L. humilis, R. Brown, prodr. 268 (1810)

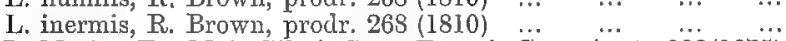

L. Mariae, F.v.M. in Giles's Geog. Trav. in Cintr. Austr. $2222(1875)$

L. australis, Martius, hist. nat. palm. III, 241 (1839) ..

\section{PANDANEAE.}

(Corypha partly).

$$
\begin{aligned}
& \text { - - - - - - N.A. B.A.VII,146 M.fr.VIII,221. } \\
& \text { - S.A. - - - - N.A. B.f.VII,146 } \\
& \text { - - - - N.A. }- \text { N.A. - M.Xr.XI,54. }
\end{aligned}
$$

R. Brown, prodr. Nov. Holl, (1810)

PANDANUS, Rumphius, herb. Amboin. IV, 139, t. 74 (1750) (Athrodactylis, Fiquetia).

P. odoratissimus, Linné, fil, suppl. 424 (1781) ... ... .. - - - - - - N.A. B.fl.VII, 148 M.fr. XI,55.

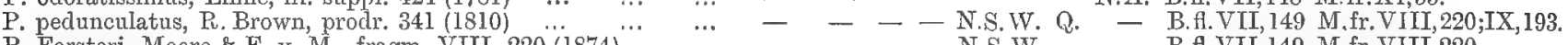

P. Forsteri, Moore \& F. v. M., fragm. VIII, 220 (1874) $^{\cdots} \quad \cdots \quad-\quad-\quad$ - - N.S.W. - - B.fl.VII,149 M.fr.VIII,220.

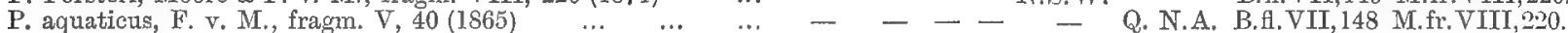

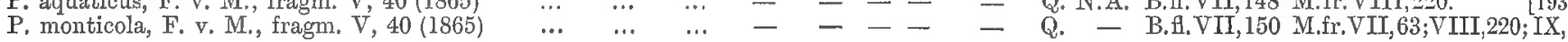

FREYCINETIA, Gaudichaud in Ammal. des scienc. natur. III, 509 (1824).

F. Gaudichaudii, Brown \& Bennett, pl. Jav, rar. 31, t.9(1838) - - - - - Q. - B.fl.VII,151

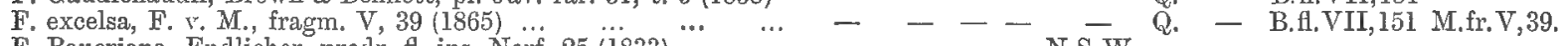

F. Baueriana, Endlicher, prodr. A. ins. Norf. 25 (1833) $\ldots \ldots \ldots$

NIPA, Wurmb in Verhandl. Batav. Genootsch. I, 349 (1779), from Camellus, Ray \& Rumphius.

N. fruticans, Wurmb in Verhandl. Batav, Genootsch, I, 349 (1779) - - - - - Q. - - M.fr.XI,12S.

\section{AROIDEAE,}

A. L. de Jussieu, gen. plant. 23 (1789), from B. de Jussieu (1759).

TYPHONIUM, Schott in Wien. Zeitschr. III, 72 (1829). (Arum partly.)

T. liliifolium, F. v. M. in Schott, prodr. syst, aroid. 107 (1860) - - - - - - N.A. B.A.VII,153 M.fr.VIII, 187.

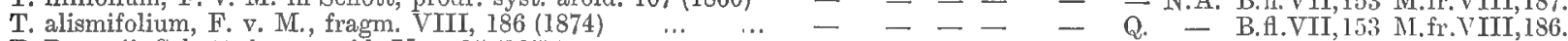

T. Brownii, Schott, icon, aroid. II, t. $15(1857) \ldots \quad \ldots \quad \ldots \quad-\quad$ - $\quad-\quad$ - N.S.W. Q. - B.f.VII,154 M.fr.VIII,187.

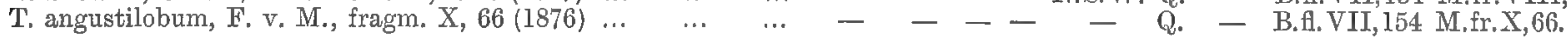

AMORPHOPHALLUS, Blume in Nouv. Ann. du Mus. III, 366 (1834). (Brachyspatha).

A. variabilis, Blume, Rumphia I, 146, t. 35 (1835) … ‥ - - _ - - - N.A. B.f.VII,155 MI.fr.VIII,S7.

COLOCASIA, Ray, Method. 157 (1682). (Calaclium partly.)

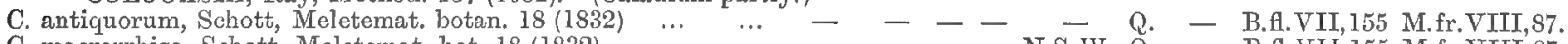

C. macrorrhiza, Schott, Meletemat. bot. $18(1832) \quad \ldots \quad \ldots \quad-\quad-\quad-\quad$ - N.S.IV. Q. $\quad$ - B.f.VII,155 M.fr.VIII,87.

RHAPHIDOPHORA, Hasskarl in Fl. Regensb. Beibl. II, 11 (1842). (Epipremnum partly.)

R. pertusa, Schott in Seem. Bonplandia V, 45 (1857) ‥ ‥ - - - - Q. - B.H.VII,156 M.fr.VIII,87.

GYMNOSTACHYS, R. Brown, prodr. fl. Nov. Holl. 337 (1810).

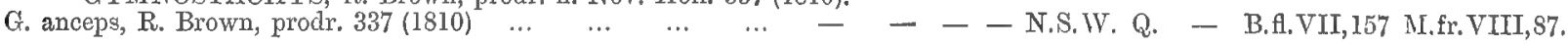

POTHOS, Linne, amon. acad. I, 410 (1747).

P. Loureiri, Hooker \& Arnott, Bot. of Beech. Voy. 220 (1841)... - - - - N.S. W. Q. - B.f.VII,158 M.fr.I,62;YIII,87.

TYPHACEAE.

A. L. de Jussieu, gener, plant, 25 (1789).

TYPHA, Tournefort, inst. 530, t. 301 (1700), from Theophrastos, Dioscorides and Plinius.

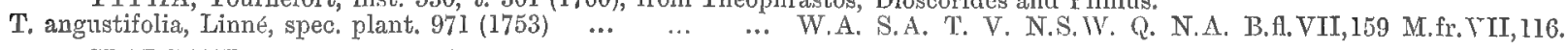

SPARGANIUM, Tonrnefort, inst. 530, t. 302 (1700).

S. angustifolium, R. Brown, prodr. $338(1810) \quad \ldots \quad \ldots . \quad \ldots \quad-\quad-\quad$ - V. N.S.W. Q. $\quad$ - B.fl.VII,160 M.fr.VII, 117. 


\section{LEMNACEAE,}

J. E. Gray, Nat, arrang. of Brit. pl. II, 729 (1821).

LEMNA, Linne, syst. nat. 9 (1735); Linne, Fl. Lappon. 351 (1737). (Telmatophace, Spirodela.)

L. trisulca, Linne, spec, plant. 970 (I753) ... ... ... ... S. A. T. V. N.S.W. Q. - B.f.VII, 162 M.fr. VIII, 188 L. minor, Linné, spec, plant. 970 (1753) $\ldots$ … $\quad \ldots \quad \ldots$ W.A. S.A. T. V. N.S.W. Q. N.A. B.fl.VI, 163 M. fr. VIII, 188,

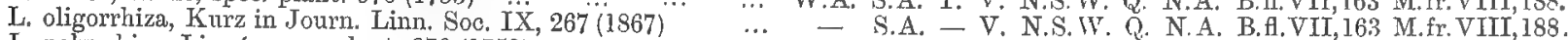

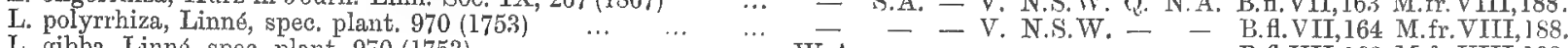

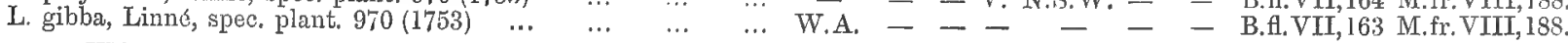

WOLFFIA, Horkel \& Schleiden in Schlecht. Linnaea XIII, 389 (1S39). (Wolfia.)

W. Michelii, Schleiden, Beitr. zur Botan. 233 (1844) .. … - - V. N.S.W. - - B.fl,VII,162 M.fr.VIII,187.

\section{FLUVIALES.}

Ventenat, Tabl. du règn. véget. II, 80, (1799).

TRIGLOCHIN, Rivinus in Ruppius, fl. Jenens. 54 et 366 (1718), from Dalechamps (1586). (Cyonogeton.)

T. centrocarpa, Hooker, icon. plant. t. 728 (I848) $\quad$... … W.A. S.A. T. V. N.S.W. -

T. striata, Ruiz \& Pavon, Fl. Per. et Chil. III 72 (1802) ... W... W. VII, 167 M.fr. VI, 82.

T mucronata, R. Brown,

$\begin{array}{llll}\cdots & \cdots & \cdots & \text { W.A. S.A. }- \text { V. }-\end{array}-$ - B.f.VII, 168 M.fr.VI,81

T. Maundii, F. v. M., fragm. VI, $83\left(18677^{\prime} \quad \ldots \quad \ldots \ldots \ldots\right.$ W.A. S.A. T. V. N.S.W. Q. N.A. B.A.VII, 168 M.fr.VI, 83. ... - - - - N.S.W. Q. - B.H.VII, 169

A. monoson, Limné fil., suppl. plant. 32 (1781).

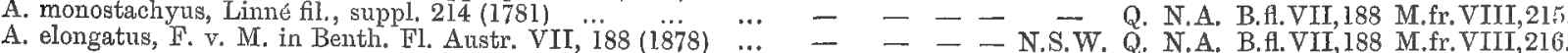
POTAMOGETON, Fuchs, histor. stirp. comment. 651 (1542).

P. natans, Linné, spec. plant. I26 (1753) ... … … … W.A. S. A. T. V. N.S.W. Q. - B.fl.VII.170 M.fr.VIII,217.

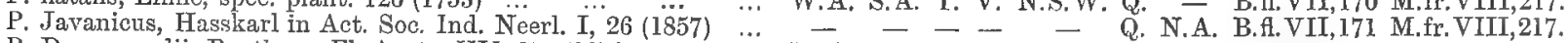

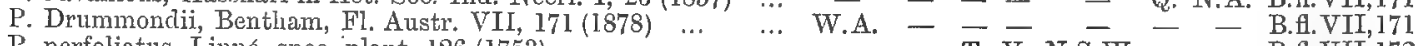

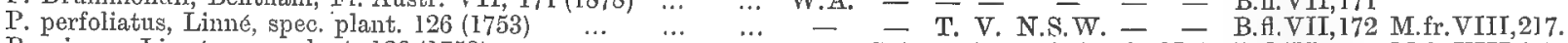

P. crispus, Limné, spec. plant. $126(1753) \ldots \ldots$... $\ldots . \ldots$ - S.A. - V. N.S.W. Q. N.A. B.A.VII,172 M.fr. VIII, 217.

P. obtusifolins, Mlertens \& Koch, Deutsch1. Fl. I, 355 (1823) $\cdots-$ - S.A. T. V. N.S.W. Q. - B.fl.VII, 172 M.fr.VIII,216.

P. aeutifolius, Link in Roem. \& Schult. syst. veg. III, $513(1818)$ - S.A. - V. N.S.W. - - B.f.VII,173 M.fr.VIII, 216.

P. pectinatus, Linne, spec. plant. 127 (1753) ‥ … ‥ - S.A. T. V. - - - B.f.VII,173 M.fr.VIII,217. RUPPIA, Linné, syst. nat. 9 (1735); Linné, gen. plant. 277 (1737).

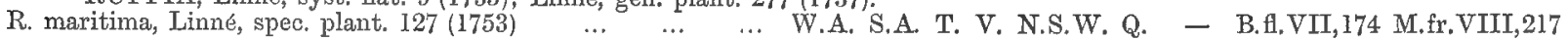
POSIDONIA, Koenig, Annals of Botany II, 95, t. 6 (1806). (Caulinia partly, Kernera partly.)

P. australis, J. Hooker, Fil. Tasman. II, $43(1860) \quad \ldots \quad \ldots$ W.A. S.A.T. V. - - - - B.f.VII,175 M.fr.VIII,21S. ZOSTERA, Linne, Waesgoeta-Resa, 166-168 et fig. (1747).

Z. nana, F. K. Mertens in Roth, enum. pl. phaner. Germ. 8 (1827) - S.A. T. V. N.S.W. - - B.fl.VII, 176 M.fr.VIII,218.

Z. Tasmanica, G. v. Mertens in Garcke, Linnaea XXXV, 68 (1867) - S.A. T. V. - - - B.fl.VII,176 M.fr.VIII,218. CYMODOCEA, C. Koenig, Annals of Botany II, 96, t. 7 (1806). (Amphibolis, Graumuellera, Kernera partly.)

C. zosterifolia, F.v. M.; Amphibolis, Agardh., spec,algar.II,477(1822)W.A. S.A. T. V. - - - B.A.VII, 177 M.fr. VIII, 218.

C. ciliata, Elhrenberg in Garcke, Linnaea XXXV, $162(1867) \ldots-$ - - - - - Q. N.A. B.fl.VII,178 M.fr.VIII,218.

C. rotundata, Ascherson \& Schweinfurth in Neumayer, Anleit. zu

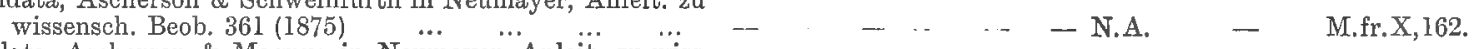

C. serrulata, Ascherson \& Magnus in Neumayer, Anleit. zu wiss.

Beob. $362(1875) \quad \ldots$
C. isoetifolia, Ascherson in Garcke, Linnaea XXXV, $163(1867) \quad$ W.A. $\quad-\quad-\quad-\quad-\quad$ Q. N.A. B.fl.VII, 178 M.fr.VIII, 218.

HALODULE, Endlicher, gen. plant. 1368 (1840). (Diplanthera, 1806.)

H. tridentata, Endlicher, gen. plant. 1368, implied (1840) $\ldots-\ldots-1-{ }_{-}-$N.A. - M.fr.VIII,2I8. LEPILAENA, Drummond \& Harvey in Hook. Kew misc. VII, 57 (1855). (Zannichellia partly, Hexatheca, Altheniae subgenus.)

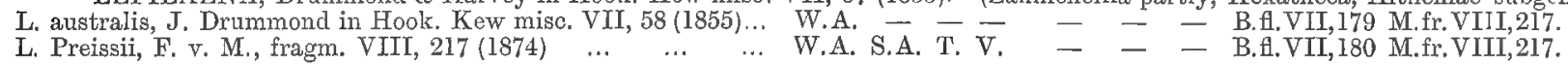

NAJAS, Linné, syst. nat. 9 (1735); Linné, gen. plant. 278 (1737). (Caulinia partly.)

N. major, Allioni, Fl. Pedem. II, 221 (1785) $\ldots$... $\ldots . .$. W.A. S.A. - - - - N.A. B.fl.VII,181 M.fr.VIII,218.

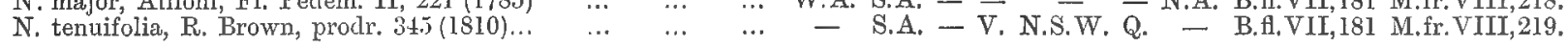

\section{ALISMACEAE.}

Ventenat, Tabl. du règn. véget. II, 157 (1799).

ALISMA, Rivinus in Ruppius, fl. Jen. 54 (1718), from Dioscorides and Plinius. (Caldesia; Echinodorus partly).

A. Plantago, Linné, spec. plant. $842(1753) \quad \ldots \quad \ldots \quad \ldots-$-.. - - V. N.S.W. - - B.H.VII, 185 M.fr. VIII, 215.

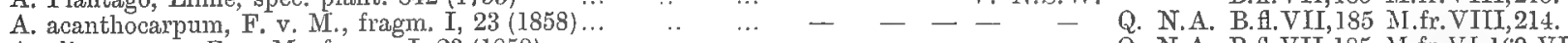

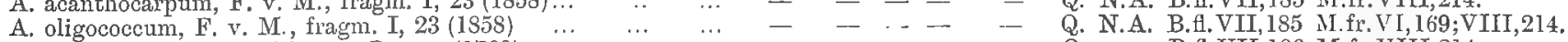

A. parnassifolium, Bassi in act. Bonon. (1768) $\quad \ldots \quad$... $\quad \ldots \quad-\quad-\quad-\quad-\quad-\quad$ - $\quad$ Q. $\quad$ - B.fl.VII,186 M.fr.VIII,214.

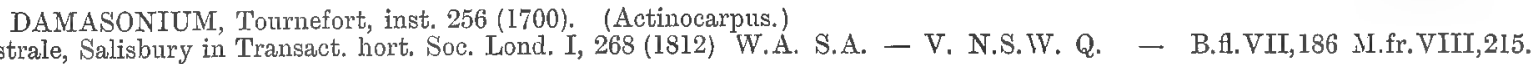

D. australe, Salisbury in Transact. hort. Soc. Lond, I, 268 (1812) W.A. S.A. - V. N.S. W.
TENAGOCHARIS, Hochstetter in Regensb. bot. Zeit. June (1841). (Butomopsis.)

T. Cordofana, Hochstetter in Regensb. bot. Zeit. June (1841) ... - - - - Q. N.A. B.A.VII,187 M.fr.X,103.

\section{PONTEDERIACEAE.}

Humboldt, Bonpland \& Kunth, nov. gen. et spec. plant. I, 265 (1815).

MONOCHORIA, Presl, reliq. Haenk. I, 127 (1827). (Limnostachys.)

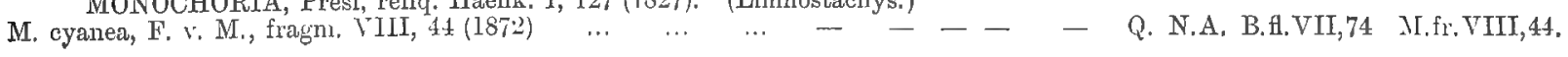




\section{PHILYDREAE.}

R. Brown in Flinders' voy. II, 57 (1814).

PHILYDRUM, Banks in Gaertner, de fruct. I, 62 t. 16 (1788). (Philhydrum.)

P. lanuginosum, Banks in Gaertn. de fruct. I. 62 t. 16 (1788) ... - - - V. N.S.W. Q. N.A. B.A.VII,73 M.fr.V,203.

PRITZELIA, F. v. M., Papuan plants 13 (1875). (Hetaeria, Philydrella.)

P. pygmaea, F. v. M., Papuan plants $13(1875) \quad \ldots \quad \ldots \quad \ldots$ W.A. $\quad-\quad-\quad-\quad-\quad$ - B.fl.VII,74 M.fr.V,203.

HELMHOLTZIA, F. v. M., fragm. V, 202 (1866).

H. acorifolia, F. v. M., fragm. V, $203(1866) \quad \ldots \quad \ldots \quad \ldots \quad-\quad-\quad-\quad-$ N.S.W. Q. $\quad$ - B.fl.VII,75 M.fr.V,203.

\section{COMMELINEAE.}

R. Brown, prodr. fl. Nov. Holl. 268 (1810).

ZYGOMENES, Salisbury in Transact. hort. Soc. I, 271 (1812). (Cyanotis).

Z. axillaris, Salisbury in Transact. hort. Soc. I, 271 (1812) $\ldots-\ldots-c_{-}-$Q. N.A. B.A.VII,82 M.fr.VIII,62.

COMMELINA, Plumier, nov. pl. Amer. gen. 48, t. 38 (1703). (Commelynia).

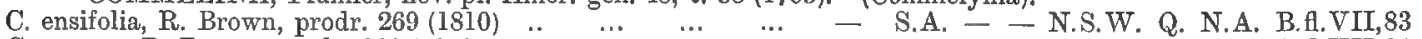

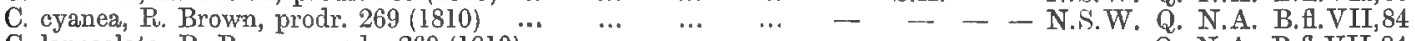

C. lanceolata, R. Brown, prodr. 269 (1810) … … … - $\quad-\quad-\quad-\quad-$ Q. N.A. B.fl.VII,84

C. agrostophylla, F.v. M. in J. Hook., FI. Tasm, p. ẌLVIİ(1860)

ANEILEMA, F. Brown, prodr. fl. Nov, Holl, 270 (1810).

A. acuminatum, R. Brown, prodr. $270(1810) \quad \ldots \quad \ldots \quad \ldots \quad-\quad-\quad-\quad$ N.S.W. Q. $\quad$ - B.fl.VII,85

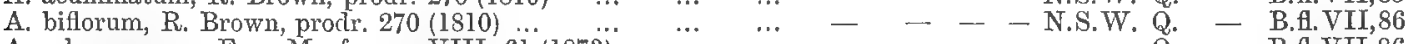

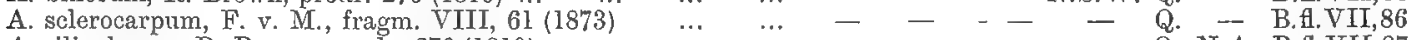

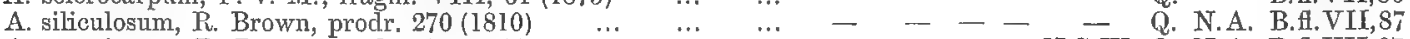

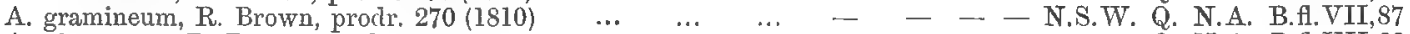

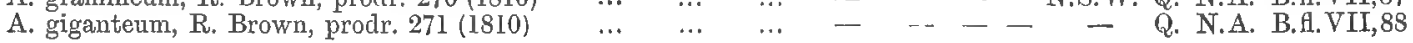

M.fr.VIII, 60;IX, 191

M.fr.VIII, $59 ; \mathrm{IX}, 191$.

M.fr.VIII,59.

M.fr.VIII,59,

FLORISCOPA, Loureiro, Fl. Cochinch. I, 192 (1790). (Floscopa, Dithyrocarpus.)

F. scandens, Loureiro, fl. Cochinch. I, 193 (1790)

$\cdots$

POLLIA, Thunberg, nov. gen. plant. I, 11 (1781).

P. cyanococca, F. v. M., fragm. V, $40(1865)$. $\quad \cdots \quad \ldots \quad-\quad-\quad-\quad-$ N.S.IV. Q. $\quad-\quad$ B.f.VII,90 M.fr.VIII,63.

CARTONEMA, R. Brown, protr. fl. Nov. Holl. 271 (1810).

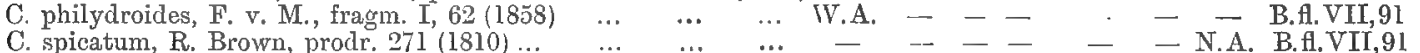

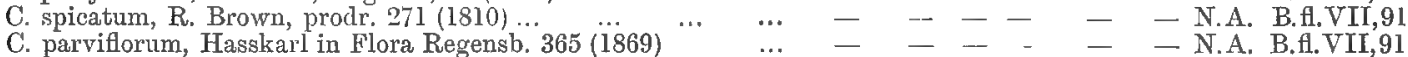

C. trigonospermum, Clarke in De Cand., mon. phaner. IIr, 24 (I881) - - - - - - N.A. B.l.V

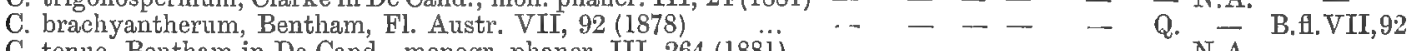

C. tenue, Bentham in De Cand., monogr, phaner. III, 264 (1881) - - - - - - N.A. -

M.fr. VIII, 61 .

M.fr.VIII, 61 .

M.fr. VIII, $61 ; \mathrm{IX}, 191$.

M.fr.VIII, 62 .

M.fr.VIII, 62.

\section{XYRIDEAE.}

Salisbury in Transact. of the Hortic. Soc. I, 326 (1812).

XYRIS, Gronovius in Linne, genera plantar. 11 (1737).

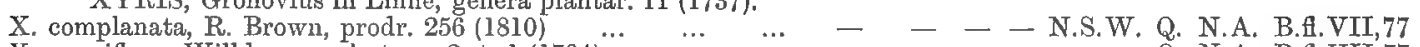

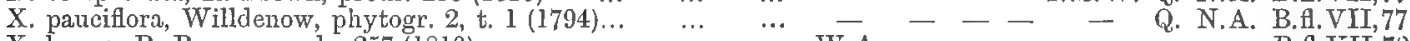

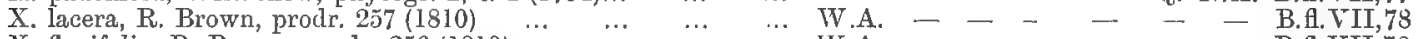

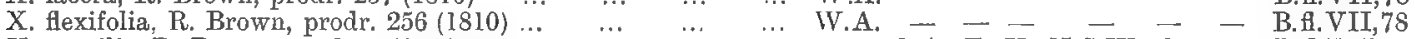

X. gracilis, R. Brown, prodr. 256 (1810) $\ldots \quad \ldots \quad \ldots . \quad \ldots \quad-\quad$ S.A. T. V. N.S.W. Q. - B.f.VII,79

X. operculata, Labillardiere, Nov. Holl. pl. spec. I, 14 t. $10(1004)$ - S.A. T. V. N.S.W. Q. - B.fl.VII,79

X. lanata, R. Brown, prodr. 257 (1810)

X. laxiflora, F, v. M. fracm. VIII, 203 (1874)

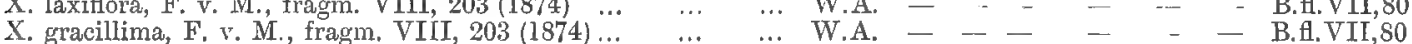

M.fr. VIII, 64.

M.fr.I,62;VIII, 64 .

M.fr.XI, 139.

M.fr. XI, 139.

\section{JUNCEAE.}

R. Brown, prodr. fl. Nov. Holl. 257 (1810).

LUZULA, De Candolle, Fl. franc. III, 158 (1805).

L. campestris, De Candolle, Fl. franc. III, 158 (1805) $\quad \ldots \quad$... $\quad$ W.A. S.A. T. V. N.S. W. Q. - B.fl.VII,123

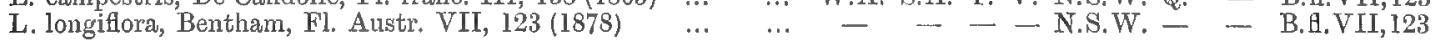

JUNCUS, Tournefort, inst. 246, t. 127 (1700), from Camerarius, J. \& C. Bauhin, Ray \& Morigon.

J. gracilis, R. Brown, prodr. 259 (1810)

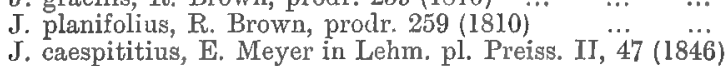

J. falcatus, E. Meyer, synops. luzilar. 34 (1823) ...

$J$. bufonius, Linné, spec. plant. 328 (1753)

$\cdots \quad \cdots$

J. homalocaulis, F. v. M., first gen. Rep. 19 (1853)

J. Brownii, F. v. M., first gen. Rep. 19 (1853) …

J. communis, E. Meyer, synops. Juncor, 12 (1822)

$J$ vaginatus, $R$. Brown, prodťr. $258(1810) \ldots \ldots \ldots$

J. pauciflorus, R. Brown, prodr. $259(1810) \quad \ldots . \quad \ldots$

J. pallidus, R. Brown, prodr. 258 (1810) ‥ $\ldots$

J. prismatocarpus, $R$. Brown, prodr. 259 (1810) ...

J. pusillus, Buchenau in Abhandl. naturw. Vereins zu Bremen V̈I,

W.A. - - - - B.fl.VII, 125

W.A. S.A. T. V. N.S.IV. - - B.A.VII,125

W.A. S.A. T. V. N.S.W. - - B.fl.VII, 126

- - T. V. N.S. WV - - B.fl.VII, 126

S. T. V. N.S.W.

- - T. V. N.S.W. - - B.fl.VII,128

W.A. S.A. T. V. N.S.TV. Q. - B.fl.VII,128

- - - - N.S.IV.Q. - B.f.VII, 129

- S.A. T. V. N.S.W. Q. - B.f.VII,129

M.fr. VIII, 205.

M.fr. VIII, 205.

M.fr. VIII, 204 .

M.fr. VIII, 204.

M.fr.VIII, 204.

M.fr. VIII, 204.

M.fr. VIIII, 204.

M.fr.VIII, 203 .

M.fr. VIII, 203.

W.A. S.A. T. V. N.S.IV, Q. - B.H.VII, 130

W.A. S.A. T. V. N.S.W. Q. - B.A.VII, 130

W.A. S.A. T. V. N.S.W. Q. -- B.fl.VII,131

M.fr.IX,78. $395(1879)$

- $\quad-$ T. V. N.S.W.

- B.fl.VII, 132 


\section{ERIOCAULEAE.}

Humboldt, Bonpland \& Kunth, nov. gen. et spec. plant. I, 251 (1815).

ERIOCAULON, Linné, gen. plant. ed. sec. 35 (1742). (Electrosperma.)

E. setaceum, Linné, spec, plant. 87 (1753)..

E. setaceum, Linné, spec, plant. $87(1753) \ldots$
E. australe, R. Brown, prodr. $254(1810)$$\ldots$

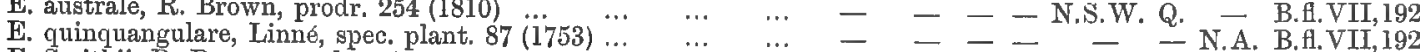

E. Smithii, R. Brown, prodr. 254 (1810)

E. nanum, R. Brown, prodr, 254 (1810)

E. cinereum, R. Brown, prodr. 254 (1810) …

E. pusillum, $R$, Brown, prodr. 254 (1810) ..

E. pallidum, R. Brown, prodr, 254 (1810) ...

E. nigricans, R. Brown, prodr. 254 (1810) ...

E. electrospermum; Electrosperma Australasicum, F. v. M. in Trans. phil. Soc. Vict, I, 24 (1854)

E. lividum, F. v. M., fragm. I, 92 (1858)

E. concretum, F. v. M., fragm. I, 92 (1858)

E. Schultzii, Bentham, Fl. Austr. VII, 195 (1878)

E. tortuosum, F. v. M., fragm. I, 94 (1858)

E. monoscapum, F. v. M., fragm, I, 94 (1858)

E. spectabile, F. v. M., fragm. I, 95 (1858)

E. scariosum, R. Brown, prodr. 255 (1810)..

E. depressum, R. Brown, prodr. 255 (1810)

$$
\begin{aligned}
& \begin{array}{llllll}
\cdots & \cdots & \cdots & - & - & - \text { V. N.S.W. }
\end{array}
\end{aligned}
$$

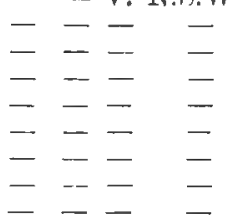

Q. - B.fl.VII, 192

Q. - B.fl.VIr,193

- N.A. B.fl.VII,193 M.fr.I,95.

Q. - B.fl.VII, 194

Q. - B.fl.VII, 194

Q. N.A. B.H.VII, 194

\section{- - B.fl.VII,195}

- N.A. B.fl.VII,195 M.fr.I,92.

- N.A. B.fl.VII, 195 M.fr.I,92.

- N.A. B.fl.VII,195

- N.A. B.H.VII,196 M.fr.I,9].

- N.A. B.f.VII, 196 M.fr. I,94.

- N.A. B.f.VII, 196 M.fr.I,95.

Q. N.A. B.fl.VII, 197

Q. N.A. B.A.VII,197 M.fr.I,92.

\section{RESTIACEAE.}

R. Brown, prodr. flor. Nov. Holl. 243 (1810).

TRITHURIA, J. Hooker, Fl. Tasman. II, 79, t. 138 (1860). (Juncella, 1854.)

T. submersa, J. Hooker, Fl. Tasman. II, 79, t. 138 (1860) ‥ W.A. S.A. T. V. N.S.W. - - B.H.VII,199 M.fr.VIII, 237. APHELTA, R. Brown, prodr. H. Nov, Holl. 251 (1810). (Brizula.)

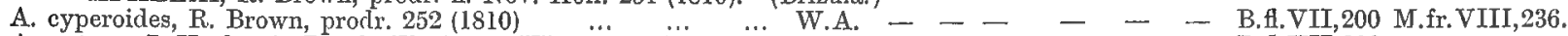
A. nutans, J. Hooker in Benth. Fl. Austr. VII, 200 (1878) $\quad \cdots$ W.A. - - - $-\quad$ - - B.fl.VII,200

A. gracilis, Sonder in Schlecht. Linnaea XXVIII, 227 (1855) $\ldots \ldots-$ - S.A. T. V. N.S.W. - — B.fl.VII,201 M.fr.VIII,237.

A. Drummondii, Bentham, FI. Austr. VII, 201 (1878) $\ldots$... W.A. - - - - - - B.fl.VII,20l

A. Pumilio, F. v. M., Schlecht. in Linnaea XXVIII, 226 (1855) $\ldots$.A. S.A. T. V. N.S. W. - - B.fl.VII,201 M.fr.VIII,237.
A. Brizula, F. v. M., fragm. V, 203 (1866)..

CENTROLEPIS, Labillardière, Nov. Holl. pl. specim. I, 7 (1804). (Desvauxia, Alepyrum.)

C. humillima, F. v. M. in Benth. Fl. Austr. VII, 203 (1878) ... W.A. - - - - - - B.fl.VII,203

C. polygyna, Hieronymus in Abh. naturf. Ges. Halle XII, 96 (1873) W.A. S.A. T. V. N.S. W. - - B.fl.VII,203 M.fr.VIII,237.

C. alepyroides, Hieronymus in Abh. nat. Ges. Halle XII, 96 (1873) W.A. - - - - - - B.fl.VII,204 M.fr.VIII,237.

C. mutica, Hieronymus in Abh. nat. Ges. Halle XII, 97 (1873)... W.A. - - - - - - B.fl.VII,204

C. glabra, F. v. M. in Abh. nat. Ges. Halle XII, 95 (1873) ‥ W.A. S.A. T. V. N.S.W. - - B.fl.VII,204 M.fr.VIII,237.

C. muscoides, Hieronymus in Abh, nat. Ges. Halle XII, 95 (1873) - - T. - - - - B.fl.VI,205

C. monogyna, Bentham, Fl. Austr. VII, 205 (1878) $\ldots \quad \ldots \quad-\quad$ - T. - - - - - B.tl.VII,205

C. pulvinata, Desvaux in Ann, des sc. nat. XIII, 42, t. $2(1828)$ - - T. - _ - - B.fl.VII,205

C. pusilla, Roemer \& Schultes, syst. veg. I, 44 (1817) ... ‥ - - - - - Q. - B.H.VII,205

C. aristata, Roemer \& Schultes, syst. veg. I, 44 (1817) $\ldots \ldots$ … IV.A. S.A. T. V. N.S.W. - $\quad$ - B.Al.VII,206 M.fr.VIII,237.

C. Drummondii, Hieronymus in Abh. nat. Ges. Halle XII, 98 (1873) W.A. - - - - - - B.fl.VII,206 M.fr.VIII,237.

C. Banksii, Roemer \& Schultes, syst. veg. I, 44 (1817) .. ‥ - - - - - Q. N.A. B.f.VII,207 M.fr.VIII,237.

C. fascicularis, Labillardiere, Nov. Holl. pl. spec. I, 7, t. I (1804) -

C. pilosa, Hieronymus in Abh, nat. Ges. Halle $102(1873) \quad \ldots$ W.A. - - - - - _ B.fl.VII,207

C. strigosa, Roemer \& Schultes, syst. veg. I, 43 (1817) $\ldots \ldots$... W.A. S.A. T. V. N.S.W. - - B.A.VII,207

C. exserta, Roemer \& Schultes, syst. veg. I, 44 (1817) $\ldots$... LYGINIA, R. Brown, prodr. fl. Nov. Holl. 248 (1810). (Schoenodum partly.)

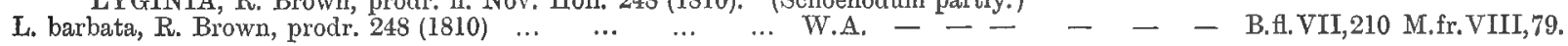
ECDEIOCOLEA, F. v. M., fragm. phytogr. Austr. VIII, 236 (1874).

E. monostachya, F. v. M., fragm. VIII, $236(1874) \quad \ldots \quad \ldots \quad$ W.A. - $\quad$ - ANARTHRIA, R. Brown, prodr. fl. Nov. Holl. 248 (1810).

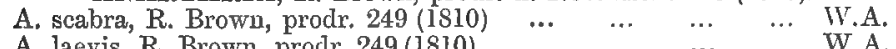

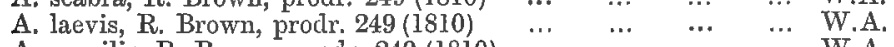

$\begin{array}{llllll}\text { A. gracilis, } R_{\text {. Brown, prodr. } 249(1810)} \ldots & \ldots & \ldots & \ldots & \text { W.A. } \\ \text { A. prolifera, } R \text {. Brown, prodr. } 249 \text { (1810) } & \ldots & \ldots & \ldots & \ldots & \text { W.A. }\end{array}$

A. polyphylla, Nees in Lehm. pl. Preiss. II, $63(1846) \quad \ldots . \quad \ldots \quad$ W.A. LEPYRODIA, R. Brown, prodr. fl. Nov. Holl. 247 (1810).

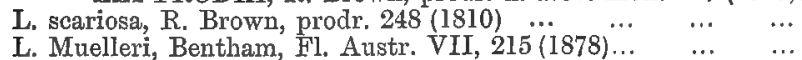

L. anarthria, F. v. M., fragm. VIII, $73(1873) \quad \ldots \quad$...

L. gracilis, R. Brown, prodr. 247 (1810) $\ldots-$. $\ldots$, $\ldots$

L. interrupta, F. v. M., fragm. VIII, 74 (1873) ...

L. hermaphrodita, R. Brown, prodr. 248 (1810) $\ldots . \quad \ldots$

L. monoica, F. v. M., fragm. VIII, 76 (1873)

L. Muirii, F. v. M., fragm. VIII, 78 (1873)

L. stricta, R. Brown, prodr. 248 (1810)

L. macra, Nees in Lehm. pl. Preiss. II, 60 (1846)...

L. Drummondiana, Steudel, syn. pl. glum. II, 248 (I855)

L. glauca, F. v. M., fragm. VIII, 77 (1873)

L. anaectocolea, F. v. M., fragm. VIII, 78 (1873)...

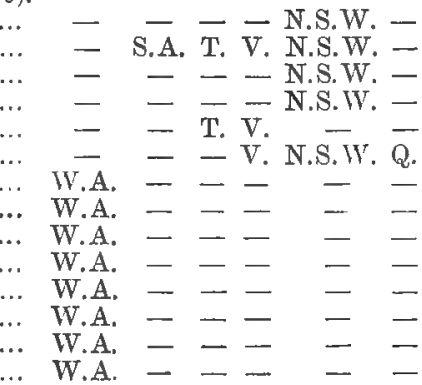

B.fl. VII,211 M.fr. VIII,236.

B.fl. VII, 212 M.fr.VIII, 80. B.fi.VII,212 M.fr.VIII, 81. - B.fl.VII,213 M.fr.VIII,72,81.

B.fl.VII, 213 M.fr.VIII, 82. B.A. VII, 214 M.fr.VIII, 83.

B.fl.VII,215 M.fr.VIII,72,

B.H.VI,215

B.H.VII, 216

B.fl.VII,216 M.fr.VIII,73.

B.fl.VII,216 M.fr.VIII,73,75;IX, 194.

B.A.VII,217 M.fr.VIII, 74 .

B.H.VII, 217 M.fr.VIII, 75 .

B.fl. VII, 217 M.fr.VIII, 76 .

B.tl.VII,218 M.fr.VIII, 76

B.Al. VII, 218 M.fr. VIII, 74.

B.H.VII,218

B.fl.VII, 219 M.fr.VIII,75.

B.H.VII,219 M.fr. VIII, 77

B.fl.VII, 220 M.fr.VIII,78. 
RESTIO, Limné, syst. nat. edit, duo dec. II, 735 (1767). (Megalotheca.)

R. fastigiatus, I., Biown, prodr. $246(1810)$
R. Megalotheca, F. T. MI, fragm. VIII, 99(1873) $\ldots \ldots$
R.

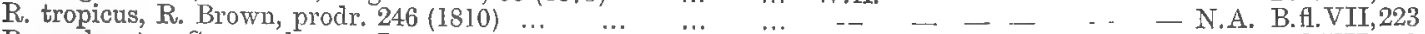

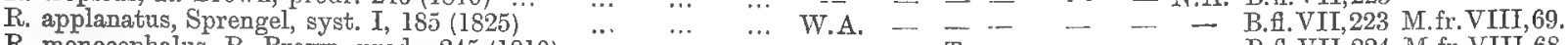

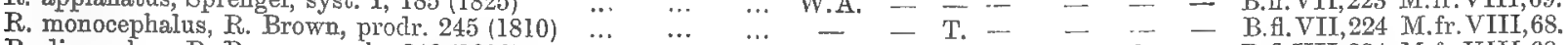

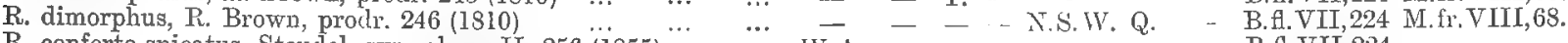

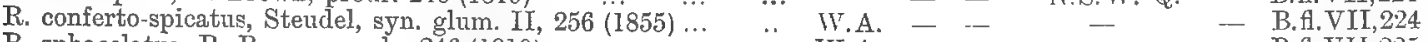

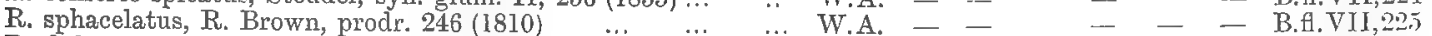

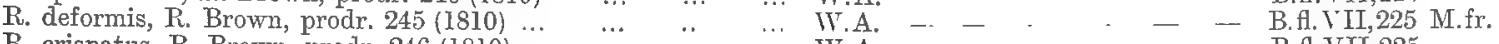

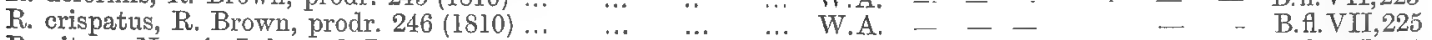

R. nitens, Nees in Lehm. pI. Preiss. II, $59(1846) \ldots$
R. gracilior, F. v. M. in Benth. Fl. Austr. VII, 226 (1878)

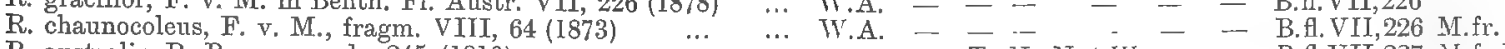

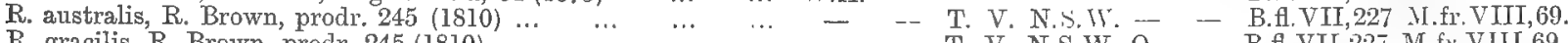

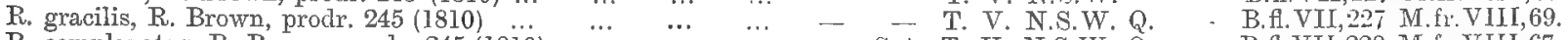

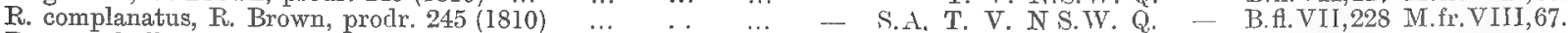

R. tetraphyllus, Labillardiere, Nov. Holl. pl. spec. II, 77 (1806) - S.A. T. V. N.S. W. Q. $\quad$ B.fl.VII,228 M.fr.VIII,66.

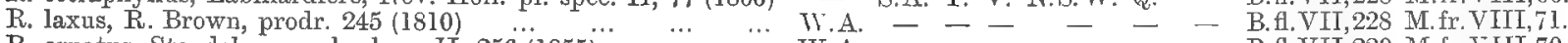

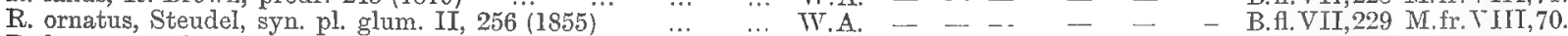

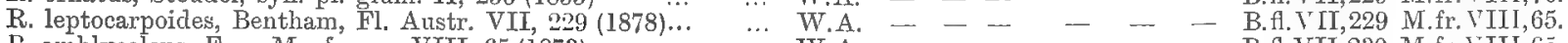

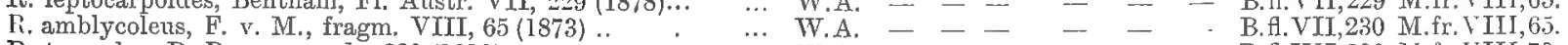

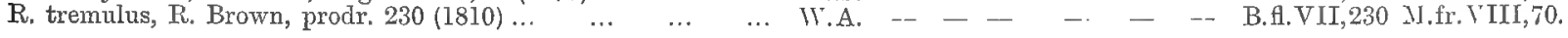

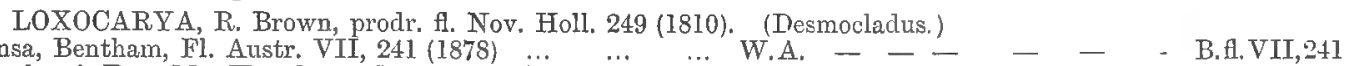

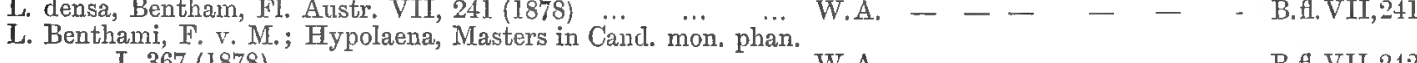

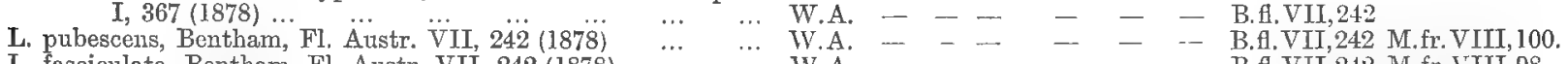

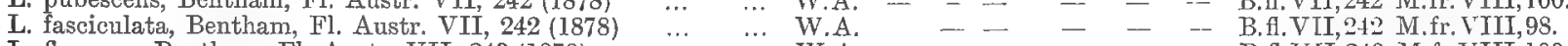

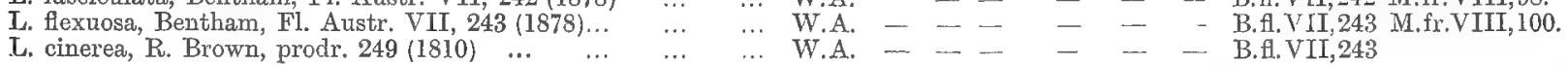

CALOSTROPHUS, Labillardière, Nov. Holl. pl. spec. II, 78 (1806). (Calorophus, Hypolaena.)

C. elongatus, F. v. M., fragm. VIII, 86 (1873)

C. lateriflorus, F. v. M., fragm. VIII, $87(1873)$.

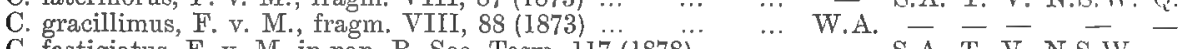

C. fastigiatus, F. v. M. in pap. R. Soc. Tasm. 117 (1878) 즌 - S.A. T. V. N.S.W.

B.fi. VII, 238 M.fr.VIII, $86 ; \mathrm{IX}, 94 ; \mathrm{X}, 120$

B.t. VII, 238 M.fr.VIII, 87.

B.fi.VII,239 M.fr.VIII, 88 .

B.fl.VII, 239 M.fr. VIII, 84.

C. exsulcus, F. v. M.; Hypolaena, R. Brown, prodr. 251 (1810) W.A. - - - - - - B.甘 VII,240 M.fr.VIII, S6. LEPTOCARPUS, R. Brown, prodr. 250 (1810). (Schoenodum partly.)

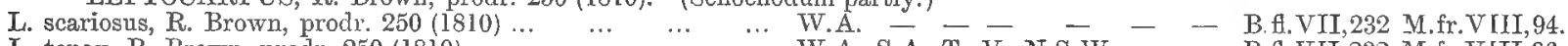

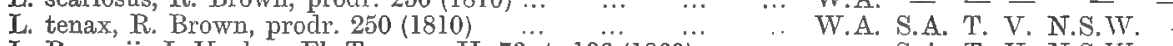

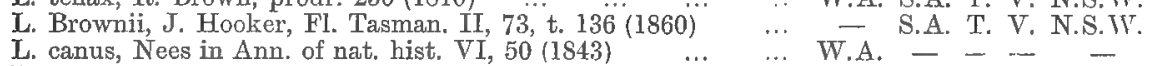

L. coangustatus, Nees in Lehm. pl. Preiss. II, 65 (1846)

L. aristatus, R. Brown, prodr. 250 (1810) $\ldots$
L. erianthus, Bentham, Fl. Austr. VII, 235 (1878)

L. erianthus, Bentham, F. Austr. VII, 23อ (18/S) ...

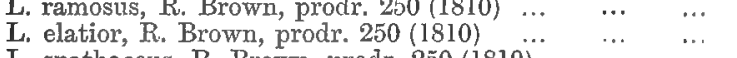

L. spathaceus, R. Brown, prodr. $250(1810)$
L. Schultzii, Bentham, Fl. Austr. VII, 237 (1878) $\quad \ldots$ LEPIDOBOLUS, Nees in Lehm. pl. Preiss. II, 66 (1846).

L. drapetocoleus, F. v. M., fragm. VIII, 84 (1873) … ...

L. Preissianus, Nees in Lehm. pl. Preiss. II, 66 (1846) $\ldots$

L. chaetocephalus, F. v. M., fragm. VIII, 84 (1873) ‥ CHAETANTHUS, R. Brown, prodr. 251 (1810). (Prionosepalum)

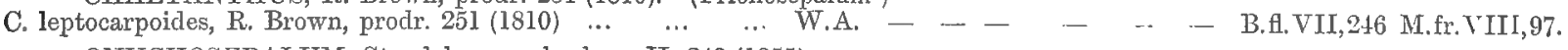
ONYCHOSEPALUM, Steudel, syn. pl. glum. II, 249 (1855).

O. laxiflorum, Steudel, syn. pl. glum. II, $249(1855) \quad \cdots \quad \ldots \quad$ W.A. $\quad-\quad-\quad-\quad--\quad \ldots \quad-$ B.f. VII,246 M.fr.IX,51.

\section{ACALYCEAE HYPOGYNEAE.}

F. v. M. in Woolls's plants of the neighb. of Sydney 48 (1880).

\section{CYPERACEAE.}

Haller, enum. stirp. Helvet. I, 234 (1742).

KYLLINGIA, Rottboell, descr. et icon. rar. et nov. plant. 13, t. 4 (1773). (Killingia.)

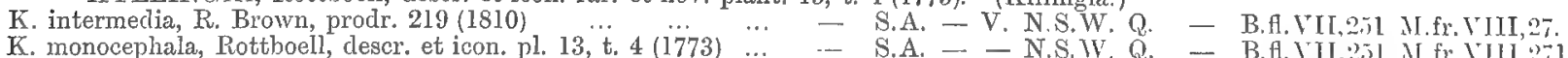

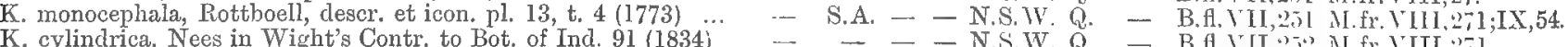

- B.fl. YII, 2.I2 M.fr. YIII, 271 .

K. triceps, Rottboell, descr, et icon. pl. 14, t. $4(1773) \ldots \ldots \ldots-\ldots-$ - .. - - Q. - B.H.VII, 25

CYPERUS, Tournefort, inst. 527, t. 299 (1700), from Hippocr., Theophr. and Plinius. (Pycreus, Mallivens, Anosporum, Sorostachys.)

C. pumilus, Linné, amoen. acad. IV, 302 (1759) ...

C. Eragrostis, Vahl, enum. plant. II, $322(1806)$...

C. Havescens, Linné, spec. plant. 46 (1753) 4 C. globosus, Allioni, auctuar. ad fl. Pedem. 49 (1789)

) - B.t.VII, 25s M.fr.VIIT,267.

C. unioloides, R. Brown, prodr. 216 (1810)

S.A. - V. N.S.W
$--\bar{Y}$ - N.S.W.

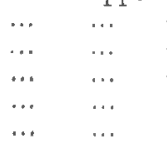

$$
\begin{aligned}
& - \\
& - \\
& -
\end{aligned}
$$

- - V. N.S.W.
- B.fl.VIl,2.8s M.fr. VIII, 260;IX,52.

- B.fH. VII, 259

- B.fl.VII, 260 M.fr. VIII, 260;IX,52. 
C. polystachyus, Rottboell, deser, et icon. pl. 39, t. 11 (1773) ...

C. flavicomus, CI. Richard in Michaux, fl. bor. Amer. I, 27 (1803)

C. pygmaeus, Rottboell, descr. et icon. pl, 20, t. 14 (1773)

C. cephalotes, Vahl, entum. II, 311 (1806)

C. laevigatus, Linné, mantissa alt. 179 (177i)

C. platystylis, R. Brown, prodr. 241 (1810)

C. alopecuroides, Rottboell, deser. et icon. pl. 38, t. 8 (1773) ..

C. pulchellus, R. Brown, prodr. 213 (1810)

C. tenellus, Linné, fil. suppl. 1.03 (1781)

C. gracilis, R. Brown, prodr. 213 (1810)

C. enervis, R. Brown, prodr, 213 (1810)

C. debilis, R. Brown, prodr. 213 (1810)

C. castaneus, Willdenow, spec. plant, I, 278 (1797)

C. cuspidatus, Humboldt, Bonpland \& Kunth, nov. gen, et sp. pl. I, 204 (1815)

C. squarrosus, Linné, amoen. acad. IV, $30 \dddot{3}$ (1759)

C. difformis, Linne, amoen. acad. IV, 302 (1759)...

C. tetraphyllus, R. Brown, prodr. 214 (1810)

C. trinervis, R. Brown, prodr. 213 (1810) ..

C. Haspan, Linné, spec. plant. 45 (1753)

C. concinnus, R. Brown, prodr. 214 (1810)."

C. filipes, Bentham, Fl. Austr. VII, 271 (1878)

C. pedunculosus, F. v. M., fragm. VIII, 266 (1874)

C. vaginatus, R. Brown, prodr, 213 (1810)...

C. Holoschoenus, R. Brown, prodr. 215 (1810) ...

C. dactylotes, Bentham, Fl. Austr. VII, 273 (1878)

C. Gilesii, Bentham, Fl. Austr. VII, 274 (1878) ...

C. fulvus, $R$. Brown, prodr. 215 (1810)

C. carinatus, R. Brown, prodr. 216 (1810)...

C. alterniflorus, R. Brown, prodr. 216 (1810)

C. pilosus, Vahl, enum. II, 354 (1806)

C. ornatus, R. Brown, prodr. 217 (1810)

C. Iria, Linné, spec. plant, 45 (1753)

C. eleusinoides, Wallich in Kunth, enum. III, 39 (ï837) '

C. distans, Linné, fil. suppl. 103 (1781)

C. tegetiformis, Roxburgh, hort. Benghal. (1814)...

C. articulatus, Linne, spec. plant. 44 (1753)

C. diphyllus, Retzius, observ. bot. V, 11 (1789) ...

C. rotundus, Linné, spec. plant. 45 (1753) ...

C. stenostachyus, Bentham, Fl. Austr. VII, 280 (1878)

C. congestus, Vahl, enum. II, 350 (1806)

C. subulatus, R. Brown, prodr. 217 (1810)...

C. sporobolus, R. Brown, prodr. 215 (1810)

C. angustatus, R. Brown, prodr. 214 (1810)

C. inornatus, Boeckeler in Fl. Regensb. 86 (1875)

C. lucidus, R. Brown, prodr. 218 (1810)

C. pennatus, Lamarck, illustr. des genr. I, 144 (1791)

C. exaltatus, Retzius, observ. bot. V, 11 (1789)

C. haematodes, Findlicher, prodr. fl. Norf. 22 (1839)

C. auricomus, Sieber in Sprengel, syst. I, 230 (1825)

C. ferax, Cl. Richard in act. soc. hist. nat. Par. I, 106 (1792)

C. Bowmanni, F. v. M. in Benth. Fl. Austr. VII, 287 (1878)

C. trichostachys, Bentham, Fl. Austr. VII, 287 (1878)

C. leiocaulis, Bentham, Fl. Austr. VII, 287 (1878)

C. scaber, Bentham, Fl. Austr. VII, 288 (1878)

C. decompositus, F. v. M., fragm. VIII, 267 (1874)

C. Armstrongii, Bentham, Fl. Austr, VII, 289 (1878)

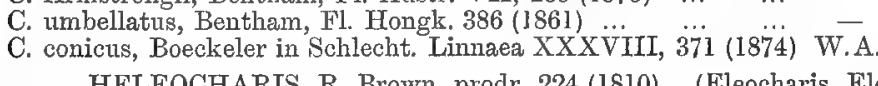
HELEOCHARIS, R. Brown, prodr. 224 (1810).

H. sphacelata, R. Brown, prodr. 224 (1810)

H. spiralis, R. Brown, prodr. 224 (1810) ..

H. compacta, R. Brown, prodr. $224(1810) \ldots$... $\ldots$...

H. tetraquetra, Nees in Wight's Contrib. Bot. of Ind. 113 (183

H. cylindrostachys, Boeckeler in Regensb. Flora, 108 (1875)

H. acuta, R. Brown, prodr. 224 (1810)

H. multicanlis, Smith, Engl. Flor, II, 64 (1824) $\ldots$

H. atricha, R. Brown, prodr. 225 (1810) $\quad \ldots \quad \ldots \quad \ldots$

H. capitata, $\mathbf{R}$. Brown, prodr. 225 (1810) $\ldots$

H. acicularis, R. Brown, prodr. 224 (1810)...

FTMBRISTYLIS, Vahl, enum, plant. II, 285 (1806).

F, acicularis, R. Brown, prodr, 226 (1810) ...

F. acuminata, Vahl, enum. II, 285 (1806) ...

F. punctata, R. Brown, prodr. 226 (1810)

F. rhyticarya, F. v. M., fragm. I, $215(1859)$

F. leucostachya, Boeckeler in Garcke, Linnaea XẌX VIII, 385 (18̈74) -

F. nutans, Vahl, enum. II, 285 (1806)

F. paucifiora, R. Brown, prodr. $225(1810) \ldots$ $-\quad-\quad-\frac{1}{\mathrm{r}}$

- N.S.W. Q. N.A. B.ff. YII, 261 M.fr.VIII, 265,270;IX, 53. - N.A. B.A.VII,261 M.fr. VIII, 265;IX, 53.

Q. N.A. B.fl. YII,262 M.fr.VIII,268;IX,53.

Q. - B.f.VII,263 M.fr.VIII,272;IX,54.

- - B.fl.VII, 263 M.fr.VII, 266;IX,53.

B.H. VII, 264

B.fl. VII,264 M.fr.VIII, $263 ; I X, 5: 2$.

Q. N.A. B.fl.TII, 265 M.fr.VIII, 271

W.A. S.A. - - N.S.W. - - B.fl.VII, 265 M.fr.VIII, 261;IX,53.

S.A. - V. N.S.W. Q

- - N.S.W.Q. - B.fl.VII,266

B.fl.VII, 265 M.fr.VIII, 264;IX, 53.

B.tl. VII, 266

Q. - B.fl.VII, 267

- - - - Q Q. B.fi.VII,267

Q. N. N.A. B.f.VII, 268 M.fr.VIII, 262;IX,53.

- - - - N.S.W. Q. - B.f.VII,269 M.fr.VIII,264.

S.A. - V. N.S.W. Q. N.A. B.fl.VII,269 M.fr.VIII,267;IX,53.

- - N.S.W. Q. N.A. B.fl.VII, 270 M.fr.VIII,260;IX,52.

$-\quad$ V. N.S.W. Q.

- - N.S.W. Q. - B.fl.VII,271

- - - - Q. B.fl.VII, 272 M.fr.

Q. N.A. B.f.VII, 272 M.fr.VIII,261;IX,53.

S.A. - - - Q. N.A. B.fl.VII,273 II.fr.I,200; VIII,262.

- - N.S.W. Q. N.A. B.f.VII, 273

S.A. - - N.S.W. Q. - B.fi.VII,274

S.A. - - N.S.W. Q. - B.fl.VII,274 M.fu.VIII,268

- - - N.S.W. Q. N.1. B.fl.VII, 274

W.A. S.A. - - - Q. - B.fl.VII,275

- - - - N.S.W. Q. - B.fl.VII,275 M.fr.VIII, 260;IX,52.

- - - - N.S.W. Q. - B.H.VII, 276

- S.A. - - N.S.W. Q. N.A. B.fl.VII,276 M.fr.VIII,266;IX,53.

- - - - Q. N.A. B.fl.VII, 277 M.fr. VIII, 264;IX,, 3

- - - - - Q. - B.fl,VII, 277 M.fr.VIII, 266;IX,53

- - - - - N.A. B.fl.VII,278 M.fr.VIII,260;IX, 52.

- - - - - - N.A. B.fl.VII, 278

W.A. S.A. - V. N.S.W. Q. N.A. B.fl.VII, 279 M.fr. VIII,269;IX,53.

W.A. - - - - - - B.fi.VII, 280

W.A. - - N.S.W. - - B.fl.VII,280 M.fr.VIII,269;IX,53.

S.A. - N.S.W.

- N.A. B.H.TIT, 281

Q. N.A. B.fl.VII, 282 M.fr.IX,54,

S.A. T. V. N.S. W. Q. N.A. B.fl.VII, 282 M.fr.IX, 54,

- - - Q. N.A. B.fl.VII,284 M.fr.VIII,263;IX,53.

S.A. - V. N.S.TV. Q. N.A. B.H.VII,285 M.fr.VIII,263;IX,53.

- - - N.S.IV. - - B.fl.VII,285 M.fr.IX,54.

- - - - Q. - B.fl.VII,286 M.fr.VIII,263.

- - - - Q. - B.f.VII,286

- - N.S.W. Q. - B.A.VII,287

- - - Q. - B.fl.VII,287

- - - N.S.T.Q. - B.fl.VII,287

- - - - Q. N.A. B.fl.VII,288

$-\quad-\quad$ Q. N.A. B.fl.VII,288
$-\quad$ Q

-- - - - Q. - - B.fl.VII, 289 M.fr.VIII, $267 ;$ IX, 53.

Eleochiton, Scirpidium.)

S.A. T. V. N.S.W. Q. N.A. B.fl.VII, 292 M.fr.VI,94;VIII,239.

- - - N.S.W. Q. N.A. B.fl.VII, 292 B.H.VII,293 M.fr.VIII, 239.

- - - Q. - B.fl.VII,293 MI.fr.VI,93;VIII,239,

- - - - N.S.W. Q. - B.fl.VII,294 MI.fr.VIII,239.

- - - N.S.IV. Q. - B.H.VII,294 II.fr.VIII, 240.

W.A. S.A. T. V. N.S.W. Q. - B.fl.VII,294 M.fr.VIII,240.

W.A. S.A. - V. N.S.W. - - B.fl.VII, 296

- - - - N.S.W. Q. N.A. B.f.VII,295 M.fr.VII, 252;IX, 100

IV.A. - - - - Q. N.A. B.fl. YII, 296 M.fr.VI,94;VIII,240.

.. - - - N.S.W. Q. N.A. B.f.VII,296 M.fr. VIII,240.

(Abildgaardia, Trichelostylis, Oncostylis)

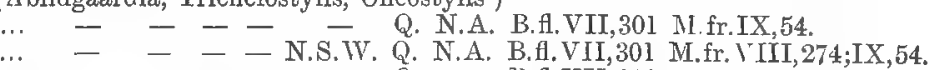

Q. N.A. B.f.VII,301

- - - - Q. N.A. B.A.VII,302 M.fr.I,215.

- - - - - N.A. B.fl.VII,302 M.fr.IX,54.

- - N.S.W. Q. N.A. B.fl.VII, 303 M.fr.VIII, 274;IX,54.

Q. N.A. B.fl, VII, 303 
F. cardiocarpa, F. r. M., fragm. I, 194 (1859) F. leucocolea, Bentham, Fl. Austr. VII, 304 (1878)

F. polytrichoides, R. Brown, prodr, 226 (1810)

F. androgyna, R. Brown, prodr. 226 (1810)

F. subbulbosa, Bentham, Fl. Austr. VII, 305 (1878)

F. tetragona, R. Brown, prodr. 226 (1810)...

F. trigastrocarya, F. v. M., fragm. I, 194 (1859) ...

F. monandra, F. \. M., fragm. I, 195 (1859)

F. pterygosperma, R. Browu, prodr. 226 (1810) $\ldots$

F. sphaerocephala, Bentham, Fl. Austr. VII, 306 (1878)...

F. xyridis, R. Brown, prodr. 226 (1810)

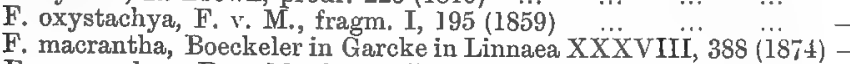

F. squarrulosa, F. v. M., fragm. I, 216 (1859)

F. monostachya, Hasskarl, pl. Jav. rar. 61-64 (1848

F. Brownii, Bentham, Fl. Austr. VII, 308 (1878)

F. Dallachyi, F. v. M. in Benth. Fl. Austr. VII, 309 (1878)

F. velata, R. Brown, prodr. 227 (1810)

F. aestivalis, Vahl, enum. II, 288 (1806) ..

F. dichotoma, Vahl, enum. II, 287 (1806) ...

F. depauperata, R. Brown, prodr. 227 (1810)

F. spirostachya, F. v. M. in Benth. Fl. Austr. vÏI, 311 (1878)...

F. communis, Kunth, enum. II, 234 (1837)

F. ferruginea, Vahl, enum. 291 (1806)

F. denudata, R. Brown, prodr. 227 (1810)...

F. elata, R. Brown, prodr. 227 (1810)

F. caespitosa, R. Brown, prodr. 228 (1810)

F. spiralis, R. Brown, prodr. 226 (1810) ...

F. subaristata, Bentham, Fl. Austr. VII, 314 (Is

F. Ieptoclada, Bentham, Fl. Austr. VII, 314 (1878)

F. debilis, F. v. M., fragm. I, 198 (1859)

F. corynocarya, F. v. M., fragm. I, 197 (1859) ...

F. solidifolia, F. v. M., fragm. I, 198 (1859)

F. obtusangula, F. v. M., fragm. I, 198 (1859)

F. miliacea, Vahl, enum. II, 287 (1806)

F. rara, R. Brown, prodr. 227 (1810)

F. microcarya, F. v. M., fragm. I, $200(1850)$

F. quinquangularis, Kunth, enum. II, 229 (1837)

F. cyperoides, R. Brown, prodr. 228 (1810)

F. furva, R. Brown, prodr. 228 (1810)

F. cymosa, R. Brown, prodr. 228 (I810)

F. multifolia, Boeckeler in Garcke, Linnaea XXXVIII, 397 (1874)

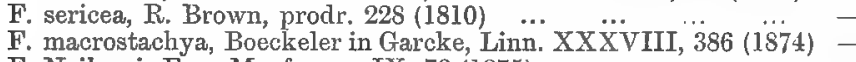

F. Neilsoni, F. v. M., fragm. IX, 79 (1875)

F. capitata, R. Brown, prodr. 228 (1810) ...

F. Schultzii, Boeckeler in Garcke, Linnaea XXX VIII, 391 (1874)

F. barbata, Bentham, Fl. Austr. VII, 321 (1878)

F. capillaris, A. Gray, Man. of Bot. N.U.S. fifth ed. 567 (1867) W.A.
-
$=$
$=$
$=$
$=$
$=$
$=$
$=$
$=$
$=$
$=$
$=$
- N.A. B.fl.VII, 303 M.fr.I,194.

- N.A. B.f.VII, 301

Q. N.A. B.H.VII, 304

- N.A. B.fl.VII,304

Q. - B.fl.VII, 305

- N.A. B.tl.VII,305 M.fr.I, 194;VIII,274.

- N.A. B.f. VII,305 M.fr.I, 194.

- N.A. B.fl.VII,306 M.fr.I, 195.

- N.A. B.f.VII,306 M.fr.I,193;IX,55.

Q. N.A. B.fl.VII, 306

Q. N.A. B.fl.vII, 307 M.fr.VIII,274;IX,54.

- N.A. B.fl.VII, 307 M.fr.VIII,272.

- N.A. B.fl.VII,307 M.fr.IX,55.

- N.A. B.fl.VII,308 M.fr.I,216.

Q. N.A. B.fl.VII, 308 M.fr.VIII,272;IX,54.

- N.A. B.f.VII,308 M.fr.VIII,273.

Q. - B.fl.VII,309 M.fr.VIII,273.

S.A. - V. N.S.W. Q. N.A. B.A.VII,309 M.fr.IX,11,54.

V. N.S.W. Q. N.A. B.fl.VII,310 M.fr.IX,11,54.

- N.S.W. Q. N.A. B.fl.VII,310 M.fr.TX, 10,54.

- N.A. B.f.VII,311

- N.A. B.fl.VII,3l1

- S.A. - V. N.S.TW. Q. N.A. B.fl.VII,311 M.fr.I, 196;IX,10,54.

IV.A. S.A. - - N.S.W. Q. N.A. B.fl.VII, 312 M.fr.I, 197;IX, 10,54.

Q. N.A. B.H.VII,313 M.fr.IX,9.

- N.A. B.fl.VII,313

Q. N.A. B.fl.VII,313 M.fr.I, 199 .

- N.A. B.H.VII,314

- N.A. B.fl.VII, 314

Q. - B.f.VII, 314

— N.A. B.fl.VII,315 M.fr.I,198.

- N.A. B.ff.VII,315 M.fr.I,187.

- N.A. B.fl.VII,315 M.fr.I, 198.

Q. N.A. B.fl.VII,315 M.fr.I, 198 .

Q. N.A. B.fl.VII,316 M.fr.I,199;IX,12,54.

- N.A. B.fl.VII, 316

Q. N.A. B.fl.VII, 316 M.fr.I,200,

- - N.A. B.H.VII, 317

- N.S.W. Q. N.A. B.fl.VII,317 MI.fr.VIII,273;IX,11,5t.

Q. - B.tl.VII,318

-- N.A. B.tl.VII,318

- N.A. B.H.VII,319 M.fr.IX,55.

- N.A. B.H.VII, 319

S.A. - - N.S.W. - N.A. B.f.TII,319 M.fr.IX,54.
B.H.VII,320 M.fr.IX,79.

- _ - Q. N.A. B.f.VII,320 M.fr.I, 196 .

S.A. - - N.S.W. Q. N.A. B.fl.VII,321 M.fr.IX,55.

Q. N.A. B.A.VII, 3222 MI.fr.IX,55.

SCIRPUS, Tournefort, inst. 528, t. 300 (1700), from Terentius. (Isolepis, Malacochaete.)

S. humillimus, Bentham, Fl, Austr. VII, 324 (1878)

S. fluitans, Linné, spec. plant. 48 (1753)

S. lenticularis, Sprengel, syst. I, 208 (1825)

S. crassiusculus, J. Hooker in Benth. Fl. Austr. $\dddot{V} I I, 326$ (1878)

S. cyperoides, Sprengel, syst. I, 208 (1825)

, Linné, spec. plant. 49 (1753)

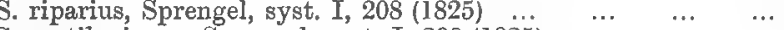

S. cartilagineus, Sprengel, syst. I, 208 (1825) ..

S. squarrosus, Linné, mant. plant. alt. 181 (1771)..

S. inundatus, Sprengel, syst. I, 207 (1825)...

S. prolifer, Rottboell, descr. et icon. pl. 55 t. 17 (1773) ...

S. articulatus, Linné, spec, plant. 47 (1753)

S. nodosus, Rottboell, descr. et icon. pl. 52, t. 8 (1773) ...

S. supinus, Linné, spec. plant. 49 (1753)

S. debilis, Pursch, flor. Amer. Sept. I, 55 (I814)

S. mucronatus, Linné, spec. plant. $50(1753) \quad \ldots$.

S. pungens, Vahl, enum. II, 255 (1806)

S. lacustris, Linné, spec. plant. 48 (1753) $\ldots \ldots$

S. litoralis, Schrader, Fl. Germ. I, 142, t. $\ddot{5}(1806)$

S. maritimus, Linné, spec. plant. 51 (1753)

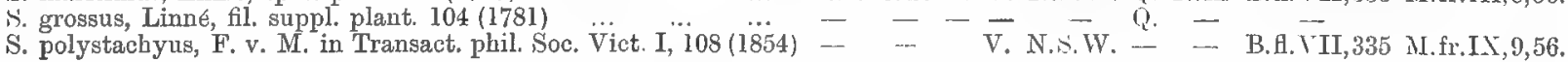

LIPOCARPHA, R. Brown in Tuckey's narrat. of an Exped to Congo, 4h9 (1818). (Hypaclyptum.)

L. argentea, R. Brown in Tuck. Congo, $459(1818)$
L. microcephala, R. Brown in Tuck. Congo, 459 (1818) ...

FUIRENA, Rottboell, descr. et icon. rar. pl. illustr, 70 (1773).

F. umbellata, Rottboell, deser, et icon. pl. 70, t. 19(1773) ‥ - - - - — - Q. N.A. B.fl.VII,337 M.fr.VIII,238;IX,57.

F. glomerata, Lamarck, Illustr. des genr. I, $150(1791) \ldots \quad \ldots \quad-\quad-\quad-\quad$ - N.S.IV. Q. N.A. B.H.VII,338 M.fr.VIII,238;IX,57. 
HYPELYTRUAI, L. C. Richard in Persoon, synops. I, 70 (1805). (Hypolytrum.)

H. latifolium, L. C. Richard in Pers. syn. I, 70 (1805) ... …-- - - - - Q. - B., .VII,339 II.fr.VIII,238;IX,57. EXOCARYA, Bentham in J. Hooker, icon. plant. t. 1206 (1877).

E. scleroides, Bentham in Hook, icon. pl. t. $1206(1877) \ldots \ldots \ldots-\ldots-$ - - - N.S.W. Q. - B.f.VII,339 M.fr.IX,12. MAPANIA, Aublet, Hist. pl. Guian. 47, t. 17 (1775). (Pandanophyllum.)

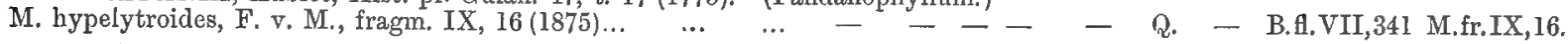
SCIRPODENDRON, Zippelius in Journ. Asiat. Soc. Beng. XXXVIII, 85 (1869).

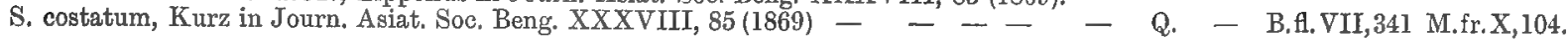
LEPIRONIA, L. C. Richard in Persoon, synops, I, 70 (1805). (Chondrachne).

L. mucronata, L. C. Richard in Pers. syn. I, 70 (1805) ... ‥ - - - - N.S.W. Q. - B.fl.VII,342 M.fr.IX,17,58. CHORIZANDRA, R. Brown, prodr. 221 (1810). (Chorisandra.)

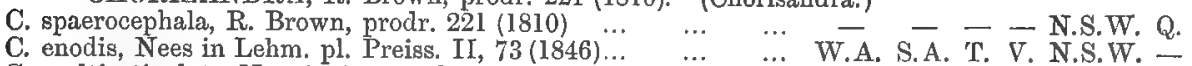

C. enodis, Nees in Lehm. pl. Preiss. II, $73(1846) \ldots$... 0 \% $\ldots$ W.A. S.A. 'T. V. N.S.W. -

C. cymbaria, R. Brown, prodr. $221(1810) \ldots \quad \ldots \quad \ldots \quad \ldots$ W.A. $\quad \ldots \quad-$ V. N.S.W. Q. OREOBOLUS, R. Brown, prodr. fl. Now, Holl. 235 (1810).

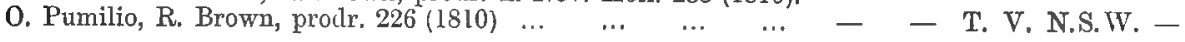
REMIREA, Aublet, Hist. des pl. de la Guian. franc. I, 44, t. 16 (1775).

R. maritima, Aublet, Hist. pl. Guian. I, 45, t. $16(1775) \quad \cdots \quad-\quad-\quad-\quad$ - Q. - B.fl.VII,347 M.fr.V,92;IX,20,58. RHYNCHOSPORA, Vahl, enum. plant. II, 229 (1806). (Morisia.)

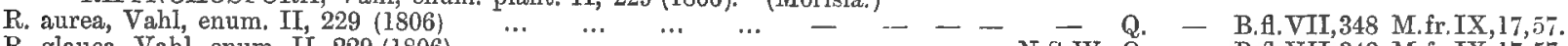

R. glauca, Vahl, enum. II, $229(1806)$
R. Wallichiana, Kunth, enum. II, $289(1837)$
R.

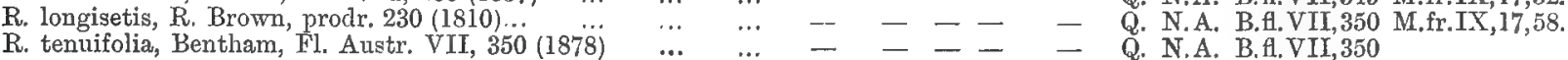
CYATHOCHAETE, Nees in Lehm. pl. Preiss. II, 86 (1846). (Tetralepsis.)

C. clandestina, Bentham, Fl. Austr. VII, 351 (1878) $\ldots \quad \ldots$ W.A. - - — - - - B.fl.VII,351 M.fr.IX,40.

C. avenacea, Bentham, Fl, Austr. VII, 351 (1878) $\quad \ldots \quad$ … W.A. - - - - - - - - B.fl.VII,351 M.fr.IX,40.

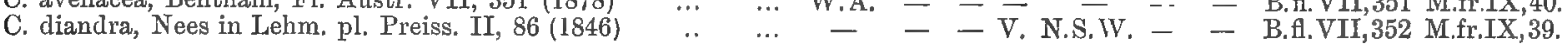
CARPHA, Banks \& Solander in R. Brown, prodr. 230 (1810).

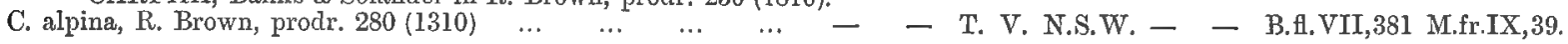
SCHOENUS, Linné, coroll. gen. 2 (1737). (Chaetospora, Elynanthus, Tricostularia, Helothrix, Isoschoenus, Gymnochaete, Gymnoschoenus, Mesomelaena, Discopodinm.)

S. cruentus, F. v. M., fragm. IX, 36 (1875) … W. W. W. A.
S. Benthami, F.v.M.; S.compressus, Benth. Fl.Austr. VII, 357 (1878) W. A.

S. lanatus, Labillard,, Nov. Holl. pl. spec. I, 19, t. 20 (1804) ... W. A.

S. curvifolius, Poiret, Encycl. méth. suppl. II, 251 (1811) ... IV.A.

S. capitatus, F. v. M., fragm. IX, $58(1875)$... $\quad \ldots . \quad \ldots$. W.A.

S. setifolius, Bentham, Fl. Austr. VII, $359(1878) \quad \ldots . \quad \ldots \quad$ TV.A.

S. Drummondii, Bentham, fl. Austr. VII, 359, non Steud. (1870) W.A.

S. turbinatus, Poiret, Encycl. méth. suppl. II, 251 (1811)

S. barbatus, Boeckeler in Garcke, Linnaea XXXVIII, 277 (1874) W.A.

S. flavus, Boeckeler in Garcke, Linnaea XXXVIII, 278 (1874) W.A.

S. brevisetis, Poiret, Encycl. méth. suppl. II, 251 (1811) ‥ W.A.

S. Armeria, Boeckeler in Garcke, Linnaea XXXVIII, 279 (1874) W.A.

S. aphyllus, Boeckeler in Garcke, Linnaea XXXVIII, 280 (1874)

S. imberbis, $R$. Brown, prodr. 231 (1810) ...

S. ericetorum, R. Brown, prodr. 231 (1810)

S. nitens, Poiret, Encycl. méth, suppl. II, 251 (1811)

S. cygneus, Nees in Lehmann, pl. Preiss. II, 81 (1846) ...

S. minutulus, F. v. M., fragm. IX, 32 (1875)

S. trachycarpus, F. v. M., fragm. IX, 33 (1875) ,.. $\quad .$.

S. Tepperi, F. v. M., fragm. XI, 106 (1880)

S. nanus, F, v. M. fragm. IX, 36 (1875)

S. pleiostemoneus, F. v. M., fragm. IX, 52 (1875)

S. brevioulis

S. breviculmis, Bentham, Fl. Austr. VII, 364 (1878) “.

S. unispiculatus, F. v. M. in Benth. FI. Austr. VII, 365 (1878

S. obtusifolius, Boeckeler in Garcke, Linnaea XXXVIII, 281 (1874)

S. grammatophyllus, F. v. M., fragm. IX, 31 (1875)

S. asperocarpus, F. v. M., fragm. IX, 29 (1875) ...

S. Moorei, Bentham, FI. Austr. VII, 367 (1878) ...

S. villosus, R. Brown, prodr. 231 (1810)

S. grandiflorus, F. v. M., fragm. IX, $30(1875) \ldots$.

S. distans ; Chaetospora, F. v. M., fragm. IX, 33 (1875)

S. calostachyus, Poiret, Encycl. méth, suppl. II, 251 (1811)

S. scabripes, Bentham, Fl. Austr. VII, 368 (1878)

S. multiglumis, Bentham, Fl. Austr. VII, 368 (1878) $\ldots$

S. efoliatus, F. v. M., fragm. IX, 32 (1875)

S. acuminatus, R. Brown, prodr. 231 (1810)

S. pedicellatus, Poiret, Encycl. méth, suppl. II, 251 (1811)

S. fascicularis, Nees in Ann. and Mag. of nat. Hist. VI, 48 (1

S. brevifolius, R. Brown, prodr. $231(1810)$
S. melanostachys, R. Brown, prodr. $231(1810)$

S. spartens, R. Brown, prodr. 231 (1810) … …

S. vaginatus, F. v. M. in Benth. Fl. Austr. VII, 371 (1878)

W.A. S.A. T. V. N.S.S.W. Q.

W.A. - - - N.S.W. Q.

W.A.

W.A.

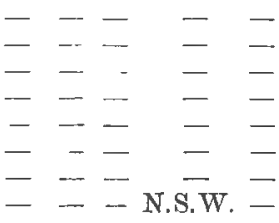

-

B.H. VII, 357 M.fr.IX,37.

B.fl.VII, 357 M.fr.IX, 37 .

B.fl.VII,357 M.fr.IX,37.

B.tl.VII,358 M.fr.IX, 36 .

B.ft.VII,358 M.fr.IX, 37.

B.fl.VII, 359

B.H.VII,359 M.fr.IX,37.

B.f.VII, 359 M.fr.IX, 33.

B.H.VII,360 M.fr.IX, 30.

B.fl.VII, 360 M.fr.IX, 30.

B.f.VII, 360 M.fr.IX, 30.

B.fl.VII, 361 M.fr.IX, 30

B.H.VII,36I M.fr.IX, 28.

B.fl.VII,361 MI.fr.IX, 28.

B.fl.VII, 362 M.fr.IX, 58

B.fl.VII,362 M.fr.IX,35.

B.fl.VII, 363 M.fr.IX, 80 .

B.fl.VII,363 M.fr.IX,32.

B. H.VII, 363 M.fr.IX, 33 .

-

B.fl.VII, 364 M.fr.XI, 106.

B.fl.VII,364 M.fr.IX,52.

B.fl.VII, 364

B.fl.VII, 364 M.fr.IX, 39.

- B.fl.VII, 365

B.fl.VII,366 M.fr.IX, 31 .

B.f.VII,366 M.fr.IX,31.

- B.fl.VII,366 M.fr.IX,29.

B.fl.VII, 366
B.fl.VII, 367

B.fl.VII,367 M.fr.IX, 28.

B.fl.VII, 367 M.fr.IX,30.

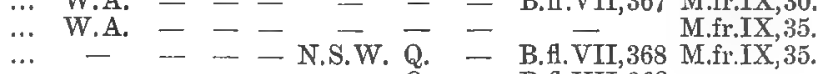

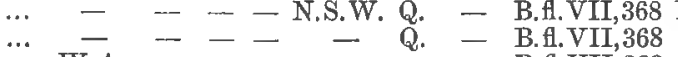

... W.A. - - - - - - B.fl.VII,368

… W.A. - - - - - - B.fl.VII,369 M.fr.IX,32.

... W.A. - - - - - - B.fl.VII,369 M.fr.IX,80.

W.A. - - - - - - B.f.VII,369

42) W.A. - - - - - - B.H.VII,370

W.A. S.A. - V. N.S.W. Q. - B.fl.VII,370 M.fr.IX,29,80,

. - - - V. N.S.W. Q. - B.f.VII,370 M.fr.IX, 29.

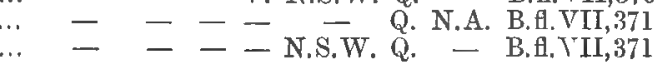


S. falcatus, R. Brown, prodr, 232 (1810)

S. punctatus, R. Brown, prodr. 232 (1810).

S. indutus, F. v. M. in Benth. Fl. Austr. VII, $3 \dddot{7} 2$ (1878)

S. bifidus, Boeckeler in Garcke, Linnaea XXXVIII, 282 (1874)

S. apogon, Roemer et Schultes, syst. veg. II, 77 (1817)

S. odontocarpus, F. v. M., fragm. IX, 32 (1875) ...

S. humilis, Bentham, Fl. Austr. VII, 374 (IS78) ...

S. sculptus, Boeckeler in Garcke, Linnaea XXXVÏII, $2 \ddot{86}$ (1874)

S. axillaris, Poiret, encycl. méth. suppl. II, 251 (1811) ..

S. tenellus, Bentham, Fl. Austr. VII, 375 (1878) ...

i. natans, F. v. M., fragm. IX, 36 (1875) ... ... ...

S. fluitans, J. Hooker, Fl. Tasman. II, S1, t. 141 (1860)...

S. octandrus, F. v. M., fragm. IX, 31 (1875)

S. capillaris, Chaetospora, F. v. M., fragm. IX, $3777(187 \ddot{7})$

S. stygius, Poiret, encycl. méth. suppl. II, 251 (1811) ‥
S. denstus, F. v. M.; Carpha deusta, R. Brown, prodr. 230 (18io)

S. tetragonus, Poiret, Encycl. inéth. suppl. II, 251 (1811)

S. sphaerocephalus, Poiret, Encycl. méth. suppl. II, ¿J1 (1811)...

S. anceps, Poiret, Encycl. méth. suppl. II, 251 (18I I)

S. paludosus, Poiret, Encycl. métl. suppl. II, 251 (1811)

S. pauciflorus; Lepidosperma, F. r. M., fragm. IX, 23 (1875) ...

S. Tricostularia, F. v. M.; Tricostularia compressa, Nees in pi.

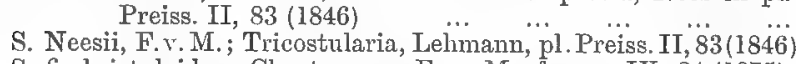

S. fimbristyloides: Chaetospora, F. v. M., fragm. IX, 34 (1875) LEPIDOSPORA, F. v. M., fragm. phytograph. Austral. IX, 34 (1875).

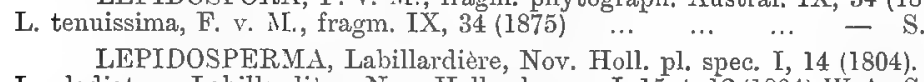

L. gladiatum, Labillardiere, Nov. Holl, pl. spec. I, 15, t. 12 (1804) W. A. S.A. T. V. N.S. TV. -

L. effusum, Bentham, Fl. Austr. VII, 387 (1878)...

I. rupestre, Bentham, Fl. Austr. VII, 388 (1878)...

I. elatius, Labillardière, Nov. Holl. pl. spec. I, 15, t. 11 (1804)

L. tetraquetrum, Nees in Lehm. pl. Preiss. II, 90 (1846)

L. Oldfieldii, J. Hooker, Fl. Tasman. II, 91, t. 146 (1860)

L. exaltatum, R. Brown, prodr. 234 (1810)...

W.A.

A. - - -

IV.A. S. A. T. $\overline{\text { V. }}-$

W.A. - - - - -

W.A. S.A. $-\overline{\text { T. }}$ N.S.W. $\bar{Q}$.

L. longitudinale, Labillardière, Nov. Holl. pl, spec. I, 16, t. 13(1804) W.A. S.A. T. V. N.S.W. -

L. concarum, R. Brown, prodr. 234 (1810)...

L. angustatum, R. Brown, prodr. 235 (1810)

L. Drummondii, Bentham, Fl. Austr. VII, 391 (1878)

L. Brunonianum, Nees in Lehm. pl. Preiss. II, 92 (1846)

L. tuberculatum, Nees in Lehm. pl. Preiss. II, 90 (1846)

L. resinosum, F. v. M. in Benth. Fl. Austr. VII, $392(1878)$
L. viscidum, R. Brown, prodr. 234 (1810) ...

L. costale, Nees in Lehm. pl. Preiss. II, 92 (1846)

L. laterale, R. Brown, prodr. 234 (1810)

L. congestum, R. Brown, prodr. 234 (1810)

L. globosum, Labillardiere, Nov. Holl. pl. spec. I, 16, t. I4 (1804)

L lineare, R. Brown, prodr. 235 (1810)

L. aphyllum, R. Brown, prodr. 235 (1810) ..

L. gracile, R. Brown, prodr. 235 (1810) L. semiteres, F. v. M. in Garcke, Linnaea XXXVIII, 327 (1974)

L. canescens, Boeckeler in Garcke, Linnaea XXXVIII, 330 (1874)

L. pubisquameum, Steudel, syn. pl. glum. II, 158 (1855)

L. scabrum, Nees in Lehm. pl. Preiss. II, 92 (1846)

L. tenue, Bentham, FI. Austr. VII, 397 (1878)

L. leptostachyum, Bentham, El. Austr. VII, 397 (1878)...

L. leptophyllum, Bentham, Fl. Austr. VII, 398 (1878) ...

L. tortuosum, F. v. M., general Report 9 (1855) ...

L. flexuosum, R. Brown, prodr. 235 (1810)

L. filiforme, Labillardière, Nov. Holl. pl. spec, I, 17, t. 15 (1804)

L. striatum, R. Brown, prodr. 235 (1810) ...

L. Neesii, Kunth, enum. II, 319 (1837)

L. carphoides, F, v. M. in Benth. Fl. Austr. VII, 400 (1878) ... CTAT
IV.A. S.A. T. V.

W.A. - - -

W.A.

IV.A.

- S.A. - V. N.S.W. -

W.A. - - - - S.A. T. $\overline{\text { V. N.S.W. W. }}$ S.A. - - - S.A. T. V. - S.A. T. V. N.S.W. -

IV.A.

W.A. S.A. - V. $-\overline{-}$

- S.A. - V. N.S.IV.

W.A.
IV.A.

W.A. - - -

W.A.

IV.A.

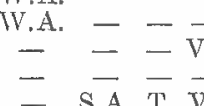

W.A.
- - - N. S.W. S.A. T. V. N.S.W. -
Q. N.A. B.fl.VIT,372 II.fr.IX, 29

- N.A. B.fl.VII,372

B.fl.VII, 373 M.fr.IX, 38,80 .

B.fl.VIT, 373 M.fr.IX, $56 ; X, 120$

B.fl.VII, 374 M.fr.IX, 32.

B. H.VII, 374

B.fl.VII, 374 MI.fr.IX, 30.

B.fl.VII,375 M.fr.IX, 34 .

B. A.VII, 375

B.fl.VII, 375 M.fr.IX, 38 .

B.fl.VII,376 M.fr.IX, 28.

B.fl.VII,377 M.fr.IX,31.

B.A.VII, 377 M.fr.IX, 34,80 .

B.A.VII,378 M.fr.IX, 36.

B.fi.VII,379 M.fr.IX, 39.

B fl. TII,380 M.fr.TX, 36.

B.fl.VII,380 M.fr.IX, 33 .

B.fi.VII, 380 M.fr.IX, 30 .

B.fl.VII,382 M.fr.IX, 35.

B.f.VII, 383 M.fr. IX, 23.

B.fl.VII,383 M.fr.IX, 37.

B.f. TII,383 M.fr, IX,37.

- N.A. B.fl.VII,384 M.fr.IX,34.

- B.fl.VII,365 M.fr IX,34.

B.fl.VII, 387

B.tl.VII,387

B.fl. VII, 388

B.fl.VII,388 MI.fr. IX, 2.5.

B.fl.VII, 388 II.fr.IX, 24 .

B. fl. VII, 389

B fl. VII, 389

B.fl. VII, 389 刃. fr. IX, 25.

B.fl.VII,390 M.fr.IX, 24 .

B.ff.VII,39I M.fr.IX, 26,80 .

B.A.VII,391

B.H.VII, 392

B. f.VII, 392 iI.fr. IX, 26.

B.fl.VII,392

B.f. YII, 393

B.fl. VII, 393

B. Al. VII, 393

B.A.YII, 394

B. fl. VII, 394

B.fl.VII, 395 M.fr.IX, 26.

B. fi. VII, 395

B.fi. YII, 395

B.f.YII.395

B.fl.VII, 396 M.fr.IX, 24

B. 1. VII, 396 II.fr.IX, 27.

B. A. VII, $: 397$ M.fr. IN, 27 .

B. f. VTI,397

B.fl.VII, 897

B.fl. VII, 398

B.fl.VII,39S MI.fr.IX,23.

B. fl.VIJ, 398

B. f. VII,39S MI.fr, IX, 27.

B.fl. III,399 MI.fr.IX, 26,27 .

B.fl. YIT,399 XI.fr. IX, 24,27 .

B.fl. YII, 400 Chapelliera, Morelotia.)

C. Mariscus, R. Brown, prodr. 236 (1810)

C. insulare, Bentham, FI. Austr. 403 (1878)

C.,articulatum, R. Brown, prodr. 237 (1810)

C. arthrophyllum, F. v. M., fragm. IX, 14 (1875)

C. glumeratum, R. Brown, prodr. 237 (1810)

C. Preissii, F. v. M. in Benth. FI. Austr. VII, 405 (1878)

C. laxum, Bentham, Fl. Austr. VII, 405 (1878) ... ...

C. riparium, Bentham, Fl. Austr. VII, 405 (1878)

C. teretifolium, R. Brown, proclr. 237 (1810)

C. teretifolium, R. Brown, prodr. 237 (1810)
C. tetraquetrum, J. Hooker, Fl. Tasman. II, 95, t. 149 (1860) ...
C. schoenoides, R. Brown, prodr. 237 (1810) $\ldots \ldots \ldots$

C. Gunnii, J. Hooker, Fl. Tasman. II, 95, t. 148 (1860)...

C. junceum, R. Brown, prodr. 237 (1810)

C. vaginale, Bentham, F'. Anstr. VIT, 409 (1878)...

C. elynanthoides, IT. v. M., fragm. LX, $31(1875) \ldots$
- S.A. - V. N.S.W. Q. N.A.

W.A. S.A. - V. N.S.W. Q. - - B.A.

B. fl. Y II, 402 MI.fr.IX, $1 \pm, 57$.

B.fl. YII,403

B. f. VII, 403 M.fr.TX, 14,57 .

IV.A. - - - - - - B.fl.VII,403 N.fr. IX.14.

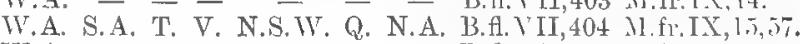

W.A. - - - - - - B.f. III,405 M.fr. IX, 13, 15,56.

W.A. - - - - - - B.fl.YII,405

W.A. - - - - - - B.fl.VII,403

- $-\overline{-}-$ N.S.W. Q. - B.f.VII, 406

W.A. S.A. T. V. N.S.W. $\overline{\text { N.S.W. Q. }}$ - B.H.VII,406
B.H.VII,407

S.A. T. V. N.S.IV. - - B.f.TII, 407 MI.fr.IX, 1.5,57.

W.A. S.A. T. V. N.S.W. Q. N.A. B.f.VII, 40 M.fr.IX, l(i,5).

IV.A. - - - - - - B fl.VII,40S

IV.A. - - - - - B.11,VII,409 Mr.fr.IX,31. 
C. Filum, R. Brown, prodr. 237 (1810)

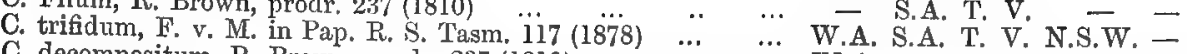

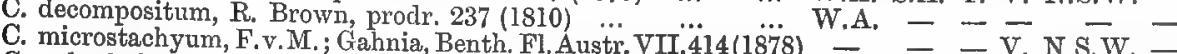

C. polyphyllum, F.v. M.; Gahnia, Benth. Fl. Austr. VII, 415(1878) W.A. - - - N.S.W. -

C. ancistrophyllum, F. v. M. in Benth. FI. Austr. VII, 415 (1878) W.A. - - -
C. lanigerum, R. Brown, prodr. 237 (1810)...

C. aristatum, F. v. M. in Benth. Fl. Austr. 416 (1878) $\ldots$... $\quad \ldots$ W. .. S.A. - - -

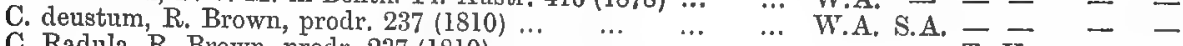

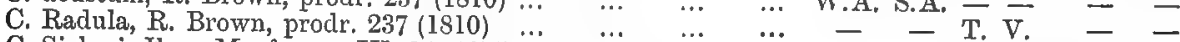

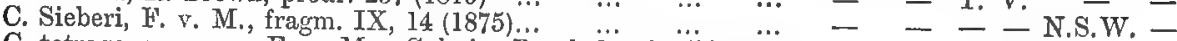

C. tetragonocarpum, F. v. M.; Gahnia, Boeckeler in Linnaea XXXVTII, 347 (1874)

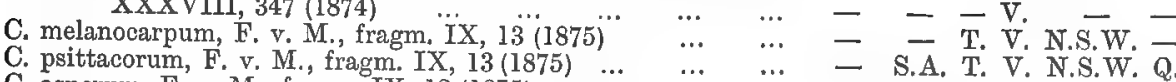

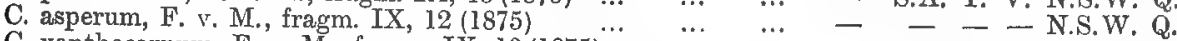

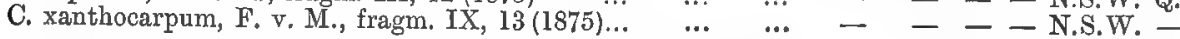
CAUSTIS, R. Brown, prodr. 239 (1810). (Eurostorrhiza.)

C. pentandra, $\mathrm{R}$. Brown, prodr. $240(1810) \ldots$

$\begin{array}{llll}\cdots & \cdots & - & - \\ & \cdots & \text { S.A. T. V. N.S.W. Q. }\end{array}$

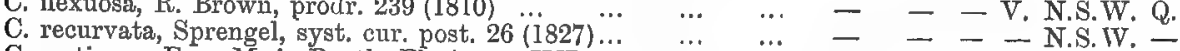

C. restiacea, F. v. M. in Benth. FI. Austr. VII, 421 (1878) $\quad \ldots \quad-\ldots-{ }_{-}-\overline{-}-\mathrm{N} . \mathrm{S}$. IV.

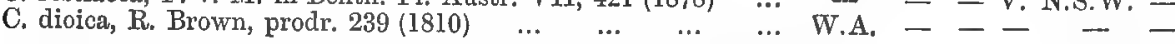
ARTHROSTYLIS, R. Brown, prodr. 229 (1810). (Arthrostyles,)

A. aphylla, R. Brown, prodr. 229 (1810) REEDIA, F. v. M., fragm. I, 240, t. 10 (1859).

R. spathacea, F. v. M., fragm. I, 240, t. 10 (1859) EUANDRA, R. Brown, prodr. 239 (1810). (Evandra.)

E. aristata, R. Brown, prodr. 239 (1810)

E. pauciflora, R. Brown, prodr. 239 (1810)

W.A.

$--$

— Q. N.A. B.fl.VII, 423 M.fr.IX, 9,55 .

S. caricina, Bentham, Fl. Austr. VII (1878)

S. pygmaea, R. Brow

S. rugosa, R. Brown, prodx. 240 (1810)

S. laxa, R. Brown, prodr. 240 (1810)

S. Brownii, Kunth, enum. II, 349 (1837)

S. lithosperma, Willdenow, spec. plant. IV, 316 “i (1805)...'

S. tessellata, Willdenow, spec. plant. IV, 315 (1805)

S. margaritifera, Willdenow, spec, plant. IV, 312 (1805)

S. Graeffeana, Boeckeler in Regensb. Flora, 121 (1875) ...

S. hebecarpa, Nees in Wight, Contrib. to Bot. of Ind. 1 i7 (1834)

S. Chinensis, Kunth, enum. II, 357 (1837)..

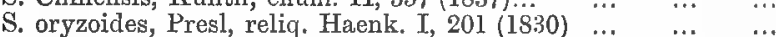

S. sphacelata, F, v. M., fragm. IX, 20 (1S75) ... ...

UNCINIA, Persoon, synops. plant. I, 534 (1807).

U. tenella, R. Brown, prodr. 24l (1810)

U. compacta, R. Brown, prodr. 241 (1810)...

U. riparia, R. Brown, prodr. 241 (1810) $\ldots$

U. debilior, F. v. M., fragm. VIII, 15L (1874)

CAREX, Ruppius, Fl. Jenens. 306 (1718).

C. cephalotes, F. v. M. in Transact. phil. Soc. Vict. I, 110 (1854)

C. acicularis, Boott in J. Hook. Fl. N. Zel. I, 280, t. 63 (1853)

C. capillacea, Boott, Illustr. of the gen. Carex I, 44, t. 110 (1858)

C. inversa, $R$. Brown, prodr. 242 (1810)

C. canescens, Linné, spec. plant. 974 (1753)

C. echinata, Murray, prodr, design. stirp. Goett. 76 (1770)

C. hypandra, F. v. M., fragm. VIII, 259 (1874) ...

C. chlorantha, $R$. Brown, prodr. 242 (1810)

C. paniculata, Linné, amoen. acad. IV, 294 (1759)

C. declinata, Boott, Ill. of the gen. Carex IV, 17, t. 580 (1867)

C. tereticaulis, F. v. M., fragm. VIII, 256 (1874)

C. fissilis, Boott, Ill, of the gen. Carex II, 86, t. 245 (1860)

C. gracilis, R. Brown, prodr. 242 (1810)

C. contracta, F. v. M., fragm. VIII, 258 (1874) ..

C. Gaudichaudiana, Kunth, enum. plant. II, 417 (I837)...

C. acuta, Linné, spec, plant. 978 (1753)

C. lobolepis, F. v. M., fragm. VIII, 258 (1874) $\ldots . \quad \ldots$

C. flava, Linné, spec. plant. 975 (1753)

C. Buxbaumii, Wahlenberg in Act. Holmiens, $139{ }^{\prime}(1803)$

C. pumila, Thunberg, Fl. Japon. 38 (1784)...

C. breviculmis, R. Brown, prodr. 242 (1810)

C. Preissii, Nees in Lehm. pl. Preiss. II, 94 (1846)

C. Gunniana, Boott in Transact. Linn. Soc. XX, 143 (1851)

C. Bichenoviana, Boott in J. Hook. Fl. Tasm. II, 101 (1860)

C. maculata, Boott in Transact. Linn. Soc. XX, 128 (1851)

C. Brownii, Tuckerman, enum. meth. Caric. 2 l (1849) ...

C. alsophila, F. v. M., fragm. VIII, 257 (1874) ...

C. longifolia, $\mathrm{R}$. Brown, prodr. 242 (1810)...

C. Pseudo-Cyperus, Linné, spec. plant. 978 (1753)
W.A.
W.A.

(1765). (Diplacrum, Hypoporum, Sphaeropus.)

- - - - - Q. - B.f.VII.426 M.fr.IX,58.

二 - - - - Q. N.A. B.f.VII, 427 M.fr.IX, 22,58.

- - - - Q. N.A. B.A.VII,428 M.fr.IX,22,58,

- - - - - Q. N.A. B.fl.VII, 428 M.fr.IX,21.

- - - - - Q. N.A. B.fl.VII,429 M.fr.IX,21,58.

- - - - Q. - B.fl.VII,430 M.fI.IX,2I,58

$\rightarrow$ Q. N.A. B.fl.VII,431

- - - N.S.W. Q. N.A. B.fl.VII,431 M.fr.IX,2I,58,

- - - - Q. - B.fl.VII,43I M.fr.IX,20,58

- $\quad-\quad-\quad$ - N.S.W. Q. $-\frac{\text { N.A. B.fl.VII, } 432}{\text { B.f.VII, } 432 \text { M.fr.IX, } 20 .}$

T. V. - - - B.fl.VII,433 M.fr.VIII,151

- T. V. V. N.S.W. -

- T. V. N.S.W. -

B.fl.VII, 434 M.fr.VIII, 152 B.fl.VII, 434 M.fr.VIII, 152 B.fl. VII, 435 M.fr. VIII, 15 l.

$-\quad-\quad-$ V. N.S.W. -

- - T. V. N.S.W. -

W.A. S.A. T. V. N.S.W. Q. - - V. N.S.W. -

- - - V. N.S.W. -

W.A. S.A. T. V. N.S.W. Q.

- S.A. T. V. N.S.W.

- - - - N.S.W. Q.

- - - V. N.S.W. Q.

- - - - NSW W

- $\quad$ - T. $\overline{\text { V. N.S.W. }}$ -

- S.A. T. V. N.S.W. Q.

- S.A. T. V. N.S.W. -

W.A. - - - - S.A. T. V. N.S.W. -

- - - - - N.S.W. Q

- - - V. N.S.W.

T. V. N.S.W. Q

B.fl.VII, 437 M.fr.VIII,251.

B.fl.VII, 437 M.fr. VIII, 251.

B.fl.VII, 438 M.fr.IX,191.

B.fl.VII, 438 M.fr.VIII, 252

B.fl.VII, 439 M.fr.VIII,253.

B.fl.VII, 439 M.fr. VIII,252.

B.fl.VII,439 M.fr.VIII, 259

B.fl.VII, 440 M.fr.VIII, 256 .

B.fl.VII, 440 M.fr.VIII, 256.

B.fl.VII,44l M.fr.VIII, 257.

B.fl.VII,441 M.fr.VIII, 256.

B.fl.VII, 441 M.fr.VIII,249;IX, 80.

B.fl. VII, 442 M.fr.VIII, 250.

B.fl.VII, 442 M.fr.VIII, 258.

B.fl.VII, 442 M.fr. VIII, 257.

B.t.VII, 443 M.fr. VIII, 259

B.fl.VII, 443 M.fr. VIII, 258

B.fl.VII, 444 M.fr.VIII, 250.

B.H. VII, 444 M.fr. VIII, 252.

B.fl.VII, 445 M.fr. VIII,251.

B.fl.VII, 445 M.fr.VIII, 255.

B.fl.VII, 446 II.fr.VIII, 251.

B.fl. VII, 446 M.fr. VIII,25l.

B.fl. VII, 446

B.fl.VII, 447 M.fr.VIII,258.

B.fl. VII, 447 M.fr.IX, 250.

B.t.VII, 447 M.fi. VIII, 257.

B.fl.VII, 448 M.fr.VIII, 250.

B.fl. VII, 448 II,fr. VIII, 249 .
- - - - - Q. N.A. B.f.VII,429 M.fr.IX,21,58.

- - - - - Q. - B.f.VII, 430

- S.A. T. V. N.S.W. N. -

W.A. S.A. T. V. N.S.W. Q.

$-\quad-\quad-$ N.S.W.

- S.A. T. V. N.S.W.Q

- - - V 


\section{GRAMINEAE.}

Haller, enum. stirp. Helv, I, 203 (1742).

ERIOCHLOA, Humboldt, Bonpland \& Kunth, nov, gen. et sp. pl. I, 94, t. 30 (1815). (Helopus.)

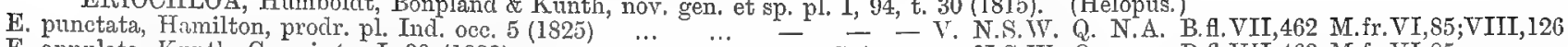

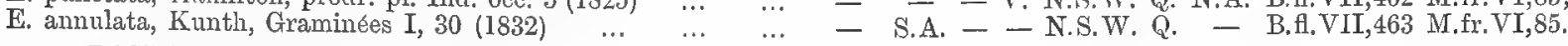

PASPALUM, Linne, syst. nat. ed. decim. 855 (1759).

P. scrobiculatum, Linne, mantiss. 29 (1767)

... - - - N.S.IW. Q. N.A. B.fl.VII, 360 M.fr.VIII, 156

P. brevifolium, Fluegge, gram. monogr. 150 (18l0) $\ldots \quad$.. W.A. $\quad$ - $\quad$ - - N.S. W. Q. - B.fl.VII,460 M.fr.VIII,156.

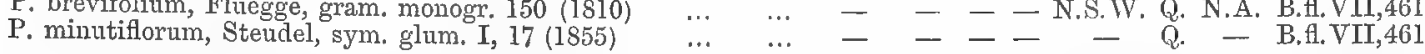

PANICUM, Tournefort, inst. 515, t. 298 (1700), from Plinius. (Isachne, Digitaria, Echinochloa, Coriclochloa, Chanıeraphis.)

P. coenicolum, F. v. M. in Transact. Vict. Inst. 45 (1854) … W.A. S.A. - V. N.S.W. - - B.fl.VII, 467

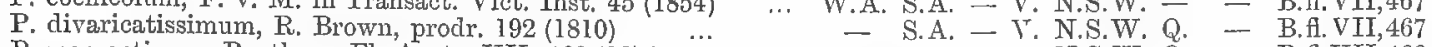

P. macractinum, Bentham, Fl. Austr. VII, 468 (1S78) $\ldots \ldots \ldots+\ldots-\ldots$ - - N.S.W. Q. - B.f.VII,468

P. papposum, R. Brown, prodr. $192(1810)$ …

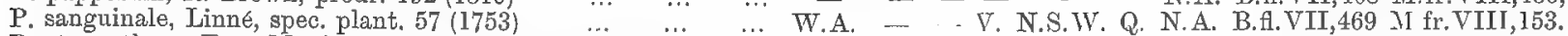

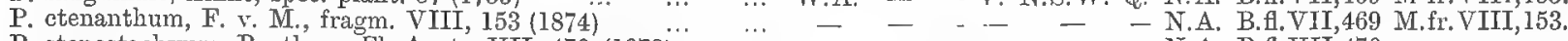

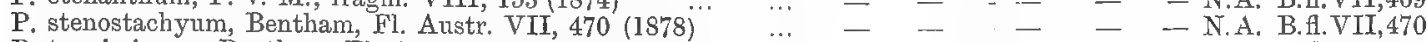

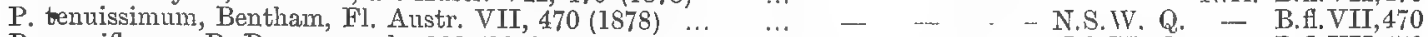

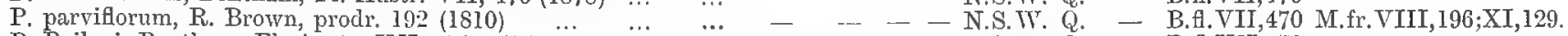

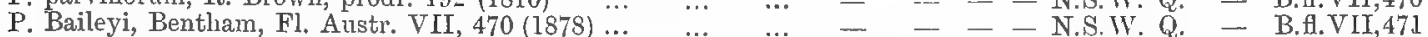

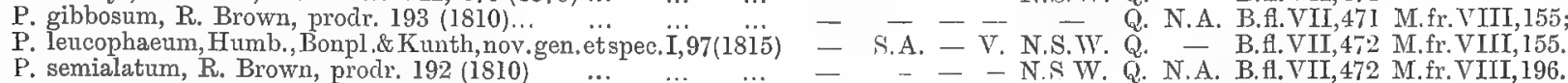

P. rarum, R. Brown, prodr. 189 (1810)

P. argenteum, R. Brown, prodr. $190(1810)$

P. holosericeum, R. Brown, prodr. 190 (1810)

P. flavidum, Retzius, observ. IV, 15 (1787)

P. gracile, R. Brown, prodr. 190 (1810)

P. prostratum, Lamarek, illustr. I, 171 (1791) $\ldots$.

P. helopus, Trinius in Spreng. Neue Entd. II, 84 (1821)

P. Gilesii, Bentham, Fl. Anstr. VII, 477 (1878)

P. piligerum, F. v. M. in Benth. Fl. Austr. VII, 477 (1878)

P. polyphyllum, R. Brown, prodr. 190 (1810)

P. distachyon, Linné, mantiss. alt. pl. 138 (1771)

P. reversum, F. v. M., fragm. VIII, 152 (1874) ...

P. colonum, Linné, syst. nat. ed. decim. $5(1758) \ldots$

P. Crus Galli, Linné, spec. plant. 56 (1753)

P. myosuroides, R. Brown, prodx. 189 (1810)

P. Indicum, Linne, mantiss. pl. alt. $184(1771) \quad \ldots$

P. Myurus, Lamarck, illustr. I, 172 (1791)

P. foliosum, R. Brown, prodr. 191 (1810) ... $\ldots$

P. adspersum, Trinius, spec. gram. t. $169(1830)$...

P. inaequale, F. v. M., fragm. VIII, 189 (1874) ...

P. uncinulatum, R. Brown, prodr. 191 (1810)

P. majusculum, F. v. M. in Benth. FI. Austr. VÏ, 482 (1878)...

P. pauciflortu, R. Brown, prodr. 483 (1810)

P. semitonsum, F. v. M. in Benth. Fl. Austr. VIÏ, 483 (1878) "

P. antidotale, Retzius, observ. bot. IV, $17(1878) \ldots$

P. repens, Liuné, spec. pl. ed. sec. 87 (1762)

P. Buncei, F. v. M. in Benth. Fl. Austr. VII, 487 (1878)

P. capillipes, Bentham, Fl. Austr. VII, 484 (1978)

P. pygmaeum, R. Brown, prodr, 191 (1810)

P. brevifolium, Linne, spec. plant. 59 (1753)

P. hermaphroditum, Steudel, syn. glum. I, 67 (1855)

P. marginatum, R. Brown, prodr. 190 (1810)

P. lachnophyllum, Bentham, Fl. Austr. VII, 486 (1878)

P. obseptum, Trinius, gram. panic. diss. alt. 149 (1826)...

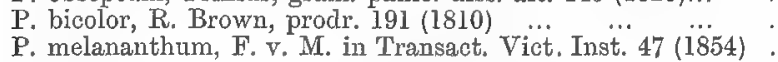

P. effusum, R. Brown, prodr. 191 (1810)

P. Mitchelli, Bentham, Fl Austr. VII 489 (187 $\because)^{\prime}$

P. decompositum, R. Brown, prodr. 191 (1810) …

P. trachyrhachis, Bentham, FI. Austr. VII, 490 (1878) ...
P. prolutum, F, v. M. in Transact. Vict. Inst. 46 (1854)

P. spinescens, $R$. Brown, prodr. 193 (1810)

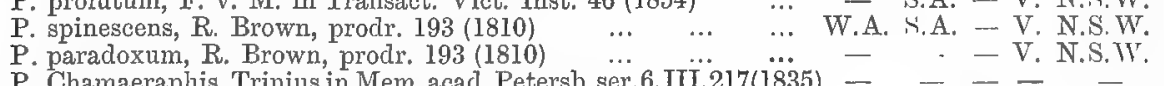

P. Chamaeraphis, Trimius in Mem, acad. Petersb. ser.6, III, 217(1835)

P. Myosotis, Steudel, syn. glum. I, 96 (1855)

OPLISMENUS, Palisot, Flore d'Oware et de Benin. II, 14 (1807). (Orthopogon.)

O. compositus, Palisot, agrostogr. 54 (1812) ... ... ... - - - V. N.S.W. Q.

N.A. B.A.VII, 473

- N.A. B.f.VII, 473

Q. N.A. B.fl.VII, 473 M.fr.VIII,155.

W.A. S.A. - V. N.S.W. Q. N.A. B.fl.VI, 474 M.fr. VIII, 159.

- - - - Q. N.A. B.fl.VII, 476 M.fr.VIII, 191 ,

- S.A. - N.S.W. Q. N.A. B.fl.VIT, 476

- S.A. - - Q. N.A. B.fl.VII,477 M.fr.XI,129.

- - - - - - N.A. B.fl.VII,477

- - - - - - N.A. B.fl.VII.477 M.fr.VIII, 194.

W.A. S.A. - N.S.TV. Q. - B.fl.VII,478 M.fr. IIII, 194.

- _ - - - L. N.A. B.fl.VII,478

T.A. S.A. -. V. N.S.IV. Q. N.A. B.fl.VII, 479 M.fr. VIII, 198.

- - - - N.W Q. N.A. B.fl.VII, 480

- - -

- - - - - Q. - B.fl.VII, $480 \mathrm{M} . \mathrm{X}, 114$.

- S.A. - N.S.W. Q. - B.fl.VII, 481 M.fr.VIII, 194.

- B.fl.VII,481

- - - - N.S.T. Q. - B.f.VII,482 M.fr.XI, 129, [129,

- - - - — - N.A. B.fl.VIT, 482

- S.A. - - - Q. N.A. B.fl.VII, 483 M.fr.VIII,193.

- . - - - N.A. B.f.VII,483

N.A. B.H.VII, 483

- V. N.S.IV. Q N.A. B.f.VII,484

- - - Q. - B.f.VII,487

- - - - N.A. B.fl.VII,484 M.fr. VIII, 193.

- N.S.W. Q. - B.A.VII,484 M.fr.VIII,193.

- - Y N.A. B.fl.VII, 485 M.fr. VIII, 193;XI, 129.

- - - Q. - B.fl.VII,485

- $-\hat{Q}$ - B.f.VII.486

- N.S.W. - B. VII,486

- - - - N.S.W.Q. - B.fl.VII,487

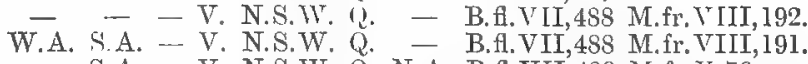

-. S.A. -- V. N.S.W. Q. N.A. B.fl.VII, 499 M.fr. X, 76.

... W.A. S.A. - V. N.S.W. (). N.A. B.fl.VII, 489 I.fr.VI, 86;VIII, 191.

... - - - - N.S.W. Q. N.A. B.fl.VII,490 M.fr.VIII, 192;XI, 129.

- S.A. - V. N.S.W.Q. - B.f.VII, 490 M.fr.VIII, 192.

A.A. - V. N.S.W. Q. N.A. B.fl.VII, 499 M.fr.VIII, 197;XI, 129.
- V. N.S.WT. - - B.f.VII,499 M.fr.VIII, 197.

- - V. N.S.W. Q. N.A. B.H.VII,500 M.f.VIII, 193;XI, 129.

B.fl.VII, 625 M.fr. VIII, 193 .

SETARIA, Palisot, Essai d'une nouv. Agrostogr. 51, t. 13 (1812).

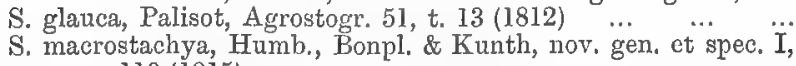
110 (1815)

S. viridis, Palisot, Agrostogr.

S.A. - V. N.S.WV. Q. N.A. B.f.VII, 492 M.fr.VIII, 110.

S. verticillata, Palisot, Agrostogr. 51 (1812)

S.A. - - N.S.W. Q. N.A. B.f.VII, 493 M.fr. VIII, 110;XI, 129.

W.A. S.A. - - - - N.A. B.f.VII, 494

IV.A. - - - - N.A. B.G.VII,494 M.fr.VIII, 110. 
PENNISETUM, L. C. Richard in Persoon, synops. plant. I. 72 (1805). (Gymnothrix, Plagiosetum.)

P. compressum, R. Brown, proclr. 195 (1810) … … … - - - N.S.W. Q. - B.fl.VII,495 M.fr.VIII, 109

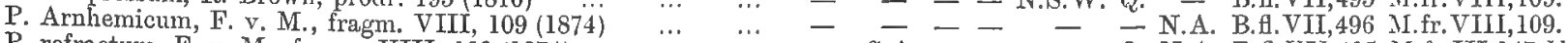

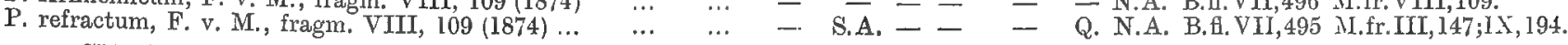

CENCHRUS, Linné, coroll. gen. 20 (i737).

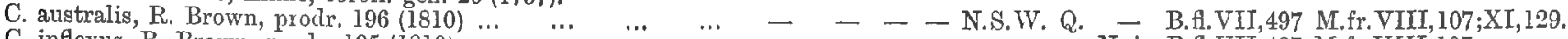

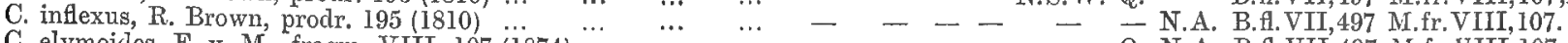

C. elymoides, F. v. M., fragm. VIII, 107 (ï874) ...

XEROCHLOA, R. Brown, prodr. fl, Nov. Holl, 196 (1810).

X. imberbis, R. Brown, prodr. $197(1810) \ldots$
X. barbata, R. Brown, prodr. $197(1810) \ldots$
$X$

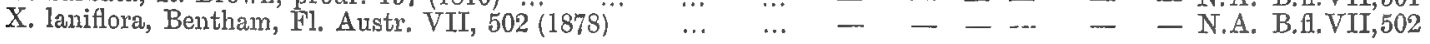

THUAREA, Persoon, synops. plant. I, 110 (1805). (Thouarsea.)

T. sarmentosa, Persoon, synops. I, $110(1805) \quad \ldots \quad \ldots \quad \ldots \quad-\quad-\quad-\quad-\quad$ Q. N.A. B.f1.VII,502 MI.fr.VI,86.

SPINIFEX, Linne, syst. nat. ed. XII, 757 (176 7 ).

S. hirsutus, Labillard., Nov. Holl. pl. spec. II, 81, t. 230 (1806) W.A. S.A. T. V. N.S.W. Q. - B.fl.VII,503 MI.fr.VIII,138.

S. longifolius, R. Brown, prodr. 198 (1810) $\quad \ldots \quad \ldots . \quad \ldots$. W.A. - - - - Q. N.A. B.fl.VII,504 M.fr,VIII,139.

S. paradoxus, Bentham in Hook. icon. plant. t. 1243 (1877) LEPTASPIS, R. Brown, prodr. fl. Nov. Holl. $2 \mathrm{H}$ (1810).

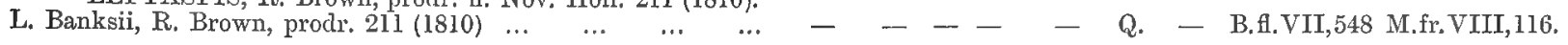
PEROTIS, Aiton, hort. Kewens. I, 85 (1789).

P. rara, R. Brown, prodr. $172(1810) \quad \ldots \quad \ldots \ldots$... $\quad \ldots \quad-\quad$ S.A. - - N.S.W. Q. N.A. B.fi.VII.509 M.fr.VIII,115. POLYPOGON, Desfontaines, flor. Atlantic. I, 66 (1798).

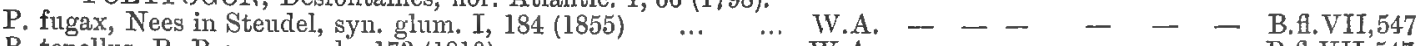

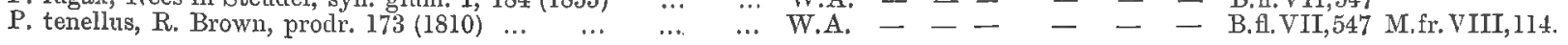
ARUNDINELLA, Raddi, agrostograph. Brasil. 37 (1823).

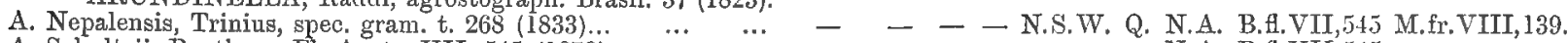

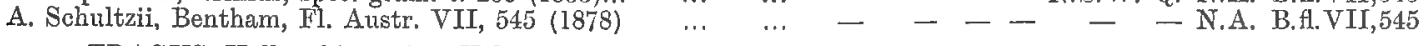
TRAGUS, Haller, hist. stirp. Helvet. u. 1413 (1768). (Lappago.)

T. racemosus, Haller, stirp. Helv. n. 1413 (I768)... … … - S.A. - V. N.S.W. Q. N.A. B.fl.VII,507 M.fr.VIII, 107. NEURACHNE, R. Brown, prodr. fl. Nov. Holl. 196 (1810).

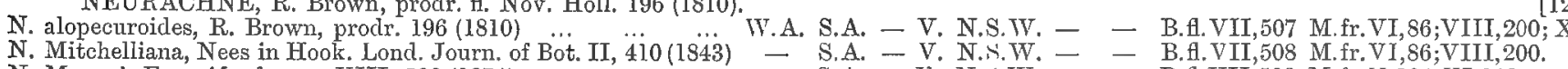

N. Munroi, F. v. M., fragm. VIII, $200(1874) \ldots \ldots \ldots \ldots$... $\quad$ - S.A. - V. N.S.W. - - B.f.VII,508 M.fi.V,204;.XI,129. ZOYSIA, Willdenow, Neue Schrift. nat. Freuncie zu Berl. III, 440 (1801).

Z. pungens, Willdenow, Neue Schrift. nat. Fr. Berl. III, 440(1S01) - - T. V. N.S. W. Q. - B.fl.VII,506 MI.fr.VIII, I16. IMPERATA, Cyrillo, plant. ral. Neap. II, t. 11 (1792).

I. arundinacea, Cyrillo, plant, rar, Neap. II, t. 11 (1792) … IV.A. S.A. T. V. N.S.W. Q. N.A. B.fl.VII,536 MI.fr.VIII,126. ERIANTHUS, L. C. Richard in Michanx, fl. bor. Amer. I, 54 (1803). (Pollinia, Eulalia, Pogonatherum, Leptatherum, Sacchainm partly.)

E. articulatus, F. v. M., fragm. VIII, $118(1874) \quad \ldots \quad \ldots \quad-\quad-\quad-\quad-$ Q. N.A. B.f.VII,52J II.fr.X,76.

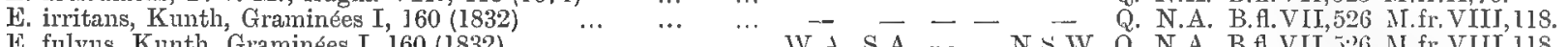

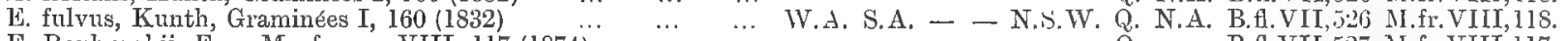

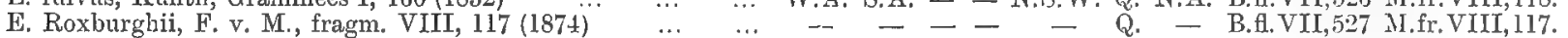

E. Mackinlayi; Pollinia, F. v. M. in Benth. fl. Austr. VII, 527 (1878) - - - - - - N.A. B.H.VII,527 M.fr.VIII,118. DIMERTA, R. Brown, prodr. fl. Nov. Holl. 204 (1810).

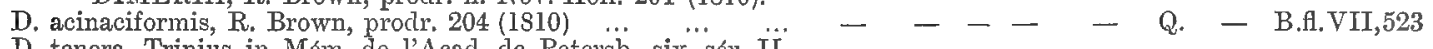

D. tenera, Trinius in Mém. de l'Acad. de Peterïb. six. "sér. Iİ, $\begin{array}{llllllllll}225 & (1833) & \ldots & \cdots & \ldots & \ldots & \ldots & \ldots & \cdots & -\end{array}-$

A. ciliare, Palisot, Agrostogr. 111, t, $11(1812) \ldots \ldots$... ... - - - - N.S.W. Q. - B.f.VII,524 M.fr.VIII,119;.X,76. ELIONURUS, Willdenow, spec. plant. IV, 941 (1805).

E. citreus, Munro in Benth. Fl. Austral. VII, 510 (1878) ‥ - - - - - Q. - B.fl.VII,510 II.fr.XI,129. LEPTURUS, R. Brown, prodr, fl. Nov. Holl. 207 (1810).

L. incurvatus, Trinius, fundam. agrostogr. $123(1820) \ldots \ldots \ldots-$ S.A. - V. N.S.W. - - - B.fl.VII,668 $1 I . f r$. TIII,117.

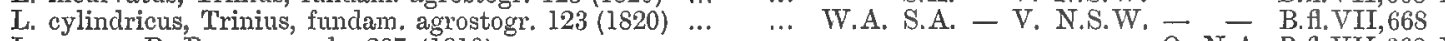

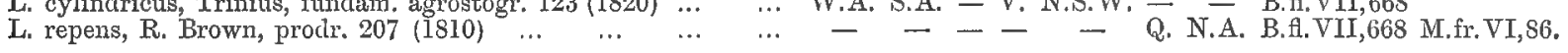
ROTTBOELLIA, Linné, fil. gram. gen. 22 (1779). (Coelorrhachis.)

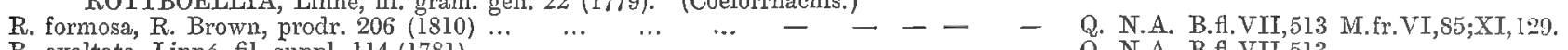

R. exaltata, Linné, fil. suppl. 114 (1781) $\ldots$ …

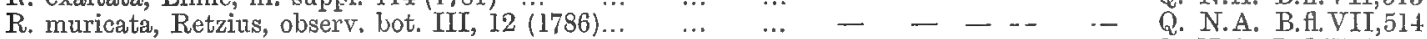

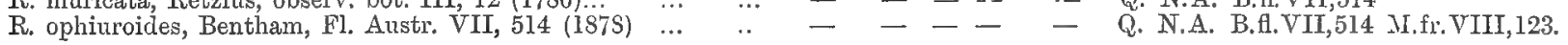
OPHIUROS, K. F. Gaertner, de fruct. III, 3, t. 181 (1805). (Ophiurus.)

o. corymbostis, K. F. Gaertner, de fruct. III, 3, t. 181 (1805).. - - - - — Q. N.A. B.A.VII,512 M.fr.VI,85. MANISURIS, Linné, syst. nat. ed. duodec, $762(1767)$.

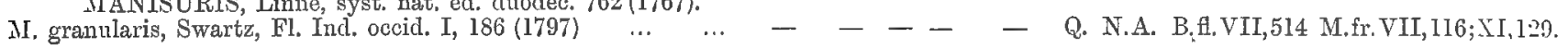
HEMARTHRIA, R. Brown, prodr. fl. Nor. Holl. 207 (1810).

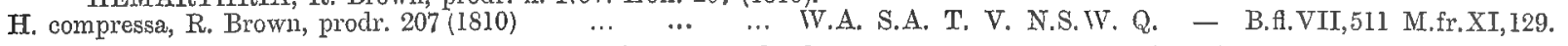
ISCHAEMUM, Linné, syst. nat. 8 (1735); Linné, gen. pl. ed. sec. 525 (1742). (Hologamium, Sportiopogon partly.)

I. truncatiglume, F. v. M. in Benth. FI. Austr. VII, 518 (1878) - - - - - - N.A. B.fl.VII,518

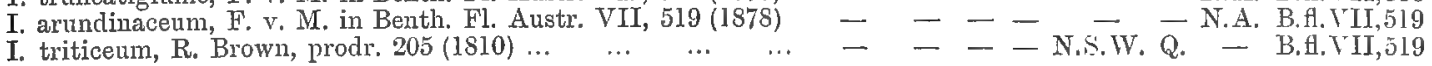


I. australe, R. Brown, prodr, 205 (1810) $\quad \ldots \quad \ldots \quad \ldots \quad \ldots \quad \ldots \quad-\quad-\quad-\quad-\quad$ N.S.W. Q. N.A. B.fl.VII,519

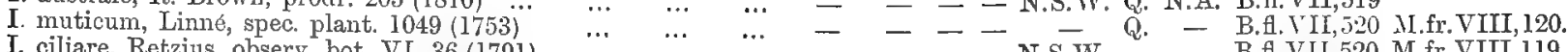

I. ciliare, Retzitus, observ, bot. VI, $36(1791)$
I. decumbens, Bentham, Fl. Austr. VII, 521 (1878)

I. pectinatum, Trinins in Mem. de l'Acad. de Petersb. six. sèr. II, 296 (I833)...

I. fragile, R. Brown, prodr. 205 (1810)

I. laxum, R. Brown, prodr. 205 (1810)

- $\quad-\quad-$ N.S.W. Q. - B.f.VII,521 M.fr.VIII,118. ANDROPOGON, Royen, prodr. exhib. pl. hort. acad. Lugd. 52 (1740). (Sorghum, Chrysopogon, Heteropogon, Schizachyrium, Trachypogon, Holcus partly, Spodiopogon partly.)

A. erianthoides, F. v. M., fragm. X, 75 (1876) … …

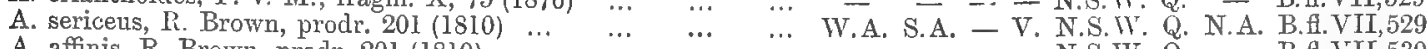

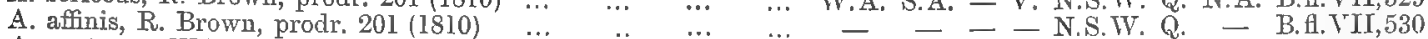

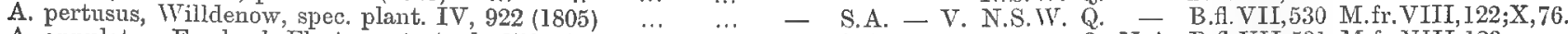

A. annulatus, Forskael, Fl. Aegypt. Arab. 173 (1775) $\ldots . . \cdots-$ S.A. - - - Q. N.A. B.f.VII,531 M.fr.VIII, 123.

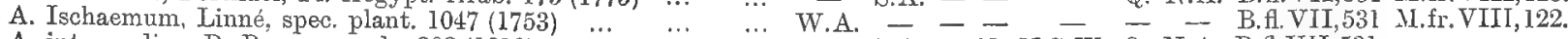

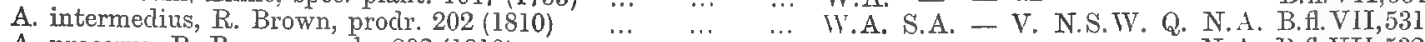

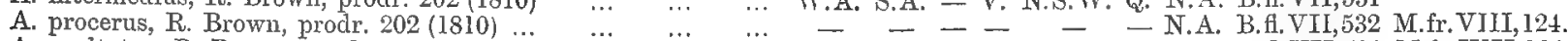

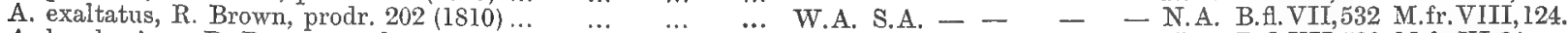

A. bombycinus, R. Brown, prodr. $202(1810) \quad \ldots . \quad \ldots . \quad \ldots$. W.A. S.A. - V. N.S.W. Q. N.A. B.f. VII,533 M.fr.XI, 20.

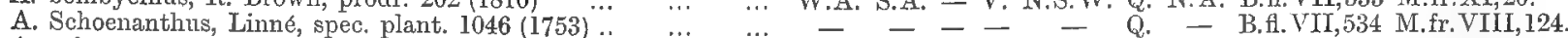

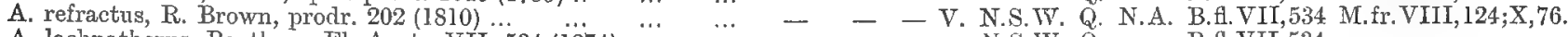

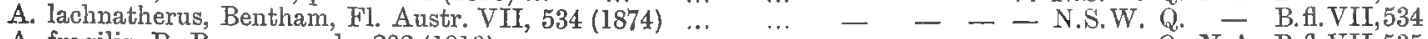

A. fragilis, R. Brown, prodr. 202 (1810) …

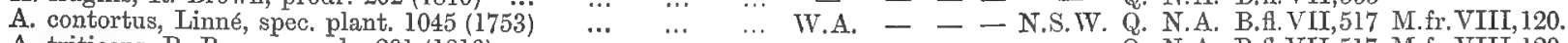

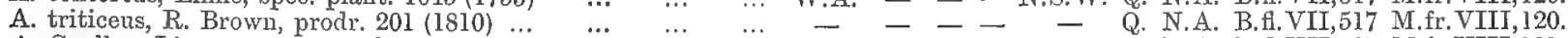

A. Gryllus, Linné, amoen. acad. IV, 332 (1̈759) $\ldots . . . . . \quad \ldots$.

A. montanus, Roxburgh, Flor. Indic. edit. Carey. I, $271(1820)-{ }_{-}-\mathrm{V}$. N.S.W. Q. N.A. B.A.VII,538 M.fr.VIII, 122;X,76;XI,

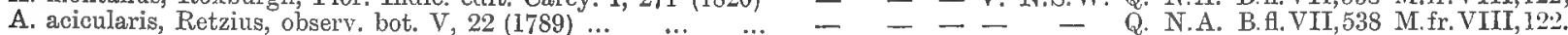

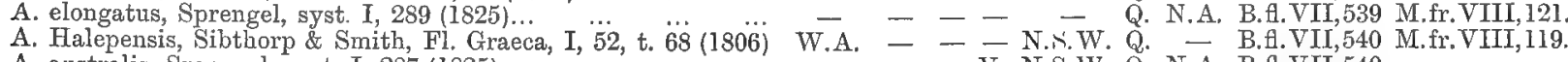

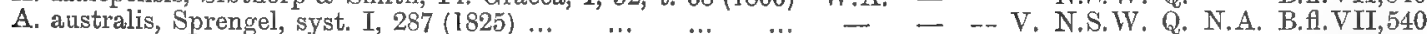

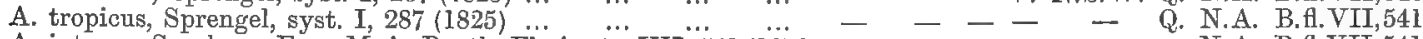

A. intrans; Sorghum, F. v. M. in Benth. Fl. Austr. VII, 541 (1878) - _ - - _ - N.A. B.fl.VII,541 ANTHISTRRIA, Linné, fil. gram. gen, 35 (1779). (Iseilema.)

A. ciliata, Linné fil., gram. gen. $35(1779) \ldots \ldots \ldots$... $\quad \ldots$ W. A. S. A. T. V. N.S.IV. Q. N. A. B.f.VIJ.,542 M.fr.V,207,VIII, 139.

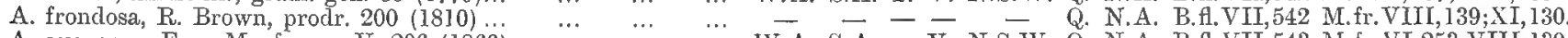

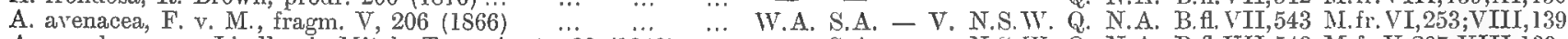

A. membranacea, Lindley in Mitch. Trop. Austr. 88 (1848) $\ldots$ - S.A. - - N.S.W. Q. N.A. B. H.VII,543 M.fr.V,207; VIII, 139. APLUDA, Linné, spec. plant. 82 (1753).

A. mutica, Linné, spec. plant. $82(1753) \quad \ldots \quad$... $\quad$.. $\quad$.. $\quad-\quad-\quad-\quad$ N.S.W. Q. - B.fl,VII, 544 M.fr.VIII, 140. CHIONACHNE, R. Brown in Horsfield's pl, Jav. rar. 15 (1838).

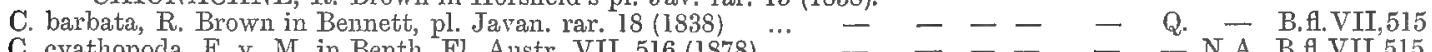

C. cyathopoda, F. v. M. in Benth. Fl. Austr. VII, $516(1878) \ldots=-\ldots=-$ N.A. B.fl.VII,515 MI.fi., VIII, 116. ALOPECURUS, Linné, syst. nat. 8 (1735); Linné, gen. plant. 18 (1737). (Perhaps immigrated.)

A. geniculatus, Linné, spec, plant. 60 (1753) … … … W.A. S.A. T. V. N.S.T. Q. - B.f. VII, 555 M.fr.VIII, 138. LEERSIA, Solander in Swartz, nov. gen. et spec, plant. 1 et 21 (1788). (Asprella, Asperella.)

L. hexandra, Swartz, nov, gen, et spec. pl. 21 (1788) ... ... - - _ N.S.W. Q. - B.t.VII,549 M.fr.VIII,115. ORYZA, Tournefort, inst. 513, t. $296(1700)$, from Theophrastos \& Dioscorides.

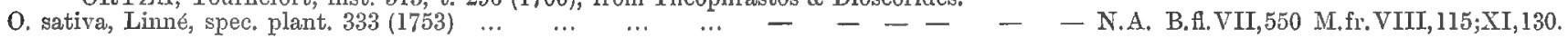
POTAMOPHILA, R. Brown, prodr, fl. Nov. Holl. 211 (1810).

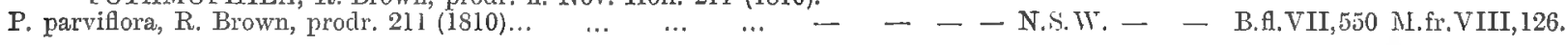
EHRHARTA, Thumberg in Svensk. Ventsk. Acad. Handl. 216, t. 8 (1779). (Tetrarrhena, Michrolaena, Diplax.)

E. distichophylla, Labill., Nov. Holl. pl, spec, 1, 90, t. $117(1804)-$ - T. V. - - - B.f.TII,554 M.fr.VII,90.

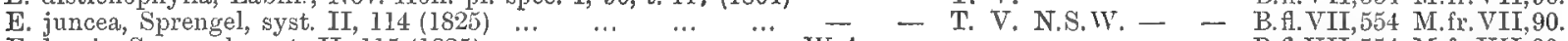

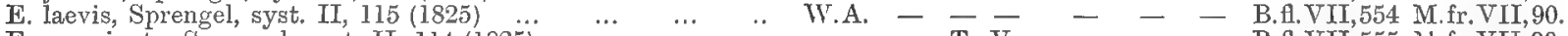

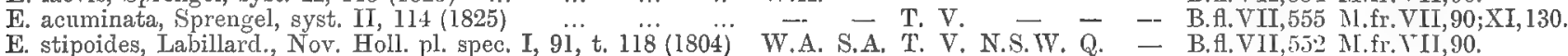

E. diarrhena, F. v. M., fragm. VII, 89 (1870) $\ldots$.. $\ldots \quad-\quad$ - T. - - - - B.A.VII,553 MI.fr.VII, 89. HIEROCHLOE, J. G. Gmelin, FI. Sibiric. I, $100(17+7)$. (Hierochloa, Disarrhenum.)

H. redolens, R. Brown, prodr. 209 (l810) ... ... .. . . - - T. V. N.S.W. - - B.fl.VII,55S M.fr. VIII, 137.

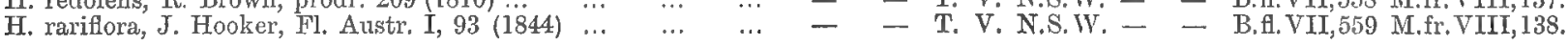

ARISTIDA, Linné, spec. plant. 82 (1753). (Chaetaria, Arthratherum.)

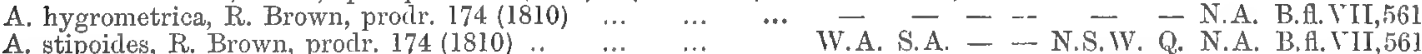

A. stipoides, R. Brown, prodr. $174(1810)$. 121026$).$.

A. arenaria, Gaudichaud in Freyc. voy. Bot. 407 (1826)...

A. leptopoda, Bentham, Fl. Anstr. VII, 562 (1878)

-... S.A. - V. N.S.W.Q.

A. vagans, Cavanilles, icont. et desor. pl. V, 45, t. 471 (1799) ... - - - - N.S.W. Q. - B.fl.VII,562 M.fr.VIII,111.

A. ramosa, R. Brown, prodr. $173(1810) \quad \ldots \quad \ldots \quad \ldots \quad \ldots \quad-\quad$ S.A. - - N.S.W. Q. - B.H.VII, 563

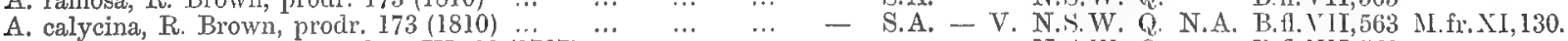

A. depressa, Retzins, observ, bot. IV, $22(1787) \ldots \quad \ldots \quad \ldots \quad-\ldots \quad-\quad-\quad$ N.S.W. Q. - 13.A.VII,563 STIPA, Linné, spec. plant. 78 (1737). (Streptachne, Uracline partly.)

S. elegantissima, Labillardiere, Nov. Holl. pl. spec. I, 23, t. 29(1804) W.A. S.A. - V. N.S.W. Q. N.A. B.fl.VII, 565 M.fr. VIII, 103.

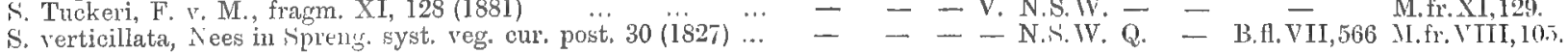


S. flavescens, Labillardière, Nov, Holl. pl. spec. I, 24, t. 30 (1804) W.A. S.A. T. V. N.S.W. S. teretifolia, Steudel, syn. glum. I, $128(1855) \quad \ldots . \quad \ldots . \quad \ldots$ W.A. - T. V. - -

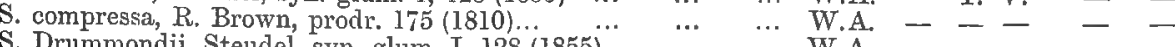

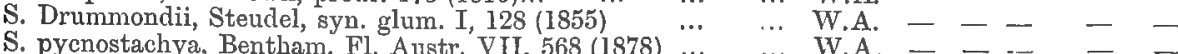

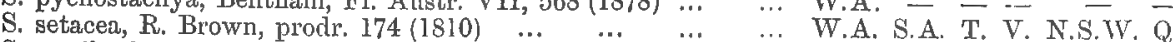

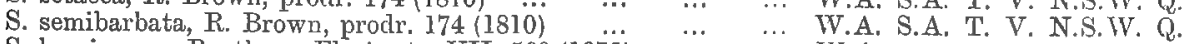

S. hemipogon, Bentham, Fl. Austr. VII, 569 (1878)

S. pubescens, R. Brown, prodr. $174(1810)$...

S. aristiglumis, F. v. M. in Transact. Vict. Inst. 43 (1854)

S. Eriopus, Bentham, Fl, Austr. VII, 570 (1878)..

S. trichophylla, Bentham, Fl, Austr. VII, 570 (1878) ‥

S. Streptachne, F. v. M. in journ. R.S. of N.S.W. 237 (1881) ... DICHELACHNE, Endlicher, prodr. fl. Norfolk. 20 (1833).

D. crinita, Hooker, Fl. N Zel, I, 293 (1853) (Muehlenbergia partly.)

D. sciurea, J. Hooker, Fl, N. Zel, I, 294 (1853) $\ldots . \quad \ldots . \quad \ldots$-.. S.A. T. V. N.S.W. Q. PENTAPOGON, R. Brown, prodr. fl. Nov, Holl. 173 (1810).

P. Billardieri, R. Brown, prodr. $173(1810) \ldots \quad \ldots \quad \ldots . \ldots-\ldots-$ S.A. T. V. N.S.W. ECHINOPOGON, Palisot, Essai d'une nouv. Agrostogr. 42, t. 9, f. 5 (1812). (Cinna partly.)

E. ovatus, Palisot, Agrostogr. 42, t. 9 (1812) $\quad \ldots \quad \ldots . \quad \ldots \quad$ W.A. S.A. T. V. N.S.W. Q. DIPLOPOGON, R. Brown, prodr. fl. Now, Holl. 176 (18io). (Dipogonia.)

D. setaceus, R. Brown, prodr. 176 (1810) ... … ... ... W.A. - AMPHIPOGON, R. Brown, prodr. 175 (1810), (Aegopogon partly, Pentacraspedion, Gamelythrum.)

A. debilis, R. Brown, prodr. $175(1810)$
A. strictus, R. Brown, prodr. $175(1810)$

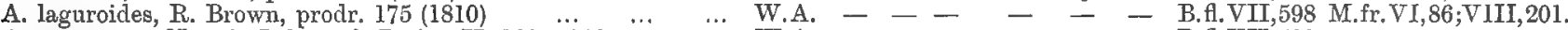

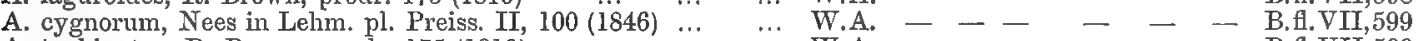

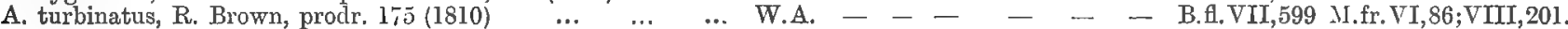
PAPPOPHORUM, Schreber, gen. pI. II, 787 (1791). (Euneapogon).

P. commune, F. v. M., plants of Gregory's Exp. 10 (1859) ‥ W.A. S.A. - V. N.S.W. Q. N.A. B.fl.VII,600 M.fr.VIII,200. SPOROBOLUS, R. Brown, prodr. fl. Nov. Holl. 169 (1810). (Vilfa partly.)

S. Virginicus, Humboldt \& Kunth, gram. I, 67 (1832) $\ldots \quad \ldots \quad$ W.A. S.A. - V. N.S.W. Q. N.A. B.fl,VII,621 M.fr.VI,84.

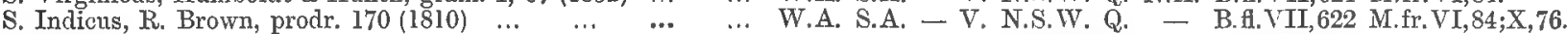

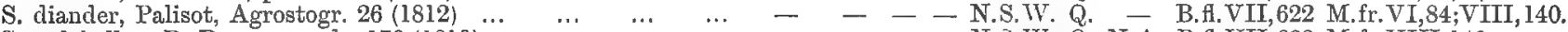

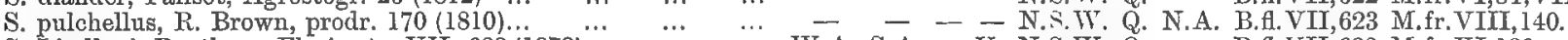

S. Lindleyi, Bentham, Fl. Austr. VII, $623(1878) \ldots \quad \ldots . \quad \ldots$, W.A. S.A. - V. N.S.W. Q. - B.fl. VII,623 M.fr.XI,130.

S. actinocladus, F. r. M., fragm. VIII, $140(1874) \quad \ldots \quad \ldots \quad-\quad$ S.A. - - N.S.T.. Q. N.A. B.fl.VII,623 M.fr.VI,84;IX, 194;XI, AGROSTIS, Linné, syst. nat. 8 (1735); Linné, gen. plant. 19 (1737). (Deyeuxia, Didymochaeta, Trichodium, Bromidium, Lachnagrostis partly).

A. Muelleri, Bentham, Fl. Austr. VII, .576 (1878)

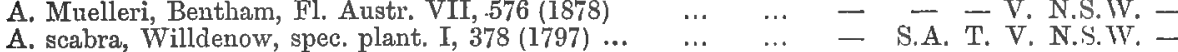

A. venusta, Trinius in Mém. de l'Acad. de Petersb. VI, 340 (1850) W.A. S.A. T. V. N.S.W. -

A. aequata, Nees in Hook. Lond. Jourm. of Bot. II, 412 (1843)..

A. Solandri, F. v. M., Veget. of the Chath. IsI. 60 (1864)

A. montana, $R$. Brown, prodr. 171 (1810) ...

A. quadriseta, R. Brown, prodr. 171 (1810)

A. cylindrica, R. Brown, prodr. 171 (1810)...

A. densa, F. v. M.; Deyeuxia, Benth. FI. Austr. VII, 582 (1878)

A. densa, $\dot{F} . \mathrm{M}$ in Benth, Fl. Austr. VII 583 (1878)

A.

A. nivalis, F, v. M. in Transact. Vict. Inst. 43 (1854)

A. Gunniana, F. v. M.; Deyeuxia, Benth. Fl. Austr. VII, 584 (1̈878)

A. breviglumis, F. v. M.; Deyeuxia, Benth. Fl. Austr. VII, 584(1878) COELACHNE, R. Brown, prodr. fl. Nov. Holl. 187 (1810).

C. pulchella, R. Brown, prodr. 187 (1810) ... MICRAIRA, F. v. M., fragm. V, 208 (1866).

M. subulifolia, F, Vo M, fragm V, $208(1866)$ AIRA, Linné, gen. plant. 335 (1737). (Aera, Deschampsia.)

A. caespitosa, Linné, spec, plant. 64 (1753) … ... TRISETUM, Persoon, synops. plant. I, 97 (1805).

T. subspicatum, Palisot, Agrostogr. 88 (1812) $\ldots . . . . \quad \ldots$ ERIACHNE, R. Brown, prodr. fl. No
acea, F. v. M., fragm. V, 206 (1866)

E. stipacea, F. v. M., fragm. V, 206 (1866)

F. squarrosa, R. Brown, prodr. 183 (1810).

E. glanca, R. Brown, prodr. 1St (1810)

E. rara, R. Brown, prodr. 183 (1810)

E. arrostidea, F. v. M., fragm. VII, $82(1870)$

E. ciliata, R. Brown, prodr. 184 (1810)

E. setacea, Bentham, Fl. Austr. VII, 629 (1878) ..'

E. avenacea, R. Brown, prodr. 184 (1810) .

E. aristidea, F. v, M., fragm. V, 205 (1866)

E. pallescens, R. Brown, prodr. 184 (1S10)

E. festucacea, F. v. M., fragm. V, 205 (1866)
- $\overline{\mathrm{A}}$ T. $\overline{\mathrm{V}}-\overline{\mathrm{T}}-\mathrm{B}$ - $\mathrm{B}$.VII,579

- S.A. T. V. N.S.W. -

A. S.A. T. V. N.S.V. Q. - B.fl.VII,581

- S.A. - V. - - - - B.fl.VII,582

- T. V. N.S.W. - - B.fl.VII,583

- T. V. N.S.W. Q. - B.fl.VII,583 M.fr.VI,85.

- - V. N.S.W. - - B.fl.VII,584

- T. - - - - B.f.VII,584

- - N.S.W. - - B.fl.VII,584

Q. - B.fl.VII, 626

- - - Q. - - M.fr.V,208.

S.A. T. V. N.S.IV. - - B.A.VI, 587 M.fr.VIII, 138.

- - T. Y. N.S.W. - - B.f.VII,588 M.fr.VII,136.

.. - - - - — Q. N.A. B.fl.VII,626 M.fr.VIII, 137;X,76.

.. - - - - - Q Q. N.A. B.fl. YII,627

- - - - - - Q. N.A. B.fl.TII,62S

- - N.A. B.fl.VII,628

- Q. - B.fl.VII,62S

- $\quad$ - N.A. B.fl.VII,628 M.fr, VIII,137.

- Q. N.A. B.f.VII,629

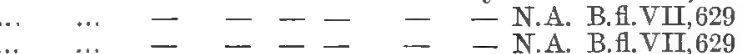

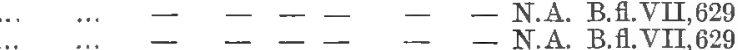

$\cdots \quad \cdots \quad$ W.A. S.A. - - N.S.W. Q. $\quad$ - B.H.VII,629 NI.fr.V,205.

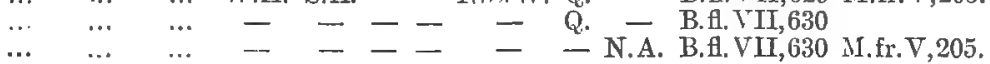

W.A. - - - - - - B.f.VII,582 

E. ovata, Nees in Hook. Lond. Journ. IT, 418 (1843)
E. meliacea, F. v. M., fragm. V, 205 (1566)
E. pallida, F. r. M. in Benth. Fl. Austr. VII, $63 \dddot{1}(1878)$
E. scleranthoides, F. v. M., fragm. VIII, 233 (1874)
E. mucronata, R. Brown, prodr. 184 (1810)
E. obtusa, R. Brown, prodr. 184 (1810)
E. capillaris, R. Brown, prodr. 184 (1810)...

$$
\text { - - - - N.S.T. Q. Q. N.A. B.fl.VII,632 M.fr.VIII, 137;XI, } 130 .
$$$$
\text { ... W.A. S.A. - - - - - N.A. B.f.VII, 630 M.fr. VIII, I37. }
$$$$
\text { ... - - - - - - N.A. B.fl.VII,631 M.fr.V,205. }
$$$$
\text { .. - }-\cdots-\ldots-\text { N.A. B.fl.VII,631 }
$$$$
\text { .. - S.A. - - - - - B.fl.VII,63I }
$$

ANISOPOGON, R. Brown, prodr. fl. Nov. Holl. 176 (1810).

A. avenaceus, R. Brown, prodr. 176 (1810)

- - N.S.W. Q. - B.f.VII,590 M.fr.VIII, 106.

DANTHONIA, De Candolle, Fl. franç. III, 32 (1805). (Amphibromus, Monacather, Plinthacanthus.)

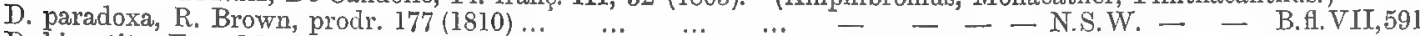

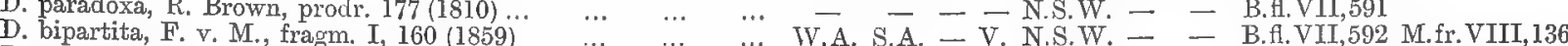

D. carphoides, F. v. M. in Benth. Fl. Austr. VII, 592 (Ï878) … - S.A. - V. N.S.IV. - - B.fl.VII,592 M.fr.XI,130.

D. penicillata, F. จ. M., fragm. VIII, 135 (1873)... $\ldots \ldots \ldots$. W.A. S.A. T. V. N.S.W. Q. - B.fl.VII,592 M.fr.VIIL,I35.

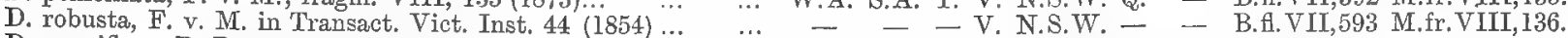

D. panciflora, R. Brown, prodr. $177(1810) \quad \ldots \quad \ldots . \quad \ldots \quad-\quad$ - T. V. N.S.WV. - - B.fl.VII,596

D. nervosa, J. Hooker, Fl. Tasman. II, 121, t. $163(1860) \quad$ … W.A. S.A. T. V. N.S.W. - - B.fl.VII,589 M.fr.VIII,135.

ASTREBLA, F. v. M., fragm. phytogr. Austr. X, 76 (1876).

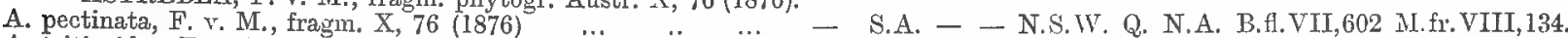

A. triticoides, F. v. M., fragm. X, $76(1876) \quad \ldots . \quad \ldots . \quad \ldots \quad-$ S.A. - - N.S.TV. Q. N.A. B.f.VII,602 M.fr.VIII,134.

MICROCHLOA, R. Brown, prodr. fl. Nov. Holl. 208 (1810).

M. setacea, R. Brown, prodr. $208(1810)$ …

CXNODON, L. C. Richard in Persoon, synops. I, 85 (1805).

C. Dactylon, L. C. Richard in Persoon, synops. I, 85 (1805) ... W.A. S.A. - V. N.S.IV. Q. - B.f.VII,609 M,fr.VIII,113.

C. tenellus, R. Brown, prodr. 187 (1810) ...

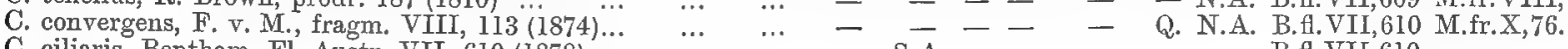

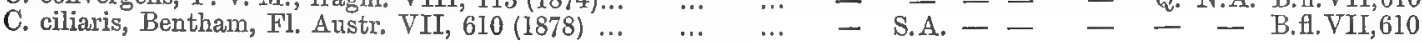

CHLORIS, Swartz, nov, gen. et spec. plant. 25 (1788).

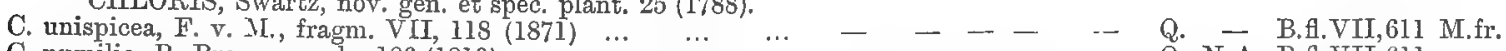

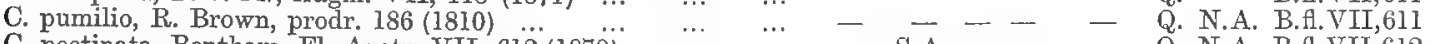

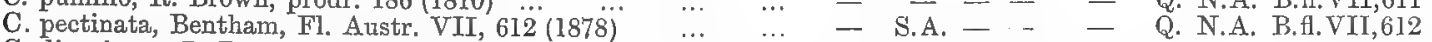

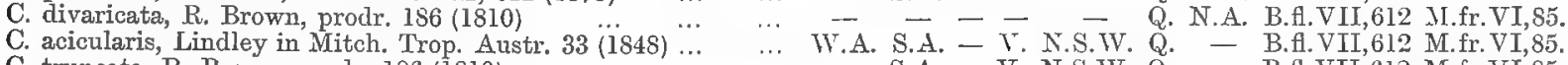

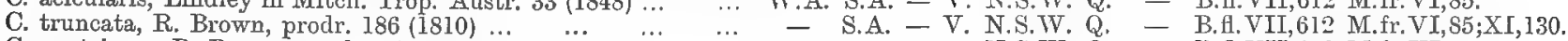

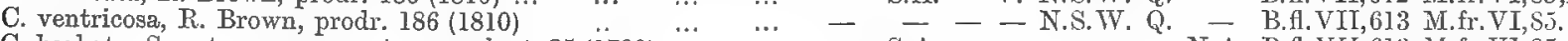

C. barbata, Swartz, nov, gen, et spec. plant. 25 (i788) $\ldots$... - S.A. - - - - N.A. B.fl.VII,613 MI.fr.VI,85.

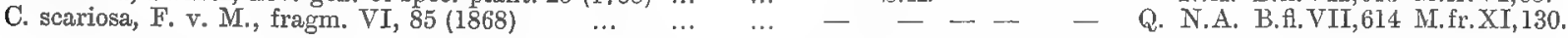

ELEUSINE, J. Gaertner, de fruct. I, 7 (1788). (Leptochloa, Dactyloctenium, Acrachne).

E. Aegyptiaca, Persoon, syn. I, 82 (1805) ... … ‥ .. - S.A. - V. N.S.WV. Q. N.A. B.fl.VII,615 MI.fr.VIII,111.

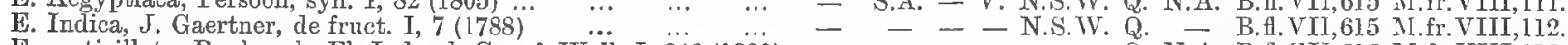

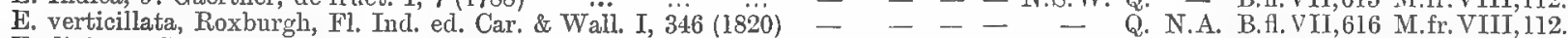

E. digitata, Sprengel, syst. cur. post. $36(1827) \ldots . \quad \ldots \quad \ldots$ W.A. S.A. -- - N.S.W. Q. N.A. B.A.VII,617 M.fr.I,216;VIII,112;XI,

E. Chinensis, F. v. M.; Poa, Koenig in Roth, nov. pi. spec. 65 (1821) - - - - N.S.W. Q. - B.Al.VII,617 NI.fr.VIII,132.

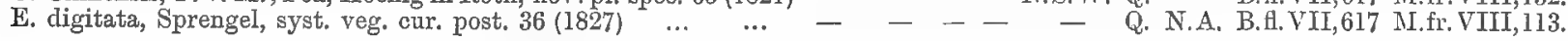

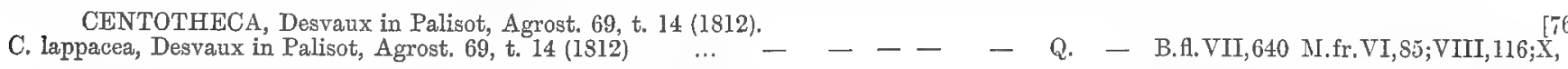

POA, Limné, gen. plant. 20 (1737). (Glyceria partly.)

P. Billardieri, Steudel, syn. glum. I, 262 (1855) ...

P. homomalla, Nees in Lehm. pl. Preiss. II, 104 (1846) ...

P. caespitosa, G. Forster, fl. ins. Austr. prodr. 89 (1786)

P. Maxwelli, Bentham, Fl. Austr. VII, 653 (1878)

P. nodosa, Nees in Lehm. pl. Preiss. II, 105 (1846)

P. saxicola, R. Brown, prodr. 654 (1810)

P. lepida, F. v. M., fragm. VIII, 130 (1874)

P. Fordeana, F. v. M., fragm. VIII, 130 (1874) $\ldots$

P. Huitans, Scopoli, flor. Carniol. 106 (1772)

P. latispicea; Festuca, F. v. M., VIII, 127 (1873)

P. syrtica, F. v. M. in Transact. Vict. Inst, 45 (1854) ...

P. dives; Festuca, F. v. M., fragm. III, 147 (1863)

P. ramigera, F. v. M. in Transact. Vict. Inst. 45 (1854)...

... IV.A. S.A. T. V. - - N.A. B.fl.VII,65I

… W.A. - - - - - - B.A.VII,651

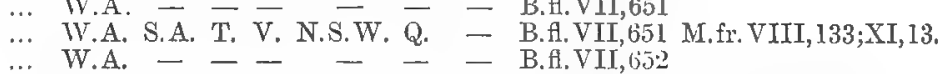

W.A. W.A. S.A. - V. N.S.IV. - - B.ff.VII, 6522 M.fr.VIII, 132,

- - T. - - B.fl.VII,654

W... W.A. S.A. $-\overline{\text { V. N.S.W. }}$

B.Hl.VII, 654 MI.fr.XI, 130 .

- S.A. - V. N.S.IV. - - B.H.VII,657 MI.fr.VIII, 130.

$\because$ W.A. S.A. T. V. N.S.W. - - B.f.VII,657 II.fr.VIII,129.

$\ldots$ W.A. S.A. T. V. N.S.W. - - B.f.VII,658 M.fr.VIII, 12S.

... - - - V - - - B.f.VII,659 M.fr.VI, 147;VIII, [130.

... W.A. S.A. - V. N.S.W. - - B.H.VII,659 MI.fr.VI, $55 ;$ VIII, 131,XI, 130.

FESTUCA, Dillenius, nov. gen. 90, t. 3 (1719). (Glyceria partly, Vulpia partly, Schedonorus, Schenorlorus, Brizopyrum.)

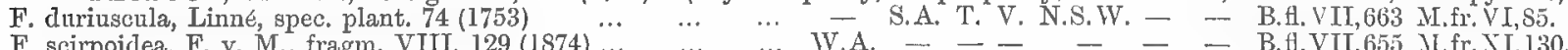

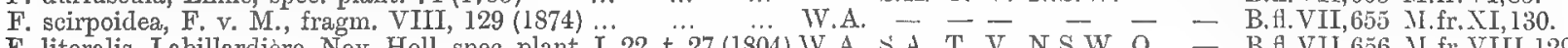

F. litoralis, Labillardiere, Nov. Holl. spec. plant. I, 22, t. 27 (1804) W.A. A.A. T. V. N.S.W. Q. - B.A.VII,656 MI.fr.VIII, 129 .

F. Hookeriana, F.v. M. in J. Hooker, Fl. Tasm. II, 122, t.115(1860) - - T. V. N.S.IV. - - B.fl.VII,656 M.fr.VIII,131.

DIPLACHNE, Palisot, Essai d'une nouv. Agrostogr. 9, t. 16 (1812). (Uralapis.)

D. loliiformis, F. v. M. in Benth. A. Austr. VII, 618 (1878) ... - S.A. - V. N.S. WV. Q. - B.f.VII,616 II.fr, VIII, l:3;XI,130.

D. Muelleri, Bentham, Fl. Austr. VII, 619 (1878)

D. fusca, Palisot, Agrost, $163(1812)$
D. parviflora, Bentham, Fl. Austr. VII, 620 $218, \ldots)$
...

- S.A. - V. N.S.IV. Q. - B.A. B.VII,616 
TRIODIA, R. Brown, prodr. fl. Nov, Holl. 182 (1810).

T. Mitchelli, Bentham, Fl. Áustr. VII, 606 (1878)

T. pungens, $R$. Brown, prodr. $182(1810) \quad \cdots \quad \cdots \quad-\quad-\quad--N . S . W . Q$ Q. - B.fl.VII,606

T Cunninh

T. irritans, R. Brown, prodr. $182(1810) \quad \cdots---1878) \cdots \cdots$

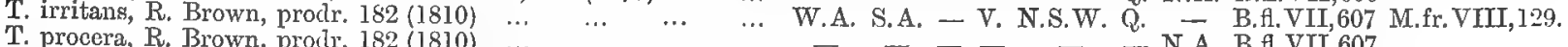

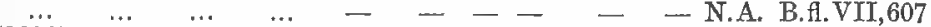

T. microdon, F. v. M.; Triraphis, Benth. Fl. Austr. vII, $605(1878)-1$ - - - N.S.W. - N.A. B.H.VII,607

DISTICHLIS, Rafinesque in Journ. de Phys. LXXXIX, 104 (1819). (Uniola partly, Brizopyrum partly.)

D. maritima, Rafinesque in Journ. de Phys. LXXXIX, 104 (1819) - S.A. T. V. N.S.W. - - B.fl.VII,637 M.fr.VIII, 129.

BROMUS, Dillenius in Linné, syst. nat. 8 (1735); Linné, gen. plant. 15 (1737).

B. arenarius, Labillardière, Nov. Holl. pl. spec. I, 23, t. 28 (1804) W.A. S.A. - V. N.S.W. Q. - B.fl.VII,660 M.fr.VI,126;XI,20. ERAGROSTIS, Palisot, Essai d'une nouv. Agrostogr. 70, t. 14 (1812). (Poa partly).

E. tenella, Palisot in Roem. et Schuit. syst. veg. II, 576 (1817) - S.A. - V. N.S.W. Q. N.A. B.Al.VII,643 MI.fr.VIII,132;XI,130

E. nigra, Nees in Steud. momencl. bot. I, 563 (1841) ... .. - - - - N.S.IV. - - B.f.VII,643

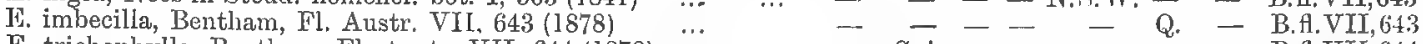

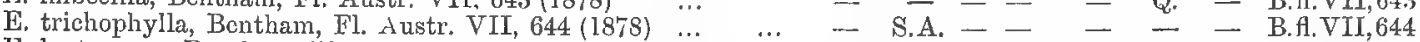

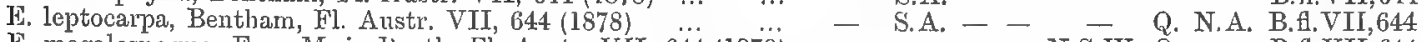

F. megalosperma, F. v. M. in Benth. Fl. Austr. VII, $644(1878)-1-2$. - - N.S.W. Q. - B.fl.VI, 644

F. pilosa, Palisot, Agrost. 71 (1812)... ... … ... $\ldots$ - S.A. - V. N.S.W. Q. - B.H.VII,645

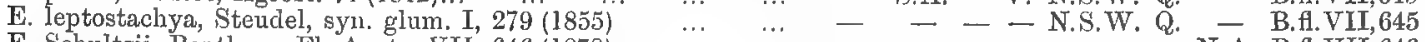

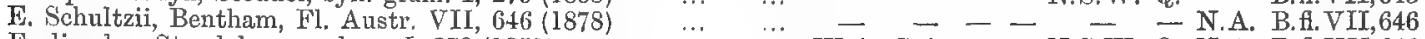

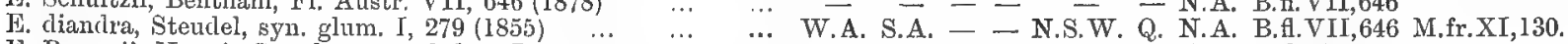

E. Brownii, Nees in Steud. nomencl. bot. I, $562(1841) \ldots . \ldots \ldots$ W.A. S.A. - V. N.S.W. Q. N.A. B.G.VII,646

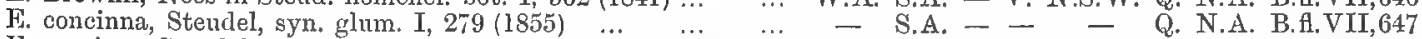

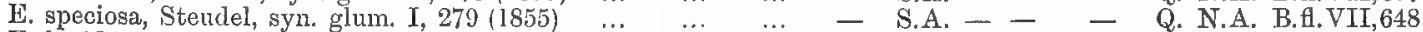

E. laniflora, Bentham, Fl. Austr. VII, $648(1878) \quad \ldots . \quad \ldots-$ - $\quad \ldots$. S.A. - - N.S.W. Q. - B.A. VII,648

F. eriopoda, Bentham, Fl. Austr. VII, $648(1878) \quad \ldots . \quad \ldots-\ldots-$ S.A. - - N.S.W. - N.A. B.f.VII,648

E. setifolia, Nees in Hook. Lond, journ. II, $419(1843) \ldots . \quad \ldots$... W.A. S.A. - - N.S.W. Q. N.A. B.Al.VII,648

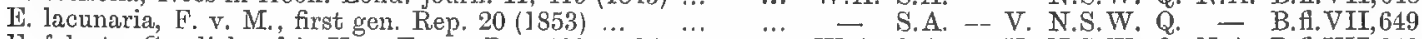

F. falcata, Gaudichaud in Voy. Freyc. Bot. $408, \ldots .25$ (1826) $\ldots$ W. W.A. S.A. - V. N.S.W. Q. N.A. B.f.VII,649

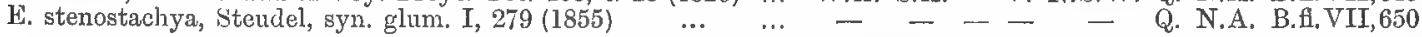
HETERACHNE, Bentham in J. Hook. icon, plant. XIII, 39 (1877).

H. Browni, Bentham in J. Hook. icon. XIII, 40 (1S77)... $\ldots-$ - - - - Q. N.A. B.fl.VII,635 M.fr.VIII, 132.

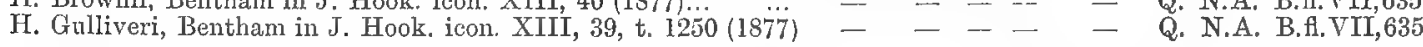
ECTROSIA, R. Brown, prodr. fl. Nov. Holl. 185 (1810).

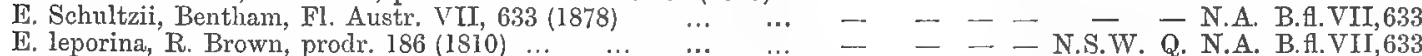

II.

... - - - - - - N.A. B.fl.VII.634

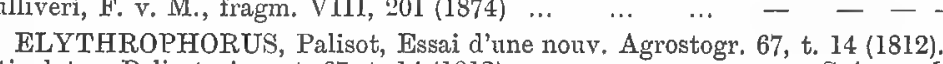

E. articulatus, Palisot, Agrost. 67, t. 14 (1812) ... ... … - S.A. - V. N.S.W. Q. N.A. B.fl.VII, 638 M.fr.VIII, 109. TRIRHAPHIS, R. Brown, prodr. fl. Nov. Holl. 185 (1810). (Triraphis.)

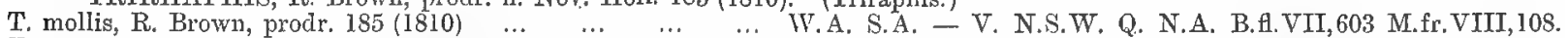

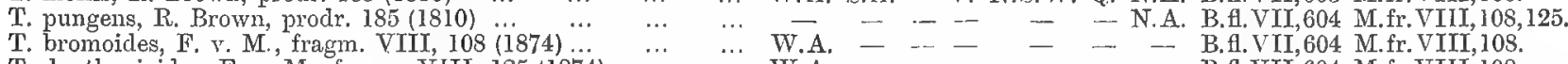

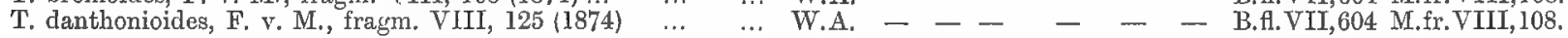

AGROPYRON, J. Gaertner, nov. comm. Petrop. XIV, 539 (1770). (Agropyrum, Triticum partly, Anthosachne, VuIpia, partly.)

A. scabrum, Palisot, Agrost. 102 (1812) ‥ $\quad \ldots \quad \ldots . \quad \ldots \quad$ W.A. S.A. T. V. N.S.W. Q. - B.fl.VII,665 M.fr.VI,85;XI,27,130,

A. velutinum, Nees in Hook. Lond. Journ. of Bot. II, $417(1843)-$ - T. V. N.S.W. - - B.fl.VII,665

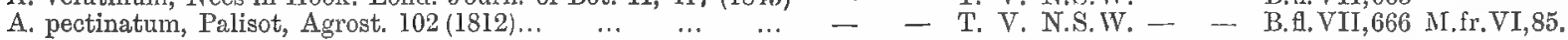
ARUNDO, Toumefort, inst. 526 (1700), from Varro. (Phragnites.)

A. Phragmites, Dodoens, stirp. hist. pemptad. $602(1583) \quad \ldots-$ - S.A. T. V. N.S. W. Q. - B.fl.VII,637

A. Roxburghii, F. v. M.; Phragmites, Kunth in nov, act. acad.

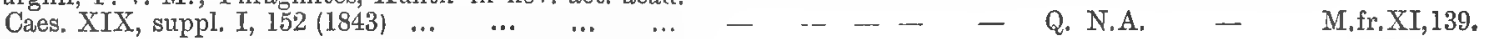

BAIBBUSA, Schreber, gener. plantar. I, 236 (1789).

Species (three, of this or allied genera, not yet found in flower) undetermined 


\title{
ACOTYLEDONEAE.
}

\author{
A. L. de Jussieu, genera plantarum l (1789).
}

ACOTYLEDONEAE VASCULARES.

Neissner, plantarum rascularium genera, 429 (1843).

RHIZOSPERMAE.

G. Weber, primit. Fl. Holsat. 74 (1780).

AZOLLA, Lamarck, Encycl. method. I, 343 (1783).

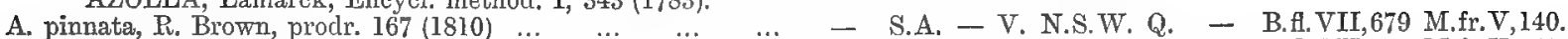

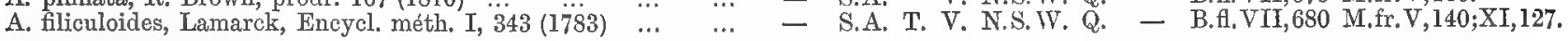
MARSILEA, Linné, syst. nat. 8 (1735); Linné, gen. plant. 326 (1737). (Marsiglia.)

M. quadrifolia, Linné, spec. plant. $1099(1753)$... $\quad . . \quad$... W.A. S.A. - V. N.S.W. Q. N.A. B.A.VII,683 M.fr.V,140. PILULARIA, Vaillant, Bot. Paris. 159, t. 15 (1727).

P. globulifera, Linné, spec. plant. $1100(1753)$... $\quad \ldots \quad$... W.A. S.A. T. V. N.S.W. - - B.A.VII,684 M.fr.V,140. ISOETES, Linne, skanska resa 420 (1751).

I. Drummondii, A. Braun in Berl. Monatsb. 593 (1863) ... ... W.A. - - V. - - - B.fl.VII,672 M.fr.V,140.

I. elatior, F. v. M. \& A. Braun in Linnaea XXV, 722(1852) ... - - - T, - - - - - - - M.fr.V,140;XI, 139

I. humilior, F. v. M. \& A. Braun in Linnaea XXV, $722(1852) \ldots-\ldots-$ - $\quad$ T. - - - - - M.fr.V,140;XI,139.

I. Gunnii, A. Braun in Berl. Monatsb. 535 (1868)

I. Muelleri, A. Braun in Berl. Monatsb. 535 (1868)

LYCOPODINEAE.

M.fr.XI, 139.

M.fr.XI, 139 .

Swartz, synops. filic. XV (1806).

PSILOTUM, Swartz in Schrader, Journ. fuer die Bot. II, 109 (1800.)

P. triquetrum, Swartz, synops. filic. $187(1806) \ldots \ldots \quad \ldots \quad \ldots \quad-\quad$ - $\quad$ - $\quad-$ N.S.W. Q. - B.f.VII,68I M.fr.V,112;X,118.

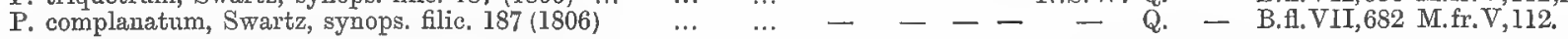

TMESIPTERIS, Bernhardi in Schrader, Journ, fuer die Bot. II, 131 (1800).
T. N.S.W. Q. - - B.fl.VII,680 M.fr.V,112;X,118.

T. Tannensis, Bernhardi in Schrad. Journ. II, 131, t. 2 (1800)... - - T. V. N.S.W. Q. - B.fl.VII,680 M.fr.V,112;X,118.
LYCOPODIUM, Ruppius, Fl. Jenens, 32 (1718), from Tabernaemontanus (1588).

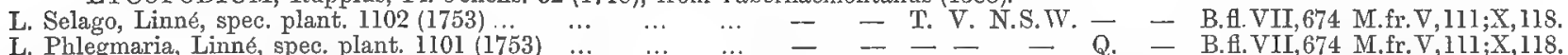

L. Phlegmaria, Linné, spec. plant. $1101(1753)$
L. clavatum, Linné, spec. plant. $1101(1753)$
L.

L. Carolinianum, Linné, spec. plant. $1104(1753) \ldots \ldots \ldots$... $\quad \ldots$ W.A. - T. V. N.S.W. Q. - B.fl.VII,675 M.fr.V,11I;X,118.

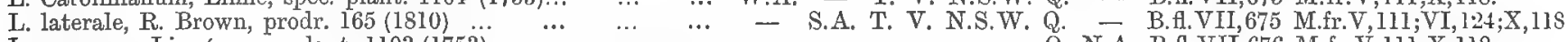

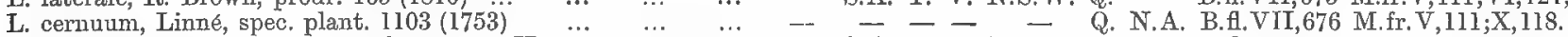

L. densum, Labill., Nov. Holl. plant. spec. II, 104, t. $251(1806)-$ S.A. T. V. N.S.T. - N. B.H.VII,676 M.fr.V,111;X,118.

L. scariosum, G. Forster, florul. ins. austr. prodr. $87(1786) \quad \ldots \quad-\quad-$ T. V. - - - - B.fl.VII,676 M.fr.V,112.

L. volubile, G. Forster, florul. ins. austr. prodr. 86 (1786) $\quad \cdots \quad-\quad-.-\quad-\quad-$ N.A. B.fl.VII,677 M.fr.V,112.

SELAGINELLA, Palisot, prodr. des fam. de l'Aetheogamie 101 (1805). (Lycopodium partly.)

S. Preissiana, Spring in Mém. de l'Ac. de Brux. 61 (1842) ‥ W.A. S.A. T. V. N.S.W. Q. - B.f1.VII,677 M.fr.V,112;VIII,275.

S. uliginosa, Spring in Mém. de l'Ac. de Brux. II, 60 (1842) … - - S.A. T. V. N.S.W. Q. - B.fl.VII,678 M.fr.V,112;VIII, 275.

S. flabellata, Spring in Mém. de l'Ac. de Brux. 174 (1849) $\quad \cdots \quad-\quad-\quad-\quad-\quad$ Q. - B.A.VII,678 M.fr.VIII,175,275.

S. concinna, Spring in Mém, de l'Ac. de Brux. 199 (1849) $\quad \cdots \quad-\quad-\quad-\quad-\quad-\quad$ Q. - B.fl.VII,678 $M I . f r . V, 112 ; V I I I, 274$.

S. Belangeri, Spring in Mém. de l'Ac. de Brux. $242(1849) \quad \ldots-1-z_{-}-$N.S.IV. Q. N.A. B.H.VII,679

PHYLLOGLOSSUM, Kunze in Mohl \& Schlechtendal, Bot. Zeitung, 721 (1843).

P. Drummondii, Kunze in der Bot. Zeit. 721 (1843) ‥ … W.A. - T. V. N.S.IV. - - B.fl.VII,672 M.fr.V,112;X,118.

\section{FILICES.}

Linné, gen. plant. 322 (1737).

OPHIOGLOSSUM, Tournefort, inst. rei herb. 548, t. 325 (1700), from Bock (1539). (Ophioderma.)

o. vulgatum, C. Bauhin, pinax theatr. bot. 354 (1623) ... ‥ - S.A. T. V. N.S.W. Q. N.A. B.fl.VII,688 M.fr.V,112;VI, I24.

O. pendulum, Limné, sp. pl. edit. sec. 1518 (1763) $\quad \ldots \quad \ldots \quad-\quad-\quad-\quad$ - N.S. V. Q. - B.f.VII,689 M.fr,V,113;VI,124.

BOTRYCHIUM, Swartz in Schrader, Journ, fuer die Bot. II, 110 (1800).

B. Lunaria, Swartz in Schrad. Journ. II, $110(1800) \quad \ldots \quad \ldots-\quad-\quad-\quad$ T. V. N.S.W. - - B.f.VII,690 MI.fr. V,113.

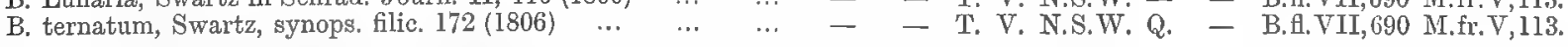

HELMINTHOSTACHYS, Kaulfuss, enum. filic. Chamiss. 28 (1824).

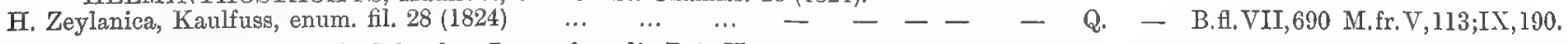

LYGODIUM, Swartz in Schrader, Journ. fuer die Bot. II, 7 \& 106 (1800). (Flydroglossum, Lygodictyon.)

L. scandens, Swartz in Schrad. Journ. II, 106 (1800) ‥ ㅠ. -- - - N.S. W. Q. N.A. B.fl.VII,691 M.fr. Y, 113;VII,84,X,118.

L. reticulatum, Schkuhr, kryptog. Gewaechse 139, t. 139 (1809) - - - - - Q. - B.f.VII,692 MI.fr.VII,S3;VIII, 157.

L. Japonicum, Swartz, syn. filic. $154(1806) \quad \cdots \quad \ldots \quad \ldots \quad-\quad-\quad-\quad-\quad$ - Q. N.A. B.fl.VII,692 M.fr.V,113;VII,84,156.

SCHIZAEA, Smith in Mem. de l'Acad. Turin. V, 149, t. 19 (1791). (Lopidium, Actinostachys.)

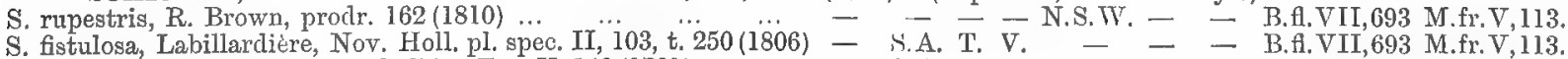

S. dichotoma, Smith in Mém, de l'Ac. Tur. V, $149(1791)$ ‥ - S.A. T. Y. N.S.IT. Q. N.A. B.fl.VII,694 MI.fr.V,113;X,118,

S. Forsteri, Sprengel, Anleit. zur Kenntn. der Gew. III, 157 (1804) - - - - - Q. - - M.fr.VIII,275.

ANGIOPTERIS, G. Hoffmann in Comment. Goett. XII, $29(1796)$.
A. evecta, G. Hoffmann in Comment. Goett. XII, $29(1796) \ldots-. \quad-\quad-\quad$ Q. - B.A.VII,694 M.fr, V,114;VII,120;X, 
MARATTIA, Swartz, nov. gen. et spec. plant. 8 \& 128 (1788).

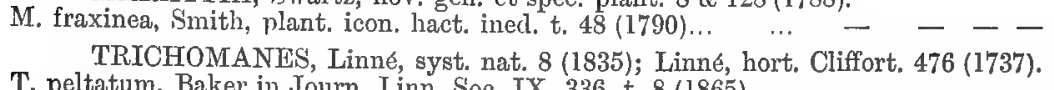

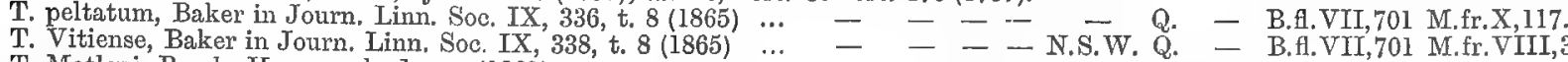

T. Motleyi, Bosch, Hymenoph. Javan (1861)

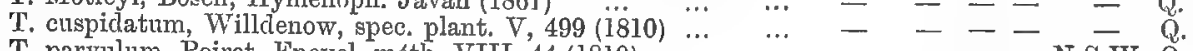

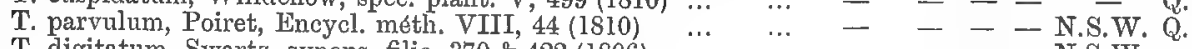

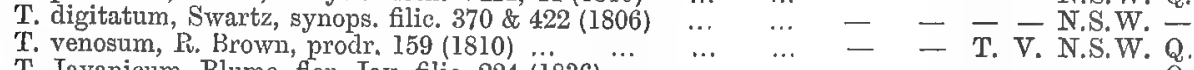

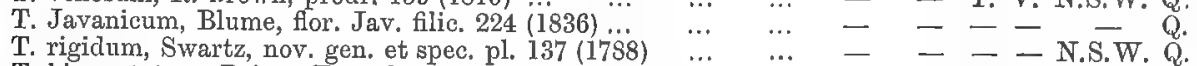

T. bipunctatum, Poiret, Encycl, méth. VIII, 44 (1810) $\cdots \cdots \cdots-$ -

T. humile, G. Forster, florul. ins. Austr. prodr. 84(1786) $\ldots-\ldots-\ldots$ - N.S.W.

T. caudatum, Brackenridge, Bot. of Wilk. U.S. Exped. fil. 256, t. 36 (1854)

T. Bauerianum, Endlicher, prodr. f.. Norf. 17 (1833) $\quad \ldots \quad \ldots-\quad-\ldots$

T. parviflorum Poiret Encyel HYMENOPHYLLUM, Smith in Roemer, Archiv I, 56 (1797).

H. marginatum, Hooker \& Greville, icon, filic.I, t. 34 (1829) ...

H. nitens, R. Brown, prodr. $159(1810)$
H. Javanicum, Sprengel, syst. veg. IV, $132(1827) \quad \ldots$.

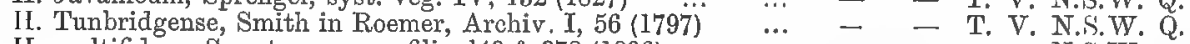

H. multifidum, Swartz, synops. filic. $149 \& 378(1806) \ldots \ldots+\ldots-\ldots$ CYATHEA, Smith in Mém. de l'Acad. Turin. V, 416 (1791). (Hemitelia, Amphicosmia.)

C. Lindsayana, Hooker, syn. filic. 25 (1865)

C. arachnoidea, Hooker, syn. filic, 24 (1866)
C. Macarthurii, F. v. M., fragm. VII, $177(1874)$

C. medullaris, Swartz, synops. filic. 140 (1806)

- $\quad$ - T. V. N.S.W.

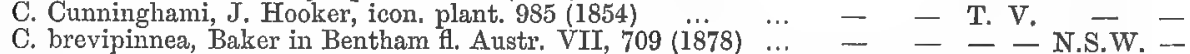

C. Moorei, F. v. M. ; Hemitelia, Baker in Gardn. Chron. $252(1872)--$ - - N.S.W. CERATOPTERIS, Brogniart in Bull. de la Soc. philom. 186 (1821). (Parkeria.)

C. thalictroides, Brogniart in Bull. Soc. philom. 186 (1821) ... - GLEICHENIA, Smith in Mem. de l'Acad. Turin. V, 418 (1791).

$\begin{array}{lllll}\text { G. platyzoma, F. v. M., fragm. V, } 114(1866) & \ldots & \ldots & \ldots & - \\ \text { G. circinata, Swartz, syn. filic. } 165 \text { et } 394(1806) & \ldots & \ldots & \ldots & -\end{array}$

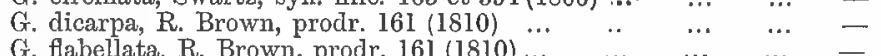

$\begin{array}{llllll}\text { G. flabellata, } R \text {. Brown, prodr. } 161(1810) \ldots & \ldots & \ldots & \ldots & - \\ \text { G. Hermanni, R. Brown. prodr. } 161(1810) & \ldots & \ldots & \ldots & -\end{array}$

G. Hermanni, R. Brown. prodr. $161(1810) \quad \ldots . \quad \ldots \quad \ldots$
OSMUNDA, Tournefort, inst. rei herb. 547 , t. $324(1700)$, from

O. barbara, Thunberg, prodr. plant. Capens, 171 (1800) ..

o. Fraseri, F. v. M. ; Todea, Hooker \& Greville, icon. filic. t. 101 (1829) -

O. Moorei, F. v. M.; Todea, Baker in Trimen, Journ. of Bot. 16(1873) ALSOPHILA, R. Brown, prodr. 158 (1810).

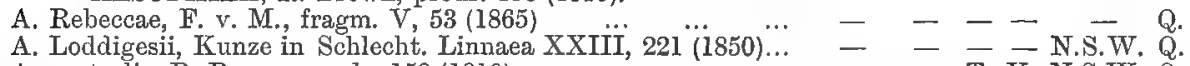

A. australis, R. Brown, prodr. $158(1810) \ldots \quad \ldots, \quad \ldots \quad \ldots, \quad-\quad-\quad$ T. V. N.S.W. Q.

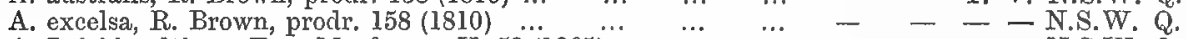

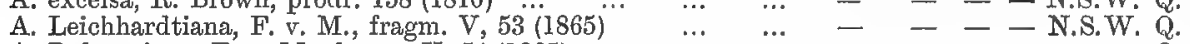

Q. N.A. B.fl.VII,695 M.fr. V,122;VIII, 158 .

(Mertensia, Platyzoma, Stromatopteris.)

\section{M.fr.XI,131\&139.}

B.fl.VII,701 M.fr.VII, ]55.

B.fl.VII, 702

B.fl.VII, $702 \mathrm{M}$. fr.V,116;IX, 191 .

B.fl.VII, 702

B.fl.VII,702 M.fr.T,115.

B.fl.VII,703 M.fr.X,117;XI, 132.

S.A. - N.S.W. Q. N.A. B.H.VII, 696 M.fr.V,114;VIII, 157;X,

V.N.W.W. Q. N.A. B.fl.VII,697 M.fr.V,115,

- T. V. N.S.W. Q. - B.fl.VII,698 M.fr.V,114;VIII, 157.

-- - N.S.W. Q. N.A. B.fl.VII,698 M.fr.V,114;X,118.

Obel (1576). (Todea, Leptopteris.)

S.A. T. V. N.S.W, Q. - B.fl.VII,699 M.fr.V,114.

- - N.S.W. - - B.f.VII,700 M.fr.V,114;VII, 156.

- - N.S.W. - - B.H.VII,700 M.fr.VIII,157.

A. Robertsiana, F. v. M., fragm. V, 54 (1865) $\quad \ldots \quad$... $\quad \ldots \quad$ -

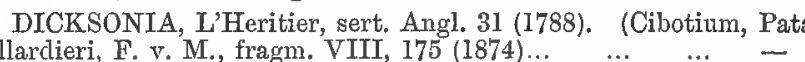

D. Billardieri, F. v. M., fragm. VIII, 175 (1874) ... … $\ldots$
D. Youngiae, C. Moore in Hook. \& Bak. syn. filic. 51 (1865) $\ldots$

D. davallioides, $R$. Brown, prodr. $158(1810) \quad \ldots$
D. nephrodioides; Deparia, Baker in Gardn. Chron. 253 (1872) ...

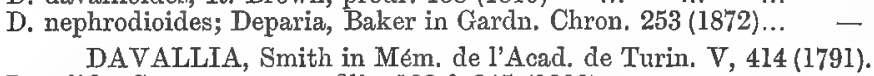

D. solida, Swartz, synops, filic. $132 \& 345$ (1806)...

D. elegans, Swartz, synops. filic. $132 \& 347$ (1806)

D. pyxidata, Cavanilles, descripcion de las plantas n. 694 (1802)

D. pedata, Smith in Mém. de I'Ac. de Turin. V, 414 (1791)

D. dubia, R. Brown, prodr. 157 (1810)

D. flaccida, R. Brown, prodr. 157 (1810)

D. tripinnata, F. v. M. in Benth. Fl. Austr. VII, 717 (1878) $\ldots$. VITTARIA, Smith in Mém. de l'Acad. de Turin. V, 414 (1791).

V. elongata, Swartz, synops. filic. 109 \& 302 (1806) $\quad \ldots \quad \ldots \quad$ -

nia, Dennstaedtia, Deparia, Balantium.)

S.A. T. V. N.S.W. Q. - B.f.VII,712 M.fr.V,117;VI, 199;VII,

$--\frac{N}{\text { N.S.W. Q. }}$

V. N.S.W. Q.

- - N.S.W. -

(Microlepia, Humata.)

- - V. N.S.W. Q.

- T. V. N.S.W. Q.

- T. V. N.S.W. Q.

$\begin{array}{ll}\text { - B.fl.VII,713 M.fr.VI,200. } & \text { [120. } \\ \text { - B.fl.VII,713 M.fr.VIII,158. } & {[104 .}\end{array}$

- B.fl.VII,7I4 M.fr.VIII,157;IX,78;X,

B.fl.VII,710 M.fr. V,117,215; VIII, 180. [VIII, 17
B.fl.VII,710
B.fl.VII,710 M.fr.V,52, 116; VII, 120;

B.f. VII,711 M.fr. V, 117;VI,200;VII, B.fl.VII,712 M.fr.V,117,213. [120

- B.f.VII,715

- B.H.VII,715 M.fr.V,118;VIII,157.

- B.fl.VII,716 M.fr.V,118.

- B.f.VII,716 M.fr.V,118.

- B.fl.VII,716 M.fr.V,118.

- B.fl.VII,717 M.fr.V,118;VII, 156.

- - - Q. - B.f.VII,717

LINDSAYA, Dryander in Mém. de l'Acad. de Turin, V, 413 (1791). (Lindsaea, Isoloma,

L. linearis, Swartz, synops. filic. 118 \& 318, t. $3(1806) \ldots \quad$... W.A. S.A. T. V. N.S.W. Q.

L. dimorpha, Bailey, Queensland Ferns 19 (1874)

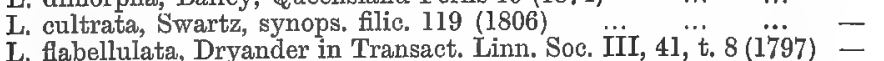

L. lobata, Poiret, Encycl. méth. XI (1813) L. trichomanoides, Dryander in Trans. Linn. Soc. III, 43, t. II(1797) -

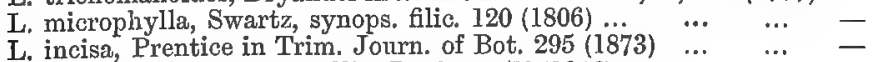

L. incisa, Prentice in Trim. Journ. of Bot. $295(1873$. Fraseri, Hooker, spec. filic. I, 221, t. $70(1846)$
L. Fra

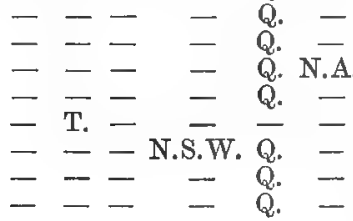

B.fl.VII,718 M.fr.V,122;VII,121.

Synophlebium, Schizoloma.)

L. Traseri, 


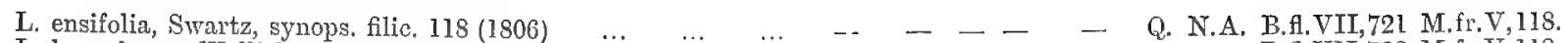

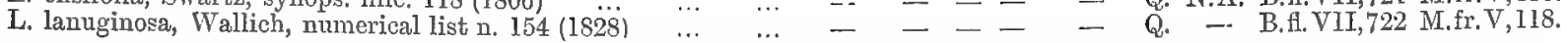
ADIANTUM, Tournefort, in 543, t. $317(1700)$, from Hippocrates, Theophrastos, Dioscorides and Plinins.

A. lunulatum, N. Burmann, Fl. Ind. 235 (1768) …

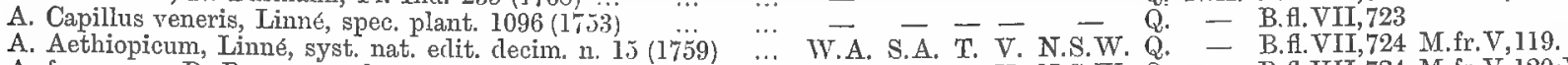

A. formosum, R. Brown, prodr. 150 (1810)

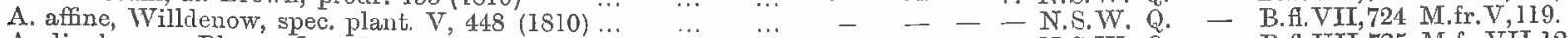

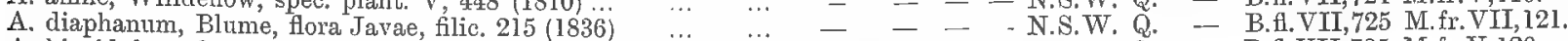

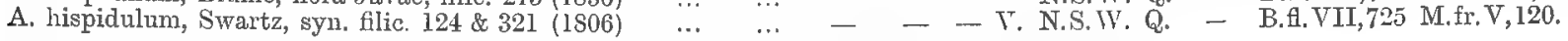

CHEILANTHES, Swartz, syn. filic. 126, t. 3 (1806). (Notholaena, Nothochlaena.)

C. Prenticei, Luerssen in Uhlw. \& Behr. Bot. Centralblatt IX, 440 (1882)

C. pumilio, F. v. M.; $\dddot{N}$ Notholaena, $\dddot{R}$. Brown, prör. $140(1810 \ddot{)}$

C. fragillima, F. v. M., fragm. $\mathrm{r}, 123(1866)$
C. vellea, F. v. M., fragm. V, $123(1866)$

C. distans, A. Braun, index sem. hort. Berol. (1859) ‥ .. W.A. S.A. - V. N.S.W. Q. - B.fl.VII,774 M.fr.V,122;VIII, 176.

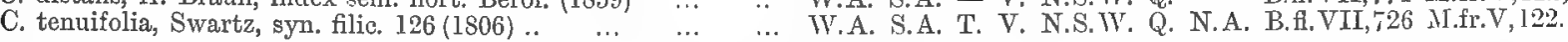
PTERIS, Linné, syst. nat. 9 (1735); Linné, gen. plant. 322 (1737.) (Pellaea, Cheiloplecton, Platyloma, Litobrochia.)

P. geraniifolia, Raddi, syn. filic. Brasil. 46 (1819)

P. paradoxa, Baker in Benth. Fl. Austr. VII, $729(1878) \quad \ldots \quad-\ldots=--$ N.S. W. Q. $\quad$ - B.H.VII,729

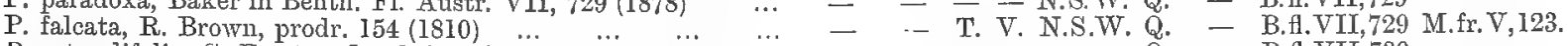

P. rotundifolia, G. Forster, florul. ins, Austr. prodr. 79 11786$) \ldots-\ldots$

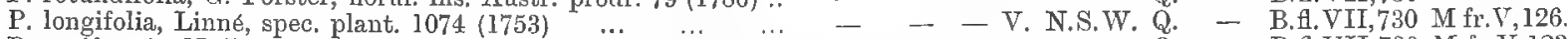

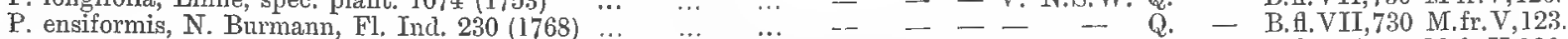

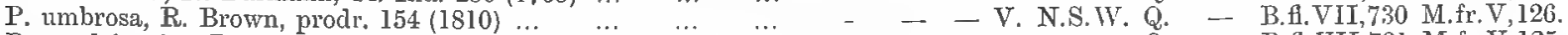

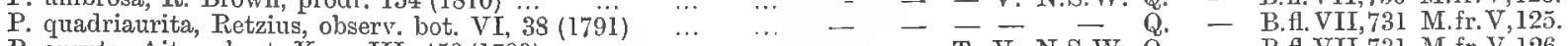

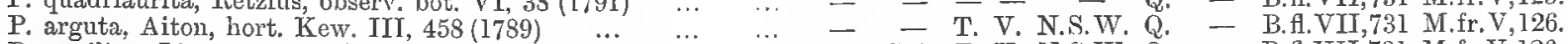

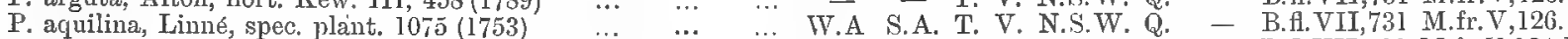

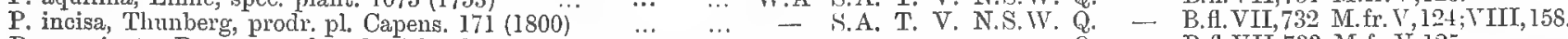

P. marginata, Bory. voy. dans les isles d'Afr. II, 192 (1804) ... - _ - - - Q. - B.fl.VII,733 M.fr.V,125.

P. comans, G. Forster, Horul. ins. Austr. prodr. 79 (1786) $\ldots .-\quad-$ T. V. N.S.W. Q. - B.fl.VII,733 M.fr.V, 125; Y'II, 121. LOMARIA, Willdenow in Magaz. der Ges. naturf. Freunde zn Berlin, III, 160 (1809.) (Stegania, Plagiogyria.)

L. Patersoni, Sprengel, syst. veg. IV 62 (1827) ... ... ... - - T. V. N.S. W. Q. - B.fl.VII,734 M.fr.V,122; Y'II, 1.38.

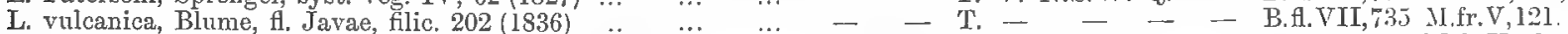

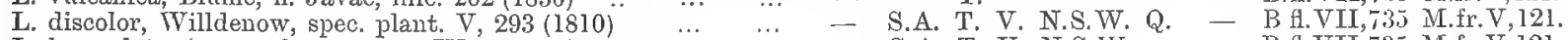

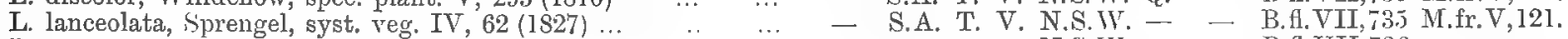

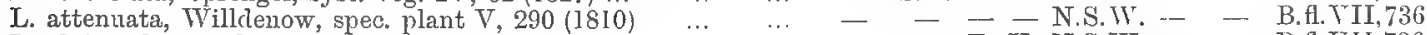

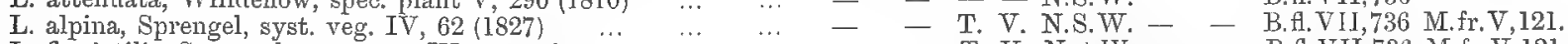

L. fluviatilis, Sprengel, syst, veg. IV, 65(1827) ... … - - T. V. N...W. - - B.甘.VII,736 M.fr.V,121.

L. Fullagari, F. v. M., fragm. VIII, 157 (1874) $\ldots \quad \ldots \quad \ldots \quad-\quad-\quad-\quad$ N.S.W. $\quad$ - B.fl.YII, 73i MI.fr.VIII, 157

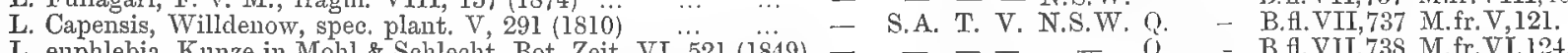

L. euphlebia, Kunze in Mohl \& Schlecht. Bot. Zeit. VI, 521 (1849) - _ _ _ - Q. - B.fl.VII,738 M.fr.VI,ll24.

BLECHNUM, Linné, spec. plant. 1077 (1753.)

B. cartilagineum, Swartz, syn. filic. 114 \& $312(1806)$

- - - V. N.S.W.Q. - B.f.VII,738 M.fr.V,120.

B. serrulatum, C. Richard in Annal. du Mus. d'Hist. nat. Par.

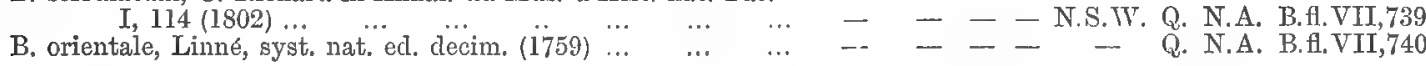

MONOGRAMMIA, Commerçon in Schkuhr's Kryptog. Gew. 82, t. 87 (1809). (Monogramme, Vaginularia, Pleurogramme, Dicliclopteris.)

M. Junghuhnii, Hooker, spec. filic. V, 123, t. $289(1864)$ ‥ - - - - Q. - B.fl.VII,740 M.ff.VII,119.

WOODWARDIA, Smith in mém. de l'Acad, de Turin. V, 411 (1791). (Doodia.)

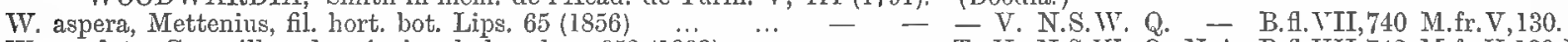

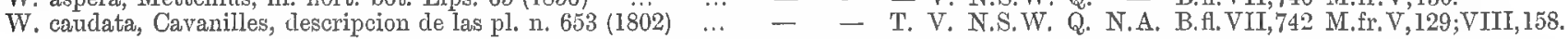

ASPLENIUM, Limé, gen. plant. 322 (1737) from C. Bauhin (1651.) (Scolopendrium, Allantodea, Diplazium, Callipteris, Anisogonium, Thamnopteris, Neottopteris, Darea, Caenopteris, Athyrium, Diplora, Asplenum.)

A. Nidus, Linné, spec. plant. 1079 (1753) ...

A. simplicifrons, F. v. M., fragm. V, $74(1865)$

A. attenuatum, R. Brown, prodr. 150 (1810)

A. Trichomanes, Linne, spec. plant. 1080 (1753)

A. flabellifolium, Cavanilles, descripcion de las pl. 1,636 (1802)

A. paleaceum, R. Brown, prodr. 150 (1810)

A. falcatum, Lamarck, Encycl. meth. I, 303 (1783)

A. Hookerianum, Colenso in Journ. of Nat. Sc. Tasm. II," 169 (18̈42)

A. furcatum, Thunberg, prodr. pl. Cap. 172 (1800)

A. laserpitiifolium, Lamarck, Encycl, méth. I, 310 (1783)

A. marinum, Linne, spec. plant. 1081 (1753) $\ldots$... 433 (1786) ...

A. pteridoides, Baker, syn. filic. sec. edit. 488 (1874)

A. umbrosum, J. Smith in Hook. Lond. Journ. IV, 174 (1842) ...

A. pallidum, Blume, fl. Javae, filic. 776 (1836) ..

A. sylvaticum, Presl, reliq. Haenk. I, 42 (1830) ...

A. maximum, D. Don, prodr. f. Nepal. 8 (1825) ...

A. polypodioides, Mettenius, fil. hort. Lips, 78 (1856)

A. melanochlamys, Hooker, spec. filic. III, 259 (1860)

A. decussatum, Swartz, spec. fil. 76 \& 260 (1806)

- - - V. N.S.IV. Q.

- - - - - N.S.W. Q.

- - T. V. N.S.W. -

W.A. S.A. T. V. N.S.W. Q.

- - - - T S. Q.

- $\quad-\quad-\overline{\text { N. N.S.W. Q. }}$

W.A. S.A. - V. N.S.W. -

- $\quad$ - T. V. N.S.W. Q.

- S.A. T. V. N.S.W. Q.

- - - - N.S.W. -

- - T. V. N.s.W. -

- - - - - $-\mathrm{Q}$.

$-\quad-\quad-\quad$ N.S.W. Q.

- $\quad-\quad-$ - N.S.W. Q.
B.fl, VII, 744 M.fr. V, 130.

B.fl. YII. 744 II.fr. V, 74,130 ; YIII, 158 ,

B.f.VII,745 M.fr.V, 130 .

B.fl.VII, 745 NI.fr.V,131

B.fl. VII, 745 M.fr. r, 131 .

B.fl.VII, 746 M.fr.V,131;VIII, 158

B.fl.VII, 746 M.fr. V',131;VII, 156.

B. A. V II, 747

B.fl.VII,748 M.fr. V,13l.

B. f.VII, 749 II.fr. V, 131 .

B,fl, VII,747 IL,fr, V, 132 .

B.fl.VII, 749 M.fr, V, 132 .

B.fl.VII, 749

B. fl, VII,749 MI.fr. T, 132.

M.fr, XI, 131 .

B.fl. VII,750 M.fr. $T, 133$.

B.fl.VII, 751

13.A.VII,751 M.fr, V,132.

B.fl. VII,75I

B. 1, VII, 751 
CYSTOPTERIS, Bernhardi in Schrader's neuem Journ. II, 26 (1806).

C. fragilis, Bernhardi in Schrad. Journ. I, $26(1806) \ldots \ldots \ldots-$-.. - T. - - - - B.fl.VII,752 M.fr.V,118.

ASPIDIUM, Swartz in Schrader's Journ. II, 429 (1000). (Nephrodium, Nephrolepis, Polystichum, Lasstraea, Sagenia, Oleandra.)

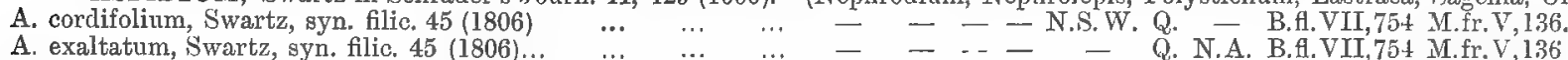

A. ramosum, Palisot, Fl. d'Oware et de Benin II, 53, t. 91 (1807) - - - - N.S.W. Q. - B.fl.VII, 7.54 M.fr. V, 135.

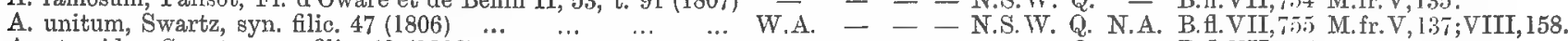

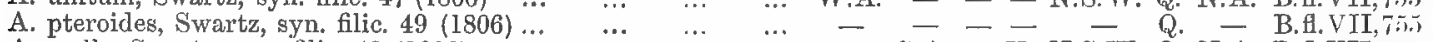

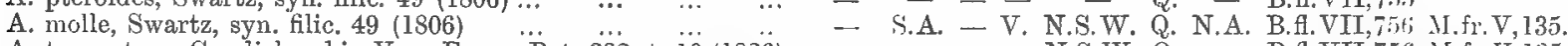

A. truncatum, Gandichaud in Voy. Freyc. Bot. 332 , t. $\dddot{10}(1826)--\quad-\quad-\quad$ N.S.W. Q. - B.fl.VII,756 M.fr.V, 1:35.

A. confluens, Mettenius in Schlecht. Linnaea XXXVI, 125(I863) - - - - - Q. N.A. B.fl.VII,757 MI.fr.V, 133;VIII, 158.

A. aculeatum, Swartz in Schrad. Journ. II, $37(1800) \ldots \ldots \ldots-$... A. T, V. N.S.W. Q. - B.fl.VII,757 M.fr.V,I34.

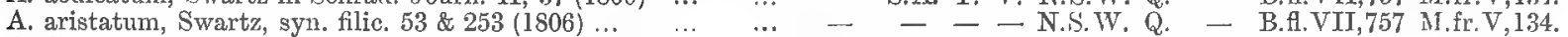

A. Capense, Willlenow, spec. plant. V, $267(1810) \quad \ldots . \quad \ldots-\ldots-\quad-\quad$ T. V. N.S.W. $-\quad-$ B.fl.VII,758 M.fr.V,134.

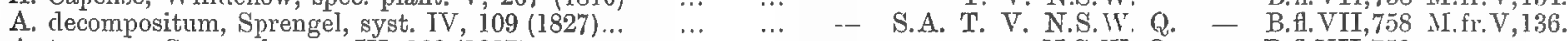

A. tenerum, Sprengel, syst. IV, 109 (1827) $\quad \ldots \quad \ldots \quad \ldots \quad \ldots \quad-\quad \ldots \quad-$ N.S.W. Q. $\quad$ - B.fl.VII,759

A. uliginosum, Kunze in Schlecht. Linnaea XX,6(1847) $\quad \ldots \quad-\quad-\quad-\quad-$ N.S.W. Q. - B.fl.VII,7.59 M.fr.V,133.

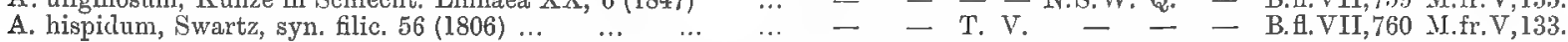

POLYPODIUM, Tournefort, inst. 540, t. 316 (1700), from Theophrastos, Dioscorides \& Plinius. (Niphobolus, Goniophlebium, Goniopteris, Phlebodium, Phegopteris, Phymatodes, Pleopeltis, Drynaria, Dictyopteris, Arthropteris, Xiphopteris, Meniscium partly; from Dodoens, l'Ecluse, Bauhin, Morison, Ray, Plumier and particularly Petiver as Polypodium.)

P. australe, Mettenius, filic. hort. bot. Lips. 36 (1856) ... ... - - T. V. N.S.W. Q.

P. Hookeri, Brackenridge, Bot. Wilk. Exped. Filic. 4 (1854) ...
P. contiguum, Brackenridge, Bot. Wilk. Exp. Fil. 6 t. (1854) ...

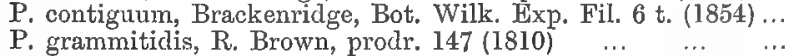

P. tenellum, G. Forster, florul. ins. Austr. prodr. 440 (1786) $\ldots$

P. proliferuin, Roxburgh in Wall. num. list 11 (1828) ...

P. urophyllum, Wallich, numerical list 11 (1828)

P. Hillii, Baker in Hook, syn. flic. sec. edit. 505 (1874)

P. poecilophlebium, Hooker, spec. filic. V, 14 (1863)

P. serpens, G. Forster, florul. insul, austr, prodr. 81 (1786)

P. confluens, R. Brown, prodr. $146(1810) \ldots$... $\ldots$. $\ldots$

P. attenuatum, R. Brown, prodr. $146(1810) \quad \ldots \quad \ldots . \ldots$

P. simplicissimum, F. v. M. in Hook. \& Bak. syn. fil. sec. edit.

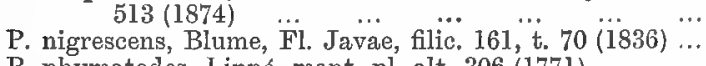

P. phymatodes, Linné, mant. pl. alt. 306 (1771) ..

P. pustulatum, G. Forster, florul. ins. Austr. pr. 81 (1786)

P. scandens, G. Forster, florul. ins. Austr. pr. 81 (1786)

P. verrucosum, Wallich, numerical list 11 (1828)...

P. subauriculatum, Blume, Fl. Javae, filic. 177, t. 83 (1836) ...

P. rigidulum, Swartz, synops. filic. 38 \& 320 (1806)

P. quercifolium, Linné, spec. plant. 1087 (1753) ...

$P$. irioides, Poiret, Encycl. méth. V, 513 (1804) ... $\quad \ldots . \quad \ldots$

P. punctatum, Thunberg, H. Japonic. 337 (1784) ...

HYPOLEPIS, Bernhardi in Schrader, neu. Journ. I, 34 (1806). H. tenuifolia, Bernhardi in Schrad. Journ. II, 24 (1800) $\cdots--1$ - - N.S.W. Q.
GRAMMITIS, Swartz in Schrarer, Journ. II, 3 \& 17 (1S00). (Gymnogramme, Selliguea).

G. Reynoldsii, F. v. M. in Benth. Fl. Austr. VIT, 775 (1878) ...

G. Muelleri, Hooker in F. v. M. fragm. V, $138(1866)$
G. rutifolia, R. Brown, prodr. $146(1810) \ldots$
.

G. leptophylla, Swartz, syn. fil. 23 et 218, t. 1 (1806) $\ldots . \quad$... W.A. S.A. T. V. N.S.W. -

G. pinnata, F. v. M., fragm. VI, 124 (1868)

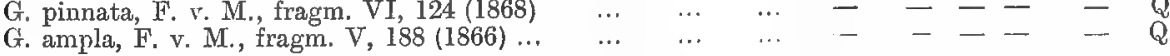
- B.fl.VII,762 M.fr. V, 127.

- - - - N.S.W.Q. - B.fi.VII,763 M.fr.V, 127.

- - T. V. N.S.TV. - - B.f.VII, 764 MI.f.V.127.

- - - N.S.W. Q. - B.fl.VII,764 M.fr.V,126;VII,121.

- - - N.S.W. Q. N.A. B.f.VII,765 M.fi.V,128.

- - - Q. - B.f.VII,765 M.fr.IV,165; V,137; VIII,

- - - - Q. - B.f.VII,766 M.fr.V,127.

- - V. N.S.T. Q. - B.fl.VII,767 M.fr.V,129.

- - N.S.W. Q. - B.fl.VII,767

- - - Q. - B.fl.VII,767 M.fr. V,129.

- - N.S.W. Q. - B.fl.VII,768 M.fr.V,128;T,121.

ANTROPHYUM, Kaulfuss, enum. filic. 197 (1824).

A. reticulatum, Kaulfuss, enum. filic. 197 (1824)...

ACROSTICHUM, Linné, gen. plant. 322 (1737), indicative. Chrysodium.)

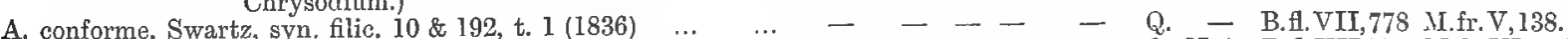

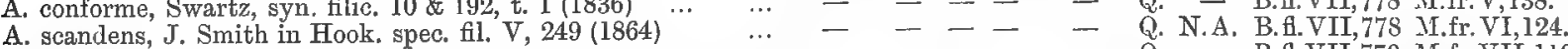

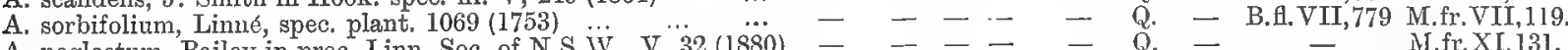

A. neglectum, Bailey in proc. Linn. Soc. of N.S. W., V, $32(1880)---\quad-\quad-\quad$ Q. -

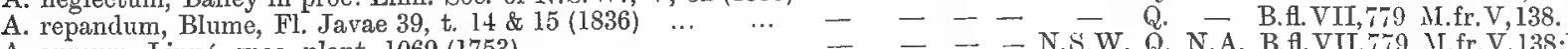

A. aureum, Linné, spec. plant. $1069(\mathbf{1 7 5 3}) \quad \ldots \quad \ldots . \quad \ldots \quad-\quad-\quad-\quad-$ N.S.W. Q. N.A. B.H.VII,779 II.fr.V,138;VII,120,

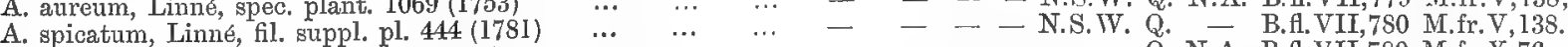

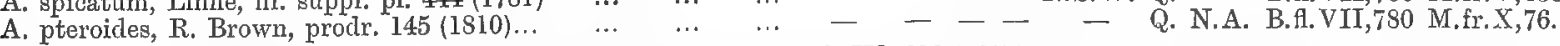

PLATYCERIUM, Desqaux in Mém, de la Soc. Linn. de Paris VI, 213 (1827).

P. alcicorne, Desvaux in Mém. Soc. Linn. Par. VI, $213(1827)$... - - - - N.S.W. Q. - B.fl.VII,780 M.fr.V, 139.

P. grande, J. Snith in Hooker, Lond. Journ. III, 402 (1841). - - - - - N.S.W. Q. - B.fl.VII,781 II.fr.V,I39. 


\section{ADDITIONAI, SPECIES.}

Pachynema sphenandrum, F. v. M. \& Tate in Transact. R. S. of S. Austr. V, 79 (1882) ... ... ... ... ...

Polyalthia Holtzeana, F. v. M. in Wing's $\mathrm{S}$. Sc. Kecord II, 230 (18S2) ...

Philotheca Hassellii, $\begin{aligned} & \ldots \\ & \mathrm{F}\end{aligned}$

Tribulus astrocarpus, F. v. MI., fragm. XII, 4 (1S52) $\quad \ldots \quad \ldots$

Phyllanthus Tatei, F. v. MI. in Wing's S. Sc. Record II, 55 (1 882 )
Ficus Pinkiana, F. v. M. in Wing's S. Sc. Record II 273 (18S2)

Ficus Pinkiana, F. v. M. in Wing's S. Sc. Record II, 273 (1882)
Peperomia Baueriana, Miquel, syst. piperac. 120 (1843)

Nepenthes Bermaysii, Bailey in proceed. L. S. of N.S. W. 185 (1S80)

Atriplex Bunburyanum, F. v. M. in Wing's S. Sc. Record II,

Kochia prosthecochaeta, F. $\begin{gathered}27 \\ \text {. }\end{gathered}$

Kochia melanocoma, F. v. M., fragm. XII, $14(1882)$
Bassia astracantha, F. T. M., fragm. XII, 12 (1882)

Bassia astracantha, F. T. M., fragm. XII, 12 (1882)
Bassia tridens, F. v. XI., fragm. XII, 12 (1882) ...

Bassia Forrestiana, F. v. M., fragm. XII, 12 (1882) $\quad \ldots$

Bossiaea Scortechinii, F. V. M. (inedit). ... ... ...

Isotropis Forrestii, F. v. M. in Wing's S. Sc. Record II, 252 (1882

Daviesia arborea, F. v. M. \& Scortechini in proc. L. S. of N.S.W.

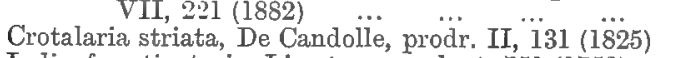

Indigofera tinctoria, Linné, spec. plant. 751 (1753)

Swainsona Oliverii, F. v. M. in Wing's S. Sc. Rec. II, 152 (1832)
Callistemon pithyoides, Miquel in Nederl. Krnidk. Arch. IV, 142

Melaleuca cylindracea, $\mathrm{R}$. Brown in Benth. Fl. Austral. III, 146 (1866) ...

Eucalyptus Foelscheana, F. $\because$ v. M. in Melb. Chemist, Nov. (1882)

Cryptandra Wayii; Trymalium, F. v. M. \& Tate in Transact. R. S. of S. Austr. V, 80 (1882)

Adenanthos Forrestii, F. v. II. in Wing's $\ddot{S}_{\text {. Sc. Record II, } 230}$

$\begin{array}{cccccc}(1882) \ldots & \ldots \\ \text { Grevillea deflexa, F. } \ldots \text {. M. inedit. } \ldots & \ldots & \ldots & \ldots & \ldots\end{array}$

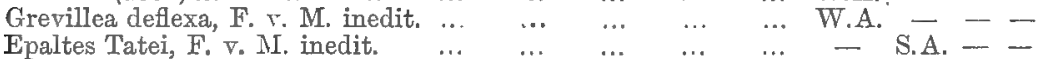

PODOSPERMIA, Labillardière, Nov. Holl. plant. specim. II, 35, t. 177 (1S06). (Podotheca.)

Podosperma Polakii, F. .. M., fragm. XII, 21 (1882) ... ... W. A. - - - _ -

Helipterum sterilescens, F. v. .I. in Wing's S. Sc. Record II,

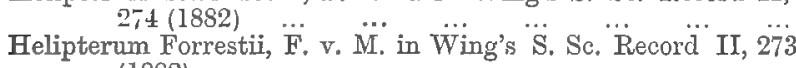

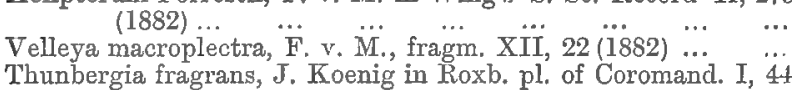

Thunbergia fragrans, J. Koenig in Roxb. pl. of Coromand. I, 44,

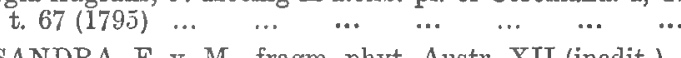

ISANDRA, F. v. M., fragm. phyt. Austr. XII (inedit.) Near Anthocercis.

Isandra Bancroftii, F. v. M., fr. XII (inedit.) … ... ... IV.A. - DICLADANTHERA, F. v. M., fragm. phytogr. Austral. XII, 23 (1882).

Dicladanthera Forrestii, F. v. M., fragm. XII, 23 (1882) …

Prostanthera Campbelli, F, v. M. in Wing's S. Sc. Record II, $252(1882)$

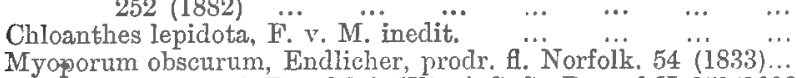

W.A.

W.A. - - - - - -

$-\quad-$

$-\quad-$ N.A.

W.A. - - -

IV.A.

IV.A. $--\infty--$

Eremophila Pantoni, F. r. M. in V'ing's S. Sc. Record II, 251(1882) TW.A. - - - N.S.T. - ERIA, Lindley in Edwards, Bot. Regist. t. 904 (1825).

Eria Fitzalani, F. v. M. in Wing's S. Sc. Record II, 252 (1882)

LUISIA, Gaudichaud in Freycin. voy. Bot. 426, t. 37 (1826).

Luisia teretifolia, Gaudichaud in Freyc. voy. 426, t. 37 (1826)

Prasophyllum Tepperi, F. r. M. in Tepp. pl. about Ardross., Oct.

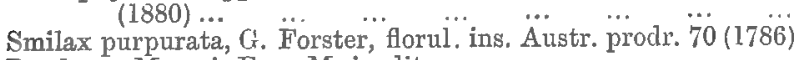

Pandanu Moorei, F. v. M. inedit. ... … … ...

Carex haematostoma, Nees in Wight, contrib. to the Bot. of Ind. 125 (1834) 


\section{ADDITIONAL ANNOTATIONS.}

Myosurus from l'Obel (1576).

$\begin{array}{lccccl}\text { Myosurus minimus } & \ldots & \ldots & \ldots & \ldots & \text { Q. } \\ \begin{array}{l}\text { Ranunculus from Plinius. } \\ \text { Ranunculus aquatilis }\end{array} \text {.. } & \ldots & \ldots & \ldots & \text { N.S.W. } \\ \text { Hibbertia hirsuta... } & \ldots & \ldots & \ldots & \ldots & \text { S.A. } \\ \begin{array}{l}\text { Doryphora Sassafras } \\ \text { Endiandra Sieberi }\end{array} & \ldots & \ldots & \ldots & \ldots & \text { Q. } \\ \text { Tristichocalyx pubescens } & \ldots & \ldots & & \text { Q. } \\ \begin{array}{l}\text { Papaver from Plinius. } \\ \text { Cleome viscosa }\end{array} & \ldots & \ldots & \ldots & \text { N.A. } \\ \text { Capparis spinosa ... } & \ldots & \ldots & \ldots & \ldots & \text { W.A. } \\ \text { Capparis lasiantha } & \ldots & \ldots & \ldots & \ldots & \text { S.A. } \\ \text { Barbarea from l'Obel (1576). } & \ldots & \ldots & \ldots & \text { W.A. } \\ \text { Barbarea vulgaris } & \ldots & \ldots & \ldots & \ldots & \text { S.A. } \\ \text { Capsella pilosula ... } & \ldots & \ldots & \ldots & \ldots & \text { W.A. }\end{array}$

Senebiera = Carara, Medicus, Pfianzen-Gattung. 34 (1792), from Cesalpini (1583). (Coronopus partly).

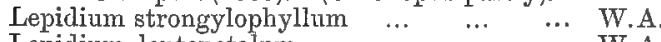

Lepidium leptopetalnm ... $\quad \ldots \quad \ldots \quad \ldots \quad \ldots \quad$ W.A.

$\begin{array}{llllll}\text { Lepidium rotundum } & \ldots & \ldots & \ldots & \ldots & \text {...A. }\end{array}$

$\begin{array}{llllll}\text { Cakile from l'Obel }(\mathrm{I} 576) . & & & & \\ \text { Hybauthus enneaspermus } & \ldots & \ldots & \text {... } & \text { W.A. }\end{array}$

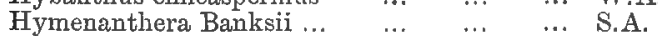

$\begin{array}{lllll}\text { Pittosporum revolutum ... } & \ldots & \ldots & \ldots & \text { M.fr.XII,4. }\end{array}$

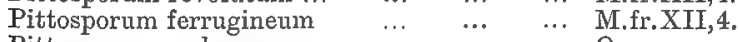

Pittosporum melanospermum $\ldots$... $\quad \ldots \quad$ Q.

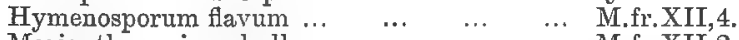

$\begin{array}{lllll}\text { Marianthus microphyllus } & \ldots & \ldots & \ldots & \text {... M.fr.XII, } 2 .\end{array}$

$\begin{array}{llllll}\text { Citriobatus pauciflorus } & \ldots & \ldots & \ldots & \ldots & \text { N.S.W. }\end{array}$

$\begin{array}{llllll}\text { Billardiera variifolia } & \ldots & \ldots & \ldots & \ldots & \text { M.fr.XII, } 2 .\end{array}$

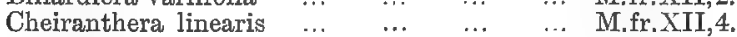

$\begin{array}{llllll}\text { Cheiranthera filifolia } & \ldots & \ldots & \ldots & \ldots & \text { M.fr.XII, } 4 \text {. }\end{array}$

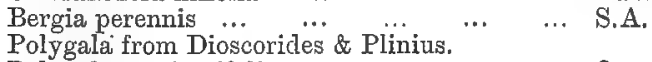

$\begin{array}{llllll}\text { Polygala persicarifolia } & \ldots & \ldots & \ldots & \ldots & \text { Q. } \\ \text { Polygala Chinensis } & \ldots & \ldots & \ldots & \ldots & \text { S.A. }\end{array}$

$\begin{array}{llllll}\text { Comesperma sylvestre } & \ldots & \ldots & \ldots & \ldots & \text { S.A.,N.S.W }\end{array}$

$\begin{array}{llllll}\text { Comesperma viscidulum... } & \ldots & \ldots & \ldots & \text { S.A. }\end{array}$

Tribulus Hystrix... $\ldots \quad \ldots \quad, \ldots$ W.A.

Hedraianthera porphyropetala ... $\quad \ldots \quad$... $\quad$ N.S.W.

$\begin{array}{llllll}\text { Tribulus macrocarpus } & \ldots & \ldots & \ldots & \ldots & \text { W.A. }\end{array}$

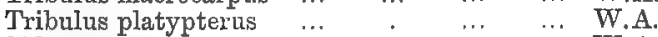

$\begin{array}{lllllll}\text { Sida virgata } & \ldots & \ldots & \ldots & \ldots & \ldots & \text { W.A. }\end{array}$

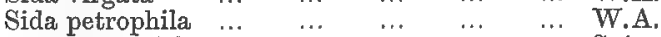

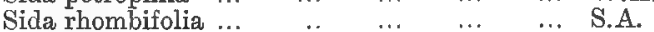

$\begin{array}{lllllll}\text { Sida physocalyx } & \ldots & \ldots & \ldots & \ldots & \ldots & \text { W.A. }\end{array}$

$\begin{array}{lllllll}\text { Sida lepida } & \ldots & \ldots & \ldots & \ldots & \ldots & \text { W.A. S.A. }\end{array}$

$\begin{array}{llllll}\text { Abutilon tubulosum } & \ldots & \ldots & \ldots & \ldots & \text { N.A. }\end{array}$

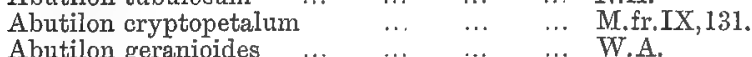

Abutilon geranioides $\ldots$... $\ldots$.... W. A.

Abutilon Avicennae, Gerard, gen. hist. of pl. 790 (1597)

$\begin{array}{llllllll}\text { Urena lobata } \ldots & \ldots & \ldots & \ldots & \ldots & \text { N.A. }\end{array}$

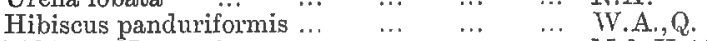

$\begin{array}{llllll}\text { Hibiscus Normani } & \ldots & \ldots & \ldots & \ldots & \text { M.fr. } V, 44 .\end{array}$

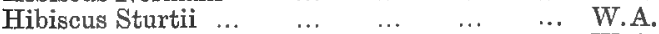

$\begin{array}{lllllll}\text { Gossypium australe } & \ldots & \ldots & \ldots & \ldots & \text { W.A. M.fr. } V, 44 .\end{array}$

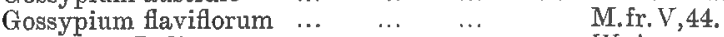

$\begin{array}{llllll}\text { Waltheria Indica } & \ldots & \ldots & \ldots & \ldots & \text { IV.A. }\end{array}$

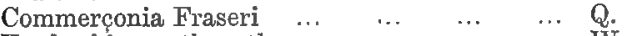

$\begin{array}{lllll}\text { Euphorbia erythrantha ... } & \ldots & \ldots & \ldots & \text { W.A. }\end{array}$

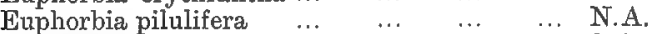

$\begin{array}{llllll}\text { Monotaxis luteiflora } & \ldots & \ldots & \ldots & \ldots & \text { S.A. }\end{array}$

Poranthera microphylla

Pseudanthus micranthus...

Beyeria viscosa

Ricinocarpus pinifolius ...

Bertya rosmarinifolia

Bertya rotundifolia

Micrantheum hexandrum

Dissiliaria tricornis

Phyllanthus rhytidospermus

Phyllanthus rigens
Phyllanthus Gastroemii ...

$\begin{array}{llll}\ldots & \ldots & \ldots & \text { M.fr.XII, } 11 \text {. }\end{array}$

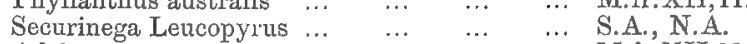

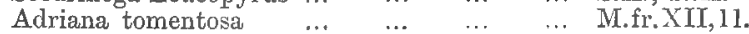

Adriana quadripartita $\ldots . \quad \ldots \quad$... $\quad \ldots$ M.fr.XII, 11 .

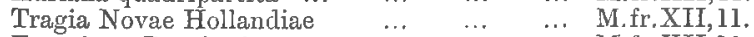

Fontainea Pancheri $\quad \ldots . \quad \ldots \quad$... M.fr.XII, II.

Omalanthus populifolius... $\quad \ldots \quad$. $\quad \ldots$ M.fr.XII,11.

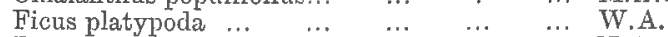

$\begin{array}{lllllll}\text { Ficus scabra } & \ldots & \ldots & \ldots & \ldots & \ldots & \text { N.A. }\end{array}$

Ficus stenocarpa $=$ Ficus subglabra, F. fragm. IX, $152(1875) \quad \ldots \quad \ldots . \quad \ldots$, N.S.W.,,

$\begin{array}{llllll}\text { Pouzolzia quinrpuenervis } & \ldots & \ldots & \ldots & \text { N.A. }\end{array}$

$\begin{array}{llllll}\text { Casuarina suberosa } & \ldots & \ldots & \ldots & \ldots & \text { S.A. }\end{array}$

$\begin{array}{llllll}\text { Casuarina inophloia } & \ldots & \ldots & \ldots & \ldots & \text { S.A. } \\ \text { C.S.W }\end{array}$

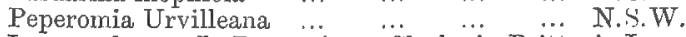

Leea aculeata $=$ L. Brunoniana, Clarke in Britten's Journ. of Bot. X, 105 (1881)

Castanospora Alphandi ... $\quad \ldots \quad \ldots \quad \ldots$. N.S.W.

$\begin{array}{llllll}\text { Atalaya variifolia } & \ldots & \ldots & \ldots & \ldots & \text { N. } \\ \text { Dolay } & \ldots & \ldots & \ldots & \text { Q. }\end{array}$

Dodonaea lanceolata $\quad \ldots \quad \ldots . \quad \ldots \quad \ldots$ S.A.

Dodonaea truncatiales $=$ D. calycina, Cunningham in A. Gray, Bot. of Wilk. Expl. Exped, I, 262 (1854).

Dodonaea platyptera

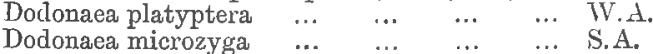

$\begin{array}{llllll} & & & & & \text { S.A. }\end{array}$

$\begin{array}{llllll}\text { Celastrus bilocularis } \quad \ldots & \ldots & \ldots & \ldots & \text { N.S.W. }\end{array}$

$\begin{array}{llllll}\text { Stackhousia muricata } & \ldots & \ldots & \ldots & \ldots & \text { S.A. }\end{array}$

$\begin{array}{llllll}\text { Plumbago Zeilanica } & \ldots & \ldots & \ldots & \ldots & \text { S.A. }\end{array}$

$\begin{array}{llllll}\text { Aegialitis annulata } & \ldots & \ldots & \ldots & \ldots & \text { N.A. }\end{array}$

Portulaca from W. Turner (1538).

Sagina apetala $\ldots .$.
Saponaria from Bock (1539).

Ptilotus incanus ... ...

Ptilotus psilotrichoides ...

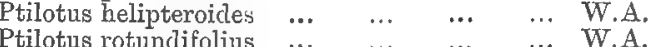

$\begin{array}{llllll}\text { Ptilotus latifolius... } & \ldots & \ldots & \ldots & \ldots & \text { W.A. } \\ \text {. } & \ldots & \ldots & \ldots & \text { W.A. }\end{array}$

Rhagodia Billardieri

Rhagodia parabolica

Rhagodia crassifolia

Rhagodia Preissii

Rhagodia Gaudichaudii ...

Rhagodia spinescens

Rhagodia nutans ...

Rhagodia linifolia

Chenopodium nitrariaceun

Chenopodium microphyllum

Chenopodium rhadinostachyum (1882).

Chenopodium cristatum ...

Chenopodium atriplicinum

Chenopodium carinatum

Dysphania plantaginella...

Dysphania litoralis

Dysphania myriocephala"

Atriplex from W. Turner (1538)

Atriplex prostratum includes A. nicrocarpum and $A$. Pumilio

Atriplex fissivalve

Atriplex paludosum

Atriplex halimoides

Atriplex vesicarium

Atriplex holocarpum

Atriplex nummularium

Atriplex leptocarpum

Atriplex crystallinum

Kochia sedifolia ...

Kochia lobiflora ...

Kochia lanosa

Kochia triptera ...

Kochia oppositifolia

Kochia brevifolia,
N.A. M.fr.XII, 16.

TV.A.,V. M.fr.XII, 15

Q. M.fr. XII, 15.

S.A. M.fr.XII, 15

.. M.fr.XII, 15.

M.fr.XII, 15.

IV.A.,N.A. M.fr.XII, 15

.. M.fr.XII, 15 .

... M.fr.XII, 16 .

.. M.fr.XII, 16 .

.. M.fr.XII, I7.

. M.fr.XII, 17

... N.A. M.fr. XII, 17 .

W... W.

... M.fr.XII, 17 .

... M.fr.XII, 17 . 
Kochia humillima

Kochia planifolia..

Kochia microphylla

Kochia brachyptera

Kochia ciliata

Kochia stelligera ...

Babbagia dipterocarpa

Bassia tricornis

Bassia carnosa

Bassia sclerolaenoides

Bassia bicomis ...

Bassia paradoxa ...

Bassia salsuginosa

Bassia lanicuspis ...

Bassia biflore

Bassia echinopsila

Bassia enchylaenoirles

Enchylaena tomentosa

Salicornia from Gerard ( 1597 ).

Salicornia robusta

Salicornia arbuscula

Salicornia cinerea...

Salicornia anstralis

Salsola Kali

Tetragonia expansa

Zaleya decandra.

Trianthema turgidifolia ...

Trianthema crystallina ...

Trianthema pilosa

Mollugo Ginus=M. Glinus.

Mollugo Cerviana...

Polygonum barbation '.

Didymotheca pleiococca

Gastrolobium grandiflorum

Mirbelia speciosa...

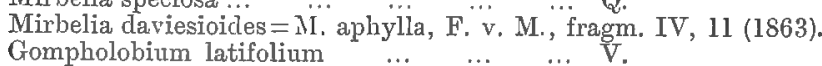

Aotus villosa

$\begin{array}{llll}\ldots & \ldots & \ldots & \text { S.A. }\end{array}$

$\begin{array}{llllll}\text { Psoralea cephalantha } & \ldots & \ldots & \ldots & \ldots & \text { N.A. }\end{array}$

$\begin{array}{llllll}\text { Psoralea balsamica } & \ldots & \ldots & \ldots & \ldots & \text { S.A. }\end{array}$

$\begin{array}{llllll}\text { Psoralea Schultzii } & \ldots & \ldots & \ldots & \ldots & \text {... } \\ \text { P.A. }\end{array}$

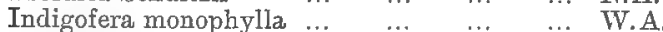

$\begin{array}{llllll}\text { Tephrosia flammea } & \ldots & \ldots & \ldots & \ldots & \text { N.A. }\end{array}$

$\begin{array}{llllll}\text { Tephrosia juncea ... } & \ldots & \ldots & \ldots & \ldots & \text { N.A. } \\ \text { Sesh. } & \ldots & \ldots & \ldots & \text { N.A. }\end{array}$

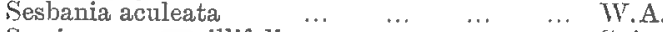

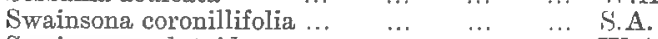

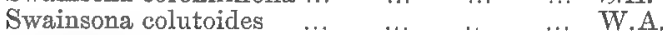

Aeschynomene Indica $\ldots \quad$.. $\quad$... S.A., N.S.W. M.fr.XII, 18.

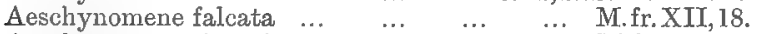

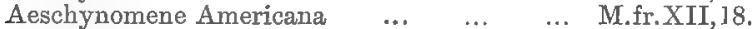

$\begin{array}{llllll}\text { Uraria lagopoides... } & \ldots & \ldots & \ldots & \ldots & \text { N.A. }\end{array}$

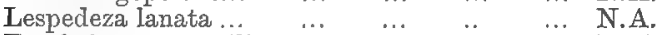

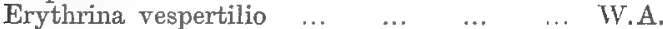

$\begin{array}{llllll}\text { Canavalia obtusifolia } & \ldots & \ldots & \ldots & \ldots & \ldots . A \\ \text { Vig } & \ldots & \ldots & \ldots & \text { W.A. }\end{array}$

$\begin{array}{llllll}\text { Vigna lanceolata ... } & \ldots & \ldots & \ldots & \ldots & \text { W.A }\end{array}$

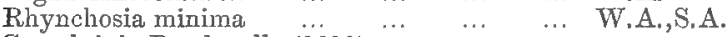

Caesalpinia Bonctucella (1810).

Labichea Buettneriana ... ... ... $\quad$ M. fr. XII,18.

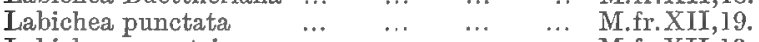

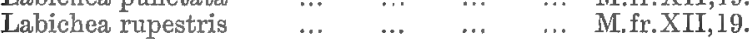

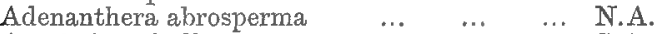

$\begin{array}{llllll}\text { Acacia iteaphylla... } & \ldots & \ldots & \ldots & \ldots & \text {... A. }\end{array}$

$\begin{array}{llllll}\text { Acacia dissonemra } & \ldots & \ldots & \ldots & \ldots & \text { N.A. }\end{array}$

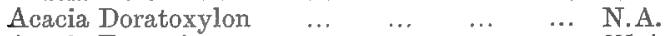

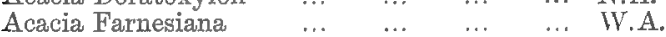

Acacia triptera, A. rhigiophylla, A. Oxycedrus, A. verticillata, A. Riceana typographically transposed from Juliferae to Plurinerves.

Albizzia Hendersoni

M.fr. I, 191.

Alchemilla from Bock (1539).

Ammannia crinipes $=$ Koelnea crinipes, F. v. M. (inedit.) =Nesaea, sect. Crinipertium, Koehne in Engler, bot. Jahrb. III, 337 (1882).

Lagerstroemia Flos Reginae

Ceratophyllum denersum

Bruguiera caryophylloicles

Thryptomene cricaea

Baeckea densifolia

Kunzea peduncularis
Melalenca armillaris

Eucalyptus amygdalina ...

Eucalyptus macrorrhyncha

Eucalyptus capitellata ...

Eucalyptus clavigera $\quad .$.

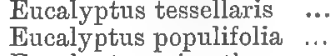

Eucalyptus microtheca ..

Pomaderris prunifolia

Pomaderris myrtilloides

Astrotricha Hamptoni

Didiscns hemicarpus=D. setulosus, F. v. M. in Proceed. R. S. Tasm III, 238 (I857).

Trachymene cirrosa (not in S.A. and V.) ,.. TY.A.

Trachymene heterophylla (not in W.A.) … V.

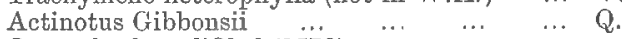

Oenanthe from l'Obel (15\%6).

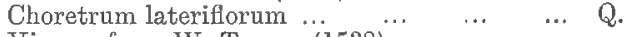

Viscum from W. Turner (1538).

Viscum angulatum

Tiscum articulatum

Isopogon ceratophyllus $\cdots \quad \cdots, \quad \cdots$

Isopogon asper (not in S. A., T., V.) ... $\quad$.. $\quad$ W.A.

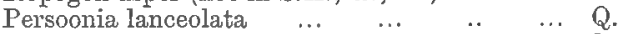

$\begin{array}{llllll}\text { Grevillea agrifolia } & \ldots & \ldots & \ldots & \ldots & \text { S.A. }\end{array}$

$\begin{array}{llllll}\text { Grevillea ilicifolia } & \ldots & \ldots & \ldots & \ldots & \text { Q. }\end{array}$

$\begin{array}{llllll}\text { Grevillea juncifolia } & \ldots & \ldots & \ldots & \ldots & \text { N.A. }\end{array}$

$\begin{array}{llllll}\text { Grevillea ramosissima } & \ldots & \ldots & & \ldots & \text { V. } \\ \text { Grevillea Chrysodendron } & \ldots & \ldots & \ldots & \text { W.A. }\end{array}$

$\begin{array}{llllll}\text { Grevillea stenobotrya } & . . & \ldots & \ldots & \ldots & \text { W.A. }\end{array}$

$\begin{array}{lllllll}\text { Grevillea striata } & \ldots & \ldots & \ldots & \ldots & \ldots & \text { IV.A. }\end{array}$

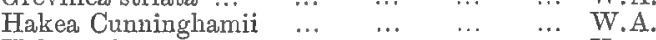

$\begin{array}{lllllll}\text { Hakea saligna } & \ldots & \ldots & \ldots & \ldots & \ldots & \text { V. }\end{array}$

$\begin{array}{llllll}\text { Hakea microcarpa } & \ldots & \ldots & \ldots & \ldots & \mathrm{Q} .\end{array}$

$\begin{array}{llllll}\text { Hakea multilineata } & \ldots & \ldots & \ldots & \ldots & \text { N.A. }\end{array}$

$\begin{array}{lllllll}\text { Banksia serrata } & \ldots & \ldots & \ldots & \ldots & \ldots & \text { Q. }\end{array}$

$\begin{array}{llllll}\text { Pimelea sanguinea } & \ldots & \ldots & \ldots & \ldots & \text { N.A. }\end{array}$

$\begin{array}{llllll}\text { Pimelea trichostachya } & \ldots & \ldots & \ldots & \ldots & \text { N.A. }\end{array}$

$\begin{array}{llllll}\text { Pimelea elachantha } & \ldots & \ldots & \ldots & \ldots & \text { S..A. }\end{array}$

$\begin{array}{llllll}\text { Pimelea ammocharis } & \ldots & \ldots & \ldots & \ldots & \text { S.A. }\end{array}$

$\begin{array}{llllll}\text { Ixora Dallachyana } & \ldots & \ldots & \ldots & \ldots & \text { N.A. }\end{array}$

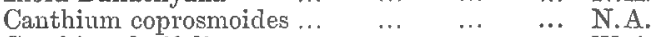

$\begin{array}{llllll}\text { Canthium latifolium } & \ldots & \ldots & \ldots & \ldots & \text { N.A. } \\ \text { O. }\end{array}$

Oldenlandia (Anotis).

Oldenlandia scleranthoides, Bentham \& J. Hooker, gen. pl. II, 8S(1873).

Oldenlandia galioides $\ldots . . . . \quad \ldots \quad \ldots$ W.A.

$\begin{array}{lllllll}\text { Opercularia varia } & \ldots & \ldots & \ldots & \ldots & \mathrm{Q} .\end{array}$

Asperula from Dalechamps (1554).

Galium umbrosum

T.A.

Sambucus from Plinius.

Trichosanthes cucumerin

$\begin{array}{llllll}\text { Cucumis trigonus .. } & \ldots & \ldots & \ldots & \ldots & \text { Q. }\end{array}$

Eupatorium from Gerard (1597).

Lagenophora Emphysopus _.. $\quad \ldots \quad$... S.A.

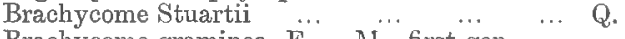

Brachycome graminea, F. . M., first gen. Rep. 14 (1853)

Brachycome ciliaris

Calotis microcephala

Aster ramulosus

Vittadinia macrorrhiza

Podocoma cuneifolia

Pterigeron liatroides

Pterigeron microglossus ...

Epaltes (Gynaphanes).

Pterocaulon sphacelatus.

Podotheca fuscescens $=$ Heliptoium, T... ... W.A. (cum, Turczaninow

Podosperma gnaphaloides $\quad \ldots \quad \ldots, \quad \ldots \quad$ MI.fr. XII, 21.

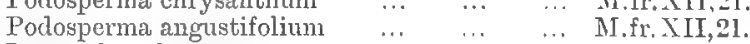

Leptorrhynchos squamatus $\quad \ldots \quad$... $\quad \ldots$ (!)

Helipterum Kendallii $=$ Podolepis Kendallii, F. r. M. (inedit.)

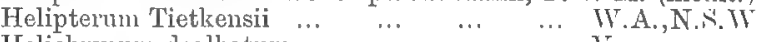

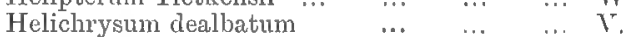

Helichrysum baccharoides $\quad \ldots, \quad \ldots . \quad \ldots, \quad$ N.S.IV.

Helichrysum pholidotum $=$ Humea squamata.

Flaveria Australasica
Eclipta alba

Wedelia verbesinoirles

Bidens bipinnatus

N.A.

S.A.

$\therefore$ A.

2 


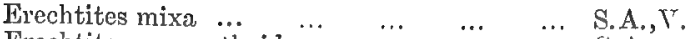

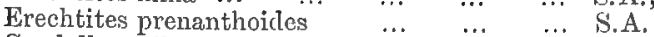

Candollea pilosa, Labillardière in Annal. du Mus. VI., 453, t. 63 (1805).

Candollea glauca, Labillardiere in Annal. du Mus. VI, 454, t. 64 (1805).

Wahlenbergia (Strzeleckia).

\begin{tabular}{|c|c|c|c|c|c|}
\hline belia Benthamii & Nia & & & & \\
\hline Isotoma petraea... & $\cdots$ & .. & . & & S.A. \\
\hline Dampiera Brownii & $\cdots$ & . & .. & $\cdots$ & $\mathbf{N}$ \\
\hline Dampiera diversifolia & $\cdots$ & $\cdots$ & $\cdots$ & $\cdots$ & \\
\hline Dampiera incana ... & $\cdots$ & $\cdots$ & $\cdots$ & $\cdots$ & M.fr.I, 120. \\
\hline $\begin{array}{l}\text { Damplera incana ...' } \\
\text { Leschenaltia hirsuta }\end{array}$ & $\cdots$ & ". & $\cdots$ & $\cdots$ & N.A. \\
\hline $\begin{array}{l}\text { Leschenaultia hirsuta } \\
\text { Leschenaultia longiloba }\end{array}$ & $\ldots$ & ... & $\cdots$ & $\ldots$ & M. fr. XII, 23. \\
\hline sschenaultia longilo ba & $a$ & .. & $\cdots$ & $\cdots$ & M.fr.XII, 23. \\
\hline tosperma Muelleri & $\ldots$ & .. & $\cdots$ & $\ldots$ & S. A. \\
\hline caevola depauperata & & & $\ldots$ & $\ldots$ & N.A. \\
\hline Scaevola globuliflora $=$ & S. glc & ulifera. & & & \\
\hline oodenia Chambersii & & $\because \because$ & $\ldots$ & $\ldots$ & N.A. \\
\hline $\begin{array}{l}\text { Goodenia Hassallii }=\mathrm{G} \text {. } \\
\text { Mitrasacme pilosa }\end{array}$ & Has & ellii. & & & \\
\hline Mitrasacme pilosa & ... & $\cdots$ & $\cdots$ & .. & S.A. \\
\hline $\begin{array}{l}\text { Logania floribunda } \\
\text { Plantago from W. Turn }\end{array}$ & $\ldots$ & & $\cdots$ & $\cdots$ & \\
\hline igustrum from $\mathrm{W}$. Tu & urne & $1538)$. & & & \\
\hline Notelaea linearis ... & $\ldots$ & $\ldots$ & $\ldots$ & $\ldots$ & \\
\hline Cynanchum floribundun & & & $\ldots$ & $\ldots$ & IV.A. \\
\hline Gymnanthera nitida & 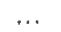 & $\cdots$ & & $\ldots$ & W.A, \\
\hline Tylophora erecta ... & ... & $\ldots$ & $\cdots$ & $\ldots$ & N.A. \\
\hline Ipomoea Turpethum & $\cdots$ & & . & . & N.A. \\
\hline Ipomoea Muelleri... & $\ldots$ & $\because$ & $\ldots$ & $\cdots$ & S.A. \\
\hline Convolvulus from W. T & Turn & (1538). & & & \\
\hline Polymeria angusta & & & & $\cdots$ & S.A. \\
\hline uta australis ... & & & & & \\
\hline & & & & & \\
\hline
\end{tabular}

Solanum viride, Solander in G. "Forster, prodr. 89 (I786).

Datura Leichhardtii $\ldots$.... .. (Pyxidaria).

$\begin{array}{lllll}\text { Peplidium Muelleri } \quad \ldots & \ldots & \ldots & \text { W.A., S.A. }\end{array}$

$\begin{array}{lllllll}\text { Buechnera parviflora } & \ldots & \ldots & \ldots & \ldots & \text { W.A. }\end{array}$

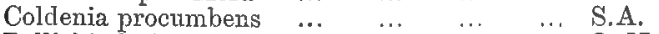

$\begin{array}{llllll}\text { Pollichia latisepalea } & \ldots & \ldots & \ldots & \ldots & \text { Q., N.A. }\end{array}$

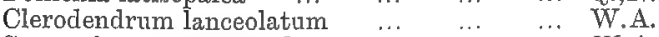

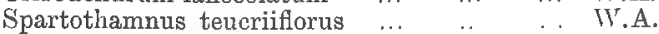

$\begin{array}{lllll}\text { Eremophila oppositifolia } & \ldots & \ldots & \ldots & \text { TW.A. }\end{array}$

$\begin{array}{llllll}\text { Eremophila Mitchelli } & \ldots & \ldots & \ldots & \ldots & \text { S.A. }\end{array}$

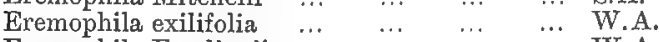

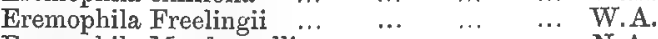

$\begin{array}{lllll}\text { Eremophila Macdonmelli... } & \ldots & \ldots & \ldots & \text { N.A. }\end{array}$

$\begin{array}{llllll}\text { Eremophila Goodwinii } \ldots & \ldots & \ldots & \ldots & \ldots & \text { N.A. }\end{array}$

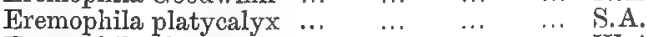

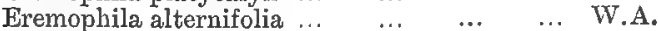

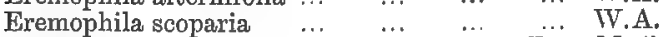

Prasophyllum Fimbria $=\dddot{P}$. Reichenbachii, F. v. M. (inedit.)

$\begin{array}{llllll}\text { Chiloglottis Gunnii } & \ldots & \ldots & \ldots & \ldots & \text { N.S.W. }\end{array}$

Astelia (Funkia 1805).

$\begin{array}{llllll}\text { Typhonium Brownii } & \ldots & \ldots & \ldots & \ldots & \text { N.A. }\end{array}$
Wolffia Michelii ... Cyperus Lragrostis Cyperus pulchellus Cyperus gracilis ... Cyperus tenellus ... Cyperus squarrosus Cyperus fulvus ... Cyperus rotundus Cyperus polystachys Cyperus Iria

Cyperus decompositus Scirpus grossus ... Scirpus supinus ... ... Scirpus litoralis ... .... Cyathochaete clandestina
Cyathochaete diandra. Schoenus aphyllus Schoenus ericetorum Schoenus sphaerocephalus Lepidospora tenuissinı ... Lepidosperma gladiatum

Lepidosperma filiforme ... Cladium junceum... Clarlium schoenoides

Cladium trifidum ...

Cladium filum ...

Cladium melanospermum

Cladium Mariscus

Cladium teretifolium

Cladium psittacorum

Cladium Rarlula ...

Caustis pentandra

Arthrostylis aphylla

Carex Brownii

Carex tereticaulis...

Carex declinata

Carex breviculmis

Carex inversa

Carex acuta

Carex pumila

Tragus racemosus...

Andropogon Gryllus

Androphogon procertus ...

Anthistiria membranacea

Chionachne cyathopoda ...

Sporobolus Lindleyi

Eriachne obtusa, W.A., S.A.

Eleusine Aegyptia=E. cruciata, Lamarck, tabl, encycl. et meth. I, 203 t. 48 (1791)

Setaria glauca

Setaria macrostachya

Eragrostis pilosa ...

Eragrostis tenella

Trirhaphis pungens

Arundo Phragmites

Botrychium ternatum

$\cdots \quad \cdots$ $\begin{array}{lllll}\ldots & \ldots & \ldots & \ldots & \text { S.A. }\end{array}$

M.fr.XII, 25

M.fr.XII, 25.

MI.fr.XII, 25.

.. M.fr.XII,25.

M.fr.XII,25.

W.A.

M.fr.XII, 25 .

.. M.fr.XII, 25.

M.fr.XII,25.

M.fr.XII, 25 .

... M.fr.XII,24.

... M.fr. XII,25.

M.fr.XII,25.

... M.fr.XII,25.

... M.fr.XII,25.

... M.fr.XII,26.

... M.fr.XII,26.

... S.A.

... M.fr.XII,26.

.. M.fr.XII, 26.

... M.fr.XII,26.

.. M.fr.XII, 25,

.. M.fr.XII, 26.

M.fr.XII, 26.

M.fr.XII, 26.

M.fr.XII,26.

M.fr.XII,25.

M.fr.XII, 25.

M.fr.XII, 25.

S.A.

.. M.fr.XII,26.

... M.fr.XII, 25 .

.. Q. Ml.fr.XII, 26 .

.. M.fr.XII, 26.

... M.fr.XII,26.

... M.fr.XII, 26 .

... M.fr.XII, 26.

... M.fr.XII, 26.

... M.fr.XII,26.

... W.A.

... W.A

... W.A.

... W.A

... Q

... N.A.

W.A.

W.A.

.. W.A.

... W.A.

... W.A

... Q.

… W. $\mathrm{W}$

... S.A. 


\section{SEQUENCE OF ORDERS ACCORDING TO PREDOMINANCE, With Indications of their Numbers of Species.}

Total number of Vasculares $=8646 . \quad$ Dicotyledoneae $=6897 . \quad$ Nonocotyledoneae $=1522 . \quad$ Acotyledoneae $=227$.

\begin{tabular}{|c|c|c|}
\hline Leguminosae & & 1058 \\
\hline Myrtaceae... & & 651 \\
\hline Proteaceae... & $\cdots$ & 586 \\
\hline Compositae & $\cdots$ & 529 \\
\hline Cyperaceae & $\ldots$ & 372 \\
\hline Gramineae... & $\ldots$ & 346 \\
\hline Epacrideae... & $\ldots$ & 273 \\
\hline Orchicleae ... & & 255 \\
\hline Euphorbiaceae & $\ldots$ & 224 \\
\hline Goodeniaceae & $\ldots$ & 212 \\
\hline Filices $\quad .$. & $\ldots$ & 200 \\
\hline Rutaceae ... & $\ldots$ & 180 \\
\hline Liliaceae ... & $\ldots$ & 161 \\
\hline Rubiaceae ... & $\ldots$ & 124 \\
\hline Labiatae $\quad .$. & $\cdots$ & 124 \\
\hline Sterculiaceae & $\cdots$ & 123 \\
\hline Salsolaceae... & $\ldots$ & 112 \\
\hline Malvaceae ... & $\ldots$ & 105 \\
\hline Umbelliferae & $\ldots$ & 103 \\
\hline Sapindaceae & $\ldots$ & 100 \\
\hline Candolleaceae & $\ldots$ & 95 \\
\hline Dilleniaceae & .. & 95 \\
\hline Amarantaceae & $\cdots$ & 4 \\
\hline Restiaceae ... & .. & 93 \\
\hline Rhamnaceae & $\ldots$ & 89 \\
\hline Amaryllideae & $\ldots$ & 6 \\
\hline Solanaceae... & . & 80 \\
\hline Scrophularinae & $\cdots$ & \\
\hline Myoporinae & $\ldots$ & 8 \\
\hline Terbenaceae & $\ldots$ & \\
\hline Thymeleae... & $\cdots$ & \\
\hline Convolvulaceae & $\cdots$ & \\
\hline Urticaceae ... & .. & 6. \\
\hline Halorageae & & \\
\hline Asclepiacleae & $\cdots$ & \\
\hline Cruciferae ... & - & \\
\hline Asperifoliae & $\because$ & \\
\hline
\end{tabular}

\begin{tabular}{|c|c|}
\hline liaceae & \\
\hline Loganiaceae & $\ldots$ \\
\hline Apocyneae... & $\ldots$ \\
\hline Droseraceae & $\ldots$ \\
\hline Santalaceae & ... \\
\hline Pittosporeae & $\ldots$ \\
\hline Lauraceae ... & $\ldots$ \\
\hline Meliaceae ... & $\ldots$ \\
\hline Saxifrageae & $\ldots$ \\
\hline Campanulaceae & $\ldots$ \\
\hline Polygaleae... & $\ldots$ \\
\hline Portulaceae & $\ldots$ \\
\hline Acanthaceae & $\ldots$ \\
\hline Coniferae ... & $\ldots$ \\
\hline Fluviales ... & $\ldots$ \\
\hline Combretaceae & $\ldots$ \\
\hline Ficoideae ... & $\ldots$ \\
\hline Palmae & $\ldots$ \\
\hline Polygonaceae & $\ldots$ \\
\hline Loranthaceae & . \\
\hline Irideae & $\ldots$ \\
\hline Caryophylleae & $\ldots$ \\
\hline Capparideae & $\ldots$ \\
\hline Castuarineae & $\ldots$ \\
\hline Cucurbitaceae & $\ldots$ \\
\hline Gentianeae... & \\
\hline Lentibularinae & $\ldots$ \\
\hline Araliaceae ... & . \\
\hline Zygophylleae & $\ldots$ \\
\hline Jasmineae ... & \\
\hline Sapotaceae... & $\ldots$ \\
\hline Commelineae & . \\
\hline Viniferae $\ldots$ & $\ldots$ \\
\hline Eriocauleae & $\cdots$ \\
\hline Lycopodinae & $\ldots$ \\
\hline Ranunculaceae & \\
\hline Tremandreae & $\cdots$ \\
\hline
\end{tabular}

Rosaceae $\ldots \quad \ldots \quad 17$

Anonacea.... ... 16

Salicarieae... $\quad . . \quad 16$

Junceae $\quad \ldots \quad \ldots \quad 16$

NLonimieae... $\quad \ldots \quad 15$

Celastrinae $\quad \ldots \quad 15$

Olacinae $\quad \ldots \quad \ldots \quad 15$

Ebenaceae... $\quad \ldots \quad 15$

Menispermeae … 14

Stackhousieae $\quad \ldots \quad 13$

$\begin{array}{llll}\text { Cycadeae ... } & \ldots & 13\end{array}$

$\begin{array}{llll}\text { Violaceae } & . . & \ldots & 12\end{array}$

$\begin{array}{lll}\text { Myrsinaceae } \quad \ldots & 12\end{array}$

Phytolacceae 11

Scitamineae ... 11

Piperaceae... $\quad \ldots \quad 10$

$\begin{array}{llll}\text { Aroideae } & \ldots & \ldots & 10\end{array}$

Pandaneae... ... 10

Anacardiaceae $\quad . .69$

Hydrocharideae $\quad . . \quad 9$

$\begin{array}{llll}\text { Xyrideae } & \ldots & \ldots & 9\end{array}$

Rhizospermeae ... 9

Flacourtieae $\quad$... 8

Simarubeae $\quad \ldots .7$

Geraniaceae $\quad \ldots .7$

Frankeniaceae $\quad . .7$

Bignoniaceae $\quad . .7$

$\begin{array}{lll}\text { Crassulaceae } & \ldots & 6\end{array}$

Rhizophoreae ... 6

Nelastomaceae $\quad . .66$

$\begin{array}{lll}\text { Primulaceae } & \ldots & 6\end{array}$

Lemnaceae... $\quad$... 6

Alismaceae $\quad \ldots .6$

Aristolochieae $\quad \ldots \quad 5$

Nyctagineae $\quad . .5$

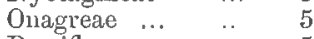

Passifloreae $\quad$... 5
Fricaceae ...

\begin{tabular}{lll} 
Nymphaeaceae & $\ldots$ & 4 \\
\hline
\end{tabular}

Magnoliaceae ... 4

Samydaceae $\quad \ldots .4$

$\begin{array}{llll}\text { Elatineae } & \ldots & \ldots & 4\end{array}$

Lineae $\quad . . \quad \ldots \quad 4$

Cupuliferae $\quad$... 4

Plumbagineae $\quad$... 4

Plantagineae $\quad \ldots \quad 4$

Dioscorideae $\quad \ldots \quad 4$

Gesneriaceae $\quad \ldots \quad 3$

$\begin{array}{lll}\text { Pedalinae ... } & \ldots & 3\end{array}$

$\begin{array}{lll}\text { Philydreae... } & \ldots & 3\end{array}$

Nepenthaceae $\quad \ldots \quad 2$

Guttiferae ... $\quad \ldots \quad 2$

Malpighiaceae $\quad$.. 2

$\begin{array}{lll}\text { Burseraceae } \quad . . & 2\end{array}$

Connaraceae … 2

Caprifoliaceae 2

Aquifoliaceae $\quad . .25$

$\begin{array}{llll}\text { Styraceae } & \ldots & \ldots & 2\end{array}$

Hydrophylleae $\quad \ldots \quad 2$

Burmanniaceae ... 2

$\begin{array}{lll}\text { Typhaceae ... } & \ldots & 2\end{array}$

$\begin{array}{lll}\text { Myristicaceae } & \ldots & 1\end{array}$

Papaveraceae $\quad . . .11$

$\begin{array}{lll}\text { Hypericinae } & \ldots & 1\end{array}$

Ochnaceae... $\quad . . .1$

Podostemoneae ... 1

Balonophoreae ... I

Hamamelidae $\quad . . .1$

$\begin{array}{lll}\text { Elaeagneae } & \ldots & 1\end{array}$

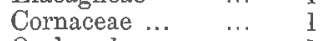

Orobancheae ... 1

Taccaceae ... ... 1

Roxburghiaceae ... 1

Total number of Genera of Vasculares $=1355$.

\section{INDEX OF ORDERS AND GENERA.}

Abbottia 74

Abelmoschus 14

Abilclgaardia 125

Abroma 15

Abrophyllum 48

Abrotanella 84

Abrus 41

Abutilon 14, 141

Acacia 43,142

Acaena 47

Acalypha 20

Acanthaceae 99

Acanthocarpus 119

Acanthocladium 81

Acanthus 99

Achilleopsis 16

Achras 91

Achromolaena 82

Achrysum 83

Achyranthes 25

Acianthus 113

Acicalyptus 59
Aciphylla 53

Ackama 48

Acmene 59

Acomis 82

Acrachne 134

Acradenia 12

Acroclinium 80

Acronychia 12

Acrophyllum 48

Acrostichum 139

Acrotriche 105

Actephila 19

Actinocarpus 121

Actinodium 51

Actinopappus 82

Actinostachys 136

Actinostigma 10

Actinostrobus 109

Actinotus 63, 14:

Aclansonia 15

Acleliopsis 4

Adcuanthera 43, 14:
Adenanthos 66, 140

Adenochilus 113

Adenosma 97

Arlenostemma 77

Adenostephanus 68

Adiantum 137

Acirastaea 1

Adriana 21, J41

Aegialinites 26

Aegialitis 26, 141

Aegiceras 91

Aegopogon 133

Aeschynomene 40,142

Afzelia 43

Agastachys 67

Agathis 109

Agati 39

Ageratum it

Aglaia 9

Aglaiopsis 9

Agnostus 71

Agonis 54
Anigozanthos 116

Agrimonia 47

Agropyron 135

Agrostis 133

Agrostocrinum $11 \mathrm{~S}$

Ailantus 12

Aillya 88

Aira 133

Aizoon 31

Ajuga 10:

Akania 24

Albizzia 47, 142

Aldrovanda 7

Alchemilla 47, 1.2

Alchornea 21

Alectryon 24

Alepyrum 123

Aleurites 20

Alisma 121

Alismaceae 121

Allania $1 \mathrm{IS}$

Allantodea 138 
Allophylus 24

Alopecurus 132

Alphitonia 60

Alpinia 114

Alsomitra 76

Alsophila 137

Althenia 121

Altingia 109

Alstonia 93

Alternanthera 28

Alysicarpus 40

Alyssum 5

Alyxia 93

Amanoa 20

Amarantaceae 28

Amarantus 29

Amaryllideae 115

Amblysperma 85

Ambrina 29

Ammannia 49, 142

Ammobium 82

Amomum 114

Amoora 9

Amorphophallus 120

Amorphospermum 91

Amperea 19

Amphibolis 121

Amphibromus 134

Amphicosmia 137

Amphipogon 133

Amphodus 41

Anacardiaceae 25

Anadenia 68

Anagallis 91

Anarthria 123

Ancana 3

Ancistrostigma 31

Ancistrum 47

Andersonia 108

Andrachne 19

Andrewsia 104

Andropogon 132,143

Androstemma I16

Androstoma 105

Aneilema 122

Anemone 1

Angianthus 82

Angiopteris 136

Angophora 57

Anguillaria 117

Anisacantha, 30

Anisandra 102

Aniseia 94

Anisogonium 138

Anisolepis 80

Anisomeles 101

Anisopogon 134

Anisotome 63

Anonaceae 3

Anodopetalum 48

Anopterus 48

Anosporum 124

Antennaria 80

Antheidosorus 82

Anthericum 118

Anthistiria 132,143

Anthobolus 64

Anthocerastes 82

Anthocercis 96

Anthotium 87

Anthotroche 96

Anthosachne 135

Antiaris 22

Anticoryne 53

Antidesma 19

Antirrhoea 75

Antrophyum 139

Aotus 35, 142

Aphananthe 21

Aphelia 123

Apiunı 63
Aplotaxis 85

Apluda 132

A pocyneae 92

Apodytes 64

Apolochlamys 82

Aponogeton 121

Apophyllum 5

Aporosa 20

Apostasia 114

Aquifoliacene 63

Arabis 5

Araliaceae 61

Araucaria 109

Archeria 108

Archidendron 47

Archontophoenix 120

Ardisia 91, 105

Areca 120

Arenaria 27

Argophyllum 47

Argyrocome 80

Argyrodendron 15

Argyroglottis 81

Argyrophanes 81

Aristida 131

Aristolochia, 23

Aristolochiaceae 23

Aristotelia 17

Arnocrinum 119

Aroideae 120

Artanema 97

Arthratherum 132

Arthraxon 131

Arthrochilus 113

Arthrocnemum 30

Arthropodium 118

Arthropteris 139

Arthrostylis 129,143

Arthrotrichum 28

Arum 120

Arundinella 130

Arundo 135, 143

Aryteria 24

Asclepiadeae 93

Asparagus 117

Asparagopsis 117

Asperella 132

Asperifoliae 99

Asperula 76,142

Aspidium 138

Asplenium 138

Asprella 132

Astartea 54

Astelia 117, 143

Aster 78, 142

Asteridea 80

Asterochiton 16

Asterolasia 10

Asteromyrtus 55

Asterotrichon 13

Astraea 52

Astrebla 134

Astroloma 105

Astrotricha 61,142

Atalantia 12

Atalaya 24,141

Atelandra 101

Atherocephala 108

Atherosperma 3

Athrixia 80

Athrodactylis 120

Athrotaxis 109

Athyrium 138

Atkinsonia 65

Atriplex 29,140,141

Atylosia 41

Australina 22

Avicennia 103

Aylmeyera 27

Azolla 136

Azorella 63
Babbagia 30,141

Babingtonia 53

Backhousia 59

Bacularia 120

Baea 98

Baeckea 53,142

Baeobotrys 91

Balanops 22

Balantium 137

Balaustion 54

Balfouria 93

Baloghia 21

Balonophora 233

Balonophoreae 2:3

Bambusa 135

Banksia 71,14?

Barbarea 5,141

Barklya 42

Barringtonia 39

Bartlingia 36,118

Basilicum 100

Bassia 30, 140,141

Batatas 94

Batratherum 131

Bauera 48

Bauhinia 42

Baumea 128

Baxteria 119

Beaufortia 56

Bedfordia 84

Beilschmiedia 3

Bellis 77

Bellendena 67

Benincasa 76

Berchemia 60

Bergia 8, 141

Berrya 17

Bertolonia 104

Bertya 19,141

Beyeria 18,141

Beyeriopsis 18

Bidaria 94

Bidens 84, 142

Bignoniaceae 99

Billardiera 7,141

Billiotia 54

Billotia 56

Bischoffia 20

Blackburnia 12

Blackwellia 6

Blancoa 116

Blandfordia 117

Bleasdalea 68

Blechnum 138

Blennodia 5

Blennospora 83

Blepharanthemum 13

Blepharocarya 25

Blitum 29

Blumea 79

Blyxa 115

Boehmeria 22

Boerhaavia 32

Bombax 15

Bonnaya 97

Boronia 10

Borya 119

Bosistoa 11

Bossiaea 37,140

Bothryodendron 61

Botrychium 136,143

Bouchardatia 12

Bowenia 110

Brachychiton 15

Brachycome 77,142

Brachyloma 107

Brachynema 48

Brachypterum 42

Brachysema, 32

Brachyspatha 120

Brachystephium 77

Brackenridgea 10

Bradleia 19

Bramia 97

Brasenia 1

Brassaia 61

Breweria 95

Breynia 20

Bridelia 20

Brizopyrum 134

Brizula 123

Brochosiphon 99

Brombya I 2

Bromidium 133

Bromus 135

Brucea 12

Bruguiera 50,14.2

Brunella 101

Brunonia 87

Bryonia 76

Bryonopsis 76

Buchanania 25

Buckinghamia 71

Buechnera 98,143

Bulbine $11 \mathrm{~s}$

Bulbopbyllum 110

Bulliardia 48

Bulweria 99

Bunnya 103

Burchardia 117

Burmannia 115

Burmanniaceae 115

Burnettia 113

Burgesia 32

Bursaria 7

Burseraceae 25, 15 I

Burtonia 34

Busbeckea 5

Butomopsis 121

Byblis 8

Byronia 63

Cabomba 1

Cadaba 5

Cadellia 13

Caesalpina 42,142

Caesia 118

Cajanus 41

Cakile 6,141

Caladenia 113 
Calycopeplus 18

Calycothrix 52

Calyptranthes 59

Calyptrostegia 73

Calyptrostigma 18

Calystegia 95

Campanulaceae 85

Camphoromyrtus 53

Camptostemon 15

Campy]anthera 7

Campynema 115

Cananga 3

Canarium 25

Canarronia 71

Canavalia 41, 142

Candollea $1,85,143$

Candolleaceae 85

C'anscora 90

Cansjera 63

Canthium 75,14:2

Capparideae 4

Capparis 5,141

Caprifoliaceae 76

Capsella 6,141

Carallia 50

Carana 9

Carara 141

Cardamine 5

Cardiospermum 24

Cardwellia $7 \mathrm{l}$

Carex 129,140, 143

Careya 60

Cargillia 92

Carissa 93

Carmichaelia 39

Carpha 127

Carpodontos 48

Carronia 4

Cartonema 12:

Carumbium 21

Caryodaphne 3

Caryophylleae 27

Caryospermum 26

Caryota 120

Casearia 6

Cassia 42

Cassinia 82

Cassiniola 80

Cassytha 4

Castanospermum 42

Castanospora 24,141

Casuarina 22,141

Casuarineae 22

Catakidozamia 110

Cathartocarpus 42

Cathormion 47

Catosperma 87,142

Caulinia 41, 121

Caustis 129

Cedrela 9

Celastrineae 26

Celastrus 26,141

Celmisia 78

Celosia 29

Celtis 21

Cenarrhenes 67

Cenchrus 130

Centaurea 85

Centella 63

Centipeda 84

Centranthera 98

Centratherum 77

Centrolepis 123

Centropappus 84

Centrotheca 134

Centunculus 91

Cephaelis 75

Cephalipterum 83

Cephalosorus 82

Cephalotus 48

Ceranthes 92
Ceratogyne 84

Ceratopetalum 48

Ceratophyllum 50,1 42

Ceratopteris 137

Cerbera 93

Cercodia 49

Ceriops 50

Ceropegia 94

Cesatia 62

Chaetanthus 124

Chaetaria 132

Chaetospora 127

Chalcas 12

Chamaeraphis 130

Chamaescilla 118

Chamaesphaerion 83

Chamaexeros 119

Chamaelancium 51

Chapelliera, 127

Charlwoodia 117

Cheilanthes 138

Cheilococca 37

Cheiloplecton 138

Cheiranthera 7,141

Cheiroloma 78

Chenolea 30

Chenopodina 30

Chenopodium 29,141

Cheynia 54

Chilocarpus 92

Chilodia 101

Chiloglottis 114,143

Chionachne 132,143

Chionanthus 92

Chithonanthus 43

Chlamydia 117

Chlamyspermum 11s

Chloanthes 103, 140

Chloris 134

Chlorophytum 118

Chondrachne 127

Choretrum 64, 142

Choriceras 19

Chorilaena 11

Choripetalum 91

('horizandra 127

Chorizema 32,33

Chromochiton 82

Chrysobactron 118

Chrysocephalum 81

Chrysocoryne 82

Chrysodium 139

Chrysodiscus 80

Chrysogonum 83

Chrysophyllum 91

Chrysopogon 132

Chrysorrhoea 51

Chitonocephalus 83

Chuncoa 50

Cibotium 137

Cinna 133

Cinnamomum 4

Cissodendron 61

Citriobatus 7,141

Citrus 12

Cladium 128, 143

Cladodes 21

Claoxylon 20

Clausena 12

Claytonia 27

Clematis 1

Cleistanthus 20

Cleome 4,141

Clerodendrum 103,143

Cleisostoma 111

Clianthus 39

Clidanthera 40

Clinostigma 120

Clitoria 40

Cloezia 59

Coatesia 12
Cocculus 4

Cochlospermum 6

Cocos 120

Codiaeum 21

Codonocarpus 32

Coelachne 133

Coelandria 110

Coelebogyne 21

Coelorrhachis 131

Coelospermum 75

Coenopteris

Coffea $7 \tilde{5}$

Coilocarpus 30

Coldenia 100, 143

Coleanthera 107

Coleocoma 79

Coleostylis 86

Coleus 100

Colobandra 101

Colobanthus 27

Colletia 61

Colmeiroa 48

Colocasia 120

Colubrina 60

Columbea 109

Combretaceae 50

Comesperma 8,141

Commelina $12: 2$

Commelineae 1.2.

Commerçonia 16,14]

Compositae 77

Conanthodium 81

Conchium 70

Coniferae 109

Connaraceae 32

Conospermum 66

Conostephiopsis 107

Conostephium 107

Conostylis 116

Conothamnus 56

Convolulaceae 94

Convolvulus 95,143

Conyza 79

Coprosma 75

Corchorus 17

Cordia 99

Cordyline 117

Corethrostylis 16

Coridochloa 130

Cornaceae 74

Coronopus 141

Correa 11

Corybas 112

Corymbis 111

Corymborchis 111

Corypha 120

Corynotheca 118

Corysanthes 11:2

Cosmelia 108

Costus 114

Cotula 84

Covellia 21

Craspedia 83

Crassulaceae 48

Crantzia 63

Crantziola 63.

Crepis 85

Cressa 95

Crinum 116

Crossolepis 83

Crossotoma 87

Crotalaria 38, 140, I42

Croton 20

Crowea 10

Cruciferae 5

Cryphia 101

Cryptandra 60, 140

Cryptocarya 3

Cryptosema 32

Cryptostomon 51

Cryptostylis 112

Cucunis 76,142

Cucurbitaceae 76

Cudrania 22

Cupania 24

Cupuliferae 22

('ureuligo 116

Curcuma 114

Cuscuta 95,143

Cuttsia 48

Cyanostegia 103

Cyanothamnus 10

Cyanotis 121

Cyathea 137

Cyathochaete 127,143

Cyathodes $10 \mathrm{~s}$

Cyathopappus 83

Cyathopsis 105

Cybele 71

Cycadeae 109

Cycas 109

Cyclogyne 39

Cyclotheca 32

Cycnogeton 121

Cylicodaphne 4

Cylindrosorus $\$ 2$

Cymbidium 111

Cymbonotus 85

Cyminosma 12

Cymodocea 121

Cymanchum 93,143

Cynoctomum 93

Cynodon 134

Cynoglossum 100,143

Cynometra 43

Cyperaceae 124

Cyperus 124, 143

Cyphanthera 96

Cyphokentia 120

Cypselocarpus 32

Cyrtostylis 113

Cystanthe 108

Cystopteris 138

Dacrydium 109

Dactyloctenium 134

Daemia 94

Dalbergia 42

Dallachya 60

Damasonium 121 
Desmocladus 124

Desmodium 40

Desvauxia 123

Deyeuxia 133

Diaspasis 87

Dianella 117

Dicarpidium 15

Dicera 17

Dicerma 40

Dichelachne 133

Dichondra 95

Dichopetalum 63

Dichopogon 118

Dichosema 33

Dichrostachys 43

Dicksonia 137

Dicladanthera 140

Diclidopteris 138

Dicliptera 99

Dicrastylis 103

Dictyopteris 139

Didiscus 62,142

Didymanthus 30

Didymeria 11

Didymochaete 133

Didymonema 128

Didymotheca 32,142

Digitaria 130

Dilivaria 99

Dillenia 2

Dilleniaceae 1

Dillwynia 37

Dimeria 130

Dimetopia 62

Dimorpholepis 80

Diodontium 84

Dioscorea 115

Dioscorideae 115

Diospyros 92

Diotosperma 84

Diplachne 134

Diplacrum 129

Diplanthera 99, []ㅡ.

Diplarrhena 114

Diplaspis 63

Diplax 132

Diplazium 138

Diploglottis 24

Diplolaena Il

Diplolobitum 39

Diplopeltis 25

Diplopogon 133

Diplora 138

Diplospora 74

Dipodium 111

Dipogonia 133

Dipteracanthus 99

Disarrhenum 232

Discaria 61

Dischidia 94

Discopodium 127

Discospermum 74

Diselma 109

Disemma 76

Disoon 104

Dissiliaria 19,141

Dissocarpus 30

Distichlis 135

Distichostemon 25

Distylis 88

Dithyrocarpus 122

Dithyrostegia 82

Dituris 112

Dodonaea 25, 141

Dolichandra 99

Dolichandrone 99

Dolichos 41

Donatia 87

Donia 39

Doodia 138

Dopatrium 97
Doryanthes 116

Doryphora 3,141

Dracaena 117

Dracophyllım 109

Drakaea 113

Drapetes 74

Drosera 7

Droseraceae 7

Drummondita 11

Dryandra 72

Drymaria 27

Drymophila 117

Drymis 2

Drynaria 139

Duboisia 96

Dumbaria 41

Duperreya 95

Duttonia 80,104

Dysophylla 100

Dysoxylum 9

Dysphania 29,141

Eadesia 96

Earlia 99

Ebelingia 13

Ebenaceae 92

Ebermayera 99

Ecdeiocolea 123

Echinocarpus 17

Echinochloa 130

Echinocroton 21

Echinodorus 121

Echinopogon 133

Echinopsilon 30

Echinospermum 100

Echinus 21

Eclipta 83,142

Ectrosia 135

Ehretia 99

Ehrharta 132

Elachanthus 84

Elachocroton 21

Elachopappus 8?

Elachothamnus 77

Elaeagneae 64

Elaeagnus 64

Elaeocarpus 17

Elaeodendron 26

Elaphoglossum 139

Elatine 8

Elatineae 8

Elatostemma 22

Elattostachys 24

Electrosperma 12:?

Eleochiton 125

Elephantopus 77

Elettaria 114

Eleusine 134, 143

Eleutranthes 76

Elionurus 131

Elynanthus 127

Elythrophorus 135

Emblingia 5

Embothrium 71

Emex 31

Emilia 84

Emmenospermum 60

Emphysopus 77

Empleurosma 25

Encephalartos 110

Enchylaena 30,142

Enchysia 85

Endiandra 4,141

Enhalus 115

Enhydra 83

Entada 43

Enterolobium 47

Epacrideae 105

Epacris 108

Epaltes 79,140,142

Epiandra 128
Epiblema 112

Epicharis 9

Epilobium 49

Epipogum 111

Epipremnum 120

Epithinia 75

Epitriche 82

Eragrostis 135, 143

Eranthemum 99

Erechtites 85, 143

Eremaea 57

Eremodendron 104

Eremophila 104,140

Eremopyxis 52

Eremosyne 48

Eria 140

Eriachne 133, 143

Erianthus 131

Ericaceae 105

Ericomyrtus 53

Erigeron 79

Eriocalia 63

Eriocauleae 123

Eriocaulon 123

Eriochilus 113

Eriochiton 30

Eriochlamys 82

Eriochloa 130

Eriocladium 82

Eriosema 41

Eriostemon 10

Eritrichum 100

Erodium 13

Erodiophyllum 77

Erycibe 94

Eryngium 63

Erysimum 5

Erythraea 90

Erythrina 41,142

Erythrorchis 111

Erythrophlaeum 43

Frythroxylum 13

Etaeria 111

Ethuliopsis 79

Euandra 129

Eucalyptus 57,141.142

Eucarya 64

Euchilopsis 37

Euchilus 36

Euchiton 79

Eucryphia 48

Eudesmia 57

Eugenia 59

Euneapogon 133

Euodia 12

Euonymus 26

Enosma 91

Eulalia 131

Eulophia 111

Eupatorium 77,141

Euphorbia 18, 140,141

Euphorbiaceae 18

Euphoria 24

Eupomatia 3

Euphrasia 98

Euroschinus 25

Eurostorrhiza 129

Eurybia 78

Eurybiopsis 79

Eurycles 116

Euryomyrtus 53

Eustrephus 117

Eutacta 109

Eutassa 109

Eutaxia 32

Euthales 89

Euxolus 29

Exarrhena 100

Excaecaria 21

Exocarpos 64

Exocarya 127

Evolvulus 95

Eyrea 79

Fabricia 54

Fagus 22

Fagraea 90

Faradaya 103

Fatolna 22

Faustula 81

Fawcettia 4

Fenzlia 59

Festuca 134

Fieldia 98

Ficoideae 31

Ficus 21,140,141

Filices 136

Fimbristylis 12.5

Fischera 62

Fisquetia 120

Fitzalania 3

Fitzroya 109

Flacourtieae 6

Flagellaria 117

Flaveria 84,142

Flemingia 41

Fleurya 22

Flindersia 9

Floriscopa 122

Floscopa 122

Flueggea 20

Fluviales 121

Fontainea 21,141

Forstera 86

Forsteropsis 85

Fragosa 63

Francisia 51

Frankenia 26

Frankeniaceae 26

Franklandia 67

Freirea 22

Fremya 59

Frenela 109

Freycinetia 120

Friesia 17

Froebelia 105

Fugosia 15

Fuirena 126

Fusanus 64

Gahnia 128

Galactia 41 
Glabaria 4

Gleichenia 137

Glinus 31

Glischocaryon 49

Glochidion 19

Glossodia 114

Glossogyne 8t

Glossostigma 97

Glyceria 134

Glycine 41

Glycosmis 12

Glycyrrhiza 40

Gmelina 103

Gnaphalium 79

Gnaphalodes 83

Gnephosis 83

Gomphandra 64

Gompholobium34,142

Gomphrena 28

Gongronema 94

Goniocarpus 49

Goniophlebium 139

Goniopogon 78

Goniopteris 139

Goniotriche 28

Goodenia 88, 143

Goodeniaceae 87

Goodenoughia 88

Goodia 38

Goodyera 111

Gossypium 15,141

Gouania 61

Gramineae 131

Grammatotheca 85

Grammitis 139

Graptophyllum 99

Gratiola 97

Graumuellera 121

Greevesia 14

Grevillea 68, 140, 142

Grewia 17

Grisebachia 120

Grumilia 75

Guettarda 75

Guettardella 75

Guichenotia 16

Guilandina 42

Gunnera 50

Gunnia 31,11

Guioia 24

Guttiferae 8

Gymnagathis 55

Gymnanthera 93,143

Gymnema 94

Gymnochaete 127

Gymnoschoenus 127

Gymnococca 73

Gymnogramme 139

Gymnogyne 84

Gymnopteris 139

Gymnopogon 93

Gymnosporia 26

Gymnostachys 120

Gymnostylis 81

Gymnothrix 131

Gynaphanes 14:

Gynura 84.

Gypsophila 27

Gyrocarpus 51

Gyrostachys 111

Gyrostemon 32

Gyrostephium 83

Habenaria 114

Haekeria 82

Haemodorum 115

Hakea 70,142

Halgania 100

Halfordia 12

Halocnemum 30

Halodule 121
Halophila 115

Halorageae 49

Haloragis 49

Halothamnus 13

Hamamelideae 48

Hamelinia 117

Hannafordia 16

Haplopappus 79

Hardenbergia 41

Harmogia 53

Harpulia 24

Harrisonia 13

Hartigshea 9

Haussmannia 99

Hearnia 9

Hedaroma 51

Hedera 61

Hedraianthera 9,14l

Hedycarya 3

Hedyotis 74

Hedyscepe 120

Heleocharis 125

Helichrysum 81,142

Helicia 68

Helicteres 15

Heliophytum 100

Heliotropium 100

Helipterum 80.140.142

Hellenia 114

Helmholtzia 12.2

Helmintostachys 136

Helophyllum 87

Helopus 130

Helostigma 85

Helothrix 127

Hemarthria $13 \mathrm{~L}$

Hemarrhena 97

Hemiandra 101

Hemicarpus 62

Hemichroa 29

Hemiclidia 72

Hemicyclia 20

Hemigenia 101

Hemiphora 103

Hemiphues 63

Hemisteirus 28

Hemistemma I

Hemistephus 1

Hemitelia 137

Heptopleurum 61

Heritiera 15

Hermannia 15

Hernandia 4

Herpestis 97

Herpolirion 118

Hetaeria 122

Heterachne 135

Heterodendron 24

Heterolaena 73

Heteropogon 132

Hewardia 117

Hexatheca 121

Hibbertia 1,141

Hibiscus 14,141

Hitrochloe 132

Hippocratea 26

Hippocrepandra 18

Hodgkinsonia 75

Hodgsonia 118

Hodgroniola 118

Holcus 132

Hologamium 131

Holotome 63

Homalium 6

Homalocalyx 52

Homalospermum 54

Homalostoma 108

Hormogyne 92

Hovea 38

Howittia 14

Hoya 94
Huanaca 63

Huegelia 62

Huenefeldia 78

Hugonia 13

Humata 137

Humea, 82,142

Hutchimsia 6

Huttia 1

Hyalochlamys 82

Hyalolepis 82

Hyalosperma 80

Hybanthus 6,141

Hydriastele 120

Hydrilla 115

Hydrocharideae 115

Hydrocharis 115

Hydrocotyle 62

Hydroglossum 136

Hydrolea 99

Hydropeltis 1

Hydrophila 99

Hydnophytum 75

Hydrophylleae 99

Hylococcus 19

Hymenanthera 6,141

Hymenolepis 139

Hymenophyllum 137

Hymenosporum 7,141

Hymenotheca 3.2

Hypaelyptum 126

Hypelytrum 1:7

Hypericinae 8

Hypericum 8

Hypocalymma 54

Hypoestes 99

Hypolepis 139

Hypoporum 129

Hypolaena 1:2t

Hypoxis 110

Hypserpa 4

Hyptiandra, 12

Hyrtandra 9.9

Iberis 6

Ichnocarpus 93

Ilex 63

Ilysanthes 97

Imbricaria 5

Imperata 131

Indigofera 39,140,142

Ionidium 6

Iphigenia 117

Ipomoea 94, 143

Iricleae 114

Iris 114

Irwingia 61

Isachne 130

Isandra 140

Ischaemum 131

Iseilema 1:i:2

Isoetes 136

Isoetopsis 84

Isolepis $12 \bar{y}$

Isoloma 137

Isopogon $65,1+2$

Isoschoenus 1:2

Isotoma $85,14^{\circ}$

Isotropis 33, 140

Ixauchenus 77

Ixiochlamys 79

Ixiolaena 80

Ixiosporum 7

Ixodia 82

Ixora $74,14: 3$

Jacksonia 34

Jambosa 59

Jansonia :3:

Jasmineae 92

Jisminum 9.?

Johnsonia 119

Josephia 72

Josephinia 105

Junceae 122

Juncella 123

Juneus 122

Jungia 53

Jussieua 49

Justicia 99

Kaleniczenkia 32

Kamphusia 87

Kamptzia 59

Kelleria 74

Kennedya 41

Kentia 120

Kentiopsis 120

Kentropsis 30

Keraudrenia 16

Kernera 121

Kibara 3

Kingia 119

Kippistia it

Kissodendron 61

Klanderia 101

Knoxia 76

Kochia $30.140,14: 2$

Koehnea 14.)

Kreyssigia 117

Kunzea $5 \pm, 1 \pm 2$

Kyllingia 124

Labiatae 100

Labichea 4:2,142

Laboucheria 43

ILaccospadix 120

Lachnagrostis 133

Lachnocephalus 103

Lachnostachys 102

Lachnothalamus 85

Lactaria 93

Lagenaria 76

Lagenophora 77,142

Lagerstroemia 49,11:

Lagrezia 29

Lagunaria 15

Lalage 37

Lamarchea 57

Lambertia 68

Lampocarya 128

Lamprochlaena 82

Lamprolobium 39

Laportea 22

Lappago 131 
Lepidobolus 124

Lepidocoma 82

Lepidosperma 128

Lepidospora 128, 143

Lepidozamia 110

Lepigonum 27

Lepironia 127

Lepistemon 94

Leptaspis 131

Leptatherum 131

Leptinella 84

Leptocarpus 124

Leptoceras 113

Leptochloe 134

Leptocyamnus 41

Leptocytisus 37

Leptolobium 41

Leptomeria 64

Leptopteris 137

Leptorrhynchos 80, 142

Leptosema 32

Leptospermum 54

Leptotriche 83

Lepturus 131

Lepyrodia 123

Leschenaultia 87,142

Lespedeza 40,142

Lestibudesia 29

Leucas 101

Leucocarpum 26

Leucolaena 62

Leucophyta 83

Leucopogon 105

Leucothamnus 16

Leuzea 85

Lhotzkia 52

Libertia 114

Licuala 120

Ligusticum 63

Ligustrum 92, 143

Liliaceae 117

Limnanthemum 90

Limnophila 97

Limnostachys 12]

Limosella 97

Lindernia 97,143

Lindsaea 137

Lindsaya 137

Lineae 13

Linkia 67

Linociera 92

Linospadix 120

Linschotenia 87

Linum 13

Liparis 110

Liparophyllum 90

Lipocarpha 126

Lippaya 74

Lippia 102

Lissanthe 105

Litobrochia 138

Litsea 4

Livistona 120

Lobelia 85,142

Lobophyllum 100

Lopopogon 107

Logania 91, 143

Loganiaceae 90

Lomandra 119

Lomaria 138

Lomariopsis 139

Lomatia 71

Lonchocarpus 42

Lopadocalyx 63

Lophoclinium 80

Lophostemon 58

Lopidium 136

Loranthaceae 64

Loranthus 65

Lotus 38

Loudonia 49
Lourea 40

Loxocarya 124

Lucuma 91

Ludwigia 49

Luffa 76

Luisia 140

Lumnitzera 50

Luzula 122

Lazuriago 117

Lycium 96

Lycopodineae 136

Lycopodium 136

Lycopus 101

Lyginia 123

Lygodictyon 136

Lygodium 136

Lyonsia 93

Lyperanthus 113

Lysanthe 68

Lysicarpus 59

Lysimachia 91

Lysinema 108

Lysiosepalum 17

Lythrum 49

Maba 92

Macadamia 68

Macaranga 21

i) Lacarthuria 31,1 42

Mactonaldia 111

Macgregoria 26

Mackinlaya 61

Macropidia 116

Macropiper 23

Macropodia 116

Macropteranthes 51

Nacrostegia 73

Macrostigma 47

Macrozamia 110

Maesa 91

Magnoliaceae 2

Mahernia 15

Maireana 30

Malacochaete 126

Malaisia 22

Malcolmia 5

Mallophora 103

Mallotus 21

Malpighiaceae 25

Malva 13

Malvaceae 13

Malvastrum 13

Manglesia 68

Manisuris 131

Mapania 127

Mappa 21

Marattia 130

Mariantlnus 7,141

Mariscus 124

Marlea 74

Marsdenia 94

Marsilea 136

Marquisia 75

Mayepea 92

Nazus 97

ILegalotheca 124

Meionectes 50

Medicosma 12

Melachne 128

Melaleuca 55, 140,142

Melanthesa 20

Melanthesiopsis 20

Melanthium 117

Melastoma 60

Melastomaceae 60

Melhania 15

Melia 9

Meliaceae 9

Melichrus 105

Melicope 12

Melochia 15
Melodinus 92

Melodorum 3

Melothria 77

Memecylon 60

Memorialis 22

Menkea 5

Meniocus 5

Meniscium 139

Menispermeae 4

Mentha 101

Merkusia 87

Mertya 61

Mesembrianthemum 31

Mesomelaena 127

Mesotriche 105

Methorinm 15

Metrosideros 59

Mezoneurum 42

Michiea 107

Micraira 133

Micrantheum 18,141

Microcachrys 109

Microcarpaea 97

Microchloa 134

Microcladium 63

Microclisia 4

Microcorys 102

Microcybe 10

Microgyne 79

Microlaena 132

Microlepirlium 6

Nicrolepia 137

Micromelum 12

Nioromyrtus 52

Micropyxis 91

Vicroseris 85

Microstachys 21

Microstemma 94

Microtis 112

Microstylis 111

Miliusia 3

Milletia 39

Milligania 50,117

Millotia 82

Milnea 9

Mimosa 43

Mimulus 97

Mimusops 92

Minuria 77

Mirbelia 33,142

Mischocarpus 2

Mitragyne 90

Mitrasacme 90, I42

Nitreola 90

Mniarum 27

Modecca 76

Molkenboeria 87

Mollinedia 3

Molloya 68

Mollugo 31, 142

Momordica 76

Monacather 134

Monencyanthes 80

Tonenteles 79

Monimieae 3

Monogramma 138

Monochoria 121

Monococcus 3:2

Monoploca 6

Monotaxis 18,14l

Monotoca 105

Montia 27

Moonia 83

Moraea 114

Morelotia 128

Morgania 97

Morinda 75

Morisia 127

Morna 80

Moschosma 100

Motherwellia 61

Wucuna 4$]$

Muehlenbeckia 31

Muehlenbergia 133

Mukia 77

Murraya 12

Musa 114

Myoporinae 104

Myoporum 104, 140

Myosotis 100

Myosurus 1, lál

Myriocephalus 82

Myriogyne 84

Myriophyllum 50

Myristica 3

Myristiceae 3

Myrmecodia 7.7

Myrsinaceae 91

Myrsine 91

Myrtaceae 5l

Myrtus 59

Nablonium 83

Nageia 109

Najas 121

Nania 59

Nasturtium 5

Needhamia 107

Negria 98

Nelitris 59

Nelsonia 99

Nelumbium 1

Nelumbo 1

Nematoceras II

Nematolepis II

Nematophyllum 38

Nematopus 83

Nematostigma 114

Nemedra 9

Neoroepera 20

Neottopteris 138

Nepenthaceae 23

Nepenthes 23, 140

Nephelium 24

Nephrodium 138

Nephrolepis 138

Neptunia 43

Nertera 75

Nesaea 49,142

Nesodaphne 3

Nesogenes 102 
Oldenlandia 74,142

Olea 92

Oleandra 138

Olearia 78

Oliganthemum 79

Oligarrhena 107

Omalanthus 21,141

Omphacomeria 64

Onagreae 49

Oncosporum 7

Oncostylis 125

Onychosepalum 124

Opercularia 75, 142

Ophioderma 136

Ophioglossum 136

Ophiorrhiza 74

Ophiuros 131

Opilia 63

Oplismenus 130

Orchideae 110

Oreobolus 127

Oreomyrrhis 63

Oreostylidium 87

Orites 68

Oritina 68

Ormocarpum 40

Ornithochilus 111

Orobanche 98

Orobancheae 98

Orthoceras 112

Orthopogon 130

Orthostemon 90

Orthosiphon 100

Orthotropis 33

Orthrosanthus 114

Oryza 132

Osbeckia 60

Osbornia 59

Oschatzia 63

Osmunda 137

Osteocarpum 30

Otanthera 60

Ottelia 114

Ourisia 97

Owenia 9

Oxalis 13

Oxleya 9

Oxycladium 33

Oxylobium :3:

Oxymyrrhine 53

Oxystelma 93

Ozothamnus 81

Pachycornia 30

Pachygone 4

Pachynema 2,140

Pachysurus 83

Pacquerina 77

Pagetia 1:

Palmae 119

Palmeria 3

Panax 61

Pancovia 24

Panaetia 80

Pandaneae 120

Pandanophylium 127

Pandanus 120, 140

Panicum 130

Panopsis 68

Papaver 4,141.

Papaveraceae 4

Pappophorum 133

Paratropia 61

Parkeria 137

Parietaria 22

Parinarium 47

Paritium 14

Parsonsia 93

Paryphantha 52

Paspalum 130

Passiflora 76
Passifloreae 76

Patania 137

Patersonia 114

Pavetta 74

Pavonia 14

Pedalinae 105

Pedilonum 110

Pelargonium 13

Pellaea 138

Pelonastes 50

Peltophrorum 42

Pemphis 49

Pennantia 64

Pennisetum 131

Pentaceras 12

Pentachondra 107

Pentacraspedion 133

Pentadactylon 67

Pentadynamis 38

Pentagonaster 54

Pentalepis 83

Pentapeltis 62

Pentataphrus 105

Pentapogon 133

Pentaptelion 105

Pentatropis 94

Peperomia 23,140.141

Peplidium 97,143

Pericalymma 54

Pericampylus 4

Pernettya 105

Peroa 105

Perojoa 105

Perotis 131

Persoonia 67,142

Petalolepis 81

Petalostigma 19

Petalostylis 42

Petermannia 115

Petrophila 65

Phacelothrix 82

Phaenopoda 80

Phajus 111

Phalangium 118

Phaleria 74

Phanerandra 105

Pharbitis 94

Phaseolus 41

Phebalium 10

Phegopteris 139

Pherosphaera 109

Philotheca 11,140

Philoxerus 28

Philydreae 122

Philydrum 122

Phlebocalymma 63

Phlebocarya 115

Phleborlium 138

Phoberos 6

Pholidia 104

Pholicliopsis 104

Pholidota 110

Phormium 117

Phragmites 135

Phreatia 110

Phyllachne 87

Phyllanthus 19, 140, 141

Phyllocalymma 82

Phyllocladus 109

Phyllodium 40

Phylloglossum 136

Phyllopappus 85

Phyllota 36

Phymatocarpus 56

Phymatodes 139

Physalis 95

Pliysolobium 41

Physopsis 103

Phytolacceae 32

Picrophyta 88

Pigea 6
Pileanthus 52

Pilitis 108

Pilularia 136

Pimelea 73,142

Piper 23

Piperaceae 23

Piptandra 53

Piptomeris 34

Piptostemma 82

Pisonia 32

Pithecolobium 47

Pithocarpa 82

Pithyrodia 103

Piptocalyx 3

Pipturus 22

Pittosporeae 6

Pittosporum 6,141

Plagianthus 13

Plagiogyria 138

Plagiolobium 38

Plagiosetum 131

Plantagineae 91

Plantago 91, 143

Platycarpidium 62

Platycerium 139

Platychilum 38

Platylobium 37

Platyloma 138

Platyptelea 45

Platysace 62

Platytheca 9

Platyzoma 137

Plectranthus 100

Pleea 117

Pleiococca I2

Pleiogyne 84

Pleogyne 4

Pleopeltis 139

Pleurancira 1

Pleurandropsis 10

Pleurocarpaea 77

Pleurogramme 138

Pleuropappus 82

Plexaure 110

Plinia 59

Plintacanthus 134

Plokiostigma 26

Pluchea 79

Plumbagineae 26

Plumbago 26,141

Poa 134

Podocarpus 109

Podocoma 79,142

Podolepis 80,142

Podolobium 32

Podopetalum 42

Podosperma 80, 140, 142

Podostemoneae 23

Podotheca 80, 140, 142

Poecilodermis 15

Pogonia 104,111

Pogonolepis 82

Pogonolobus 75

Pogonatherum 131

Pogonetes 87

Pogostemon 100

Poiretia 38, 108

Polanisia 4

Pollia 1 02

Pollichia 100,143

Pollinia 131

Polyalthia 3,140

Polycalymma 82

Polycarpaea 27

Polycarpon 27

Polycnemon 29

Polygala 8,141

Polygaleae 8

Polygonaceae 31

Polygnnum 31,14?

Polyneria 95, 143

Polyosma 48

Polyphragmon 75

Polypodium 139

Polypogon 131

Polypompholyx 98

Polystichum 138

Polyzone 51

Pomaderris 60,142

Pomatotheca 31

Pomax 76

Ponceletia 108

Pongamia 42

Pontederiaceae 121

Popowia 3

Porana 95

Poranthera 18,141

Porospermum 61

Portulaca 27,141

Portulaceae 27

Posidonia 121

Potamogeton 121

Potamophila 132

Potentilla 47

Pothos 120

Pouzolzia 22,141

Pozoa 63

Pozoopsis 63

Prasophyllum 112,140,14?

Pratia 85

Premna 103

Primulaceae 91

Prionosepalum 124

Prionotes 108

Pritzelịa 62,122

Procris 22

Pronaya 7

Prostanthera 101,140

Proteaceae 65

Prunella 101

Pseudalangium 74

Pseudanthus 18,141

Pseudatalaya 24

Pseudomorus 22

Pseudopholidia 104

Psilotrichum 28

Psilotum 136

Psoralea 38, 142

Psychotria, 75

Psydrax 75 
Ratonia 24

Reedia 129

Regelia 56

Remirea 127

Renealmia 114

Restiaceae 123

Restio 124

Rhabdothamnus 98

Rhagodia 29,141

Rhamnaceae 60

Rhamnus 60

Rhamphicarpa 98

Rhamphidia 111

Rhaphidophora 120

Rhipogonum 117

Rhizophora 50

Rhizophoreae 50

Rhizospermeae 136

Rhodamnia 59

Rhodanthe 80

Rhodomyrtus 59

Rhodosphaera 25

Rhopala 68

Rhus 25

Rhyncharrhena 94

Rhynchosia 41,142

Rhynchospora 127

Rhychostemon 16

Rhytidandra 74

Rhytidanthe 80

Rhytidosporum 7

Richea 83,108

Ricinocarpus 19,141

Riedleya 15

Rinzia 53

Rochelia 100

Roea 35

Roeperia 5, 19

Rosaceae 47

Rostellaria 99

Rostellularia 99

Rotala 49

Rottboellia 131

Rottlera 21

Roupala 68

Rourea 32

Roxburghia 117

Roxburghiaceae 117

Rubiaceae 74

Rubus 47

Ruelingia 16

Ruellia 99

Rumex 31

Ruppia 121

Rutaceae 10

Rutidochlamys 80

Rutidosis 82

Ryssopteris 25

Saccharum 131

Saccolabium 111

Saccopetalum 3

Sagenia 138

Sagina 27,141

Salacia 26

Salicarieae 49

Salicornia 38, 142

Salisia 54

Salomonia 8

Salsola 31,142

Salsolaceae 29

Salvia 101

Samara 91

Sambucus 76, 142

Samolus 91

Samydaceae 6

Sandfordia 10

Santalaceae 64

Santalum 64

Sapindaceae 24

Sapindus 24
Saponaria 27,141

Sapota 91

Sapotaceae 91

Sarcocephalus 74

Sarcochilus 111

Sarcopetalum 4

Sarcostemma 94

Sarcozygium 13

Sarotes 16

Saussurea 85

Saxifrageae 47

Scaevola 87,142

Scalia 80

Scaliopsis 80

Schelhammera 117

Schenodorus 134

Schidiomyrtus 53

Schizaea 136

Schizachyrium 132

Schizeilema 63

Schizolema 137

Schizomeria 48

Schizopleura 56

Schmidelia 24

Schoberia 30

Schoenia 81

Schoenodum 123

Schoenolaena 62

Schoenus 127,143

Scholtzia 53

Schuemannia 51

Schwaegrichenia 116

Scirpidium 125

Scirpodendron 127

Scirpus 126,143

Scitamineae 114

Scleranthus 27

Scleria 129

Sclerochlamys 30

Solerolaena 30

Scleroleima 84

Sclerothamnus 37

Scolopendrium 138

Scolopia 6

Scoparia 97

Scorzonera 85

Scottia 37

Scrophularinae 97

Scutellaria 101

Scyphiphora 75

Scyphocoronis 82

Seaforthia 120

Sebaea 90

Sebastiania 21

Secamone 93

Securinega 20,141

Selaginella 136

Selliera 88

Selliguea 139

Semecarpus 26

Senebiera 6,141

Senecio 84

Sentis 104

Sequoia 109

Serianthes 47

Seringea 16

Sersalisia 91

Sesbania 39,142

Seseli 63

Sesuvium 31

Setaria 130,143

Selwynia 4

Sheffieldia 91

Sicyos 77

Sida 14,141

Sideroxylon 91

Siebera 62

Siegesbeckia 83

Siemssenia 80

Sieversia 47

Siloxerus 82
Silphiospermum 77

Simarubeae 12

Simsia 66

Siphonodon 26

Sisymbrium 5

Sisyrinchium 114

Sium 63

Skinnera 94

Skirrhophorus 82

Sloanea 17

Smilax 117, 140

Smithia 40

Solanaceae 95

Solanum 95, 143

Soleniscia 105

Solenogyne 77

Solenostigma 21

Soliva 84

Sollya 7

Sonneratia 60

Sonzaya 25

Sophora 42

Sopubia 98

Sorghum 132

Sorostachys 124

Sowerbaea 118

Spadostyles 36

Spanoghea 24

Sparganium 120

Spartothamnus 102,143

Spathoglottis 111

Spathodea 99

Spergularia 27

Spermacoce 76

Spermalepis 59

Spermaxyrum 63

Sphaeranthos 79

Sphaerolobium 35

Sphaeromorphaea 79

Sphaeropus 129

Sphenotoma 109

Sphincterostoma 108

Spiculaea 113

Spinifex 131

Spilanthes 84

Spiranthes 111

Spirodela 121

Spiropodium 79

Spodiopogon 131

Spondias 26

Sponia 21

Sporobolus 133,143

Sprengelia 108

Spyridium 60

Stachystemon 18

Stackhousia 26,141

Stackhousieae 26

Statice 26

Stawellia 119

Steetzia 78

Stegania 138

Steiroglossa 77

Stekovia 88

Stellaria 27

Stemodia 97

Stenanthemum 60

Stenanthera 105

Stenocarpus 71

Stenochilus 104

Stenochlaena 138

Stenodiscus 60

Stenopetalum 5

Stenosperma 84

Stephania 4

Sterculia 15

Sterculiaceae 15

Stipa 132

Stirlingia 66

Stomarrhena 105

Strangea 68

Stravadium 59

Streblorrhiza 39

Streptachne 132

Streptothamnus 6

Streptocarpus 98

Streptoglossa 79

Strobilanthes 99

Stromatopteris 137

Strongylodon 41

Strongylospermum 84

Strychnos 90

Strzeleckia 9,143

Stuartina 79

Sturmia 110

Sturtia 15

Stylidium 74, 85

Stylobasium 47

Stylocoryne 74

Stylolepis 80

Styloncerus 82

Stylurus 68

Stypandra 118

Styphelia 105

Styraceae 92

Suaeda 30

Suriana 13

Symphyomera 84

Symphyomyrtus 57

Symphyonema 67

Symphyopetalum 11

Symplocos 92

Synaphea 66

Synaptantha 74

Syncarpia 59

Synophlebium 137

Synostemon 19

Synoum 9

Syzygium 59

Swainsona $39,141.142$

Swammerdamia 81

Tabernaemontana 93

Tacea 115

Taccaceae 115

Taeniophyllum 111

Talinum 27

Tamarindus 42

Tapeinocheilos 114

Tarrietia 15

Tasmania, 2

Tecoma 99 
Thesium 64

Thespesia 15

Thespidium 79

Thlaspi 6

Thomasia 16

Thouarsea 131

Thozetia 94

Threlkeldia 30

Thrixpermum 111

Thryptomene 52,142

Thuarea 131

Thunbergia 99, 140

Thymeleae 73

Thyrsacanthus 99

Thysanotus $11 \mathrm{~s}$

Tiaridium 100

Tiliaceae 17

Tillaea 48

Timonius 75

Tinospora 4

Titania 110

Tittmannia 97

Tmesipteris 136

Todea 137

Torenia 97

Tournefortia 100

Toxanthus 82

Trachycaryon 21

Trachymene 62,142

Trachypogon 132

Tragia 21,141

Tragus 131,143

Trema 21

Tremandra 9

Tremandreae 9

Trianthema 31, 142

Tribonanthes 116

Tribulopsis 13

Tribulus 13,140,141

Trichanthodium 83

Trichelostylis 125

Trichinium 28

Trichobasis 56

Trichobolus 32

Trichocline 85

Trichodesma 100

Trichodium 133

Tricholoma 97

Trichomanes 137

Trichosanthes 76,142

Trichosiphon 15
Trichostegia 80

Tricondylus 71

Tricoryne 118

Tricostularia 127

Trigastrotheca 31

Triglochin 121

Trigonella 38

Trineuron 84

Triodia 135

Triphelia 51

Tripladenia 117

Tripetelus 76

Tripterococcus 26

Triptilodiscus 80

'Trirhaphis $13 \overline{0}, 143$

Trisetum 133

Tristania 58

Tristaniopsis 58

Tristellateia 25

Tristichocalyx 4,141

Trithuria 123

Triticun 135

Triumfetta 17

Trochocarpa 107

Trollius 1

Trymalium 60

Turraea 9

Turritis 5

Tylophora 94,14:3

Typha 120

Typhaceae 120

Typhonium 120,143

UImus 21

Umbelliferae 62

Uncinia 129

Ungeria 15

Urachne 13:

Uragoga 75

Uralepis 134

Uraria 40,142

Urena 14,141

Urocarpus 10

Urodon 36

Urostigma 21

Urtica 22

Urticaceae 21

Utricularia 98

Uvaria 3

Uvedallia, 97
Vachellia 43

Vaginularia 138

Vallisneria 115

Vandellia 97

Veitchia 120

Velleya 89, 140

Ventenata 85

Ventenatia 105

Ventilago 60

Verbena 102

Verbenaceae 102

Vernonia 77

Veronica 97

Verreauxia 87

Verticordia 5

Vigna 41,142

Vilfa 133

Villarsia 90

Villaresia 64

Viminaria 35

Vinca 93

Vincetoxicum 93

Viniferae 23

Yiola 6

Violaceae 6

Viraya 80

Viscum 64,142

Vitex 103

Vitis 23

Vittaria 137

Vittadinia 79,142

Vlamingia 6

Vulpia 134

WTahlenbergia 85,143

Waitzia 80

Walcottia 102

Waltheria 15,141

Wartmannia 21

Webera 74

Wedelia 83,142

Wehlia 53

Weinmannia 48

IVestonia 38

Westringia 102

Wichurea 60

Wickstroemia 74

Wilkia 5

Wilkiea 3

IVilsonia 95
Wistaria 39

Wittsteinia 105

Wolffia 121,143

Wollastonia 83

Woodwardia 138

Woollsia 108

Wormia 1

Wrightia 93

Wrixonia 101

Wurmbea 117

Xanthochrysum 81

Xanthophyllum 8

Xanthorrhoea 119

Xanthosia 62

Xanthostemon 59

Xanthoxylum 12

Xerochloa 131

Xerosollya 7

Xerotes 119

Ximenia 63

Xiphopteris 139

Xylocarpus 9

$X$ ylomelum 68

Xylosma 6

Xyridanthe 80

Xyrideae 122

$\mathrm{X}$ yris 122

Youngia 85

Zaleya 31,142

Zamia 110

Zannichellia 121

Zanonia 76

Zapania 102

Zehneria 77

Zichya 41

Zieria 10

Zizyphus 60

Zornia 40

Zostera 121

Zosterostylis 112

Zoysia 131

Zygia 47

Zygomenes 122

Zygophylleae 13

Zygophyllum 13 





